Franz von Kutschera

Grundifragen der Erkenntnistheorie 



\section{Franz von Kutschera}

\section{Grundfragen der}

Erkenntnistheorie

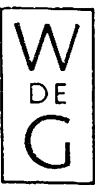

Walter de Gruyter · Berlin · New York 1982

$$
17 / 5254
$$




\section{$17 / C C \quad 4400 \quad k 97$}

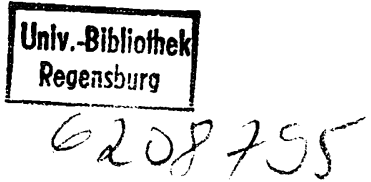

CIP-Kurztitelaufnabme der Deutschen Bibliothek

Kutschera, Franz von

Grundfragen der Erkenntnistheorie / Franz von Kutschera.

- Berlin; New York: de Gruyter, 1981.

(De Gruyter-Studienbuch)

ISBN 3-11-008777-4 brosch.

ISBN 3-11-008663-8 geb.

(C) Copyright 1981 by Walter de Gruyter \& Co., vormals G. J. Göschen'sche Verlagshandlung - J. Guttentag, Verlagsbuchhandlung - Georg Reimer Karl J. Trübner - Veit \& Comp., Berlin 30. Printed in Germany -

Alle Rechte des Nachdrucks, der photomechanischen Wiedergabe, der Herstellung von Photokopien - auch auszugsweise vorbehalten.

Satz: Dörlemann-Satz GmbH \& Co. KG, Lemförde, Druck: Werner Hildebrand, Berlin 65, Einband: Lüderitz \& Bauer, Berlin, 61 


\section{Meinem Vater \\ Kurt Freiherr von Kutschera \\ * 28. 2. $1900 †$ 13.9. 1943 \\ zum Gedenken}





\section{Vorwort}

Mit menschlicher Erkenntnis, ihren Voraussetzungen, ihrer Natur, Leistung und Entwicklung befassen sich viele wissenschaftliche Disziplinen. Neurophysiologie und physiologische Psychologie untersuchen die physiologischen Grundlagen von Erkenntnisvorgängen. Wahrnehmungs-, Denk- und Lernpsychologie studieren Typen und Strukturen von Erkenntnisleistungen, ihre Zusammenhänge untereinander und ihre Beziehungen zum Verhalten. Die Entwicklungspsychologie erforscht die Onto- wie Phylogenese der Erkenntnis, die Entwicklungsphysiologie die Entstehung des menschlichen Zentralnervensystems und der Sinnesorgane, die Biologie ordnet die Entwicklung des menschlichen Erkenntnisapparats in den größeren Horizont der Evolution kognitiver Funktionen bei anderen Lebewesen ein. Die allgemeine Sprachwissenschaft befaßt sich mit den Zusammenhängen zwischen Sprache, Denken und Erfahrung, die Soziologie mit der sozialen Konstitution und Vermittlung von Erkenntnisinhalten. Was bleibt angesichts dieser Vielzahl einzelwissenschaftlicher Zuständigkeiten der Erkenntnistheorie als philosophischer Disziplin an Themen, was an Legitimation?

Noch für Kant stellten sich solche Fragen überhaupt nicht. Zu seiner Zeit gab es keine eigenständige Psychologie oder Soziologie, keine biologische Anthropologie, wie sie seit Darwin möglich geworden ist. Es gab keine Disziplinen, die der Philosophie ihre Zuständigkeit für Fragen menschlichen Erkennens hätten bestreiten können. Die Philosophie ging ferner weithin von einem Ideal wissenschaftlicher Erkenntnis aus, nach dem ,echte“ Erkenntnis nur da vorliegt, wo die Notwendigkeit des erkannten Sachverhalts eingesehen wird. $\mathrm{Da}$ empirische Untersuchungen nur kontingente Tatsachen, aber keine Notwendigkeiten aufweisen, wurde ihnen eine geringere Dignität zugesprochen als den apriorischen Einsichten der Philosophie. Heute hat sich das Verhältnis zwischen Philosophie und Naturwissenschaften umgekehrt: Durch deren große Erfolge seit dem Beginn des 19. Jahrhunderts - in deren Verlauf sich manche ,apriorischen Einsichten" der Philosophie als falsch erwiesen - ist das philosophisch 
Erkenntnisideal durch das naturwissenschaftliche abgelöst worden. Als ,wissenschaftlich“ gelten weithin nur mehr die Methoden und Ergebnisse der Naturwissenschaften, nicht die der Philosophie. Und jene universale Zuständigkeit für alle wichtigen Fragen über die Welt und den Menschen, die früher viele Philosophen für sich reklamierten, nehmen heute mit ähnlich naiver Unbefangenheit manche Naturwissenschaftler für sich in Anspruch.

Mit dem Wandel des Erkenntnisideals hat sich auch das Selbstverständnis des Menschen grundlegend verändert. Die idealistische Konzeption, nach welcher er ein Geistwesen ist, das gewissermaßen zufällig auch einen Körper hat, ist durch eine naturalistische Konzeption abgelöst worden, nach der er Teil der Natur ist und sich seine geistigen und kulturellen Leistungen biologisch aus Struktur und Evolution seines Organismus und der ihm angeborenen Verhaltensweisen erklären lassen. Die ,genetische Erkenntnistheorie“ von J. Piaget und die „evolutionäre Erkenntnistheorie“ von K. Lorenz sind Beispiele für Versuche, Erkenntnistheorie im Rahmen der Biologie zu betreiben. Angesichts dieser Lage hat heute die Frage nach Zuständigkeit und Legitimation der Philosophie für Fragen der Erkenntnistheorie nichts Absurdes mehr an sich.

Auch wenn man wissenschaftliche Erkenntnis nicht allein den $\mathrm{Na}$ turwissenschaften zuspricht und keinen Naturalismus vertritt, wird man sagen müssen, daß Philosophie sicher nicht für alle Fragen zuständig ist, die sich mit menschlichem Erkennen verknüpfen. Es gibt eine Fülle von Problemen, die sich nur durch einzelwissenschaftliche Untersuchungen klären lassen. Andererseits gibt es aber auch Fragen, die sich in den Einzelwissenschaften nicht behandeln lassen, weil sie Voraussetzungen dieser Disziplinen betreffen. Jede Spezialdisziplin bestimmt sich in jedem Stadium ihrer Entwicklung durch gewisse Grundannahmen, durch Untersuchungsmethoden und durch einen theoretischen Ansatz - kurz durch ein Paradigma im Sinne von Thomas Kuhn. ${ }^{1}$ In ihren Untersuchungen wird vorausgesetzt, daß diese Annahmen richtig und daß diese Methoden ein zuverlässiges Werkzeug zur Gewinnung von Erkenntnissen sind. So geht z. B. die Biolo-

\footnotetext{
${ }^{1}$ Vgl. dazu Kuhn (62) und den Abschnitt 9.6. - Die in Klammern nach den Autorennamen angeführten Zahlen verweisen auf das Erscheinungsdatum, Buchstaben auf Ausgaben der im Literaturverzeichnis angegebenen Arbeit. Zitate sind der letzten dort angegebenen Auflage entnommen.
} 
gie von einer realistischen Konzeption aus, nach der es eine vom menschlichen Bewußtsein, vom Erleben und Erkennen in ihrer Existenz wie Beschaffenheit unabhängige Außenwelt gibt, und sie beurteilt menschliche Wahrnehmung danach, in welchen Grenzen und wie genau unser Erleben den objektiven physikalischen Gegebenheiten entspricht. Auf die Frage, ob ein solcher Realismus haltbar ist - eine der zentralen Fragen der Erkenntnistheorie -, kann man von ihr keine Antwort erwarten. Allgemein fallen die Fragen nach der Gültigkeit der Voraussetzungen einer Disziplin und nach der Zuverlässigkeit ihrer Methoden außerhalb ihres Horizonts, der sich eben durch diese Voraussetzungen und Methoden definiert. Der Versuch, darüber mit den Mitteln der Disziplin selbst zu entscheiden, würde offensichtlich zu zirkulären Argumenten führen. Indem der Biologe unreflektiert voraussetzt, daß er mit seinen Methoden die Wirklichkeit so erfaßt, wie sie an sich ist, blendet er ferner einen Teil der Erkenntnisprozesse aus dem Horizont seiner Untersuchungen aus, nämlich jene, die er selbst vollzieht. Es ist zwar methodisch korrekt, bei der Untersuchung spezieller Erkenntnisleistungen gewisse andere hypothetisch als unproblematisch vorauszusetzen. Methodisch unkorrekt ist es aber, Aussagen über das menschliche Erkennen insgesamt machen zu wollen, ohne auch jene Verfahren in die Untersuchung einzubeziehen, auf die sich diese Aussagen stützen. In diesem Sinn sind allgemeine Aussagen über das Erkennen von Seiten der Einzelwissenschaften in der Regel naiv. Sie übersehen, daß auch ihr eigenes Erkennen einen Teil des Gegenstandsbereichs ausmacht, über den sie reden. Sie tun, als ob der Standpunkt ihrer Wissenschaft einen von den Bedingungen menschlichen Erkennens freien und damit von erkenntnistheoretischen Problemen unbelasteten Blick auf die Natur und die Bedingtheiten menschlichen Erlebens und Erkennens ermöglichte. ${ }^{2}$ Es ist eine

${ }^{2}$ Nicht alle Aussagen von Naturwissenschaftlern, die sich mit menschlicher Erkenntnis befassen, sind in diesem Sinn naiv. So sieht z. B. K. Lorenz in (73) die naturwissenschaftlichen Methoden nicht einfach als unproblematisch an, sondern begreift die Voraussetzung ihrer Zuverlässigkeit als eine hypothetische Grundlage für die Erforschung der Erkenntnisleistungen, und sagt, diese Voraussetzung habe sich dadurch zu bewähren, daß sich von ihr aus eine umfassende und kohärente Theorie dieser Leistungen ergibt, innerhalb derer sich dann auch die vorausgesetzten naturwissenschaftlichen Verfahren rechtfertigen lassen. Mit solchen Überlegungen verläßt er aber erstens das 
der grundlegendsten Einsichten der Erkenntnistheorie, die durch keinen Fortschritt der Naturwissenschaften überholt ist, daß sie sich nicht ,,von außen“ oder ,,von einem höheren Standpunkt aus" betreiben läßt, sondern daß sie nur als eine immanente Selbstkritik des Erkenntnisvermögens möglich ist, als eine Reflexion des Denkens auf sich selbst; daß ihre eigenen Einsichten einen Teil ihres Gegenstandes bilden und nicht aus der Reflexion ausgeklammert werden können. Allgemeine Aussagen über menschliches Erkennen müssen in diesem Sinn selbstanwendbar sein. Begründet man z. B. den Satz, daß es keine voraussetzungslosen Begründungen gibt, so muß er auf die Begründung selbst anwendbar sein; es muß also auch diese Begründung Voraussetzungen haben. Und man kann es nur dann als evident bezeichnen, daß Evidenz keine Wahrheitsgarantie enthält, wenn das auch für diese Evidenz gilt.

Die Reflexion auf die Methoden einer Einzelwissenschaft und die Prüfung des Geltungsanspruchs der damit gewonnenen Resultate gehört zur Wissenschaftstheorie und Grundlagenforschung dieser Disziplin. Wer sich damit befaßt, verläßt die normalen Pfade dieser Disziplin. In Wissenschaftstheorie und Grundlagendiskussion geht es um

Gebiet der Biologie und bestätigt, daß eine biologische Theorie des Erkennens Voraussetzungen macht, für deren Klärung die Philosophie zuständig ist. Zweitens ist dieser „hypothetische Realismus“ zwar ein durchaus diskutabler Ansatz, er wird aber nicht durchgeführt; nirgends wird gezeigt, daß sich auch biologische Erkenntnis im Rahmen der Biologie abhandeln läßt. Die bisherigen Ergebnisse der „evolutionären Erkenntnistheorie“ nehmen sich angesichts ihres Ziels, eine umfassende Theorie menschlichen Erkennens zu liefern doch sehr bescheiden aus, und man muß sich mit dem Optimismus des Autors trösten, das weitere werde sich schon finden. Da Erkenntnis ein geistig-seelisches Phänomen ist, kann man es mit naturwissenschaftlichen Mitteln auch nur dann aufklären, wenn sich Psychisches und Geistiges auf naturwissenschaftlich Faßbares, d. h. auf Physisches reduzieren lassen. Eine solche Reduzierbarkeitsthese, wie sie explizit oder implizit heute wohl von den meisten Biologen und Psychologen vertreten wird, ist aber eine philosophische These - sie fällt nicht in den Horizont der Physik, weil diese von vornherein nur für Physisches zuständig ist - , und sie ist, wie wir im 6. Kapitel sehen werden, auch höchst problematisch. Lorenz stellt eine solche Reduzierbarkeitsthese nicht auf, sondern erklärt im Gegenteil das Leib-SeeleProblem, eins der zentralen erkenntnistheoretischen Probleme, für unlösbar. Es ist aber nicht einzusehen, wie dann sein Programm einer rein biologischen Analyse des Erkennens durchführbar sein soll. 
philosophische Fragen. Und die allgemeinen und grundlegenden Fragen, auf die mar bei dieser Reflexion zurïckgeführt wird, sind die Fragen der philosophischen Disziplin, die sich mit Erkenntnis befaßt, der Erkenntnistheorie im üblichen Verständnis dieses Wortes. Diese Disziplin leidet also weder unter einem Mangel an Themen noch fehlt - es ihr an Legitimation. Nun ist sicher auch Philosophie nicht voraussetzungslos. Auch in ihr kann man nicht alles begründen, sondern muß mit Unbegründetem beginnen. (Das heißt natürlich noch nicht, $\mathrm{da} ß$ es philosophische Voraussetzungen gibt, die sich nicht begründen lassen, sondern nur, daß sich in ein und derselben Untersuchung nicht alles begründen läßt.) Im Gegensatz zu den Einzelwissenschaften gehören aber die Voraussetzungen, die bei philosophischen Aussagen gemacht werden, selbst zum Gegenstand der Philosophie, und man bleibt auf ihrem Boden, wenn man sie einer Kritik unterzieht. Der Preis, den die Philosophie dafür bezahlen muß, daß sie ihre eigenen Grundlagen mitreflektiert, besteht darin, daß sie nicht die Gestalt einer normalen, paradigmengeleiteten Wissenschaft annehmen kann, daß sie sich nicht, wie Kant meinte, auf den ,,sicheren Weg der Wissenschaft" bringen läßt.

Im Blick auf die Eigenart der Erkenntnistheorie, daß ihre Untersuchungen selbst einen Teil ihres Gegenstands ausmachen, ist es keineswegs von vornherein klar, welcher Erfolg ihr beschieden sein wird. Aus der Logik ist bekannt, daß selbstanwendbare Aussagen zu Paradoxien führen können ${ }^{3}$ und $d a ß$ keine formale Theorie (die in einer hinreichend ausdrucksfähigen Sprache formuliert ist) ihre eigene $\mathrm{Me}$ tatheorie enthalten kann - also eine vollständige Beschreibung der Theorie -, wenn sie widerspruchsfrei ist. ${ }^{4}$ Man kann zwar solche Resultate nicht ohne weiteres auf die Erkenntnistheorie übertragen, sie legen aber jedenfalls Vorsicht und Bescheidenheit bei den Zielsetzungen für erkenntnistheoretische Untersuchungen nahe. Da es insbesondere keine voilständige Theorie der Erkenntnis geben kann, da sie

${ }^{3}$ A. Tarski hat folgende Antinomie angegeben (vgl. (44), Anm. 11): Ein Satz der Gestalt ,Alle Sätze haben die Eigenschaft F" soll selbstanwendbar heißen genau dann, wenn er die Eigenschaft $\mathrm{F}$ hat. Es ergibt sich dann der Widerspruch: Der Satz ,Alle Sätze sind nicht selbstanwendbar“ ist genau dann selbstanwendbar, wenn er nicht selbstanwendbar ist.

${ }^{4}$ Vgl. dazu Gödel (31), sowie den Abschnitt 6.4. 
sonst ihre eigene Metatheorie enthalten müßte, ist die Bezeichnung „Erkenntnistheorie“ eigentlich unpassend, und man sollte besser und bescheidener von einer „Erkenntniskritik“ reden.

Für Bescheidenheit spricht auch die Tatsache, daß jede Reflexion auf Methoden, Möglichkeiten und Grenzen der Erkenntnis von den gegebenen Voraussetzungen, vom erreichten Entwicklungsstand des Erkenntnisvermögens und der Erkenntnismethoden ausgehen muß und sich im Rahmen der begrifflichen und methodologischen Möglichkeiten ihrer Zeit bewegt - was natürlich nicht heißt, daß sie nichts zur Weiterentwicklung des Vorhandenen beitragen könnte. Die Geistesgeschichte beweist die Entwicklungsfähigkeit des Erkenntnisvermögens, und es erscheint daher nicht als sinnvoll, vom heute erreichten Stand aus generelle Aussagen über künftige Erkenntnismöglichkeiten zu machen. Damit würde man im Effekt den eigenen Horizont zur Grenze künftiger Erkenntnisfortschritte erklären. Ein Blick auf die Geschichte der Erkenntnistheorie zeigt ferner, wie wenig gesicherte Resultate es hier gibt. Die verwirrende Vielfalt der Meinungen und die Tatsache, daß wohl jede denkbare Ansicht, und sei sie noch so abwegig, vertreten wurde, ${ }^{5}$ läßt die Möglichkeit definitiver Einsichten auf diesem Gebiet als zweifelhaft erscheinen. So ist denn auch die These von der Unmöglichkeit der Erkenntnistheorie vertreten worden. ${ }^{6}$ Dabei hat man aber der Erkenntnistheorie eine ganz unrealistische Zielsetzung unterschoben, und man hat übersehen, daß auch diese These und ihre Begründung ein Stück Erkenntnistheorie ist. Anspruch und Zielsetzung der Erkenntnistheorie sind also bescheiden zu formulieren. Was aber im einzelnen möglich ist, kann allein ein systematischer Versuch der Ausführung zeigen.

Wir haben schon betont, daß die Erkenntnistheorie als philosophische Disziplin nicht für alle Probleme zuständig ist, die sich mit menschlichem Erkennen verbinden. Die traditionellen Themen der Erkenntnistheorie, auf die wir uns in dieser Arbei beschränken, bilden aber auch nur einen Teil jener Fragen, die eine philosophische Theo-

\footnotetext{
SSchon Cicero meinte, daß es keine überhaupt denkbare Ansicht gebe, die Philosophen nicht vertreten hätten, und seitdem hat man fast zweitausend Jahre weiterphilosophiert.

${ }^{6}$ In neuerer Zeit z. B. von L. Nelson in (8). Vgl. dazu den Abschnitt 1.6.
} 
rie der Erkenntnis im umfassenden Sinn dieses Wortes zu behandeln träite. Erkenntnistheorie befaßt sich nicht mit allen Formen des Erkennens, sondern nur mit Erkenntnissen, deren Inhalt sich begrifflich hinreichend präzise bestimmen läßt. Begriffliche Erkenntnis spielt sicher eine besondere Rolle, da dazu insbesondere auch wissenschaftliche Erkenntnis gehört. Daneben gibt es aber auch andere kognitive Leistungen, die nicht an begriffliche Bestimmungen oder sprachlichen Ausdruck gebunden sind. Sie bleiben üblicherweise außerhalb des Horizonts der Erkenntnistheorie, und damit auch die wichtige Frage nach Eigenart und Grenzen begrifflicher Erkenntnis. Das liegt daran, daß wir begriffliche, insbesondere wissenschaftliche Erkenntnis als höchste Form der Erkenntnis ansehen, und z. B. anschauliches, intuitives oder gefühlsmäßiges Erkennen als primitive Vorformen davon betrachten. Erkenntnistheorie beschäftigt sich ferner nicht, oder doch nur am Rande, mit der Geschichte der Erkenntnisbegriffe, -ideale und -modelle, die für das Verständnis unserer eigenen Konzeptionen wichtig sind. Sie untersucht auch nicht alle Formen begrifflicher Erkenntnis, sondern befaßt sich hauptsächlich mit Erfahrungserkenntnis. Apriorische Erkenntnis wird im wesentlichen in der Philosophie von Logik und Mathematik, Werterkenntnis in Ethik und Ästhetik, religiöse Erfahrung in der Religionsphilosophie behandelt. Wegen der tiefgreifenden Unterschiede zwischen diesen Erkenntnisarten ist eine getrennte Behandlung sicher gerechtfertigt. Eine umfassende Theorie der Erkenntnis müßte aber all diese Themen behandeln.

Die Untersuchungen dieser Arbeit zerfallen in zwei Teile: Im ersten allgemeinen Teil, der die Kapitel 1 und 2 umfaßt, beschäftigen wir uns mit Fragen, die für alle Formen (begrifflicher) Erkenntnis relevant sind. Im 1. Kapitel wird dieser Erkenntnisbegriff analysiert, und es werden dann die Thesen des universellen Skeptizismus erörtert, welche die Möglichkeit von Erkenntnis generell infrage stellen. Das 2. Kapitel befaßt sich mit Formen des Verstehens. Ziel der Erkenntnisbemühung ist ja nicht nur, einzusehen, welche Sachverhalte bestehen, sondern auch, warum sie bestehen. Eine Analyse von Typen des Verstehens ist also ein wichtiges Thema der Erkenntnistheorie, auch wenn sie heute meist zur Wissenschaftstheorie gerechnet wird. Allgemein ist zu betonen, daß sich die Erkenntnistheorie mit anderen philosophischen Disziplinen thematisch überschneidet. So ist die Analyse der Begriffe Erkennen, Wissen und Glauben auch Gegenstand der 
epistemischen Logik. Da man jedoch z. B. über die Grenzen der Erkenntnis - ein Hauptthema der Erkenntnistheorie - nicht sinnvoll diskutieren kann, ohne sich zunächst über den Begriff der Erkenntnis zu verständigen, kommen wir an einer solchen Analyse nicht vorbei.

Der zweite Teil, der die restlichen Kapitel umfaßt, beschäftigt sich mit Problemen der (natürlichen, nicht-normativen) Erfahrungserkenntnis. Das zentrale Problem ist die Natur der äußeren Erfahrung. Es läßt sich ganz grob durch zwei Fragen charakterisieren:

1. Was sind die Gegenstände der Erfahrung? Sind es im Sinn des Idealismus Sinnesdaten, mentale Bilder oder Erscheinungen? Oder sind es Gegenstände der Außenwelt, deren Existenz und Beschaffenheit unabhängig von der Erfahrung durch uns ist, wie das der Realismus annimmt? Und wenn ja: Welchen Anteil haben subjektive Faktoren (unsere Sinnes- und Verstandesorganisation) an der Art und Weise, wie wir die Gegenstände wahrnehmen?

2. Was ist das Verhältnis zwischen Geistig-Seelischem und Körperlichem (Materiellem)? Das ist das Leib-Seele-Problem im weiteren Sinn.

Beide Fragen hängen eng zusammen: Erfahrungen sind Vorgänge, an denen Psychisches wie Physisches beteiligt ist. Eine Stellungnahme zur Realismus-Idealismus-Kontroverse muß also einem ontologischen Modell vom Verhältnis von Psychischem und Physischem entsprechen. Man kann z. B. nicht einerseits einen Realismus vertreten, nach dem eine physische Außenwelt Gegenstand unserer Erfahrung ist, und andererseits einen ontologischen Phänomenalismus, für den die Wirklichkeit nur aus Sinnesdaten besteht. Umgekehrt sind ontologische Aussagen erkenntnistheoretisch zu rechtfertigen, und daher läßt sich, wie schon Berkeley betont hat, auf der Basis eines erkenntnistheoretischen Idealismus, nach dem uns in der Erfahrung nur Sinnesdaten gegeben sind, keine ontologische These von der Existenz einer bewußtseinsunabhängigen Außenwelt vertreten. Wir erörtern daher beide Fragen, die Realismus-Idealismus-Kontroverse und das LeibSeele-Problem, zusammen, und diese Erörterung in den Kapiteln 4 bis 8 macht den Hauptteil dieser Arbeit aus. Das 3. Kapitel dient im wesentlichen der Vorbereitung dieser Diskussion. Das 9. Kapitel befaßt sich mit dem Aufbau der empirischen Sprache und der Begründung von Theorien durch Beobachtungen, und dem Zusammenhang zwischen Sprache und Theorien über die Natur, also mit Themen, die heute meist der Wissenschaftstheorie zugerechnet werden. Auch an 
ihnen kommt man aber nicht vorbei, wenn man - jedenfalls in groben Umrissen - eir Bild empirischer Erkenntnis entwerfen will, wie das Aufgabe der Erkenntnistheorie ist.

In dieser Arbeit nehmen sprachliche und logische Analysen einen verhältnismäßig breiten Raum ein, da sich Sachfragen mit Aussicht auf Erfolg nicht ohne hinreichend präzise Begriffe behandeln lassen und die Alltagssprache nun einmal nicht für wissenschaftliche Zwecke gemacht ist. Für die Lektüre sind daher logische Grundkenntnisse nützlich, wenn auch nicht unentbehrlich. Ich möchte freilich nicht verhehlen, daß ich mit Leibniz - indem ich seine Rede von „Mathematik" so verstehe, daß damit auch und vor allem Logik gemeint ist - der Meinung bin: „Sans les Mathématiques on ne pénètre point au fond de la philosophie. Sans la philosophie on ne pénètre point au fond de Mathématiques. Sans les deux on ne pénètre au fond de rien".

Das Buch ist keine „Einführung“ in die Erkenntnistheorie. In der Erkenntnistheorie gibt es kaum allgemein anerkannte und gesicherte Resultate, die man einfach referieren könnte. Jede systematische Arbeit zur Erkenntnistheorie ist eine Auseinandersetzung des Autors mit strittigen Fragen, fordert also den Leser zu aktiver und kritischer Teilnahme an der Diskussion heraus. So ist auch mein primäres Ziel nicht, den Leser mit den Problemen der Erkenntnistheorie und gängigen Meinungen dazu bekannt zu machen, sondern zu einer sachlichen Klärung der Fragen beizutragen. Das Buch hat nur insofern ,,einführenden" Charakter, als es ohne spezielle Vorkenntnisse gelesen werden kann.

Meinen Mitarbeitern, Frau Brigitte Weininger, Herrn Dr. Matthias Schirn und Herrn Uwe Meixner, danke ich für ihre wertvolle Hilfe bei der Erstellung des Manuskripts, der Literatur-, Namen- und Stichwortverzeichnisse und beim Lesen der Korrekturen. 


\section{Inhalt}

1 Gewißheit und Wabrheit . . . . . . . . . . . . . . 1

1.1 Glauben . . . . . . . . . . . . . . . . . . . 1

1.2 Wissen und Erkennen . . . . . . . . . . . . . 9

1.3 Wissen als wahre oder als wahre und fundierte Überzeugung . . . . . . . . . . . . . . 16

1.4 Perfektes Wissen . . . . . . . . . . . . . . . . 28

1.5 Evidenz . . . . . . . . . . . . . . . 36

1.6 Wahrheit .................... 42

1.7 Skepsis ......................... 52

2 Verstehen .................... 79

2.1 Verstehen, Erklären und Begründen . . . . . . . . 79

2.2 Kausale Erklärungen . . . . . . . . . . . . . . 95

2.3 Teleologische Erklärungen . . . . . . . . . . . . 107

2.4 Das Verstehen von Handlungen . . . . . . . . . . . . . . 121

2.5 Zum Problem des Verstehens in den Geisteswissenschaf-

3 Wabrnebmung und Wirklichkeit ............. 151

3.1 Wahrnehmung ................. 151

3.2 Wirklichkeit und Existenz . . . . . . . . . . . . . 160

3.3 Reduktionen und Modelle . . . . . . . . . . . . . . 165

3.4 Realismus . . . . . . . . . . . . . . . . . . . 179

$4 \quad$ Repräsentationstheorie und Idealismus . . . . . . . . . . . . 189

4.1 Repräsentationstheoretische Konzeptionen . . . . . . . 189

4.2 Argumente für den erkenntnistheoretischen Idealismus . . 202

4.3 Der ontologische Idealismus . . . . . . . . . . 213

5 Der Pbänomenalismus . . . . . . . . . . . . . 223

5.1. Formen und Thesen des Phänomenalismus . . . . . . . . . 223

5.2 Argumente des Phänomenalismus . . . . . . . . . . 226

5.3 Sinnesdaten . . . . . . . . . . . . . 231 
5.4 Kritik der Reduzierbarkeitsthese . . . . . . . . . . . . . 236

5.5 Die Intentionalität des Erlebens . . . . . . . . . . . 243

6 Der Physikalismus . . . . . . . . . . . . . . . . 249

6.1 Formen und Thesen des Physikalismus . . . . . . . . . . 249

6.2 Die Übersetzbarkeitsthese . . . . . . . . . . . . . . . . 254

6.3 Die Reduzierbarkeitsthese . . . . . . . . . . . . . . . . . 274

6.4 Determinismus . . . . . . . . . . . . . . . . . 279

$7 \quad$ Der Dualismus. . . . . . . . . . . . . . . . . . . . 289

7.1 Grundgedanken ... . . . . . . . . . . . . . . 289

7.2 Argumente des Dualismus . . . . . . . . . . . . . . 296

7.3 Das Problem der psycho-physischen Wechselwirkung . . 303

7.4 Die wechselseitige Bezogenheit von Psychischem und Physischem ................. 311

7.5 Seele und $\mathrm{Ich} \ldots \ldots \ldots \ldots \ldots \ldots \ldots$

$8 \quad$ Subjekt und Objekt . . . . . . . . . . . . . . . 337

8.1 Das polare Verhältnis von Psychischem und Physischem . 337

8.2 Die Differenzierung von Physischem und Psychischem . . 343

8.3 Raum und Zeit . . . . . . . . . . . . . . . . . . . 353

8.4 Grenzen der Objektivierbarkeit physikalischer Aussagen . 371

8.5 Das Leib-Seele-Problem . . . . . . . . . . . . . . . . . . . . . . . 384

8.6 Objektivität und Realismus . . . . . . . . . . . . . . . . 395

8.7 Objektivität und Idealismus . . . . . . . . . . . 414

9 Theorie und Wirklichkeit . . . . . . . . . . . . 435

9.1 Die Beobachtungssprache .............. 435

9.2 Theoretische Terme . . . . . . . . . . . . . . . . 447

9.3 Beobachtungssätze . . . . . . . . . . . . . . . . 455

9.4 Das Induktionsproblem . . . . . . . . . . . . . . 461

9.5 Kognitivistische und nichtkognitivistische Deutungen von Theorien . . . . . . . . . . . . 478

9.6 Holismus . . . . . . . . . . . . . . . . . . . . . . . . . . . . . 593

9.7 Relativismus . . . . . . . . . . . . . 512

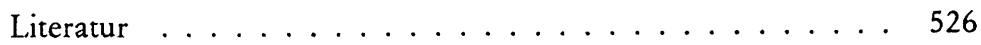

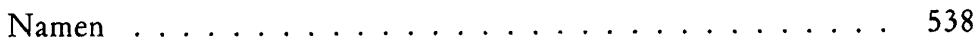

Stichwörter . . . . . . . . . . . . . . . 542 



\section{Gewißheit und Wahrheit}

\subsection{Glauben}

Wir wollen uns in diesem Kapitel mit der Analyse einiger Begriffe befassen, die in der Erkenntnistheorie eine wichtige Rolle spielen, insbesondere mit dem Erkenntnisbegriff selbst, und wollen dann mit diesen begrifflichen Unterscheidungen ein erstes erkenntnistheoretisches Grundproblem, den Skeptizismus, erörtern. Zunächst beschäftigen wir uns mit den Begriffen ,Glauben' und,Wissen', die auch in der epistemischen Logik untersucht werden. ${ }^{1}$ Hier gehen wir aber nicht auf technische Details ein, sondern beschränken uns auf die Erörterung grundlegender Unterscheidungen. ${ }^{2}$

Die Grundform von Aussagen mit dem Prädikat ,,glauben“ ist ,a

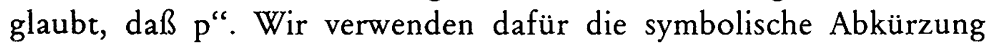
„G(a,p)". Dabei steht ,a" für eine Person und ,,p" für einen Sachverhalt. Auch Formulierungen wie ,Der Richter glaubt der Versicherung des Angeklagten“ oder „Fritz glaubt an den Sieg von Hans" lassen sich auf diese Normalform bringen, denn sie sind synonym mit den Sätzen „Der Richter glaubt, daß die Versicherung des Angeklagten korrekt ist", bzw. „Fritz glaubt, daß Hans siegen wird“. Damit ist die kategoriale Form des Glaubensbegriffs bestimmt: Glauben ist eine Relation zwischen Personen und Propositionen oder Sachverhalten. ${ }^{3}$

\footnotetext{
${ }^{1}$ Man bezeichnet gelegentlich nur die Logik des Wissensbegriffs als ,epistemische Logik", die des Glaubensbegriffs hingegen als ,doxastische Logik“. Obwohl das dem Sinn der griechischen Termini zweifellos besser entspricht, ziehen wir die einheitliche Bezeichnung „,epistemische Logik“ vor, da beide Disziplinen sehr eng zusammenhängen und von den beiden Termini dieser der gebräuchlichere ist.

${ }^{2}$ Die epistemische Logik ist von J. Hintikka in (62) begründet worden. Für eine Diskussion neuerer Arbeiten vgl. Lenzen (78). Systematische Darstellungen finden sich in Kutschera (76), Kap. 4 und sehr viel ausführlicher in Lenzen (80).

${ }^{3}$ Das Wort „Proposition“ verwenden wir synonym mit "Sachverhalt". Sach-
} 
Wenn wir sagen, die Person a glaube, daß der Sachverhalt $\mathrm{p}$ besteht, so ist damit noch nicht bestimmt, welchen Grad von (subjektiver) Wahrscheinlichkeit $p$ für a hat. Es kann sein, daß p für a nur wenig wahrscheinlicher ist als nicht- $\mathrm{p}$, daß also a nur vermutet, daß $\mathrm{p}$ gilt. Oder $\mathrm{p}$ kann für a eine sehr viel höhere Wahrscheinlichkeit haben als nicht-p, so daß a sich ziemlich sicher ist, daß p gilt. Oder $\mathrm{p}$ kann für a maximale Wahrscheinlichkeit haben, so daß sich a gewiß ist, fest davon überzeugt ist, daß p gilt. Man kann die Logik der verschiedenen Glaubensbegriffe aus der Theorie der subjektiven Wahrscheinlichkeit ableiten, einer vor allem von B. de Finetti begründeten mathematischen Theorie, ${ }^{4}$ indem man mit ihnen Wahrscheinlichkeitsgrade assoziiert. Dabei bietet sich vor allem der scbwache Glaubensbegriff (,Glauben“ im Sinn von ,mindestens vermuten“) an, nach dem a einen Sachverhalt $\mathrm{p}$ genau dann glaubt, wenn $\mathrm{p}$ für ihn wahrscheinlicher ist als nicht-p, also eine Wahrscheinlichkeit $>\frac{1}{2}$ hat, und der starke Glaubensbegriff ("Glauben“ im Sinn von „,fest davon überzeugt sein", „,sich sicher sein"), nach dem a den Sachverhalt p genau dann glaubt, wenn p für a die Wahrscheinlichkeit 1 hat. Für das folgende ist nur der starke Glaubensbegriff wichtig. Daber verwenden wir „Glauben" hier immer im Sinn von „Überzeugt sein".

Die epistemische Logik interessiert sich nicht für die Natur des Glaubens, ob z. B. der Glaube einer Person a, daß p der Fall ist, einen neurophysiologischen Zustand darstellt, eine psychische Disposition oder eine Verhaltensdisposition. Sie geht vielmehr davon aus, daß es einen für viele $Z$ wecke hinreichend eindeutigen umgangssprachlichen Gebrauch von ,glauben“ gibt, und unternimmt es, dieses Prädikat für gewisse philosophische, speziell erkenntnistheoretische Zwecke aufzubereiten, indem sie dafür präzise und systematisch fruchtbare Kriterien seiner Verwendung angibt. Dabei muß nicht die Frage beantwortet werden: „Was ist, oder worin besteht Glauben?"،, sondern es genügt, die logische Struktur des Prädikats „Glauben“, d. h. seine grundlegenden Eigenschaften durch Bedeutungspostulate festzulegen.

verhalte sind von Tatsachen als bestehenden Sachverhalten oder wahren Propositionen $\mathrm{zu}$ unterscheiden.

${ }^{4}$ Die bahnbrechende Arbeit von de Finetti war (37). Zur Theorie subjektiver Wahrscheinlichkeit vgl. a. de Finetti (70) und (72), sowie die kurzen Darstellungen in Stegmüller (73), Kutschera (72), Kap. 1, (76), 4.4 oder Lenzen (80). 
Ziel der Angabe solcher Bedeutungspostulate ist also nicht, den alltäglichen Begriff des Glaubens möglichst getreu wiederzugeben, sondern eine Explikation dieses Begriffs für wissenschaftliche, hier insbesondere für erkenntnistheoretische Zwecke vorzunehmen. Im Blick auf Präzision und systematische Fruchtbarkeit sind dabei gewisse Abweichungen der Bedeutung des Explikats, des Wortes „Glauben“ in seinen epistemisch-logischen und erkenntnistheoretischen Verwendungen, von der Bedeutung des Explikandums, der alltäglichen Bedeutung von "Glauben“, in Kauf zu nehmen. Solche Abweichungen ergeben sich für uns daraus, daß wir einen Begriff rationalen Glaubens angeben wollen, so daß sich die Bedeutungspostulate als Rationalitätsforderungen verstehen lassen. Es ist also wichtig, zwischen dem, wie wir hier auch sagen wollen, deskriptiven Glaubensbegriff des Alltags und dem rationalen Glaubensbegriff der epistemischen Logik klar $\mathrm{zu}$ unterscheiden. Im üblichen, deskriptiven Sinn verstanden machen die Sätze der Form ,,a glaubt, daß p“ Aussagen darüber, was die Person a tatsächlich glaubt. Wenn man jemand z. B. eine Liste von Sätzen vorlegt und ihn bittet, jene anzukreuzen, von deren Wahrheit er überzeugt ist, kann es sich herausstellen, daß er zugleich glaubt, daß ein Sachverhalt $\mathrm{p}$ besteht, wie daß $\mathrm{p}$ nicht besteht, oder $\mathrm{da}$ er glaubt, daß $\mathrm{p}$, aber nicht, daß q, obwohl q eine logische Folge von $\mathrm{p}$ ist. Dem Mangel an logischer Intelligenz sind keine ersichtlichen Schranken gesetzt. Es gibt keine apriorisch begründbaren generellen Prinzipien über den Zusammenhang der Überzeugungen der Leute; was sie glauben, ist eine empirische Frage, also auch welche logischen Strukturen die Systeme ihrer Annahmen haben. Daher gibt es auch keine Logik des deskriptiven Glaubensbegriffs.

Für die Erkenntnistheorie sind aber weniger Systeme faktischer Annahmen von Interesse als Systeme von Annahmen, die gewissen einfachen Rationalitätsbedingungen genügen. Die Rationalität wird dabei allein mit einem logischen Maßstab gemessen, und man geht von folgenden grundlegenden Prinzipien für einen rationalen Glauben aus:

G1: Gilt p analytisch, so gilt auch $G(a, p)$ - Analytische Wahrheiten werden geglaubt. ${ }^{5}$

\footnotetext{
${ }^{5}$ Ein Satz heißt analytisch genau dann, wenn sich sein Wahrheitswert (seine Wahrheit, bzw. Falschheit) allein aus den semantischen Regeln der Sprache ergibt. Analytisch wahre Sätze bezeichnet man auch als „Bedeutungswahr-
} 
G 2: $G(a, p \supset q) \wedge G(a, p) \supset G(a, q)$ - Jeder glaubt die Sachverhalte, von denen er selbst glaubt, daß sie Konsequenzen eigener Annahmen sind. ${ }^{6}$

G 3: $G(a, p) \supset \neg G(a, \neg p)-$ Man kann nicht zugleich p und nicht-p glauben.

Würden wir ,G“ als deskriptiven Glaubensbegriff verstehen, so wären die Prinzipien G1 bis G3 sämtlich empirisch falsch. Als Bedeutungspostulate für den rationalen Glaubensbegriff sind sie hingegen analytisch wahre Sätze. Durch sie wird der deskriptive Glaubensbegriff so modifiziert, daß sich mit dem Prädikat ,,glauben“ nur mehr Annahmen beschreiben lassen, die diesen Rationalitätsbedingungen genügen. Der Wert dieses so modifizierten Glaubensbegriffs liegt nicht darin, daß damit die Annahmen rationaler Personen beschrieben werden können, sondern darin, daß er logische Zusammenhänge zwischen Glaubensannahmen ausdrückt, die jeder vernünftigerweise jedenfalls dann berücksichtigen wird, wenn er darauf aufmerksam gemacht wird. Die epistemische Logik ist nicht deswegen interessant,

heiten " im Gegensatz zu Tatsachenwahrheiten. Oft nennt man nur analytisch wahre Sätze ,,analytisch“. Analytische Sachverhalte sind die durch analytische Sätze ausgedrückten Sachverhalte. W. V. Quine hat in (51) auf die Problematik der Unterscheidung analytischer und synthetischer (d. h. nicht analytischer) Sätze in natürlichen Sprachen hingewiesen. Vgl. dazu auch die Diskussion im Abschnitt 9.1. Sie ergibt sich u. a. daraus, daß ein Satz genau dann analytisch wahr ist, wenn er unter allen möglichen Umständen wahr ist (in allen "möglichen Welten“ wie man in der intensionalen Semantik auch sagt), während die Bedeutungen umgangssprachlicher Ausdrücke nicht für alle möglichen, sondern in der Regel nur für alle normalerweise vorkommenden Umstände erklärt sind (für die möglichen Welten, die unserer hinreichend ähnlich sind). Dieses Problem tritt für die Kunstsprachen der epistemischen Logik nicht auf, so daß hier ein präziser Begriff der Analytizität angegeben werden kann.

"Wir verwenden hier und im folgenden die logischen Symbole „־“ für „nicht", „^ “ für ,,und“, , $\vee$ “ für das nichtausschließende ,oder“, ,, $\supset$ “ für ,wenn-, dann" (genauer: für die materiale Implikation), „”" für ,genau dann, wenn" (genauer: für die materiale Äquivalenz), , $\wedge$ " für ,,alle" und „V“ für „es gibt“. Vgl. dazu z. B. Kutschera und Breitkopf (71). Der Gebrauch logischer Symbole erlaubt eine präzisere und übersichtlichere Formulierung vieler Aussagen. Wir geben jedoch bei den wichtigen Prinzipien eine normalsprachliche Übersetzung der Formeln an. 
weil es rationale Personen gibt, deren faktische Annahmen sich damit beschreiben lassen, sondern weil sich jeder von uns bemühen wird, eine rationale Person zu sein und sich als solche zu verhalten. Da jedermann Grund hat, analytisch wahre Sätze zu glauben, mit einem Sachverhalt auch das zu glauben, was nach seiner Ansicht daraus folgt, und wenn er glaubt, daß ein Sachverhalt $p$ besteht, nicht zugleich anzunehmen, daß nicht-p besteht - da also jedermann bestrebt sein wird, den Prinzipien G 1 bis G 3 für seine Annahmen Geltung zu verschaffen, ignorieren wir mit der Verwendung des rationalen Glaubensbegriffs nur erkenntnistheoretisch irrelevante logische Inkompetenzen.?

Wenn man eine prädikatenlogische Sprache zugrundelegt, in der auch die logischen Ausdrücke ,,alle“ und ,einige“ vorkommen, muß man zu G1 bis G3 das folgende Prinzip hinzunehmen:

G 4: $\wedge \mathrm{xG}(\mathrm{a}, \mathrm{F}(\mathrm{x})) \supset \mathrm{G}(\mathrm{a}, \wedge \mathrm{xF}(\mathrm{x}))$ - Wenn a von jedem Ding glaubt, daß es die Eigenschaft $F$ hat, so glaubt a auch, daß alle Dinge diese Eigenschaft haben.

Auch das ist eine Rationalitätsforderung, die sich intuitiv so erläutern läßt: Aus $G 1$ und $G 2$ folgt $G(a, p) \wedge G(a, q) \supset G(a, p \wedge q)-$ Glaubt a, daß p, und zugleich, daß q, so glaubt a auch, daß p und q gilt. $\mathrm{Da}$ sich $\wedge \mathrm{xF}(\mathrm{x})$ als ,große Konjunktion" $\mathrm{F}\left(\mathrm{b}_{1}\right) \wedge \mathrm{F}\left(\mathrm{b}_{2}\right) \wedge \mathrm{F}\left(\mathrm{b}_{3}\right) \wedge \ldots$ auffassen läßt, wobei für jedes Ding $b_{i}$ ein Konjunktionsglied $F\left(b_{i}\right)$ vorkommt (also als ,und“-Verbindung aller Instanzen des Allsatzes), wird man auch fordern, daß aus $\mathrm{G}\left(\mathrm{a}, \mathrm{F}\left(\mathrm{b}_{1}\right)\right) \wedge \mathrm{G}\left(\mathrm{a}, \mathrm{F}\left(\mathrm{b}_{2}\right)\right) \wedge \ldots$ folgt $\mathrm{G}\left(\mathrm{a}, \mathrm{F}\left(\mathrm{b}_{1}\right) \wedge \mathrm{F}\left(\mathrm{b}_{2}\right) \wedge \ldots\right)$, also $\mathrm{G}(\mathrm{a}, \wedge \mathrm{xF}(\mathrm{x})){ }^{8}{ }^{8}$

Mit $G 1$ bis $G 4$ haben wir Prinzipien für $G$ betrachtet, in denen keine iterierten Anwendungen des Operators $G$ vorkommen. Es sind aber auch Sätze sinnvoll wie

$\mathrm{G}(\mathrm{a}, \mathrm{G}(\mathrm{b}, \mathrm{p}))$ - a glaubt, daß b glaubt, daß p, $\mathrm{G}(\mathrm{a}, \mathrm{G}(\mathrm{b}, \mathrm{G}(\mathrm{a}, \mathrm{p})))$ - a glaubt, daß b glaubt, daß er (a) glaubt, daß p, usw.

\footnotetext{
' Für den Übergang vom deskriptiven zum rationalen Glaubensbegriff vgl. auch Kutschera (76), S. 79-82.

${ }^{8}$ Zur Diskussion dieses Prinzips vgl. Kutschera (76), S. $92 \mathrm{f}$. - Die Axiome G 2 und G 4 gelten für den schwachen Glaubensbegriff nicht. Vgl. dazu Lenzen (80), 3.2 und 8.1 .
} 
Für die Verknüpfung der Annahmen verschiedener Personen bieten sich dabei keine rationalen Prinzipien an: Was b glaubt, ist für a eine empirische Frage, und a kann durch rein logische Überlegungen über die Annahmen von b nichts ausmachen, was nicht schon in G 1 bis G 4 steckt. Dagegen wird man annehmen, daß gilt

G 5: $G(a, p) \supset G(a, G(a, p))$ - Wenn die Person a glaubt, daß p, so glaubt sie auch, daß sie es glaubt.

Wenn die Person a im Zeitpunkt t glaubt, daß p, so kann sie zwar zu einem früheren oder späteren Zeitpunkt $t$ ' bezweifeln, ob sie in $t$ glauben wird, bzw. geglaubt hat, daß p; aber in $t$ ist ihr das evident. Unsere Annahmen verändern sich natürlich mit der Zeit; wir bilden aufgrund neuer Erfahrungen neue Annahmen und verwerfen alte. Diese Zeitabhängigkeit könnte man explizit deutlich machen, indem man von Sätzen der Form ,a glaubt zur Zeit $t$, daß p“ - symbolisch $G_{t}(a, p)$ - ausgeht. Wir verzichten in der Regel aber aus Gründen der Kürze darauf, den Parameter t explizit anzugeben. Fehlt er, so sollen sich alle Glaubensaussagen auf denselben Zeitpunkt beziehen. ${ }^{9}$

Neben G 5 tritt das Prinzip

G6: $\neg \mathrm{G}(\mathrm{a}, \mathrm{p}) \supset \mathrm{G}(\mathrm{a}, \neg \mathrm{G}(\mathrm{a}, \mathrm{p}))$ - Glaubt die Person a nicht, daß p, so glaubt sie auch, daß sie das nicht glaubt.

G 5 und $\mathrm{G} 6$ besagen zusammen: Wir können uns darüber, ob wir etwas glauben oder nicht, nicht täuschen. Aussagen über eigene Überzeugungen sind für uns problemlos.

Die Prinzipien G 1 bis G6 bilden die Axiome der Logik des Glaubensbegriffs $G$. Es gibt nun in der epistemischen Logik kaum ein Prinzip, dessen Geltung nicht bestritten worden wäre, und das gilt auch für unsere Prinzipien. Die Einwände gegen G1 bis G 4 beruhen im wesentlichen darauf, daß ,G“ nicht im Sinn eines rationalen Glaubensbegriffs, sondern deskriptiv verstanden wird. Wir wollen darauf hier nicht eingehen, da diese Einwände in Kutschera (76), 4.1, 4.2 und Lenzen (78) und (80) ausführlich diskutiert werden. Hier wollen wir

${ }^{9}$ Auch für die Veränderung subjektiver Wahrscheinlichkeiten gibt es logische Gesetze. Vgl. dazu z. B. Kutschera (72), 2.1.3. Für den Glaubensbegriff ergibt sich daraus z. B.: gilt $\neg G_{t}(a, \neg p)$ und ändern sich die Annahmen von a im Übergang von $t$ zu t' so, daß a nun annimmt, daß p gilt, so ist $G_{t}(a, q)$ $\equiv G_{t}(a, p \supset q)$ für alle Sachverhalte $q$. 
nur kurz auf die Einwände gegen G5 und G6 hinweisen, da diese Prinzipien für das folgende besonders wichtig sind. Gegen sie hat man erstens eingewendet, daß diese Prinzipien eine fragwürdige Fähigkeit untrüglicher Introspektion voraussetzen. Nun, man kann natürlich die problembeladene Redeweise von einer "Introspektion" vermeiden, aber es bleibt doch das Faktum, das in folgendem einfachen Experiment deutlich wird: Man versuche einmal zu glauben, daß ein Sachverhalt besteht, bzw. das nicht zu glauben, und zugleich daran zu zweifeln, ob man das glaubt oder nicht! Das faktische Glauben oder Nichtglauben allein ist schon der zureichende wie notwendige Real- wie Erkenntnisgrund für die Überzeugung, daß man glaubt, bzw. nicht glaubt. Es ist nicht nur so, daß Äußerungen der Form „Ich glaube, daß p, glaube aber nicht, daß ich das glaube" oder „Ich glaube, daß ich glaube, daß p, glaube aber nicht, daß $\mathrm{p}$ “ ganz offensichtlich absurd sind, weil ich mit einer Behauptung auch ausdrücke, daß ich vom Bestehen des behaupteten Sachverhalts überzeugt bin. Das wäre nur ein pragmatisches Phänomen bei Äußerungen in ,Ich"-Form. Vielmehr kann man auch von einer anderen Person Entsprechendes nicht behaupten.

Gegen diese Argumentation könnte man einwenden, daß dabei der Glaubensbegriff so verwendet wird, daß die Aussage „Die Person a glaubt (im Zeitpunkt $t$ ), daß p“ impliziert, daß a (in t) über $p$ nachdenkt, so daß ,glauben" hier einen momentanen geistigen Akt ausdrücken würde, während das Wort gewöhnlich so verwendet wird, $\mathrm{da} ß$ es eine Disposition von a bezeichnet. ${ }^{10}$ Dieser Einwand ist aber nicht stichhaltig: Im dispositionellen Sinn von ,glauben“ gilt: Solange ich glaube, daß p, sehe ich p als wahr an, wenn ich die Geltung von $\mathrm{p}$ prüfe. Im gleichen Sinn gilt: Solange ich glaube, daß ich glaube, daß $\mathrm{p}$, sehe ich den Sachverhalt, daß ich p glaube, als wahr an, wenn ich seine Geltung prüfe. Die Geltung des Sachverhalts, daß ich p glaube, prüfen, heißt aber nichts anderes als p prüfen. Es gilt also: Solange ich

10 Zum normalen Gebrauch des Wortes ,glauben“ gehört, daß die Aussage ,a glaubt, daß $\mathrm{p}$ " nicht impliziert, daß a jetzt gerade an den Sachverhalt $\mathrm{p}$ denkt. Ich glaube auch dann, daß $2+2=4$ ist, wenn ich an ganz andere Dinge denke. Mit ,glauben" bezeichnen wir also keinen momentanen Akt, sondern Zustände oder Dispositionen. Diese Bestimmung soll auch für den rationalen Glauben gelten. 
glaube, daß p, glaube ich auch, daß ich glaube, daß p. Und entsprechendes gilt für $\mathrm{G} 6 .^{11}$

Ein zweiter Einwand geht von der auf den ersten Blick plausiblen These aus „Wenn ich mir über $\mathrm{p}$ noch keine Meinung gebildet habe (wenn ich insbesondere noch nik über $\mathrm{p}$ nachgedacht habe), so glaube ich nicht, daß $\mathrm{p}$ “. Dann gilt aber: Wenn ich noch nie drüber nachgedacht habe, ob ich glaube, daß p, so gilt: ich glaube nicht zu glauben, daß $p$, selbst wenn ich glaube, daß p. Es kann also gelten $G(a, p) \wedge$ $\neg \mathrm{G}(\mathrm{a}, \mathrm{G}(\mathrm{a}, \mathrm{p}))$, und entsprechend auch $\neg \mathrm{G}(\mathrm{a}, \mathrm{p}) \wedge \neg \mathrm{G}(\mathrm{a}, \neg \mathrm{G}(\mathrm{a}, \mathrm{p}))$. Hier wird nun gerade der dispositionelle Charakter des Glaubens verfehlt: Glauben daß p heißt, so haben wir gerade gesagt, $\mathrm{p}$ als wahr ansehen, wenn man sich überlegt, ob $\mathrm{p}$ gilt. Daher ist die obige These falsch: Es kann sein, daß ich mir noch nie die Frage gestellt habe, ob Hans ein besserer Tennisspieler ist als Fritz. Wenn ich aber weiß, daß Hans die allermeisten Spiele gegen Fritz gewonnen hat, und wenn ich die Ergebnisse der Spiele miteinander als direkten Maßstab dafür ansehe, wer von zwei Spielern der bessere ist, so liegt das Ergebnis der Überlegung schon fest, d.h. ich glaube dispositionell schon, daß Hans der bessere Tennisspieler ist, bevor ich noch über diese Frage nachgedacht habe. Im Sinn des rationalen Glaubensbegriffs gilt nach G1 und G 2 ferner, daß ich mit einem Sachverhalt $p$ auch alle Sachverhalte $q$ glaube, die aus $p$ analytisch folgen. $D a$ es aber unendlich viele solche Sachverhalte $\mathrm{q}$ gibt, kann ich nicht über sie alle nachgedacht haben.

Eine dritte Gruppe von Einwänden gegen $G 5$ und $G 6$ beruht darauf, daß man die beiden Vorkommnisse von , $G$ “ in "G(a, $G(a, A))$ “, bzw. , $G(a, \neg G(a, A))$ “ in verschiedener Weise deutet. So wird z. B. darauf hingewiesen, daß man sich einreden kann, etwas zu glauben, was man tatsächlich nicht glaubt, bzw. etwas nicht zu glauben, wovon man im Grunde doch überzeugt ist. Dann würde also gelten: man glaubt etwas zu glauben, was man tatsächlich nicht glaubt $(\mathrm{G}(\mathrm{a}, \mathrm{G}(\mathrm{a}, \mathrm{p})) \wedge \neg \mathrm{G}(\mathrm{a}, \mathrm{p}))$, oder man glaubt nicht, etwas zu glauben, was man tatsächlich glaubt $(\neg \mathrm{G}(\mathrm{a}, \mathrm{G}(\mathrm{a}, \mathrm{p})) \wedge \mathrm{G}(\mathrm{a}, \mathrm{p}))$. Man kann sich

${ }^{11}$ Man kann den Einwand geradezu umkehren: Ginge man von einem ,,aktuellen" Glaubensbegriff aus, so könnte man nicht mit $p$ zugleich auch glauben, $\mathrm{da}$ man $\mathrm{p}$ glaubt, denn sonst müßte man zugleich unendlich viele Sachverhalte aktuell glauben: mit $p$ müßte die Person a auch $G(a, p)$ glauben, damit $G(a, G(a, p))$, usf. 
nun zwar etwas einreden, wovon man zunächst nicht überzeugt ist, ater wenn man es sich erfolgreich eingeredet hat, so giaubt marı es auch, und glaubt nicht nur fälschlich, es zu glauben. Man kann freilich sagen: Jemand hat keine guten Gründe, etwas zu glauben, glaubt aber, sie zu haben. Dann deutet man jedoch in,$\neg G(a, A) \wedge$ $G(a, G(a, A))^{\prime \prime}$ das erste und das dritte Vorkommnis von " $G$ " in anderer Weise (im Sinn von ,hat gute Gründe, zu glauben") als das zweite Vorkommnis (,,glaubt").

Insgesamt kann man sagen, daß G 1 bis G6 unverzichtbare Bedingungen für rationale Überzeugungen darstellen, so daß wir im folgenden von diesen Prinzipien ausgehen können.

\subsection{Wissen und Erkennen}

Der zweite epistemische Grundbegriff neben Glauben ist Wissen. Wie die Grundform von Aussagen mit dern Prädikat „,glauben“ Sätze der Gestalt ,,a glaubt, daß p“ sind, so können wir als Normalform von Aussagen mit dem Prädikat ,wissen" Sätze der Gestalt ansehen ,a weiß, daß $\mathrm{p}^{\text {“ }}$ - symbolisch W(a,p). Auch das Prädikat ,wissen“ drückt also eine Relation zwischen Personen und Sachverhalten aus. Wie „Glauben“ ist auch „Wissen“ zeitabhängig. Wollen wir ausdrükken, daß die Person a im Zeitpunkt $t$ weiß, daß p gilt, so können wir dafür $W_{t}(a, p)$ schreiben. In der Regel lassen wir aber den Zeitparameter auch hier weg, wenn sich alle betrachteten epistemischen Aussagen auf denselben Zeitpunkt beziehen.

Den Begriff Erkennen kann man durch den des Wissens erklären, denn Erkennen ist der Schritt vom Nichtwissen zum Wissen. Man kann also sagen:

D 1.2-1: Die Person a erkennt, im Zeitpunkt $t$, daß p, genau dann, wenn $a$ in $t$ von einem Zustand des Nichtwissens, daß $p$, in einen Zustand des Wissens, daß p übergeht.

$\mathrm{Da}$ es nichts gibt, was wir, ohne es einmal erkannt zu haben, schon immer wüßten, ${ }^{12}$ kann man umgekehrt auch ,,wissen“ durch ,,erkennen" erklären und sagen:

I) a weiß, daß p, genau dann, wenn a erkannt hat, daß p. 
Man muß geglaubte oder gewußte Sachverhalte, d. h. Glaubensoder Wissensinhalte, von Sachverhalten des Glaubens oder des Wissens unterscheiden, d.h. die Sachverhalte $p$ von den Sachverhalten $G(a, p)$, bzw. W(a,p). Diese Sachverhalte sind verschieden: $p$ kann bestehen, ohne daß G(a,p), bzw. W(a,p) gilt (es kann etwa Hans erkältet sein, ohne daß Fritz das glaubt oder weiß), und umgekehrt kann auch $G(a, p)$ gelten, ohne daß p gilt (Fritz kann überzeugt sein, daß Hans erkältet ist, obwohl das tatsächlich nicht der Fall ist). Daher ist diese Unterscheidung wichtig, zumal sie sich sprachlich nicht immer in verschiedenen Formulierungen ausdrückt: Wenn wir von einem Glauben oder Wissen einer Person a sprechen, kann damit sowohl ein Sachverhalt des Typs $G(a, p)$, bzw. W(a,p) gemeint sein als auch der Sachverhalt p selbst, d. h. der Inhalt des Glaubens, bzw. Wissens. Das Wissen von a ist die Gesamtheit der von a gewußten Sachverhalte, der Wissensinhalte von a. Entsprechende Unterscheidungen gelten für das Wort „Erkenntnis“: eine Erkenntnis von a kann ein Sachverhalt des Erkennens durch a sein wie der von a erkannte Sachverhalt (Erkenntnisinhalt). Die Erkenntnis von a ist die Gesamtheit aller von a erkannten Sachverhalte, deckt sich also nach I mit dem Wissen von a. Die Erkenntnisse der Physik sind z. B. alle Sachverhalte, die durch physikalische Forschung erkannt worden sind.

$\mathrm{Da}$ es nun in der Erkenntnistheorie weniger um Akte des Erkennens als um Kriterien für die Unterscheidung von Erkenntnissen von bloßen Annahmen oder Überzeugungen, also von Wissen und Glauben geht, können wir bei unseren Erörterungen auch vom Begriff des Wissens ausgehen. Nach D 1.2-1 läßt sich ,,erkennen“ durch ,,wissen" definieren, so daß eine Analyse des Wissensbegriffs für eine Diskussion des Erkenntnisbegriffs ausreicht. Die Analyse des Erkenntnisbegriffs, das erste zentrale Thema der Erkenntnistheorie, nimmt also die Form einer Analyse des Wissensbegriffs an.

Wir beschränken uns in diesem Kapitel auf die Erörterung von Erkenntnissen, die sich durch Sätze der Form „Die Person a erkennt, daß $\mathrm{p}$ “ ausdrücken lassen. Mit dem Erkennen warum, wozu und wie,

${ }^{12}$ Auch Rationalisten, die ,eingeborene“ Prinzipien annehmen, glauben nicht, daß wir gewissermaßen von Geburt an wissen, daß sie gelten, sondern nehmen nur an, daß wir sie apriorisch, d. h. ohne Rückgriff auf Erfahrungstatsachen erkennen können. Vgl. dazu z. B. Descartes (MP), 171 und (PP), 293 ff. oder Leibniz (WP), IV, S. 451 und (WP), V, S. 62 ff. 
das ein Verstehen der Ursachen, Gründe, Zwecke oder Funktionsweisen darstellt, befassen wir uns im. 2. Kapitel. Aussagen über das Erkennen oder Wiedererkennen von Gegenständen oder Personen, wie z. B. , Als er näher kam, erkannte Hans seinen Bruder", lassen sich in Aussagen über ein Erkennen-daß übersetzen - im Beispiel in „Als er näher kam, erkannte Hans, daß es sein Bruder war".

Wir wollen in diesem Abschnitt noch nicht auf spezielle Wissensbegriffe eingehen, sondern zunächst einige generelle Adäquatheitsbedingungen für Wissensbegriffe formulieren. Dabei zielen wir, ähnlich wie im Fall des Glaubens, auf einen rationalen Wissensbegriff ab, den wir wieder von dem deskriptiven Wissensbegriff des Alltags unterscheiden. Die bestehenden Zusammenhänge zwischen Wissen und Glauben (vgl. die Prinzipien W6 bis W 9* unten) sind entweder für beide Begriffe als rationale oder für beide als deskriptive zu formulieren. Da wir uns für die Verwendung eines rationalen Glaubensbegriffs entschieden haben, müssen wir also auch einen rationalen Wissensbegriff verwenden. Wir ignorieren damit wieder die für erkenntnistheoretische Zwecke irrelevanten logischen Inkompetenzen der Personen.

\section{Prinzipien für den Wissensbegriff}

W 1: W(a,p) つp - Wenn a weiß, daß p gilt, so gilt $\mathrm{p}$.

Diese Bedingung ist kaum bestritten. ${ }^{13} \mathrm{Da}$ Shakespeare nicht der Autor von ",Tamburlaine the Great" ist, kann man auch nicht wissen, daß er der Autor dieses Stückes ist. ${ }^{14}$ Im Deutschen präsupponiert der Satz ,a weiß, daß p“, daß der Sachverhalt p besteht; d. h. der Satz wird ebenso wie seine Verneinung ,,a weiß nicht, daß p" nur dann als sinnvoll angesehen, wenn $\mathrm{p}$ besteht. ${ }^{15} \mathrm{Da}$ wir hier auf die logische $\mathrm{Be}-$ handlung solcher Präsuppositionen aber nicht eingehen wollen, schließen wir uns dem in der epistemischen Logik üblichen Verfahren

${ }^{13}$ Es gibt natürlich auch hier Einwände, die aber darauf beruhen, daß ,,wissen“ mit „zu wissen glauben“ verwechselt wird. Für eine ausführliche Diskussion dieser Einwände, wie auch der Einwände gegen die folgenden Prinzipien sei wieder auf Lenzen (78) und (80), 3.3 verwiesen.

${ }^{14}$ Das Gesetz W(a,p) $\supset \neg \mathrm{W}(\mathrm{a}, \neg \mathrm{p})$, das $\mathrm{G} 3$ entspricht, folgt direkt aus W 1 .

${ }^{15}$ Daher ist es auch nicht sinnvoll zu sagen: „Ich weiß nicht, daß p“. Denn mit einer solchen Behauptung drückt man einerseits aus, daß man die Präsuppo- 
an und fassen die Präsupposition als Teil der Assertion auf. Der Satz $\mathrm{W}(\mathrm{a}, \mathrm{p})$ ist dann im Falle $\neg \mathrm{p}$ nicht sinnlos, sondern falsch. Das ist bei der Übertragung natursprachlicher Sätze in unsere Symbolik und bei der Beurteilung der intuitiven Adäquatheit epistemologischer Prinzipien zu beachten.

Im Gegensatz zu W 1, das auch für den deskriptiven Wissensbegriff gilt, sind die folgenden drei Prinzipien typische Rationalitätspostulate.

W 2: $W(a, p \supset q) \wedge W(a, p) \supset W(a, q)-W e i ß a, d a ß$ q eine Folge von $\mathrm{p}$ ist, und weiß a, daß p gilt, so weiß a auch, daß q gilt.

W 3: $\Lambda \mathrm{xW}(\mathrm{a}, \mathrm{F}(\mathrm{x})) \supset \mathrm{W}(\mathrm{a}, \Lambda \mathrm{xF}(\mathrm{x}))$ - Weiß a von jedem Ding, daß es die Eigenschaft $F$ hat, so weiß a auch, daß alle Dinge die Eigenschaft $\mathrm{F}$ haben. ${ }^{16}$

W 4: Gilt p analytisch, so gilt W(a,p) - Analytisch wahre Sätze werden gewußt.

Die Rationalitätsbedingungen W 2 bis W 4 brauchen wir hier nicht näher zu erörtern, da Einwände gegen sie immer von einem deskriptiven Wissensbegriff ausgehen.

Ein Prinzip, das auch für den deskriptiven Wissensbegriff gilt, ist dagegen

W 5: W(a,p) つW(a,W(a,p)) - Weiß die Person a, daß p gilt, so weiß sie auch, daß sie das weiß.

Einwände gegen W 5 stützen sich meist auf Beispiele der folgenden Art: Ein Prüfling wird gefragt, wann Aristoteles gestorben ist. Er ist sich nicht sicher und antwortet probeweise ,322 v. Chr.". Die Antwort ist richtig, er wußte es also, wußte aber nicht, daß er es wußte. Diese Darstellung ist aber offenkundig unkorrekt: Die richtige Antwort belegt kein Wissen des Prüflings. Der Prüfer freilich kann nicht unterscheiden, ob die Antwort nur geraten oder bekannt war; er wird daher die Antwort so bewerten, als habe der Prüfling gewußt, daß sie richtig ist.

sition, daß p, als erfüllt ansieht, daß also $\mathrm{p}$ gilt, behauptet andererseits aber explizit, daß man das nicht weiß. Man kann nur sagen „Ich weiß nicht, ob p“.

${ }^{16}$ Dieses Prinzip stellt wieder eine Generalsierung des aussagenlogischen Prinzips $W(a, p) \wedge W(a, q) \supset W(a, p \wedge q)$ dar, das aus W2 und W4 folgt. 
Wenn ich nicht weiß, daß ich weiß, daß p gilt, so weiß ich entweder, daß ich p nicht weiß, dann weiß ich aber nach W 1 p tatsächlich nicht, oder ich weiß nicht, ob ich $p$ weiß (d.h. ich weiß weder, daß ich $p$ weiß, noch daß ich $\mathrm{p}$ nicht weiß). Ein derartiger Zustand der Unsicherheit bzgl. meines eigenen Wissens, ob p gilt, ist aber nach üblichem Sprachgebrauch nicht mit einem Wissen verträglich, daß p gilt.

\section{Prinzipien über den Zusammenbang von Wissen und Glauben}

W 6: W(a,p) つG(a,p) - Wenn a weiß, daß p, so glaubt a auch, daß p.

Auch diese Bedingung ist keine Rationalitätsforderung, sie würde auch für W und $G$ als deskriptive Begriffe gelten. Sie ist aber ebenfalls nicht unbestritten. So hat man verschiedentlich darauf hingewiesen, daß wir oft sagen: „Ich glaube das nicht, ich weiß es“. Das darf man aber nicht durch einen Satz der Gestalt $\neg \mathrm{G}(\mathrm{a}, \mathrm{p}) \wedge \mathrm{W}(\mathrm{a}, \mathrm{p})$ übersetzen. Gemeint ist vielmehr: „Ich glaube es nicht nur, sondern ich weiß es auch", und das ist zu übersetzen durch $G(a, p) \wedge W(a, p)$. Ferner hat man darauf hingewiesen, daß es unkorrekt wäre, zu sagen , IIch glaube, daß p“, wenn ich weiß, daß p. Diese Inkorrektheit ist aber nicht semantischer, sondern pragmatischer Natur. Wenn ich weiß, daß

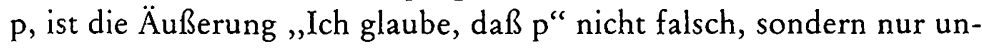
korrekt in dem Sinn, daß sie die Konversationsmaxime verletzt, hinreichend informativ zu sein. ${ }^{17}$ Wenn ich jemand auf die Frage, wie spät es ist, antworte „Ich glaube 12 Uhr 15“, obwohl ich weiß, daß diese Angabe korrekt ist, so teile ich ihm weniger mit, als er wissen wollte und ich ihm hätte sagen können, ähnlich als wenn ich sagte „etwa 12 Uhr", obwohl ich weiß, daß es genau 12 Uhr 15 ist. Im übrigen wird in beiden Einwänden das Wort ,glauben" wohl eher im schwachen als im hier zugrundegelegten starken Sinn verstanden.

Für einen generellen Wissensbegriff, nach dem es insbesondere ein Wissen von eigenen Überzeugungen geben kann, wird man auch fordern:

W 7*: $G(a, p) \supset W(a, G(a, p))$ - Wenn die Person a glaubt, daß p gilt, so weiß sie auch, daß sie das glaubt.

W 8*: $\neg \mathrm{G}(\mathrm{a}, \mathrm{p}) \supset \mathrm{W}(\mathrm{a}, \neg \mathrm{G}(\mathrm{a}, \mathrm{p}))-$ Wenn die Person a nicht glaubt, daß p gilt, so weiß sie auch, daß sie das nicht glaubt.

${ }^{17}$ Vgl. dazu die Theorie der Konversationsmaximen von H. P. Grice in (68). 
Diese beiden Prinzipien besagen, daß uns die Tatsachen, daß wir von etwas überzeugt, bzw. nicht überzeugt sind, immer bekannt sind. Sie stellen also eine Verstärkung der Prinzipien G 5 und G6 dar. Glaubt die Person a, daß p, so gilt nach G 5, daß sie auch glaubt, das zu glauben. Sie hat damit auch recht, und der über $G 5$ hinausgehende Inhalt von $\mathrm{W} 7 *$ ist nur, daß man ihr dann nicht ein Wissen davon absprechen kann, daß sie glaubt, daß p. Gegen W $7 *$ kann man entweder einwenden, daß jemand von gewissen eigenen Annahmen nichts wissen könne. Es ist aber völlig uneinsichtig, wie eine solche These begründet werden soll, da Annahmen bewußt sind, bzw. in der Reflexion darauf zu Bewußtsein kommen, also zweifellos evident sind, und $\mathrm{da}$ ferner andere Leute das, was ich glaube, nur sehr viel schwieriger und indirekter feststellen können als ich selbst. Oder man geht von einem speziellen Wissensbegriff aus, nach dem es von gewissen Sachverhalten - in unserem Fall: von eigenen Überzeugungen - von vornherein kein Wissen geben kann. Entsprechendes gilt für W 8*. Nach dem unten auch solche speziellen Wissensbegriffe diskutiert werden sollen, versehen wir diese Prinzipien mit einem Stern, um anzudeuten, daß sie nicht generell gelten.

W 9*: $G(a, p) \supset G(a, W(a, p))$ - Was wir glauben, glauben wir auch zu wissen.

Wenn wir glauben, daß p gilt, aber nicht zu wissen glauben, daß p gilt, so drückt ,,zu wissen glauben“ entweder eine stärkere Form des Fürwahr-Haltens aus als ,,glauben“, 18 oder eine Form des Für-wahr-Haltens, die sich auf eine spezielle Klasse von Sachverhalten bezieht. Nun verstehen wir hier aber ,glauben“ immer im starken Sinn von „,Überzeugtsein“; ,glauben“ drückt also die stärkste Form des Für-wahrHaltens aus. Daher kann der erste Fall nicht vorliegen. ${ }^{19}$ Nur in ihrem

${ }^{18}$ Aus $G(a, W(a, p))$ folgt umgekehrt ja wegen W 1 und $G 1, G 2$ G(a,p).

${ }^{19} \mathrm{~W}$. Lenzen weist in (80),3.3 darauf hin, daß man im Sinne des normalen (allgemeinen) Wissensbegriffs nicht sagen kann: „Ich bin mir völlig sicher, daß p gilt, weiß aber nicht, ob p gilt" , das also auch nicht glauben kann. Danach gilt allgemein $\neg \mathrm{G}(\mathrm{a}, \mathrm{G}(\mathrm{a}, \mathrm{p}) \wedge \neg \mathrm{W}(\mathrm{a}, \mathrm{p}))(*)$. Daraus erhält man aber W ${ }^{*}$ mit W $8 *$. Aus $\neg \mathrm{G}(\mathrm{a}, \mathrm{p})$ folgt nach W $8 * \mathrm{~W}(\mathrm{a}, \neg \mathrm{G}(\mathrm{a}, \mathrm{p}))$, nach W 6 also $\mathrm{W}(\mathrm{a}, \neg \mathrm{W}(\mathrm{a}, \mathrm{p}))$; es gilt also $\neg \mathrm{W}(\mathrm{a}, \neg \mathrm{W}(\mathrm{a}, \mathrm{p})) \supset \mathrm{G}(\mathrm{a}, \mathrm{p})$. Aus $(*)$ folgt aber $\mathrm{G}(\mathrm{a}, \mathrm{p}) \supset \neg \mathrm{G}(\mathrm{a}, \neg \mathrm{W}(\mathrm{a}, \mathrm{p}))$, also auch $\mathrm{G}(\mathrm{a}, \mathrm{p}) \supset \neg \mathrm{W}(\mathrm{a}, \neg \mathrm{W}(\mathrm{a}, \mathrm{p}))$. Mit $(*)$ $\mathrm{G}(\mathrm{a}, \mathrm{p}) \equiv \neg \mathrm{W}(\mathrm{a}, \neg \mathrm{W}(\mathrm{a}, \mathrm{p}))$ ergibt sich aber aus $\mathrm{G}(\mathrm{a}, \mathrm{p}) \neg \mathrm{W}(\mathrm{a}, \neg \mathrm{W}(\mathrm{a}, \mathrm{p}))$, mit W 5 also $\neg \mathrm{W}(\mathrm{a}, \neg \mathrm{W}(\mathrm{a}, \mathrm{W}(\mathrm{a}, \mathrm{p})))$, mit $(*)$ also $\mathrm{G}(\mathrm{a}, \mathrm{W}(\mathrm{a}, \mathrm{p}))$. 
Anwendungsbereich beschränkte, spezielle Wissensbegriffe können also W 9* verletzen.

Aus W 6 bis W9* folgen die Sätze:

TW 1": $G(a, p) \equiv \neg W(a, \neg W(a, p))-$ Glaubt die Person a, daß p, so weiß sie nicht, daß sie nicht weiß, daß p, und umgekehrt. ${ }^{20}$ TW $2^{*}: \neg \mathrm{W}(\mathrm{a}, \neg \mathrm{W}(\mathrm{a}, \mathrm{p})) \supset \mathrm{W}(\mathrm{a}, \neg \mathrm{W}(\mathrm{a}, \neg \mathrm{W}(\mathrm{a}, \mathrm{p})))-$ Weiß a nicht, $\mathrm{da} ß$ er $\mathrm{p}$ nicht weiß, so weiß er, daß er das nicht weiß." ${ }^{21}$

TW $2 *$ ist ein reines W-Prinzip, das wir deshalb nicht unter den anderen reinen $W$-Prinzipien aufgeführt haben, weil es ohne den Umweg über W 6 bis W 9* intuitiv kaum plausibel zu machen ist. Wie TW 1* aus W 6 bis W 9* folgt, so folgen diese Prinzipien umgekehrt auch aus TW 1* mithilfe von W 1 bis W 5 und TW $2 * .22$ Nach TW $1 *$ läßt sich der Begriff des Glaubens durch den des Wissens definieren, und damit (wie allgemein über die Prinzipien, die beide Begriffe verbinden) lassen sich aus der Logik des Wissens Prinzipien für die Logik des Glaubens ableiten. Das ist für uns jedoch weniger interessant, da erstens der Glaubensbegriff wesentlich einfacher ist als der Wissensbegriff und man diesen auch inhaltlich als abgeleiteten Begriff aufzufassen hat, und da zweitens W $8 *$ und W 9*, und damit TW $1 *$, eben nicht für alle Wissensbegriffe gelten. ${ }^{23}$

Nach dieser Erörterung genereller Adäquatheitsbedingungen für rationale Begriffe des Wissens wenden wir uns der Diskussion spezieller Wissensbegriffe zu.

${ }^{20}$ Aus $W$ 8* folgt nach der letzten Anmerkung $\neg W(a, \neg W(a, p)) \supset G(a, p)$.

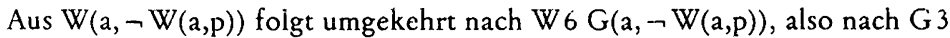
$\neg \mathrm{G}(\mathrm{a}, \mathrm{W}(\mathrm{a}, \mathrm{p}))$, also nach W 9* $\neg \mathrm{G}(\mathrm{a}, \mathrm{p})$. Durch Kontraposition erhält man daraus $G(a, p) \supset \neg W(a, \neg W(a, p))$.

${ }^{21}$ Aus $\neg \mathrm{W}(\mathrm{a}, \neg \mathrm{W}(\mathrm{a}, \mathrm{p}))$ folgt nach $T W 1^{*} \mathrm{G}(\mathrm{a}, \mathrm{p})$, daraus nach $\mathrm{W} 7 *$ $\mathrm{W}(\mathrm{a}, \mathrm{G}(\mathrm{a}, \mathrm{p}))$, mit TW $1 *$ also $\mathrm{W}(\mathrm{a}, \neg \mathrm{W}(\mathrm{a}, \neg \mathrm{W}(\mathrm{a}, \mathrm{p})))$.

${ }^{22} \mathrm{Vgl}$. dazu und zur Erörterung der beiden Prinzipien Lenzen (80).

${ }^{23}$ Aus W 1 bis W 5 und TW $2 *$ folgen mit TW $1 *$ die Axiome G1,G2,G3,G 5 (vgl. Lenzen (80)), nicht jedoch G4. Um G4 zu erhalten, muß man ein WAxiom W 5*a: $\Lambda \mathrm{x} \neg \mathrm{W}(\mathrm{a}, \neg \mathrm{W}(\mathrm{a}, \mathrm{A}(\mathrm{x}))) \supset \neg \mathrm{W}(\mathrm{a}, \neg \mathrm{W}(\mathrm{a}, \Lambda \mathrm{xA}(\mathrm{x})))$ annehmen. 


\subsection{Wissen als wahre oder als wahre und fundierte Überzeugung}

$\mathrm{Zu}$ einem ersten Begriff des Wissens gelangt man, wenn man die beiden notwendigen Bedingungen $W 1$ und W 6 für Wissen zusammen als hinreichend ansieht. Wir wollen diesen Wissensbegriff durch $W_{0}$ symbolisieren. Dann können wir also definieren

D 1.3-1: $W_{o}(a, p):=G(a, p) \wedge p-a$ weiß, daß p, genau dann, wenn a glaubt, daß $\mathrm{p}$, und damit recht hat.

Wieder sei daran erinnert, daß wir ,glauben“ hier im starken Sinn von „Überzeugtsein" verwenden. Richtige Vermutungen stellen also nach D 1.3-1 noch kein Wissen dar. Aus D 1.3-1 und den G-Prinzipien folgen die angegebenen W-Prinzipien, einschließlich $W 7^{*}$ bis W 9*, damit also auch TW $1 *$ und TW 2*.

Als Argument für die Definition kann man anführen: Überzeugung ist nach W9* ein hinreichendes subjektives Kriterium für Wissen. Eine Suche nach stärkeren subjektiven Kriterien für Wissen ist also illusorisch: Sicherer als ganz sicher kann man nicht sein. Stärkere objektive Kriterien für Wissen als die Wahrheit des Sachverhalts sind aber ebenfalls nicht denkbar: Richtiger als wahr kann ein Satz ebenfalls nicht sein. Wissen wird hier also in zwei Komponenten aufgespalten: in die subjektive Komponente der Überzeugung und in die objektive Komponente der Wahrheit, und beide sind einer Steigerung nicht fähig.

Gegen D 1.3-1 wendet man nun aber gewöhnlich ein, daß wahre Überzeugungen noch kein Wissen darstellen. Wenn ein Hellseher z. B. davon überzeugt ist, daß im nächsten Jahr in Thessaloniki ein Erdbeben stattfindet und das tatsächlich eintritt, so wird man nicht sagen, er habe das gewußt. Man würde vielmehr sagen: Er konnte das gar nicht wissen, da ihm die nötigen Daten und Theorien für eine verläßliche Voraussage fehlten. Platon, der im Theäthet, der ersten systematischen Abhandlung über den Wissensbegriff, diesen Begriff des Wissens als wahrer Überzeugung ( $\dot{\alpha} \lambda \eta \vartheta \dot{\eta} \varsigma \delta \delta \xi \alpha)$ ebenfalls diskutiert, verwirft ihn mit einem ähnlichen Argument: Wenn ein Kläger den Richter von der Schuld des Angeklagten überzeugt, so kann man auch dann, wenn diese Überzeugung richtig ist, nicht sagen, der Richter wisse, daß der Angeklagte schuldig sei, weil man der Aussage des Klä- 
gers als Beteiligtem allein die Schuld des Angeklagten nicht zuverlässig entnehmen kann. ${ }^{24}$

Theätet schlägt dann jene Definition des Wissens vor, die bis heute als ,klassisch“ gilt und den Begriff des Wissens ergibt, den die meisten Erkenntnistheoretiker verwenden, obwohl ihn Platon selbst verwirft: Wissen ist wahre und begründete Überzeugung ( $\dot{\varepsilon} \tau \iota \sigma \tau \eta \dot{\eta}$ als $\dot{\eta}$

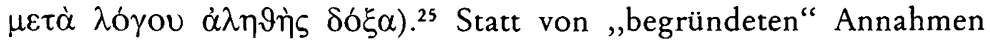
spricht man heute vielfach von ,fundierten" Annahmen, wobei es verschiedene inhaltliche Festlegungen darüber gibt, was unter ,Fundiertheit" zu verstehen ist.

Symbolisieren wir einen solchen Wissensbegriff durch $W_{F}$ und die Aussage „Die Annahme von a, daß p, ist fundiert" durch $F(a, p)$, so können wir also setzen:

D 1.3-2: $W_{F}(a, p):=G(a, p) \wedge F(a, p) \wedge p-a$ weiß genau dann, daß $\mathrm{p}$, wenn a glaubt, daß $\mathrm{p}$, wenn diese Annahme fundiert ist und wenn $p$ wahr ist.

Nach W 1 und W6 kann kein Wissensbegriff weiter sein als $W_{0}$. D 1.3-2 können wir daher als generelles Schema für Wissensbegriffe ansehen, die enger sind als $\mathrm{W}_{\mathrm{o}}$, nämlich dann, wenn nicht gilt $\mathrm{G}(\mathrm{a}, \mathrm{p}) \wedge \mathrm{p} \supset \mathrm{F}(\mathrm{a}, \mathrm{p}), \mathrm{d}$. h. wenn die Wahrheit einer Überzeugung nicht schon deren Fundiertheit impliziert, was wir im folgenden voraussetzen wollen. Alle engeren Wissensbegriffe erhält man also durch entsprechende Deutungen der Fundiertheitsbedingung. Eine davon ist die Deutung, nach der ,F(a,p) “ besagt, daß die Annahme $p$ von a begründet ist. Da diese Deutung aber nicht die einzige ist, wollen wir zunächst davon absehen und einige erkenntnistheoretisch besonders wichtige Typen von Fundiertheitsbegriffen herausstellen, bevor wir auf inhaltliche Festlegungen eingehen.

Aus den W-Prinzipien folgen schon gewisse Postulate für den Fundiertheitsbegriff. ${ }^{26}$ Aus $W 5$ folgt insbesondere $p \wedge G(a, p) \wedge F(a, p) \supset$ $\mathrm{G}(\mathrm{a}, \mathrm{F}(\mathrm{a}, \mathrm{p}))$. Dieses Prinzip können wir verstärken zu

\footnotetext{
24 Theätet $187 \mathrm{~b}$ und $201 \mathrm{~b}, \mathrm{c}$.

${ }^{25}$ Theätet $201 \mathrm{c}, \mathrm{d}$.

${ }^{26}$ Man wird die Bedingung hinzunehmen: Gilt $p \equiv q$ analytisch, so auch $F(a, p) \equiv F(a, q)$. Denn wenn $p \equiv q$ analyrisch gilt, stellen , $p$ “ und , $q$ " denselben Sachverhalt dar.
} 
F1: $G(a, p) \wedge F(a, p) \supset G(a, F(a, p))$ - Ist der Glaube der Person a, daß p gilt, fundiert, so glaubt a das auch.

Andernfalls müßten wir annehmen, daß bei $G(a, p) \wedge F(a, p)$ die Überzeugung $G(a, F(a, p))$ vom Bestehen von $p$ abhängt, was völlig unplausibel wäre. Im Fall $\neg p \wedge G(a, p) \wedge F(a, p)$ gegenüber $p \wedge G(a, p)$ $\wedge F(a, p)$ bleiben die Überzeugungen von a ja gleich, also kann sich auch nichts an der Überzeugung von a bzgl. der Frage ändern, ob a fundiert glaubt, daß p gilt. Wir wollen daher auch das Prinzip F 1 im folgenden voraussetzen. ${ }^{27}$ Die Aussage, daß die Person a in fundierter Weise glaubt, daß p gilt, ist durch $G(a, p) \wedge F(a, p)$ darzustellen; wir schreiben dafür auch $G_{F}(a, p)$.

Die Fundiertheit einer Überzeugung kann nun erstens eine subjektive Eigenschaft dieser Überzeugung sein, die für das Subjekt selbst problemlos ist. Wir wollen allgemein sagen:

D 1.3-3: Die Sachverhalte einer Menge $\mathcal{A}$ sind für die Person a problemlos genau dann, wenn für alle Elemente $p$ von $\mathcal{A}$ gilt $\mathrm{p} \supset \mathrm{G}(\mathrm{a}, \mathrm{p})$ und $\neg \mathrm{p} \supset \mathrm{G}(\mathrm{a}, \neg \mathrm{p}) .^{28}$

Die Person a ist dann über das Bestehen, bzw. Nichtbestehen der Sachverhalte aus $\mathbb{A}$ vollständig informiert. Die Menge der Sachverhalte, die durch Sätze der Form $G(a, p)$ ausgedrückt werden, ist nach G 5 und G6 für a in diesem Sinn problemlos. In einem schwächeren Sinn könnte man auch Sachverhalte als „unproblematisch“ bezeichnen, bzgl. derer wir uns nicht irren, also sagen: Die Sachverhalte einer Menge $A$ sind für die Person a unproblematisch genau dann, wenn für alle Elemente $p$ von $A$ gilt $G(a, p) \supset p$ und $G(a, \neg p) \supset \neg p .{ }^{29}$ Problemlose Sachverhalte sind unproblematisch, aber nicht umgekehrt. Die Aussage, die Sachverhalte aus A seien unproblematisch für a, ist inso

${ }^{27}$ Aus W 9* folgt F 1, W 9* können wir aber nicht generell voraussetzen.

${ }^{28}$ Daraus folgen mit den G-Axiomen die Gesetze $p \equiv G(a, p),-p \equiv G(a, \neg p)$ und $G(a, p) \vee G(a, \neg p)$. Sollen die Bedingungen im Definiens analytisch gelten, so genügt es, $p \equiv G(a, p)$ zu fordern. Denn mit $G 1$ erhält man daraus dann $G(a, \neg G(a, p) \supset \neg p)$, also mit $G 2 G(a, \neg G(a, p)) \supset G(a, \neg p)$, mit $G 6$ also $\neg \mathrm{G}(\mathrm{a}, \mathrm{p}) \supset \mathrm{G}(\mathrm{a}, \neg \mathrm{p})$; daraus ergibt sich aber mit $\neg \mathrm{p} \supset \neg \mathrm{G}(\mathrm{a}, \mathrm{p})$ $\neg \mathrm{p} \supset \mathrm{G}(\mathrm{a}, \neg \mathrm{p})$.

${ }^{29}$ Daraus folgt weder $p \equiv G(a, p)$, noch $\neg p \equiv G(a, \neg p)$ oder $G(a, p) v$ $\mathrm{G}(\mathrm{a}, \neg \mathrm{p})$ - auch dann nicht, wenn die beiden Definitionsbedingungen analytisch gelten. 
fern recht schwach, als sie nicht impliziert, daß a irgendwelche Überzeugungen (und damit Informationen) über sie hat.

Legen wir also die Bedeutung des Fundiertheitsbegriffs so fest, daß die Sachverhalte $G_{F}(a, p)$ für a problemlos sind, so heißt das, es solle gelten:

A.) $G_{F}(a, p) \equiv G\left(a, G_{F}(a, p)\right)$.

Daraus folgt unmittelbar F1. (A) gilt insbesondere dann, wenn fundierter Glauben eine Weise des Glaubens ist, wenn also $G_{F}$ die GAxiome erfüllt.

Soll die Fundiertheit einer Überzeugung hingegen eine objektive Eigenschaft dieser Überzeugung sein, so wird man fordern, daß fundierte Überzeugungen verläßlich sind:

B) $G_{F}(a, p) \supset p$.

Danach fällt $W_{F}$ mit $G_{F}$ zusammen. Auch aus (B) folgt $F 1$.

Wenn man nun die Forderungen (A) und (B) zusammen nimmt, so ergibt sich das Prinzip:

W 10*: $G(a, W(a, p)) \supset W(a, p) \cdot{ }^{30}$

Nach W 5 und W 6 gilt auch W(a,p) $\supset \mathrm{G}(\mathrm{a}, \mathrm{W}(\mathrm{a}, \mathrm{p}))$, zusammen also $\mathrm{W}(\mathrm{a}, \mathrm{p}) \equiv \mathrm{G}(\mathrm{a}, \mathrm{W}(\mathrm{a}, \mathrm{p}))$. Und da das analytisch gelten soll, ergibt sich damit, daß auch die Sachverhalte W(a,p) für a problemlos sind. ${ }^{31}$ Aus W 10* folgen umgekehrt auch (A) und (B) mit F 1.32 Gilt W 10*, so kann W9* nicht gelten, denn nach W9* folgt aus $\neg p \wedge G(a, p)$ $\mathrm{G}(\mathrm{a}, \mathrm{W}(\mathrm{a}, \mathrm{p}))$, nach $\mathrm{W} 10^{*}$ jedoch $\neg \mathrm{G}(\mathrm{a}, \mathrm{W}(\mathrm{a}, \mathrm{p}))$, so daß analytisch gelten müßte $G(a, p) \supset p$, was falsch ist.

Das Postulat (A) der Problemlosigkeit fundierter Überzeugungen ohne (B), das Postulat (B) der Verläßlicbkeit fundierter Überzeugungen ohne (A), und die Postulate (A) und (B) zusammen charakterisie-

${ }^{30}$ Aus $\neg W(a, p)$ folgt nach $(B) \neg G_{F}(a, p)$, nach $(A)$ also $\neg \mathrm{G}\left(\mathrm{a}, \mathrm{G}_{\mathrm{F}}(\mathrm{a}, \mathrm{p})\right)$, nach (B) also $\neg \mathrm{G}(\mathrm{a}, \mathrm{W}(\mathrm{a}, \mathrm{p}))$. Durch Kontraposition erhält man daraus W $10 *$

${ }^{31}$ Das würde sich auch aus dem Prinzip $\neg \mathrm{W}(\mathrm{a}, \mathrm{p}) \supset \mathrm{W}(\mathrm{a}, \neg \mathrm{W}(\mathrm{a}, \mathrm{p}))$ ergeben, das jedoch stärker ist als W 10*, wenn man von Begriffen fundierten Wissens ausgeht. (Für $W_{0}$ folgt es aus W 10*, jedoch gelten beide Prinzipien für $W_{0}$ nicht.) Für unsere Überlegungen ist es jedoch weniger relevant als W $10^{*}$.

${ }^{32}$ Aus $\neg \mathrm{p} \wedge \mathrm{G}_{\mathrm{F}}(\mathrm{a}, \mathrm{p})$ folgt nach $\mathrm{F} 1$ und $\mathrm{G} 5 \mathrm{G}\left(\mathrm{a}, \mathrm{G}_{\mathrm{F}}(\mathrm{a}, \mathrm{p})\right)$, nach W 10 * aber $\neg \mathrm{G}\left(\mathrm{a}, \mathrm{p} \wedge \mathrm{G}_{\mathrm{F}}(\mathrm{a}, \mathrm{p})\right)$, wegen $\mathrm{G}(\mathrm{a}, \mathrm{p})$ also $\neg \mathrm{G}\left(\mathrm{a}, \mathrm{G}_{\mathrm{F}}(\mathrm{a}, \mathrm{p})\right)$, so daß (B) gelten muß. Nach (B) gilt $W(a, p) \equiv G_{F}(a, p)$, so daß aus W $10 *$ und W 5 , W 6 auch (A) folgt. 
ren die für die folgende Diskussion wichtigsten Typen von Fundiertheits- und damit Wissensbegriffen.

Wir gehen nun zur Erörterung inhaltlicher Bestimmungen des Fundiertheitsbegriffs über. Eine erste Bestimmung lautet so:

I) $F(a, p)$ besagt, daß die Person a für den Sachverhalt $p$ eine nach Überzeugung von a korrekte Begründung angeben kann. ${ }^{33}$

Diese Definitionsbedingung kann man durch die Forderung verstärken:

Ia) die Begründung soll auch tatsächlich korrekt sein (d. h. p aus wahren Prämissen mit gültigen Schlußweisen ableiten) ${ }^{34}$

Im Sinn von (I) diskutiert E. Gettier den Fundiertheitsbegriff in (63); (Ia) ist der oben erwähnte dritte Vorschlag des Theätet in Platons gleichnamigen Dialog.

Nach (I) gilt $F(a, p)$ also genau dann, wenn es Sachverhalte $\mathrm{q}_{1}, \ldots, \mathrm{q}_{\mathrm{n}}$ gibt, aus denen $\mathrm{p}$ logisch folgt, und von denen die Person a überzeugt ist. ${ }^{35}$ Nach (I) wie nach (Ia) gilt $F(a, p) \supset G(a, p)$. Im Sinne von (I) ist anzunehmen, daß $G_{F}$ der Bedingung (A), aber nicht (B) genügt und daß daher $W_{F}$ auch nicht $W 10 *$ erfüllt. Nach (Ia) wird $G_{F}$ (B), aber nicht (A) und damit auch nicht W 10* erfüllen. Denn aus der Annahme, eine Begründung sei korrekt, folgt nicht, daß sie tatsächlich korrekt ist.

${ }^{33}$ Wir setzen im folgenden voraus, daß eine korrekte Begründung für einen Sachverhalt $\mathrm{p}$ in der Angabe von Tatsachen $\mathrm{q}_{1}, \ldots, \mathrm{q}_{\mathrm{n}}$ besteht, aus denen $\mathrm{p}$ logisch folgt. An diesem einfachen Begründungstyp lassen sich die Mängel des Wissensbegriffs nach (I) bereits hinreichend deutlich machen. Wir setzen ferner voraus, daß es Kriterien für solche Begründungen gibt - bislang gibt es sie nicht - , die Trivialisierungen ausschließen, wie z. B. eine „Begründung" von $p$ mit $p$ selbst, mit $\neg \neg p$, etc. Insbesondere soll, wo die Tatsachen $\mathrm{q}_{1}, \ldots, \mathrm{q}_{\mathrm{n}}$ aus $\mathrm{p}$ logisch folgen, die Ableitung von $\mathrm{p}$ aus diesen Tatsachen nicht als Begründung von $p$ gelten. Sonst würde aus $G(a, p)$ nach unserem rationalen Glaubensbegriff sowohl im Sinne von (I) wie von (Ia) F(a,p) folgen, so daß sich $W_{F}$ mit $W_{o}$ deckte.

${ }^{34} \mathrm{Im}$ Fall, daß a eine Begründung für $\mathrm{p}$ angeben kann, die zwar tatsächlich korrekt ist, von deren Korrektheit a aber nicht überzeugt ist, würde man kaum von einem fundierten Wissen sprechen.

${ }^{35}$ Folgt $\mathrm{p}$ logisch aus $\mathrm{q}_{1}, \ldots, \mathrm{q}_{\mathrm{n}}$, so glaubt a das nach unserem rationalen Glaubensbegriff auch, und glaubt a umgekehrt, daß $p$ aus $q_{1}, \ldots, q_{n} \operatorname{logisch}$ folgt, so ist das im Sinne diese Begriffs auch richtig. 
Gegen (I) ist einzuwenden, daß eine wahre Überzeugung der Person a nicht dadurch einen anspruchsvolleren Status erhalten kann, $\mathrm{da} \mathrm{a}$ in der Lage ist, dafür Gründe anzugeben, von denen sie fälschlich glaubt, sie seien wahr. Wenn schon wahre Überzeugungen allein kein Wissen darstellen sollen, so können das auch nicht wahre Überzeugungen tun, die durch falsche begründet sind. Die Kritik an (Ia) hat Platon selbst im „,Theätet" gegeben. Seine Argumentation ist zwar auf Begriffserkenntnis zugeschnitten, sie läßt sich aber so auf den Fall der Erkenntnis von Sachverhalten übertragen: Eine Begründung liefert offenbar nur dann eine Erkenntnis des begründeten Sachverhalts, wenn die begründenden Sachverhalte erkannt sind. Nun hat aber jede Begründung ein Ende; sie beginnt mit selbst nicht mehr begründeten Sätzen, die also in dem hier erörterten Sinn nicht erkannt sind. Daher kann sie dem begründeten Satz keinen Erkenntnischarakter vermitteln. Man kann auch nicht definieren: a weiß, daß p, genau dann, wenn $\mathrm{p}$ gilt, a davon überzeugt ist, und wenn a diese Überzeugung mit Sachverhalten begründen kann, von denen a weiß, daß sie gelten. Denn das wäre offensichtlich zirkulär. ${ }^{36}$

In unseren Tagen wissenschaftlichen Fortschritts hat E. Gettier gegenüber Wissensdefinitionen im Sinne von (I) in (63) betont, daß eine durch unkorrekte Gründe fundierte wahre Überzeugung kein besseres Wissen darstellt als andere wahre Überzeugungen. Daher wird man fordern, daß die fundierenden Gründe auch korrekt sein müssen, und gelangt in diesem Sinn dann zu dem Ansatz (Ia). Aber auch

${ }^{36} \mathrm{Vgl}$. Theätet 201cff. Für den Fall der Begriffserkenntnis argumentiert Platon etwa so: Ein Begriff soll ,,erkannt" heißen, wenn wir eine richtige Ansicht von seinen Merkmalen haben und diese auch begründen können. Begründung beruht in diesem Fall auf einer Begriffsanalyse. Da nun jede Begriffsanalyse ein Ende hat, d. h. von nicht mehr analysierten Begriffen ausgeht, und da das Begründete nur dann erkannt ist, wenn das Begründende erkannt ist, so gibt es danach keine Begriffserkenntnis. Die Definition: Ein Begriff ist erkannt, wenn wir eine richtige Ansicht von seinen Merkmalen haben und diese mit einer Analyse des Begriffes begründen können, bei der wir alle analysierenden Begriffe erkennen, wäre wiederum zirkulär. - Beide Ideen beruhen also auf der Tatsache, daß sich weder alle Sätze begründen, noch alle Begriffe definieren lassen. Diesen Gedanken hat auch Pascal in dem Fragment „Esprit de géométrie“ (W,Bd.XI) benützt, um die Unmöglichkeit einer ,,perfekten Wissenschaft" nachzuweisen. 
das genügt noch nicht: Wenn man wahre Überzeugung nicht als zureichende Bedingung für Wissen ansieht, so wird man auch korrekte Annahmen nicht als hinreichendes Fundament für ein Wissen ansehen können; man wird also, wie M. Clark in (64) betont - einer der vielen Arbeiten, die sich an den Aufsatz von Gettier anschlossen -, auch die Fundiertheit der fundierenden Gründe verlangen müssen. Die begründete Annahme ist ja nicht besser begründet als ihre schwächste Prämisse. Aber auch das genügt nicht: Man wird durch ähnliche Einwände wiederum dazu geführt, die Fundiertheit der fundierenden Gründe für die fundierenden Gründe der Überzeugung zu fordern, usf. Mit anderen Worten: Man kann nicht zulassen, daß die fundierenden Gründe für ein Wissen selbst kein Wissen darstellen. Bestimmt man aber den Fundiertheitsbegriff so, daß die fundierenden Gründe ein Wissen darstellen, so wird die Definition des Wissens, wie wir sahen, zirkulär. Man hat so schließlich in mühsamen Erörterungen nach zweieinhalb Jahrtausenden die Einsicht Platons wieder erreicht.

Wenn so die Bestimmungen (I) und (Ia) daran scheitern, daß Begründungen ein Ende haben, so wird man den Ansatz dadurch zu erweitern suchen, daß man auch wahre Überzeugungen als "Wissen“ bezeichnet, die auf Evidenz beruhen, die also in sich so klar sind, daß sie einer Begründung nicht bedürfen. Solche Evidenzen können z. B. durch direkte Beobachtungen entstehen, sie können aber auch allgemeine Sätze betreffen, wie z. B. mathematische Axiome. Auf den Evidenzbegriff werden wir erst im Abschnitt 1.5 eingehen. Dort werden wir aber zeigen, daß Evidenzen nur spezielle Überzeugungen sind, die nicht generell verläßlich sind. Die Bezugnahme auf Evidenzen und Begründungen im Wissensbegriff ergibt also zwar einen engeren, aber nicht einen anspruchsvolleren Begriff des Wissens als $W_{0}$. Ein solcher Begriff entginge auch nicht dem Einwand gegen $W_{\mathrm{o}}$ : Auch unserem Hellseher mag es aufgrund seiner Intuition evident sein, daß das Erdbeben stattfinden wird. Wenn wir seine wahre Überzeugung trotzdem nicht als Wissen ansehen, so führt es auch nicht weiter, wenn man Wissen auf Begründungen und Evidenzen stützt - es sei denn, man beschränkt den Bereich der Evidenzen, die ein Wissen fundieren können. Dann begegnet man aber beim Evidenzbegriff den gleichen Problemen wie beim Wissensbegriff.

Für eine Modifikation des Ansatzes (I) bietet sich folgender Gedanke an: Es seimfleine Methode zur Auszeichnung von Sätzen (bzw. Sachverhalten), die z. B. gewisse Sätze kategorisch auszeichnet sowie 
die Resultate von Beobachtungen, die in bestimmter Weise vorgenommen worden sind, und die ferner gewisse Schlußweisen angibt, mit denen sich aus nach $2 \mathbb{7}$ ausgezeichneten Sätzen andere, nach $2 \mathfrak{A}$ dann ebenfalls ausgezeichnete Sätze gewinnen lassen. Als eine solche Methode können wir insbesondere wissenschaftliche Methoden ansehen. Dabei wären die kategorisch ausgezeichneten Sätze die Grundannahmen dieser Wissenschaft, z. B. die von ihr zur Interpretation von Meßresultaten vorausgesetzten Theorien, ${ }^{37}$ die Beobachtungsmethoden wären ihre experimentellen Verfahren, und die Schlußweisen logisch-mathematische und etwa statistische Verfahren.

Der Fundiertheitsbegriff ist dann bzgl. einer solchen Methode so zu deuten:

II) $F_{m}(a, p)$ besagt, daß die Person a ihre Überzeugung, daß p gilt, durch eine (korrekte) Anwendung der Methode2Al gewonnen hat. Nach (II) gilt wieder $F(a, p) \supset G(a, p)$. Nehmen wir an, daß (korrekte) Anwendungen der Methode nur richtige Überzeugungen liefern, so ist zusätzlich zu fordern

IIa) $F_{2 \sharp n}(a, p) \supset p$, so daß (B) gilt.

Solange nun $\mathbf{m}$ nicht genau festgelegt ist, kann man weder beweisen, daß der damit nach D 1.3-2 definierte Wissensbegriff $W_{F}$ den Axiomen W 1 bis W6 genügt, noch prüfen, ob (A) gilt. Es ist aber anzunehmen, daß F nach (II), bzw. (IIa) dieselben Bedingungen erfüllt wie nach (I), bzw. (Ia), d. h. daß nach (II) (A) gilt, aber nicht (B), nach (IIa) hingegen (B), aber nicht (A).

Ist 27 der Inbegriff der gegenwärtigen wissenschaftlichen Methoden, so erfaßt man mit II den Begriff der wissenschaftlichen Erkenntnis. Da wissenschaftliche Erkenntnis nur ein Spezialfall dessen ist, was wir im allgemeinen Sinn als „Erkenntnis“ bezeichnen - wenn auch für das heutige Verständnis der Idealfall - so wird man W9* nicht annehmen: Es gibt Überzeugungen, die wir nicht als wissenschaftlich ausgewiesene Überzeugungen ansehen. Unser Hellseher wird z. B. seine Überzeugung bzgl. des Erdbebens in Thessaloniki selbst nicht als eine wissenschaftlich fundierte Überzeugung ansehen. Tatsächlich sind die allermeisten unserer Überzeugungen nicht wis-

${ }^{37}$ Was unter den Grundannahmen einer Wissenschaft zu verstehen ist, diskutieren wir im Kapitel 9. 
senschaftlich fundiert. Wenn Introspektion keine wissenschaftliche Methode ist, so wird man auch W $7 *$ und W $8 *$ nicht annehmen können.

Gegen die Bestimmung II lassen sich nun analoge Einwände vorbringen wie gegen I, bzw. Ia: Die mithilfe von 2 Hl gewonnenen Sätze sind nicht besser begründet als die Prinzipien von $2 \mathfrak{A}$ und nicht verläßlicher als die Beobachtungs- und Schlußverfahren von 272 . Da diese Prinzipien und Verfahren die Methode $2 \mathfrak{A}$ definieren, sind sie mit der Methode 27l selbst nicht begründbar; eine solche Begründung wäre offenbar zirkulär. Es sind also keine $2 ¥$-Erkenntnisse, daß die Prinzipien von $2 \mathfrak{F l}$ wahr sind oder daß die Verfahren von $2 \mathfrak{A l}$ zuverlässig sind. Will man diese Aussagen methodisch begründen, so ist dazu eine andere Methode 2Al' erforderlich, für die sich dieselben Probleme stellen. Es läßt sich also nicht alles methodisch begründen.

Tatsächlich sind ja z. B. die heute akzeptierten wissenschaftlichen Theorien keineswegs unbezweifelbar; sie sind die Falsifikationskandidaten von morgen. Auch die Grundlagen statistischer Argumente sind umstritten, und selbst strenge Experimente sind nicht immer zuverlässig. ${ }^{38}$ Unsere Überzeugung, die wissenschaftlichen Methoden seien zuverlässig, ist also eine Überzeugung, für die wir zwar sicherlich Gründe haben, die wir aber doch nicht mehr wissenschaftlich begründen können; sie ist also auch keine wissenschaftlich fundierte Überzeugung, sondern, wenn sie richtig ist, nur ein Wissen im Sinne von $W_{0}$.

Man kann daher zwar den Begriff des Wissens als wahrer Überzeugung im Sinne von (II) einschränken auf wahre Überzeugungen, die auf gewisse Weise gewonnen sind - und das entspricht manchen umgangssprachlichen Verwendungen des Wortes "Wissen“ besser als $\mathrm{W}_{\mathrm{o}}-$, aber man gelangt dadurch nicht zu einer qualitativ höheren Art des Wissens. Vielmehr beruhen wie im Fall (I), wenn man Wissen zirkelfrei definieren will, fundierte Überzeugungen nur auf anderen (wahren) Überzeugungen; es sind also nur durch (wahre) Überzeu-

${ }^{38}$ Es gibt sorgfältige, nach allen Regeln wissenschaftlichen Experimentierens gewonnene Meßresultate, wie z. B. den positiven Ausgang des MichelsonExperiments von D.C. Miller 1921-26 am Mount Wilson, die man nicht anerkennt, obwohl man Fehlerquellen nie nachweisen konnte. Daher spricht Popper in (66), S. 19 vom hypothetischen Charakter der Beobachtungsaussagen. 
gungen vermittelte wahre Überzeugungen; sie sind also erkenntnistheoretisch nicnt von höherer Dignität als diese.

Damit soll natürlich nicht impliziert werden, wissenschaftliche Methoden leisteten nicht mehr als irgendwelche anderen Verfahren, mit denen wir zu Überzeugungen gelangen. Für ein methodisch geleitetes Erkenntnisbemühen sprechen zwei wichtige Gründe. Erstens kann Erkennen nur dann zu einem gemeinsamen Unternehmen werden und damit die Grenzen individueller Horizonte überwinden, wenn es allgemein anerkannte Methoden der Erkenntnisgewinnung gibt, allgemein anerkannte Voraussetzungen, empirische Verfahren und Begründungsformen. Damit einer auf den Ergebnissen des anderen aufbauen kann, müssen diese nach auch von ihm selbst anerkannten Maßstäben gewonnen sein. Gemeinsame, intersubjektiv anerkannte Formen des Erkennens gibt es natürlich auch ohne Wissenschaft. Wir sprechen immer schon eine gemeinsame Sprache, haben einen gemeinsamen Schatz von Erfahrungen, gemeinsame Kriterien für zuverlässige Beobachtungen und gemeinsame Argumentationsweisen. Das Entstehen einer Wissenschaft bedeutet aber einen.entscheidenden Fortschritt, bei dem die Methoden der Erkenntnisgewinnung enger umschrieben und expliziter formuliert werden, so daß ihre Anwendung genauer kontrolliert und kritisch reflektiert werden kann.

Die fortlaufende strenge, intersubjektive Erfolgskontrolle, der wissenschaftliche Methoden unterworfen sind, bewirkt zweitens, daß wir sie als besonders zuverlässig ansehen. Die Überzeugung, daß eine Methode nur richtige Annahmen auszeichnet, ermöglicht uns aber definitive Urteile auch in Fragen, die sich unmittelbarer Einsicht entziehen. Überzeugung ist in vielen Fällen vermittelt, sei es durch Argumente wie z. B. im Fall komplizierter mathematischer Sätze, oder durch Meßverfahren in Bereichen, in denen direkte Beobachtung nicht möglich ist. Sie hängt also davon $a b$, daß die verwendete $\mathrm{Me}-$ thode als zuverlässig angesehen wird. Jeder, der sich in komplexeren Fragen um Erkenntnis bemüht hat, ist sich bewußt, daß man ohne ein gesichertes methodisches Verfahren nicht weit kommt. Jede Erweiterung des Erkenntnishorizonts beruht auf der Ausbildung und Absicherung von Erkenntnismethoden.

Auch wir bemühen uns ja hier um eine den Standards der Wissenschaftlichkeit entsprechende Einsicht. Trotzdem ist zu betonen, daß eine methodisch gewonnene wahre Überzeugung weder subjektiv sicherer noch objektiv wahrer ist als jede andere wahre Überzeugung. 
Die Tatsache, daß wir in vielen Fällen nur methodisch gesicherten oder wissenschaftlich begründeten Annahmen vertrauen, spricht nicht für eine Wissensdefinition nach D 1.3-2 und (II) gegenüber D 1.3-1. Denn das besagt nur: in diesen Fällen sind wir von unseren Annahmen überhaupt nur dann überzeugt und vermuten nicht nur ihre Richtigkeit, wenn sie methodisch begründet sind. Überhaupt nur von Annahmen überzeugt zu sein, die mit bestimmten wissenschaftlichen Methoden gewonnen sind, wäre aber eine ganz unwissenschaftliche Einstellung. Die Überzeugung von der Verläßlichkeit einer wissenschaftlichen Methode läßt sich ja erstens, wie wir sahen, nicht selbst wieder mit dieser Methode rechtfertigen, und zweitens würde man mit dieser Haltung die Wissenschaft gegenüber Falsifikationen durch Erfahrung immunisieren, also einen wissenschaftlichen Fortschritt unmöglich machen. Eine Methode zur Auszeichnung von Sätzen läßt sich nur dann testen, wenn man nicht alle Sätze, die sie auszeichnet, allein von ihr her beurteilt, sondern wenn es von ihr unabhängige Beurteilungskriterien gibt, wie z. B. solche schlichter Beobachtung. ${ }^{39}$ Die Feststellung, daß sich bisher ein höherer Prozentsatz von wissenschaftlich fundierten Überzeugungen als richtig erwiesen hat als von anderen Überzeugungen, kann endlich keinen Zweifel an der Richtigkeit einer gegenwärtigen, wissenschaftlich nicht fundierten Überzeugung bedingen. Es liegt im Begriff der Überzeugung, daß jemand, wenn er davon überzeugt ist, daß p gilt, auch davon überzeugt ist, daß er sich mit der Annahme von $p$ nicht irrt. Hätte er Gründe, daran zu zweifeln, wäre er eben nicht von $p$ überzeugt, sondern sähe $p$ nur als mehr oder minder wahrscheinlich an.

Während die Deutung (II) des Fundiertheitsbegriffs eine Erweiterung der Deutung (I) ist, beruht die folgende Interpretation auf einem anderen Gedanken:

III) $\mathrm{F}(\mathrm{a}, \mathrm{p})$ besagt, daß die Person a den Sachverhalt $\mathrm{p}$ kompetent beurteilen kann.

Man wird dabei von Kompetenz nicht bzgl. einzelner Sachverhalte reden, sondern bzgl. Klassen von Sachverhalten. Andernfalls müßte man wohl sagen, daß a einen Sachverhalt p kompetent beurteilt, wenn

${ }^{39}$ Auf das komplexe Problem des Zusammenhangs von wissenschaftlichen Theorien und Erfahrung gehen wir im 9. Kapitel näher ein. 
a entweder wei $\beta_{o}$, daß p, oder wei $\beta_{0}$, daß nicht $p$. Weiß $\beta_{o}$ a, daß p gilt, sc wüßte a damit aber auch im Sinne von (III), daß p gilt. Man wird also die Kompetenz von a bzgl. p so bestimmen, daß es eine $p$ enthaltende Klasse von Sachverhalten gibt, die a in der Regel richtig beurteilt; Kompetenz soll also nicht eine generelle Unfehlbarkeit bzgl. der fraglichen Sachverhalte beirhalien. Urteile von Fachleuten sind zwar in der Regel korrekt, Irrtümer aber nicht ausgeschlossen. Auch ein Verständnis von „Wissen“ im Sinn von D 1.3-2 und (III) entspricht vielen umgangssprachlichen Verwendungen des Wortes.

Gegen die Deutung (III) ist einzuwenden, daß sie keinen engeren Wissensbegriff ergibt als $W_{0}$. Das gilt zumindest, solange man diejenigen Klassen von Sachverhalten nicht einschränkt, bzgl. derer von „Beurteilungskompetenz" gesprochen werden soll. Dann definiert ja im Falle von $W_{o}(a, p)$ jede Klasse von Sachverhalten, die p enthält, $\neg \mathrm{p}$, und evtl. irgendwelche weiteren Sachverhalte q, die für a unproblematisch sind, und ihre Negationen - insbesondere also die Klasse $\{p, \neg p\}-$ einen Begriff $F(a, p)$, für den gilt $p \wedge G(a, p) \wedge F(a, p)$, also $W_{F}(a, p)$ im Sinne von III. ${ }^{40}$ Auch wenn man nur den Fachleuten im üblichen Sinn (dem Zahlentheoretiker, dem Kernphysiker, dem Lungenfacharzt, dem Steuerberater, dem Automechaniker) ein Wissen auf ihren Fachgebieten zuspricht, also den Wissensbegriff enger faßt als nach D 1.3-1, erhält man mit (III) keinen anspruchsvolleren Wissensbegriff. Die Kompetenz des Fachmanns besteht ja nur darin, daß er in der Regel weiß $\beta_{0}$, daß ein für seinen Bereich einschlägiger Sachverhalt $p$ besteht, wenn er das glaubt. Sein Wissen, daß p gilt, beinhaltet in diesem Sinn also nicht mehr, als daß er weiß $\beta_{o}$, daß p besteht, und $\mathrm{da} ß$ auch seine Überzeugungen bzgl. anderer Sachverhalte seines Arbeitsgebietes in der Regel richtig sind.

Die bisher diskutierten Explikationsvorschläge für den Fundiertheitsbegriff fassen also zwar den Wissensbegriff enger als $W_{o}$ und werden manchen umgangssprachlichen Verwendungen des Wortes „Wissen" besser gerecht, führen aber nicht zu einem in erkenntnistheoretisch relevanter Weise von $W_{0}$ abgehobenen Wissensbegriff, zum Begriff eines Wissens in einem qualitativ höheren Sinn. ${ }^{41}$

${ }^{40} \mathrm{Da}$ jeder mit einem Sachverhalt $\mathrm{p}$ auch den Sachverhalt $\neg \mathrm{p}$ kompetent beurteilen kann, gilt $F(a, p) \supset F(a, \neg p)$.

${ }^{41}$ Es gibt in der Literatur Explikationsvorschläge für die Fundiertheitsbedingung $F(a, p)$, die von den hier erörterten abweichen. Sie sind aber, soweit ich 


\subsection{Perfektes Wissen}

Aus der Tatsache, daß jemand davon überzeugt ist, daß ein Sachverhalt $p$ besteht, folgt nicht, daß $p$ tatsächlich besteht. Überzeugungen können falsch sein, wir erleben das leider immer wieder. Irrtümer, d. h. Fälle, in denen wir Falsches glauben, sind also nicht ausgeschlossen. Wenn wir wissen, daß p gilt, so folgt daraus zwar nach W 1 , daß $p$ dann tatsächlich gilt, aber wir können uns mit der Annahme irren, wir

sehe, ohne tieferes Interesse. Als Beispiel greifen wir den Vorschlag von K. Lehrer in (74) S. $195 \mathrm{ff}$. heraus. Dort wird zunächst eine Menge G(a) bestimmt als Menge der wahren Sätze $G(a, p)$. (Lehrer versteht dabei belief im Sinne von conviction, also im Sinn unseres starken Glaubens vgl. z. B. S. 12,209). Genauer wird G(a) bestimmt als Menge der wahren Sätze $G(a, p)$, die eine wohlerwogene, objektive Überzeugung von a ausdrücken (S. 189). Nachdem für diese Einschränkung aber keine exakten Kriterien angegeben werden, wollen wir davon absehen. Es wird dann $F(a, p)$ - Lehrer liest das als ,,a is completely justified in believing that $\mathrm{p}^{\text {" }}$ - definiert durch:

a) die subjektive Wahrscheinlichkeit $\mathrm{w}_{\mathrm{a}}(\mathrm{p})$, die a dem Sachverhalt $\mathrm{p}$ zumißt, ist größer als $w_{a}(\neg p)$, und für alle Sachverhalte $r$, die für a mit $p$ konkurrieren, gilt $\mathrm{w}_{\mathrm{a}}(\mathrm{p})>\mathrm{w}_{\mathrm{a}}(\mathrm{r})$. b) $\mathrm{r}$ konkurriert mit $\mathrm{p}$ für a genau dann, wenn $\mathrm{r}$ stark negativ relevant ist für $p$ in $G(a)$. (c) $r$ ist stark negativ relevant für $p$ in $G(a)$ genau dann, wenn $w_{a}(p, r)<w_{a}(p)$ ist, und wenn für alle Disjunktionen $d$ von Gliedern der vollständigen disjunkten Normalform von $p$, die zugleich Glieder der vollständigen disjunkten Normalform von $\mathrm{r}$ sind, gilt $\mathrm{w}_{\mathrm{a}}(\mathrm{p}, \mathrm{d}) \neq$ $\mathrm{w}_{\mathrm{a}}$ (p). (Die Definition auf S. 201 stimmt nicht mit der auf S. $195 \mathrm{f}$. überein; auf S. 201 muß es in der 12. Zeile von unten , r" statt ,h" heißen.)

Diese Definition ist aber unbrauchbar, denn gilt $G(a, p)$, also $w_{a}(p)=1$, so ist $\mathrm{w}_{\mathrm{a}}(\mathrm{p}, \mathrm{r})\left(\right.$ für $\left.\mathrm{w}_{\mathrm{a}}(\mathrm{r})>0\right)=1$. Es gibt dann also keine stark negativ relevanten Propositionen für p. Es gilt daher $G(a, p) \supset F(a, p)$, d. h. die Fundiertheitsbedingung ist überflüssig. (Im übrigen folgt nach (c) $p$ aus $d$, so daß $\mathrm{w}_{\mathrm{a}}(\mathrm{p}, \mathrm{d})=1$ ist. Ist also $\mathrm{r}$ stark negativ relevant für $\mathrm{p}$, so gilt $\mathrm{w}_{\mathrm{a}}(\mathrm{p})<1$. Die zweite Bedingung von (c) ist daher äquivalent mit $\mathrm{w}_{2}(\mathrm{p})<1$, und das folgt schon aus der ersten Bedingung von (c). Ferner ist die Bezugnahme auf $G(a)$ überflüssig, denn im Effekt geht es um die Wahrscheinlichkeitsbewertung $\mathrm{w}_{\mathrm{a}}$ von a, nicht nur um das, was a glaubt; $G(a)$ ergibt sich aber aus $w_{a}$, während das Umgekehrte nicht gilt.)

Auf S. 224 wird die Bedingung, ,a is completely justified in believing that $p$ in a way that does not depend on any false statements" - symbolisch $F^{*}(a, p)$ - so definiert, daß im Effekt gilt $G(a, p) \wedge p \supset F^{*}(a, p)$. Auch dieser Fundiertheitsbegriff ist also überflüssig; er sollte das Gettier-Beispiel ausschließen. 
wüßten, daß p gilt. Nach W 5 und W 6 gilt zwar: Weiß die Person a, daß p gilt, so glatb: sie auch, daß sie das weiß - W(a,p) つ $\mathrm{G}(\mathrm{a}, \mathrm{W}(\mathrm{a}, \mathrm{p}))$. Es gilt aber nicht generell, insbesondere nicht für $\mathrm{W}_{\mathrm{o}}$, das Prinzip, das aus W 10* folgt:

$\neg W(a, p) \supset G(a, \neg W(a, p))-W e i ß$ die Person a nicht, daß p gilt, so glaubt sie auch, daß sie das nicht weiß.

In diesem Punkt unterscheidet sich der Wissensbegriff $W_{\mathrm{o}}$ vom Glaubensbegriff, für den nach G6 ein solches Prinzip gilt. Die Frage, was er glaubt, ist für jedermann problemlos, nicht aber die Frage, was er weiß. Es gilt zwar nach $\mathrm{G} 6$ und $\mathrm{W} 6$

$\neg \mathrm{G}(\mathrm{a}, \mathrm{p}) \supset \mathrm{G}(\mathrm{a}, \neg \mathrm{W}(\mathrm{a}, \mathrm{p}))-$ Wenn die Person a nicht glaubt, daß $\mathrm{p}$, so glaubt sie, daß sie nicht weiß, daß p.

Es gilt aber nicht generell

$\neg \mathrm{p} \wedge \mathrm{G}(\mathrm{a}, \mathrm{p}) \supset \mathrm{G}(\mathrm{a}, \neg \mathrm{W}(\mathrm{a}, \mathrm{p}))-$ Wenn die Person a fälschlich davon überzeugt ist, daß $p$, so weiß sie, daß sie nicht weiß, daß p.

Das würde gerade dem Prinzip W $9 *$ widersprechen, das für allgemeine Wissensbegriffe höchst plausibel ist.

Es ist nun das Ziel einiger von D 1.3-1 abweichender Wissensdefinitionen, einen Wissensbegriff anzugeben, nach dem auch Sachverhalte des Wissens problemlos sind, der also das Prinzip W 10* erfüllt. Dazu muß man nach den Bemerkungen in 1.3 von einer Definition nach dem Schema D 1.3-2 ausgehen und die Bedingungen (A) und (B) annehmen. Wir wollen sagen:

D 1.4-1: W ist ein Begriff perfekten Wissens genau dann, wenn er W 1 bis W 6 und W 10* erfüllt.

Die Annahme perfekten Wissens bedeutet nicht, daß wir generell wahre von falschen Überzeugungen zuverlässig unterscheiden könnten; denn da wir von einem Sachverhalt, von dem wir wissen, daß er falsch ist, nicht überzeugt sind, würde daraus analytisch folgen, daß alle unsere Überzeugungen korrekt sind. Wohl aber impliziert ein solcher Wissensbegriff, daß wir zuverlässig zwischen bloßen Überzeugungen und Wissen unterscheiden können.

Es gibt zwei Klassen von Sachverhalten, für die eine perfekte Erkenntnis möglich ist. Die erste ist die der analytischen (d. h. der analytisch wahren wie der analytisch falschen) Sachverhalte. 
IV) Es besage $F(a, p)$, daß der Sachverhalt $p$ analytisch ist.

Dann gelten im Sinne unseres rationalen Glaubensbegriffs die $\mathrm{Ge}$ setze:

$F(a, p) \supset(p \equiv G(a, p)) \quad$ - Analytische Sachverhalte werden genau dann geglaubt, wenn sie wahr sind.

$\mathrm{F}(\mathrm{a}, \mathrm{p}) \supset \mathrm{G}(\mathrm{a}, \mathrm{F}(\mathrm{a}, \mathrm{p})) \quad$ - Ist $\mathrm{p}$ analytisch, so ist man davon auch überzeugt.

$\neg \mathrm{F}(\mathrm{a}, \mathrm{p}) \supset \mathrm{G}(\mathrm{a}, \neg \mathrm{F}(\mathrm{a}, \mathrm{p}))$ - Ist $\mathrm{p}$ nicht analytisch, so ist man auch davon überzeugt.

Daraus folgen die Bedingungen (A) und (B) also auch W 10*. Dieser Wissensbegriff erfüllt dagegen keines der Prinzipien $W 7^{*}$ bis $W 9^{*}$. Denn die Sätze ,G(a,p)“ und „, $\neg(a, p)$ “ sind nicht analytisch, und daß ein Wissensbegriff nicht zugleich W $9 *$ und W 10* erfüllen kann, haben wir schon wiederholt bemerkt.

In der Philosophiegeschichte hat ein Erkenntnisbegriff eine wichtige Rolle gespielt, nach dem nur notwendigerweise bestehende Sachverhalte erkannt werden können. Schreiben wir „,Np“ für „,Es ist notwendig, daß $\mathrm{p}$ ", so gilt für einen solchen Begriff notwendigen Wissens also

W 11*: W(a,p) つ Np. - Nur notwendige Sachverhalte können gewußt werden.

Es gibt nun verschiedene Begriffe der Notwendigkeit, z. B. analytische Notwendigkeit, die sich mit analytischer Wahrheit deckt, und naturgesetzliche Notwendigkeit. Auch der Wissensbegriff nach IV und D 1.3-2 ist also ein Begriff notwendigen Wissens. Bei den meisten Autoren, die einen Begriff notwendigen Wissens verwenden, bleibt aber unklar, wie sie Notwendigkeit verstehen. Unklar bleibt auch, welche Eigenschaften der Wissensbegriff im übrigen haben soll.

Zwei Gedanken waren es vor allem, die zu diesem Erkenntnisideal führten:

1. Soll Erkenntnis in begründetem Wissen bestehen, so kann die Unendlichkeit im Regreß auf die Gründe, die Gründe der Gründe usw. nur dann vermieden werden, wenn er auf Gründe führt, die notwendigerweise gelten. Sehe ich ein, daß ein Sachverhalt notwendigerweise besteht, so bedarf er keiner weiteren Erklärung. Sind aber die ersten Prämissen einer Kette von Begründungen notwendig, so auch die letzte Konklusion. Hinreichend begründetes Wissen gibt es also nur von notwendigen Sachverhalten. 
Dieser Gedanke kommt bei Leibniz besonders klar zum Ausdruck. Für Leibniz ist das Prinzip vom zureichenden Crund „Alles, was ist, muß einen zureichenden Grund haben, warum es ist" ein fundamentales Vernunftprinzip. Er deutet es aber nicht einfach so, daß sich danach jede Tatsache mit anderen erklären läßt. Da man bei kontingenten Gründen wieder nach ihrem Grund fragen kann, müssen die letzten Gründe einer zureichenden Erklärung für ihn mit Notwendigkeit gelten. Daraus ergibt sich: Was wahr ist, gilt mit Notwendigkeit, und die Erkenntnis einer Tatsache besteht in der Erkenntnis ihrer Notwendigkeit. Leibniz versteht Notwendigkeit (in diesem Sinn) sogar als analytische Wahrheit. ${ }^{42}$

Dieses Argument für W 11* ist aber nicht haltbar. Wir können uns auch bzgl. der Notwendigkeit eines Sachverhalts irren (jedenfalls dann, wenn wir „Notwendigkeit“ nicht als analytische Wahrheit verstehen und den rationalen Glaubensbegriff zugrunde legen). Auch die Behauptung, ein Sachverhalt gelte notwendigerweise, bedarf oft einer Begründung, ebenso wie die einfache Behauptung seiner Wahrheit. Der Rekurs auf notwendige Prämissen stellt also keinen Ausweg aus dem infiniten Regreß der Gründe dar. Deutet man W(a,p) im Sinne von $W_{o}(a, N p)$, so entgeht man nicht dem Einwand gegen $W_{o}$ : Es kann sein, daß jemand von der Notwendigkeit eines Sachverhalts überzeugt ist, z. B. von der Geltung der Großen Fermatschen Vermutung, damit auch recht hat, wir ihm aber trotzdem im normalen Sinn des Wortes ein Wissen davon absprechen, weil er seine Behauptung nicht rechtfertigen kann, im Beispiel also keinen Beweis dieser Vermutung hat.

2. Erkenntnis liegt nur da vor, wo wir uns nicht irren können. Die Behauptung, p gelte, kann aber nur dann nicht falsch sein, wenn p notwendigerweise gilt. Wissen impliziert also die Notwendigkeit des $\mathrm{Ge}$ wußten, d. h. es muß W 11* gelten.

Auch diese Argumentation ist unkorrekt. Nach W 1 gilt zwar analytisch, also auch notwendig, daß das, was jemand weiß, auch wahr ist. Es ist also unmöglich, daß wir uns irren, wenn wir etwas wissen (und nicht nur glauben). Daraus folgt aber nicht W $11 \%$; der Schluß von $\mathrm{N}(\mathrm{W}(\mathrm{a}, \mathrm{p}) \supset \mathrm{p})$ auf $\mathrm{W}(\mathrm{a}, \mathrm{p}) \supset \mathrm{Np}$ ist falsch.

Bei der Rekonstruktion von Begriffen notwendigen Wissens, bei denen Notwendigkeit nicht mit analytischer Wahrheit identifiziert

${ }^{42}$ Vgl. dazu Kutschera (79). 
wird, bietet es sich an, den Begriff rationalen Glaubens zu verwenden und entsprechend die Axiome W 1 bis W6, für den Notwendigkeitsbegriff aber nur die Standardaxiome anzunehmen, also z. B. jene des modallogischen Systems S5. Nach $W 11^{*}$ gilt nun $W(a, p) \supset N p \wedge$ $\mathrm{G}(\mathrm{a}, \mathrm{Np})$. Da p aus $\mathrm{Np}$ folgt, wird man den Begriff der Fundiertheit so festlegen:

V) $F(a, p):=N p \wedge G(a, N p)-$ Die Annahme von a, daß p gilt, ist genau dann fundiert, wenn $p$ notwendig und a davon überzeugt ist.

Damit erhalten wir nach D 1.3-2 wieder $W(a, p) \equiv W_{o}(a, N p)-W i s-$ sen besteht in wahrer Überzeugung von der Notwendigkeit von $p$. Damit W 10* gilt, also im Sinn des zweiten Motivs für W 11* ein Begriff perfekten Wissens entsteht, ist jedoch auch das Prinzip $\mathrm{G}(\mathrm{a}, \mathrm{Np}) \supset \mathrm{Np}$ anzunehmen. Das ist aber höchst unplausibel, wenn man, wie hier vorausgesetzt, nicht an analytische Notwendigkeir denkt. In Annahmen über naturgesetzliche Notwendigkeit können wir uns sicher täuschen, und ebenso, wie Kants synthetisch-apriorische Begründung der Newtonschen Mechanik zeigt, mit Annahmen über das, was aus apriorischen Gründen notwendig ist. Man kann also diesen Ansatz als eine Verlagerung des naiven Vertrauens in die eigenen Überzeugungen von der Ebene kontingenter Sachverhalte auf jene notwendiger Sachverhalte ansehen. Er ergibt jedenfalls keinen brauchbaren Wissensbegriff.

Die zweite Klasse von Sachverhalten neben der analytischer Sätze, für die es tatsächlich perfekte Erkenntnis gibt, ist die Menge der eigenen Überzeugungen.

VI) Besagt $F(a, p)$, daß p ein Sachverhalt ist, der sich auf Überzeugungen von a bezieht,

so läßt sich bei geeigneter Präzisierung dieser Aussage zeigen, daß der damit nach $D$ 1.3-2 definierte Wissensbegriff die Bedingungen (A) und (B), bzw. W 1 bis W $8 *$ und W 10*, nicht aber W 9* erfüllt. ${ }^{43}$

${ }^{43}$ Wir können definieren:

a) $F(a, p):=\operatorname{VqN}(p \equiv G(a, q))$.

Dabei drücke $\mathrm{N}$ eine analytische Notwendigkeit aus. Wenn man von einer Sprache der Prädikatenlogik ausgeht und sie durch die Festlegung erweitert, daß mit A auch $G(a, A)$ (für ein festes a) ein Satz ist (man erhält so die in Kutschera (76), S. 91 angegebene Sprache $G$ der epistemischen Logik, wobei 
Wo ein Begriff perfekten Wissens als der Wissensbegriff verwendet wird, stiitzt man sich entweder darauf, daß nur perfektes Wissen ein „,echtes" Wissen sei - Argumente dafür haben wir schon oben kennengelernt - , oder auf die These, daß analytische Sachverhalte oder solche, die sich auf problemlose eigenseelische Zustände beziehen, eire hinreichende Basis für die Erkenntnis aller (im normaien Sinn erkennbarer) Tatsachen bilde. Das ist der Ansatz jener Form des Rationalismus, die alle wahren Sätze als analytisch wahr und alle falschen Sätze als analytisch falsch ansieht, wie jener Form des Idealismus, die versucht, alle Aussagen über die Welt auf Aussagen über das eigene Bewußtsein zu reduzieren. Auf den Idealismus gehen wir in späteren

die Ausdrücke GA dieser Sprache hier die Form G(a,A) haben), so kann man den Begriff ,A ist ein reiner G-a-Satz' definieren durch die Bestimmungen:

1) Ist $A$ ein Satz von $G$, so ist $G(a, A)$ ein reiner $G-a-S a t z$.

2) Ist $A$ ein reiner $G-a-S a t z$, so auch $\neg A$.

-) Sind $A$ und $B$ reine G-a.-Sätze, so ist auch $(A \supset B)$ ein reiner $G-a-S a t z$.

4) Ist $A[b]$ ein reiner $G-a-S a t z$, so auch $\wedge x A[x]$.

Reine G-a-Sätze drücken dann Sachverhalte aus, die sich nur auf Überzeugungen von a beziehen. Es gilt nun, wie man leicht beweist:

b) $\mathrm{Zu}$ jedem reinen $\mathrm{G}-\mathrm{a}-\mathrm{Satz} \mathrm{A}$ gibt es einen Satz $\mathrm{B}$, so daß gilt $N(A \equiv G(a, B))$.

Wegen (b) besagt $F(a, p)$ nach (a), daß - in den Grenzen der Genauigkeit, mit der man in der intensionalen Semantik Sachverhalte charakterisieren kann - p ein Sachverhalt ist, der sich nur auf Überzeugungen von a bezieht. Diese Grenze bewirkt freilich, daß auch $2+2=4$ ein solcher Sachverhalt ist, weil gilt $\mathrm{N}(2+2=4 \equiv \mathrm{G}(\mathrm{a}, \mathrm{p} \vee \neg \mathrm{p}))$. Das ist nun weniger störend, als die Tatsache, daß man daraus, daß A ein reiner G-a-Satz ist, zwar schließen kann, daß auch die logischen Komponenten reine G-a-Sätze sind, also bei $G(a, B) \wedge G(a, C)$ z. B. die Komponenten $G(a, B)$ und $G(a, C)$, daß man jedoch aus $N(p \wedge q \equiv G(a, r))$ nicht darauf schließen kann, daß es auch Sachverhalte $s$ und $t$ gibt mit $N(p \equiv G(a, s))$ und $N(q \equiv G(a, t))$. Diesen Mangel an Differenzierungsvermögen der intensionslogischen Sprache muß man beim Beweis der Behauptung (d) mit einer Annahme über die zugelassenen Interpretationen ausgleichen, die zwar im gegenwärtigen Kontext plausibel, aber nicht allgemeingültig ist:

c) $N(p \equiv G(a, p)) \wedge N(p \supset q \equiv G(a, p \supset q)) \wedge p \supset N(q \equiv G(a, q))$

Mit (c) läßt sich dann beweisen:

d) Nach D 1.3-2 ergibt sich mit (a) ein perfekter Wissensbegriff, der zusätzlich die Axiome W 7* und W 8*, aber nicht W9* erfüllt. 
Kapiteln ein. Einen Rationalismus dieser Art hat insbesondere Leibniz vertreten. Neben dem schon oben referierten Argument über das Prinzip vom zureichenden Grund benutzt er dabei folgenden Gedanken: Für die traditionelle Logik hatten alle Aussagen Subjekt-Prädikat-Gestalt. Da nun Leibniz Substanzen als Mengen ihrer Eigenschaften auffaßte, war für ihn eine solche Aussage genau dann wahr, wenn der Prädikatsbegriff im Subjektbegriff enthalten, also dessen definierendes Merkmal ist. ${ }^{44}$ Daraus ergibt sich unmittelbar, daß alle wahren Sätze analytisch wahr sind.

Abgelöst vom Hintergrund der Leibnizschen Metaphysik, auf die wir hier nicht eingehen können, ${ }^{45}$ wirkt dieser Gedanke sicher reichlich unplausibel. Es sei daher jedenfalls angemerkt, daß es natürlich auch Leibniz klar war, daß wir nicht durch eine Bedeutungsanalyse der Wörter feststellen können, ob Sätze wie ,In Peking regnet es jetzt" wahr sind oder nicht. Er unterscheidet zwischen Sätzen, die sich ausgehend von Tautologien (,identischen Sätzen") durch endlich viele Substitutionen von definitorisch äquivalenten Ausdrücken ableiten, d. h. als analytisch erweisen lassen - nur sie gelten für ihn mit Notwendigkeit - und Sätzen, für die unendliche Beweisketten erforderlich wären, bzw. Schlüsse mit unendlich vielen Prämissen. Auch sie gelten in seiner Terminologie ,, analytisch“, werden aber als ,kontingent" bezeichnet. Der Satz „In Peking regnet es jetzt“ wäre danach zwar analytisch, aber wir könnten nicht feststellen, ob er analytisch wahr oder analytisch falsch ist; er wäre also nicht erkennbar (beweisbar) analytisch. Leibniz verwendet also das Wort ,, analytisch" anders als das heute üblich ist. Seine Argumente laufen im Effekt auf einen universellen logischen Determinismus hinaus, aus dem natürlich mit W 1 W 11* folgt, sie besagen aber nichts über den Sinn von Aussagen über Wissen und Erkennen, der unabhängig von solchen ontologischen Thesen analysiert werden muß. Und nur darum geht es uns hier.

Bevor wir uns endgültig auf einen bestimmten Wissensbegriff festlegen, müssen wir zunächst über Evidenz und Wahrheit, sowie über die skeptischen Thesen sprechen. Als Fazit der bisherigen Erörterun-

44 "Semper igitur praedicatum seu consequens inest subjecto seu antecedenti; et in hoc ipso consistit natura veritatis in universum . . " (OF), 518.

${ }^{45} \mathrm{Vgl}$. dazu z. B. Rescher (67) oder Kutschera (79). 
gen in den Abschnitten 1.2 bis 1.4 können wir aber folgendes festhalten: Es ging uns um die Explikation eines Wissensbegriffs für erkenntnistheoretische Zwecke. Dabei haben wir uns auf einen Begriff rationalen Wissens festgelegt, der die Prinzipien W 1 bis W6 erfüllt. Der einfachste und weiteste Begriff solchen Wissens ist der wahrer Überzeugung nach D 1.3-1. Was gegen ihn spricht, ist zunächst nur, daß er - gemessen am normalen Gebrauch des Wortes ,,wissen“ $\mathrm{zu}$ weit ist. $\mathrm{Da}$ es uns aber nicht primär um eine möglichst genaue Entsprechung zum normalen Wissensbegriff geht, ist das allein kein Grund, „Wissen“ in der Erkenntnistheorie nicht so zu verwenden. Daher haben wir untersucht, ob erkenntnistheoretisch relevante Gründe für eine andere Explikation sprechen und ob es eine anspruchsvollere Form von Wissen gibt, der man diesen Namen vorbehalten sollte. Alle engeren Wissensdefinitionen lassen sich mit dem Schema D 1.3-2 der Definition fundierten Wissens erfassen. Man kann drei Gruppen von Fundiertheitsbegriffen unterscheiden, solche, die der Bedingung (A) aus 1.3 genügen, nach der Fundiertheit problemlos ist, solche die der Bedingung (B) genügen, nach der fundierte Annahmen verläßlich sind, und solche, die beiden Bedingungen genügen. (A) besagt, daß Fundiertheit eine subjektive Qualität des Glaubens ist. Da aber "Glauben“ schon den stärksten Grad subjektiver Gewißheit ausdrückt, ergibt sich dabei keine anspruchsvollere, sondern nur eine engere Umschreibung der für Wissen hinreichenden subjektiven Komponente. Wir haben aber unter den intuitiven Argumenten für eine solche Einengung (vgl. I,II,III) keins gefunden, das eine solche Einengung erkenntnistheoretisch als sinnvoll erscheinen ließe. (B) besagt, daß Fundiertheit eine objektive Qualität des geglaubten Sachverhalts ist. $\mathrm{Da}$ aber Wahrheit nicht steigerungsfähig ist, ergab sich auch hier kein Argument für eine Einengung der Sachverhalte, von denen es ein Wissen geben kann (vgl. die Vorschläge I a und II a). Fundiertheitsbegriffe, die sowohl (A) wie (B) genügen, ergaben zwar Wissensbegriffe, die in erkenntnistheoretisch relevanter Weise von wahrer Überzeugung abgehoben sind, aber diese Begriffe sind wiederum zu eng, um als generelle Wissensbegriffe dienen zu können. Die Abweichung vom normalen Sprachgebrauch ist hier noch sehr viel gravierender als nach D 1.3-1. Im Sinn perfekten Wissens müßte ich sagen, daß ich weder wissen kann, wie ich heiße, noch daß ich zwei Hände und einen Kopf habe. Die bisherigen Erörterungen sprechen also dafür, das Wort "Wissen" in der Erkenntnistheorie im Sinn 
wahrer Überzeugung zu verwenden. Wo nichts anderes gesagt wird, wird ,wissen“, bzw. „W“ daher im folgenden immer im Sinn wahrer Überzeugung (im Sinne von $W_{0}$ ) verstanden.

\subsection{Evidenz}

Unsere Überzeugung, daß ein Satz gilt, stützt sich oft auf eine Begründung, im Idealfall auf einen Beweis des Satzes. Nun lassen sich aber nicht alle Sätze begründen. Denn jede Begründung beginnt mit Prämissen und vollzieht sich mit Schlüssen, von deren Wahrheit, bzw. Gültigkeit wir schon überzeugt sein müssen, damit die Begründung uns von der Wahrheit des begründeten Satzes, der letzten Konklusion überzeugt. Begründungen gibt es also nur, wo es Überzeugungen gibt, die nicht selbst wieder durch Begründungen vermittelt sind, die als einer Begründung nicht bedürftig angesehen werden. Begründeter Glaube beruht also auf unbegründetem, begründetes Wissen auf nicht begründetem.

Das heißt natürlich nicht, daß es Sätze gibt, die einer Begründung überhaupt nicht fähig oder bedürftig wären, die in jeder Begründung nur als erste Prämissen, nicht aber als Konklusion vorkommen. Vielmehr sind in jedem Kontext von Begründung Voraussetzungen zu machen, die in diesem Kontext nicht mehr hinterfragt werden, in anderen Kontexten aber sehr wohl zum Gegenstand einer Begründung gemacht werden können. Es ist also möglich, daß es zu jedem Satz eine Begründung gibt, ohne daß sich alle Sätze als Glieder einer großen Deduktion auffassen ließen. Auch Schlußweisen können problematisiert und begründet werden. Die Einsicht, daß sich nicht alles begründen läßt, bedingt also keinen Dogmatismus, nach dem bestimmte Sätze nicht infrage gestellt und in keinem Kontext als einer Begründung bedürftig angesehen werden.

Sachverhalte, von denen wir überzeugt sind, ohne uns auf eine Begründung zu stützen, die uns unmittelbar einleuchten, nennt man evident. Als Normalform einer Aussage über Evidenz sehen wir Sätze der Gestalt an: „Der Person a ist es evident, daß der Sachverhalt $p$ besteht" - symbolisch $V(a, p)$. Solche Sätze sind z. B. „Es ist mir evident, daß 2+2=4 ist“ oder "Inspektor Schmidt ist es evident, daß Herr Müller der Mörder ist". Eine Evidenz ist dann entweder ein 
Sachverhalt, daß jemandem etwas evident ist - so gebraucht man das Wort meistens -, oder ein Sachverhalt, der jemandem evident ist.

Wir wollen nun nicht versuchen, eine Logik des Evidenzbegriffs zu formulieren, sondern werden nur einige Bedingungen für diesen Begriff erörtern. Klar ist jedenfalls, daß es hier nicht sinnvoll wäre, in Analogie zum Glaubens- und Wissensbegriff so etwas wie einen rationalen Evidenzbegriff zu bestimmen, nach dem alle analytisch wahren Sätze jedermann evident wären und mit einem Sachverhalt auch dessen analytische Folgen. Denn mithilfe des Evidenzbegriffs will man ja gerade zwischen unmittelbar einsichtigen, d.h. in der Regel mehr oder minder einfachen Sachverhalten und daraus erschlossenen unterscheiden.

Nach dem üblichen Verständnis von ,evident“ wird man das Prinzip annehmen:

V1: $V(a, p) \supset G(a, p)$ - Wenn es a evident ist, daß p, so glaubt a, daß p. ${ }^{46}$

Das Wort ,evident" wird aber so verwendet, daß die Umkehrung $\mathrm{G}(\mathrm{a}, \mathrm{p}) \supset \mathrm{V}(\mathrm{a}, \mathrm{p})$ nicht allgemein gilt. Ich glaube $\mathrm{z}$. B., daß München etwa 1,3 Millionen Einwohner hat, ohne daß mir das evident wäre; ich vertraue den Angaben eines Lexikons, ohne mich selbst davon überzeugt zu haben, daß sie richtig sind.

Soweit ist die Sache klar. Das eigentliche Problem bei der Analyse des Evidenzbegriffes liegt darin, ob man das Bedeutungspostulat annehmen soll:

$V 2^{*}: V(a, p) \supset p-$ Evidente Sachverhalte bestehen,

d. h. ob Evidenzen auch verläßlich sind.

Ist es mir jetzt evident, daß $\mathrm{p}$, und stelle ich später fest, daß $\mathrm{p}$ falsch ist, soll ich dann sagen: „Es war mir evident, daß p, aber $p$ ist falsch“,

${ }^{46}$ Nach V 1 verwenden wir das Wort ,evident" hier im starken Sinn, in dem gilt, daß wir vom Bestehen uns evidenter Sachverhalte fest überzeugt sind, d. h. ihnen die Wahrscheinlichkeit 1 zuordnen. Das entspricht dem üblichen Verständnis von ,evident“. Man kann aber auch einen schwächeren Evidenzbegriff einführen, für den anstelle von $\mathrm{V} 1$ nur gilt: Wenn es a evident ist, daß p, so glaubt a im schwachen Sinn, daß p. Auch Vermutungen stützen sich nicht immer nur auf Argumente. Es gibt z.B. Beobachtungen, die einen Sachverhalt nur mehr oder minder wahrscheinlich machen. 
oder soll ich sagen: „Es schien mir evident zu sein, daß p, aber es war mir tatsächlich nicht evident, da ja $\mathrm{p}$ falsch ist"? Von Vertretern der Deutung V 2* wird Evidenz in diesem Sinn auch oft als ,,echte Evidenz" bezeichnet. Daneben muß man dann noch eine "Scheinevidenz" einführen, bei deren Vorliegen wir uns bzgl. der Evidenz eines Sachverhalts irren.

Es sind also zwei Deutungen von ,evident“ zu unterscheiden: Nach der ersten ist Evidenz ein rein subjektives Kriterium für Wahrheit, so daß Sachverhalte des Evidentseins immer problemlos sind. Es gilt dann im Sinn von D 1.3-3:

V3: $V(a, p) \equiv G(a, V(a, p))$ - Ist es der Person a evident, daß p gilt, so glaubt sie auch, daß ihr das evident ist, und umgekehrt.

Nähme man daneben auch V $2 *$ an, so würde gelten $G(a, V(a, p)) \supset p$, es gäbe also keine "Scheinevidenz". Das widerspricht nun aber der Erfahrung: Es kommt immer wieder einmal vor, daß wir überzeugt sind, ein Sachverhalt sei evident, der sich hinterher als falsch erweist. Daher liegt V 2* ein anderes Verständnis von Evidenz zugrunde als V3; nach V 2* ist Evidenz kein rein subjektives Kriterium für Wahrheit. Man muß sich also zwischen den Bedeutungspostulaten V 3 und $V 2 *$ entscheiden, d. h. ob Evidenz ein glaubensartiger oder ein wissensartiger Begriff sein soll. Wir entscheiden uns dafür, das Wort „evident" im Sinne von V 3 zu verwenden, fordern also nicht V 2*, da wir die Problemlosigkeit im Sinne von V 3 als charakteristisch für den Evidenzbegriff ansehen, so wie er üblicherweise gebraucht wird, nicht dagegen die Verläßlichkeit im Sinne von V $2 *$. Im normalen Verständnis ist es unsinnig zu sagen ,Ich weiß nicht, ob mir der Sachverhalt $p$ evident ist", und dazu paßt, daß solche Aussagen nach V 3 kontradiktorisch sind. Nach V $2 *$ ist Evidenz hingegen durchaus problematisch, so daß die fragliche Aussage sinnvoll und in vielen Fällen richtig sein müßte. Nur wenn Evidenz unproblematisch ist, kann sie auch als letzter Berufungsgrund für Überzeugungen dienen, der Begründungen überflüssig macht. In diesem Sinn ist dann auch der Begriff einer Scheinevidenz leer, d. h. man kann sich in den Annahmen über eigene Evidenzen nicht irren.

Descartes geht in den „Meditationes de Prima Philosophia“ von einem Evidenzbegriff im Sinne von V3 aus, versucht dann aber auf dem Wege über die Existenz eines vollkommenen Gottes, der uns nicht täuscht, zu beweisen, daß Evidenz auch verläßlich, daß jede klare und 
distinkte Perzeption richtig ist. Das spricht dafür, daß er einen, wie wir in Analogie zum entsprechenden Wissensbegriff nach D 1.4-1 sagen können, perfekten Evidenzbegriff annimmt, der sowohl V2* wie V 3 erfüllt. Wenn er aber auf Einwände, daß wir uns auch bei Annahmen irren können, die für uns evident sind, antwortet, daß dann eben keine wirkliche Evidenz vorgelegen habe, nimmt er wieder ,Scheinevidenzen" an, gibt also V 3 auf. ${ }^{47}$ Man muß daher sagen, daß Descartes das Wort ,evident" nicht konsistent verwendet. Perfekte Evidenz läßt sich nur für eng umgrenzte Klassen von Sachverhalten in Anspruch nehmen. Als Beispiele können wir auch hier, wie beim Begriff perfekten Wissens, manche analytischen Sachverhalte ansehen wie Sachverhalte, die sich auf einfache eigene Überzeugungen beziehen. Uns kommt es aber hier auf einen allgemein anwendbaren Begriff der Evidenz an, und dafür ist der perfekter Evidenz bei weitem zu eng.

$\mathrm{Daß}$ die Forderungen der Verläßlichkeit und der Problemlosigkeit für den Evidenzbegriff unverträglich sind, hat auch M. Schlick betont. ${ }^{48}$ Zur Darstellung seines Arguments schreiben wir ,evident"*“ (symbolisch V*) für ,,evident im Sinne von V $2 *$ statt V3". Ausgehend vom normalen Evidenzbegriff $V$ kann man setzen $V^{*}(a, p):=V(a, p)$ $\wedge p$; daraus folgt sofort $V 2^{*}$. Es gilt dann $V(a, p) \equiv G\left(a, V^{*}(a, p)\right){ }^{49}$ Evidenzen zerfallen dann in echte und scheinbare Evidenzen*, wobei $V(a, p) \wedge p, d . h . V^{*}(a, p)$, eine echte und $V(a, p) \wedge \neg p$ (oder äquivalent $\left.\mathrm{G}\left(\mathrm{a}, \mathrm{V}^{*}(\mathrm{a}, \mathrm{p})\right) \wedge \neg \mathrm{V}^{*}(\mathrm{a}, \mathrm{p})\right)$ eine scheinbare Evidenz $\mathrm{z}^{*}$ darstellt.

Schlick argumentiert nun so: Bei Evidenzen ist uns der Unterschied zwischen echter und scheinbarer Evidenz* entweder gegeben, oder er ist uns nicht gegeben. Daß dieser Unterschied ,,gegeben“ ist, soll heißen, daß wir feststellen können, ob eine echte Evidenz* vorliegt oder nicht, und daß diese Feststellung problemlos ist, d. h. keiner weiteren Begründung bedarf ( $\mathrm{daß}$ der Unterschied also z. B. in einem in der Evidenz selbst mitgegebenen qualitativen Unterschied echter und scheinbarer Evidenz* besteht). Im ersten Fall, in dem also für V* V3 gelten soll, würden wir die scheinbaren Evidenzen* ohne weiteres als

\footnotetext{
${ }^{47}$ Vgl. dazu die Erwiderung von Descartes auf den 13. Einwand von Hobbes. ${ }^{48} \mathrm{Vgl}$. Schlick (18), S. $129 \mathrm{ff}$.

${ }^{49}$ Aus $V(a, p)$ folgt nach $V 1$ und $V 3 G(a, V(a, p) \wedge p)$, also $G\left(a, V^{*}(a, p)\right)$, und aus $G\left(a, V^{*}(a, p)\right)$ folgt $G(a, V(a, p))$, nach $V 3$ also $V(a, p)$.
} 
solche erkennen, würden uns also diesbezüglich nie irren. Es gäbe also keine Schein-Evidenzen*, weil sie von vornherein als solche erkannt würden. Das widerspricht aber der Erfahrung der Fehlbarkeit unserer mit Evidenz gefällten Urteile. Im zweiten Fall hingegen ist es, wenn uns ein Sachverhalt $p$ evident ist, problematisch, ob eine echte Evidenz* vorliegt oder nicht. D. h. es ist nun die Echtheit der Evidenz* zu begründen. Dann liegt aber keine Evidenz vor im üblichen Sinn eines subjektiven Wahrheitskriteriums, das Begründungen überflüssig macht.

Die Konfusionen, die in Diskussionen des Evidenzproblems häufig auftreten, ergeben sich daraus, daß man sich meist nicht die Mühe solcher begrifflichen Unterscheidungen macht. So wird Evidenz mit scheinbarer Evidenz verwechselt, und perfekte Evidenz mit Evidenz*\% W. Stegmüller hält z. B. dem angegebenen Argument von Schlick entgegen, daß es kontradiktorisch sei: Denn es solle doch zur Evidenz gebracht werden, die sicher keine Scheinevidenz sein solle, daß es echte, von Scheinevidenzen unterscheidbare Evidenzen nicht gebe..$^{50}$ Das Argument von Schlick will aber nur zur Evidenz bringen, daß man V 3 nicht für Evidenzen* fordern kann, daß also die Unterscheidung von echten und scheinbaren Evidenzen* nicht problemlos ist, daß es keine perfekte Evidenz gibt. Eine ,bloße“ Evidenz im Sinne unserer Explikation ist aber keine Scheinevidenz", sondern sie impliziert vielmehr, daß wir allen Grund haben, den evidenten Sachverhalt für wahr zu halten. Widersprüchlich wäre Schlicks Argument nur dann, wenn er zur Evidenz (oder zur Evidenz*) bringen wollte, daß es keine Evidenz* gibt. Das ist aber nicht der Fall.

Für Stegmüller ist das Evidenzproblem, die Frage, ob es Evidenz gibt, unentscheidbar: „Denn alle Argumente für die Evidenz stellen einen circulus vitiosus dar und alle Argumente gegen sie einen Selbstwiderspruch".51 D. h.: Wer die Existenz von Evidenzen begründen will, setzt dabei schon Sachverhalte und Schlußweisen als evident voraus. Wer ihre Nichtexistenz begründen will, setzt ebenfalls Evidenzen voraus. Das letztere ist sicher richtig: Jede Begründung stützt sich auf Sachverhalte, die als evident angesehen werden. Aber dieser Hinweis rechtfertigt die These von der Unentscheidbarkeit des Evidenzproblems noch nicht. Aus ihrer Unbegründbarkeit folgt nicht die Unent-

50 Vgl. Stegmüller (69a), S. 180.

51 Stegmüller (69a), S. 168 f. 
scheidbarkeit der Behauptung, es gebe Evidenzen. Denn eine einzige Evidenz, die ich tatsächlich habe, entscheidet die Frage für mich schon ohne weitere Begründungen und Argumente. Für Stegmüller sind „Evidenzen“ immer Evidenzen”. Ob sie auch problemlos sein sollen, bleibt offen, wenn auch manche Äußerungen darauf hinauszulaufen scheinen. Daß es keine problemlosen Evidenzen* gibt, zeigt Schlicks Argument. Daß es aber Evidenzen* gibt, nehmen wir wegen $\mathrm{V} 1$ immer an, wenn uns etwas evident ist - nach V 1 folgt ja, wie wir sahen, $G\left(a, V^{*}(a, p)\right)$ aus $V(a, p)$. Auch die Behauptung „Es gibt Evidenzen*" ist also durch eine einzige Evidenz gerechtfertigt, die ich habe. Für die These „Alle evidenten Sachverhalte sind falsch" hingegen kann man sicher keine Evidenz beanspruchen, denn daraus würde ein Widerspruch folgen; eine Begründung dafür müßte sich aber, wie Stegmüller betont, auf Evidenzen stützen, würde sie also widerlegen. Rational ist also eine universelle Evidenzskepsis sicher nicht haltbar. Wem freilich nichts evident ist - auch dies nicht - , den kann man durch Argumente nicht überzeugen.

Gegen die Auffassung, unser Wissen beruhe auf Evidenz, hat sich Wittgenstein in (74) gewendet. „Ein inneres Erlebnis kann es mir nicht zeigen, daß ich weiß“" (569). Das heißt aber nur: Die verläßliche Evidenz ist wie Wissen nicht generell problemlos, nicht subjektiv entscheidbar. Mit der Aussage „Es ist mir evident, daß p“ bezeichne ich auch nicht ein ,inneres Erlebnis“, sondern die Tatsache, daß sich meine Überzeugung von $p$ nicht auf Argumente stützt. ${ }^{52}$ - Interessanter ist, daß Wissen für Wittgenstein ein soziales Phänomen ist, das auf einer Lebensform beruht, zu der es gehört, ein System von Annahmen als gültig anzusehen. Dieses System konstituiert erst die Unterscheidung wahr - falsch, und legt die Form von Argumenten fest, insbesondere das, was man ohne Begründung als Prämissen verwenden kann. Er sagt: „Mein Weltbild habe ich nicht, weil ich mich von seiner Richtigkeit überzeugt habe; auch nicht, weil ich von seiner Richtigkeit überzeugt bin. Sondern es ist der überkommene Hintergrund, auf welchem ich zwischen wahr und falsch unterscheide" (94). "Wenn wir anfangen, etwas zu glauben, so nicht einen einzelnen Satz,

52 Ein Satz kann mir aufgrund eines Beweises evident geworden sein. Wenn er mir aber evident ist, bin ich auch dann von seiner Richtigkeit überzeugt, wenn ich in diesem Beweis einen Fehler entdecke, wenn also die Stütze des Beweises wegfällt. 
sondern ein ganzes System von Sätzen“ (141). Dem Einwand, daß ich ja zumindest erkennen muß, wie die Lebensform beschaffen ist, und das System der Annahmen, um im Einklang damit urteilen und argumentieren zu können, will Wittgenstein dadurch entgehen, daß er die Beherrschung eines solchen „Sprachspiels“ als Kompetenz begreift, die nicht auf einer Kenntnis der Regeln beruht, sondern durch Erziehung und Übung vermittelt wird. „Die Begründung aber, die Rechtfertigung der Evidenz kommt zu einem Ende, - das Ende aber ist nicht, daß uns gewisse Sätze unmittelbar als wahr einleuchten, also eine Art Sehen unsererseits, sondern unser Handeln, welches am Grunde des Sprachspiels liegt" (204). Diese Auskunft genügt aber nicht, denn um durch Erziehung und Überzeugung zu lernen, muß ich zumindest feststellen, d. h. erkennen können, was andere mir sagen und ob mein Verhalten von ihnen als korrekt anerkannt wird. Erkenntnis läßt sich nicht auf einsichtsloses Verhalten reduzieren. Richtig ist nur, daß unser Urteilen und Argumentieren erstens nicht voraussetzungslos ist, sondern sich immer schon im Rahmen vorgegebener Überzeugungen bewegt, und daß es zweitens insofern eine soziale Praxis ist, als ein großer Teil unserer Überzeugungen durch andere vermittelt ist und Übereinstimmung mit anderen ein wichtiges, wenn auch keineswegs das einzige Kriterium für die Richtigkeit unserer Urteile ist.

\subsection{Wahrheit}

Die Erörterung erkenntnistheoretischer Fragen erfordert auch eine Verständigung über den Wahrheitsbegriff. Man wendet heute in der Logik das Wort ,wahr" meist auf Sätze, nicht auf Propositionen an. Umgangssprachlich ist es dagegen gebräuchlicher zu sagen: „Es ist

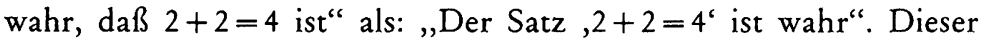
Unterschied ist aber unerheblich, denn man kann von der einen Verwendungsweise zur anderen übergehen: Ein Satz ist wahr genau dann, wenn die Proposition wahr ist, die er ausdrückt.

Der wichtigste Wahrheitsbegriff der traditionellen Philosophie ist der der Adäquationstheorie. Er geht auf Platon und Aristoteles zurück und wird in der philosophischen Tradition etwa so formuliert: „Ein Satz ist wahr genau dann, wenn die Proposition, die er ausdrückt, mit 
der Wirklichkeit übereinstimmt“. Die Kurzformel dafür lautet: veritas est adcequatio intellectus ad rem.

An dieser Bestimmung von Wahrheit ist mit Recht Kritik geübt worden, denn was soll hier ,Übereinstimmung“ heißen? Wann stimmt eine Proposition mit der Wirklichkeit überein? Den Begriff der Wahrheit mit dem der Übereinstimmung zu erklären, bedeutet, ihn durch einen dunkleren Begriff zu ersetzen. Aufgrund dieser Schwierigkeit und anderer vermeintlicher Mängel des Adäquationsbegriffs ${ }^{53}$ hat man diesen Wahrheitsbegriff durch andere zu ersetzen versucht. So will z. B. Franz Brentano die Adäquationstheorie durch eine Evidenztheorie ersetzen, nach der ein Urteil (Satz) genau dann wahr ist, wenn es mit Evidenz gefällt wird, oder wenn jemand, der mit Evidenz über den Gegenstand urteilte, das Urteil akzeptieren würde. ${ }^{54}$ Hier wird (mögliche) Evidenz als hinreichende und notwendige Bedingung für Wahrheit verstanden. Wir haben aber im letzten Abschnitt gesehen, daß Evidenz im üblichen Sinn kein hinreichendes Wahrheitskriterium ist. Es gibt zudem Sachverhalte, die wahr sind, die man aber nicht mit Evidenz beurteilen kann, sondern die nur erschlossen werden können, wie z. B. die Temperatur im Sonnenkern oder Wechselwirkungen zwischen Elementarteilchen. Daher ist Evidenz auch keine notwendige Bedingung für Wahrheit.

${ }^{53}$ Franz Brentano übt z. B. daran Kritik, daß

a) mathematische Sätze, die nichts über reale, sondern nur etwas über ideale Gebilde wie Zahlen, Funktionen, Mengen oder algebraische Strukturen aussagen, nach dieser Definition nicht wahr wären (das gilt aber nur, wenn man den Begriff ,Wirklichkeit“ im Sinn von ,physischer Realität", also in einem sehr engen Sinn versteht);

b) wahre Sätze des Inhalts, daß es ein Objekt a nicht gibt, nur mit nicht existenten Dingen übereinstimmen könnten, die andererseits jedoch real sein müßten, damit von einer Übereinstimmung mit der Wirklichkeit die Rede sein könnte (Propositionen sollen jedoch nicht mit Dingen, sondern mit Tatsachen übereinstimmen, und es ist eine Tatsache, daß es a nicht gibt);

c) nach dieser Definition die Feststellung der Wahrheit eines Satzes auf einen unendlichen Regreß hinauslaufen würde, da man, um die Übereinstimmung einer Proposition p mit der Wirklichkeit festzustellen, schon ein wahres Urteil über die Wirklichkeit formulieren muß (die Adäquationstheorie will aber nur eine Analyse des Wahrheitsbegriffs angeben, nicht ein Wahrheitskriterium).

${ }^{54}$ Vgl. dazu Brentano (30). 
Man hat der Adäquationstheorie auch eine Kobärenztheor der Wahrheit gegenüber gestellt, nach der die Wahrheit von Sätzen nicht durch ihre Übereinstimmung mit der Wirklichkeit erklärt wird, sondern durch ihre (logische) Verträglichkeit miteinander. Da nun sowohl ein Satz A wie seine Negation $\neg$ A mit den Sätzen einer Menge verträglich sein kann, man aber nicht beide als wahr erklären kann, läuft dieser Gedanke darauf hinaus, daß man im Sinne der Konventionstheorie der Wahrheit eine (widerspruchsfreie) Menge von Sätzen kraft Konvention als wahr auszeichnet und dann die Wahrheit anderer Sätze nach Maßgabe ihrer Verträglichkeit mit den bereits als wahr akzeptierten Sätzen durch neuerliche Konventionen festlegt. Eine solche Theorie wurde z. B. im Wiener Kreì vertreten. Wir nennen aber nicht einen Satz wahr, weil wir ihn kraft Konvention akzeptieren, sondern wir akzeptieren ihn, weil wir ihn für wahr halten. Außerdem soll die Frage, ob ein Satz mit anderen verträglich ist, wohl nicht ihrerseits wieder eine Frage der Konvention sein. Und die Frage, ob eine Konvention vorliegt und wie sie lautet, soll wohl auch nicht nur durch Konvention entschieden werden, denn das liefe auf einen unendlichen Regress hinaus. Die Konventionstheorie, und damit auch Kohärenztheorie, setzt also stillschweigend einen anderen Wahrheitsbegriff voraus.

Ein typisches Argument für den Wahrheitskonventionalismus ist dies: Ob ein Satz wahr ist oder nicht hängt von seiner Interpretation ab. Die semantischen Regeln für eine Sprache sind aber Konventionen der Leute, die diese Sprache sprechen, d. h. subjektive Festlegungen. Somit ist auch die Wahrheit eine Sache subjektiver Konventionen. Diese Argumentation ist aber nicht haltbar. Es ist zwar richtig, daß ein Satz, verstanden als ein rein syntaktisch bestimmter Ausdruck, als eine Folge phonetischer oder graphischer Zeichen, keinen Wahrheitswert hat; das ist erst dann der Fall, wenn ihm durch semantische Regeln eine Bedeutung zugeordnet worden ist. Es könnte z. B. sein, daß dasselbe graphische oder phonetische Gebilde in zwei Sprachen als Satz vorkommt, dabei aber in verschiedener Weise gedeutet wird und aufgrund dieser Deutungen verschiedene Wahrheitswerte erhält. Dann würde man aber üblicherweise nicht sagen, es sei derselbe Satz, der einmal wahr, das andere mal aber falsch ist. Denn ein Satz wird im normalen Verständnis durch seine syntaktischen und semantischen Eigenschaften bestimmt. Der entscheidende Punkt ist jedoch, daß einem Satz mit seiner Bedeutung nicht zugleich ein Wahrheitswert zu- 
geordnet wird - es sei denn, er gelte analytisch. Die semantischen Regeln legen vielmehr mit der Bedeutung eines Satzes nur seine Wahrheitsbedingungen fest; über seinen Wahrheitswert, d. h. darüber, ob diese Wahrheitsbedingungen erfüllt sind, entscheiden nicht die semantischen Regeln, sondern Tatsachen. D. h. der Wahrheitswert eines Satzes als eines rein syntaktischen Gebildes hängt von der semantischen Festlegung seiner Wahrheitsbedingungen und von Tatsachen $a b$; der Wahrheitswert eines Satzes im üblichen Sinn des Wortes hängt nur von Tatsachen ab. In jedem Fall wird der Wahrheitswert nicht allein durch Konventionen bestimmt, wenn es sich nicht um analytische Sätze handelt.

Ein anderer Einwand gegen die Adäquationstheorie ist von P. F. Strawson in (49) und (50) vorgebracht worden. Danach ist das Prädikat ,wahr" kein deskriptives Prädikat, sondern es dient umgangssprachlich meist zum Ausdruck der Bestätigung eigener oder fremder Aussagen, wie z. B. in dem Satz: ,Was ich gesagt habe, ist wahr", oder: „Was Fritz gesagt hat, ist wahr“. In diesen Sätzen behaupte ich nichts von Sätzen, sondern bestätige sie. In der deskriptiven Verwendung hingegen besagt der Satz „Der Satz A ist wahr“ nicht mehr als der Satz A selbst; die behauptende Kraft des Satzes liegt nicht im Wort ,wahr", sondern, wie Frege sagt, in der Form des Satzes selbst. Auch Strawsons Gedanke ist aber nicht überzeugend. Zweifellos gibt es nichtdeskriptive Verwendungen des Wortes „,wahr“, daneben hat das Wort aber auch eine wichtige deskriptive Funktion, so daß ein umgangssprachliches Explanandum für eine philosophische Erklärung keineswegs fehlt, wie Strawson meint. Wir teilen die Sätze ja in wahre und falsche in und sagen z. B. ,Sind A und B wahr, so ist auch $A \wedge B$ wahr", ,Alle logischen Folgerungen aus wahren Sätzen sind wahr" usw., und dabei verwenden wir das Wort ,wahr" rein deskriptiv. In erkenntnistheoretischen wie semantischen Untersuchungen ist allein dieser deskriptive Gebrauch relevant.

Die erwähnte Schwierigkeit der traditionellen Formulierung der Adäquationstheorie vermeidet man am besten dadurch, daß man, statt nach der Natur der Wahrheit zu fragen, nach der Bedeutung, bzw. dem Gebrauch des Prädikats ,wahr" fragt. Denn ebenso, wie uns die Frage nach dem Wesen der Röte in Schwierigkeiten bringt, obwohl wir den Sinn des Prädikats ,,rot“" kennen und es korrekt zu gebrauchen wissen, ist die Frage nach dem Wesen der Wahrheit nur eine irre- 
führende Formulierung der Frage nach dem Gebrauch des Prädikats „wahr". Wenn wir nach dem Gebrauch fragen, können wir im Sinne der Adäquationstheorie sagen: Ein Satz A wird wahr genannt genau dann, wenn es sich in Wirklichkeit so verhält, wie A es darstellt. Es verhält sich aber genau dann in Wirklichhkeit so, wie es der Satz A darstellt, wenn der Sachverhalt besteht, den A ausdrückt. D. h. wir können sagen:

WK: Ein Satz ist wabr genau dann, wenn der Sachverbalt besteht, den er ausdrückt.

Danach ist z. B. der Satz „Fritz hat Masern“ wahr genau dann, wenn Fritz Masern hat. Damit ist der Gebrauch des Wortes „wahr" festgelegt, und die Konvention WK ist der einfache Rest der Adäquationstheorie der Wahrheit, wie er von A. Tarski formuliert wurde..$^{55}$

Diese Erklärung des Wahrheitsbegriffes ist weder zirkulär noch trivialisiert sie das Wahrheitsproblem, wie oft behauptet wird. Die Rede vom „Bestehen eines Sachverhalts", ist nicht wieder durch dessen Wahrheit zu erklären, sondern es gilt einfach: der Sachverhalt $\mathrm{p}$ besteht genau dann, wenn p. Man braucht ferner keine besondere Beziehung anzunehmen, die Sätze irgendwie mit der Wirklichkeit verbindet. Behauptungssätze werden gebraucht, um Aussagen über die Wirklichkeit zu machen. Der fragliche Wirklichkeitsbezug liegt also in ihnen selbst, in ihrem behauptenden Charakter. Daher besagt auch der Satz „A ist wahr" nicht mehr als der Satz A selbst, und der Wahrheitsbegriff reduziert sich in der Konvention WK darauf, die Wahrheitsbedingung für A durch A selbst oder einen synonymen Satz zu charakterisieren. Darüber hinaus gibt es keine allgemeine und selbständige Bedeutung des Prädikats ,wahr": Was es heißt, daß A wahr ist, läßt sich nur durch $\mathrm{A}$ oder durch mit $\mathrm{A}$ synonyme Bedingungen erläutern, die von Satz zu Satz anders lauten. ${ }^{56}$

55 Vgl. Tarski (35).

${ }^{56}$ Der Wahrheitsbegriff verhält sich also ähnlich wie der Begriff des Zutreffens einer Eigenschaft auf ein Objekt. Wenn man nach der Beziehung fragt, die zwischen einer Eigenschaft $F$ und einem Gegenstand a besteht, wenn a die Eigenschaft $F$ hat, so kann man nur sagen: $F$ trifft genau dann auf a zu, wenn a ein $F$ ist. Also z. B.: Die Eigenschaft, ein Regensburger zu sein, trifft auf Kuno genau dann zu, wenn Kuno ein Regensburger ist. Die fragliche 
Wir haben schon oben betont, daß die Wahrheitskonvention WK nichi als ein allgemeines Wahrheitskriterium angesehen werden kann, sondern nur der Explikation des Wortes, ,wahr" dient. Ein hinreichendes, bzw. notwendiges Kriterium für das Bestehen eines Sachverhalts $\mathrm{p}$ ist ein von $\mathrm{p}$ verschiedener Sachverhalt $\mathrm{q}$, der nur dann, bzw. immer dann besteht, wenn p besteht, aus dessen Bestehen bzw. Nichtbestehen man also auf $p$, bzw. nicht-p schließen kann. Im üblichen Verständnis des Wortes ist ein Kriterium für das Bestehen von p auch meist ein Sachverhalt, den man unabhängig von $\mathrm{p}$ oder leichter als $\mathrm{p}$ selbst überprüfen kann - so, wie man z. B. durch die Rotfärbung eines Lackmuspapiers leichter erkennen kann, ob eine Säure vorhanden ist, als durch eine chemische Analyse. In der Erkenntnistheorie spielt nun die Frage-eine wichtige Rolle, ob es generelle Wahrheitskriterien gibt, ja man hat in der Angabe eines solchen Wahrheitskriteriums die zentrale Aufgabe der Erkenntnistheorie gesehen.

Ein hinreichendes Kriterium für das Bestehen des Sachverhalts $p$ ist eine Bedingung, die nur dann erfüllt ist, wenn $p$ gilt. Ein allgemeines hinreichendes Wahrheitskriterium wäre eine Funktion $\mathrm{K}$, die jedem Sachverhalt $\mathrm{p}$ einen Sachverhalt K(p) zuordnet, der ein hinreichendes Kriterium für $\mathrm{p}$ darstellt. Es müßte also gelten:

1) $\wedge p(K(p) \supset p)$ - Für alle Sachverhalte $p$ gilt: Besteht der Sachverhalt $\mathrm{K}(\mathrm{p})$, so auch $\mathrm{p}$.

Der Satz (1) drückt aus, daß $\mathrm{K}$ ein allgemeines Kriterium darstellt, ist aber selbst kein solches Kriterium.

$\mathrm{Daß}$ es ein allgemeines Wahrheitskriterium gibt, kann man aber nicht annehmen. Denn Kriterien für das Bestehen von p sehen je nachdem, worauf sich der Sachverhalt $\mathrm{p}$ bezieht, ganz verschieden aus: Ursachen oder Symptome dafür, daß es regnet, sehen anders aus als Ursachen oder Symptome dafür, daß ein Stoff Eisen enthält, und diese wieder anders als Gründe dafür, daß es unendlich viele Primzahlen gibt. Man kann also die Funktion $\mathrm{K}$ sicher nicht so erklären, daß man von einem generellen und einheitlichen Kriterium sprechen könnte.

Ein Grundproblem der Erkenntnistheorie ist die Frage: „Was können wir wissen?". Diese Fragestellung wird oft so verstanden, als sei

Beziehung des Zutreffens einer Eigenschaft $\mathrm{F}$ auf ein Objekt a ist also nicht ein drittes, das a und $F$ verbindet, sondern sie liegt in der pädikativen Natur des Begriffs F selbst. 
es Aufgabe der Erkenntnistheorie, ein allgemeines Kriterium anzugeben, mit dem wir vermeintliches Wissen von echtem Wissen unterscheiden können. Ausgehend von diesem Verständnis hat Leonard Nelson in (08) einen „Beweis für die Unmöglichkeit der Erkenntnistheorie" angegeben. Er schreibt dort: „Die Erkenntnistheorie ist nach allgemeinem Sprachgebrauch - die Wissenschaft, die die Untersuchung der objektiven Gültigkeit der Erkenntnis überhaupt zur Aufgabe hat. Die Stellung dieser Aufgabe setzt voraus, daß man an der objektiven Gültigkeit der Erkenntnis zweifelt, d. h. daß ihr Vorhandensein ein Problem bildet. Ich behaupte nun, daß eine wissenschaftliche Auflösung dieses Problems unmöglich ist" ".57

Nelsons Beweis für diese Behauptung lautet: „Angenommen nämlich, es gäbe ein Kriterium, das zur Auflösung des Problems dienen könnte. Dieses Kriterium würde entweder selbst eine Erkenntnis sein, oder nicht. - Nehmen wir an, das fragliche Kriterium sei eine Erkenntnis. Dann gehörte es gerade dem Bereiche des Problematischen an, über dessen Gültigkeit erst durch die Erkenntnistheorie entschieden werden soll. Das Kriterium, das zur Auflösung des Problems dienen soll, kann also keine Erkenntnis sein. - Nehmen wir also an, das Kriterium sei nicht eine Erkenntnis. Es müßte dann, um zur Auflösung des Problems dienen zu können, bekannt sein; d. h. es müßte selbst Gegenstand der Erkenntnis werden können. Ob aber dieser Erkenntnis, deren Gegenstand das fragliche Kriterium ist, eine gültige ist, müßte entschieden sein, damit das Kriterium anwendbar ist. Zu dieser Entscheidung müßte aber das Kriterium schon angewendet werden. - Eine Begründung der objektiven Gültigkeit der Erkenntnis ist also unmöglich". 58

Nelson fährt dann fort: „Aber vielleicht ließe sich zeigen, daß unserer Erkenntnis teilweise objektive Gültigkeit zukommt und teilweise nicht? Auch dies ist unmöglich. Denn man nehme an, es gäbe ein Kriterium zur Entscheidung, ob eine Erkenntnis ( $z$. B. die fragliche Behauptung von der teilweisen Gütligkeit unserer Erkenntnis) in die Klasse der gültigen oder in die der ungültigen gehört. Dies Kriterium müßte, um anwendbar zu sein, erkannt werden können. Um aber zu wissen, daß diese Erkenntnis des Kriteriums eine gültige ist, müßte ich das Kriterium schon angewendet haben. - Wollte man, um diese

57 Nelson (08), S. 444.

${ }^{58}$ Nelson (08), S. 444. 
Widersprüche zu vermeiden, vorschlagen, zur Prüfung der Erkenntnis des Kriterilims ein nevies, d. h. von ihm verschiedenes Kriteriun anzuwenden, so wäre damit nichts gewonnen. Denn auch dieses Kriterium müßte, um anwendbar zu sein, erkannt werden, und diese Erkenntnis würde, um als gültig angenommen zu werden, wieder ein weiteres Kriterium voraussetzen, so daß wir auf einen unendlichen Regreß geführt wären. Dieser Regreß müßte vollendet vorliegen, ehe irgend eine Erkenntnis als gültig angenommen werden könnte. Die Annahme der Vollendung eines unendlichen Regresses schließt aber einen Widerspruch ein". ${ }^{59}$

Wenn Nelson hier von „Erkenntnis“ spricht, kann nicht schon eine „gültige" Erkenntnis, d.h. ein Wissen gemeint sein, sonst wäre die Frage nach der Gültigkeit von Erkenntnissen nach dem Axiom W 1 gegenstandslos. Also ist unter "Erkenntnis“ eine vermeinte Erkenntnis zu verstehen. Eine vermeinte Erkenntnis, daß ein Sachverhalt $p$ besteht, ist entweder (a) die schlichte Überzeugung, daß p besteht, oder aber (b) die Überzeugung, zu wissen, daß p besteht. ${ }^{60}$. Für einen generellen Wissensbegriff ist das Axiom W 9* höchst plausibel. Danach gilt aber $G(a, p) \equiv G(a, W(a, p))$, so daß wir hier eine einfachere Deutung (a) zugrunde legen können. Nimmt man W9* nicht an, so lassen sich die folgenden Überlegungen für die Deutung (b) entsprechend formulieren. Eine ,gültige Erkenntnis“ ist dann eine wahre Überzeugung, so daß $W_{o}$ der hier verwendete Wissensbegriff ist. Das Wort „Erkenntnis“ wird also von Nelson in recht irreführender Weise verwendet. Wenn im Beweis von ,,der Erkenntnis“ die Rede ist, so ist das universell zu verstehen: Die Erkenntnis ist objektiv gültig, wenn alle vermeinten Erkenntnisse richtig, d.h. wahre Überzeugungen sind.

Nelsons Beweis läßt sich dann so darstellen:

1. Die Fragestellung der Erkenntnistheorie geht davon aus, daß wir uns in unseren Überzeugungen irren können. Aus $G(a, p)$ folgt nicht allgemein $p$, sondern (im Sinn unseres rationalen Glaubensbegriffs)

59 Nelson (08), S. 445.

${ }^{60}$ Vermute ich nur, daß p besteht, so glaube ich es nicht, weiß also nach W6, daß ich nicht weiß, daß p. Bloße Vermutungen sind also keine vermeinten Erkenntnisse. Und vermute ich nur, daß ich weiß, daß p, so weiß ich ebenso, $\mathrm{da} ß$ ich nicht weiß, daß ich $\mathrm{p}$ weiß. Bloße Vermutungen zu wissen sind also ebenfalls keine vermeinten Erkenntnisse. 
nur für eine enge Klasse von Sachverhalten wie solche, die analytisch sind, und solche, die sich auf eigene Überzeugungen beziehen. Für andere Sachverhalte p - die wir hier als ,problematisch“ bezeichnen wollen - ist die Korrektheit der Überzeugungen zu prüfen, kann also nicht vorausgesetzt werden.

2. Es werde nun ein Kriterium $\mathrm{K}(\mathrm{a}, \mathrm{p})$ angegeben, von dem behauptet wird, daß für alle Sachverhalte $\mathrm{p}$ - insbesondere die problematischen - gelte

*) $G(a, p) \wedge K(a, p) \supset p$.

3. Ist nun $K(a, p)$ selbst eine vermeinte Erkenntnis, also nach (a) ein Sachverhalt des Glaubens von a, so ist die Feststellung, ob das Kriterium im Fall von $p$ erfüllt ist, ob also $\mathrm{K}(\mathrm{a}, \mathrm{p})$ gilt, für a problemlos. Problematisch ist dann aber, ob $\left(^{*}\right)$ gilt. Denn $(*)$ gilt dann weder analytisch - sonst wäre ja mit $K(a, p)$ auch p selbst unproblematisch für a (aus $\mathrm{G}(\mathrm{a}, \mathrm{K}(\mathrm{a}, \mathrm{p})$ ) würde $\mathrm{p}$ analytisch folgen) - noch ist (*) ein Sachverhalt des Glaubens.

4. Ist hingegen die Geltung von (*) nicht problematisch, ist also z. B. $\mathrm{K}(\mathrm{a}, \mathrm{p})$ eine Bedingung, aus der $\mathrm{p}$ logisch folgt, so ist die Feststellung, ob $K(a, p)$ gilt, für a nicht einfacher als jene von $p$ selbst. Es ist dann also für problematisches $\mathrm{p}$ auch der Sachverhalt $\mathrm{K}(\mathrm{a}, \mathrm{p})$ problematisch.

Nelsons Ergebnis ist also, daß wir den Anspruch der „,objektiven Gültigkeit der Erkenntnis" nicht legitimieren können. Er verwickelt sich damit nicht in einen Widerspruch: Er behauptet nicht, daß es kein Wissen gib́t, sondern er sagt nur: Wenn man die Gültigkeit von ,Erkenntnissen" generell infrage stellt, so läßt sie sich nicht begründen.

Sein Argument enthält zwei Gedanken, die für die Erkenntnistheorie wichtig sind. Erstens gilt trivialerweise: Wenn kein Wissen vorausgesetzt wird, so läßt sich auch nicht begründen, daß es Wissen gibt. Denn in jeder Begründung ist der begründete Satz nicht besser gesichert als die begründenden Sätze und Schlußweisen. Wenn alles in Zweifel gezogen wird, so fehlt den Begründungen die Basis. Daß man auch in der Erkenntnistheorie nicht alles begründen kann und nicht ohne Voraussetzungen auskommt, ist also klar. Auch die cartesische Zweifelsbetrachtung in den „Meditationen“ ist nicht so zu verstehen, daß dabei alles in Zweifel gezogen wird, sondern Descartes will dabei prüfen, was sich alles sinnvoll bezweifeln läßt. Und dabei zeigt sich, $\mathrm{daß}$ z. B. die Sachverhalte eigenen Überzeugtseins nicht in Zweifel gezogen werden können. Dieser Ansatz kommt deutlich in der Erwiderung auf die Einwände Bourdins zum Ausdruck. 
Das methodische Postulat, alles zu begründen, ist nicht nur unrealisierbar, es ist auch deswegen unsinnig, weil es Sachverhalte gibt, die uns evident sind. Evidenz ist nicht immer verläßlich, aber würde man sie generell in Zweifel ziehen, so wäre aller Argumentation und allen Behauptungen der Boden entzogen. Evidenz ist unser letztes subjektives Wahrheitskriterium, und daher ist der Versuch verfehlt, die Zuverlässigkeit von Evidenz begründen zu wollen.

Der zweite wichtige Gedanke in Nelsons Beweis ist: Ein allgemeines Wissens- oder Wahrheitskriterium kann nicht zugleich verläßlich und problemlos sein. Perfektes Wissen gibt es nur in eng umschriebenen Bereichen. Das zeigen Erfahrungen der Fehlbarkeit unserer Überzeugungen, etwas zu wissen, die leider gar nicht seltenen Entdeckungen, daß sich etwas als falsch erweist, was wir zu wissen glaubten. Diese Erfahrung bildet ja gerade den Anstoß zu der Problemstellung der Erkenntnistheorie, wie sic Nelson versteht, zur Frage nach Kriterien zur Unterscheidung von vermeintlichen und tatsächlichen Erkenntnissen, die sich für einen perfekten Wissensbegriff gar nicht stellen würde.

Aus all dem folgt nicht die Unmöglichkeit der Erkenntnistheorie. Ihre Aufgabe ist es nicht, Wissen erst zu begründen, allgemeine Wahrheits- oder Wissenskriterien anzugeben (über die analytischen Bedingungen hinaus, mit denen wir den Gebrauch dieser Begriffe festlegen) und ihre Zuverlässigkeit zu begründen. Erkenntnistheorie setzt Wissen voraus, sie stützt sich auf Überzeugungen, speziell Evidenzen. Sie beansprucht weder Voraussetzungslosigkeit noch Unfehlbarkeit für ihre Aussagen. Sie will nicht die Existenz von Wissen nachweisen, sondern in ihr bemühen wir uns um eine immanente Erkenntniskritik, eine Selbsterhellung des Erkenntnisvermögens. Auch die Erkenntnistheorie kann nicht die Grenze überwinden, die uns damit gezogen ist, $\mathrm{da} ß$ unsere Überzeugungen unser letztes subjektives Wahrheitskriterium sind, daß wir Erkenntnisansprüche immer nur auf der Basis von Überzeugungen beurteilen können und daß Überzeugungen keine unbezweifelbare Wahrheitsgarantie enthalten. Daß Erkenntnistheorie nicht einem unrealisierbaren und also unvernünftigen Anspruch genügen kann, heißt nicht, daß sie unmöglich wäre. Kant sagt:

„Was ist Wahrheit? Die Namenerklärung der Wahrheit, daß sie nämlich die Übereinstimmung der Erkenntnis mit ihrem Gegenstande sei, wird hier geschenkt, und vorausgesetzt; man verlangt aber zu wissen, welches das allgemeine und sichere Kriterium der Wahrheit einer 
jeden Erkenntnis sei. Es ist schon ein großer und nötiger Beweis der Klugheit oder Einsicht, zu wissen, was man vernünftigerweise fragen solle. Denn, wenn die Frage an sich ungereimt ist, und unnötige Antworten verlangt, so hat sie, außer der Beschämung dessen, der sie aufwirft, bisweilen noch den Nachteil, den unbehutsamen Anhörer derselben zu ungereimten Antworten zu verleiten, und den belachenswerten Anblick zu geben, daß einer (wie die Alten sagten) den Bock melkt, der andere ein Sieb unterhält. Wenn Wahrheit in der Übereinstimmung einer Erkenntnis mit ihrem Gegenstande besteht, so muß dadurch dieser Gegenstand von anderen unterschieden werden; denn eine Erkenntnis ist falsch, wenn sie mit dem Gegenstande, worauf sie bezogen wird, nicht übereinstimmt, ob sie gleich etwas enthält, was wohl von anderen Gegenständen gelten könnte. Nun würde ein allgemeines Kriterium der Wahrheit dasjenige sein, welches von allen Erkenntnissen, ohne Unterschied ihrer Gegenstände gültig wäre. Es ist aber klar, daß, da man bei demselben von allem Inhalt der Erkenntnis (Beziehung auf ihr Objekt) abstrahiert, und Wahrheit gerade diesen Inhalt angeht, es ganz unmöglich und ungereimt sei, nach einem Merkmale der Wahrheit dieses Inhalts der Erkenntnisse zu fragen, und daß also ein hinreichendes, und doch zugleich allgemeines Kennzeichen der Wahrheit unmöglich angegeben werden könne" ${ }^{61}$

\subsection{Skepsis}

Die wichtigste Form der Skepsis ist die Erkenntnisskepsis. In ihrer universellen Form wird sie meist so ausgedrückt:

$\mathrm{SE}_{1}$ : Es gibt kein Wissen - symbolisch $\wedge \mathrm{xp} \neg \mathrm{W}(\mathrm{x}, \mathrm{p})$.

Bei dieser wie den folgenden Formulierungen können wir keinen speziellen Wissensbegriff voraussetzen, „Wissen“ also insbesondere nicht als wahre Überzeugung deuten, da die Skeptiker sich in aller Regel nicht die Mühe machen, den von ihnen vorausgesetzten Begriff des Wissens genauer zu erläutern. Darin liegt ein grundsätzlicher Mangel dieser. Thesen, denn Behauptungen über die Existenz von Wissen lassen sich natürlich erst dann prüfen, wenn man weiß, was

${ }^{61}$ Kant (KrV), $100 \mathrm{f}$. 
„Wissen" bedeuten soll. Wir werden daher die Thesen der Erkenntnisskepsis in all den Deutungen zu prüfen haben, die sich bei Zugrundelegung der in 1.3 und 1.4 besprochenen Wissensbegriffe ergeben. Manches läßt sich aber auch schon aufgrund der allgemeinen Axiome für den Wissensbegriff sagen.

- Oft wird die Behauptung, es gebe kein Wissen, nur auf ein Wissen des Wissens bezogen. Man gesteht dann zu, daß es tatsächlich Wissen geben mag, behauptet aber, daß wir nie wissen, daß wir wissen $\wedge \mathrm{xp} \neg \mathbb{W}(\mathrm{x}, \mathrm{W}(\mathrm{x}, \mathrm{p}))$. Wegen $\mathrm{W} 1$ und $\mathrm{W} 5$ gilt aber $\mathrm{W}(\mathrm{a}, \mathrm{p}) \equiv$ $\mathrm{W}(\mathrm{a}, \mathrm{W}(\mathrm{a}, \mathrm{p}))$, so daß diese Behauptung sich mit $\mathrm{SE}_{1}$ deckt. Gibt es kein Wissen des Wissens, so auch kein Wissen. Daher brauchen wir solche Thesen nicht eigens zu prüfen. ${ }^{62}$

In einer zweiten Formulierung besagt die Erkenntnisskepsis

$\mathrm{SE}_{2}$ : Es ist immer möglich, daß wir uns mit unseren Überzeugungen irren, etwas zu wissen $-\wedge \mathrm{xpM}(\mathrm{G}(\mathrm{x}, \mathrm{W}(\mathrm{a}, \mathrm{p})) \wedge \neg \mathrm{W}(\mathrm{x}, \mathrm{p}))$.

Dabei bedeute „,Mp“ soviel wie ,p ist möglich“.63

Partielle Formen der Erkenntnisskepsis behaupten nicht, Wissen sei für uns auf allen Gebieten unerreichbar, sondern sie leugnen nur, daß es in bestimmten Bereichen Wissen, bzw. unfehlbare Überzeugungen, etwas zu wissen, gibt; daß wir z. B. erkennen können, ob es eine AuBenwelt gibt und wie sie an sich beschaffen ist. Auf solche Thesen gehen wir in späteren Kapiteln ein; hier beschränken wir uns auf die Diskussion universeller Formen der Skepsis.

${ }^{62}$ Die Behauptung, wir wüßten nie, ob wir wissen $-\wedge \mathrm{xp}(\neg \mathrm{W}(\mathrm{x}, \mathrm{W}(\mathrm{x}, \mathrm{p}))$ $\Lambda \neg \mathbb{W}(x, \neg \mathbb{W}(x, p)))-$ ist ebenfalls äquivalent mit $S_{1}$. Denn daraus folgt einerseits die Behauptung, daß wir nie wissen, daß wir wissen, also $\mathrm{SE}_{1}$. Und aus $\mathrm{SE}_{1}$ folgt umgekehrt auch die fragliche Behauptung.

${ }^{63}$ Die Deutung von $\mathrm{SE}_{2}$ als $\wedge \mathrm{xp}(\mathrm{G}(\mathrm{x}, \mathrm{W}(\mathrm{x}, \mathrm{p})) \supset \mathrm{M}-\mathrm{W}(\mathrm{x}, \mathrm{p}))$ ist ohne Interesse. Sachverhalte $G(a, p)$ des Glaubens sind - mit Ausnahme der epistemologisch beweisbaren - kontingent, so daß wegen $W / 6$ auch die entsprechenden Sachverhalte des Wissens möglicherweise falsch sind. Insofern ist diese Behauptung trivial. - Die Behauptung, es gelte auch $\wedge \mathrm{xpM}(\mathrm{G}(\mathrm{a}, \neg \mathrm{W}(\mathrm{a}, \mathrm{p}))$ $\wedge \mathrm{W}(\mathrm{a}, \mathrm{p}))$, wäre stärker, und ist auch nicht haltbar, wenn man W 9* akzeptiert. - Ersetzt man die Rede von objektiver (alethischer) Möglichkeit durch eine Aussage über subjektive Möglichkeit, über ein Für-möglich-Halten (wobei a den Sachverhalt p für möglich hält, wenn gilt $\neg \mathrm{G}(\mathrm{a}, \neg \mathrm{p})$ ), so lautet die Behauptung: Wir können nie sicher sein, daß wir wissen - 
Die klassische Argumentation für eine Erkenntnisskepsis innerhalb der neuzeitlichen Philosophie ist die von David Hume. ${ }^{64}$ Sie läßt sich so zusammenfassen: Hume geht davon aus, daß unser empirisches Wissen auf Beobachtungen und Kausalschlüssen beruht. Bei Beobachtungen können wir uns aber irren, wie Fälle von Sinnestäuschungen zeigen. Und Kausalschlüsse stützen sich auf Kausalgesetze. Aussagen über das Bestehen von Kausalgesetzen haben aber immer nur hypothetischen Charakter; sie lassen sich nicht vollständig durch Beobachtungen verifizieren. Es ist also nicht auszuschließen, daß wir uns in unseren empirischen Annahmen irren. Es gibt daher kein Tatsachenwissen, keine definitive empirische Erkenntnis.

Nach Hume können wir uns aber auch in unseren logisch-mathematischen Urteilen irren. Denn sie stützen sich auf Beweise. Daß ein Beweis korrekt ist, ist jedoch eine empirische Aussage. Ein Beweis ist eine Folge von Sätzen, die wir z. B. auf ein Blatt Papier schreiben. Daß die Sätze, die wir dabei als Axiome verwenden, tatsächlich mit den mathematischen Axiomen übereinstimmen, wie sie etwa in einem Lehrbuch stehen, und daß wir bei den Übergängen von Sätzen zu anderen die logischen Schlußregeln korrekt angewendet haben, ist eine Annahme, die wir nur durch Überprüfung der Zeichenreihen kontrollieren können. Wenn wir den Beweis nicht auf dem Papier, sondern im Kopf geführt haben, so verlassen wir uns auf unsere Erinnerung, wenn wir behaupten, er sei korrekt. Logisch-mathematisches Wissen gibt es also nur, wenn es Tatsachenwissen gibt. Das gibt es aber, wie gezeigt wurde, nicht. Also gibt es auch keine logisch-mathematische Erkenntnis.

$\wedge \mathrm{xp} \neg \mathrm{G}(\mathrm{x}, \mathrm{W}(\mathrm{x}, \mathrm{p}))$. Das ist aber falsch, da es tatsächlich Leute gibt, die etwas zu wissen glauben. $D a$ aus $G 5$ und $G 6$ folgt $G(a, G(a, p) \supset p)$, gilt auch $\wedge \mathrm{xpG}(\mathrm{x}, \mathrm{G}(\mathrm{x}, \mathrm{W}(\mathrm{x}, \mathrm{p})) \supset \mathrm{W}(\mathrm{x}, \mathrm{p}))$ - jedermann glaubt, daß er all das tatsächlich weiß, was er zu wissen glaubt. - Im Fall von $\mathrm{SE}_{1}$ ergibt die Abschwächung zur Behauptung objektiver Möglichkeit den Satz $\wedge \mathrm{xp} M-\mathrm{W}(\mathrm{x}, \mathrm{p})$, der für analytische Sachverhalte falsch, im übrigen aber wegen W 6 und der Kontingenz der Glaubenssachverhalte trivial ist. Die Abschwächung von SE $E_{1}$ zur Behauptung subjektiver Möglichkeit ergäbe wieder die faktisch falsche Behauptung $\wedge \mathrm{xp} \neg \mathrm{G}(\mathrm{x}, \mathrm{W}(\mathrm{x}, \mathrm{p}))$. - Es bieten sich also neben $\mathrm{SE}_{1}$ und $\mathrm{SE}_{2}$ keine weiteren für die Diskussion relevanten Formulierungen einer universellen Erkenntnisskepsis an.

${ }^{64} \mathrm{Vgl}$. dazu insbesondere Hume (T) Buch 1, Teil IV, Abschn. 1 und 2, sowie (EHU), Abschn. XII. 
Neben dieses allgemeine Argument treten bei Hume eine Reihe von partiellen skeptischen Thesen, die sich z. B. auf Induktionsschlüsse, die Existenz einer bewußtseinsunabhängigen Außenwelt und die personale Identität beziehen, und die dem Skeptizismus Humes seinen konkreten Inhalt und seine praktische Relevanz verleihen. Auf diese Argumente wollen wir aber hier, wo es um den universellen Skeptizismus geht, nicht eingehen.

Hume begründet seine Skepsis also mit der Fehlbarkeit unserer Überzeugungen, speziell mit der Fehlbarkeit von Überzeugungen, etwas zu wissen. Daß bloße Überzeugungen fehlbar sind, ist trivial. Es ist eine schlichte Folge der Tatsache, daß die Implikation $G(a, p) \supset p$ nicht notwendigerweise für alle Sachverhalte $\mathrm{p}$ und Personen a gilt; daß Überzeugungen nicht ihrem Begriff nach, oder aus naturgesetzlichen Gründen, unfehlbar sind. Und daß es darüber hinaus auch tatsächlich Irrtümer gibt, ist eine ebenso schlichte Erfahrungstatsache. Beides begründet noch keinen Skeptizismus.

Von einer Fehlbarkeit von Überzeugungen, etwas zu wissen, kann man sowohl dann sprechen, wenn $\mathrm{SE}_{2}$ gilt, als auch dann, wenn gilt: a) $\operatorname{Vxp}(\mathrm{G}(\mathrm{x}, \mathrm{W}(\mathrm{x}, \mathrm{p})) \wedge \neg \mathrm{W}(\mathrm{x}, \mathrm{p}))-$ Es gibt falsche Überzeugungen etwas zu wissen..$^{65}$

Auch (a) ist keine spektische These, sondern eine Feststellung, die jeder akzeptieren wird. Denn wir alle haben wohl schon einmal geglaubt, etwas zu wissen, was sich später als falsch erwiesen hat, nach W 1 also nicht Inhalt eines Wissens sein konnte. Humes Argument zielt denn auch auf die Aussage $\mathrm{SE}_{2}$ ab. Er will zeigen, daß in allen Bereich Annahmen, etwas zu wissen, irrig sein können.

Aus (a) wie aus $\mathrm{SE}_{2}$ folgt, daß W 10* nicht gilt, daß also der in diesen Aussagen verwendete Begriff des Wissens kein Begriff perfekten Wissens ist. Da nun (a) im normalen Sinn von ,wissen" richtig ist, ist der normale Wissensbegriff kein Begriff perfekten Wissens. Das haben wir schon in 1.4 betont. Diese Feststellung kann man aber nicht so ausdrücken:

${ }^{65}$ Aus (a) folgt nicht $\mathrm{SE}_{2}$, und aus $\mathrm{SE}_{2}$ nicht (a). Gibt es Irrtümer bei manchen Überzeugungen, etwas zu wissen, so folgt daraus nicht, daß jede Überzeugung etwas zu wissen, falsch sein kann. Und kann jede Überzeugung etwas zu wissen, falsch sein, so folgt daraus noch nicht, daß einige solche Überzeugungen tatsächlich falsch sind. 
b) Es gibt kein perfektes Wissen.

Denn das wird man so verstehen: Es gibt keinen Fall, in dem jemand etwas zu wissen glaubt, und sich mit dieser Annahme unmöglich irren kann. ${ }^{66}$ Aus $\mathrm{SE}_{2}$ folgt nun aber, daß (b) gilt, und darin besteht die erkenntnistheoretische Relevanz von $\mathrm{SE}_{2} \cdot{ }^{67}$

Wir haben in 1.4 zwei Klassen von Sachverhalten angegeben, von denen es perfektes Wissen gibt: die Menge der analytischen Sachverhalte und die Sachverhalte, die sich auf eigene Überzeugungen beziehen. ${ }^{68}$ Diese Aussagen ergaben sich aus unserem rationalen Glaubensund Wissensbegriff. Bei einer Prüfung der Frage, ob es entgegen der These (b) perfekte Erkenntnisse analytischer Sachverhalte gibt, wird man nun nicht solche rationalen Begriffe voraussetzen können, da das z. B. auch Hume nicht tut. Bezieht man sich aber auf den deskriptiven Glaubensbegriff, so kann man kein perfektes Wissen aller analytischen Sachverhalte annehmen. Es hat Überzeugungen, auch begründete Überzeugungen ausgezeichneter Logiker oder Mathematiker gegeben, die sich als falsch erwiesen haben, ${ }^{69}$ von kleineren Geistern ganz zu schweigen. Die These einer perfekten Erkenntnis im gesamten Bereich analytischer Sätze ist also nicht haltbar. Geht man von natürlichen Sprachen und nicht von Kunstsprachen aus, so ist ferner zu

${ }^{66}$ Das wäre so dazustellen $\neg \operatorname{Vxp}(G(x, W(x, p)) \wedge N(G(x, W(x, p)) \supset W(x, p)))$. Wegen W 5 , W 6 gilt allgemein $W(a, p) \supset G(a, W(a, p))$. Daher ist die Behauptung äquivalent mit $\neg \operatorname{Vxp}(W(x, p) \wedge N(G(x, W(x, p)) \supset W(x, p)))$. - Eine andere Deutung von (b) wäre: Es gibt keinen Begriff W des Wissens, der nicht leer ist (für den also gilt $V_{x p W}(x, p)$ ) und W 10* erfüllt. Die folgende Kritik an (b) in der ersten, hier zugrundegelegten Deutung beinhaltet auch eine Kritik an dieser These in der zweiten Deutung. Daher beschränken wir uns auf jene.

${ }^{67}$ (b) folgt nicht aus der Negation von W 10*. Die besagt ja nur, daß es Fälle gibt, in denen kein perfektes Wissen vorliegt.

${ }^{68}$ Daneben sind auch sonstige eigene doxastische Einstellungen wie Wahrscheinlichkeiten, oder Vermutungen problemlos, so daß es auch von ihnen perfekte Erkenntnis gibt.

${ }^{69}$ Ein prominentes Beispiel ist etwa Freges Beweis, daß alle Sätze seiner mengentheoretischen Sprache genau einen Wahrheitswert haben. Vgl. (GGA) Bd. $1, \mathbb{1} 31 . \mathrm{Zu}$ den Fehlern bedeutender Mahematiker vgl. die zahlreichen Belege in Lecat (35). 
betonen, daß wir uns häufig nicht einmal darüber klar sind, ob ein bestimmter Satz, wie z. B. ,Alle Körper sind ausgedehnt", als analytisch anzusprechen ist oder nicht. ${ }^{70}$ Es besteht jedoch ein erheblicher Unterschied zwischen der Behauptung, alle analytischen Sachverhalte seien perfekt erkennbar und der These, kein solcher Sachverhalt sei perfekt erkennbar. Von einer einfachen Tautologie wie z. B. ,Wenn p,

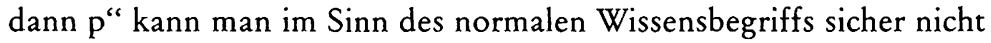
behaupten, jemand wüßte nicht, daß sie gilt, wenn er überzeugt ist zu wissen, daß sie gilt. Aus zu wissen glauben folgt glauben; glauben, $\mathrm{da} \beta \mathrm{p}$, setzt aber voraus, daß man weiß, was für ein Sachverhalt $\mathrm{p}$ ist; wissen, welchen Sachverhalt eine elementare Tautologie darstellt, heißt aber wissen, daß sie wahr ist. Man wird also auch dann, wenn man nicht schon von einem rationalen Wissensbegriff ausgeht, die perfekte Erkennbarkeit gewisser elementarer analytischer Sachverhalte kaum leugnen können.

Die Bedingungen G 5 und G6 drücken auch Eigenschaften des deskriptiven Glaubensbegriffs aus. Daher ist es nicht möglich, daß eine Person a nicht perfekt erkennt, ob sie glaubt, daß ein Sachverhalt $\mathrm{P}$ besteht. Für den deskriptiven Glaubensbegriff ist es hingegen denkbar, daß a verschiedenes glaubt, bzw. nicht glaubt, ohne die Richtigkeit eines komplexen Satzes zu glauben, der aus diesen Sachverhalten des Glaubens folgt. Auch hier wird man also, wenn man nicht von einem rationalen Glaubensbegriff ausgeht, die Grenzen des perfekt Erkennbaren erheblich enger ziehen müssen, ohne aber zu leugnen, daß in einfachen Fällen perfekte Erkenntnis möglich ist.

Die These (b), und damit auch $\mathrm{SE}_{2}$, ist also nicht haltbar, obwohl es richtig ist, daß es perfekte Erkenntnisse nur in eng begrenzten Bereichen gibt.

Wie steht es nun mit der These $\mathrm{SE}_{1}$ ? Versteht man den dabei verwendeten Wissensbegriff im Sinn eines perfekten Wissens, so deckt sie sich mit (b), ist also, wie wir sahen, falsch.

Versteht man im Sinne von D1.3-1 unter ,Wissen“ wahre Überzeugung, so ist $\mathrm{SE}_{1}$ äquivalent mit der Behauptung

c) Alle Überzeugungen sind falsch $-\wedge \mathrm{xp}(\mathrm{G}(\mathrm{x}, \mathrm{p}) \supset \neg \mathrm{p})$.

$70 \mathrm{Vgl}$. den Abschnitt 9.1. 
Das ist nun schlicht absurd..$^{71} \mathrm{Da}$ verschiedene Leute oft Gegenteiliges glauben, und da auch ich heute von einigen Sachverhalten überzeugt bin, von deren Falschheit ich früher überzeugt war, ergäben sich aus (c) Widersprüche; denn eine der beiden Alternativen muß ja gelten. Zudem sind nach $G 5, G 6$ die Sätze $G(a, G(a, p)) \supset G(a, p))$ analytisch wahr. Endlich könnte man von (c) nicht überzeugt sein, ohne in einen Selbstwiderspruch zu geraten. Denn glaubt a, daß (c) gilt, so weiß er nach $\mathrm{W} 8^{*}$, daß er das glaubt. Ja man kann konsistenterweise nicht einmal vermuten, daß (c) richtig ist, denn aus ,,a vermutet, daß $p^{\prime \prime}$ folgt ebenso ,a weiß, daß a vermutet, daß p“. Es lohnt also nicht, diese These eingehender zu erörtern. Sie ist auch nie ernsthaft als skeptische These vorgetragen worden. Es gibt also Wissen im Sinne wahrer Überzeugung.

Wird die These $\mathrm{SE}_{1}$ mit einem Wissensbegriff nach $\mathrm{D} 1.3-2$, einem Begriff fundierten Wissens formuliert, so ist sie äquivalent mit der Behauptung:

d) Alle fundierten Annabmen sind falsch $-\wedge \mathrm{xp}(\mathrm{G}(\mathrm{x}, \mathrm{p}) \wedge \mathrm{F}(\mathrm{x}, \mathrm{p}) \supset$ $\neg \mathrm{p}$ ).

Das ist nun ebenso unplausibel wie (c), denn fundiert sollten ja gerade solche Überzeugungen sein, die einen Wissensanspruch legitimieren. Würde also (d) für einen Fundiertheitsbegriff $F$ gelten, so wäre das nur ein Anlaß, ihn als unbrauchbar aufzugeben. Nach allen in 1.3 diskutierten inhaltlichen Bestimmungen der Fundiertheit ist (d) aber falsch. Im übrigen reicht auch hier ein Hinweis auf die Fallibilität unserer Überzeugungen - sei es im Sinn von $\mathrm{SE}_{2}$ oder von (a) - nicht aus, eine solche Behauptung wie (d) zu rechtfertigen.

Plausibel ist also die These $\mathrm{SE}_{1}$ nur, wenn man von einem Begriff perfekten Wissens ausgeht - plausibel, da es perfektes Wissen nur in eng begrenzten Bereichen gibt, aber nicht richtig, da es eben zumindest in einigen Fällen doch perfektes Wissen gibt. Dieses Verständnis von ,wissen" wird auch - meist stillschweigend - von den Verfechtern dieser These in aller Regel vorausgesetzt. Es war insbesondere das Ideal notwendigen Wissens, das zum Skeptizismus Anlaß gab. Es

"Wir lassen hier wie oben und im folgenden der Einfachheit halber die Generalisierung über die Zeitmomente weg (vgl. dazu die Bemerkung im Abschnitt 1.1). Die These $\wedge \operatorname{xpt}\left(G_{t}(x, p) \supset \neg p\right)-$ Was immer irgendwer jemals geglaubt hat, glaubt oder glauben wird, ist falsch - ist noch absurder als (c). 
war das Ideal eines Wissens, bei dem die Überzeugung zu wissen jede Möglichkeit des Irrtums ausschließt, das die Skepsis auf den Plan ru-. fen mußte. Indem sie diesen Begriff des Wissens als den Wissensbegriff beibehielt, gab sie sich den Anschein weit größerer Bedeutung, als sie ihr zukommt. Heute ist unser Erkenntnisbegriff weitgehend von den empirischen Naturwissenschaften her bestimmt, und da ist es uns ganz geläufig, daß unsere Wissensansprüche fehlbar sind, daß auch gut begründete Theorien sich als falsch erweisen können. Da wir also nicht mehr von dem Ideal notwendiger oder perfekter Erkenntnis ausgehen, hat die Diskussion skeptischer Thesen für uns heute nicht mehr dieselbe Bedeutung wie früher.

Man könnte nun sagen: Die Einsicht in die Fallibilität unserer Überzeugungen in weiten Bereichen muß doch bewirken, daß wir unsere Annahmen in diesen Bereichen nicht mehr als sicher ansehen werden, sondern nur als mehr oder minder wahrscheinlich. Überzeugt sein können wir nicht mehr, wenn wir wissen, daß wir uns irren können. Die Einsicht, daß es falsche Überzeugungen gibt, ist aber kein besserer Grund, von nichts mehr überzeugt zu sein, als die Erkenntnis, daß es faule Eier gibt, für den Entschluß, keine Eier mehr zu kaufen. Die Einsicht, daß wir uns mit unseren Überzeugungen irren können, legt nur eine gewisse Vorsicht bei unseren Annahmen nahe, Gründlichkeit bei ihrer Überprüfung und Strenge in den Anforderungen an das, was wir als evident oder als begründet gelten lassen. Die Erfahrung, daß manches, was wir annahmen, sich hinterher als falsch erwiesen hat, und die Erwartung, daß auch einiges, was wir jetzt annehmen, sich in Zukunft als falsch erweisen wird, ist kein Grund, eine bestimmte oder gar alle unsere gegenwärtigen Annahmen in Zweifel zu ziehen. Ein Zweifel ist erst dann sinnvoll, wenn er begründet ist, und wenn wir von der Richtigkeit der Gründe überzeugt sind. In diesem Sinn sagt L. Wittgenstein in (74): „,Wer an allem zweifeln wollte, der würde auch nicht bis zum Zweifel kommen. Das Spiel des Zweifelns selbst setzt schon Gewißheit voraus" (115).

Impliziert denn aber Überzeugtsein nicht, die Falschheit dessen, wovon wir überzeugt sind, für nicht möglich $\mathrm{zu}$ halten? Und folgt daraus nicht, daß wir nur von Sachverhalten überzeugt sein, sie also nach W6 auch nur wissen können, die wir für notwendig halten? Dann könnten wir aber nur in wenigen Fällen Wissen beanspruchen.

Dieses Argument beruht auf einer Äquivokation. Im doxastischen, subjektiven Sinn heißt ,,p für möglich halten“ soviel wie „,nicht glau- 
ben, daß nicht-p gilt“. Die Person hält also in diesem Sinn nicht-p für nicht möglich, wenn gilt $G(a, p) .^{72}$ In diesem Sinn gilt also trivialerweise: Wenn ich glaube, daß p, so halte ich nicht-p für nicht möglich. Im alethischen, objektiven Sinn von ,möglich“ heißt jedoch ,,p für möglich halten" soviel wie ,glauben, daß p möglich ist". In diesem Sinn hält a nicht-p für nicht möglich, wenn gilt $G(a, \neg M \neg p)$, oder äquivalent - ,p ist möglich" heißt ja soviel wie ,es ist nicht notwendig, daß $\neg \mathrm{p}$ " $-\mathrm{G}(\mathrm{a}, \mathrm{Np})$. Die beiden Deutungen des Ausdrucks ,, für möglich halten" sind inhaltlich ganz verschieden; weder folgt $\mathrm{G}(\mathrm{a}, \mathrm{Mp})$ aus $\neg \mathrm{G}(\mathrm{a}, \neg \mathrm{p})$, noch gilt das Umgekehrte. Ich kann es z. B. subjektiv für möglich halten, daß es Telepathie gibt, ohne bzgl. ihrer naturgesetzlichen Möglichkeit oder Unmöglichkeit feste Überzeugungen zu haben. Und umgekehrt kann man von der Möglichkeit von $\mathrm{p}$ überzeugt sein und $\mathrm{p}$ doch für falsch halten. So sind wir davon überzeugt, daß die Erde kleiner ist als die Sonne, halten aber das Umgekehrte für möglich, da wir diese Tatsache als kontingent ansehen. Insbesondere folgt aus $G(a, p)$ - a hält nicht-p im subjektiven Sinn für nicht möglich - nicht $\mathrm{G}(\mathrm{a}, \mathrm{Np})$ - a hält $\neg \mathrm{p}$ (im objektiven Sinn) für nicht möglich. ${ }^{3}$

In der Verwechslung beider Deutungen von „, für möglich halten“ kann man auch ein Motiv für das Ideal notwendiger Erkenntnis sehen: Würde gelten $G(a, p) \supset G(a, N p)$, so könnten wir nach W 6 nur das wissen, was wir als notwendig ansehen.

${ }^{72}$ Man beachte: Im Ausdruck ,,a hält p für möglich“ kann man an drei Stellen ein Negationszeichen setzen: ,,a hält nicht-p für möglich“, ,,a hält p nicht für möglich“ (d. h. ,Es ist nicht der Fall, daß a p für möglich hält" $)$, ,, a hält p für nicht möglich" (d. h. ,,a hält p für unmöglich“). Bei der subjektiven Deutung besagen die letzten beiden Aussagen dasselbe, nicht hingegen bei der folgenden objektiven.

${ }^{73} \mathrm{Vgl}$. dazu auch Lenzen (80a). Lenzen zeigt dort, daß auch Wittgenstein in (74) diesem Fehlschluß erlegen ist. Hier zeigt sich, daß die Idee notwendigen oder perfekten Wissens auch in der gegenwärtigen Philosophie noch wirksam ist. Ein zweites Beispiel bilden Poppers wiederholte Aussagen, alle Hypothesen und Theorien könnten sich als falsch erweisen, also könnten wir nicht wissen, daß sie richtig sind. Dieses ,also“ ist nur dann korrekt, wenn man von einem Begriff perfekten Wissens ausgeht. 
Wir können also vom Bestehen eines Sachverhalts überzeugt sein, zugleich aber auch davon, daß wir uns mit dieser Annahme irren können: Die Aussage $G(a, p) \wedge G(a, M \neg p)$ ist nicht kontradiktorisch. Es gilt zwar epistemologisch der Satz $G(a, G(a, p) \supset p)$ - Wir sind überzeugt, daß alle unsere (gegenwärtigen) Überzeugungen richtig $\operatorname{sind}^{74}$ -; also auch $\mathrm{NG}(\mathrm{a}, \mathrm{G}(\mathrm{a}, \mathrm{p}) \supset \mathrm{p})$, aber nicht der Satz $\mathrm{G}(\mathrm{a}, \mathrm{N}(\mathrm{G}(\mathrm{a}, \mathrm{p}) \supset \mathrm{p}))$ - Wir glauben, daß ein Irrtum in unseren (gegenwärtigen) Überzeugungen unmöglich ist. Die Überzeugung, daß Irrtümer möglich sind - $\mathrm{G}(\mathrm{a}, \mathrm{M}(\mathrm{G}(\mathrm{a}, \mathrm{p}) \wedge \neg \mathrm{p}))$ - impliziert also nicht die Überzeugung, daß einige unserer Überzeugungen falsch sind. Wenn wir auch die Richtigkeit unserer (gegenwärtigen) Überzeugungen als kontingent ansehen, so halten wir sie doch für eine Tatsache. Wir halten es also für möglich, daß unsere (gegenwärtigen) Überzeugungen falsch sind. Das gilt aber nur im objektiven Sinn von ,für möglich halten", d. h. im Sinn von $G(a, M(G(a, p) \wedge \neg p))$. Im subjektiven Sinn von $\neg \mathrm{G}(\mathrm{a}, \neg(\mathrm{G}(\mathrm{a}, \mathrm{p}) \wedge \neg \mathrm{p}))$ ist die Behauptung jedoch falsch.

Argumente gegen $\mathrm{SE}_{1}$ begegnen nun dem Einwand, daß sie ein Wissen von den Gründen, die sie gegen diese These anführen, beanspruchen, damit aber gerade das voraussetzen, was der Skeptiker leugriet. In diesem Sinn sagt W. Stegmüller, die These $\mathrm{SE}_{1}$ sei einerseits unwiderlegbar, sofern sie nicht den Anspruch erhebe, selbst eine Erkenntnis darzustellen, andererseits aber auch unbegründbar. ${ }^{75}$ Denn jeder Begründungsversuch führe zu einem Selbstwiderspruch, jeder Widerlegungsversuch laufe aber auf eine petitio principii hinaus. Nun ist natürlich jede These mit Argumenten unangreifbar, die Argumente nicht gelten läßt. Das heißt aber nicht, daß sie vernünftig wäre. Der Skeptiker, der nichts zu wissen behauptet, sagt damit auch, daß er nicht weiß, was seine These bedeutet, und daß wir das ebenfalls nicht wissen können. Man wird aber kaum eine Behauptung ernst nehmen können, von der der Proponent zugesteht, keiner wisse, was sie beinhaltet. Im übrigen gilt im Sinn von „Wissen“ als wahrer Überzeugung, daß eine einzige Überzeugung, die ich habe, eo ipso die Überzeugung ist, dies zu wissen, also die Überzeugung, daß die

${ }^{74}$ Gilt $\neg G(a, p)$, so nach $G 6 G(a, \neg G(a, p))$, also $G(a, G(a, p) \supset p)$. Und gilt $G(a, p)$, so gilt $G(a, G(a, p) \supset p)$ ebenfalls.

${ }^{75} \mathrm{Vgl}$. Stegmüller (69a), S. $39 \mathrm{f}$. 
These $\mathrm{SE}_{1}$ falsch ist. Für jeden, der irgend etwas glaubt, ist sie also ohne weitere Argumente evidentermaßen falsch. ${ }^{76}$

Neben der Erkenntnisskepsis haben wir noch drei weitere Formen erkenntnistheoretischer Skepsis zu erörtern, die jedoch eine weit geringere Rolle spielen. Wir betrachten wieder nur universelle Formulierungen dieser Thesen.

Die Evidenzskepsis besagt:

SV: Es gibt keine (korrekten) Evidenzen,

und impliziert damit, da jede Begründung sich auf evidente erste Prämissen stützen muß, daß es weder ein unmittelbares noch ein mittelbares Wissen gibt.

Dazu ist Analoges zu sagen wie im Fall der These $\mathrm{SE}_{1}$, und es ist auch schon in 1.5 gesagt worden. Wegen der Fehlbarkeit auch der Evidenz ist es richtig, daß der normale Evidenzbegriff nicht der einer perfekten Evidenz ist, falsch dagegen, daß es keine perfekten Evidenzen gibt, und erst recht, daß es keine (korrekten) Evidenzen im normalen Sinn gibt. $\mathrm{Da}$ uns gewisse Sachverhalte evident sind, ist einfach eine empirische Tatsache, ebenso wie die, daß wir glauben, daß manche Sachverhalte wahr sind; daß sie bestehen, nehmen wir dabei auch immer an, und wegen V1 haben wir keinen Grund, daran zu zweifeln.

Wabrheitsskepsis drückt sich in der These aus:

SW: Es gibt keine Wabrheit.

Soll das nicht soviel heißen wie „Die Wahrheit ist für uns nicht erkennbar", d. h. soviel wie $\mathrm{SE}_{1}$, so besagt die These entweder ,Alle

${ }^{76}$ Eine Widerlegung der skeptischen These ist für den Skeptiker selbst sicher nicht akzeptabel. Jede dabei verwendete Prämisse ist ihm problematisch. Das heißt aber nicht, daß die Widerlegung tatsächlich unkorrekt wäre oder einen Zirkel enthielte. Der Schluß ,G(a,p), also $W(a, G(a, p))$, also $\neg \wedge \mathrm{px} \neg \mathrm{W}(\mathrm{x}, \mathrm{p})$ “ ist im normalen Sinn sicher keine petitio principii. Begründungen für einen in Frage gestellten Satz wären überhaupt unmöglich, wenn mit dem Satz generell alle Prämissen infrage gestellt werden müßten, aus denen er folgt. Es scheitern also Versuche, den Skeptiker zu überzeugen, nicht aber Widerlegungen seiner These. 
Sätze sind falsch", was offenbar absurd ist. Denn ist A ein falscher Satz, so ist $\neg$ A ein wahrer Saiz. Gibt es also falsche Sätze, so auch wahre. Da zudem diese Behauptung wohl wahr sein soll, widerspricht sie sich selbst. Oder die These SW ist im Sinn eines Wabrbeitsrelativismus zu verstehen, nach dem Wahrheit und Falschheit nicht den Sachverhalten selbst zukommen. Wahrheit ist dann entweder Sache der Konvention - gegen diese Auffassung hatten wir uns aber schon im Abschnitt 1.6 gewendet - oder Wahrsein fällt mit Für-Wahr-gehalten werden zusammen. Eine solche Auffassung wird oft aus dem Satz des

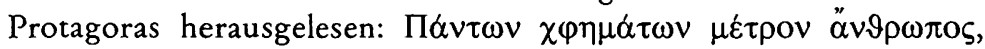

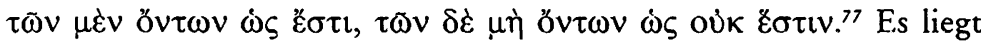
aber wohl näher, diesen Satz im Sinn eines Wahrheitskonventionalismus zu verstehen.

Wir können eine entsprechende These so formulieren:

WR: Wabr sind Sachverhalte nur für Personen; „der Sachverhalt $p$ ist wabr für die Person a" bedeutet, daß a davon überzeugt ist, daß $p$ besteht.

Die Auffassung, das Prädikat „wahr“ drücke keine Eigenschaft von Sachverhalten aus, sondern eine Beziehung zwischen ihnen und Personen, widerspricht nicht der Tatsache, daß das Wort ,wahr" syntaktisch wie ein einstelliges Prädikat verwendet wird. Es gibt auch andere Fälle, in denen Wörter oberflächensyntaktisch von anderer Kategorie sind als tiefensyntaktisch. So wird z. B. auch das Wort ,vermutlich“ wie ein einstelliges Satzprädikat verwendet, obwohl es so viel bedeutet wie „Ich vermute, daß ...", d. h. eine Relation zwischen dem Sprecher und einer Proposition ausdrückt. Gegen WR ist aber einzuwenden, daß diese Definition von ,,wahr" ebenso wie die Konventionstheorie der Wahrheit entweder einen anderen Wahrheitsbegriff voraussetzt oder zu einem unendlichen Regreß führt. Das ergibt sich daraus, daß ,glauben, daß p besteht" gleichbedeutend ist mit ,glauben, daß p wahr ist". Versteht man nur ,wahr" im Kontext von Glaubenssätzen im Sinne der Wahrheitskonvention WK aus 1.6, so verwendet man neben dem relativen auch den normalen Wahrheitsbegriff. Die Behauptung ist dann also falsch. Versteht man das Wort

7 Diels (51) Bd. 2, 263. - „Der Mensch ist das Maß von allem, des Seienden, $\mathrm{da}$ (wie) es ist, des Nichtseienden, daß (wie) es nicht ist". 
dagegen im Sinn von WR, so bedeutet ,glauben, daß p wahr ist" soviel wie ,glauben, daß man glaubt, daß p wahr ist", und das soviel wie „glauben, daß man glaubt, daß man glaubt, daß p wahr ist", usf. Der normale Wahrheitsbegriff ist auch deswegen nicht entbehrlich, weil sich mit Behauptungen, die nicht durch ,vermutlich“, ,,wahrscheinlich" etc. eingeschränkt werden, immer der Anspruch verbindet, der behauptete Satz sei wahr im üblichen objektiven Sinn des Wortes. AuBerdem widerspricht sich die These selbst, wenn sie Wahrheit im üblichen Sinn beansprucht. Wird aber nur behauptet, man glaube, daß die These wahr sei, so ist auch das im normalen Verständnis eine Aussage, die objektive Wahrheit beansprucht, die These also widerlegt. Ferner würde nach WR das tertium von datur nicht gelten, da nicht für alle Sachverhalte $p$ gilt $G(a, p)$ v $G(a, \neg p)$. Wir könnten nach WR auch nicht zwischen $\neg \mathrm{G}(\mathrm{a}, \mathrm{p})$ (a glaubt nicht, daß $\mathrm{p}$ ) und $\mathrm{G}(\mathrm{a}, \neg \mathrm{p}$ ) (a glaubt, daß $p$ nicht gilt) unterscheiden, und schließlich wäre der Wahrheitswert eines Sachverhalts nach WR nicht nur personen-, sondern auch zeitabhängig. Wenn ich gestern glaubte, daß der Satz „Lincoln wurde 1866 ermordet" wahr sei, und das heute nicht mehr glaube, so werde ich aber nicht sagen, dieser Satz sei gestern wahr gewesen, heute hingegen falsch. ${ }^{78}$

Ein typisches Argument für den Wahrheitsrelativismus ist folgendes: Überzeugungen sind unser einziges Wahrheitskriterium, der einzige Maßstab, mit dem wir Wahrheit beurteilen können. Wir können unsere Überzeugungen nicht direkt mit Tatsachen vergleichen, sondern nur mit anderen Überzeugungen über Tatsachen. Ein objektiver Wahrheitsbegriff ist daher nutzlos, weil uns objektive Wahrheit verborgen bleibt, sofern sie sich von unseren (gegenwärtigen) Überzeugungen unterscheidet. - Man muß aber unterscheiden zwischen Überzeugungen im Sinne von Sachverhalten des Überzeugtseins $G(a, p)$ und Sachverhalten $p$, von denen wir überzeugt sind. Nur die letzteren stellen Realgründe, Erkenntnisgründe oder Kriterien für die Wahrheit anderer Sachverhalte q dar. ${ }^{79}$ Aus der Tatsache, daß ich q nur dann als Grund für $\mathrm{p}$ ansehe, wenn ich glaube, daß q gilt, folgt nicht, daß $G(a, q)$ der Grund für $p$ ist. Sachverhalte des Über-

${ }^{78}$ Entsprechendes gilt natürlich auch für die These, wahr sei, was allgemein für wahr gehalten wird.

${ }^{79} \mathrm{Vgl}$. dazu die Unterscheidungen in 2.2, S. $107 \mathrm{f}$. 
zeugtseins sind also sicher nicht die einzigen Wahrheitskriterien. Der objektive Wahrheitsbegriff ist, wie wir sahen, nicht nur nicht überflüssig, sondern unentbehrlich. Wir müssen zwischen dem Wahrheitswert von $G(a, p)$ und dem von $p$ selbst unterscheiden. Die Behauptung schließlich, die Wahrheit eines Satzes bleibe uns verborgen, wenn wir nicht von ihr überzeugt sind, ist unhaltbar. Man kann nur sagen: Die Wahrheit ist uns verborgen. Daraus folgt nicht, daß wir sie nicht später erkennen könnten.

\section{Die Sinnskepsis endlich besagt}

SS: Es gibt keine Aussagen, die so präzise sind, daß sich ibnen in eindeutiger Weise ein Wabrheitswert zuordnen ließe.

Wenn das eine Aussage sein soll, die so präzise ist, daß man sie eindeutig als wahr bezeichnen kann, so widerspricht sie sich selbst. Andernfalls darf man sie auch als falsch ansehen, ein Anerbieten, das wir gerne akzeptieren. In welchem Sinn könnte man denn z. B. auch die Sätze , $2+2=4$ “ oder „Abraham Lincoln wurde 1865 ermordet" als falsch ansehen?

Wie wir sahen, wendet man gegen Widerlegungen des Skeptizismus oft ein, daß sie das voraussetzen, was der Skeptiker leugnet, und so für ihn ohne Überzeugungskraft bleiben müssen. ${ }^{80}$ Kein Argument ist voraussetzungslos, und das gilt natürlich auch für unsere Argumente gegen den Skeptizismus. Wir haben z. B. die Begriffe „Glauben“, „Wissen“, „Wahrheit“ und „Evidenz" in einer bestimmten Weise verwendet und haben einfache logische und epistemologische Prinzipien benützt. All das könnte der Skeptiker bezweifeln und damit würden für ihn unsere Argumente ihre Überzeugungskraft verlieren. Der entscheidende Punkt ist aber, daß der Skeptiker sich selbst als jemand erklärt, mit dem man nicht mehr argumentieren kann. Denn er stellt nicht irgendwelche speziellen Annahmen in Frage, sondern unverzichtbare Voraussetzungen allen Argumentierens, als da sind: feste Gebrauchsregeln für die verwendeten Wörter (die üblichen oder anzugebende neue), elementare logische Zusammenhänge (die normalen oder anzugebende und zu begründende andere), die Unterscheidung

${ }^{80}$ M. Heidegger z. B. spricht von ,formal-dialektischen Überrumpelungsversuchen gegenüber dem Skeptizismus". 
wahr - falsch, ohne die Behauptungen ihren Sinn verlieren. Der universelle Skeptizismus stellt insofern eine „Verknotung des Verstandes" dar, wie Wittgenstein sagt, die, konsequent durchgehalten, durch Argumente nicht mehr auflösbar ist.

Nach Wittgenstein behandelt der Philosoph oft eine Frage wie eine Krankheit. ${ }^{81}$ Der konsequente Skeptizismus ist aber eine sich gegen philosophische Heilmittel, d. h. gegen Argumente, selbst immunisierende Krankheit. Wenn wir also den Skeptizismus oben nur ,von auBen" diskutiert, und dabei vorausgesetzt haben, daß der Leser sich nicht bereits in eine radikal skeptische Position eingegraben hat, so ist das die einzig mögliche Argumentationsebene, wenn man über den Skeptizismus noch rational diskutieren will. Die Voraussetzungen, die wir dabei gemacht haben, kann man natürlich kritisch prüfen. Wenn man das aber unter Angabe von Gegengründen tut, so hat man die skeptische Position bereits verlassen. Ein Zweifel ist, wie wir sahen, nur sinnvoll, wo er sich auf Gründe stützt, d. h. auf Annahmen, die jedenfalls im Moment - nicht bezweifelt werden. Ein universeller Zweifel ist also unsinnig. Wenn man die skeptischen Thesen rational verstehen will, so kann man sie nur als inkonsistent verstehen. Tut man das nicht, so stellen sie den absurden, weil rational nicht mehr artikulierbaren Ausdruck eines fundamentalen Erkenntnismißtrauens dar. ${ }^{82}$

Auch der Skeptiker selbst muß sich auf rationales Argumentieren und damit auf Voraussetzungen einlassen, wenn er andere von seiner These überzeugen will. Er versucht dann die Annahme, es gebe Wissen, durch eine reductio ad absurdum zu widerlegen. Um sich dabei nicht in einen Selbstwiderspruch zu verwickeln, wird er, wie das auch Hume betont, ${ }^{83}$ diese reductio nicht als Beweis seiner These verstehen, sondern als ein Verfahren, dem Gegner die Unhaltbarkeit seiner Position deutlich zu machen und ihn zu einer skeptischen Haltung zu bewegen.

${ }^{81}$ Wittgenstein (53), 255.

82 Wie in der Kunst das Absurde als legitimer Ausdruck des Protests gegen díe gängigen Normalitätsstandards gilt, so könnte man auch manche skeptische Äußerungen als Protest gegen die Standards der Rationalität ansehen.

${ }^{83} \mathrm{Vgl}$. (T), Buch 1, Teil IV, $₫ 1$. 
Dem konsequenten Skeptiker, für den Argumentationen kein Gewicht haben, kann man mit Hume erwidern, daß seine Haltung sich praktisch, d. h. im normalen Leben nicht konsequent durchhalten läßt. Denn Leben heißt handeln; Handeln erfordert aber Entscheidungen, und diese müssen sich an Tatsachen orientieren. Wenn man sinnvoll handelt, geht man zumindest von Wahrscheinlichkeitsannahmen über die gegebenen Umstände und die Konsequenzen der möglichen Handlungsweisen aus und zieht daraus seine Schlüsse. Man weiß also zumindest, daß man diese Annahmen selbst macht und sieht die Schlüsse als richtig an. Die Versicherung, man glaube nicht, etwas zu wissen, bleibt so rein akademisch und wird durch die Tat widerlegt. Die Natur, sagt Hume, zwingt uns zum Urteilen wie zum Atmen. ${ }^{84}$ Eine universelle Skepsis ist aber nicht nur, wie Hume und auch Pascal meinten, ${ }^{85}$ praktisch undurchführbar, sondern sie ist eben auch, wie wir zu zeigen versuchten, keine theoretisch haltbare Position.

Erkenntnis wurde erstmals zum philosophischen Problem angesichts des Umbruchs überkommener Anschauungen über Welt, Gesellschaft und Religion, der sich mit den tiefgreifenden sozialen und politischen Veränderungen in der griechischen Welt im 6. und 5. Jahrhundert v. Chr. vollzog. Die Frage drängte sich auf, was an den bisherigen Anschauungen richtig und was bloß historisch bedingte Meinung war. Man suchte nach einer Abgrenzung dessen, was sich aus

${ }^{84}$ Hume schreibt (Treatise 1, Teil IV, $\mathbb{\$} 1$, S. $178 \mathrm{f}$ ), nachdem er seine allgemeinen Argumente für den Skeptizismus zusammengefaßt hat: "Should it here be asked me, whether I sincerely assent to this argument ... and whether I be really one of those sceptics who hold that all is uncertain and that our judgement is not in any thing possessed of any measure of truth and falsehood; I should reply, that this question is entirely superfluous, and that neither I, nor any other person, was ever sincerely and constantly of that opinion. Nature, by an absolute and uncontrollable necessity, has determined us to judge as well as to breathe and feel...". Damit richtet sich Hume gegen den phyrrhonischen Skeptizismus - benannt nach Pyrrho von Elis (ca. $360-270$ v. Chr.) -, der aus seiner theoretischen Einstellung praktische Konsequenzen für das Leben ziehen wollte, die auf ein Bemühen um völlige Indifferenz, ja Apathie gegenüber äußeren Ereignissen abzielten. Auch von Pyrrho wird jedoch berichtet, daß er vor einem bissigen Hund davonlief.

${ }^{85}$ Pascal, (W), XIII, Fr. 434. 
menschlichen Konventionen (vóuos) ergibt, von dem, was in der $\mathrm{Na-}$

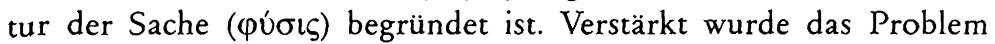
durch die Vielzahl der nun auftretenden neuen Ansichten über Mensch und Welt und der politischen Ordnungsentwürfe. Als Reakliun auf den naiver Glauben an dia Wahrheit dessen, was allgemeiner Überzeugung entspricht, kann man die erste Form der Skepsis ansehen, nach der es keine objektiven Kriterien zur Unterscheidung wahrer und falscher Meinungen gibt, so daß eine Meinung so gut ist wie die andere. Versteht man die oben erörterten drei ersten skeptischen Thesen so, daß darin die Wörter „Wissen“, „Evidenz" und ,Wahrheit" ihre normale Bedeutung haben, so ergibt sich in etwa die Form der Skepsis, die nach den uns erhaltenenen Äußerungen von den Sophisten vertreten wurde.

Die Auseinandersetzung mit dieser Form der Skepsis geschah nun aber nicht so, daß man, wie wir das oben getan haben, die Fallibilität unserer Überzeugungen anerkannte, aber betonte, daß sie keine hinreichende Grundlage für eine universelle Skepsis ist. Vielmehr suchte man nach Erkenntnissen, die unbezweifelbar gültig und damit dem Streit der Meinungen entrückt sind. Und man fand solche Erkenntnisse im Feld von Mathematik und Logik, in der Erkenntnis des Notwendigen, als dessen, was wahr sein muß, weil es gar nicht anders sein kann. ${ }^{86}$ Dieser Erkenntnistyp gab nun den Standara' wahrer Erkenntnis ab, der $\varepsilon \pi \iota \sigma \tau \eta \dot{\eta} \eta$ gegenüber der bloßen $\delta \delta \xi \alpha$, der Meinung. Wenn auch schon Aristoteles für eine Liberalisierung dieses Erkenntnisbegriffs eintrat und sagte, verschiedenen Gegenstandsbereicherı enisprächen verschiedene Erkenntnisarten und es sei nicht sinnvoll, überall Erkenntnis von mathematischer Qualität zu fordern, so blieb das Ideal perfekter Erkenntnis in der speziellen Form der Erkenntnis des Notwendigen doch weithin bestimmend. Und als man sich am Beginn der neuzeitlichen Philosophie, von F. Bacon an, wiederum aufgrund einer Kritik an der Tradition, die Aufgabe einer Neubegründung der Philosophie und der Wissenschaften stellte, da war, speziell bei Hobbes, Descartes, Spinoza und Leibniz, wieder die Mathematik das große Vorbild, und Descartes stellte das methodologische Postulat auf, nur Sätze, die ebenso evident sind oder mit ebenso evidenten

\footnotetext{
${ }^{80}$ Das erste bedeutende Zeugnis von dem tiefen Eindruck, den die Entdeckung dieser Erkenntnisart machte, ist das große Parmenides-Fragment 1-8, Diels (51) Bd. 1, $228 \mathrm{ff}$.
} 
Prämissen ebenso streng begründet sind wie mathematische, dürften als wissenschaftliche Sätze anerkannt werden: „Omnis scientia est cognitio certa et evidens ... Atque per hanc propositionem rejicimus illas omnes probabiles tantum cognitiones, nec nisi perfecte cognitis, et de quibus dubitari non potest, statuimus esse credendum ... Jam vero ex his omnibus est concludendum, non quidem solas Arithmeticam et Geometriam esse addiscendas, sed tantummodo rectum veritatis iter quaerentes circa nullum objectum debere occupari, de quo non possint habere certitudinem Arithmeticis et Geometricis demonstrationibus aequalem “ ${ }^{87}$

Perfekte Erkenntnis war nicht nur das Erkenntnisideal der rationalistischen Philosophie, sondern auch der empiristischen und idealistischen, mit dem Unterschied, daß hier - wie schon bei Descartes auch die unbezweifelbare Evidenz der eigenen cogitationes (der Sachverhalte des Überzeugtseins, Vermutens, Für-Wahrscheinlich-Haltens, Beobachtens, Denkens, Fühlens, Wollens etc.) neben jener mathematisch-logischer Säize als Basis für einen methodisch gesicherten Aufbau der Philosophie wie der Wissenschaften dienen sollte. Mit der Berufung auf die eigenen cogitationes wird zwar das Ideal notwendiger Erkenntnis aufgegeben, aber das Ideal perfekter Erkenntnis beibehalten. Dieses Ideal stellt eines der wichtigsten Motive für die rationalistischen, phänomenalistischen und idealistischen Reduktionsversuche dar. Deren Scheitern einerseits wie die Entwicklung exakter, methodisch gefestigter und erfolgreicher Naturwissenschaften, die einen liberaleren Erkenntnisbegriff verwendeten, andererseits haben erst im 19. Jahrhundert zu einer Ablösung dieses großen Erkenntnisideals geführt.

Auf einer zweiten Stufe stellt sich die Skepsis - mit den Thesen $\mathrm{SE}_{1}$ und $\mathrm{SV}$ - als Kritik an diesem Ideal perfekter Erkenntnis dar, als Kritik, die durchaus berechtigt ist, wenngleich dann die Formulierung beider Thesen (zumindest für das heutige Verständnis von „Wissen“ und „Evidenz") irreführend ist und man die Existenz perfekten Wissens jedenfalls in engen Bereichen anerkennen muß. Entscheidend ist aber, daß das Ideal perfekter Erkenntnis eine radikale Verengung des Erkenntnishorizonts zur Folge hat: Fordert man subjektiv problemlose, d. h. entscheidbare Kriterien für Wissen, so beschränkt man das,

${ }^{87}$ Regulae ad Directionem Ingenii, Regel 2, Abs. 1, 6. 
was sich erkennen läßt, auf den Inhalt von Sätzen, deren Wahrheit von uns selbst abhängt: auf analytische Sätze, deren Geltung eine Folge unserer sprachlichen Konventionen ist, und auf die eigenen cogitationes. Die gegenständliche Realität, so kann man - wenn auch nur ganz grob - sagen, ${ }^{88}$ ist die Menge der Sachverhalte, deren Bestehen von uns selbst, von unseren cogitationes und Konventionen unabhängig ist. Diese Außenwirklichkeit kann danach nicht perfekt erkennbar sein, denn für die Richtigkeit unserer Annahmen über sie gibt es eben keine hinreichenden subjektiv entscheidbaren Kriterien. Für uns sind aber nicht so sehr unsere eigenen cogitationes von Interesse als die Außenwirklichkeit. Und von ihr gibt es Erkenntnis nur dann, wenn man auf die Sicherheit subjektiv entscheidbarer Wissenskriterien verzichtet; wenn man sich auf das Wagnis einläßt zu scheitern; wenn man sich der Gefahr des Irrtums aussetzt. In diesem Sinn sagt Frege: „Mit dem Schritte, mit dem ich mir eine Umwelt erobere, setze ich mich der Gefahrt des Irrtums aus".89

Hier wird nun ein drittes Niveau der Problematik deutlich, auf die Skepsis abzielt, und das wir oben noch nicht erörtert haben: Es wird deutlich, daß wir uns in der Erkenntnisbemühung auf ein Wagnis einlassen, das getragen wird von dem Vertrauen, daß unsere sorgfältig geprüften Überzeugungen uns in der Regel nicht trügen; daß die Welt für uns (jedenfalls partiell) erkennbar ist. Dieses Vertrauen läßt sich rational nicht mehr rechtfertigen, weil alle rationale Begründung sich immer auf Evidenzen stützt, und Evidenzen schon Überzeugungen darstellen.

Wir wollen Fragen, deren Beantwortung aus theoretischen oder praktischen Gründen wichtig ist, die sich, wie Kant sagt, der Vernunft unvermeidlich aufdrängen, sich aber nicht entscheiden lassen, als Grenzfragen bezeichnen. Als Entscheidung der Frage, ob eine Behauptung A gilt, sehen wir dabei nicht nur hinreichende Argumente für oder gegen $\mathrm{A}$ an - Fragen lassen sich auch ohne Argumente durch Evidenzen und Beobachtungen entscheiden - und auch nicht nur „,definitive“ Entscheidungen, die jede Irrtumsmöglichkeit ausschließen. Ob A gilt, ist nicht schon dann eine Grenzfrage, wenn sich perfekt weder erkennen läßt, daß $A$ gilt noch daß $A$ nicht gilt, und auch

\footnotetext{
${ }^{88} \mathrm{Vgl}$. dazu das Kapitel 8.

${ }^{89}$ Frege (KS), 358.
} 
nicht schon dann, wenn wir gegenwärtig nicht wissen, ob A gilt, sondern erst dann, wenn es auf diese Frage eine wohlbegründete Anwori im normalen Sinn dieses Wortes nicht geben kann.

Die Frage, ob es verläßliche Evidenzen gibt, ist daher zunächst keine Grenzfrage. ${ }^{90}$ Denn es ist mir evident, daß mir einige Sachverhalte evident sind, und ich bin davon überzeugt, daß das, was mir (jetzt) evident ist, auch richtig ist. Die Frage ist also, wie wir früher gesagt haben, durch eine einzige Evidenz entschieden, die wir tatsächlich haben. In dem Sinn, in dem wir sie jetzt diskutieren, will die Skepsis aber deutlich machen, daß alle unsere Erkenntnisansprüche auf einem Evidenzvertrauen beruhen, das sich nicht mehr rechtfertigen läßt. Stellt man die Verläßlichkeit von Evidenzen generell infrage, dann wird die Frage, ob es verläßliche Evidenzen gibt, in der Tat zu einer Grenzfrage, weil dann weder unser Argument noch irgendein anderes brauchbar ist. Für die ersten Prämissen jedes Arguments müssen wir ja schon Evidenzen in Anspruch nehmen, und deren Verläßlichkeit wird nun gerade bezweifelt. Die Skepsis weist so darauf hin, daß ein Akt der Anerkennung vorliegt, wenn wir einen Sachverhalt als evident gelten lassen. „Das Wissen gründet sich am Schluß auf der Anerkennung" sagt Wittgenstein,91 und auch für Fichte gilt: „Das Element der Gewißheit ist der Glaube".92 Glaube ist aber für ihn der Entschluß, eine Annahme als wahr anzuerkennen. Wir verlassen uns auf das, was uns einleuchtet, erkennen es als richtig an, und wir kommen auch gar nicht umhin, das zu tun, weil das die conditio sine qua non aller Erkenntnis ist. ${ }^{93}$ In der Skepsis, speziell in der Evidenzskepsis, artikuliert sich so ein fundamentales Erkenntnismißtrauen, das

90 Verläßliche Evidenzen in diesem Sinn sind echte Evidenzen im Sinn von 1.5, also Sachverhalte, die zugleich evident und wahr sind, nicht aber Evidenzen von einer Art, für die das Prinzip V2* aus 1.4 als genereller, analytischer Satz gilt.

91 Wittgenstein (74), 378.

92 Fichte (W), Bd. V, 182.

${ }^{93}$ Der Hinweis, daß sich unser Vertrauen in Überzeugungen dadurch rechtfertigen lasse, daß sie sich in der Praxis des Lebens bewährt haben, wie er oft vorgebracht wird, ist zwar nicht unrichtig: Wenn wir Urteile über die praktische Bewährung von Annahmen nicht als problematisch ansehen, können wir damit natürlich andere Urteile begründen. Wenn aber, wie in der universellen Skepsis, alle Annahmen in Zweifel gezogen werden, hilft er uns offenbar nicht weiter. 
sich zwar nicht konsequent durchhalten läßt; die Bedeutung der Skepsis liegt aber darin, daß sie deutlich macht, daß das Gebäude aller unserer Annahmen über die Welt auf einer vorgängigen, rational nicht mehr zu rechtfertigenden Haltung beruht.

Es ist charakteristisch, daß Descartes, der die Verläßlichkeit von Evidenzen rational zu begründen, also das Wagnis zu eliminieren suchte, das auf dem Weg über den Beweis der Existenz eines vollkommenen und damit auch benevolenten Gottes tat, in dessen Absicht es nicht liegen kann, uns zu täuschen, wie umgekehrt auch der fundamentalste Zweifel an der Verläßlichkeit von Evidenz bei Descartes in der ersten Meditation mit dem Hinweis auf die Möglichkeit der Existenz eines trügerischen Dämons begründet wird. Beide Gedanken liefern keine Argumente zur Entscheidung der Frage: Abgesehen von den bekannten Problemen des ontologischen Gottesbeweises stützt sich Descartes dabei bereits auf Evidenzen, kann also allenfalls die Verläßlichkeit von Evidenzen bzgl. der Außenwelt begründen. Und auch die Annahme, es könne einen bösen Dämon geben, stützt sich auf die Evidenz dieser Behauptung und ist im übrigen allein kein Argument, die Verläßlichkeit aller Evidenzen in Zweifel zu ziehen. Solche „Argumente" sind also nur zur Illustration des Problems brauchbar. So betont denn auch Pascal, unser Anspruch auf Wissen bleibe prinzipiell rational unbegründbar. Die Vernunft müsse hier ihre Grenze erkennen, und die Größe der Vernunft beweise sich darin, daß sie diese Grenze annimmt: „La dernière démarche de la raison est de reconnaître qu'il y a une infinité de choses qui la surpassent; elle n'est que faible, si elle ne va jusqu'à connaître cela“".94 Die Grenzfragen der Erkenntnistheorie ergeben sich daraus, daß wir uns in ihr auf eine immanente Selbstkritik der Erkenntnisleistungen beschränken müssen, und, wie wir früher sagten, keinen Standpunkt ,,über uns“ einnehmen können, von dem aus sich ein freier, von den Bedingtheiten menschlichen Erkennens unbelasteter Blick auf die Wirklichkeit eröffnet und damit ,,absolute" Feststellungen über Wahrheit und Falschheit menschlicher Überzeugungen möglich werden. In diesem Sinn sagt Pascal: ,Si l'homme s'étudiait le premier, il verrait combien il est incapable de passer outre. Comment se pourrait-il qu'une partie connût le tout? ‘95

94 Pascal (W) XIII, S. 196, Fr. 267.

${ }_{95}$ Pascal (W) XII, S. 87, Fr. 72. 
Nach Pascal erkennen wir die Wahrheit mit dem Herzen, nicht nur mit der Vernunft. Coeur ist bei Pascal das Organ der Evidenz und zugleich das Organ religiöser Erfahrung. Raison ist dagegen das Vermögen logischen Schließens. Schlußfolgerungen ergeben immer nur hypothetische Sätze der Form "Wenn dies der Fall ist, so auch jenes", aber nicht kategorische Sätze der Form „Dies ist der Fall“. Durch Beweise vermittelte Erkenntnis beruht also auf Evidenz, die nicht mehr begründbar ist. ,Nous connaissons la vérité, non seulement par la raison, mais encore par le coeur; c'est de cette dernière sorte que nous connaissons les premiers principes". 96

Der Streit zwischen Skeptikern und Dogmatikern ist daher für Pascal rational nicht auflösbar. ${ }^{97}$,Wir haben keine Gewähr für die Wahrheit unserer Prämissen, nur Überzeugungen, die auch falsch sein können“, sagen die einen, „Man kann an den natürlichen Prinzipien nicht zweifeln, wenn man aufrichtig und konsistent spricht" die anderen. Die Überzeugungen der Dogmatiker beruhen aber auf einem Evidenzvertrauen, das sie nicht mehr begründen können. Auch bei Pascal wird das Problem durch eine Berufung auf Gott gelöst, aber die Existenz Gottes kann nicht bewiesen, sondern muß geglaubt werden, so daß damit die Grenze der Vernunft nicht aufgehoben wird. Pascal bezieht sich auf die These von Augustinus, nach der der Glaube der Erkenntnis vorausgeht und sie begründet, indem er dem Erkenntnisvertrauen Halt gibt: „Credidimus ut cognosceremus, nam si prius cognoscere, et deinde credere vellemus, nec cognoscere, nec credere valeremus". ${ }^{98}$ Die Berufung auf Gott ist freilich eher ein $A$ Asdruck als eine Rechtfertigung des Erkenntnisvertrauens, denn bzgl. religiöser Überzeugungen stellt sich dasselbe Problem, ja es stellt sich hier mit noch größerer Schärfe.

All das kann nun nicht bedeuten, daß die Frage: „Sollen wir unseren wohlerwogenen Annahmen vertrauen?" nur eine willkürliche, nicht mehr erhellbare, irrationale Entscheidung zuläßt. Wir haben erstens gar keine andere Wahl, da ein völliger Verzicht auf Überzeugungen praktisch undurchführbar ist. Und zweitens gehört es zum Phänomen der Evidenz, daß sie nicht blinde Überzeugung ist, sondern

\footnotetext{
${ }^{96}$ Pascal (W) XIII, S. 203, Fr. 282.

${ }^{97} \mathrm{Vgl}$. Pascal (W) XIII, S. $341 \mathrm{ff}$, Fr. 434.

98 Tractatus in Joannis Evangelium 27,9.
} 
Überzeugung, die ihre Rechtfertigung in sich trägt; daß sie unmittelbare Einsicht ist, in der sich zeigt, wie es sich tatsächlich verhält. Die Erfahrung der Fallibilität auch der mit Evidenz gefällten Urteile zwingt uns zwar, zwischen Evidentsein und Wahrsein zu unterscheiden, aber damit wird die Korrektheit der Evidenzen nicht generell infrage gestellt; vielmehr stützen sich alle sinnvollen Zweifel auf Überzeugungen und diese letztlich wieder auf Evidenzen. Es ist wichtig zu sehen, daß die Berufung auf bestimmte Evidenzen nicht alle Möglichkeit sinnvollen Zweifels an ihrer Verläßlichkeit ausschließt, und daß auch ein Zweifel an aller Evidenz möglich ist. Aber dieser letztere Zweifel ist kein begründbarer Zweifel im normalen Sinn, sondern hat nur die Funktion, uns auf die Grenze hinzuweisen, die aller Rechtfertigung und Begründung gezogen ist, auf die Grenzen immanenter Erkenntniskritik.

Nachdem wir nun die wichtigsten Probleme erörtert haben, die sich mit dem Erkenntnisbegriff verbinden, wollen wir zum Abschluß die Ergebnisse zusammenfassen. Die erste Feststellung ist

A) Als genereller Wissensbegriff kommt ein Begriff perfekter Erkenntnis nicht infrage.

Der Bereich der Sachverhalte, von denen es - auch im Sinn unseres rationalen Wissensbegriffs - perfekte Erkenntnis gibt, ist viel zu eng begrenzt, als daß sich Wissen im normalen Sinn darauf beschränken ließe. Das entspricht auch dem heutigen Verständnis wissenschaftlicher Erkenntnis. Keine Wissenschaft erhebt heute den Anspruch, über perfekte Erkenntnis zu verfügen. Das gilt insbesondere für die Naturwissenschaften, für deren Methodologie die Auffassung vom hypothetischen Charakter ihrer Theorien bestimmend ist, und deren Arbeit sich im Entwurf von Hypothesen und Theorien und deren fortlaufender Überprüfung durch Experimente vollzieht. Es gilt aber auch für die Geisteswissenschaften, deren methodologische Konzeption heute vom Bewußtsein der historischen Bedingtheit ihrer Interpretation geprägt wird.

Die zweite Feststellung ist

B) Als genereller Wissensbegriff kommt auch ein Begriff wabrer und fundierter Überzeugung nicht infrage.

Wir haben bei der Erörterung solcher Wissensbegriffe im Abschnitt 1.3 gesehen, daß die dort angegebenen Bestimmungen I und II des Fundiertheitsbegriffs F die Sachverhalte, von denen es ein Wis- 
$\operatorname{sen}_{\mathrm{F}}$ gibt - also ein gemäß $\mathrm{F}$ fundiertes Wissen - , auszeichnen als Folgen von Sachverhalten, von denen es kein Wissen $\mathrm{F}$ gibt, bzw. nach Kriterien, deren Zuverlässigkeit kein Sachverhalt ist, von dem es ein

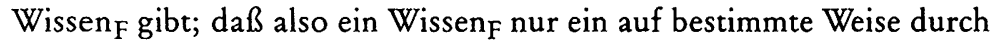
anderes Wissen vermitteltes oder begründetes Wissen ist. Man kann daher von einem $W_{i s s e n_{F}}$ nur sprechen, wo man auch eine andere Art von Wissen anerkennt. Diese Fundiertheitsbegriffe geben daher zu Wissensbegriffen Anlaß, die schon deswegen nicht als generell angesehen werden können, weil sie selbst andere Typen von Wissen voraussetzen. Sie sind aber auch inhaltlich zu eng: Nicht alles Wissen ist begründet - es gibt auch Wissen aufgrund von Evidenz. Und wenn man auch wahre Evidenzen als fundiertes Wissen bezeichnet, so läßt sich, wie wir gesehen haben, fundiertes Wissen nicht mehr klar von wahrer Überzeugung abgrenzen. Jemand, dem die Große Fermatsche Vermutung evident ist, wird man noch kein fundiertes Wissen ihrer Richtigkeit zuschreiben wollen. Nicht alles Wissen ist nicht mit wissenschaftlichen Methoden gewonnen - den weitaus größten Teil dessen, was wir im normalen Sinn wissen, haben wir nicht mithilfe wissenschaftlicher Methoden erworben. Man sagt auch, ein Wissenschaftler wisse etwas, worüber er sich nur in einer Fachzeitschrift informiert hat. Zeitschriftenlektüre ist jedoch im engen Sinn des Wortes sicher keine wissenschaftliche Methode. Aber wie weit käme der einzelne ohne sie?

Aus der Feststellung (A) ergibt sich ferner, daß Sachverhalte des Fundiertseins nicht zugleich verläßlich und problemlos sein können. Und die Diskussion des „Beweises für die Unmöglichkeit der Erkenntnistheorie" von Nelson in 1.6 hat gezeigt, daß Sachverhalte des Fundiertseins einerseits, wenn sie verläßlich, aber nicht problemlos sind, kein subjektiv brauchbares Kriterium für Wahrheit und Wissen abgeben, da es dann nicht einfacher ist zu erkennen, daß eine Annahme fundiert ist, als daß sie wahr ist, und daß solche Sachverhalte andererseits, wenn sie problemlos, aber nicht verläßlich sind, nicht verläßlicher sind als schlichte Überzeugungen.

Mit Fundiertheitsbegriffen kann man also zwar spezielle Formen von Wissen auszeichnen, aber nicht den allgemeinen Wissensbegriff erfassen, und fundiertes (aber nicht perfektes) Wissen ist auch keine Form eines anspruchsvolleren Wissens als wahre Überzeugung.

Daher ergibt sich als dritte Feststellung: 
C) Als genereller Wissensbegriff kommt nur der Begriff wabrer Überzeugung infrage.

Dieser Begriff genügt den beiden Minimalbedingungen W 1 und W6 über den Zusammenhang von Wissen, Wahrheit und Glauben. Überzeugung ist unser letztes subjektives Kriterium für Wissen, das nicht steigerungsfähig ist, und Wahrheit ist das einzige notwendige objektive Kriterium, das sich auch nicht überbieten läßt. Der einzige qualitativ anspruchsvollere Wissensbegriff ist der perfekten Wissens, der aber nach (A) nicht als genereller Begriff infrage kommt. Perfektes Wissen unterscheidet sich von wahrer Überzeugung dadurch, daß die Korrespondenz zwischen Überzeugtsein und Wahrheit garantiert ist, da Wahrheit hier Folge des Überzeugtseins ist. Problematisch ist allein, daß wahre Überzeugung einen sehr weiten Begriff von Wissen darstellt, nach dem wir oft auch das als ,Wissen" zu bezeichnen haben, was wir normalerweise nicht so nennen.

Wenn wir im folgenden „Wissen“ als wahre Überzeugung, also im Sinne von $W_{0}$ verstehen, so ist das eine Explikation des umgangssprachlichen Ausdrucks. An eine Explikation sind allgemein folgende Forderungen zu stellen: ${ }^{99}$

1. Sie muß dem Wort in den wissenschaftlichen Kontexten, auf die sie zugeschnitten ist, eine hinreichend präzise Bedeutung zuordnen.

2. Diese Bedeutung soll in etwa der umgangssprachlichen Bedeutung des Ausdrucks entsprechen.

3. Die Explikation soll einen für die angezielten Verwendungen systematisch fruchtbaren Begriff ergeben.

Dabei gehen Präzision und systematische Fruchtbarkeit oft auf Kosten der Forderung (2). Die Abweichung unseres Wissensbegriffs $W_{0}$ vom ungangssprachlichen wird besonders deutlich erstens in den Fällen, in denen die Rationalitätspostulate W 2 bis W 4 zum Tragen kommen, und zweitens in Fällen, in denen eine Begründung fehlt, uns aber erforderlich erscheint, um ein definitives Urteil abzugeben. Beidesmal können wir uns auf die größere systematische, d. h. hier: erkenntnistheoretische Fruchtbarkeit unseres Begriffs berufen. Die entsprechenden Argumente haben wir in 1.1, 1.2 und 1.3 dargestellt. Wir haben auch gesehen, daß sich das, was einer Begründung bedarf, nicht zugleich allgemein und scharf abgrenzen läßt. Man könnte na-

${ }_{99}$ Vgl. dazu z. B. Hempel (52), $\mathbb{\int} 3$. 
türlich, um Konflikte mit dem umgangssprachlichen Gebrauch von „Wissen" zu vermeiden, auí dieses Wort in der Erkenntristheorie verzichten und nur von ,wahrer Überzeugung “ reden, würde sich damit aber im Effekt nur um die Analyse des Erkenntnisbegriffs herumdrükken.

- Die Suche nach einem engeren Wissensbegriff geht von der Fallibilität unserer Überzeugungen aus, und zielt letztlich auf subjektiv entscheidbare hinreichende Bedingungen für Wahrheit ab. Daß dieses Ziel nicht generell erreichbar ist, haben wir unter (A) noch einmal betont. Irrtumsmöglichkeiten lassen sich nicht eliminieren. Dieser Einsicht wird unser Wissensbegriff gerecht. Man könnte ihm aber entgegenhalten, daß er Wissen in zwei Komponenten, subjektive Überzeugung und objektive Wahrheit zerlege, die unabhängig von einander sind, so daß ihre Übereinstimmung nur zufällig und darüber hinaus für uns auch nicht definitiv feststellbar ist, so daß wir wissen können, bzw. nicht wissen, ohne das zu wissen. ${ }^{100}$ Das Grundphänomen der Erkenntnis, daß sich in ihr die Wirklichkeit zeigt, werde so verfehlt. - Aufgrund der Fallibilität unserer Annahmen, etwas zu wissen, die man nicht leugnen kann, muß man aber beide Komponenten unterscheiden und zwar so, daß die eine keine notwendige Folge der anderen ist. Die Übereinstimmung ist dann sicherlich kontingent. Man kann jedoch nicht behaupten, sie sei bloß zufällig. Denn wenn wir von der Wahrheit eines Sachverhalts überzeugt sind, ist sein Bestehen für uns eben nicht problematisch, sondern gewiß. Das Grundphänomen der Erkenntnis wird nicht verfehlt, denn in ihr sind unsere Überzeugungen nach W 1 immer richtig. Und nach W 5 gilt jedenfalls: Wir können nicht wissen, ohne zu wissen, $\mathrm{da} ß$ wir wissen. Sieht man von

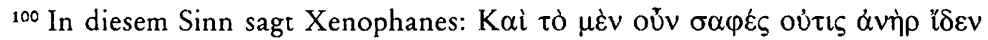

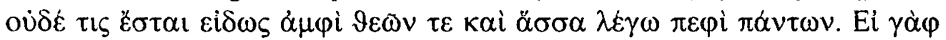

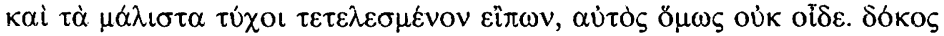

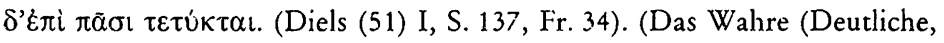
Genaue, Unzweifelhafte) aber erblickte kein Mensch, und es wird auch nie jemand sein, der es weiß in Bezug auf die Götter und alle Dinge, die ich immer erwähne; denn selbst wenn es einem im höchsten $M a ß$ gelänge, ein Vollendetes auszusprechen, so hätte er selbst doch kein Wissen davon; Schein haftet an allem.) - Über Annahmen kommen wir danach also nicht hinaus, und selbst wenn sie zufällig richtig sind, können wir das nicht feststellen. 
dem durch die Skepsis aufgewiesenen Grenzproblem ab, so kann man im Sinn des gesunden Menschenverstands auch sagen: Wir haben aufgrund langer Erfahrung eine recht gute Vorstellung davon, worauf wir uns verlassen können, und urteilen - wenn wir das mit Überlegung und Sorgfalt tun - entsprechend; solche Urteile erweisen sich in der Regel auch als richtig. Bewähren sich unsere Überzeugungen aber in unserem Leben, so haben wir diejenige Sicherheit, die wir brauchen und die wir allein erwarten können. 


\section{Verstehen}

\subsection{Verstehen, Erklären und Begründen}

Im ersten Kapitel haben wir den Erkenntnisbegriff analysiert, der in Sätzen der Form „Die Person erkennt, daß p“ verwendet wird. Wir haben schon darauf hingewiesen, daß es neben dem Erkennen, daß ein Sachverhalt besteht, auch andere Formen des Erkennens gibt, z. B. ein Erkennen, warum oder wozu etwas so ist, wie es ist; wie es entstanden ist, usf. Ziel unserer Erkenntnisbemühung ist nicht nur das Konstatieren von Tatsachen, sondern auch das Erfassen von Ursachen, Gründen, Zwecken und Zielen, ein Verstehen der Tatsachen. Wenn wir dem Erkennen von Tatsachen Formen des Verstehens gegenübersteilen, so behaupten wir damit nicht, daß Verstehen kein Erkennen sei. Erkenntnis ist vielmehr der Obertitel, unter den auch alle Formen des Verstehens zu subsumieren sind, für die wir uns im Kontext dieser erkenntnistheoretischen Arbeit interessieren. Die Unterscheidung Verstehen - Tatsachenerkenntnis bedeutet auch nicht, daß ein Verstehen keine Tatsachenerkenntnis einschließt oder über den Rahmen von Tatsachenerkenntnis hinausgeht. Ich kann nur dann verstehen, warum ein Wasserrohr geplatzt ist, wenn ich zunächst erkannt habe, daß es so ist, und das Verständnis der Ursache dieses Ereignisses besteht z. B. in der Erkenntnis, daß es über Nacht gefroren hat und $\mathrm{da}$ sich Wasser beim Gefrieren ausdehnt. Wenn ich also verstehe, warum es so kam, so weiß ich mehr, als wenn ich nur erkenne, daß es so kam, aber dies Mehr besteht lediglich in weiteren Tatsachenerkenntnissen. In diesem Sinn stellt das Erkennen-daß die Grundform allen Erkennens dar.

Die Frage, was das Wort „Verstehen“ bedeutet, worin Verstehen besteht, ist in der philosophischen Literatur heiß umstritten, und es gibt eine Vielzahl gegensätzlicher und oft auch abstruser Bestimmungen seines Inhalts. Wir wollen auf diese Diskussionen - insbesondere auf jene über Begriff und Funktion des Verstehens in den Geistesund Sozialwissenschaften - zunächst nicht eingehen, sondern uns erst in den folgenden Abschnitten dieses Kapitels damit befassen. 
Hier gehen wir vom Gebrauch des Wortes in der Umgangssprache aus. Unsere erste Aufgabe besteht darin, uns eine Übersicht über die verschiedenen Verwendungsweisen von ,,verstehen “ zu verschaffen. ${ }^{1}$

Es gibt ein praktisches und ein theoretisches Verstehen. Im praktischen Sinn versteht man sein Handwerk, man versteht Deutsch, versteht sich auf etwas. Praktisches Verstehen besteht in einer Kompetenz, und da wir uns hier nur für Formen des Verstehens interessieren, die ein Erkennen darstellen, wollen wir darauf nicht eingehen.

Formen theoretischen Verstehens sind:

1. Determinatives Verstehen. Wir verstehen einen Vorgang, wenn wir ihn näher bestimmen können, wenn wir erkennen, um was für einen Vorgang es sich handelt. Beispiele:

a) Ich beobachte in einem Zulu-Dorf einen Tanz. Ich verstehe, was vorgeht, wenn ich erkenne, daß es sich um einen Regenzauber handelt.

b) Ich beobachte, wie Herr X auf einen Knopf drückt. Ich verstehe, was er tut, wenn ich erkenne, daß er damit dem Besuch die Haustür öffnet.

c) Ich höre, wie Herr Y zu Frau Z sagt ,Ich halte nichts von Leuten, die über ihre Nachbarn tratschen ". Ich verstehe, daß das ein bewußter Affront ist, wenn ich weiß, daß Herr Y mit seiner Äußerung einen längeren Bericht der Frau $Z$ über das Eheleben ihrer Nachbarsleute unterbricht.

In allen drei Beispielen beinhaltet das Verstehen des Vorgangs, daß zu der Erkenntnis, daß der fragliche Vorgang stattfindet (daß getanzt wird, daß Herr X auf einen Knopf drückt, daß Herr X zu Frau Z das und das sagt), die Einsicht hinzukommt, um was für eine Art Vorgang es sich näherhin handelt. Von einem determinativen Verstehen von Objekten redet man dagegen nicht. Wenn man z. B. erkennt, daß ein

\footnotetext{
${ }^{1}$ Vorweg ist anzumerken, daß ,,verstehen“ wie ,erkennen" ebenso einen Prozeß bezeichnet (wir sprechen von schnellem und langsamen Verstehen) wie das Ergebnis dieses Prozesses, das wir im Fall von ,erkennen' durch ,,wissen" ausdrücken. Man unterscheidet umgangssprachlich nicht klar zwischen „verstehen“ und „,verstanden haben“. Wenn wir Verstehen als Erkenntnis oder als Wissen bezeichnen, so meinen wir das Resultat des Verstehensprozesses. Für den Prozeß, wie man zum Verstehen gelangt, interessieren wir uns hier ebensowenig wie für das Erkennen als Prozeß. - Für eine Übersicht über Formen des Verstehens vgl. a. Passmore (62).
} 
Schmetterling ein Pfauenauge ist, so sagt man nicht, man verstehe den Schinetterling.

2. Bedeutungsversteben. Wir verstehen ein Zeichen, eine Geste, ein Wort, einen Text, eine Äußerung, wenn wir erkennen, was sie bedeuten. Beispiel:

d) Ich verstehe das Wort $\alpha \lambda \dot{\eta} \vartheta \varepsilon \iota \alpha$, wenn ich weiß, daß es etwa soviel wie ,Wahrheit" bedeutet.

Wenn von „Bedeutungsverstehen“ die Rede ist, so nehmen wir das Wort „Bedeutung“ im engeren Sinn, also z. B. nicht im Sinn von „Relevanz“.

3. Kausales Verstehen. Wir verstehen, warum ein Ereignis stattfand, wenn wir seine Ursachen erkennen. Beispiel:

e) Die Quecksilbersäule eines kalten Thermometers fällt zuerst, wenn ich es in heißes Wasser halte. Ich verstehe das, wenn mir klar wird, daß die Wärme zuerst das Glasgefäß erreicht und ausdehnt, und erst dann das Quecksilber. ${ }^{2}$

4. Rationales Versteben. Wir verstehen, warum jemand etwas tut, wenn wir erkennen, daß es im Sinn seiner Überzeugungen und Präferenzen rational ist, oder wenn wir die Beweggründe seiner Handlungen erkennen. Hier geht es wie beim kausalen Verstehen um ein Verstehen von Gründen. Während aber dort die Gründe Ursachen (causae) sind, sind es hier Beweggründe (rationes, reasons), und daher sprechen wir von ,,rationalem" Verstehen.

f) Ich verstehe, warum sich Fritz scheiden ließ, wenn ich weiß, daß ihm klar wurde, daß seine Frau ihn ständig betrog.

g) Ich verstehe, warum Hans mit der Eisenbahn und nicht mit dem Auto in den Urlaub nach Italien fährt, wenn ich weiß, daß er einen Diebstahl seines Wagens befürchtet.

5. Intentionales Verstehen. Wir verstehen die Absichten oder Ziele, die jemand mit einer Handlung verfolgt, wenn wir erkennen, was er damit erreichen will. Ein Beispiel:

h) Ich verstehe, wozu Max im Herbst damit beginnt, täglich einen Dauerlauf zu absolvieren, wenn ich weiß, daß er sich damit für den winterlichen Skilauf in Form bringen will.

Absichten sind keine Gründe, daher ist ein intentionales Verstehen kein rationales Verstehen. Aus Absichten können sich aber Gründe er-

${ }^{2}$ Das Beispiel stammt von Hempel. 
geben, d. h. ein intentionales Verstehen kann ein rationales implizieren, wie wir später sehen werden.

6. Funktionsverstehen. Wir verstehen, wozu etwas dient, wenn wir seinen Zweck, seine Funktion erkennen. Und wir verstehen, wie etwas funktioniert, wenn wir den Wirkungszusammenhang der Teile erkennen. Beispiele:

i) Ich verstehe, wozu ein Thermostat dient, wenn ich erkenne, daß er die Zimmertemperatur konstant hält.

j) Ich verstehe, wie ein Thermostat funktioniert, wenn ich weiß, daß er aus einem Bimetallstreifen besteht, der bei Erwärmung über die Richttemperatur hinaus einen Stromkreis unterbricht und damit die Heizung ausschaltet.

Rationales, intentionales und funktionales Verstehen faßt man auch unter dem Obertitel teleologisches Verstehen zusammen. Zum teleologischen Verstehen rechnet man auch das Sinnverstehen. Das Wort "Sinn" hat verschiedene Bedeutungen. In Kontexten wie „Gehörsinn" oder "der Sinn für das Schöne“ bedeutet es etwa soviel wie „Empfänglichkeit", „Auffassungsgabe“. So einen Sinn kann man aber nicht verstehen. In Kontexten wie "Sinn eines Wortes (einer Aussage, eines Textes)“ ist es mit „Bedeutung“ synonym, und ein Sinnverstehen ist dann ein Bedeutungsverstehen. Mit einem teleologischen Verstehen hat Sinnverstehen nur etwas zu tun, wenn "Sinn“ in Kontexten wie „Sinn einer Handlung“, ,,Sinn einer Institution“, ,,Sinn einer Vorrichtung" vorkommt. Dann bedeutet es soviel wie „Absicht", „Zweck“, „Funktion“, „Nutzen“ oder „,Wert“. Sinnverstehen ist dann ein intentionales oder funktionales Verstehen.

7. Genetisches Versteben. Wir verstehen, wie etwas entstanden ist, wenn wir erkennen, welche Umstände und Ereignisse dazu führten. Beispiel:

k) Wir verstehen, wie das Gesetz über die Mitbestimmung entstanden ist, wenn wir die politische Konstellation kennen, die dazu führte, die Absichten und Überlegungen der Regierung, die den Gesetzentwurf einbrachte, die Mehrheitsverhältnisse im Parlament, usw.

8. Versteben als Interpretation und hypothetisches Verstehen. Das Wort „,verstehen" wird in Wendungen wie "Ich verstehe das als..." oder "Ich verstehe das so und so" im Sinn von ,deuten“ oder ,,interpretieren" verwendet. Im Beispiel (a) verstehe ich den Tanz als Regentanz, wenn ich ihn so deute. Im Beispiel (d) verstehe ich $\alpha \lambda \eta \dot{\eta} \vartheta \varepsilon \_$in einem Satz als ,Wahrheit", wenn ich es so interpretiere. Ein determinatives, 
bzw. Bedeutungsverstehen liegt nur dann vor, wenn diese Deutung richtig ist; nur dann liegt eine Erkenntnis vor. ${ }^{3}$ Von dieser Verwendungsweise des Wortes ,,verstehen" sehen wir ab, da wir uns hier nur für Formen des Verstehens interessieren, die ein Erkennen darstellen. Man kann statt ,,so und so verstehen“ auch sagen ,, sich so und so verständlich machen". Auch beim hypothetischen Verstehen wird aufgrund von Annahmen oder Deutungen ein Sachverhalt verständlich gemacht. Man kann z. B. einen Brauch in seiner sozialen Funktion verständlich machen mit gewissen psychologischen Annahmen; man kann seine Entstehung mit geschichtlichen Hypothesen verständlich machen; und man kann den negativen Ausfall eines physikalischen Experiments verständlich machen mit einer Annahme über Störfaktoren. Ein Verstehen als Erkennen liegt aber nur dann vor, wenn die Annahmen richtig sind.

Diese Unterscheidungen und Beispiele zeigen schon, daß es ein breites Spektrum von Formen des Verstehens gibt, und wir können nicht einmal behaupten, damit schon alle erfaßt zu haben. ${ }^{4}$ Wir streben das hier auch nicht an, sondern beschränken uns im folgenden

${ }^{3}$ Interpretationen können verschiedene Ziele verfolgen. Bei der Interpretation eines Textes kann es $z$. B. darum gehen, seinen objektiven (sprachlichen) Sinn aufzuhellen; oder zu erkennen, was der Autor damit sagen wollte; oder das Problem, das darin behandelt und die Lösung, die vorgeschlagen wird, dem Verständnis dadurch aufzuschließen, daß man sie mithilfe moderner Begriffe formuliert. Im letzteren Fall könnte man auch von einer Rekonstruktion sprechen, aber die Grenzen zwischen Interpretation und Rekonstruktion sind fließend.

${ }^{4}$ Oft sieht man die Leistung des Verstehens darin, daß Unbekanntes, Erstaunliches oder Merkwürdiges auf Bekanntes und Gewohntes reduziert wird. Es gibt aber auch ein Verstehen von Tatsachen, die uns keineswegs als erstaunlich oder merkwürdig erscheinen, z. B ein kausales Verstehen des freien Falls. Zudem würde durch diese Charakterisierung das Verstehen zu einem rein psychologischen Phänomen, denn was den einen erstaunt, kann einem anderen als normal erscheinen. Die Erkenntnis, daß ein Phänomen einer Norm oder Regularität entspricht, kann man freilich oft als „Verstehen“ bezeichnen. Man kann sagen, man verstehe eine zunächst als ungewöhnlich erscheinende Handlung, wenn man erkennt, daß sie einer Sitte oder Norm entspricht. Oder man verstehe den plötzlichen Tod von Herrn Müller, wenn man erfährt, daß er von einer Kobra gebissen wurde und daß so etwas in $80 \%$ aller Fälle tödlich ist. 
auf die Analyse von einigen besonders wichtigen Formen. Die Typen des Verstehens, die wir aufgewiesen haben, sind ferner nicht disjunkt. Ein Beispiel:

1) Im Verlauf eines Gesprächs mit anderen wirft Herr $X$ seiner Frau einen Blick zu. Ich verstehe, daß dieser Blick eine Warnung an seine Frau ist, das nicht zu sagen, was sie offenbar gerade sagen wollte.

Ist das ein determinatives Verstehen (der Blick ist eine Warnung), ein Bedeutungsverstehen (mit dem Blick drückt Herr X seine Warnung aus) oder ein intentionales Verstehen (Herr $\mathrm{X}$ will seine Frau damit warnen)? Offenbar fällt hier Bedeutung, Absicht und Bestimmung der Handlung zusammen. ${ }^{5}$

In einer Verstehensleistung können auch verschiedene Verstehensweisen zusammenwirken. Ein Beispiel:

m) Wenn jemand das Schematismus-Kapitel der „Kritik der reinen Vernunft" versteht, so besagt das nicht nur, daß er es sprachlich versteht, sondern auch, daß ihm der gedankliche Aufbau klar geworden ist, die Rolle des Kapitels im Ganzen der „Kritik“, seine systematische Relevanz, die Konsequenzen der Aussagen; das, was Kant mit dem meinte, was er sagte, und das, was ihn zur Annahme führte, daß jedem Begriff ein Schema zugeordnet ist, usw.

In dieser Art von Verstehen verbinden sich also Bedeutungsverstehen, determinatives Verstehen, Funktionsverstehen, Absichtsverstehen und genetisches Verstehen.

Wir können ferner dasselbe auf verschiedene Weise verstehen. Wir können z. B. dieselbe Handlung determinativ verstehen als Handlung einer bestimmten Art, wir können sie kausal verstehen, indem wir sie z. B. als durch bestimmte physiologische Vorgänge bewirkt begreifen, wir können sie intentional aus der sie leitenden Absicht verstehen, und wir können sie genetisch verstehen, indem wir erkennen, wie es zu dieser Handlung kam. Die Aussage „Ich verstehe diese Handlung“ kann also ganz verschiedenes beinhalten. An ein und derselben Sache können wir verschiedenes verstehen.

Nicht jede Erkenntnis und jedes Verstehen von Sachverhalten, die irgendwie mit einer gegebenen Sache zusammenhängen, stellt jedoch ein Verstehen dieser Sache dar. Wenn ich z. B. erfahre, aus welcher indogermanischen Wurzel unser Wort „Milch“ entstanden ist, verstehe

${ }^{5}$ Vgl. dazu auch die Bemerkung auf S. 135 zur Bedeutungstheorie von H. P. Grice. 
ich es nicht besser als vorher. Interessanter ist folgender Fall: Wir verstehen, wie ein zunächsi überraschendes oder ungewöhnlich scheinendes Ereignis eintreten konnte, oder warum es zu erwarten war, wenn wir erkennen, daß Umstände vorlagen, die es möglich oder wahrscheinlich machten. Beispiele:

n) Wir verstehen, wie es möglich war, daß der im Vereinsleben bisher kaum hervorgetretene Herr X zum Präsidenten seines Clubs gewählt wurde, wenn wir erfahren, daß zwischen den beiden anderen profilierteren Kandidaten ein unauflösliches Patt entstanden war.

o) Wir verstehen, warum es zu erwarten war, daß Frau Y eine schwere Erkrankung überstand, wenn wir erfahren, daß sie mit einem Medikament behandelt wurde, das sich in einem hohen Prozentsatz solcher Erkrankungen als wirksam erwiesen hat.

Dieses Verstehen, warum ein Ereignis p möglich oder zu erwarten war, wird in der Literatur oft als eine eigene Form des Verstehens von p bezeichnet. Es ist aber wohl angemessener, ein solches Verstehen nicht als Verstehen von $\mathrm{p}$ selbst anzusehen, sondern als Erkenntnis der Tatsache, daß $\mathrm{p}$ möglich oder wahrscheinlich war. Ich kann auch einsehen, daß $\mathrm{p}$ möglich oder wahrscheinlich ist, wenn $\mathrm{p}$ tatsächlich nicht besteht. Verstehen lassen sich aber nach üblichem Sprachgebrauch nur Tatsachen (und tatsächlich existierende Dinge). Man müßte also, wenn man erkennt, daß p möglich ist, sagen, daß ein Verstehen von $p$ nur dann vorliegt, wenn $p$ eine Tatsache ist; die Erkenntnisleistung ist davon aber unabhängig. Daher sehen wir solche Einsichten hier nicht als Formen des Verstehens von $\mathrm{p}$ an.

Ein begründetes Wissen von einer Tatsache $p$, bei dem die Gründe weder Ursachen noch Beweggründe sind, sondern irgendwelche verläßliche Kriterien, stellt ebenfalls kein Verstehen von $p$ dar. Wenn ein Arzt am Patienten gewisse Symptome beobachtet und weiß, daß sie ein untrügliches Anzeichen einer Krankheit sind, so hat er zwar ein begründetes Wissen davon, daß der Patient unter dieser Krankheit leidet, wir würden aber nicht sagen, die Kenntnis der Symptome vermittle ihm ein Verständnis der Erkrankung. Von einem Verstehen der Erkrankung kann man nur reden, wenn der Arzt sie in ihrer Eigenart (z. B. in ihrem typischen Verlauf oder ihren Folgen) näher bestimmen kann oder wenn er ihre Ursachen kennt.

Im Alltag redet man oft schon dann von einem „Verstehen“, wenn einem etwas klarer geworden ist. Wir befassen uns hier aber nicht mit einem komparativen, sondern nur mit einem klassifikatorischen Be- 
griff des Verstehens. In diesem Sinn ist ein Verstehen immer ein im Sinne der Fragestellung vollständiges, oder besser: hinreichendes Verstehen. Ein partielles Verstehen ist sicher nicht wertlos, und es spielt praktisch eine wichtige Rolle, da uns für ein vollständiges Verstehen oft die nötigen Kenntnisse fehlen. Bei der Analyse der verschiedenen Typen des Verstehens kann man sich aber auf vollständiges Verstehen beschränken.

Aufgabe einer Analyse des Verstehens ist es, Formen des Verstehens $z u$ unterscheiden und Kriterien dafür anzugeben, wann ein Verstehen der einen oder anderen Art vorliegt. Das generelle Kriterium dafür, daß jemand etwas verstanden hat, ist, daß er es erklären kann. „Etwas erklären" heißt, es verständlich machen, und ,etwas verstehen" heißt, es erklären können. ${ }^{6}$ Wenn jemand behauptet, er hätte etwas verstanden, es aber nicht erklären kann, so werden wir ihm keinen Glauben schenken. Wegen dieser engen Parallelität zwischen Verstehen und Erklären, entsprechen den angegebenen Formen des Verstehens Formen des Erklärens. ${ }^{7}$ Es gibt zunächst ein praktisches Erklären, wie man etwas macht, bei dem man jemand eine Kompetenz vermittelt, und ein theoretisches Erklären, mit dem man jemand Erkenntnis vermittelt. Unter dem Obertitel ,theoretisches Erklären“ gibt es ein determinatives Erklären, bei dem ein Vorgang näher bestimmt wird, ein Erklären der Bedeutung, bei dem diese angegeben wird, ein kausales Erklären, bei dem die Ursachen eines Ereignisses dargelegt werden, ein rationales Erklären mit Beweggründen, ein Erklären von Zwecken und Funktionsweisen. Es gibt genetische und hypothetische Erklärungen.

\footnotetext{
"Neben dieser Verwendung des Wortes „Erklärung“ im Sinn von explanatio gibt es noch eine andere Verwendung im Sinn von declaratio. In diesem Sinn redet man von einer Regierungserklärung, einer Steuererklärung, einer Erklärung vor Gericht. Für diese Bedeutung des Wortes interessieren wir uns hier nicht.

${ }^{7}$ Verstehen und Erklären wird oft als Gegensatz begriffen, so daß man das, was man verstehen kann, nicht erklären kann, und umgekehrt. Das widerspricht aber dem normalen Sprachgebrauch und beruht auf einer zu engen Deutung des Verstehens- wie des Erklärungsbegriffs. Erklären wird auch oft dem Beschreiben gegenübergestellt (vgl. z. B. Stegmüller (69), 76 ff). Dabei werden dann alle Erklärungen als Begründungen angesehen. Determinative und Bedeutungserklärungen sind aber Beschreibungen. Nicht alle Beschreibungen sind freilich Erklärungen: Wenn ich jemandem beschreibe, wie mein neues Auto aussieht, so ist das keine Erklärung.
} 
Wir können also die Kriterien für Verstehen als Kriterien für die Korrekthıit der entsprechenden Erklärungen formulieren.

Neben objektiven Kriterien für Korrektheit oder Vollständigkeit von Erklärungen gibt es auch subjektbezogene Maßstäbe für sie. So sind Erklärungen für jemand akzeptabel, überzeugend oder verständlich. Wenn jemand nicht glaubt, daß die in einer Erklärung angeführten Argumente korrekt sind, ist sie für ihn nicht akzeptabel, auch wenn sie tatsächlich korrekt ist. Wir interessieren uns hier jedoch vor allem für objektive Kriterien. ${ }^{8}$

Viele Erklärungen stellen Begründungen dar oder enthalten Begründungen. Daher müssen wir im Zusammenhang mit Erklärungen auch etwas über Begründungen sagen. In einer Begründung wird der begründete Satz - wir nennen ihn E - als Folge eines oder mehrerer begründender Sätze $A_{1}, \ldots, A_{n}$ ausgewiesen. ${ }^{9}$ Nach der Natur dieser Folgebeziehung unterscheiden wir zwei Typen von Begründungen: deduktive Begründungen, in denen gezeigt wird, daß $\mathrm{E}$ eine analytische Foige der Sätze $A_{1}, \ldots, A_{n}$ ist, und modale Begründungen, die in einer Aussage der Gestalt „E, weil A“ bestehen. Folgt $E$ analytisch aus den Sätzen $A_{1}, \ldots, A_{n}$, so gilt der Konditionalsatz ,Wenn $A_{1}$ und ... und $A_{n}$, so $E^{\prime \prime}$; sind also die Sätze $A_{1}, \ldots, A_{n}$ wahr, so gilt auch der Kausalsatz ,E, weil $A_{1}$ und $\ldots$ und $A_{n}$ ". ${ }^{10}$ Modale Begründungen umfas-

${ }^{8}$ Unter einer Erklärung kann man eine Tätigkeit und das Produkt dieser Tätigkeit verstehen. Wir befassen uns hier nur mit Produkten. Auch in der Logik wird nicht die Tätigeit des Beweisens behandelt, sondern nur die Struktur von Beweisen. Entsprechendes gilt für Begründungen.

9 Es gibt auch Begründungen mit unendlich vielen begründenden Sätzen. Der Einfachheit halber beschränken wir uns hier auf den endlichen Fall. - Da Folgebeziehungen in der Logik als Beziehungen zwischen Sätzen diskutiert werden, ist es praktisch, sich dieser Redeweise anzuschließen und von Begründungen von Sätzen mit anderen Sätzen zu reden. Man sagt aber auch oft, ein Sachverhait werde mit Tatsachen begründet. Wir brauchen hier nicht streng zwischen diesen beiden Ausdrucksweisen zu unterscheiden, denn eine Begründung des Satzes $E$ mit den Sätzen $A_{1}, \ldots, A_{n}$ ist nichts anderes als eine Begründung des Sachverhalts, den $E$ ausdrückt, mit den Sachverhalten, welche die Sätze $A_{1}, \ldots, A_{n}$ ausdrücken.

${ }^{10}$ Ein Kausalsatz im grammatischen Sinn des Wortes ist jeder Satz der Form „E, weil A“, unabhängig davon, ob A eine Ursache von E darstellt oder nicht. Auch die Aussagen „Der Luftdruck steigt, weil das Barometer steigt“ 
sen daher deduktive. Als „,modal“ im engeren Sinn kann man solche Begründungen bezeichnen, in denen $E$ keine analytische Folge von $A_{1}, \ldots, A_{n}$ ist. Meist hat man nur den deduktiven Fall im Auge, wenn von Begründungen die Rede ist, und sieht diese daher allgemein als Schlüsse an. Auch Kausalsätze wie „,Fritz hat sich ein Bein gebrochen, weil er auf einer Bananenschale ausgerutscht ist" stellen aber im normalen Sinn Begründungen dar, und man würde den Begründungsbegriff zu sehr einengen, wenn man solche Sätze ausschlösse. Es ist aber zu betonen, daß die Wahrheitsbedingungen für Konditionalsätze auf Normalitätsstandards beruhen, und daß das, was wir als normale Umstände, Folgen oder Abläufe ansehen, sich mit unseren Annahmen und Theorien ändert. Beispiele dafür bringt Passmore in (62). Da das, was nach allgemeiner Ansicht normal ist, nicht exakt festliegt, liegt auch der Wahrheitswert von Konditional- und Kausalsätzen oft nicht eindeutig fest. Das zeigt sich insbesondere bei irrealen Konditionalsätzen. Formal lassen sich die Wahrheitsbedingungen für Konditionalsätze zwar exakt bestimmen; was sich aber nicht genau sagen läßt, ist, welche formalen Bestimmungen dem üblichen Gebrauch solcher Sätze entsprechen.

Eine Begründung ist nur dann korrekt, wenn die begründenden Sätze wahr sind und die zwischen ihnen und der begründeten Aussage hergestellte Folgebeziehung gültig ist - wenn also im deduktiven Fall der Schluß von $A_{1}, \ldots, A_{n}$ auf $E$ gültig ist und im modalen Fall die Aussagen „Wenn A, dann E“. In beiden Fällen ergibt sich daraus die Wahrheit des begründeten Satzes. ${ }^{11}$ Man wird von einer Begründung von $\mathrm{E}$ auch nur dann sprechen, wenn die angegebenen Gründe $A_{1}, \ldots, A_{n}$ hinreichen um die Wahrheit von $E$ zu belegen. Daher bezeichnet man ein Argument, mit dem E nur als wahrscheinlich ausgewiesen wird, nicht als Begründung von E. Wenn z. B. ein Meteorologe die Daten angibt, auf die sich seine Wettervorhersage stützt und die sie wahrscheinlich machen, so spricht man zwar von einer Begründung seiner Vorhersage, aber nicht von einer Begründung des vorher-

und „Die Länge dieses Pendels beträgt $6.20 \mathrm{~m}$, weil seine Schwingungsdauer 5 sec beträgt" sind korrekt, obwohl der begründende Sachverhalt keine Ursache des begründeten ist. - Zur Logik der Konditional- und Kausalsätze vgl. Kutschera (76), Kap. 3.

${ }^{11}$ Aus „Wenn A, dann E“ folgt die materiale Implikation A $\supset E$; mit A muß also auch $E$ wahr sein. 
gesagten Wetters. Im üblichen Sinn des Wortes „,begründen“ lassen sich nur wahre Sätze begründen. Wenn sich der Meteorologe mit seiner Vorhersage irrt, liegt also keine Begründung des vorhergesagten Wetters vor. Da er aber mit der Angabe der Daten etwas korrekt begründet hat, kann es nur die Vorhersage sein, seine Vermutung über das morgige Wetter. ${ }^{12}$

Da solche induktiven Argumente jedoch eine wichtige Rolle spielen und auch als „Erklärungen" bezeichnet worden sind, wollen wir hier einen kurzen Exkurs darüber einschieben. In solchen Argumenten wird behauptet, daß ein Satz $E$ aufgrund gewisser Daten $A_{1}, \ldots, A_{n}$ wahrscheinlich ist, wobei ,wahrscheinlich sein" hier mindestens soviel heißt wie ,,eine Wahrscheinlichkeit größer 1/2 haben“, in einem anspruchsvolleren Sinn aber auch soviel bedeuten kann wie „eine Wahrscheinlichkeit nahe bei 1 haben". Wir beschränken uns der Kürze wegen auf den ersten Fall. Dann ist das induktive Argument für $\mathrm{E}$ also ein Satz der Gestalt $w\left(E, A_{1} \wedge \ldots \wedge A_{n}\right) \geq 1 / 2, d$. h. eine Aussage über eine bedingte Wahrscheinlichkeit von $E$. w stellt dabei eine subjektive Wahrscheinlichkeitsbewertung dar, die über eine Menge von Ereignissen definiert ist, $z u$ denen $E$ wie $A_{1}, \ldots, A_{n}$ gehören. Damit $w\left(E, A_{1} \wedge \ldots \wedge A_{n}\right)$ definiert ist, muß $w\left(A_{1} \wedge \ldots \wedge A_{n}\right)>0$ sein. ${ }^{13} U m$ $\mathrm{w}\left(\mathrm{E}, \mathrm{A}_{1} \wedge \ldots \wedge \mathrm{A}_{\mathrm{n}}\right)$ als Wahrscheinlichkeit von $\mathrm{E}$ aufgrund von $A_{1}, \ldots, A_{n}$ bezeichnen zu können, müssen $A_{1}, \ldots, A_{n}$ wahre Sätze sein. ${ }^{14}$ Ist $\mathrm{w}$ die Wahrscheinlichkeitsbewertung der Person a im Zeitpunkt $t$, so bestimmt die durch $A_{1}, \ldots, A_{n}$ bedingte Wahrscheinlichkeitsbewertung die Wahrscheinlichkeiten, die a in einem späteren 'Zeitpunkt t' den Ereignissen zumißt, wenn sich die Informationen, die a zwischen $t$ und ' $t$ ' erhält, darauf beschränken, daß die Sätze $A_{1}, \ldots, A_{n}$ gelten. Die Wahrscheinlichkeit, die a dem Satz E zumißt,

12 Wir bezeichnen daher auch induktive Bestätigungen vom Hypothesen durch einzelne Beobachtungen nicht als Begründungen dieser Hypothesen, sondern nur als Argumente dafür, daß diese Hypothesen so und so wahrscheinlich sind.

${ }^{13}$ Andernfalls müßte w von vornherein als bedingte Wahrscheinlichkeit erklärt sein.

${ }^{14}$ Man kann auch Prinzipien angeben, nach denen sich eine modifizierte Wahrscheinlichkeit für $E$ aus neuen Wahrscheinlichkeiten für $A_{1}, \ldots, A_{n}$ errechnet. Darauf wollen wir hier aber nicht eingehen. Vgl. dazu Jeffrey (65), Kap. $11,12$. 
ändert sich dann im Übergang von $t \quad z u$ ' von $w(E) \quad z u$ $w\left(E, A_{1} \wedge \ldots \wedge A_{n}\right)$. w $\left(E, A_{1} \wedge \ldots \wedge A_{n}\right)$ ist also die Wahrscheinlichkeit, die a $E$ zumißt aufgrund der Information, daß $A_{1}, \ldots, A_{n}$ gelten.

Von einem induktiven Argument für $E$ wird man nur dann reden, wenn $w\left(E, A_{1} \wedge \ldots \wedge_{n}\right)>w(E)$ ist - wenn also $E$ aufgrund von $A_{1}, \ldots, A_{n}$ wahrscheinlicher ist als vorher. Das schließt nicht aus, daß es weitere Tatsachen gibt, deren Kenntnis bewirken würde, daß man den Satz $E$ als wenig wahrscheinlich ansehen würde. Ein induktives Argument ist also für die Person a im Zeitpunkt t nur dann akzeptabel, wenn $w$, bedingt durch $A_{1}, \ldots, A_{n}$, die Wahrscheinlichkeitsbewertung von a im Zeitpunkt $t$ ist. ${ }^{15}$ Die Korrektheit induktiver Argumente kann man unabhängig davon aber so erklären:

D 2.2-1: Der Satz $w\left(E, A_{1} \wedge \ldots \wedge A_{n}\right)>1 / 2$ ist ein korrektes induktives Argument für $E$ mit $A_{1}, \ldots, A_{n}$ bzgl. der Wahrscheinlichkeitsbewertung $w$, wenn gilt:

a) $A_{1}, \ldots, A_{n}$ und $w\left(E, A_{1} \wedge \ldots \wedge A_{n}\right)>1 / 2$ sind wahre Sätze, b) $w\left(E, A_{1} \wedge \ldots \wedge A_{n}>w(E)\right.$.

Was in der Literatur unter dem Stichwort „statistische Erklärungen“ diskutiert wird, ${ }^{16}$ sind solche induktiven Argumente. Wir können hier von dem formalen Unterschied absehen, der darin besteht, daß statistische Erklärungen bei Hempel als Schlüsse formuliert sind, in denen die Sätze $A_{1}, \ldots, A_{n}$ als Prämissen und die Aussage w(E) $>1 / 2$ als Konklusion auftritt, denn diese Konstruktion beruht auf einem Mißverständnis. Dann sind Hempel statistische Erklärungen induktive Argumente, in denen unter den Bedingungen $A_{1}, \ldots, A_{n}$ auch statistische Gesetze vorkommen. Statistische Gesetze sind z. B., daß der Biß einer Kobra in $80 \%$ aller Fälle tödlich ist, oder daß in einem Zeitraum von 1590 Jahren im Mittel die Hälfte aller Radiumatome zerfallen. Solche statistischen Gesetze werden mithilfe objektiver Wahrscheinlichkeitsaussagen formuliert. Objektive Wahrscheinlichkeiten charakterisieren nicht Erwartungen von Personen, sondern die Natur der Sache. Es ist z. B. eine objektive Eigenschaft des Radiums, eine Halbwertszeit von 1590 Jahren zu haben, und diese Eigenschaft hängt mit anderen ob-

${ }^{15}$ Darauf bezieht sich Hempels Forderung, $A_{1}, \ldots, A_{n}$ müsse alle verfügbaren Informationen umfassen, die nicht schon in $w$ enthalten sind.

${ }^{16}$ Vgl. dazu Hempel (62) und (65), Abschn. 3 und 12, und Stegmüller (69), Kap. IX. 
jektiven Eigenschaften des Radiums, mit der Struktur der Radiumatome zusammen. ${ }^{17}$ Auch statistischen Hypothesen lassen sich subjektive Wahrscheinlichkeiten zuordnen, so daß man die subjektive Wahrscheinlichkeit eines Ereignisses unter der Bedingung berechnen kann, daß ein statistisches Gesetz gilt. Die sogenannten statistischen Erklärungen bilden also einen Spezialfall induktiver Argumente. Wir bezeichnen sie hier aber nicht als ,Erklärungen“, da wir oben gesagt haben, eine Erkenntnis, daß ein Sachverhalt $p$ wahrscheinlich oder zu erwarten ist, stelle kein Verstehen von $p$ dar.

Nach diesem kurzen Exkurs kehren wir zu unserem Thema „Begründung" zurück. Eine Begründung ist eine Antwort auf eine Warum-Frage. Diese Frage kann erstens eine Frage nach Ursachen sein. Dann lautet sie: „Warum ist dieses Ereignis eingetreten?" Begründungen, die eine solche Frage beantworten, bezeichnen wir als kausale Begründungen. Die begründenden Sätze oder Gründe stellen dann Ursachen für den begründeten Sachverhalt dar. Ursachen bezeichnet man auch als Seins-oder Realgründe. Die Warum-Frage kann zweitens auf Gründe in einem weiteren Sinn des Wortes abzielen, in dem alle Tatsachen, aus denen sich ergibt, daß ein Sachverhalt $p$ besteht, Gründe für das Bestehen von $p$ sind. Da man mit solchen Gründen erkennen kann, daß p besteht, bezeichnet man Gründe in diesem weiten Sinn auch als Erkenntnis- oder Vernunftgründe. Begründungen, die sich auf Erkenntnisgründe stützen, wollen wir hier epistemische Begründungen nennen.

Diese Terminologie ist nicht unproblematisch, da sie leicht zu Mißverständnissen Anlaß geben kann. Unter einem „Erkenntnisgrund“ könnte man ja auch einen Grund dafür verstehen, daß jemand etwas weiß. Auf die Frage ,Woher weiß Hans, daß p gilt?" kann die Antwort aber nicht lauten: „Weil q gilt, und weil q eine hinreichende Bedingung für $\mathrm{p}$ ist", sondern nur so: „Weil Hans weiß, daß q gilt und daß q eine hinreichende Bedingung für $p$ ist". Es läge also nahe, als „Erkenntnisgründe“ Gründe zu bezeichnen, die jemand bekannt sind. ${ }^{18}$

${ }^{17}$ Zum Begriff der objektiven Wahrscheinlichkeit vgl. z. B. Stegmüller (73), 1. Halbbd. und Kutschera (72), 2.2.

18 Entsprechend könnte man einen Sachverhalt q, von dem jemand glaubt, daß er besteht und daß er eine hinreichende Bedingung für $p$ ist, als „,Glaubensgrund" für $p$ bezeichnen. 
Wir halten hier trotzdem an der üblichen Terminologie fest und verstehen unter „Erkenntnisgründen“ allgemein wahre und hinreichende Gründe, wobei wir ,, hinreichend“ im (weiteren) modalen Sinn verstehen, so daß q eine hinreichende Bedingung für $p$ ist, falls gilt: wenn q, dann p. ${ }^{19}$ Alle Ursachen oder Seinsgründe sind also auch Erkenntnisgründe, aber nicht umgekehrt. So ist z. B. das Fallen des Luftdrucks eine Ursache für das Fallen des Barometers, aber nicht umgekehrt; das Fallen des Barometers ist nur ein Erkenntnisgrund für das Fallen an Luftdrucks.

Wir können auch sagen: Seinsgründe sind Gründe von Ereignissen, Erkenntnisgründe sind Gründe für Ereignisse, aber nicht notwendig Gründe, die jemand für eine Überzeugung hat, nicht Gründe von jemand. Ein Sachverhalt $q$ ist ein Grund von jemand für den Sachverhalt $p$, wenn er glaubt, daß q ein Grund für $p$ ist, wen sich also seine Überzeugung, daß p gilt, aus seiner Überzeugung ergibt, daß q gilt. Ein Grund für $p$ ist für die Person a nur dann akzeptabel, wenn er auch ein Grund von a für $p$ ist; aber auch von niemand akzeptierte Gründe für $p$ können korrekte Gründe für $p$, also Erkenntnisgründe für $p$ sein. Daher sei noch einmal betont, daß wir ,epistemisch“ nicht solche Begründungen nennen, die irgendetwas mit Wissen zu tun haben, die ein Wissen belegen oder zu einem Wissen führen. Es sind nicht Antworten auf die Frage „Woher weißt du, daß p?“", sondern Antworten auf die Frage nach hinreichenden und wahren Bedingungen für p. ${ }^{20}$

Sowohl deduktive wie modale Begründungen können ebenso kausal wie epistemisch sein. Kausale Begründungen sind immer auch epistemische, aber nicht umgekehrt. Als ,epistemisch“ im engeren Sinn kann man nicht-kausale Begründungen bezeichnen.

${ }^{19}$ Als „Grund“ für p bezeichnet man oft auch Tatsachen, die dafür sprechen, $\mathrm{daß}$ der Sachverhalt $\mathrm{p}$ besteht, aufgrund derer $\mathrm{p}$ also wahrscheinlich ist. Das sind dann Prämissen eines induktiven Arguments, aber nicht Prämissen von Begründungen in dem Sinn des Wortes, in dem wir es hier verwenden.

${ }^{20}$ Eine korrekte Antwort auf die Frage: ,, Woher weißt du, daß p?" ergibt natürlich auch eine korrekte epistemische Begründung für $p$, denn man weiß ja nur dann, daß die Gründe bestehen und die Geltung von $p$ garantieren, wenn das tatsächlich so ist. Und wenn ich behaupte, dies und jenes seien Gründe für $p$, drücke ich damit auch aus, daß ich sie akzeptiere. Das berührt aber nicht die Tatsache, daß korrekte Gründe nicht immer Gründe von jemand sind und umgekehrt. 
Wir haben oben gesagt, eine Begründung von $E$ mit Sätzen $A_{1}, \ldots, A_{n}$ sei nur dann korrekt, wenn $A_{1}, \ldots, A_{n}$ wahr sind und wenn im deduktiven Fall $E$ eine analytische Folge von $A_{1}, \ldots, A_{n}$ ist, und im modalen Fall der Satz gilt ,Wenn $A_{1}$ und ... und $A_{n}$, dann $E^{\prime}$. Korrekte Begründungen müssen ferner zirkelfrei sein. Der Schluß von $E$ auf $E$ ist sicher gültig, aber er steilt keine korrekte Begründung dieses Satzes dar. Eine Begründung enthält keinen formalen Zirkel, wenn unter den begründenden Sätzen $A_{1}, \ldots, A_{n}$ kein mit $E$ synonymer Satz vorkommt. Die drei genannten Bedingungen garantieren die formale Korrektheit einer Begründung. Wir können also sagen:

D 2.1 - 2: Eine (deduktive oder modale) Begründung von $\mathrm{E}$ mit den Sätzen $A_{1}, \ldots, A_{n}$ ist genau dann formal korrekt, wenn gilt:

a) Die Sätze $A_{1}, \ldots, A_{n}$ sind wahr,

b) Unter diesen Sätzen kommt keine mit E synonyme Aussage vor,

c) E folgt (analytisch oder modal) aus $A_{1}, \ldots, A_{n}$.

Formale Korrektheit ist aber nicht hinreichend dafür, daß eine Begründung brauchbar ist. Eine epistemische Begründung i.e.S. von $\mathrm{E}$ mit den Sätzen $A_{1}, \ldots, A_{n}$ dient dazu, einsichtig zu machen, daß E gilt. Sie ist also nur dann brauchbar, wenn sich die Wahrheit der Sätze $A_{1}, \ldots, A_{n}$ einsehen läßt, ohne daß man sich dabei schon auf die Geltung von $E$ stützt. Eine Begründung von $E$ mit einem Satz $A \wedge E$ ist zwar formal zirkelfrei, aber unbrauchbar, wenn man nicht erkennen kann, daß dieser Satz gilt ohne zu erkennen, ob E selbst gilt - und das wird in der Regel der Fall sein. In solchen Fällen kann man von einem Begründungszirkel in einem weiteren, pragmatischen Sinn sprechen. Solche Zirkel kann man nun nicht mit rein logischen Mitteln erfassen. Man kann z. B. nicht fordern, daß die Sätze $A_{1}, \ldots, A_{n-}$ zusammen mit $\neg E$ verträglich sein sollen, denn aus ihnen soll ja $E$ folgen. Man kann auch nicht verlangen, daß die Sätze $A_{1}, \ldots, A_{n}$ einzeln mit $\neg E$ verträglich sein sollen, da man damit Ein-Prämissen-Begründungen ausschließen würde und sich jede Begründung mit $A_{1}, \ldots, A_{n}$ als Begründung mit der Konjunktion $A_{1} \wedge \ldots \wedge A_{n}$ darstellen läßt. Endlich können Argumente derselben logischen Struktur in einem Fall brauchbare Begründungen, im anderen Fall aber im weiteren Sinn zirkulär sein. ${ }^{21}$

${ }^{21} \mathrm{Vgl.} \mathrm{dazu}$ das Beispiel von U. Blau in Stegmüller (69), 769. 
Ein prominentes Beispiel für Begründungen sind Beweise. Ein Beweis eines Satzes $E$ in einer Theorie $T$ ist eine Folge von Sätzen, deren letztes Glied E ist und für deren sämtliche Glieder gilt: sie sind Axiome von $\mathrm{T}$ oder sie folgen logisch aus vorhergehenden Gliedern der Folge. Hier werden Beweiszirkel im engeren wie im weiteren Sinn dadurch vermieden, daß die Axiome von T von vornherein als Grundlage der Beweise ausgezeichnet sind, daß ferner $E$ kein Axiom von $T$ ist, und daß in jedem Beweisschritt nur solche Sätze als Prämissen auftreten, die entweder Axiome von $T$ sind oder bereits in $T$ bewiesene Sätze darstellen, so daß man sich also auch auf sie stützen kann. Dieses Beispiel zeigt, daß man nur dann sagen kann, welche Begründungen brauchbar sind, wenn man angibt, auf welche Sätze sie sich stützen können. Von was aber eine Begründung ausgehen kann, hängt vom Kontext der Untersuchung ab. In einem Fall kann man z. B. eine Theorie voraussetzen und damit ihre Anwendungen begründen. In einem anderen Kontext hingegen, in dem die Geltung der Theorie problematisiert wird, kann man das nicht tun. Was man also bei Begründungen voraussetzen kann, ist von Fall zu Fall verschieden, und daher kann man nicht allgemein definieren, welche Begründungen brauchbar sind. Dieses Problem des Ausschlusses von Begründungszirkeln i.w.S. hat in der Diskussion des sogenannten DN-Modells der wissenschaftlichen Erklärung eine zentrale Rolle gespielt..22 Für die im folgenden diskutierten kausalen und teleologischen Erklärungen wird es sich aber als harmlos erweisen.

Nicht jede Erklärung stellt eine Begründung dar, denn in determinativen und Bedeutungserklärungen werden keine Gründe angegeben. ${ }^{23}$ Und wenn eine Erklärung sich vermittels einer Begründung vollzieht, so ist nicht gesagt, daß der erklärte Sachverhalt selbst begründet wird. Wir haben oben betont, daß Erklärungen eines Sach-

${ }^{22}$ Vgl. dazu die Diskussion in Stegmüller (69), Kap. X sowie in Käsbauer (76).

${ }^{23}$ Man muß unterscheiden zwischen Begründungen, die Bestandteil einer Erklärung sind, und solchen für die Korrektheit dieser Erklärung, z. B. für die in der Erklärung verwendeten Prämissen oder für die Gültigkeit eines in ihr verwendeten Schlusses. Wenn ich jemandem wie im Beispiel (b) einen Vorgang erkläre, so kann er Zweifel haben, ob diese Erklärung korrekt ist. Das ist dann eigens zu begründen, ohne daß diese Begründung Teil der Erklärung wäre. 
verhalts verschiedenes beinhalten können, und daher können Erklärungen desselben Sachverhalts in Begründungen verschiedener Sachverhalte bestehen. Wenn ich eine Handlung kausal erkläre, so begründe ich, daß sie stattfand. Erkläre ich sie aber rational, so begründe ich nicht, daß sie stattfand, sondern daß sie im Sinne der Präferenzen und Annahmen des Handelnden vernünftig war. Umgekehrt stellt auch nicht jede Begründung eine Erklärung dar. Kausale Begründungen sind immer kausale Erklärungen, aber epistemische Begründungen i.e.S. stellen nie Erklärungen der begründeten Sachverhalte dar, sondern allenfalls Erklärungen anderer Sachverhalte. Wenn z. B. die Länge eines Pendels mit seiner Schwingungsdauer begründet wird, so ist das keine Erklärung der Tatsache, daß der Pendel diese Länge hat. Und wenn ich die Rationalität einer Handlung begründe, so ist das keine Erklärung ihrer Rationalität, sondern eine Erklärung der Handlung selbst.

Nach diesen allgemeinen Vorbemerkungen zu den Begriffen, Verstehen', ,Erklären' und ,Begründen' wollen wir uns in den folgenden Abschnitten mit den wichtigsten Typen von Erklärungen näher befassen.

\subsection{Kausale Erklärungen}

Kausale Erklärungen stellen Antworten auf Fragen nach den Ursachen von Ereignissen dar. In einer kausalen Erklärung wird also das zu erklärende Ereignis als Wirkung bestimmter vorhergehender Ereignisse ausgewiesen. Was sind nun Ursachen? Die übliche Antwort ist: „Ein Ereignis q ist Ursache eines Ereignisses p genau dann, wenn es eine kausale Erklärung von $\mathrm{p}$ mit $\mathrm{q}$ gibt". Wir wollen daher nicht mit einer Analyse des Begriffs ,Ursache' beginnen und damit dann kausale Erklärungen definieren, sondern zunächst den umgekehrten Weg versuchen.

Anknüpfend an Gedanken, die sich z. B. schon bei Hume und J. St. Mill finden, haben C. G. Hempel und P. Oppenheim in (48) ein Modell wissenschaftlicher Erklärung entwickelt. Danach ist eine Erklärung eine formal korrekte deduktive Begründung im Sinn von D 2.1-2, die zwei zusätzlichen Bedingungen genügt: Unter den erklärenden Tatsachen sollen Naturgesetze vorkommen, und diese $\mathrm{Na-}$ 
turgesetze sollen zur Ableitung der erklärten Tatsache notwendig sein. Wegen dieser Bezugnahme auf Gesetze bezeichnet man solche Erklärungen als deduktiv-nomologische Erklärungen, kurz DN-Erklärungen. Es gilt also

D 2.2-1: Eine DN-Erklärung des Satzes E (des Explanandums) ist eine formal-korrekte deduktive Begründung von $\mathrm{E}$ mit Sätzen $G_{1}, \ldots, G_{m}$, die Naturgesetze ausdrücken, und Sätzen $\mathrm{A}_{1}, \ldots, \mathrm{A}_{\mathrm{n}}$ (den Antezedensbedingungen), wobei $\mathrm{E}$ keine analytische Folge der Sätze $A_{1}, \ldots, A_{n}$ allein ist. ${ }^{24}$

Die erklärenden Sätze bilden das Explanans. Es gibt eine Reihe von Vorschlägen für Spezifikationen dieses Grundmodells der DN-Erklärung, mit denen auch Begründungszirkel im weiteren Sinn des Wortes ausgeschaltet werden sollten. Wir haben jedoch im vorigen Abschnitt. gesehen, daß sich solche Zirkel mit logischen Mitteln nicht allgemein ausschließen lassen. Im folgenden wird sich auch zeigen, daß bei kausalen Erklärungen Zirkel automatisch verhindert werden. Daher wollen wir auf diese Spezifikationen des Grundmodells nicht eingehen.

Kausale Erklärungen werden nun im Anschluß an Hempel und Oppenheim gewöhnlich so bestimmt, daß die dabei verwendeten Gesetzesaussagen Kausalgesetze sind. Was also sind Kausalgesetze? Wir können hier nicht sagen, ein Kausalgesetz sei eine Aussage, die eine generelle Beziehung zwischen Ursachen eines Typs und ihren Wirkungen beinhaltet, also z. B. eine Aussage der Gestalt „Alle Ereignisse vom Typ X bewirken Ereignisse vom Typ Y“. Denn die Begriffe ,Ursache' und ,Wirkung' sollen ja erst mithilfe des Begriffs der kausalen Erklärung definiert werden. Kausalgesetze sind spezielle Naturgesetze. Die erste Frage ist daher, was ein Naturgesetz ist. In der wissenschaftstheoretischen Literatur findet sich keine allgemein akzeptierte Antwort darauf; was Naturgesetze sind, sieht man meist als offenes Problem an. ${ }^{25}$ Naturgesetze sind wahre gesetzesartige Aussagen.

${ }^{24}$ Allgemeiner könnte man fordern, daß alle erklärenden Sätze zur Ableitung des erklärten erforderlich sind; daß also $E$ nicht schon aus einer echten Teilmenge der Sätze $A_{1}, \ldots, A_{n}, G_{1}, \ldots, G_{m}$ folgt. - Wie in Abschnitt 2.1 verwenden wir auch hier die beiden Redeweisen „Ein Satz wird mit Sätzen erklärt" und „Eine Tatsache wird mit Tatsachen erklärt" nebeneinander.

${ }^{25}$ Vgl. dazu N. Goodman (55) sowie die Darstellung in Stegmüller (69), Kap. V. 
Das philosophische Problem ist nicht, welche gesetzesartigen Aussagen wahr sind - das festzustellen ist Sache der Naturwissenschaften -, sondern was gesetzesartige Aussagen sind. Da Naturgesetze synthetisch sind, muß das auch für gesetzesartige Aussagen gelten. Und da Naturgesetze generelle Aussagen sind, müssen gesetzesartige Aussagen die Gestalt von Allsätzen haben. Nun kann man auch dem

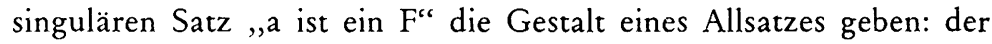
Satz ,Alle Dinge, die mit a identisch sind, haben die Eigenschaft $F^{\text {“ }}$ ist mit ,,a ist ein $F^{“}$ logisch äquivalent. Man wird also fordern müssen, $\mathrm{da}$ gesetzesartige Aussagen wesentlich generell sind, $d . h$. nicht logisch äquivalent mit einem Satz ohne Quantoren (,,alle“, ,einige"). Soweit ist die Sache klar. Es gibt nun aber viele synthetische, wesentlich generelle Sätze, die im Fall ihrer Wahrheit keine Naturgesetze darstellen, wie z. B.

a) Alle Studenten, die sich jetzt in diesem Hörsaal befinden, sind im Juni geboren.

Dieser Satz ist zwar faktisch, aber nicht logisch äquivalent mit einem Satz ohne Quantoren: Befinden sich im Hörsaal die Studenten $a_{1}, \ldots, a_{n}$, so ist der Satz faktisch, aber nicht logisch äquivalent mit ,, $a_{1}$ ist im Juni geboren und ... und $a_{n}$ ist im Juni geboren“. Auf faktische statt logische Äquivalenz können wir jedoch bei der Definition gesetzesartiger Aussagen nicht rekurrieren, da es im Fall der Endlichkeit der Welt sonst überhaupt keine gesetzesartigen Aussagen gäbe.

Man hat nun folgende Bedingungen für gesetzesartige Aussagen vorgeschlagen:

1. Gesetzesartige Aussagen dürfen nicht auf bestimmte Orte, Zeitpunkte oder Objekte Bezug nebmen.

Danach wäre zwar (a) keine gesetzesartige Aussage, aber die Forderung ist erstens zu restriktiv, denn auch Aussagen über alle Schmetterlinge auf der Erde, oder über die Bewegung der Planeten im Sonnensystem können Gesetzescharakter haben. Zweitens gibt es zu jedem Satz A logisch äquivalente Sätze A' (wie z. B. $A \wedge a=a$ ), die also im Sinn der üblichen logischen Bestimmungen des Wortes „Sachverhalt" denselben Sachverhalt ausdrücken wie A, aber die Bedingung (1) verletzen. Daher ist auch Carnaps Vorschlag, die Forderung (1) auf fundamentale Gesetze zu beschränken, nicht brauchbar. ${ }^{26}$

${ }^{26} \mathrm{Vgl}$. dazu auch die Kritik von N. Goodman an diesem Vorschlag in (55). 
2. Gesetzesartige Aussagen sollen durch Beobachtungen induktiv bestätigungsfähig sein.

Auch danach wäre (a) keine gesetzesartige Aussage. Denn die Feststellung, daß alle Studenten bis auf einen im Juni Geburtstag haben, macht es nicht wahrscheinlicher, daß auch sein Geburtstag in den Juni fällt. Die induktive Bestätigungsfähigkeit von wesentlich generellen Aussagen hängt jedoch erstens von der Anfangswahrscheinlichkeit ab, von der man ausgeht. Und zweitens sind viele wesentlich generelle Sätze induktiv bestätigungsfähig, die wir im Fall der Wahrheit nicht als Gesetze ansehen, wie z. B. der Satz „Alle Kugeln in dieser Urne sind rot".

Der beste Ansatz zur Lösung des Problems liegt in der Forderung 3. Ein Satz der Form „Alle F's sind G's" ist nur dann gesetzesartig, wenn für Objekte a, die keine $F$ 's sind, der irreale Konditionalsatz gilt „Wäre a ein $F$, so auch ein $G$ ".

Auch nach dieser Bedingung ist (a) keine gesetzesartige Aussage, denn man kann nicht behaupten ,Wäre Fritz in diesem Hörsaal, so wäre er im Juni geboren“. N. Goodman hielt in (55) diesen Vorschlag für nicht praktikabel, da es damals noch keine brauchbare Logik der Konditionalsätze gab. Nach der Entwicklung einer solchen Logik durch R. Stalnaker und D. Lewis zu Beginn der 70er Jahre ${ }^{27}$ haben aber Goodmans Einwände keine Geltung mehr. Ein indikativischer Konditionalsatz ist ein Satz der Form ,Wenn p, dann q“ - symbolisch $\mathrm{p} \rightarrow \mathrm{q}$. Ein irrealer Konditionalsatz ist ein Konditionalsatz mit falschem Antezedens. Ein Satz der Form ,Alle F's sind G's“ ist also nach (3) als gesetzesartige Aussage anzusehen, wenn er synthetisch ist und wenn gilt ,Für alle Dinge $x$ gilt: Wenn $x$ ein $F$ ist, dann ist $x$ ein G“ - symbolisch $\wedge \mathrm{x}(\mathrm{F}(\mathrm{x}) \rightarrow \mathrm{G}(\mathrm{x}))$. Wir wollen hier nicht auf die Frage eingehen, wie die Bedingung (3) für Gesetze komplexerer Struktur zu formulieren ist, z. B. für Differentialgleichungen. Das ist ein technisches Problem. Auch aus den Differentialgleichungen der klassischen Physik folgen jedoch generelle Aussagen vom Typ ,,Wenn das und das der Fall ist, so auch dies und jenes", und der intuitiv höchst plausible Gedanke ist, daß Aussagen nur dann gesetzesartig sind, wenn aus ihnen generelle konditionale Wenn-Dann Aussagen folgen, so daß wir behaupten können: „Dies und jenes ist passiert,

${ }^{27}$ Vgl. vor allem D. Lewis (73). 
weil das und das der Fall war" und „Dies und jenes wäre passiert, wenn das und das der Fall gewesen wäre".

Wir bestimmen also den Begriff der gesetzesartigen Aussage hier so, daß aus solchen Aussagen synthetische, wesentlich generelle Wenn-Dann-Sätze folgen. Damit liegt auch der Begriff des Gesetzes fest, und wir können uns nun der Frage zuwenden, was Kausalgesetze sind.

Betrachten wir folgende Gesetze:

1. Das Ohmsche Gesetz V=I·R. (Die an einen Stromkreis angelegte Spannung ist gleich dem Produkt aus der Stärke des Stroms, der in ihm fließt, und dem Widerstand.)

2. Das Fallgesetz $\mathrm{v}(\mathrm{t})=\mathrm{v}\left(\mathrm{t}_{\mathrm{o}}\right)+\mathrm{g} \cdot\left(\mathrm{t}-\mathrm{t}_{\mathrm{o}}\right)$. (Ein zur Zeit $\mathrm{t}_{\mathrm{o}}$ mit der Geschwindigkeit $v\left(t_{\mathrm{c}}\right)$ senkrecht nach unten fallender Körper hat im Zeitpunkt $\mathrm{t}>\mathrm{t}_{\mathrm{o}}$ die Geschwindigkeit $\mathrm{v}(\mathrm{t})$; $\mathrm{g}$ ist die Fallbeschleunigung.)

3. Die Scbrödingersche Wellengleichung $\mathrm{H} \psi=\frac{\mathrm{h}}{\mathrm{i}} \cdot \frac{\delta \psi}{\delta \mathrm{t}}$ (Nach dieser Gleichung ergibt sich z. B. aus der Wahrscheinlichkeitsverteilung von Teilchen im Orts- und Impulsraum zur Zeit t die entsprechende Verteilung in späteren Zeitpunkten.)

(1) und (2) sind deterministische Gesetze. Sie stellen einen Zusammenhang zwischen mehreren Zustandsgrößen (Spannung, Stromstärke und Widerstand, bzw. den Geschwindigkeiten in $t_{o}$ und $t$ ) her. (1) ist ein Koexistenzgesetz, das etwas über die Beziehungen zwischen gleichzeitigen Zuständen aussagt, (2) ein Sukzessions- oder Verlaufsgesetz, das die Abhängigkeit späterer Zustände von früheren angibt. ${ }^{28}$ (3) ist hingegen ein statistisches oder indeterministisches Gesetz, genauer: ein statistisches Sukzessionsgesetz. Es ermöglicht keine Bestimmung der

${ }^{28}$ Die Unterscheidung von Koexistenz- und Sukzessionsgesetzen ist allerdings nicht scharf. So kann man das Ohmsche Gesetz, wenn man die Größen V und I als zeitabhängig ansieht, in der Form schreiben: $\frac{d V}{d t}=R \cdot \frac{d I}{d t}$, in der es als Verlaufsgesetz erscheint. Und man kann umgekehrt das Fallgesetz in der Form $\frac{d^{2} s(t)}{d t^{2}}=g$ angeben $(s(t)$ sei der Weg, den der fallende Körper in $t z u-$ rückgelegt hat): Die Beschleunigung, die ein frei fallender Körper erfährt, hat den Wert g. Bei der Bestimmung kausaler Erklärungen kommt es aber nur darauf an, daß aus den Kausalgesetzen Aussagen über die Abfolge von Zuständen folgen. 
Orte und Impulse der Teilchen aus früheren, sondern besagt nur etwas über die Wahrscheinlichkeiten dieser Zustände bei künftigen Messungen aus. Dieses Beispiel zeigt, daß die Unterscheidung von deterministischen und statistischen Gesetzen von den Zustandsgrößen abhängt, die man ins Auge faßt: (3) ist ein deterministisches Gesetz, was die Wahrscheinlichkeitsverteilungen betrifft, ein statistisches Gesetz, was die einzelnen Orte und Impulse anlangt. Als Kausalgesetze bezeichnet man nun deterministische Sukzessionsgesetze. ${ }^{29}$

Damit haben wir Kausalgesetze ohne Rückgriff auf die Begriffe ,Ursache" und ,Wirkung' charakterisiert, und wir könnten nun diese Begriffe so erklären: Ein Ereignis p ist eine Ursache eines Ereignisses q - und $\mathrm{q}$ ist Wirkung von $\mathrm{p}$-, wenn es eine kausale Erklärung von $\mathrm{p}$ mithilfe von q gibt. Da Ursachen ihren Wirkungen stets vorausgehen und man mit Kausalgesetzen wie jenen der Newtonschen Mechanik auch frühere Zustände aus späteren ableiten kann - man kann z. B. mit dieser Mechanik aus der gegenwärtigen Konstellation der Planeten ihre Konstellation für ein Datum des Jahres 1153 errechnen müssen wir für kausale Erklärungen fordern, daß sich die Antezedensbedingungen $A_{1}, \ldots, A_{n}$ auf Ereignisse oder Zustände in früheren Zeitpunkten beziehen als das Explanandum. Zeitlose Sachverhalte, wie z. B. Naturgesetze, haben keine Ursachen. Man kann zwar Gesetze aus anderen ableiten, sie also deduktiv begründen, aber das ist keine kausale Erklärung der Gesetze; die allgemeinen Gesetze sind nicht Ursachen, sondern nur (mögliche) Erkenntnisgründe für die spezielleren. Das Explanandum einer kausalen Erklärung ist also immer ein Ereignis, das zu einem bestimmten Zeitpunkt eintritt, bzw. die Tatsache, daß ein Zustand zu einer bestimmten Zeit besteht.

Mit der Forderung, das Explanandumereignis sollte später stattfinden als die Antezedensereignisse sind nun auch Begründungszirkel i.w.S. ausgeschlossen: keins dieser Antezedensereignisse läßt sich dann zugleich kausal mithilfe des Explanandumereignisses erklären. Es kann freilich sein, daß wir eine erklärende Tatsache aus der zu erklärenden erschlossen haben. Ein Mechaniker kann z. B. feststellen, $\mathrm{da} ß$ ein Motorschaden nur dadurch entstanden sein kann, daß der Öldruck abgesunken ist, und kann dann den Schaden kausal durch

${ }^{29}$ Auf die Frage, ob der Begriff des Kausalgesetzes noch durch weitere Bedingungen zu bestimmen ist, gehen wir hier nicht ein. Für das folgende genügt die angegebene Bestimmung. Zur Diskussion dieser Frage vgl. Feigl (53) und Stegmüller (69), Kap. VII, 5. 
das Absinken des Öldrucks erklären. Dadurch wird die kausale Begrïndung nicht zirkelhaft: Das Absinken des Öldrucks wird epistemisch mit dem Motorschaden begründet, der Motorschaden kausal mit dem Absinken des Drucks. Es liegen also zwei verschiedene Begründungen vor, und keine von beiden enthält einen Zirkel.

Das DN-Modell kausaler Erklärung ist aber zu eng. Wir haben im letzten Abschnitt betont, daß es neben deduktiven auch modale Begründungen gibt, und die meisten kausalen Erklärungen, die wir im Alltag angeben, sind modale, nicht aber deduktive Erklärungen. Wenn der Mechaniker den Motorschaden erklärt oder wenn jemand sagt: „Fritz hat sich verletzt, weil er die Treppe herunter gefallen ist", so gibt er keine deduktive kausale Begründung an. In seiner Erklärung werden weder Naturgesetze angegeben, noch besteht eine Beziehung analytischer Folge zwischen Explanans und Explanandum. Man kann aber nicht sagen, all das seien nur unvollständige Erklärungen. Eine Explikation von „Erklärung“" wäre inadäquat, nach der fast alles, was wir normalerweise so nennen, keine Erklärung ist.

Ein Kausalsatz ,,p, weil q“ ist genau dann wahr, wenn gilt ,Wenn

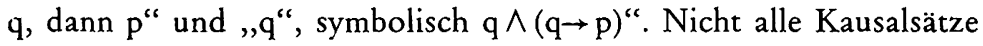
im grammatischen Sinn des Wortes geben Ursachen an. Wann stellt aiso $q \rightarrow p$ einen kausalen Nexus zwischen $q$ und $p$ her? Wir können nicht sagen: ,, Wenn q eine mögliche Ursache von $p$ ist", falls wir Ursachen wieder über kausale Erklärungen definieren wollen. Auf Kausalgesetze können wir uns hier nicht beziehen. So bleibt zunächst nur die Forderung, daß q ein früheres Ereignis sein soll als p. Genügt das? Kann man $q$ immer dann als Ursache von $p$ bezeichnen, wenn gilt $q \wedge(q \rightarrow p)$ ? Zunächst einmal schließt die Geltung von $q \rightarrow p$ nicht aus, daß auch $\neg \mathrm{q} \rightarrow \mathrm{p}$ gilt, daß also $\mathrm{p}$ stattfindet, egal ob das Ereignis $\mathrm{q}$ stattfindet oder nicht. In diesem Fall kann man aber $q$ nicht als Ursache von $p$ bezeichnen. Diese Schwierigkeit kann man dadurch eliminieren, $d a ß$ man anstelle der Bedingung $q \rightarrow p$ die stärkere Bedingung $\mathrm{q} \rightarrow \mathrm{s}$ setzt, die durch $(\mathrm{q} \rightarrow \mathrm{p}) \wedge \neg(\neg \mathrm{q} \rightarrow \mathrm{p})$ definiert ist. ${ }^{30}$ Auch mit

${ }^{30}$ Vgl. dazu Kutschera (76), S.63. - Dagegen wird man nicht fordern, daß neben $q \rightarrow p$ auch $\neg q \rightarrow \neg p$ gilt. Denn dasselbe Ereignis $p$ kann mehrere Ursachen haben, die jede für sich schon bewirken, daß p stattfindet. Dieselben Symptome können z. B. durch zwei verschiedene Krankheiten bewirkt werden; sie würden also auch dann auftreten, wenn der Patient nur eine der beiden Krankheiten hätte. 
dieser Verstärkung der Konditionalaussagen erfassen wir den üblichen Sinn des Wortes „Ursache“ noch nicht. In diesem Sinn bezeichnet man ein Ereignis q genau dann als Ursache eines Ereignisses p, wenn es vor dem Eintritt von q (im Sinn eines normalen Ablaufs der Geschehnisse) noch möglich war, daß p nicht eintreten würde, hinterher aber nicht mehr. ${ }^{31}$ Versteht man „Ursache“ freilich so, dann gibt es in einer Welt, in der alles Geschehen determiniert ist, keine Ursachen, und was selbst Wirkung ist, kann nicht Ursache sein. Die obige Explikation von Ursachen unter Rückgriff auf deduktive kausale Erklärungen ist dann inadäquat. Danach hat ja z. B. die gegenwärtige Planetenkonstellation unendlich viele Ursachen: jede Konstellation in einem früheren Zeitpunkt stellt eine solche Ursache dar; die Konstellation vor einem Jahr, einem Tag, einer Minute, einer Sekunde, einer Millisekunde usf. - all das sind Ursachen der gegenwärtigen Konstellation, und jede dieser Ursachen ist zugleich Wirkung unendlich vieler anderer Ursachen. Der Ursachenbegriff des Alltags unterscheidet sich also erheblich von dem in der Wissenschaftstheorie üblichen. Der erstere ist nur dann nicht leer, wenn der Lauf der Welt nicht von deterministischen Gesetzen beherrscht ist, wenn also das Kausalprinzip nicht gilt, ${ }^{32}$ während der letztere sich gerade auf deterministische Gesetze stützt. Wir müssen also zwei Verwendungsweisen des Wortes „Ursache“ unterscheiden: im alltäglichen Sinn gilt: „Was selbst Wirkung ist, kann nicht Ursache sein", im wissenschaftstheoretischen Sinn gilt das nicht.

Wenn wir von der Restriktion absehen, daß Ursachen keine Wirkungen sind, können wir aber den oben unter Rekurs auf deduktive kausale Erklärungen eingeführten Begriff der Ursache so erweitern, daß wir sagen:

D 2.2-2: Das Ereignis $q$ ist Ursache von p genau dann, wenn q dem Ereignis $\mathrm{p}$ vorangeht und wenn gilt $\mathrm{q} \wedge(\mathrm{q} \rightarrow \mathrm{p})$.

und

D 2.2 - 3: Eine modale kausale Erklärung des Ereignisses p ist ein wahrer Satz der Gestalt , $\mathrm{q} \wedge(\mathrm{q}-\overrightarrow{\mathrm{s}} \mathrm{p})$ “, wobei $\mathrm{q}$ ein Ereignis ist, das zu einem früheren Zeitpunkt als p stattfindet.

\footnotetext{
${ }^{31}$ Für eine genauere Charakterisierung dieses Ursachenbegriffs vgl. Åqvist (74) und Kutschera (80).

${ }^{32}$ Zum Kausalprinzip und Determinismus vgl. den Abschnitt 6.4.
} 
Zielt man hingegen auf den alltäglichen Begriff der Ursache ab, so sind beide Definitionen durch den Zusatz zu modifizieren, daß es kein Ereignis $r$ gibt, das $q$ vorausging, und für das gilt $r \rightarrow q$.

Im Alltag werden viele Argumente als kausale Erklärungen angesehen, die das auch im Sinn von D 2.2-3 nicht sind. Die Aussage „Fritz hat sich ein Bein gebrochen, weil er auf einer Bananenschale ausgerutscht ist" ist keine kausale Erklärung in unserem Sinn, da wir nicht behaupten können, daß es die normale Folge eines derartigen Ausrutschers sei, daß man sich nun gerade ein Bein bricht. Und im Fall, daß Fritz tatsächlich nicht ausgerutscht ist, können wir auch nicht behaupten ,Wäre er auf einer Bananenschale ausgerutscht, so hätte er sich ein Bein gebrochen". Solche Sätze werden aber im Alltag durchaus als Erklärungen von Ursachen akzeptiert. Sie machen eine zunächst überraschende Erscheinung - Fritz kommt eines Morgens mit einem Gipsverband ins Büro - verständlich. Ist also nicht auch die Definition D 2.2-3 noch zu eng? Und würde man nicht sagen, das Ausrutschen von Fritz sei die Ursache seines Beinbruchs? Das sind zwei verschiedene Fragen, die verschiedene Antworten erfordern. Die erste Antwort lautet: Es handelt sich hier nicht um eine vollständige, sondern um eine unvollständige kausale Erklärung im Sinn der Bemerkungen in 2.1. Die zweite Antwort ist: Nicht der Sachverhalt, daß Fritz auf der Bananenschale ausrutschte, ist Ursache dafür, daß er sich das Bein gebrochen hat, sondern die spezielle Art, in der das geschah. Das Ausrutschen von Fritz ist ein spezieller Fall des allgemeinen Vorgangs „Ausrutschen auf einer Bananenschale", der sich in vielfacher Weise vollziehen kann (einfache Bauchlandung, Salto rückwärts, usw.). Der Satz „Fritz brach sich ein Bein, weil --““ wird erst dann zu einem Kausalsatz, wenn man für ,---“ einen Satz einsetzt, der einen sehr viel spezielleren Sachverhalt ausdrückt, als die Aussage „Fritz rutschte auf einer Bananenschale aus“. Beide Sätze beziehen sich aber auf denselben Vorgang, und daher kann man sagen, dieser Vorgang sei die Ursache des Beinbruchs.

Modale kausale Erklärungen können im Weil-Satz auf Naturgesetze Bezug nehmen, tun das aber in der Regel auch dann nicht, wenn uns einschlägige Naturgesetze bekannt sind. $\mathrm{Da}$ Naturgesetze generelle Wenn-Dann-Sätze sind oder Aussagen, aus denen solche Sätze folgen, bestimmen sie schon den „normalen Lauf der Dinge“, nach dem wir die Geltung von Konditionalsätzen beurteilen. Nur wenn uns 
ein Naturgesetz bekannt ist, auf das sich unsere Erklärung stützen kann, dem Adressanten unserer Erklärung hingegen nicht, werden wir es in der Erklärung anführen, und z. B. sagen: Das Wasserrohr ist geplatzt, weil es in der Nacht gefroren hat und sich Wasser beim Gefrieren ausdehnt. Modal können wir viele Ereignisse kausal begründen, die wir deduktiv nicht begründen können, weil uns passende Naturgesetze nicht bekannt sind oder nicht all die Antezedensbedingungen, die wir zu einer deduktiven Begründung benötigen würden. Es ist oft unklar, wie sich singuläre Konditionalsätze generalisieren lassen und welche Zusatzbedingungen dabei in die Wenn-Bedingung aufzunehmen wären.

Erklärungen ohne Gesetze werden in der Literatur unter dem Stichwort ,dispositionelle Erklärungen“ behandelt. ${ }^{33}$ Eine solche Erklärung ist z. B. ,Die Fensterscheibe ist zerbrochen, weil sie zerbrechlich ist und von einem Stein getroffen wurde". Man hat nun behauptet, solche Erklärungen lieferten kein Argument gegen das DN-Modell der Erklärung, weil sie sich in DN-Erklärungen umformen ließen.

Man unterscheidet dispositionelle und manifeste Eigenschaften. Dispositionelle Eigenschaften charakterisieren Fähigkeiten, Anlagen oder Neigungen, auf gewisse Bedingungen in bestimmter Weise zu reagieren oder sich unter gewissen Umständen in bestimmter Weise zu verhalten. Sie stellen also keine unmittelbar wahrnehmbaren Eigenschaften dar, sondern treten erst unter den fraglichen Bedingungen oder Umständen in Erscheinung. Dispositionsprädikate sind z. B. ,zerbrechlich sein" (zerbrechlich sind Dinge, die schon bei verhältnismäBig geringer Gewalteinwirkung zerbrechen), ,elastisch sein" (ein fester Körper ist elastisch, wenn er seine Formänderung unter äußerer Kraftwirkung nach deren Aufhören rückgängig macht), ,magnetisch sein" (ein Körper ist magnetisch, wenn er in seiner Nähe befindliches Eisen anzieht), ,hilfsbereit sein“, usf. Die Prädikate ,zerbrechen“, „,kugelförmig sein“, ,,jemandem helfen“ drücken hingegen manifeste Eigenschaften aus. Die Unterscheidung der Eigenschaften in manifeste und dispositionelle ist nun recht vage. Blau sein ist $\mathrm{z}$. B. eine manifeste Eigenschaft, da man sie direkt beobachten kann. Man kann sie aber auch dispositionell so erklären ,Blau ist eine Oberfläche, die bei

${ }^{33}$ Vgl. dazu z. B. Hempel (65), IV 12, $₫ 9$ und Stegmüller (69), I,7. 
Bestrahlung mit physikalisch weißem Licht, Licht in dem und dem Frequenzbereich reflekciert". ${ }^{34}$ Wenn man genau zwischen analyzischen und empirischen Sätzen einer Sprache unterscheiden kann, und damit zwischen Bedeutungspostulaten für ein Prädikat und empirischen Aussagen mit diesem Prädikat, so kann man sagen: Dispositionsprädikate $D$ sind durch Bedingungen erklärt, die im einfachsten Fall die Gestalt haben:

1. Das Objekt $x$ hat die Eigenschaft $D$ genau dann, wenn es die Reaktion $R$ zeigt, falls es der Testbedingung $T$ unterworfen wird.

Also z. B. „,x ist wasserlöslich genau dann, wenn sich $\mathrm{x}$ auflöst, falls man $x$ in Wasser gibt". Allgemeiner kann es sich um Systeme notwendiger oder hinreichender Bedingungen für das Vorliegen von $D$ handeln. ${ }^{35}$ Hempel hat darauf hingewiesen, daß man nicht alle solche $\mathrm{Be}-$ dingungen für ein Dispositionsprädikat als Bedeutungspostulate ansehen kann. Aus den beiden Kriterien: „, $\mathrm{x}$ ist genau dann magnetisch, wenn $x$ Eisenspäne anzieht falls man diese in die Nähe von $x$ bringt" und , $\mathrm{x}$ ist genau dann magnetisch, wenn $\mathrm{x}$ in einem Kreisleiter einen Strom induziert falls man es durch diesen Leiter hindurch führt" folgt z. B. der Satz: ,Wenn man Eisenfeilspäne in die Nähe von $x$ bringt und $\mathrm{x}$ durch einen Kreisleiter führt, so zieht $\mathrm{x}$ die Späne genau dann an, wenn $x$ im Leiter einen Strom induziert". Dieser Satz, in dem das Prädikat ,magnetisch“ nicht mehr vorkommt, ist offenbar empirisch. Höchstens eines der beiden Kriterien kann also analytisch gelten. In natürlichen Sprachen ist aber meist nicht klar, welche Sätze Bedeutungspostulate und welche empirische Behauptungen sind. ${ }^{36}$ Man kann also nur sagen:

${ }^{34}$ Stegmüller bestimmt in (70), 1. Halbbd., S. $213 \mathrm{ff}$ dispositionelle Eigenschaften so: „Als Kriterium dafür, ob wir mit einem Ausdruck eine Disposition oder eine manifeste Eigenschaft bezeichnen wollen, kann man folgendes benützen: Sofern der Satz ,,a ist P“ einen anderen Sinn hat als ,, a scheint P zu sein", so bezeichnet „P" eine Disposition. Wenn hingegen Sinngleichheit besteht, so liegt eine manifeste Eigenschaft vor" (S. 214). Danach würde aber kein Prädikat eine manifeste Eigenschaft ausdrücken, da ,,a scheint (mir) $\mathrm{P}$ zu sein" immer einen anderen Sinn hat als ,,a ist P“.

${ }^{35}$ Auch beim Bestehen statistischer Korrelationen zwischen Testbedingungen und Reaktionen spricht man oft von Dispositionen.

${ }^{36}$ Vgl. dazu den Abschnitt 9.1. 
2. Wenn ein Prädikat durch Bedingungen wie (a) erklärt ist, oder wenn solche Bedingungen analytisch gelten, ist es ein Dispositionsprädikat.

In der Literatur ist die Frage, ob und ggf. wie sich Dispositionsprädikate durch manifeste definieren lassen, ausführlich diskutiert worden. ${ }^{37}$ Das Ergebnis ist, wie im Fall der gesetzesartigen Aussagen: (1) ist nur dann eine adäquate Definition des Dispositionsprädikats D, wenn die Wenn-Dann-Bedingung im Definiens konditional interpretiert wird. Deutet man das ,,wenn-dann“ nur als materiale Implikation, so haben alle Objekte die Eigenschaft D, die der Testbedingung nicht unterworfen werden - es wären also z. B. alle Stoffe wasserlöslich, die nie in Wasser gegeben wurden. Aus (1) soll aber für $D(x)$ auch folgen „Würde man $x$ der Testbedingung $T$ unterwerfen, so würde $\mathrm{x}$ die Reaktion $\mathrm{R}$ zeigen".

Die Behauptung, dispositionelle Erklärungen ließen sich in DN-Erklärungen umformen, stützt sich nun darauf, daß es passende empirische Gesetze über Dispositionsprädikate gibt, mit denen man eine DN-Erklärung formulieren kann. Das Argument: „Die Scheibe ist zerbrochen, weil sie zerbrechlich ist und von einem harten Gegenstand getroffen wurde" ließe sich z. B. umformen in die DN-Erklärung:

Die Scheibe ist zerbrechlich.

Was zerbrechlich ist, zerbricht, wenn es von einem harten Gegenstand getroffen wird.

Die Scheibe wurde von einem harten Gegenstand getroffen.

Die Scheibe ist zerbrochen.

Hier wird man jedoch die generelle Prämisse nicht als Naturgesetz, sondern eher als Bedeutungserklärung von ,zerbrechlich“, also als analytischen Satz ansehen, so daß dieses Argument kaum haltbar ist. Freilich werden sich viele solche dispositionelle Erklärungen in DNErklärungen umformen lassen, wenn man das Explanans entsprechend modifiziert und z. B. von den Sätzen „Die Scheibe ist aus Glas" und „Alles, was aus Glas besteht, ist zerbrechlich“ ausgeht.

Läßt man auch modale Erklärungen zu, so bilden dispositionelle Erklärungen kein Problem. Für die Korrektheit einer Erklärung ist es dann nicht erforderlich, daß im Explanans Gesetze vorkommen.

${ }^{37}$ Für eine Darstellung dieser Vorschläge vgl. z. B. Stegmüller (70), 1. Halbbd. IV,1. 


\subsection{Teleologische Erklärungen}

In der Literatur wird fast alles, was mit Zielen, Absichten, Beweggründen, Zwecken, Funktionen oder Funktionsweisen zusammenhängt, unter den Titel „Teleologie“ gestellt. Da wir in 2.1 als teleologišche Erklärungen nur funktionale, intentionale und rationale aufgeführt haben, werden wir uns also im folgenden auch zu überlegen haben, ob damit die wichtigsten Typen dessen erfaßt sind, was man sonst als ,,teleologische Erklärungen“ bezeichnet. Erklärungen können, wie wir in 2.1 sahen, sowohl Feststellungen sein als auch Begründungen. Teleologische Erklärungen, die nur in Feststellungen bestehen, könnte man als determinativ-teleologische Erklärungen bezeichnen, denn sie sind determinative Erklärungen im Sinne von 2.1. Sie beschränken sich auf die Behauptung, das und jenes sei Ziel oder Zweck der infrage stehenden Handlung oder Sache. Sie sind also korrekt, wenn diese Behauptung richtig ist, und daher werfen solche Erklärungen von ihrer Struktur her keine Probleme auf. Determinativteleologisch sind insbesondere funktionale und intentionale Erklärungen.

Als funktionale Erklärungen haben wir Erklärungen bezeichnet, wie etwas funktioniert oder welche Funktion es hat. Erklärungen von Funktionsweisen geben Auskunft, wie ein Maschinenteil oder eine ganze Maschine, wie ein Organ oder eine Institution funktioniert. Eine funktionale Erklärung liegt aber auch vor, wenn nicht dargelegt wird, wie etwas funktioniert, sondern welche Funktion es für etwas anderes hat, welche Rolle es z. B. in einem großen Zusammenhang spielt, wozu es dient. So kann man einerseits die Funktionsweise eines Ventils erklären, andererseits aber auch die Funktion, die es für das Funktionieren einer Dampfmaschine hat. Und die Erklärung der Funktionsweise der Nieren ist etwas anderes als die Erklärung der Funktion, welche die Nieren für den menschlichen Stoffwechsel haben.

Wir sagen gewöhnlich nur dann, eine Sache habe eine gewisse Funktion, Rolle oder Aufgabe für etwas anderes, wenn sie einen positiven Effekt dafür hat. In der Rede von „Funktionen“ und „Aufgaben" liegt also eine Wertung. Ein Krebsgeschwür hat Wirkungen auf den Organismus, wir sagen aber nicht, es hätte eine „Funktion“ für ihn. Angaben über bloße Wirkungen sind keine teleologischen Erklärungen. Die Wertungen kommen besonders klar zum Ausdruck bei 
der Rede von Zwecken. Wenn etwas einen Zweck hat, zweckmäßig ist, so hat es einen Nutzen. Das Ventil an einem Dampfkessel dient dazu, das Entstehen eines Überdrucks zu verhindern. Entsteht ein Überdruck im Kessel, so kann dieser platzen. Das Ventil ist also wichtig für die Erhaltung und das Funktionieren des Dampfkessels, bzw. der ganzen Anlage, zu der er gehört. Vom Nutzen einer Sache spricht man in der Regel in einem relativen Sinn, in dem etwas unter einem Aspekt oder für etwas nützlich, unter einem anderen Aspekt oder für eine andere Sache hingegen schädlich sein kann. Für das Funktionieren der Anlage ist das Ventil nützlich, unter einem anderen Aspekt kann es aber nützlich sein, wenn die Anlage nicht funktioniert. Unter diesem Aspekt ist also das Ventil nicht nützlich. Den Aspekt, unter dem ein Nutzen behauptet wird, geben wir bei funktionalen Erklärungen in der Regel nicht an; er ist meist durch den Kontext bestimmt. Wenn man sagt „Verwaltungsgerichte haben die Aufgabe, den Einzelnen gegen fehlerhafte Verwaltungsakte zu schützen", so ist klar, daß der Nutzen der Verwaltungsgerichtsbarkeit ein Nutzen für den einzelnen Bürger ist.

Funktionserklärungen sind also für uns Zweck-Erklärungen. Wenn wir das Wort „Zweck“ so verwenden, daß ein Zweck einer Sache ein Nutzen dieser Sache für etwas oder jemand ist, der ihr aufgrund ihrer Natur, ihrer Wirkung oder Funktionsweise zukommt, so ist klar, daß Zweckmäßigkeit nicht immer Zweckmäßigkeit für Personen ist, und Zweckmäßigkeit auch nicht immer aus planvollem Handeln entsteht. Zweckmäßigkeit gibt es dann nicht nur da, wo ein zwecksetzender Wille am Werk ist. In der Literatur wird die Rede von ,Zweckmäßigkeit" hingegen oft in diesem engeren Sinn verwendet. Das entspricht aber nicht dem üblichen Gebrauch des Wortes „Zweck", nach dem z. B. Nieren nicht nur beim Menschen, sondern auch bei Tieren einem Zweck dienen; Nieren sind auch nicht Produkte planmäßigen Handelns. Der „Nutzen“ einer Sache im engeren Sinn des Wortes ist sicherlich immer ein Wert, den sie für eine Person hat, und dieser Nutzen ergibt sich aus den Präferenzen der Person. Im weiteren Sinn spricht man aber auch vom Nutzen einer Sache für einen Gegenstand, ein Lebewesen, einen Zustand, wenn sie dessen Erhaltung dient, wenn sie seiner Entwicklung oder Entfaltung förderlich ist. ${ }^{38}$ Bezeichnet

${ }^{38}$ Kant unterscheidet in der ,Kritik der Urteilskraft" „Zwecke der Freiheit“ und „Zwecke der Natur", meint aber, Zweckmäßigkeit ließe sich nur be- 
man alle Aussagen über Funktionsweisen oder Wirkungen als ,,teleologisch", so verwendet man das Wort in einem weiteren Sinn als das üblich ist. Solche Aussagen kommen ja auch in der Physik vor, die nach allgemeinem Verständnis außerhalb der Domäne der Teleologie liegt. Im normalen Sinn haben nur solche Sätze einen teleologischen Géhalt, in denen sich Aussagen über Funktionsweisen oder Wirkungen mit Aussagen über deren Nutzen verbinden, also mit Wertaussagen. In einem noch engeren Sinn ist von teleologischen Aussagen die Rede, wenn dieser Nutzen ein Nutzen für Personen ist oder ein objektiver Wert, z. B. ein moralischer oder ästhetischer Wert. Es gibt also ein breites Spektrum von Verwendungsweisen des Wortes ,teleologisch", und daher ist bei jedem Autor zu prüfen, wie seine Behauptungen über den teleologischen Charakter von Sätzen, Erklärungen etc. gemeint sind. Wir verwenden das Wort hier wie gesagt so, daß nur Aussagen ,teleologisch" heißen, die Behauptungen über den Nutzen einer Sache im weiteren Sinn dieses Wortes implizieren.

Bei intentionalen Erklärungen geht es nicht um den Zweck, dem eine Handlung dient, sei es ein Zweck für den Handelnden selbst oder für etwas anderes (z. B. für die Gesellschaft), sondern um die Absicht, bzw. die Absichten, die sie leiten. Eine Handlung kann absichtlich, aber zwecklos sein - nämlich dann, wenn sie ihr Ziel nicht erreicht. Eine intentionale Erklärung ist eine korrekte Antwort auf die Frage nach der oder den Absichten oder Zielen einer Handlung. Im Beispiel (h) von 2.1 erkläre ich jemandem, wozu Max im Herbst damit beginnt, täglich einen Dauerlauf zu absolvieren, wenn ich ihm mitteile, daß sich Max damit für den winterlichen Skilauf in Form bringen will. Mit derselben Handlung kann man mehrere Absichten verfolgen. Es kann sein, daß Max seine Läufe auch unternimmt, um sein Gewicht zu reduzieren. Eine intentionale Erklärung kann also die Absichten einer Handlung mehr oder minder vollständig darlegen. Auch eine unvollständige Angabe der Absichten ist aber eine korrekte intentionale Erklärung, wenn auch sicherlich nicht immer eine korrekte Antwort auf die Frage nach den Absichten, oder nach der Absicht, wenn darunter die leitende Absicht verstanden wird.

greifen als hervorgebracht durch eine planende Intelligenz (vgl. WW 8, $165 \mathrm{ff})$. 
Das einzige Problem, das sich im Zusammenhang mit intentionalen Erklärungen stellt, ist das einer Explikation der Ausdrücke „Absicht“ und „Ziel“. Da wir diese beiden Begriffe in den folgenden Abschnitten verwenden, wollen wir etwas näher auf sie eingehen.

Die Aussage

1. Ich beabsichtige mit der Handlungsweise $F$ zu erreichen, daß ein $Z u$ stand (Ereignis) $p$ eintritt

gilt nur unter folgenden Bedingungen:

2. Ich tue $F$.

Wir̀ sprechen zwar auch von Absichten, die sich nicht mit gleichzeitigen Handlungen verbinden; ich kann z. B. heute die Absicht haben (planen), meinen nächsten Urlaub auf Mykonos zu verbringen. Das sind dann aber Absichten, etwas zu tun, nicht Absichten von (oder bei) Handlungen, für die wir uns hier allein interessieren.

3. Ich glaube nicht, daß p obnebin eintreten wird, egal ob ich $F$ tue oder nicht. ${ }^{39}$

Denn was ohnehin sein wird, wird nicht durch mein Handeln erreicht.

4. Ich bin davon überzeugt, daß p eintritt, wenn ich F tue.

Glaube ich, daß p nicht eintritt, wenn ich $F$ tue, so kann es nicht Absicht dieser Handlung sein zu erreichen, daß p eintritt, da ich ja dann überzeugt bin, daß das nicht der Fall sein wird. Ich muß es also mindestens für möglich halten, daß $\mathrm{p}$ eintritt, wenn ich $\mathrm{F}$ tue. ${ }^{40}$ Sehe ich es aber darüber hinaus nicht auch als wahrscheinlicher an, daß p eintritt, wenn ich $F$ tue, als wenn ich das nicht tue, so kann ich mit meinem F-Tun nicht erreichen wollen, daß p; ich glaube ja dann, daß mein F-Tun hinderlich oder zumindest nicht förderlich ist für das Eintreten von p. Auch das genügt aber nicht. Ist es nur wahrscheinlicher, daß p eintritt, falls ich $F$ tue, als wenn ich das nicht tue, so wird man nicht sagen, ich täte $F$ in der Absicht zu erreichen, daß p, son-

${ }^{39}$ Absichtliche Handlungen gehen aus einer Entscheidung hervor. Daher sind Aussagen über Wahrscheinlichkeiten, Präferenzen (die auch von Wahrscheinlichkeiten abhängen, vgl. 2.4) und Überzeugungen, die solchen Handlungen zugrundeliegen, auf den Zeitpunkt der Entscheidung zu beziehen, nicht auf den Moment der Handlung. Im Moment der Handlung ändern sie sich, da man nun z. B. weiß, daß man so handelt, daß also nicht all das, was vorher möglich war, auch jetzt noch möglich ist.

${ }^{40}$ Die Redeweise ,für möglich halten“ wird hier im subjektiven Sinn verstanden. Vgl dazu S. 60. 
dern nur, ich täte $\mathrm{F}$ in der Hoffnung, daß dann p eintritt. Wenn jemand Lotio spielt, beabsichtigt er damit nicht, den Hauptgewinn zu erzielen - er vermutet ja nicht einmal, daß er gewinnen wird, da er weiß, wie minimal seine Chancen sind - , sondern er tut es in der Hoffnung zu gewinnen. Ich kann also (1) nur dann behaupten, wenn ich mir bzgl. des Resultats p sicher bin. Das entspricht auch der Rede von „Absichten" im Recht."

5. Ich will, daß p eintritt.

Damit grenzt man das, was beabsichtigt wird, von dem ab, was nur in Kauf genommen wird. Wenn z. B. Herr X ins Theater geht, obwohl er weiß, daß er dort seinen Kollegen $Y$ treffen wird, dem er nur ungern begegnet, so würde man sagen: $\mathrm{X}$ geht nicht ins Theater in $\operatorname{der} A b$ sicht, Y zu treffen; seine Absicht ist, das Stück zu sehen, und die Begegnung mit $Y$ nimmt er nur in Kauf als eine unter den gegebenen Umständen unvermeidliche Begleiterscheinung seines eigentlichen Ziels. Man sagt auch, bei einer Handlung nehme man das in Kauf, was man nur als mögliches Resultat der Handlung ansieht. In diesem Sinn würde $\mathrm{X}$ es bei seinem Theaterbesuch in Kauf nehmen, den Kollegen $Y$ zu treffen, wenn er es nur für möglich hielte, ihm im Theater zu begegnen. Was in diesem Sinn in Kauf genommen wird, kann aber schon nach (4) nicht als Absicht gelten. Als ,,beabsichtigt" bezeichnet man auch im juristischen Sprachgebrauch nur ein Resultat, von dem der Handelnde will, daß es eintritt. Die Forderung (5) bewirkt freilich, daß man nicht allen Handlungen, bei denen sich der Handelnde bzgl. ihres Resultats sicher ist, eine Absicht zuordnen kann. Das gilt aber nur für nicht-rationale Handlungen, und solchen Handlungen läßt sich nicht immer in sinnvoller Weise eine Absicht zuordnen. In der Bedingung (5) taucht nun das Wort ,,wollen“ auf, das selbst erklärungsbedürftig ist. Dieses Wort wird in drei verschiedenen Bedeutungen verwendet: Wenn ich am nächsten Sonntag nach München fahren will, so plane ich, das zu tun. Wenn ich meinen Freund durch einen

${ }^{41}$ Bei Entscheidungen unter Risiko, bei denen die Resultate der Handlungsalternativen nach Einschätzung des Handelnden von Umständen abhängen, denen er nur mehr oder minder große Wahrscheinlichkeiten zuzuordnen vermag, kann man auch deswegen nicht von Absichten sprechen, weil hier rationale Entscheidungen nicht Entscheidungen für gewisse Resultate sind, sondern Entscheidungen mit dem Ziel, den zu erwartenden Nutzen zu maximieren. Vgl. dazu den Abschnitt 2.4. 
Anruf an eine Verabredung erinnern will, so beabsichtige ich das mit dem Anruf zu tun. Und wenn ich will, daß mein Freund mich besucht, so wünsche ich das. Für (5) kommt nur die letzte Bedeutung in Betracht. Auf diesen Begriff des Wollens werden wir aber erst im nächsten Abschnitt eingehen, wenn wir uns näher mit Präferenzen beschäftigt haben.

6. Das Ereignis $p$ ist verschieden von dem Ereignis, daß ich F tue.

Mit der F-Handlung kann ich nicht zu erreichen beabsichtigen, daß ich $\mathrm{F}$ tue. Einer Handlung kann man nur dann eine Absicht zuordnen, wenn sie für den Handelnden ein Mittel ist, etwas zu erreichen. Wenn ich spazieren gehe, einfach um spazierenzugehen, so ist das zwar eine willentliche Handlung, aber keine absichtliche.

Sind nun die Bedingungen (2) bis (6) hinreichend für die Geltung von (1)? Als ,Absichten“ einer Handlung bezeichnet man oft nur das, woran der Handelnde bei seiner Entscheidung gedacht hat. Es kann sein, daß jemand im dispositionellen Sinn des Wortes ,glauben“ glaubte, daß seine Handlung das Resultat $p$ haben würde, daran aber nicht gedacht hat. Wenn wir also (1) durch die Bedingungen (2) bis (6) erklären, so stellt das wegen dieser Abweichung vom normalen Gebrauch eine Explikation des Ausdrucks ,,beabsichtigen“ dar. Ob es eine sinnvolle, fruchtbare Explikation ist, muß sich in den Anwendungen zeigen. Sie genügt jedenfalls für diejenigen Fälle, in denen wir den Begriff im folgenden verwenden.

Als Ziel einer Handlung bezeichnen wir das, was der Handelnde damit zu erreichen beabsichtigt. Das Ereignis $\mathrm{p}$ ist also ein Ziel des FTuns einer Person a, wenn sie mit dieser Handlung beabsichtigt zu erreichen, daß p eintritt. Statt ,Ziel“" sagt man auch ,Absicht“. Dann ist eine Absicht nicht ein Sachverhalt des Beabsichtigens, sondern ein beabsichtigter Sachverhalt. Das Ziel einer Handlung ist nicht immer ein Zweck dieser Handlung. Erreicht sie ihr Ziel nicht, so erweist sie sich, wie schon gesagt wurde, für den Handelnden als zwecklos.

In einer Reihe von Arbeiten hat H. P. Grice eine Konzeption der Bedeutung sprachlicher Äußerungen entwickelt, nach der Bedeutungsverstehen eine spezielle Art intentionalen Verstehens ist. ${ }^{42}$ Dieser

${ }^{42} \mathrm{Vgl}$. insbesondere Grice (57) und (68) sowie Lewis (69). - Für ein einfaches Modell zur Rekonstruktion dieser Ideen vgl. Meggle (81) und Kutschera (79a). 
Ansatz ist sprachphilosophisch außerordentlich interessant; er ist auch der einzige, mit dem man nicht-konventionalisierte Ausdrucksweisen beschreiben und den Unterschied erfassen kann zwischen dem, was jemand sagt, und dem, was er damit meint. Wir erwähnen diese Ideen - näher können wir hier nicht darauf eingehen - um die Wichtigkeit des Begriffs intentionaler Erklärungen zu unterstreichen.

Als determinativ-teleologische Erklärungen, die nicht funktionale oder intentionale Erklärungen sind, kommen nur Beschreibungen von Beweggründen infrage, die jemand für eine Handlung oder Verhaltensweise hat. Was unter Beweggründen zu verstehen ist, soll jedoch erst im nächsten Abschnitt erörtert werden.

In Begründungen können teleologische Aussagen (Aussagen über Ziele, Absichten, Gründe, Funktionen, Zwecke und Funktionsweisen) als Prämissen oder als Konklusionen auftreten. Mit Begründungen von teleologischen Aussagen brauchen wir uns hier nicht weiter zu befassen; es sind begründete determinativ-teleologische Erklärungen, über deren Sinn wir schon gesprochen haben. Was läßt sich nun miı Zielen, Zwecken, Funktionen etc. erklären oder begründen? Lassen sich insbesondere nicht-teleologische Aussagen mit teleologischen erklären? Das ist die zentrale Frage der Diskussion teleologischer Erklärungen, und wir wollen uns auf die Erörterung dieser Frage beschränken.

Im Abschnitt 2.1 haben wir betont: Eine Erklärung der Tatsache p, die in einer Begründung besteht, ist nicht immer eine Begründung von $p$, sondern kann auch in der Begründung einer Aussage über $p$ bestehen. Die Frage nach der Möglichkeit teleologischer Erklärungen nicht-teleologischer Aussagen hat also zwei Aspekte:

1. Gibt es teleologische Erklärungen von nicht-teleologischen Tatsachen, die Begründungen dieser Tatsachen darstellen?

2. Gibt es teleologische Erklärungen von nicht-teleologischen Tatsachen, die Begründungen von Aussagen über diese Tatsachen darstellen?

Meist denkt man nur an den ersten Fall, da man Erklärungen von Tatsachen allgemein als Begründung dieser Tatsachen ansieht.

Die erste Frage ist nun zu bejahen. Beispiele solcher Erklärungen sind etwa folgende:

a) Diese Dampfmaschine kann nur dann funktionieren, wenn das Entstehen eines Überdrucks im Dampfkessel verhindert wird. Allein 
ein Ventil am Kessel erfüllt diesen Zweck. Nun funktioniert die Maschine. Also muß am Kessel ein Ventil angebracht sein.

b) Die Burg konnte nur erobert werden, wenn die Belagerer über eine Vorrichtung zum Schleudern schwerer Geschosse verfügten. So etwas konnte damals nur ein Katapult sein. Die Burg wurde erobert. Also müssen die Belagerer Katapulte gehabt haben.

Bei solchen Erklärungen ist zu beachten: Die Tatsache allein, daß ein Gegenstand der Art X geeignet ist, einen Zweck, eine Funktion oder Aufgabe zu erfüllen, genügt neben der Prämisse, daß diese Aufgabe erfüllt worden ist, noch nicht für eine Begründung, daß ein $\mathrm{Ge}$ genstand der Art $\mathrm{X}$ vorhanden war. Vielmehr muß auch gezeigt werden, daß unter den gegebenen Umständen nur ein $X$ diese Funktion erfüllen konnte. (a) und (b) sind Beispiele epistemischer Begründungen. Es gibt aber auch kausale Begründungen mit Zwecken, z. B.:

c) In der Gemeinde $Y$ ist der Strom ausgefallen, weil ein Generator im Elektrizitätswerk nicht mehr funktionierte.

Alle drei Beispiele könnte man nun insofern als fragwürdig ansehen, als sich die Prämissen durch Aussagen ersetzen lassen, die nur von Wirkungen, nicht aber von Nutzenswerten und damit auch nicht von Zwecken reden. Diese Kritik ist aber nicht haltbar. Aus Aussagen über Zwecke und Absichten folgen analytisch ,natürliche“ Aussagen, in denen von Werten, Zwecken und Absichten nicht mehr die Rede ist. Aus „Fritz beabsichtigt mit seinem Anruf bei Max, diesen daran zu erinnern, daß sie für morgen verabredet sind“, folgt „Fritz ruft Max an“. Und aus „Der Zweck der Verordnung ist es, den Energieverbrauch einzudämmen" folgt, daß der Erlaß der Verordnung diese Wirkung hat. Daher gilt umgekehrt auch: Aus einigen natürlichen, d. h. nicht-teleologischen Aussagen folgen analytisch teleologische. Die bloße Umformulierung beseitigt also nicht den teleologischen Gehalt einer Aussage; auch natürliche Aussagen können einen teleologischen Gehalt haben. Eine bloß syntaktische Unterscheidung teleologischer und nicht-teleologischer Aussagen nach dem Kriterium, ob in ihr Ausdrücke wie ,Zweck" und ,Absicht" vorkommen oder nicht, ist also irrelevant. Und wegen dieser analytischen Abhängigkeiten zwischen teleologischen und nicht-teleologischen Aussagen kann man Sätze der einen Art mit Sätzen der anderen begründen.

Teleologische Erklärungen einer nicht-teleologischen Tatsache, die Begründungen von Aussagen über diese Tatsachen darstellen, sind 
z. B. Erklärungen des Zwecks oder der Absicht von Handlungen. Wie schon in 2.1 erwähnt wurde, kann man eine Handlung auch durch den Hinweis erklären, daß sie mit geltenden rechtlichen oder gesellschaftlichen Normen übereinstimmt. Man kann z. B. einem Fremden ein für ihn merkwürdiges Verhalten eines Einheimischen dadurch erklären, daß man darauf hinweist, daß es hierzulande Sitte ist, sich so zu benehmen. Damit begründet man nicht, daß er sich so verhält niemand ist gezwungen, geltende Verhaltensregeln zu befolgen -, sondern daß sein Verhalten gewissen Konventionen entspricht. Auch das ist aber eine Erklärung der Handlung, und diese Erklärung kann sich in Form einer Begründung vollziehen. Den weitaus wichtigsten Typ von Begründungen von Aussagen über nicht-teleologische Tatsachen mit teleologischen Prämissen bilden jedoch rationale Erklärungen. Auf sie gehen wir aber erst im nächsten Abschnitt ein.

Als finale Erklärungen bezeichnet man oft Erklärungen von Ereignissen durch andere Ereignisse, die später stattfinden; Erklärungen also, bei denen die zeitliche Ordnung zwischen Explanans und Explanandum gegenüber kausalen Erklärungen umgekehrt ist. ${ }^{43}$ Solche Begründungen sind sicher nicht kausal, sondern epistemisch; sie sind aber von teleologischen Begründungen zu unterscheiden. Die Begründung einer Handlung mit Absichten oder Beweggründen sind nicht final, da sie nicht die gegenwärtige Handlung mit dem künftigen Ereignis begründen, das der Handelnde herbeiführen will, sondern mit gegenwärtigen oder vorausgehenden Beweggründen. Und eine Begründung mit dem Zweck oder der Funktion einer Sache ist nicht eine Begründung mit ihren künftigen, sondern mit ihren gegenwärtigen oder vergangenen Wirkungen.

Finale Begründungen können auch dort vorliegen, wo weder die Prämissen, noch die Konklusionen etwas mit Zwecken oder Absichten zu tun haben. Wenn man z. B. die Konstellation der Planeten zu Christi Geburt mit den Gesetzen der Mechanik aus ihrer heutigen Konstellation ableitet, so ist das in diesem Sinn eine finale Begründung; sogar eine finale Begründung mit Kausalgesetzen. Es gibt auch finale Erklärungen mit statistischen Gesetzen. Wenn z. B. der Ablauf

${ }^{43} \mathrm{~W}$. Stegmüller verwendet in (69) die Ausdrücke ,teleologisch" und „final" in gleichem Sinn und bezeichnet das, was wir hier ",finale Begründungen“ nennen als ,formal-teleologische“. 
eines Systems, das gewisse diskrete Zustände $Z_{1}, Z_{2}, \ldots$ annehmen kann, durch die statistischen Übergangswahrscheinlichkeiten von einem Zustand in den anderen charakterisiert wird, so kann es vorkommen, daß das System den $Z$ ustand $Z_{i}$ nur dann annehmen kann, wenn es vorher im Zustand $Z_{j}$ war. Obwohl man evtl. nicht mit Sicherheit sagen kann, ob es in $Z_{i}$ übergeht, wenn es in $Z_{j}$ ist (weil die Wahrscheinlichkeit des Übergangs in einen anderen Zustand größer als Null ist), kann man dann doch sagen: Wenn es in $Z_{i}$ ist, so muß es vorher in $Z_{j}$ gewesen sein. Es gibt ferner Naturgesetze, die ein Geschehen so beschreiben, daß es auf einen bestimmten Zustand hin verläuft; daß z. B. ein Gleichgewichtszustand erreicht wird. Solche ,,finalen" Gesetze finden sich in der Physik. Nach dem vermutlich von Leibniz entdeckten, aber nach Maupertuis benannten Wirkungsprinzip verläuft das mechanische Geschehen in einem abgeschlossenen System so, daß die Wirkung minimal ist. ${ }^{44}$ Auch in der geometrischen Optik finden sich ,finale“ Gesetze. So nimmt das Licht beim Durchgang durch Medien verschiedener Dichte den Weg, auf dem es am schnellsten zum Ziel gelangt. Aus dem Maupertuisschen Prinzip kann man die Grundgesetze der Mechanik ableiten, und umgekehrt. Da es also mathematisch mit einer ,,kausalen“ Formulierung der mechanischen Gesetze äquivalent ist, betrifft die Unterscheidung kausal - final hier nicht die Sache selbst, sondern nur die Formulierung. Kausale und finale Beschreibungen schließen sich also nicht aus, und ebenso wenig kausale und finale Begründbarkeit von Tatsachen.

In der Biologie spielen funktionale Erklärungen und Zweckerklärungen eine besonders wichtige Rolle, da es hier um das Verstehen der Funktionsweise von Organen und Organismen geht, um ein Verständnis der Funktion von Merkmalen oder Verhaltensweisen einer Spezies für ihr Leben und Überleben. Die beeindruckende ZweckmäBigkeit der körperlichen Konstitution wie der angeborenen Verhaltensweisen von Lebewesen legte den Gedanken nahe, diese ZweckmäBigkeit durch die Annahme von Kräften oder Anlagen verständlich zu machen, welche die Entwicklung des Lebendigen in Richtung auf Ziele steuern. Solche zielgerichteten Kräfte oder Anlagen wurden als

${ }^{44}$ Die Bezeichnung ,final“ ist für solche Integralprinzipien insofern nicht ganz passend, als sie den gesamten Ablauf eines Geschehens charakterisieren, also Vergangenheit, Gegenwart und Zukunft gleichermaßen berücksichtigen. 
„Entelechien“ bezeichnet. Kausale Erklärungen von Zweckmäßigkeiten sah man lange Zeit als unmöglich an. Auch Systeme, deren Verhalten durch kausale Gesetze bestimmt wird, können aber zweckmäßig funktionieren. Beispiele dafür sind sich selbst regulierende Automaten, wie z. B. eine Heizung, deren Temperatur über einen Thermostaien geregelt wird. Hier kann man sagen, die Heizung arbeite so, daß eine konstante Raumtemperatur eingehalten wird, und das ist dann eine teleologische oder finale Beschreibung. Die Funktionsweise der Heizung läßt sich aber mit kausalen physikalischen Gesetzen erklären. Das Modell für eine kausale Erklärung der Zweckmäßigkeiten im biologischen Bereich ist heute die darwinsche Evolutionstheorie. Danach treten in den Genen spontan oder durch äußere Einwirkung Mutationen auf, die unter bestimmten Umständen einen Vorteil im Kampf ums Dasein bedingen. Dieser Kampf bewirkt eine Selektion derjenigen Formen, die der Lebensumwelt am besten angepaßt sind. Was in diesem Selektionsprozeß besteht, ist daher durch seine Zweckmäßigkeit ausgezeichnet. Das ist ein Modell einer nicht-teleologischen Erklärung von Zweckmäßigkeit: ${ }^{45}$ Die bestehenden Arten sind zweckmäßig, weil das Unzweckmäßige im Selektionsprozeß untergegangen ist.

Ein bekanntes Beispiel der Erklärung von Anpassungsleistungen ist folgendes: Die normale Farbe des Birkenspanners, die Farbe seiner Wildform, ist ein helles Grau. Diese Farbe schützt den Falter auf den hellen Flechten, die normalerweise auf den Baumstämmen leben. Bei Luftverschmutzung sterben die Flechten $a b$, und der helle Birkenspanner ist dann auf den dunklen Baumstämmen auffällig und wird so eine leichte Beute von Vögeln. In Gebieten starker Luftverschmutzung hat sich nun ein Typ des Falters mit dunkler Farbe durchgesetzt. Das kann man teleologisch so ausdrücken: Der Birkenspanner hat

${ }^{45}$ Von einer kausalen Erklärung kann man freilich nur dann sprechen, wenn sich alle Mutationen kausal-deterministisch und nicht nur statistisch erklären lassen, was im Blick auf die Rolle mikrophysikalischer Ereignisse bei solchen Mutationen und die Tatsache, daß die Grundgesetze der Mikrophysik statistischen Charakter haben, fraglich ist. Den gesamten Vorgang der Mutation wie der Selektion kann man als Massenphänomen sicher nur statistisch beschreiben. Statistische Beschreibungen wie jene der statistischen Mechanik schließen aber nicht aus, daß die Einzelvorgänge kausal determiniert sind. 
seine Farbe den veränderten Umweltgegebenheiten angepaßt, er hat sie so verändert, daß sie ihm in der neuen Umgebung Schutz bietet. Diese Leistung läßt sich aber kausal so verstehen, daß zufällig auftretende dunkle Mutanten, die auf den hellen Flechten geringe Überlebenschancen hatten, in der veränderten Umwelt nun einen Vorteil im Kampf ums Überleben haben, sich verbreiten können, weil sie besser geschützt sind, während hier die helle Form ausstirbt.

Die Evolutionstheorie erklärt zunächst nur die Entwicklung der Arten, nicht aber die Entstehung des Lebens. Auch dafür gibt es aber heute plausible kausale Modelle.

Es wird oft behauptet, nur kausale Erklärungen seien wissenschaftliche Erklärungen; nur sie vermittelten echte Erkenntnis und ein wirkliches Verständnis der Phänomene. Diese Auffassung ist aber nicht haltbar. Es ist nicht einzusehen, wieso Begründungen eines Phänomens mit früheren Ereignissen von höherer Dignität sein sollten als z. B. Begründungen mit späteren Ereignissen. Auch eine Erkenntnis der Ursachen ist, wie schon früher betont wurde, keine "definitive“ Erkenntnis der Wirkung, die keine weiteren Fragen offenläßt. Wir können nach den Ursachen der Ursachen fragen, nach dem Grund der Geltung von Kausalgesetzen usf. Für Kausalgesetze gibt es keine kausale Erklärung, denn sie bilden die Grundlage kausaler Erklärungen. Es könnte dafür aber teleologische Erklärungen geben. So sah es jedenfalls Leibniz, der versuchte, aus dem Perfektionsprinzip, nach dem unsere Welt die beste aller möglichen Welten ist, über das Wirkungsprinzip die physikalischen Gesetze abzuleiten. Wie teleologische und finale Erklärungen kausale nicht ausschließen, so gilt auch das Umgekehrte. Welche Erklärungen jeweils brauchbar sind, hängt vom Ziel der Untersuchung ab. Die Bevorzugung kausaler Erklärungen erklärt sich vor allem aus ihrer praktischen Bedeutung: ${ }^{46}$ Kausale Gesetze geben uns Auskunft darüber, wie das, was sein wird, von dem

${ }^{46}$ Das läßt sich geistesgeschichtlich gut aus den Schriften von F. Bacon und Hobbes belegen, die neben Descartes als Begründer der neuzeitlichen Wissenschaftsidee angesehen werden können. Beide haben teleologische Erklärungen als wertlos erklärt, und diese Auffassung war bis zu Leibniz prägend für die Philosophie der Aufklärung. Hobbes definiert in De Corpore geradezu Philosophie (für ihn der Inbegriff aller Wissenschaften) als Erkenntnis der Wirkungen oder Phänomene aus ihren Ursachen. 
abhängt, was jetzt ist. Diese Information ermöglicht es uns, durch passencle Veränderung der gegenwärtigen Bedingungen das künftige Geschehen im Sinn unserer Ziele zu steuern. Für die Behauptung, auch unter theoretischem Aspekt seien kausale Erklärungen teleologischen grundsätzlich vorzuziehen, ist aber kein brauchbares Argument in Sicht. Gesetze dienen zur Ordnung der Erfahrungen, und der Wert eines Gesetzes hängt von seinem Informationsgehalt ab, von der Menge der Phänomene, die sich damit erfassen lassen; daneben auch von seiner Einfachheit. Gesetze dienen also nur der Beschreibung der Welt. Diese Kriterien zeichnen kausale nicht grundsätzlich vor teleologischen oder finalen Gesetzen aus. In der Physik spielt daher auch die Frage der Kausalität keine Rolle. Das Problem, wie Kausalgesetze von anderen abzugrenzen sind, wird in der Physik selbst nicht diskutiert; es ist ein rein philosophisches Problem, und die Exaktheit der Physik leidet nicht darunter, daß man bisher keine präzisen Kriterien zur Abgrenzung solcher Gesetze gefunden hat. Nur die Einsicht, daß etwas notwendigerweise so ist, wie es ist, wäre eine „definitive“ Antwort auf die Frage, warum es so ist. Kausale Erklärungen, die sich auf kontingente Naturgesetze stützen, sind kein Ersatz dafür. Von einer „,definitiven" teleologischen Erkenntnis könnte man wohl nur dann sprechen, wenn die dabei zugrundegelegten Werte nicht nur relative, sondern intrinsische Werte sind. Für Leibniz waren die Merkmale, durch die er Perfektion definiert, Merkmale intrinsischer Werthaftigkeit. Seine Erkenntnisbemühung richtete sich also darauf, den Wert der Welt zu erkennen, und in dieser Weise zu einer definitiven Erkenntnis zu gelangen. Damit vertritt er im Rahmen der Philosophie der Aufklärung als einziger ein teleologisches Wissenschaftsideal. Allgemein kann man von einem teleologischen Erkenntnisideal sprechen, wenn die Zielsetzung auf teleologische Erklärungen geht. Teleologisch - wenn auch in ganz anderer Weise - sind auch die Entwürfe von Schelling und Hegel. Man kann wohl sagen, daß das Streben nach einem teleologischen Weltverständnis in der Geschichte der Philosophie eine bedeutendere Rolle gespielt hat als das Bemühen um ein kausales Verständnis der Wirklichkeit. Auch ein „,definitives“ teleologisches Verständnis wäre aber kein Verständnis der Phänomene, das keine weiteren Fragen offen ließe. Aus der Werthaftigkeit einer Sache folgt ja nicht, daß sie existiert. Ein teleologisches Verstehen macht also z. B. ein kausales Verstehen nicht überflüssig. Bei Handlungen kann zwar der Beweggrund gerade der damit realisierte Wert sein; je- 
mand kann etwas Gutes tun, weil es gut ist. Aber auch damit sind nicht alle Fragen beantwortet; es bleiben Fragen wie ,Wieso konnte die Handlung gelingen?" oder „Warum hatte der Betreffende die entsprechenden Präferenzen?"،

Als Kausalitätsprinzip bezeichnet man die These, daß sich alles Geschehen kausal erklären läßt. Als Finalitätsprinzip kann man entsprechend die These bezeichnen, daß sich alles final erklären läßt. Diese Thesen schließen sich nicht aus. Unverträglich wären nur die Behauptungen, daß sich alles nur kausal, bzw. final erklären läßt. ${ }^{47}$ Diese Behauptungen sind aber falsch, wie wir gesehen haben. Aufgrund der Auffassung, daß die Grundgesetze der Physik, die Gesetze der Quantentheorie, statistische Gesetze sind, sehen wir heute auch das Kausalitätsprinzip als ungültig an. Und für die Annahme, das Finalitätsprinzip sei richtig, gibt es im Blick auf die Form quantenphysikalischer Gesetze ebenfalls keinen Anhalt. Unter einem Teleologieprinzip hätte man die These zu verstehen, daß in der Welt alles zweckmäßig eingerichtet ist; daß es nichts gibt, was keinen Zweck hätte; daß sich also alles teleologisch, d. h. als zweckmäßig erklären läßt. Eine solche Auffassung vertrat Leibniz, während das Prinzip für Kant nur eine regulative Idee war. Es gibt weder ein brauchbares apriorisches Argument für ein Teleologieprinzip, noch läßt es sich durch Beobachtungen entscheiden - als universellen Satz kann man es durch endlich viele Beobachtungen nicht verifizieren, und um zu sagen, etwas sei zu gar nichts gut gewesen, müßte man den gesamten Lauf der Welt überblikken können. Dieses Prinzip ist aber auch ohne tieferes Interesse, wenn der Wert der Dinge nicht mit einem einheitlichen Wertmaßstab gemessen wird, also nicht behauptet wird, alles sei unter einem bestimmten Aspekt zweckmäßig oder alles diene der Verwirklichung eines Endzwecks. Eine Welt, in der zwar alles unter irgendeinem Aspekt zweckmäßig, unter anderen Aspekten aber unzweckmäßig wäre, eine Welt, in der es neben den partikulären und relativen Zwecken keinen allgemeinen, übergeordneten Zweck von allem gäbe, würde kaum der Idee einer teleologischen Weltordnung entsprechen. Ein Endzweck der Welt kann aber, wie schon Kant betont hat, ebensowenig in ihr zu finden sein, wie eine Erstursache.

${ }^{47}$ Vgl. dazu auch Rescher (63) und Stegmüller (69), $527 \mathrm{f}$. 


\subsection{Das Verstehen von Handlungen}

In allen Wissenschaften, die sich mit dem Menschen, insbesondere mit menschlichem Handeln und Verhalten und menschlichen Werken befassen, also in den Geisteswissenschaften im umfassenden Sinn dieser Bezeichnung, in dem dazu nicht nur historische oder vorwiegend historisch orientierte Disziplinen zu rechnen sind (Geschichte und Kulturwissenschaften), sondern auch die Sozialwissenschaften und Rechtswissenschaft, spielt naturgemäß das Verstehen und die Erklärung von Handlungen eine zentrale Rolle. Das Verstehen von Handlungen kann, wie wir früher sahen, ein determinatives Verstehen sein (ein Verstehen, was für eine Art von Handlung vorliegt), ein Bedeutungsverstehen (bei sprachlichen Äußerungen oder Gesten), ein genetisches Versteben (wie es dazu kam), ein funktionales Versteben (wie eine Handlung dieser Art funktioniert, wie sie vollzogen wird, welchem Zweck sie dient), ein intentionales Versteben (ein Verstehen der sie leitenden Absichten und Ziele), ein kausales Versteben ihrer Ursachen oder ein rationales Verstehen ihrer Beweggründe. Hier wollen wir uns mit dem rationalen Verstehen von Handlungen, d. h. mit der Struktur rationaler Erklärungen befassen, da wir auf die anderen Erklärungsformen oben schon eingegangen sind. Rationale Erklärungen sind teleologische Erklärungen. Wir behandeln sie hier aber gesondert einmal wegen der großen systematischen Bedeutung, die ihnen zukommt, zum anderen im Blick auf die Tatsache, daß gerade ihre Struktur und Leistung in der Literatur besonders heftig umstritten ist.

Für ein Verständnis der Leistung rationaler Erklärungen kommt es entscheidend darauf an, daß man beachtet, was oben schon mehrfach betont wurde: Eine Erklärung einer Tatsache $p$ ist nicht immer eine Begründung von $p$. Weder bestehen Erklärungen immer in Begründungen, noch ist eine Erklärung von $\mathrm{p}$, die sich in Form einer Begründung vollzieht, immer eine Begründung der Tatsache $\mathrm{p}$ selbst; sie kann vielmehr in der Begründung einer Aussage über $p$ bestehen. Da rationale Erklärungen in Begründungen bestehen, ist hier der letztere Punkt wichtig, denn eine rationale Erklärung einer Handlung bestebt nicht in einer Begründung dafür, daß diese Handlung stattfand, sonderm in einer Begründung dafür, daß sie rational war. Das ist in den Diskussionen um Möglichkeit und Wert rationaler Erklärungen immer wieder übersehen worden. Wir kommen darauf unten zurück. Zunächst wollen wir uns aber überlegen, wie eine Begründung für die Behaup- 
tung auszusehen hat, eine Handlung sei rational. Das ergibt sich nun, wie in früheren Fällen, aus der Analyse des Begriffs ,,rational“ in Anwendung auf Handlungen, und dazu können wir uns auf die Entscheidungstheorie beziehen. ${ }^{48}$

In der Entscheidungstheorie betrachtet man Situationen $S$, in denen eine Person a zwischen mehreren Handlungsweisen $F_{1}, \ldots, F_{n}$ wählen kann, die sich gegenseitig ausschließen. ${ }^{49}$ a muß also in $S$ mindestens eine dieser Handlungsweisen vollziehen - eine Handlungsweise F zu unterlassen zählt auch als Handlungsweise - und a kann nur eine dieser Alternativen vollziehen. Nehmen wir zunächst an, a sei überzeugt, daß jede der möglichen Handlungen $F_{i}$ zu einem bestimmten Resultat $\mathrm{p}_{\mathrm{i}}(\mathrm{i}=1, \ldots, \mathrm{n})$ führt. In solchen Fällen spricht man von einer Entscheidung unter Sicherbeit. Die Resultate $\mathrm{p}_{\mathrm{i}}$ haben nun für a einen gewissen Wert. Die Person a kann mindestens sagen, welches von zwei Resultaten sie vorzieht oder ob sie beide Resultate als gleich wertvoll ansieht. Wir nehmen hier an, daß a die Nutzenswerte der Resultate $\mathrm{p}_{\mathrm{i}}$ sogar in Zahlen $\mathrm{u}_{\mathrm{a}}\left(\mathrm{p}_{\mathrm{i}}\right)$ ausdrücken kann. ${ }^{50}$ Eine $\mathrm{F}_{\mathrm{i}}$-Handlung ist nun eine rationale Handlung von a in $\mathrm{S}$, wenn ihr Resultat $\mathrm{p}_{\mathrm{i}}$ - verglichen mit allen möglichen Resultaten - optimal ist für a, wenn also für alle $k=1, \ldots, n$ gilt $u_{a}\left(p_{i}\right) \geq u_{a}\left(p_{k}\right)$. Zu jeder anderen Handlung gibt es eine bessere Alternative, es wäre also für a nicht vernünftig, sie zu wählen.

Für die Rationalität einer Handlung kommt es nicht darauf an, ob die Überzeugungen des Handelnden richtig sind - ob also in unse-

${ }^{48}$ Eine gute Einführung in die Entscheidungstheorie ist Luce und Raiffa (57).

${ }^{49}$ Es gibt natürlich auch Situationen, in denen man zwischen unendlich vielen Alternativen wählen kann. Man kann z. B. in unendlich vielen Weisen einen Strich der Länge $\leq 1$ auf einer Tafel ziehen. Der Einfachheit halber betrachten wir hier jedoch nur Entscheidungen zwischen endlich vielen Alternativen; wir wollen ja nicht Entscheidungstheorie treiben, sondern nur den Grundgedanken rationaler Erklärung an einem einfachen Modell verdeutlichen.

${ }^{50}$ Von einem komparativen Wertbegriff ,,p ist für a höchstens so wertvoll wie

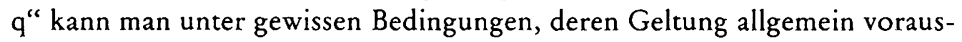
zusetzen intuitiv plausibel ist, zu einem metrischen Wertbegriff $u_{a}(p)$ übergehen, wobei diese Funktion nur bis auf positiv-lineare Transformationen (d. h. bis auf Wahl eines Nullpunkts wie einer Einheit) eindeutig ist. Vgl. dazu z. B. Jeffrey (65). 
rem Fall die Alternativen $F_{i}$ wirklich zu den Resultaten $p_{i}$ führen -, und ob die Präferenzen von a in irgendeinem objektiven Sinn korrekt sind oder ob sie den ,wahren Interessen" von a auf lange Sicht entsprechen. Rationalität bemißt sich allein an den Überzeugungen und Präferenzen des Handelnden im Moment der Entscheidung. ${ }^{51}$

Eine Entscheidung unter Risiko liegt vor, wenn die Person a sich bzgl. der Resultate der verschiedenen Handlungsweisen $F_{i}$ nicht sicher ist. Dann gibt es zu jeder Alternative $F_{j}$ mehrere mögliche Resultate $\mathrm{P}_{\mathrm{i}_{1}}, \ldots, \mathrm{P}_{\mathrm{im}}$, die alle für a eine nicht verschwindende Wahrscheinlichkeit haben. $w_{a}\left(p_{i j}\right)(j=1, \ldots, m)$ sei die Wahrscheinlichkeit, die a dem Resultat $\mathrm{P}_{\mathrm{ij}}$ unter der Bedingung zuordnet, daß a in der Situation $S F_{i}$ tut. $^{52}$ Es ist also $\sum_{j=1}^{m} w_{a}\left(p_{i j}\right)=1$, da ja eins der Resultate $p_{i j}$ bei der Handlung $F_{i}$ eintreten muß. Während bei einer Entscheidung unter Sicherheit der Wert $u_{a}\left(F_{i}\right)$ der Handlungsweise $F_{i}$ mit dem Wert $u_{a}\left(p_{i}\right)$ des Resultats $\mathrm{P}_{\mathrm{i}}$ identisch ist, das a als sicheres Resultat seines $\mathrm{F}_{\mathrm{i}}$-Tuns ansieht, ist bei einer Entscheidung unter Risiko $u_{a}\left(F_{i}\right)$ der Erwartungswert des Nutzens bei $F_{i}$, also $u_{a}\left(F_{i}\right)=\sum_{j=1}^{m} u_{a}\left(p_{i j}\right) \cdot w_{a}\left(p_{i j}\right)$. Dann gilt wieder: In $S$ ist es für a rational, eine optimale Alternative $F_{i} z u$ wählen, für die also gilt: für alle $k=1, \ldots$,n ist $u_{a}\left(F_{k}\right) \leq u_{a}\left(F_{i}\right)$.

Dazu ein Beispiel: Die Person a muß nach Frankfurt fahren. Sie hat die Wahl, mit dem Wagen zu fahren $\left(F_{1}\right)$ oder zu fliegen $\left(F_{2}\right)$. Der Nutzen dieser Alternativen für a bestehe in der Kürze der Fahrzeit. Diese hängt vom Wetter ab: Ist gutes Wetter (q), so dauert der Flug 1 Stunde, die Autofahrt 3 Stunden. Ist es neblig (nicht-q), so muß das Flugzeug umgeleitet werden; die Flugzeit beträgt dann 5 Stunden, und die Autofahrt möge 4 Stunden dauern. Setzen wir die Nutzenswerte gleich dem negativen Betrag der Fahrtzeit, so erhalten wir folgende Tabelle:

${ }^{5 i}$ Es kann auch sein, daß sich die Person a über die Alternativen täuscht, die ihr in einer Entscheidungssituation offenstehen; daß sie z. B. glaubt, sie hätte gewisse Möglichkeiten nicht, die sie tatsächlich hat. Dann heißt Rationalität Optimalität unter jenen Alternativen, die a zu haben glaubt. Von solchen Fällen wollen wir hier jedoch der Einfachheit halber absehen.

52 Wir können ohne Beschränkung der Allgemeinheit annehmen, jede Alternative hätte dieselbe Zahl m von möglichen Resultaten. Man kann ja einfach für $m$ die größte Zahl möglicher Resultate einer Alternative wählen und, wenn $F_{i}$ eigentlich nur $r<m$ mögliche Resultate $p_{i 1}, \ldots, p_{i r}$ hat, $p_{i r}+1, \ldots, p_{i m}$ gleich $\mathrm{P}_{\text {ir }}$ setzen. 


\begin{tabular}{c|c|c} 
& $\mathrm{q}$ & $\neg \mathrm{q}$ \\
\hline $\mathrm{F}_{1}$ & -3 & -4 \\
\hline $\mathrm{F}_{2}$ & -1 & -5
\end{tabular}

Die Zahlen geben die Nutzenswerte der möglichen Resultate an. Die Wahl, die a trifft, wird von der Wahrscheinlichkeit abhängen, die a dem Sachverhalt q zuordnet, daß gutes Wetter ist. Diese Wahrscheinlichkeit ist gleich, egal ob a fliegt oder mit dem Auto fährt. Ist z. B. $\mathrm{w}_{\mathrm{a}}(\mathrm{q})=0,5$ (also auch $\left.\mathrm{w}_{\mathrm{a}}(\neg \mathrm{q})=0,5\right)$, so ist $\mathrm{u}_{\mathrm{a}}\left(\mathrm{F}_{1}\right)=-3,5$, $\mathrm{u}_{\mathrm{a}}\left(\mathrm{F}_{2}\right)=-3$, a wird also fliegen. Ist hingegen $\mathrm{w}_{\mathrm{a}}(\mathrm{q})=0,25$ (also $\left.\mathrm{w}_{\mathrm{a}}(\neg \mathrm{q})=0,75\right)$, so ist $\mathrm{u}_{\mathrm{a}}\left(\mathrm{F}_{1}\right)=-3,75, \mathrm{u}_{\mathrm{a}}\left(\mathrm{F}_{2}\right)=-4$, a wird also rationalerweise mit dem Auto fahren.

Als dritten Fall sieht man oft Entscheidungen unter Unsicherheit an, bei denen sich die Person a bzgl. der möglichen Resultate $\mathrm{p}_{\mathrm{i} 1}, \ldots$. $\mathrm{P}_{\mathrm{im}}$ der Alternativen $F_{i}$ unsicher ist, ihnen aber nicht einmal Wahrscheinlichkeiten zuordnen kann. Dann kann a z. B. nach dem Prinzip der Minimierung des möglichen Schadens verfahren und eine Alternative wählen, bei der das schlechteste mögliche Resultat im Vergleich mit den schlechtesten möglichen Resultaten anderer Alternativen optimal ist.

Im Fall

\begin{tabular}{l|c|c} 
& $\mathrm{q}$ & $\neg \mathrm{q}$ \\
\hline $\mathrm{F}_{1}$ & 10 & -3 \\
\hline $\mathrm{F}_{2}$ & 2 & 4
\end{tabular}

wird a also $F_{2}$ wählen. $\mathrm{Da}$ ein solches Prinzip aber nicht immer vernünftig ist, zeigt das Beispiel:

\begin{tabular}{c|c|c} 
& $\mathrm{q}$ & $\neg \mathrm{q}$ \\
\hline $\mathrm{F}_{1}$ & 100 & -1 \\
\hline $\mathrm{F}_{2}$ & 0,5 & 0,5
\end{tabular}

Hier müßte a nach dem Maximin-Prinzip $F_{2}$ wählen. Das ist aber kaum sinnvoll, da $F_{1}$ im günstigen Fall ein sehr viel besseres Resultat ergibt als $\mathrm{F}_{2}$, im ungünstigen hingegen nur ein geringfügig schlechte- 
res Resultat als $F_{2}$. J. Harsanyi und andere haben zurecht betont, daß Fälle, in denen wir den möglichen Resultaten unserer Alternativen nicht einmal ungefähre oder komparative Wahrscheinlichkeiten zuordnen können, kaum vorkommen. Eine ungefähre Abschätzung der Erfolgsaussichten einer Alternative wird immer möglich sein. Da man ferner auch Entscheidungen unter Sicherheit als Spezialfälle von Entscheidungen unter Risiko ansehen kann (mit $\mathrm{m}=1$ ), kann man Rationalität generell so bestimmen:

R) Eine Handlung ist genau dann rational, wenn der Erwartungswert des Nutzens bei ibr maximal ist.

Daraus ergibt sich unmittelbar, wie Behauptungen des Inhalts, eine Handlung sei rational, zu begründen sind. Es ist dabei nicht immer erforderlich, die Wahrscheinlichkeits- und die Nutzensbewertung des Handelnden genau anzugeben. Es genügen oft wesentlich allgemeinere Informationen. Im Ailtag können wir ja auch in der Regel nicht einmal unsere eigenen Wahrscheinlichkeiten und Nutzensfunktionen genau angeben, geschweige denn die anderer.

Wir können sagen, die Person a wolle, daß der Zustand p eintritt, genau dann, wenn $\mathrm{p}$ für a in jedem Fall mindestens ebenso gut ist wie nicht-p. ${ }^{53} \mathrm{p}$ kann ja unter verschiedenen Bedingungen eintreten. Nur dann, wenn $p$ unter all diesen Bedingungen mindestens so gut ist wie nicht-p, kann man sagen, a wolle $\mathrm{p}$; andernfalls kann man nur behaupten, a wolle $\mathrm{p}$ unter den und den Bedingungen. ${ }^{54}$ Es gilt nun: Will a, daß p eintritt, und glaubt a, daß p genau eintritt, wenn a $F$ tut, so ist es für a im Sinne von ( $R$ ) rational, F zu tun. Wir erhalten somit ein oft

${ }^{53}$ Genauer: a will, daß $p$, wenn gilt: $O<w_{a}(p)<1$ und für alle Bedingungen $q$ mit $w_{a}(p \wedge q)>O$ und $w_{a}(\neg p \wedge q)>0 u_{a}(p, q) \geq u_{a}(\neg p, q)$ ist. Dabei ist $u_{a}(p, q)$ der Nutzenswert von $p$ für a unter der Bedingung, daß q gilt; dieser Wert stellt also den erwarteten Nutzen von $p$ unter der Annahme dar, $q$ gelte. Er wird mit den durch $\mathrm{q}$ bedingten Wahrscheinlichkeiten gebildet. Falls es nur endlich viele Welten i gibt und wir Propositionen als Mengen der Welten darstellen, in denen sie gelten, erhalten wir also: $u_{a}(p, q)=\frac{1}{w_{a}(p, q)}$. $\sum_{i \varepsilon p} u_{a}(i) \cdot w_{a}(i, q)=\frac{1}{w_{a}(p \wedge q)} \sum_{i \varepsilon p} \wedge u_{q} u_{a}(i) \cdot w_{a}(i)=u_{a}(p \wedge q)$ für $w_{a}(p \wedge q)>0$. Zur Logik ähnlicher Wollensbegriffe vgl. Lenzen (81).

54 Das Wort ,wollen" wird hier in der letzten der drei in 2.3 aufgewiesenen Bedeutungen verwendet. 
anwendbares Argument einfachen Typs, mit dem sich die Rationalität einer Handlung begründen läßt.

Will jemand, daß $p$, und glaubt er, daß p genau dann eintritt, wenn er $\mathrm{F}$ tut, so beabsichtigt er mit $\mathrm{F}$ auch zu erreichen, daß p eintritt. Rationale Erklärungen dieser Form von Handlungen implizieren also auch intentionale Erklärungen der Handlungen. Wie schon früher gesagt wurde, läßt sich aber nicht jeder rationalen Handlung eine Absicht zuordnen. Es gibt also auch rationale Erklärungen von Handlungen, die sich intentional nicht erklären lassen. Umgekehrt ist nicht jede absichtsvolle Handlung rational; man kann also nicht generell mit der oder den Absichten einer Handlung ihre Rationalität begründen. Absichten sind keine Beweggründe - sie können allenfalls Beweggründe implizieren -, und daher haben wir begründete intentionale Erklärungen von rationalen Erklärungen zu unterscheiden.

Die Mißdeutungen rationaler Erklärungen beruhen darauf, daß man Erklärungen eines Ereignisses immer als Begründungen dafür ansieht, daß dieses Ereignis stattfindet, bzw. stattgefunden hat oder stattfinden wird. Aufgrund dieser, meist stillschweigend gemachten Annahme, sagt man dann, rationale Erklärungen einer Handlung seien keine ,echten“ oder ,,vollständigen“ Erklärungen dieser Handlung, da aus den Prämissen ja nicht folge, daß die Handlung stattfindet. Dazu bräuchte man vielmehr eine Prämisse des Inhalts, daß der Handelnde nur tut, was für ihn rational ist, daß er eine ,rationale Person ist". ${ }^{55}$ Generell wird man das aber von niemandem behaupten können. Wir alle handeln immer einmal wieder anders, als wir das bei genauer Erwägung unserer Wahrscheinlichkeitsannahmen und Präferenzen tun sollten. Daß aber in der fraglichen Situation rational gehandelt wird, läßt sich oft nicht anders begründen als so, daß der Handelnde etwas tut, was für ihn rational ist. Damit wird die Begründung der Handlung aber insofern zirkulär, als sich eine der Prämissen (daß er rational handelt) nur auf dem Wege über die Konklusion begründen läßt. Auf dieses Problem haben z. B. C. G. Hempel und W. Stegmüller in ihrer Kritik an dem Modell rationaler Erklärung hingewiesen, das W. Dray in (57) entwickelt hat. ${ }^{56}$ Dieses Problem

${ }^{55}$ Alles zu tun, was rational ist, ist gar nicht immer möglich, weil es viele Situationen gibt, in denen mehrere Handlungsalternativen rational sind, von denen man aber nur eine wählen kann.

${ }^{56}$ Vgl. dazu Hempel (65), $463 \mathrm{ff}$ und Stegmüller (69), Kap. IV, bes. S. $379 \mathrm{ff}$. 
entsteht aber nur - um es noch einmal zu betonen - aus einer Mißdeutung der Absicht und Struktur rationaler Erklärungen. Daher gehen auch die Bemühungen von $\mathrm{G}$. $\mathrm{H}$. von Wright in (71), einen „praktischen Syllogismus" anzugeben, in dem allein aus Aussagen über Annahmen und Präferenzen des Handelnden irgendwie erschiossen werden soll, daß er so und so handelte, von falschen Voraussetzungen aus. Wir werden freilich unten sehen, daß eine Begründung von Handlungen mit Präferenzen und Annahmen durchaus möglich ist. Man benötigt dabei allerdings Prämissen, die der Rationalitätsannahme entsprechen. Solche Prämissen bewirken nicht immer, daß das Argument zirkulär wird. Auch kausale Erklärungen stützen sich auf die Geltung allgemeiner Gesetze, die falsch wären, wenn das Explanandum nicht wahr wäre. Wie solche Gesetze unabhängig von der Geltung des Explanandums gut bestätigt oder bewährt sein, also plausible Hypothesen darstellen können, so kann auch die Annahme, jemand verhalte sich in Situationen eines gewissen Typs rational, eine unabhängig vom Explanandum gut bestätigte Hypothese sein.

Eine rationale Erklärung einer Handlung ist eine Begründung ihrer Rationalität aus den Gegebenheiten der Entscheidungssituation, aus der sie hervorgeht. Diese Gegebenheiten sind subjektive Faktoren: Annahmen und Präferenzen des Handelnden; ${ }^{57}$ sie sind mögliche $\mathrm{Be}$ -

Dort wird unter Bezugnahme auf die Tatsache, daß es für Entscheidungen unter Unsicherheit neben dem Maximin-Kriterium auch andere Kriterien rationalen Verhaltens gibt, auch behauptet, es gäbe keinen einheitlichen Rationalitätsbegriff. Nach den obigen Bemerkungen ist das einerseits nicht richtig, andererseits aber auch irrelevant: Man könnte auch von rationalen Erklärungen verschiedener Typen sprechen, die auf verschiedene Arten von Entscheidungssituationen anwendbar sind und begründen, daß die Handlung in einem speziellen Sinn rational ist. Ferner wird dort gesagt, tatsächlich rationale Handlungen seien nicht immer als solche intendiert, ihnen gingen z. B. nicht immer rationale Überlegungen voraus. Das wird aber bei einer rationalen Erklärung gar nicht behauptet; der Anspruch einer rationalen Erklärung ist nicht, die Überlegungen des Handelnden wiederzugeben. Wir handeln oft intuitiv, ohne weitere Überlegungen rational. Eine rationale $\mathrm{Er}$ klärung will nur zeigen, daß die Handlung tatsächlich rational war, daß sie also einer rationalen Überlegung entspringen konnte.

${ }^{57}$ Wir haben oben betont, daß es nicht auf die tatsächlich bestehenden Alternativen ankommt, sondern auf die Annahmen des Handelnden über diese Alternativen. 
weggründe für den Handelnden. Als „,Beweggründe“ einer Handlung bezeichnet man gewöhnlich das, was den Handelnden dazu bewogen hat, was also bei seinen Überlegungen den Ausschlag gab. Annahmen und Präferenzen lassen sich daher generell nur als mögliche Beweggründe bezeichnen. Tatsächliche Beweggründe sind sie, sofern sie vom Handelnden berücksichtigt worden sind. Als „Beweggrund“ in einem weiteren Sinn könnte man auch das bezeichnen, was die Entscheidungssituation bestimmt, also z. B. Informationen, die zu einer Entscheidung führen.

Sind nun Beweggründe Ursachen? Lassen sich also mit Beweggründen Handlungen auch kausal erklären? Und ist rationale Erklärbarkeit mit kausaler verträglich? Diese Fragen sind heftig umstritten, zumal sie auf so schwierige Probleme führen wie das der Handlungsund Willensfreiheit und das Leib-Seele-Problem. ${ }^{58}$ Zunächst könnte es so scheinen, als seien sie einfach deswegen zu verneinen, weil Beweggründe immer Beweggründe von Handlungen sind, wir das Verhalten einer Person aber nur dann als Handlung bezeichnen, wenn sie es auch hätte unterlassen können. Dann kann aber die Handlung nicht durch Ursachen determiniert sein. Diese Bestimmung des Handlungsbegriffs ist durchaus sinnvoll. Wir bezeichnen sicher nicht jedes Verhalten einer Person in dem weiten Sinn, in dem wir auch vom Verhalten von Tieren und physikalischen Objekten sprechen, als Handlung. Stolpern oder Fallen sind z. B. keine Handlungsweisen. Die naheliegenden Alternativen zur Abgrenzung von Handlungen, sie als bewußtes oder absichtliches Verhalten zu charakterisieren, sind aber nicht brauchbar. Weder die Bewußtheit eines Verhaltens noch seine Absichtlichkeit ist notwendig, um es als „Handeln“ bezeichnen zu können. Handlungen haben nicht immer ein Ziel, wie wir gesehen haben. Sie sind auch nicht immer absichtlich in dem Sinn, daß wir sie tun wollen. Ein großer Teil der Verben, die Handlungsweisen bezeichnen, sind Erfolgsverben, die nur dann auf ein Verhalten anwendbar sind, wenn es zu einem bestimmten Resultat führt. Solche Verben sind z. B. ,,schließen“ oder ,töten“. Man kann nur sagen, daß jemand eine Tür schließt, wenn sie danach geschlossen ist; und man kann nur sagen, daß jemand einen anderen tötet, wenn dieser hinterher tot ist.

${ }^{58}$ Vgl. dazu z. B. Hempel (65), $254 \mathrm{f}$, Stegmüller (69), $530 \mathrm{ff}$ und Davidson (63). 
Den Erfolg kann man aber auch unabsichtlich herbeiführen. Ebenso sind nicht alle Handilungen bewußt: Man kann jemand töten, ohne es zu wissen. Bewußtheit oder Konformität eines Verhaltens mit Absichten sind auch nicht hinreichend für den Handlungscharakter. Auch ein bewußtes Fallen ist kein Handeln. Und ein Geschehen kann im Sinne meiner Wünsche und Pläne liegen - ich kann z. B. gefesselt dorthin transportiert werden, wo ich ohnedies hin wollte - , ohne ein Handeln darzustellen. Das entscheidende Kriterium dafür, daß ein Verhalten ein Handeln ist, liegt also darin, daß der Betreffende es auch hätte unterlassen können.

$\mathrm{Daß}$ jemand etwas tun kann, bzw. nicht tun kann, können wir unter verschiedenen Aspekten behaupten. Wenn jemand gefesselt oder gelähmt ist, kann er sich nicht bewegen; es ist ihm physisch unmöglich, sich zu bewegen; er steht unter einem physischen Zwang. Wenn jemand unter einer Phobie leidet, ist es ihm psychisch unmöglich, etwas zu tun; er steht unter einem psychischen Zwang, der für ihn praktisch unwiderstehlich ist. Von physischem Zwang ist dabei keine Rede. Wenn jemand mit vorgehaltener Waffe zu etwas gezwungen wird, so liegt weder physischer noch psychischer Zwang vor, sondern es gilt nur: er kann nicht anders handeln, wenn er am Leben bleiben will. Die Grenzen zwischen diesen Fällen sind sicher fließend und undeutlich. ${ }^{59}$ Wenn aber gesagt wird, eine Handlung liege nur dann vor, wenn der Betreffende sie auch hätte unterlassen können, so ist nur gemeint, daß Handlungen nicht durch physischen oder psychischen Zwang determiniert sind, nicht aber, daß sie dem Handelnden nicht eindeutig durch seine Präferenzen vorgezeichnet sind. Unsere Präferenzen zwingen uns nicht zu etwas; man kann sich nur aufgrund seiner Präferenzen in einer Situation gezwungen sehen, etwas zu tun, was man sonst lieber unterlassen hätte. ${ }^{60}$

Handlungen lassen sich demnach nicht ohne Beweggründe kausal erklären. Und da Beweggründe immer nur Beweggründe von Handlungen sind, und rationale Erklärungen Erklärungen mit Beweggrün-

${ }^{59}$ Das gilt insbesondere dann, wenn man im Sinne des Physikalismus das Psychische als Teilbereich des Physischen ansieht oder als durch Physisches bewirkt. Vgl. dazu die Kapitel 6 und 7.

${ }^{60} \mathrm{Vgl}$. dazu auch Kutschera (81), Kap. 6. Dort wird auch das Freiheitsproblem diskutiert, auf das wir uns hier nicht einlassen wollen. 
den, besteht eine Unverträglichkeit rationaler Erklärbarkeit mit der Existenz kausaler Erklärungen, die nicht auf Beweggründe Bezug nehmen. Es ist aber nicht ausgeschlossen, daß es Gesetze gibt, die besagen, daß jemand mit den und den Präferenzen unter gewissen Umständen immer so und so handelt. Das sind dann deterministische Sukzessionsgesetze, also Kausalgesetze im Sinn von 2.2. Es kann also durchaus kausale Erklärungen von Handlungen mit Beweggründen geben. Unsere Annahmen und Präferenzen bestimmen in vielen Fällen (wenn nämlich nicht mehrere Handlungsalternativen optimal sind), was wir tun, wenn wir rational handeln. Nun kann man sicher nicht behaupten, die Leute handelten immer rational. Aber es gibt wohl gewisse Minimalstandards vernünftigen Verhaltens, nach denen sich die Leute normalerweise richten. Es gibt Verhaltensdispositionen, die auf solchen Standards beruhen, ebenso wie es angeborene und erworbene Verhaltensdispositionen gibt. Die Annahme solcher Dispositionen bei anderen bildet die Grundlage unserer Erwartungen bzgl. ihres künftigen Verhaltens; der Voraussagbarkeit ihres Verhaltens, ohne die ein Zusammenleben kaum möglich wäre. Wenn auch diese Dispositionen meist nur statistischen Charakter haben, so daß wir das Verhalten auch bei Kenntnis der Umstände nicht mit Sicherheit, sondern nur mit mehr oder minder großer Wahrscheinlichkeit vorhersagen können, so ist doch die Existenz deterministischer Dispositionen nicht auszuschließen. Und Aussagen über solche Dispositionen implizieren, wie wir gesehen haben, Gesetzesaussagen, die kausale Erklärungen ermöglichen. ${ }^{61}$

Im Alltag geben wir häufig kausale Begründungen von Handlungen an, meist in modaler Form. Wir behaupten, Hans habe sich scheiden lassen, weil (er erfuhr, daß) seine Frau ihn betrog, oder daß Max seinen Freund besuchte, weil (er erfuhr, daß) dieser krank ist. Hier werden gewisse Verhaltensdispositionen als normal (zumindest als normal bei dem Handelnden) vorausgesetzt; es wird als normal angesehen, $\mathrm{da} ß$ die Leute in gewissen Situationen so und so reagieren. Es wird

${ }^{61}$ Auch D. Davidson vertritt in (63) die Ansicht, es gebe kausale Erklärungen von Handlungen mit Beweggründen. Er gèht dabei aber von einer behavioristischen Deutung von Präferenzen und Überzeugungen aus, nach der sie selbst schon Verhaltensdispositionen darstellen. Zur Problematik solcher Deutungen vgl. den Abschnitt 6.2. 
vorausgesetzt, daß jemand beim Eintreten gewisser Umstände davon erfährt, daß jemand gewisse Neigungen oder Informationen hat, etc. Und daher kann man sich meist mit einer oder mit nur wenigen Informationen im ,,weil"“-Satz begnügen.

Beweggründe können also Ursachen sein. Nicht alle Ursachen eines Verhaltens sind Beweggründe, aber die Ursachen von Handlungen sind Beweggründe. ${ }^{62}$ (Die Tatsache, daß Emils Freund krank ist, ist keine Ursache dafür, daß Emil ihn besucht, sondern nur die Tatsache, $\mathrm{da} \beta$ er davon erfuhr.) Wenn wir fragen, warum jemand etwas getan hat, so erwarten wir die Angabe von Beweggründen. Wenn andere Ursachen angegeben werden, so sehen wir das Verhalten nicht mehr als Handlung an. Wenn wir erfahren, daß jemand wegen einer Klaustrophobie einen Raum nicht betritt, so betrachten wir ihn nicht mehr als handelndes Subjekt mit Beweggründen. Nicht alle Beweggründe sind aber auch Ursachen, denn wenn die passenden Verhaltensgesetze oder -dispositionen fehlen, kann man mit ihnen die Handlung nicht kausal erklären. Auch bei Annahmen der Rationalität des Handelns und Kenntnis der Präferenzen und Wahrscheinlichkeitsbewertung einer Person kann es sein, daß sich die Handlung nicht begründen läßt, weil es mehrere gleich gute Alternativen gab.

Die Tatsache, daß Fragen nach den Ursachen einer Handlung Fragen nach den Beweggründen und nicht nach physischen Ursachen sind, läßt die Kritik an Erklärungen mit Beweggründen - seien sie rational oder kausal - als ebenso verfehlt erscheinen wie den Versuch, sie durch kausale Erklärungen mit physischen Ursachen zu ersetzen. Diese Kritik findet sich schon in Platons „Phaidon“: Sokrates ist unschuldig zum Tode verurteilt und sitzt nun im Gefängnis in Erwartung seiner Hinrichtung, obwohl man ihm (offenbar mit offizieller Duldung) die Gelegenheit zur Flucht gegeben hatte. Warum ist Sokrates im Gefängnis geblieben und nicht geflohen? Die rationale Begründung lautet: ,,Sokrates sitzt im Gefängnis, weil er lieber den Tod auf sich nimmt, als die Gesetze Athens zu brechen“. Die Antwort der

$62 \mathrm{Zu}$ den Ursachen können natürlich auch physische Umstände zählen, die es verhinderten oder ermöglichten, daß dem Handelnden gewisse Alternativen offenstanden. Die ,Warum"-Frage zielt aber meist auf Gründe ab, die dazu führten, daß er in dieser äußeren Situation das und jenes getan hat, und das sind dann Beweggründe. 
Naturphilosophen aber ist: „Sokrates sitzt im Gefängnis, weil seine Beine aus Knochen und Sehnen bestehen und die Sehnen gelockert sind, so daß die Knochen in den Knien gebeugt sind“. - „Aber", sagt Sokrates darauf, „, derartiges Gründe zu nennen, ist gar zu unsinnig

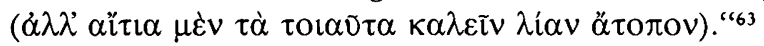

Wir haben uns in diesem Abschnitt nur mit der Erklärung einzelner Handlungen durch Beweggründe befaßt. Mit Beweggründen lassen sich aber auch Verhaltensweisen oder -strategien einzelner wie allgemeine Konventionen erklären, sowohl im Sinne einer rationalen wie einer kausalen Erklärung. Besonders interessant ist der Fall der Konventionen. Dazu können wir aber auf das Buch "Convention" von D. Lewis verweisen.

\subsection{Zum Problem des Verstehens in den Geisteswis- senschaften}

Die Bezeichnung „Geisteswissenschaften“ hat sich erst in der zweiten Hälfte des 19. Jahrhunderts eingebürgert, und erst mit W. Dilthey setzt die Reflexion auf die methodologische Eigenart dieser Disziplinen ein. ${ }^{64}$ Wissenschaftstheoretische Untersuchungen zu einzelnen geisteswissenschaftlichen Fächern wie z. B. Geschichts- oder Rechts-

${ }^{63}$ Phaidon, 99a. Nachdem ich dieses Beispiel in (72), 381 anführte, habe ich entdeckt, daß schon Leibniz sich bei seiner Unterscheidung zwischen Ursachen und Gründen darauf bezogen hat. Er gibt dort auch folgenden Vergleich eines Begründungsversuchs von Handlungen mit physischen Ursachen: „Dies ist ebenso, als wollte ein Historiker bei der Darstellung einer Eroberung, die ein großer Fürst durch die Einnahme einer wichtigen Stadt gemacht hat, sagen, das sei deshalb geschehen, weil die kleinen Teilchen des Kanonenpulvers der Berührung mit einem Funken ausgesetzt waren und mit einer Geschwindigkeit entwichen seien, daß sie in der Lage waren, einen harten und schweren Körper gegen die Mauern der Stadt zu schleudern, während die Haken der kleinen Teilchen, aus denen das Kupfer der Kanone besteht, fest genug miteinander verbunden waren, um sich durch diese Geschwindigkeit nicht voneinander zu lösen -, statt zu zeigen, wie die Voraussicht des Eroberers ihn die richtige Zeit und die geeigneten Mittel wählen ließ und wie sein Können alle Hindernisse überwunden hat" (DM,51). 
wissenschaft gab es natürlich schon viel früher, neu ist hingegen das Beinühen, dem zunächst nur als Sammelbezeichnung eingeführter, Ausdruck "Geisteswissenschaften" einen einheitlichen methodologischen Inhalt zu geben. Für Dilthey bestand der Unterschied zwischen Geistes- und Naturwissenschaften vor allem darin, daß die ersteren „,verstehende:", die letzteren hingegen ,erklärende“ Wissenschaften sind. Da seine Aussagen für die heutigen Diskussionen über Wesen und Leistung des Verstehens in den Geisteswissenschaften noch weithin prägend sind und da sie in vielen Punkten von unseren Aussagen abweichen, wollen wir darauf kurz eingehen. Unser Thema ist dabei weder die Methodologie der Geisteswissenschaften noch die Hermeneutik im allgemeinen, sondern allein der hermeneutische Verstehensbegriff.

Für das Verständnis der Gedanken Diltheys ist es nützlich, kurz auf die Versuche zu einer inhaltlichen Charakterisierung der Geisteswissenschaften einzugehen - eine Bestimmung durch Auflistung von Disziplinen ist kaum brauchbar, da man üblicherweise nur Teile solcher Disziplinen wie der Psychologie oder der Soziologie als ,,geisteswissenschaftlich" bezeichnet. Eine Bestimmung der Geisteswissenschaften kann sich auf ihren Gegenstandsbereich, ihre Methoden oder ihre Ziele beziehen. Vom Gegenstandsbereich her bestimmt Dilthey sie als das „Ganze der Wissenschaften, welche die geschichtlich gesellschaftliche Wirklichkeit zu ihrem Gegenstand haben" ${ }^{65}$ E. Rothacker charakterisiert ihren Gegenstand als ,,die Ordnungen des Lebens in Staat, Gesellschaft, Recht, Sitte, Erziehung, Wirtschaft, Technik und die Deutungen der Welt in Sprache, Mythos, Kunst, Religion, Philosophie und Wissenschaft" ${ }^{\text {" }}{ }^{66}$ Und H. Rickert spricht von den Geisteswissenschaften als Kultur-oder Geschichtswissenschaften. Die Stichwörter „Gesellschaft", „Kultur“ und „Geschichte“ tauchen auch in allen anderen Bestimmungen des Gegenstands der Geisteswissenschaften auf. Geht man von einem Gegensatz von Geistes- und Naturwissenschaften aus, so wird man zunächst einmal sagen: Gegen-

${ }^{64}$ Zur Geschichte des Ausdrucks „,Geisteswissenschaften“ vgl. Rothacker (27), 6 ff., zur Geschichte der methodologischen Reflexion auf die Geisteswissenschaften Rothacker (19) und Wach (26).

${ }^{65}$ Dilthey (W), I, S. 4.

${ }^{66}$ Rothacker (27), 3. 
stand der Geisteswissenschaften sind Mensch, Gesellschaft und die Produkte menschlicher Tätigkeit. All das läßt sich, wie die Natur, sowohl systematisch wie historisch untersuchen. Die Unterscheidung zwischen Wissenschaften vom Menschen und solchen von der Natur ist freilich insofern problematisch, als der Mensch auch Teil der $\mathrm{Na}$ tur ist und daher z. B. auch Gegenstand der Biologie, während die Medizin, die es nur mit Menschen zu tun hat, üblicherweise nicht als „Geisteswissenschaft" bezeichnet wird. Die Natur ist ihrerseits teilweise vom Menschen geprägt, so daß sich z. B. in der Geographie rein naturwissenschaftliche Untersuchungen mit solchen über Bevölkerungs- und Wirtschaftsstruktur verbinden. Die Geisteswissenschaften befassen sich also nicht mit dem Menschen unter all den Aspekten, unter denen er Gegenstand wissenschaftlicher Untersuchung sein kann, sondern mit dem Menschen als handelndem, erlebendem, denkendem Subjekt, mit menschlicher Gesellschaft, Geschichte und Kultur. $\mathrm{Zu}$ betonen ist freilich, daß sich die Geisteswissenschaften nicht ausschließlich, sondern nur primär mit dem Menschen befassen - da sich sein Leben in einer natürlichen Umwelt vollzieht und zum großen Teil in der Auseinandersetzung mit ihr, ist es allein aus sich selbst heraus nicht verständlich. Diese Abgrenzung des Gegenstandsbereichs der Geisteswissenschaften ist sicher nicht scharf, aber jedenfalls nicht weniger klar als die Abgrenzungen zwischen Einzeldisziplinen wie Physik und Chemie, Chemie und Biologie oder Biologie und Psychologie.

Sehr viel schwieriger ist es, einen methodologischen Unterschied zwischen Geistes- und Naturwissenschaften anzugeben. Ein solcher Unterschied kann sich auf die Verwendung verschiedener Formen von Begriffen und Erklärungen, oder verschiedener Verfahren der Beobachtung oder Begründung beziehen. Die Diskussion hat sich vor allem auf die beiden ersten Punkte konzentriert. Man hat $\mathrm{z}$. B. behauptet, geisteswissenschaftliche Begriffe seien qualitativ, naturwissenschaftliche quantitativ. Aber erstens kommen in den Naturwissenschaften auch qualitative Begriffe vor und in den Geisteswissenschaften (z. B. der Psychologie) auch quantitative, und zweitens lassen sich alle qualitativen Begriffe (wenn auch nicht immer mit Gewinn), unabhängig von ihrem Gegenstandsbereich, metrisieren. ${ }^{67}$ Man kann nur

67 Für eine erste Einführung in die Theorie der Metrisierung von Begriffen vgl. z. B. Stegmüller (70), 1. Halbbd., Kap. I, oder Kutschera (72), Kap. 1. 
sagen, daß die Begriffe, die in den Geisteswissenschaften verwendet werden, mit wenigen Ausnahmen eine Metrisierung (noch) nicht als sinnvoll erscheinen lassen. Aber das gilt ebenso für viele Begriffe der Biologie. Auch die Unterscheidung „,dynamischer" geisteswissenschaftlicher von „,statischen" naturwissenschaftlichen Begriffen ist nicht haltbar. Sind physikalische Begriffe wie „Beschleunigung“, „Kraft“, „Impuls“ nicht dynamisch? Außerdem gibt es keine dynamischen Begriffe, sondern nur Begriffe zur Beschreibung dynamischer Phänomene, und diese kommen sowohl in den Natur- als auch in den Geisteswissenschaften vor. Vage und unbrauchbar ist endlich auch die Rede von ,organologischen“ Begriffen in den Geisteswissenschaften. Gemeint sind Begriffe wie Organismus, Ganzheit, Gestalt, Wachstum, Leben usw., die aus dem biologischen Bereich entnommen sind und oft auf Kulturen oder kulturelle Phänomene angewendet werden. Gerade die biologische Herkunft dieser Termini beweist aber, daß sie nicht typisch für die Geisteswissenschaften sind. Insgesamt kann man sagen, daß der Versuch, Begriffsformen aufzuweisen, die nur in den Geisteswissenschaften vorkommen, bislang erfolglos geblieben ist. Natürlich gibt es in jeder Wissenschaft Begriffe, die nur in ihr vorkommen - das ist eine Trivialität. So kommen z. B. Begriffe wie „Handlung', ,Sprache' oder ,politische Institution' nur in den Geisteswissenschaften vor. Begriffsformen, wie Klassifikatorisch, Komparativ, Metrisch oder Typenbegriff sind aber so umfassend, daß sie sich nicht nur einzelnen wissenschaftlichen Disziplinen oder Gruppen solcher Disziplinen zuordnen lassen. Die meisten Thesen über einen prinzipiellen methodologischen Unterschied zwischen Geistes- und Naturwissenschaften beziehen sich denn auch darauf, daß wir geisteswissenschaftliche Phänomene in einer prinzipiell anderen Weise verstehen können als naturwissenschaftliche. Darauf gehen wir unten ein.

Was endlich die Erkenntnisziele angeht, so hat man einen Unterschied zwischen Geistes- und Naturwissenschaften erstens darin sehen wollen, daß diese nomothetisch, jene aber idiographisch seien. Diese Terminologie geht auf Windelband zurück, während Rickert in gleichem Sinn sagt, die Geisteswissenschaften seien individualisierend, die Naturwissenschaften hingegen generalisierend. Nach Windelband interessiert in den Naturwissenschaften das Einzelne nur als Exemplar einer Gattung, in den Geisteswissenschaften ist hingegen gerade die individuelle Persönlichkeit, das einmalige historische Ereignis Objekt der Erkenntnisbemühung. Der Biologe interessiert sich nicht für ein 
bestimmtes Pferd, sondern will allgemeine Aussagen über Körperbau und Verhaltensweisen von Pferden machen. Dem Historiker jedoch geht es nicht darum, wie sich Menschen in Situationen einer bestimmten Art allgemein verhalten, sondern was z. B. Alexander der Große in einer bestimmten historischen Situation getan hat. An diesem Gedanken ist sicher etwas Richtiges, aber in dieser Allgemeinheit ist die Behauptung nicht haltbar, in den Naturwissenschaften spiele nur das Allgemeine, in den Geisteswissenschaften nur das Besondere eine Rolle. Auch der Naturwissenschaftler interessiert sich für einzelne Gegenstände, für den Mars oder unsere Milchstraße, und auch der Geisteswissenschaftler bemüht sich um allgemeine Aussagen, wie z. B. über das Verhältnis von Malerei, Plastik und Architektur im Rokoko. Auch der Psychologie geht es nicht um Herrn Meier, sondern sie will allgemeine Aussagen über den Menschen machen. Das Interesse für das Einmalige, Unwiederholbare ergibt sich zum Teil einfach aus einer historischen Betrachtung, während systematische Untersuchungen immer auf generelle Aussagen abzielen. Man kann auch nicht behaupten, die Geisteswissenschaften stellten keine eigenen Gesetze auf - seien also in diesem Sinn nicht nomothetisch - , sondern benützen nur Gesetze anderer Disziplinen. Denn dann müßte man im Blick auf psychologische, soziologische und ökonomische Gesetze zumindest diese Disziplinen aus dem Feld der Geisteswissenschaften ausschließen. Richtig ist, daß es in den Geisteswissenschaften, die sich mit menschlichem Handeln (im weitesten Sinn des Wortes) befassen, die Zahl von Gesetzen im Blick auf die Rolle menschlicher Individualität, Kreativität und Spontaneität erheblich geringer ist als in den Naturwissenschaften, und daß zudem die Allgemeinheit solcher Gesetze beschränkt ist. Der Mensch ist, wie A. Gehlen sagt, von Natur ein Kulturwesen. Sein Verhalten ist sehr stark durch die kulturelle Umgebung geprägt, in der er lebt, und der Mensch - in seinen kulturunabhängigen Zügen - ist Objekt der Biologie, aber nicht der Geisteswissenschaften. Richtig ist auch, daß die Geisteswissenschaften in vielen Fällen auf ein Verstehen abzielen, das nur von Einzelphänomenen möglich ist. Darauf kommen wir unten zurück.

Ein zweiter Versuch, das Erkenntnisziel der Geisteswissenschaften von dem der Naturwissenschaften abzuheben, ist in der Behauptung zu sehen, in diesen gehe es um eine kausale Erklärung, in jenen aber um ein teleologisches Verständnis der Phänomene. Wir sehen zunächst einmal von dem Unterschied Erklären - Verstehen ab, der dabei vor- 
ausgesetzt wird, und sprechen von kausaler und teleologischer Erklärung. Dann ist zu dieser Unterscheidung zu sagen, daß sich in den Naturwissenschaften nicht alles kausal erkären läßt, und daß sich andererseits in den Geisteswissenschaften manches auch kausal erklären läßt - das gilt insbesondere auch für Handlungen, wie wir im letzten Abschnitt sahen. Es gibt ferner in der Biologie funktionale, insofern also teleogische Erkärungen, und solche Erklärungen spielen auch in den Geisteswissenschaften eine wesentliche Rolle, z. B. für das Verständnis sozialer, ökonomischer und politischer Institutionen. ${ }^{68}$

In den Naturwissenschaften gibt es keine intentionalen und rationalen Erklärungen, aber das ist eine Folge der Abgrenzung ihres Gegenstandsbereichs. Richtig ist, daß die Geisteswissenschaften dadurch zur Domäne teleologischer Betrachtungen geworden sind, daß diese aus den modernen Naturwissenschaften verdrängt wurden.

Nach all dem ist die zentrale These zur Methodologie der Geisteswissenschaften in der schon von Dilthey vertretenen Behauptung zu sehen, diese seien verstehende, die Naturwissenschaften hingegen erklärende Wissenschaften: „Die Natur erklären wir, das Seelenleben verstehen wir" ${ }^{69}$ Hier wird ein Gegensatz Erklären - Verstehen konstruiert, während wir in 2.1 gesagt hatten, alles was man verstanden habe, könne man auch erklären, und alle Erklärungen dienten dem Verstehen. Was für eine Art von Verstehen ist also in dieser These ge-

${ }^{68}$ Aus dem teleologischen Charakter vieler Erklärungen ergibt sich, daß Aussagen über Werte und Normen in den Geisteswissenschaften eine große Rolle spielen. Daraus folgt aber nicht, daß die Geisteswissenschaften im Gegensatz zu den Naturwissenschaften normative Wissenschaften wären, in denen Wertungen vorgenommen und Normen aufgestellt werden. Aussagen über Werte und Normen können normativ sein, wie z. B. das Verbot ,Keiner darf einen anderen töten, es sei denn in Notwehr"; sie können aber auch nur behaupten, daß in gewissen Wert- oder Normensystemen bestimmte normative Sätze gelten, wie z. B. der Satz „Nach dem in der Bundesrepublik geltenden Recht darf keiner einen anderen töten, es sei denn in Notwehr". Solche Aussagen bezeichnet man auch als deskriptiv. (Vgl. dazu Kutschera (81), 2.1.) In den Geisteswissenschaften kommen zweifellos auch normative Aussagen vor wie z. B. solche über den Rang einer historischen Persönlichkeit oder über die Qualität eines Kunstwerks; ein Großteil der geisteswissenschaftlichen Aussagen über Werte und Normen ist aber deskriptiver Natur.

${ }^{69}$ Dilthey (W), Bd. 5, 144. 
meint? Das herauszufinden ist nun nicht ganz einfach, da die Aussagen Diltheys und seiner Nachfolger sich nicht gerade durch Präzision auszeichnen. Die Reflexionen zur Methodologie der Geisteswissenschaften sind aus einer ganz anderen philosophischen Tradition hervorgegangen als jene zur Methodologie der Naturwissenschaften. Vergleicht man z. B. H. G. Gadamers "Wahrheit und Methode" oder die Arbeiten von E. Betti (54) und (55) mit C. G. Hempels „Aspects of Scientific Explanation", so wird der immense Unterschied im Präzisionsniveau deutlich. Von daher ist es verständlich, daß sich die analytische Philosophie lange Zeit kaum ernsthaft mit den Arbeiten der Hermeneutiker auseinandergesetzt hat und sich die Kritik daran recht leicht machen konnte. ${ }^{70}$ In den letzten Jahren zeigt sich aber ein zunehmendes Interesse der analytischen Philosophie an der Methodologie der Geisteswissenschaften, und dabei wird deutlich, daß Vagheit und Dunkelheit der Aussagen über diesen Gegenstand keineswegs von der Sache selbst erzwungen werden.

Worin also soll jenes für die Geisteswissenschaften typische Verstehen bestehen? Es ist sicher keine Methode, wie oft behauptet wird. Verstehen im normalen Sinn ist eine - evtl. vermittels einer Methode erzielte - Leistung, nicht aber ein Verfahren. Eine Kunstlehre des Verstehens, speziell eine Hermeneutik als Kunst des Auslegens oder Erklärens ist vom Verstehen selbst zu unterscheiden. ${ }^{71}$ Für das fragliche Verstehen sollen nun vor allem drei Merkmale charakteristisch sein:

1. Es ist ein einfüblendes („empathetisches“) Verstehen.

Wir verstehen die Handlung eines anderen, indem wir uns in seine Situation hineinversetzen und einsehen, daß wir in dieser Situation ebenso gehandelt hätten. Nach Dilthey haben wir zu den Gegenständen der Geisteswissenschaften, speziell zu menschlichen Handlungen,

70 Zur analytischen Kritik am hermeneutischen Verstehensbegriff vgl: z. B. Abel (48), Hempel (65), IV und Stegmüller (69), Kap. VI,5 und (79), $29 \mathrm{ff}$.

${ }^{71}$ Nach Betti ist in diesem Sinn Auslegung ein Verfahren mit dem Ziel des Verstehens. - Unter „Hermeneutik“ versteht man nicht nur eine Kunstlehre oder Technik der Auslegung, sondern auch eine Theorie der Interpretation. Nur in diesem Sinn kann sie ein Teil der geisteswissenschaftlichen Methodologie sein. Eine Interpretationskunst ist ebensowenig Thema dieser Methodologie wie eine Kunst des Experimentierens Teil der Methodologie der Naturwissenschaften ist. 
Erlebnis- und Denkweisen einen unmittelbaren Zugang, sie haben für uns eine unmittelbare Einsichtigkeit, die physische Phänomene nicht haben. Die Natur können wir nur ,,von außen“ betrachten. Meine eigenen Erlebnis- und Reaktionsweisen, Ziele und Motivationszusammenhänge sind mir hingegen unmittelbar ,,von innen her" einsichtig. Ick habe ein unmittelbares Wissen davon, wie ich etwas erlebe, wie ich darauf reagiere, was ich will, und auf diesem Wissen beruhen meine Annahmen über Fremdpsychisches. Ich weiß, wie mir in gewissen Situationen zumute ist, wie ich mich verhalte, und so kann ich verstehen, wie anderen in solchen Situationen zumute ist und wie sie sich verhalten. Als Grunddisziplin der Geisteswissenschaften sah Dilthey daher zuerst eine verstehende Psychologie an.

2. Es ist ein anschauliches, erlebnismäßiges Verstehen.

Die Einsicht, die sich mit diesem Verstehen verbindet, ist nicht von der Art, daß sie sich sprachlich vollständig ausdrücken ließe. Auch das Verstehen, von dem die Hermeneutiker reden, soll in einer Einsicht, einer Erkenntnis bestehen, aber nicht in einer Erkenntnis, die sich adäquat in Sätzen der Form „Ich habe erkannt, daß..." mitteilen läßt. Wie eine auch noch so detaillierte Beschreibung von etwas, das ich wahrnehme, für den anderen kein Äquivalent einer eigenen Anschauung ist, so ist auch eine noch so ausführliche Schilderung dessen, was ich anschaulich an einem Gegenstand oder Vorgang verstehe, für andere kein Ersatz eines eigenen erlebnismäßigen Verstehens. Verstehen in diesem Sinn kann man freilich nicht nur Objekte oder Ereignisse, die man wahrnimmt, sondern z. B. auch historische Vorgänge, die man sich durch Phantasie und Einbildungskraft anschaulich machen, die man erlebnismäßig nachvollziehen kann. Am fraglichen Verstehen sind auch emotionale Kräfte, besonders Gefühl und Empfindung beteiligt. Ein solches Verstehen kann sich nicht auf generelle, sondern immer nur auf individuelle Sachverhalte oder Objekte beziehen. Daher spielt es auch nur in den ,,idiographischen“ Geisteswissenschaften eine Rolle.

3. Es handelt sich um ein absolutes Verstehen, nicht nur um ein relatives. Die physikalischen Vorgänge folgen einer ,,blinden Naturkausalität“ (Dilthey) und müssen somit aus Ursachen und Naturgesetzen erklärt werden. Diese Gesetze und Ursachen sind uns nicht einsichtiger als die Wirkung, daher verstehen wir diese nur als durch anderes bedingt. So entsteht die Frage nach dem Grund der Naturgesetze und dem Bestehen der Ursachen, usf. Der Zusammenhang von Erleben und 
Handeln als Motivationszusammenhang ist uns hingegen unmittelbar und ohne weitere Erklärungen und Begründungen verständlich. Die Natur können wir daher nur beschreiben, aber nicht verstehen. Nur im Bereich menschlichen Verhaltens und Erlebens gibt es ein Verständnis, in dem die Phänomene selbst, in ihrem Warum und Wozu, einsichtig werden.

An dieser Charakterisierung eines geisteswissenschaftlichen Verstehens ist nicht so sehr der erste Punkt von Interesse, auf den sich die Diskussion fast ausschließlich konzentriert hat, als der zweite Punkt. Es ist also zu fragen, ob es so etwas wie ein anschauliches oder erlebnismäßiges Verstehen gibt, und worin ggf. seine Eigenart und seine Leistung besteht. Alles Verstehen ist ein Erkennen, hatten wir in 2.1 gesagt, und das ist auch hier anzunehmen - ein erlebnismäßiges Verstehen, das kein Erkennen wäre, hätte jedenfalls für die Geisteswissenschaften keine Bedeutung. Wir fragen daher zunächst allgemeiner nach Existenz, Eigenart und Leistung eines erlebnismäßigen oder anschaulichen Erkennens. Zweifellos gibt es Erkenntnis aufgrund von Erleben, durch (äußere) Wahrnehmung. ${ }^{22}$ Und die Rolle der Anschauung in der Geometrie zeigt auch, daß man von einem Erkennen aufgrund von Anschauungen reden kann, die keine Erlebnisse sind. Erleben ist aber nicht nur eine Quelle von Erkenntnissen und ein Mittel zur Überprüfung von Aussagen über eine Sache, es liefert uns nicht nur Erkenntnis über eine Sache (cognitio circa rem), sondern Erkenntnis der Sache selbst (cognitio rei). Auch noch so viele und noch so detaillierte Informationen über das Bild „Die Erschießungen des 3. Mai“ von Goya, von deren Richtigkeit ich überzeugt bin, können eine eigene Anschauung von diesem Bild nicht ersetzen. Und das nicht nur, weil allein die eigene Anschauung es ermöglicht, die Informationen zu prüfen und zu ergänzen - auch das ist natürlich wichtig - , also nicht, weil die eigene Anschauung mir zuverlässigere, genauere und zahlreichere Informationen über das Bild liefert, sondern weil allein sie mir nicht nur Erkenntnisse über das Bild vermitteit, sondern die Kenntnis des Bildes selbst. Ebenso ersetzen auch noch so viele kor-

${ }^{2}$ Die Rede von innerer Wahrnehmung und dem Erleben eigenseelischer Vorgänge ist schief. Vgl. dazu den Abschnitt 3.1. Wir beschränken uns daher nur auf äußere Wahrnehmung und das Erleben von Gegenständen und äuBeren Vorgängen. 
rekte Informationen über einen Menschen nicht die persönliche Bekanntschaft mit ihm. Der konkrete Mensch und seine Individualität erschließen sich uns erst, wenn wir ihm begegnen.

Erkenntnis in dem Sinn, in dem wir das Wort bisher immer verwendet haben, ist Erkenntnis, daß etwas der Fall ist. Erkenntnis eines Gegenstands kann in diesem Sinn nur in Erkenntnissen bestehen, daß er diese und jene Eigenschaften hat, in Erkenntnissen über ihn. Davon verschieden ist eine Kenntnis des Gegenstands, die man nicht schon mit solchen Erkenntnissen über ihn hat, sondern erst dann, wenn man ihn wahrgenommen hat. ${ }^{73}$ Wir kennen einen Gegenstand, wenn wir ihn (unter normalen Bedingungen) wiedererkennen, wenn wir ihn von anderen unterscheiden können. Diese Kenntnis verbindet sich mit gewissen Erkenntnissen über ihn - wir können z. B. kaum behaupten, wir kennten einen Menschen, wenn wir nicht wissen, ob er ein Mann oder eine Frau ist - , impliziert aber keine bestimmten Erkenntnisse über ihn. Jemand, der einen Menschen nicht kennt, kann sehr viel mehr über ihn wissen als ein anderer, der ihn kennt. Und viele Leute, die das Bild von Goya im Prado gesehen haben, wissen sehr viel weniger darüber als einer, der es nie gesehen, aber viel darüber gelesen hat. Man kann also nicht sagen, der kognitive Wert des Erlebens bestehe allein in den Erkenntnissen über Gegenstände, die man daraus gewinnt. Die Fähigeit, diesen Gegenstand wiederzuerkennen und ihn von anderen zu unterscheiden, wird man sicher als kognitive Leistung ansprechen müssen. Sie ergibt sich aber nicht aus Erkenntnissen über ihn, sondern aus der Kenntnis von ihm.

Die kognitive Leistung des Erlebens besteht aber nicht nur in der Kenntnis des Gegenstands, die sie vermittelt, und in Erkenntnissen über ihn, die wir daraus schöpfen. Man kann vielmehr sagen, daß der Inhalt des Erlebens über den Inhalt der begrifflichen Bestimmungen hinausgeht, die sich damit verbinden. Im Erleben ist vieles mehr oder minder deutlich mitgegeben, was wir nicht in Form gedanklicher oder sprachlicher Urteile explizit auffassen; es ist uns vieles bewußt, was

${ }^{73}$ Wenn wir hier Kenntnis von Erkenntnis unterscheiden, so ist $\mathrm{zu}$ betonen, daß beide Bezeichnungen oft im gleichen Sinn verwendet werden. Statt ,Ich weiß, was das Wort bedeutet" kann man auch sagen „Ich kenne die Bedeutung des Wortes“, statt „Ich weiß, was er beabsichtigt" auch „Ich kenne seine Absicht". Es gibt also im normalen Sinn des Wortes auch Kenntnis von Sachen, die man nicht erleben oder sich anschaulich vorstellen kann. 
wir begrifflich nicht bestimmen. Jede begriffliche Charakterisierung eines Gegenstands trifft auf viele Gegenstände zu, erfaßt ihn also nicht in seiner Individualität, in der wir ihn erleben. Das Netz unserer Begriffe bildet ein verhältnismäßig grobes Raster zur Klassifikation der Phänomene. Unsere Sprache hat z. B. nicht die Ausdrucksmittel, um alle Farbnuancen, die wir erlebnismäßig unterscheiden können, zu benennen. Nun gibt es zwar keine erlebnismäßigen Unterschiede, die sich sprachlich prinzipiell nicht erfassen ließen, es gibt keine prinzipielle Grenze der Genauigkeit sprachlicher Unterscheidungen, aber es bleibt doch immer ein deutlicher Abstand zwischen der Charakterisierung eines Gegenstands durch eine detaillierte Beschreibung und der Art und Weise seiner Bestimmtheit im Erleben. Beschreibungen können den Gehalt von Wahrnehmungen nicht ausschöpfen. ${ }^{74}$ Erst im Erleben einer Sache - eines Gegenstands oder eines Vorgangs - verbinden sich unsere Informationen über sie zu einem Bild der Sache selbst; erst im Erleben ist sie uns präsent. Daher ist die Idealform des Erkennens oft nach dem Muster der Anschauung, der Wahrnehmung, speziell des Sehens bestimmt worden. Besonders deutlich wird das z. B. bei Platon, Goethe, Schelling oder Hegel. ${ }^{75}$

Unser Erleben ist nicht nur die Grundlage unserer Annahmen über die Welt, sondern auch die Grundlage unserer Sprache. Wir können Eigennamen nur dadurch einen Bezug zuordnen, daß wir die Gegenstände kennen, die sie bezeichnen, oder vermittels der Beziehung der bezeichneten Objekte zu Gegenständen, die wir kennen. Und wir erlernen Prädikate, mit denen wir über die Dinge reden, durch Beispiele

${ }^{74}$ Es ist nicht nur so, daß wir bei längerer Beobachtung eines Gegenstands unsere Aufmerksamkeit auf immer neue Eigenschaften richten und so immer neue begriffliche Bestimmungen angeben, also die Reihe seiner begrifflichen Bestimmungen fortsetzen können. Vielmehr bilden in jedem Moment der Wahrnehmung die begrifflichen Feststellungen über den Gegenstand nur einen Teil der Bestimmungen, mit denen er uns erlebnismäßig erscheint.

${ }^{75}$ Hinzu kommt, daß am Erleben und der Anschauung neben den Kräften des Verstandes auch emotionale Kräfte beteiligt sind. Wenn auch nach der heute gängigen Auffassung Gefühle dem subjektiven Bereich zugehören und nichts für die kognitive Erfassung der Sache selbst leisten, so ist doch zu betonen, daß es einen breiten Strom der Kritik an dieser engen Auffassung des Kognitiven gibt; daß sie nicht so selbstverständlich ist, wie sie uns aufgrund langer Gewöhnung scheint. 
ihrer Anwendung auf Dinge, die wir kennen; dadurch erfassen wir ihren anschaulichen Sinn, und nur aufgrund ihres anschaulichen Sinns - Kant spricht von einem Schema - können wir die empirischen Prädikate sinnvoll verwenden. ${ }^{7}$

Ähnliches gilt zum Teil auch für Anschauungen, die nicht in Wahrnehmungen bestehen. Wir können uns historische Persönlichkeiten oder Vorgänge veranschaulichen, uns ein Bild von ihnen machen; wir können uns eine Vorstellung von einem Tempel machen, von dem kaum mehr als die Fundamente erhalten sind. Eine Menge von Informationen über eine Sache erlaubt es uns nicht immer, uns ein Bild von ihr zu machen. Gelingt uns das aber, so bedeutet das für uns einen Gewinn. Wir haben dann zwar nicht mehr Erkenntnisse über sie als vorher, aber sie ordnen sich uns nun zu einem Ganzen, das wir aufgrund unserer Bekanntschaft mit Ähnlichem vervollständigen können. Einen kognitiven Wert hat eine solche anschauliche Vorstellung natürlich nur insoweit, als sie ein korrektes Bild der Sache darstellt. Ein Bild, das wir uns machen, kann aber auch einen heuristischen Wert dadurch haben, daß wir aufgrund dieses Bildes sagen: „So könnte es (gewesen) sein" und so Hypothesen über die Sache formulieren, die wir dann überprüfen.

Unser kognitives Interesse beschränkt sich oft darauf, bestimmte Informationen über Gegenstände zu erhalten. Oft zielt es aber auch auf eine Kenntnis der Sachen selbst oder auf eine Anschauung von ihnen. Letzteres gilt sicher für viele Gegenstände, mit denen sich die Geisteswissenschaften befassen, z. B. für Kunstwerke, historische Persönlichkeiten oder Vorgänge. Es gilt aber weder für alle Gegenstände der Geisteswissenschaften, noch ausschließlich für deren Gegenstände. Nicht alles, womit sich die Geisteswissenschaften befassen, ist überhaupt einer Wahrnehmung oder Veranschaulichung zugänglich. Das gilt nur für individuelle Personen, Gegenstände oder Vorgänge, nicht aber für allgemeine Sachverhalte (die kann man sich nur exemplarisch an Einzelfällen veranschaulichen) oder für Abstraktes, wie z. B. die Bedeutung wissenschaftlicher Texte. Auch das kognitive Interesse an der Natur kann sich auf Anschauung richten. Das Interesse der Naturwissenschaften freilich gilt seit Beginn der Neuzeit nicht den Dingen selbst, sondern ihren Zusammenhängen; man unter-

${ }^{76} \mathrm{Vgl}$. dazu auch den Abschnitt 7.4. 
sucht nicht, was die Dinge sind, sondern nach welchen Gesetzen sie sich verhalten. ${ }^{7}$

Die Kenntnis einer Sache, ihr Erleben läßt sich nun aber nicht mitteilen. Mitteilen lassen sich nur Erkenntnisse über die Sache, und da wissenschaftliche Erkenntnis sich in Urteilen ausdrückt, ist sie immer nur Erkenntnis über Dinge. Es gibt also keine geisteswissenschaftliche Erkenntnis der Dinge, sondern nur eine Erkenntnis der Dinge mithilfe geisteswissenschaftlicher Erkenntnisse. ${ }^{78} \mathrm{Im}$ gleichen Sinn gibt es auch eine Erkenntnis z. B. von Pflanzen oder Tieren mithilfe naturwissenschaftlicher Einsichten. Wie man aufgrund kunstgeschichtlicher Erkenntnisse zu einer klareren Anschauung von Kunstwerken kommen kann, so auch aufgrund biologischer Erkenntnisse zu einer detaillierteren Wahrnehmung des Lebens in der Natur. Selbst wenn alle wissenschaftliche Erkenntnis nur Erkenntnis über Dinge ist, so sind Erleben und Anschauung doch auch wissenschaftlich nicht wertlos; wir haben ja schon oben betont, daß es ohne Kenntnis der Dinge, ohne Erleben keine Erkenntnis über die Dinge gäbe.

Wie steht es nun mit einem erlebnismäßigen, anschaulichen Versteben? Man kann sicher von einem Verstehen aufgrund von Wahrnehmung reden. Hier geht es aber um etwas sehr viel Spezielleres, um ein Verstehen, das in einem erlebnismäßigen oder anschaulichen Erkennen besteht oder darauf beruht, und das sich daher nicht in Form einer Erklärung vollständig oder angemessen mitteilen läßt. Wenn erlebnismäßige Erkenntnis eine cognitio rei im Gegensatz zu einer cognitio circa rem ist, so kann sie kein Verstehen von Sachverhalten beinhal-

" Das ist der Kernpunkt der Kritik Goethes an den Naturwissenschaften. Zum Erkenntnisinteresse der Naturwissenschaften vgl. a. den Abschnitt 3.4. Mit der ansonsten unbrauchbaren Unterscheidung idiographisch - nomothetisch ist wohl auch diese Unterscheidung zwischen einem Interesse an einer cognitio rerum und einem Interesse lediglich an einer cognitio circa res gemeint.

${ }^{78}$ Wie wir eine Sache erleben, hängt auch davon ab, welche Informationen wir über sie schon haben. Die Aspekte, unter denen man das Bild von Goya betrachtet, und das, was man dabei erfaßt, ergeben sich auch aus dem, was man über dieses Bild weiß (z. B. über seine Entstehungszeit, Komposition und kunstgeschichtliche Bedeutung), über Goya und sein Werk, über die Erhebung der Spanier gegen die Franzosen und die grausame Unterdrückung des Aufstands in Madrid durch Murat, die zu den Erschießungen am 2. und 3. Mai 1808 führte. 
ten oder begründen. Das gesuchte Verstehen muß also wie das erlebnismäßige Erkennen ein Verstehen von Gegenständen oder Vorgängen sein, und so etwas läßt sich wohl nur in Analogie zum Bedeutungsverstehen auffassen. Wir sagen zwar auch ,Ich kann Fritz gut verstehen“ oder „Ich verstehe diesen Mechanismus“, aber damit ist doch ein Verstehen von Sachverhalten gemeint: ich verstehe, warum sich Fritz so verhält, oder wie der Mechanismus funktioniert. Wie Sätze etwas ausdrücken, so drücken auch Kunstwerke, z. B. Bilder etwas aus. Wenn man sagt, Sätze drückten begrifflich bestimmte Inhalte aus, Propositionen des Inhalts z. B., daß Dinge bestimmte Attribute haben, so ist das in gewisser Weise tautologisch: Propositionen sind eben das, was Sätze, Attribute das, was Prädikate bedeuten. Propositionen und Attribute werden nicht in der Erfahrung vorgefunden und nur sprachlich abgebildet, sondern die sprachlichen Bestimmungen stellen eine Deutung der Erfahrung dar. Kunstwerke drücken nun keine begrifflich bestimmten Inhalte aus. Ein Bild ist keine Übersetzung eines gedanklichen Inhalts in Farben und Formen durch den Künstler, und man kann daher auch das, was man versteht, wenn man es versteht, nicht adäquat und vollständig in Sprache übersetzen, also sprachlich erklären. Die Sprache ist ein Ausdrucksmittel, die verschiedenen Medien künstlerischer Gestaltung sind andere Ausdrucksmittel, und wie sich der Text einer Sprache nicht ohne Modifikationen seiner Bedeutung in eine andere Sprache übersetzen läßt, so erst recht nicht ein malerischer oder musikalischer Ausdruck in einen sprachlichen. Natürlich kann man, wenn wir wieder an das Bild von Goya denken, eine ganze Menge über seinen Gehalt aussagen; man kann die dargestellte Szene im Detail schildern, etwas über die Art der Darstellung sagen, die Farben, die Beleuchtung, die Komposition, das was Goya damit zum Ausdruck bringen wollte, und all das ist Teil einer Interpretation des Bildes. Aber das Gemälde ist keine Erzählung, und eine Schilderung des Bildes kein Kunstwerk. Es sind hier keine gedanklichen Inhalte zum Ausdruck gebracht, sondern der dargestellte Vorgang ist malerisch, anschaulich begriffen, und man versteht das Bild nur, wenn man es anschaulich auffaßt. Eine Interpretation kann seinen Gehalt nicht ausdrücken, das anschauliche Verstehen nicht ersetzen, sondern diesem nur dadurch dienen, daß sie uns mehr und genauer zu sehen lehrt.

Es gibt auch ein erlebnismäßiges Verstehen von Dingen und Vorgängen in der Natur. Darauf wollen wir hier jedoch nicht eingehen. 
Uns genügen die bisherigen skizzenhaften Hinweise, denn wichtig ist für uns nur, daß es auch so etwas wie ein erlebnismäßiges, anschauliches Verstehen gibt, das sich nicht adäquat in sprachlichen Erklärungen mitteilen läßt. Eine genauere Analyse des Erlebens, des erlebnismäßigen Erkennens und Verstehens wäre Aufgabe der Ästhetik, nicht der Erkenntnistheorie.

Wie nur Erkenntnis über Dinge mitteilbar ist, so ist auch ein Verständnis nur in Form von Erklärungen mitteilbar. Als wissenschaftliches Verstehen kann also auch nur gelten, was in Erklärungen formuliert werden kann. Es gibt daher zwar ein erlebnismäßiges Verstehen, das für manche Gegenstände der Geisteswissenschaften wichtig ist, aber es ist kein geisteswissenschaftliches Verstehen, und es ist weder von allen noch ausschließlich von Gegenständen der Geisteswissenschaften möglich.

Vieles und bei weitem das Interessanteste, was von Seiten der Hermeneutiker über das Verstehen in den Geisteswissenschaften gesagt worden ist - über die Rolle emotionaler Kräfte, über ihr eigentümliches Gegenstandsverhältnis - betrifft nicht das wissenschaftliche, sondern allein das erlebnismäßige, anschauliche Erkennen und Verstehen. Dem genauer nachzugehen wäre wie gesagt Aufgabe der Ästhetik.

Zum ersten Punkt der Charakterisierung des ,,geisteswissenschaftlichen Verstehens": Bei einem erlebnismäßigen oder anschaulichen Verstehen spielt eine Art von Einfühlung sicher eine wichtige Rolle, wobei es sich aber nicht immer nur um Einfühlung in Fremdseelisches handelt, sondern z. B. auch um Veranschaulichung von Lebensbedingungen. Wo es um ein wissenschaftliches Verstehen geht, ist aber der Wert einer Einfühlung in Fremdpsychisches zurecht bestritten worden. Wir können das, was andere erleben oder anstreben oft intuitiv, aufgrund von Wahrnehmungen oder Veranschaulichungen erkennen, und dieses intuitive Erfassen beruht häufig auf einer Art von Einfühlung. Die Tatsache, daß ich nachfühlen kann, was jemand empfinden oder beabsichtigen mag, garantiert mir aber nicht, daß er tatsächlich diese Empfindungen und Absichten hat. Wir neigen oft dazu, unsere eigenen Einstellungen, Motive und Erlebnisweisen anderen unkritisch zu unterstellen. Daß ein anderer in einer Situation ähnlich empfindet oder Ähnliches will wie ich, ist eine Hypothese, die begründet oder bestätigt werden muß. Daß ein Vorgang aufgrund gewisser Hypothesen verständlich wird, impliziert nicht, daß er so zu verstehen ist. Das 
ist nur dann der Fall, wenn diese Hypothesen richtig sind. Dasselbe Phänomen kann sich mit sehr verschiedenartigen Annahmen erklären lassen, dieselbe Handlung mit verschiedenen Annahmen über Präferenzen und Überzeugungen des Handelnden. Einfühlung ist auch aus dem Grund nicht zuverlässig, weil wir oft gegenteiliges Verhalten „,verstehen“ können: Wir können ,verstehen“, wenn jemand sich über den Erfolg eines Kollegen freut, wir haben aber auch keine Schwierigkeit zu ,,verstehen“, wenn er sich darüber ärgert - nichts Menschliches ist uns fremd. Daher hat Hempel betont, daß die Tatsache, daß man das Verhalten eines anderen von den eigenen Präferenzen her verstehen kann, nur heuristischen Wert hat: sie legt die Hypothese nahe, daß der andere ähnliche Präferenzen hat, aber diese $\mathrm{Hy}$ pothese ist dann eigens zu prüfen. ${ }^{79}$ Das Verständlichwerden von Handlungen anderer aufgrund einer Einfühlung ist also nicht hinreichend für ein Verstehen dieser Handlung. Es ist aber auch nicht notwendig dafür: Man kann auch Handlungen verstehen, die aus Präferenzen hervorgehen, die einem selbst fremd sind. ${ }^{80}$ Andernfalls gäbe es kaum ein Verständnis von Persönlichkeiten, die ganz andérs geartet sind als wir selbst oder die einem fremden Kulturkreis angehören. Würde man seine eigenen Einstellungen und Ansichten oder die seiner Zeit zum Maßstab aller Dinge machen, so würde man sich auch den Blick auf andere Lebenswelten verstellen. Dilthey fordert

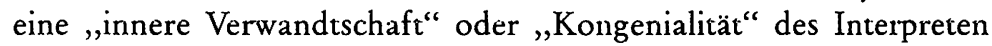
eines Textes mit dem Autor. ${ }^{81}$ Damit wird der Horizont des Verstehens aber zu sehr beschränkt; man braucht, wie Max Weber betont, nicht Cäsar sein, um ihn zu verstehen. Ebenso ist die Rede von einer „Identifizierung“ mit anderen Personen vage und irreführend. Gadamer spricht von einer „Horizontverschmelzung“. All das sind metaphorische Ausdrücke, mit denen sich kein genauer Sinn verbinden läßt. Was man fordern kann, ist eine Aufgeschlossenheit für die Viel-

${ }^{79}$ Das hat auch schon $M$. Weber in (68), 428, 437 betont. Vgl. dazu Hempel (65), $238 \mathrm{f}$., $257 \mathrm{f}$. und Stegmüller (69), $360 \mathrm{ff}$. Ähnliches hatten wir oben zum kognitiven Wert einer Veranschaulichung von historischen Vorgängen gesagt.

${ }^{80}$ Auch darauf hat schon Max Weber hingewiesen, vgl. (66), 6.

81 E. Betti spricht von der Notwendigkeit einer ,kongenialen Aufgeschlossenheit". 
falt menschlicher Charaktere und Kulturen. Oft ist es außerordentlich schwierig, in die Vorstellungswelt anderer Völker und Zeiten einzudringen, und man muß dabei auch in der Lage sein, eigene Vorurteile und Denkgewohnheiten infrage zu stellen. Darin liegt ja gerade ein wichtiger Nutzen der Beschäftigung mit Geschichte. Einfühlung ist also kein Ersatz für ein wissenschaftliches Verstehen von Handlungen, insbesondere für ein intentionales oder rationales Verstehen.

Die Behauptung endlich, ein einfühlendes Verstehen von Fremdseelischem sei ,absolut", ist naiv, denn sie beruht letztlich darauf, daß wir unser eigenes Erleben und Streben als selbstverständlich ansehen und uns also nicht wundern, wenn sich andere Leute so verhalten, wie wir das in ihrer Situation täten. In diesem naiven Sinn gibt es natürlich auch ein ,,absolutes" Verstehen physikalischer Phänomene. Wir wissen, daß Gegenstände, die nicht festgehalten werden, zur Erde fallen; es ist uns selbstverständlich; also verstehen wir es ,absolut". Warum will man also hier von einer uneinsichtigen ,blinden Naturkausalität" sprechen? Fragen nach dem Grund eigener Präferenzen und Überzeugungen haben freilich leicht den Anschein des Absurden, sind aber keineswegs immer abwegig. Daß wir dies tun wollen, läßt sich oft damit begründen, daß wir jenes erreichen wollen; daß wir dies glauben, damit, daß wir jenes glauben und überzeugt sind, daß sich dies daraus ergibt. Auch die Frage, warum wir die Präferenzen haben, die wir tatsächlich haben, ist keinesfalls absurd. Man kann sich z. B. überlegen, welchen Einfluß Erziehung und Erfahrung darauf haben; ob Präferenzen Produkte der Natur und der gesellschaftlichen Umwelt sind, in der wir leben, oder ob wir sie selbst bestimmen können. ${ }^{82}$ Wahrscheinlichkeiten ergeben sich aus Informationen mithilfe früherer Wahrscheinlichkeiten. Woher stammt aber die apriori-Wahrscheinlichkeit, die man annehmen wird, wenn man die Entwicklung unserer Überzeugungen als kohärenten Prozess der Bildung bedingter Wahrscheinlichkeiten rekonstruieren will? Daß wir dies und jenes glauben und wollen, sind kontingente Tatsachen, hinter die wir ebenso zurückfragen können wie hinter die kontingenten Tatsachen der Physik.

${ }^{82}$ Solche Fragen führen auf das Problem der Willensfreiheit. Vgl. dazu Kutschera (81), Kap. 6. 
Der Grundgedanke, auf den sich die These stützt, menschliches Verhalten habe ein höheres $M a ß$ an Verständlichkeit urd Einsichtigkeit als die Vorgänge in der Natur, ist wohl der, daß ein teleologisches Verstehen befriedigender ist als ein kausales. Teleologische Antworten sind aber keine Antworten auf Fragen nach den Ursachen für die Be-dingungen, denen unser Handeln unterworfen ist, und solange wir die nicht verstehen, verfügen wir auch nicht über ein ,,absolutes" Verständnis menschlichen Handelns.

Abschließend können wir sagen: Das, was von Dilthey und seinen Nachfolgern als „Verstehen“ charakterisiert wurde, ist bei weitem nicht die einzige Art und Weise, in der wir die Phänomene verstehen, von denen die Geisteswissenschaften handeln, und es ist kein Verstehen, dessen Inhalt sich in Form wissenschaftlicher Erklärungen darstellen ließe. Sofern aber das Verstehen erklärbar ist, fällt es mit kausalem, teleologischem, genetischem Erklären zusammen. Das Erkenntnisinteresse der Geisteswissenschaften zielt auch keineswegs nur auf solches Verstehen ab. Das, was in der Quellenkritik der Geschichtswissenschaft oder der Textkritik der Philologie geleistet wird, hat mit solchem Verstehen wenig zu tun. Ein großer Teil der geisteswissenschaftlichen Arbeit dient einfach der Beschreibung, Klassifikation und Ordnung der Phänomene. In dieser Arbeit spielt auch schlichtes Bedeutungsverstehen wie kausales, funktionales und genetisches Verstehen eine wichtige Rolle. Dem anschaulichen, erlebnismäßigen Verstehen kommt zweifellos eine wichtige Rolle zu, aber das gilt nicht nur für die Phänomene, mit denen sich die Geisteswissenschaften befassen, sondern auch für die Phänomene der Natur. Zur Abgrenzung der Geistes- von den Naturwissenschaften leistet also die Unterscheidung anschaulichen und erklärbaren Verstehens nichts. 



\section{Wahrnehmung und Wirklichkeit}

\subsection{Wahrnehmung}

In den ersten beiden Kapiteln haben wir uns mit Erkenntnis im allgemeinen befaßt. Mit diesem Kapitel wenden wir uns der Erfahrungserkenntnis $\mathrm{zu}$, die, wie schon in der Einleitung gesagt wurde, das Hauptthema der Erkenntnistheorie bildet. Das Wort „Erfahrung“ verstehen wir dabei im ,natürlichen“, d. h. im nicht-normativen Sinn. Werterfahrung und Werterkenntnis untersuchen wir hier nicht; sie sind Gegenstand der Ethik und Ästhetik.

Will man Erfahrungserkenntnis von anderen Erkenntnistypen abgrenzen, so kann man sich entweder auf den Gegenstandsbereich oder auf den Begründungsmodus jener Sätze beziehen, mit denen die Inhalte von Erfahrungserkenntnissen ausgedrückt werden. Da die Mannigfaltigkeit der Gegenstände der Erfahrungserkenntnis kaum übersehbar ist, wird man pauschal sagen: Gegenstand der Erfahrungserkenntnis ist unsere Welt; Erfahrungssätze sagen etwas über die Welt aus. „Welt" ist dabei nicht nur im Sinn von „Außenwelt" oder von „,physischer Welt" zu verstehen, denn es gibt auch Erfahrung von Seelischem. Spricht man mit Leibniz und der modernen intensionalen Logik von ,möglichen Welten“, so machen also Erfahrungssätze nicht Aussagen über alle möglichen Welten, sondern über unsere Welt, über die wirkliche unter den vielen möglichen Welten. Das läßt sich auch so ausdrücken: Ein Erfahrungssatz ist ein Satz, der nicht in allen, sondern nur in einigen möglichen Welten gilt, und er besagt, daß unsere Welt zu diesen Welten gehört. Erfahrungssätze gelten also nicht analytisch, d. h. nicht in allen möglichen Welten; sie sind synthetische Sätze. ${ }^{1}$

${ }^{1}$ Kants Bestimmung der analytischen Sätze ist zu eng, wie oft bemerkt worden ist. Er bezeichnet erstens nur wabre Sätze als analytisch, während es nach unserer Bestimmung auch falsche analytische Sätze gibt, Sätze also, die in allen Welten falsch sind. Und er bestimmt zweitens nur für Sätze der Form „Alle $S$ sind P", wann sie analytisch sind; sein Kriterium dafür ist, daß der Prädikatbegriff $P$ im Subjektbegriff $S$ enthalten sein muß. 
Nach dem Begründungsmodus kann man mit Kant empirische (aposteriorische) und apriorische Aussagen unterscheiden. Eine Aussage soll apriorisch heißen, wenn sich ihr Wahrheitswert (d. h. ihre Wahrheit oder Falschheit) ohne Rückgriff auf Erfahrungen (Beobachtungen, Experimente) feststellen läßt. Eine Aussage heißt empirisch, wenn sie nicht apriorisch ist. ${ }^{2}$ Alle analytischen Erkenntnisse sind apriorisch. Gibt es aber auch synthetische Erkenntnisse apriori, wie Kant das annahm? Diese Frage wird heute meist mit dem Hinweis verneint, $\mathrm{da}$ nicht einzusehen sei, wie sich apriorisch etwas über unsere Welt ausmachen lassen sollte, was nicht zugleich in allen möglichen Welten gilt. Daher gebraucht man heute auch weithin die Ausdrücke ,,empirisch" und ,synthetisch" als Synonyma. Wir werden diese Frage erst im 9. Kapitel diskutieren. Der Abgrenzung der Erfahrungserkenntnis legen wir das erste, semantische Kriterium zugrunde. Im Sinne des heutigen Sprachgebrauchs werden wir freilich in Fällen, in denen klar ist, daß beide Begriffe sich decken, gelegentlich auch "empirisch“ statt ,,synthetisch“" sagen. ${ }^{3}$

Man unterscheidet üblicherweise zwischen innerer und äußerer Erfahrung. Kant charakterisiert äußere Erfahrung als Erfahrung durch äußere Sinne (den optischen, akustischen, haptischen Sinn, etc.), innere

${ }^{2}$ Es ist nicht auszuschließen, daß sich derselbe Satz sowohl mit Beobachtungen (also empirisch) als auch ohne Beobachtungen (also apriorisch) begründen läßt. Daher orientiert sich die Unterscheidung an möglichen Begründungen. Es kann auch Sätze geben, deren Wahrheitswerte sich überhaupt nicht erkennen lassen; sie sind nach unserer Definition empirisch. Empirische Aussagen sind für uns also nicht Sätze, die sich aufgrund von Beobachtungen als wahr, bzw. falsch erkennen lassen, sondern Sätze, deren Wahrheitswert sich (wenn überhaupt, dann) nur durch Beobachtungen ermitteln läßt. Diese Definition empfiehlt sich auch im Blick auf manche gesetzesartigen Aussagen, die sich durch Beobachtungen weder als wahr noch als falsch erweisen lassen. Vgl. dazu den Abschnitt 9.4.

${ }^{3}$ Zum Problem der Unterscheidung analytischer und synthetischer Sätze in natürlichen Sprachen vgl. 9.1. Zu den Versuchen des logischen Empirismus empirische Sätze mit empiristischen Sinnkriterien zu bestimmen vgl. Hempel (65), S. 99 ff., Stegmüller (70), 1. Halbbd., Kap. III oder Kutschera (72), 3.4. Es handelt sich dabei im wesentlichen um Kriterien der Begründbarkeit, bzw. der Verwendbarkeit von Sätzen in Begründungen. Da sich diese Kritierien als unbrauchbar erwiesen haben, gehen wir darauf hier nicht ein. 
Erfahrung als Erfahrung mit einem inneren Sinn. Die Annahme eines inneren Sinns ist aber problematisch und für die Bestimmung innerer Erfahrung auch wenig hilfreich. Von vielen Autoren, wie z. B. von L. Wittgenstein und G. Ryle, ist die Existenz einer eigenständigen inneren Erfahrung sogar völlig geleugnet worden. Es gibt nun zweifellos syhthetische Sachverhalte, deren Bestehen wir feststellen können, ohne unsere äußeren Sinne zu gebrauchen. Gegenwärtige Überzeugungen, Gefühle, Empfindungen oder Präferenzen sind uns unmittelbar bewußt und sind für uns unproblematisch, wie wir in Abschnitt 1.4 gesagt haben. Solche Sachverhalte können also als typische Gegenstände ,,innerer Erfahrung“ angesehen werden, wobei freilich die Rede von „Erfahrung" hier nach dem allgemeinen Sprachgebrauch fragwürdig ist. Man ,erfährt" nicht, daß man etwas glaubt oder will, und man ,,erfährt" auch nicht eigene Empfindungen und Gefühle, sondern man hat sie. Auf der anderen Seite sind physikalische Sachverhalte typische Gegenstände äußerer Erfahrung. Man kann nun aber nicht behaupten, jede Erfahrung sei entweder eine innere oder eine äußere Erfahrung. Es gibt viele Erfahrungen, in denen sich beides verbindet. Mit der äußeren Wahrnehmung der Farbe eines Gegenstandes verbindet sich z. B. eine bestimmte Erlebnisweise, die ich ,innerlich erfahre“. Die Aussage „Dieser Gegenstand ist rot" beschreibt den Inhalt einer äußeren Erfahrung, der Satz ,Ich empfinde diesen Gegenstand als rot" den einer inneren. Es liegt aber nur eine Erfahrung vor, und daß ich das Objekt als rot empfinde, ist für mich in der Regel das Kriterium dafür, daß er rot ist. Innere und äußere Erfahrung sind also nur Typen, und es gibt ein breites Feld von Verbindungen und Übergängen zwischen ihnen. Darauf werden wir in den Kapiteln 7 und 8 genauer eingehen. Zunächst wollen wir von der sogenannten, ,inneren "Erfahrung absehen und uns nur mit dem befassen, was man als ,äußere Erfahrung“ bezeichnet, wobei wir speziell an das Beispiel der Erfahrung von Physischem denken. Wenn im folgenden von „Erfahrung“ die Rede ist, so ist also äußere Erfahrung gemeint. Die zentralen Probleme der Erfahrungserkenntnis betreffen diese Erfahrung.

Eine bestimmte Deutung äußerer Erfahrung können wir aber für das folgende nicht voraussetzen, insbesondere keine Deutung, nach der sie Erfahrung einer bewußtseinsunabhängigen Außenwelt ist. Im folgenden geht es vielmehr gerade um die Analyse äußerer Erfahrung. Die Frage nach der Natur dieser Erfahrung steht im Mittelpunkt der Erörterung der folgenden Kapitel. Sie umfaßt drei Teilfragen: 


\section{Was ist der Gegenstand der Erfabrung?}

Darauf gibt es zwei Antworten: Nach dem erkenntnistheoretischen Realismus bildet die Außenwelt den Gegenstand der Erfahrung. Nach dem erkenntnistheoretischen Idealismus sind dagegen allein seelische Zustände oder Objekte (Empfindungen, Sinnesdaten, mentale Bilder etc.) Gegenstände äußerer wie innerer Erfahrung. Die erste Frage führt uns so auf die Realismus-Idealismus-Kontroverse.

Ontologisch kann man die Frage nach der Natur der Erfahrung so formulieren:

2. Was ist das Verbältnis zwischen Geistig-Seelischem und Körperlichem (Materiellem)?

Das ist das Leib-Seele-Problem im weitesten Sinn des Wortes, und dazu gibt es vier Meinungen: Im Sinn des Dualismus, wie ihn z. B. Descartes vertrat, bilden Geistig-Seelisches und Körperliches zwei eigenständige Realitätsbereiche. Nach dem ontologischen Idealismus, wie er u. a. von Berkeley vertreten wurde, gibt es keine eigenständige materielle Wirklichkeit; materielle Objekte sind nichts anderes als Konstruktionen aus Sinnesdaten. Demgegenüber behauptet der Materialismus oder Pbysikalismus, alle seelischen Phänomene ließen sich physikalisch erklären, es gäbe also keine eigenständige seelische Wirklichkeit. Eine dritte monistische Position ist endlich der neutrale Monismus, wie ihn z. B. Spinoza vertrat und nach dem Seelisches wie Materielles zwei Aspekte derselben Realität sind.

Bei einer Erörterung der Natur der Erfahrung stellt sich drittens auch die Frage:

3. Welchen Anteil haben subjektive Faktoren am Erfahrungsinhalt?

Erfahren wir die Welt so, wie sie an sich ist, oder hängt die Art und Weise, in der wir sie erfahren von unserer Wahrnehmungsorganisation, unseren Denkschemata oder unserer Sprache ab? Hier stehen sich realistische und relativistische Konzeptionen gegenüber: Realistisch ist die Auffassung, daß wir - in den Grenzen unserer Wahrnehmungsmöglichkeiten und sofern unsere Sinnesorgane normal funktionieren - die Welt so wahrnehmen, wie sie an sich ist. Verstandestätigkeit beschränkt sich danach auf die begrifflich-logische Systematisierung der Erfahrungsinhalte; Sprache ist nur ein Beschreibungsmittel für sprachunabhängige Erfahrungsgegebenheiten. Relativistisch sind hingegen Ansichten, nach denen die Erfahrungsinhalte Produkte einer Wechselwirkung zwischen erlebten Dingen und erlebendem Subjekt sind (Locke) oder nach denen unsere Verstandesorganisation 
(Kant) oder unsere Sprache (W. v. Humboldt, B. Whorf) unsere Erfahrung prägt, so daß wir die Dinge nicht selbst zu Gesicht bekommen, so wie sie unabhängig von menschlicher Wahrnehmung sind, sondern immer nur in einer von uns selbst abhängigen Gestalt.

Die Probleme (1) bis (3) hängen naturgemäß eng zusammen. Der erkenntnistheoretische Realismus setzt z.B. einen ontologischen Realismus voraus, die Existenz einer Außenwelt; der ontologische Idealismus hingegen impliziert einen erkenntnistheoretischen, und der erkenntnistheoretische Idealismus beruht gerade auf der These vom Anteil der Wahrnehmungs- oder Vernunftorganisation an der Erfahrung. Wir werden daher im folgenden diese drei Probleme zusammen erörtern, und diese Erörterungen bilden mit den Kapiteln 4 bis 8 den Hauptteil dieser Arbeit.

Der zweite große Problemkreis der Erfahrungserkenntnis bezieht sich auf den Aufbau der empirischen Sprache und die Begründung empirischer Sätze. Beide Fragen sind, wie wir im 9. Kapitel sehen werden, eng miteinander verbunden; wir werden insbesondere zu untersuchen haben, ob nicht in alle sprachlichen Beschreibungen empirischer Phänomene schon Annahmen über die Welt eingehen, wie das eine Form des Holismus behauptet. Lassen sich ferner Sätze - sog. Beobachtungssätze - angeben, die durch direkte Beobachtungen entscheidbar sind, und läßt sich das Begründungsproblem auf die Frage reduzieren, wie sich andere Sätze, z. B. gesetzesartige Hypothesen, mit solchen Beobachtungssätzen rechtfertigen lassen? Kann man Theorien durch Beobachtungen als wahr oder jedenfalls als wahrscheinlich wahr erweisen, oder kann man sie durch Beobachtungen nur widerlegen? Reduzieren sich empirische Begründungen auf Beobachtungen plus Logik und Mathematik, oder stützen sie sich auch auf synthetisch-apriorische Prinzipien? All diese Fragen spielen eine Rolle, wenn man ein Modell der Erfahrungserkenntnis entwickeln will, das nicht nur einfache Beobachtungen erfaßt, sondern den gesamten Bereich unserer empirischen Annahmen. Hier stehen sich die verschiedenen Versionen empiristischer, rationalistischer und holistischer Konzeptionen gegenüber. Auf diese Themen werden wir erst im letzten Kapitel eingehen.

Dort werden wir auch den dritten Problemkreis der Erfahrungserkenntnis behandeln, der sich durch die Frage andeuten läßt: Ist, und wenn ja: in welchem Umfang ist empirische Erkenntnis möglich? Dazu 
gehört insbesondere die Erörterung skeptischer und relativistischer Thesen zur Erfahrungserkenntnis. Die empirische Skepsis, nach der sich die Existenz einer Außenwelt nicht beweisen oder ihre Beschaffenheit nicht erkennen läßt, behandeln wir im Zusammenhang mit dem Realismus. Eine bedeutendere Rolle spielt - zumindest in der gegenwärtigen Diskussion - der Relativismus. Da seine Erörterung voraussetzt, daß man sich über die Natur der Erfahrung und das Begründungsproblem Klarheit verschafft hat, gehen wir darauf erst im letzten Kapitel ein.

Mit dieser kurzen Skizze der Hauptprobleme der Erfahrungserkenntnis ist das Programm der folgenden Untersuchungen vorgezeichnet. Bevor wir uns ihnen zuwenden, müssen wir jedoch in diesem Kapitel noch einige vorbereitende begriffliche Festlegungen und Unterscheidungen vornehmen. Die ersten Bestimmungen betreffen den Begriff der Wabrnebmung.

Eine empirische Erkenntnis stellt ein empirisches Wissen dar, d. h. ein Wissen von der Geltung eines empirischen Satzes. Ein solches Wissen kann sich auf Begründungen stützen, d. h. es kann auf deduktivem oder induktivem Weg aus anderem empirischen Wissen gewonnen werden. $\mathrm{Da}$ sich aber nicht alles begründen läßt, und da sich auch bei Annahme synthetischer Erkenntnisse apriori sicher nicht alle empirischen Erkenntnisse apriorisch begründen lassen, muß es auch empirische Evidenzen geben, d. h. Sachverhalte, die uns aufgrund einer Beobachtung oder Wahrnehmung evident sind.

Das Wort ,wahrnehmen“" bezeichnet eher einen momentanen Akt, „,beobachten“ meist eine länger andauernde Tätigkeit. Von diesem Unterschied wollen wir hier jedoch absehen und ,,beobachten “ wie ,,wahrnehmen" auch auf momentane Erfahrungen anwenden. Beide Prädikate verwenden wir in zwei kategorial verschiedenen Formen. Wir sprechen einmal davon, daß wir einen Gegenstand beobachten - dann ist ,, beobachten“ ein zweistelliges Prädikat der Form ,Die Person a beobachtet den Gegenstand b". Wir sagen aber auch, wir beobachteten, daßetwas der Fall ist - dann ist das Wort ein zweistelliges Prädikat der Form ,,Die Person a beobachtet, daß der Sachverhalt $\mathrm{p}$ besteht" - symbolisch B(a,p). Wir verwenden das Wort ,, beobachten“ im folgenden nur in der zweiten Form, und entsprechend auch das Wort,,wahrnehmen“. Die Erkenntnis, die wir aus der Beobachtung eines Gegenstands gewinnen, drückt sich ja immer in einem Satz über diesen Gegenstand aus. ${ }^{4}$ 
„Wahrnehmen“ ist ein Oberbegriff für spezielle Erfahrungsweisen, wie Sehen oder Hören. Diese Verben sind, ebenso wie das Wort „Wahrnehmen“" selbst, sogenannte Erfolgsverben, ${ }^{5}$ d. h. wir können nicht sagen, jemand sehe, (höre, nehme wahr), daß $p$, wenn $p$ nicht gilt. Sie verhalten sich also ähnlich wie das Verb ,wissen“, und wie wir zwischen ,,wissen" und ,glauben" unterschieden haben, so empfiehlt es sich hier, neben ,,wahrnehmen“ einen Ausdruck einzuführen, der diese Implikation nicht enthält. Wir wollen dafür der Einfachheit halber das Prädikat ,,beobachten“ wählen, obwohl diese Verwendung dem normalen Gebrauch nicht entspricht.

Wir verwenden im folgenden das Wort ,beobachten“ ferner in dem starken Sinn, in dem eine Beobachtung der Person a es ihr evident macht, daß der Sachverhalt $\mathrm{p}$ besteht, fordern also

B 1: $B(a, p) \supset V(a, p)$ - Was wir beobachten ist uns evident.

Daraus folgt nach dem Postulat V1 in $1.5 \mathrm{~B}(\mathrm{a}, \mathrm{p}) \supset \mathrm{G}(\mathrm{a}, \mathrm{p})$ - wir sind von dem überzeugt, was wir beobachten. ${ }^{6}$

Wir definieren dann den Begriff der Wabrnebmung - symbolisch $\mathrm{P}(\mathrm{a}, \mathrm{p})$ - in Analogie zum Begriff des Wissens durch

D 2.2-1: $\mathrm{P}(\mathrm{a}, \mathrm{p}):=\mathrm{B}(\mathrm{a}, \mathrm{p}) \wedge \mathrm{p}-$ Wahrnehmung ist Beobachtung von Tatsachen.

${ }^{4}$ Man kann auch sagen, daß man eine Eigenschaft an einem Gegenstand oder eine Beziehung zwischen zwei oder mehreren Gegenständen beobachtet. Das heißt aber nichts anderes, als daß wir beobachten, daß der Gegenstand die fragliche Eigenschaft hat, bzw. daß die Gegenstände in der fraglichen Beziehung zueinander stehen.

' G. Ryle spricht in (49) von achievement words.

- Die Verwendung eines schwachen Evidenzbegriffs in B1, von dem wir in der Anmerkung 46 zu S. 37 gesprochen haben, würde zu einem schwachen Beobachtungsbegriff passen. Beobachtungen sind ja nicht immer so deutlich und klar, daß sie feste Überzeugungen von der Wahrheit des beobachteten Sachverhalts begründen können; sie weisen diesen Sachverhalt oft nur als mehr oder minder wahrscheinlich aus. Das gilt z. B. auch für physikalische Messungen: Die nicht völlig auszuschließenden Meßfehler bewirken, daß wir nur Wahrscheinlichkeitsaussagen über den ,wahren“ Wert der gemessenen Größe machen können. Für das folgende genügt aber der starke Beobachtungsbegriff, der sich, wie die starken Begriffe des Glaubens und der Evidenz, durch seine Einfachheit empfiehlt. 
„Beobachten“ und „,wahrnehmen“ verhalten sich also zueinander wie „Glauben“ und „Wissen”. Beobachtungen sind problemlos, aber nicht verläßlich, Wahrnehmungen dagegen verläßlich, aber nicht problemlos. $^{7}$

Für die späteren Diskussionen insbesondere des Phänomenalismus, benötigen wir noch ein weiteres Prädikat. Wenn ich einen Stock ins Wasser halte, so sieht es so aus, als sei er an der Stelle geknickt, an der er ins Wasser eintaucht. Dieses und andere Phänomene von Sinnestäuschungen sind uns bekannt, so daß wir uns dadurch nicht irreführen lassen. In einem derartigen Fall können wir nicht sagen, wir beobachteten, daß der Stock geknickt ist, weil wir davon überzeugt sind, $\mathrm{da}$ es sich nicht so verhält. Wir können nur sagen: „Es erscheint uns (beobachtungsmäßig, aufgrund von Sinneseindrücken), als ob der Stock geknickt sei“. Schreiben wir ,S(a,p)" für ,Es erscheint der Person a, als ob $p^{\text {", }}$, so gilt dann weder generell $S(a, p) \supset G(a, p)$, noch $\mathrm{S}(\mathrm{a}, \mathrm{p}) \supset \mathrm{V}(\mathrm{a}, \mathrm{p})$. Es gilt aber analytisch:

S1: $S(a, p) \wedge G(a, p) \supset B(a, p)-$ Wenn es uns so scheint, als ob $p$, und wir davon auch überzeugt sind, so beobachten wir, daß $p-$

und

S 2: $B(a, p) \supset S(a, p)-W e n n$ wir beobachten, daß $p$, so erscheint es uns auch, als ob $\mathrm{p}^{8}$

Danach gilt also $S(a, p) \wedge G(a, p) \equiv B(a, p)$. Wir könnten also $B(a, p)$ durch $S(a, p) \wedge G(a, p)$ definieren. Die Sätze $S(a, p)$ sind für a wieder unproblematisch, d. h. es gilt

S 3: $S(a, p) \equiv G(a, S(a, p))$ - Erscheint es $a$, als ob p gelten würde, so glaubt a auch, daß es ihm so erscheint, und umgekehrt.

In der Literatur findet man eine Reihe von Einwänden gegen das Prinzip S3. So hat A. J. Ayer behauptet, man könne für Sätze der Form $S(a, p)$ keine untrügliche Evidenz in Anspruch nehmen, denn ein

\footnotetext{
7 Für B, bzw. P gelten natürlich nicht alle G-, bzw. W-Axiome. So sind Tautologien wie $2+2=4$ nicht beobachtbar und es gilt auch nicht $B(a, p) \supset$ $\mathrm{B}(\mathrm{a}, \mathrm{B}(\mathrm{a}, \mathrm{p}))$ - Sachverhalte des Beobachtens werden im normalen Sinn dieses Wortes nicht beobachtet.

8 Wie im Falle von B sehen wir Aussagen wie $S(a, S(a, p))$ nicht als sinnvoll an.
} 
Satz könne nicht nur deswegen falsch sein, weil er einen nicht bestehenden Sachverhalt beschreibt, sondern auch weil er einen bestehenden Sachverhalt falsch beschreibt - wenn nämlich Wörter falsch gebraucht werden. Wenn es mir scheint, daß dieses Stück Papier weiß ist, ich aber das Wort ,schwarz" fälschlich im Sinne von ,,weiß" verwende und sage ,Es erscheint mir, als ob es schwarz ist", so ist dieser Satz falsch.9 Dazu ist wie schon früher zu sagen: Wahrheit und Falschheit kommen einem Satz nicht als einem bloßen syntaktischen Gebilde zu, sondern immer nur bzgl. einer bestimmten Interpretation der Sprache, in der er formuliert ist. Der fragliche Satz ist zwar bzgl. der üblichen Interpretation der deutschen Sprache falsch, nicht aber bzgl. der Interpretation, die ich ihm zugrunde gelegt habe. Bei unserer Diskussion ist aber natürlich Voraussetzung, daß die fraglichen Sätze in der vom Sprecher intendierten Weise interpretiert werden. Wir haben auch von der Evidenz von Sachverbalten gesprochen, und bei Sachverhalten tritt dieses Problem nicht auf.

Ein anderer Einwand gegen die Problemlosigkeit der Sachverhalte $\mathrm{S}(\mathrm{a}, \mathrm{p})$ besagt, die Erfahrung zeige ja, daß wir uns über unsere seelischen Zustände irren können. ${ }^{10}$ So kann es passieren, daß sich ein willensschwacher Mensch für willensstark hält, ein krasser Egoist für altruistisch, usw. Diese Beispiele treffen aber nicht Sachverhalte des Typs $S(a, p)$, und unsere Behauptung, gewisse Sätze über Eigenseelisches seien problemlos, impliziert keinesfalls, daß alle Sätze über Eigenpsychisches problemlos sind. Bei Argumenten gegen S3 kann man auch nicht die Unzuverlässigkeit der Erinnerung ins Feld führen. Wie schon mehrfach betont wurde, wird nur behauptet, Aussagen der Form ,,a erscheint es jetzt, als ob p“ seien jetzt für a entscheidbar.

P. Feyerabend meint in (63), die Problemlosigkeit von S-Sätzen habe denselben Grund wie die Problemlosigkeit analytischer Aussagen: ihren mangelnden synthetischen Gehalt. S-Sätze sind aber synthetische Sätze, sie sagen etwas über die Welt aus, wenn auch nichts über die Außenwelt. Man kann sie daher auch nicht als ,,inhaltsleer“ bezeichnen. M. Black folgert in (52) - ohne das näher zu begründen - aus der Tatsache, daß S-Sätze nicht direkt intersubjektiv überprüfbar sind, daß solche Sätze keinen deskriptiven Gehalt hätten. Aber

${ }^{9}$ Vgl. dazu Ayer (36). Im gleichen Sinn äußert sich auch Austin in (62), S. $112 \mathrm{f}$.

${ }^{10} \mathrm{Vgl}$. dazu z. B. Stegmüller (58), 68. 
wenn ich sage „Es erscheint mir, als ob diese Rose rot ist“, so sage ich doch etwas anderes, als wenn ich sage „Es erscheint mir, als ob diese Rose gelb ist“. Black behauptet weiterhin, der Satz ,, a erscheint es, als

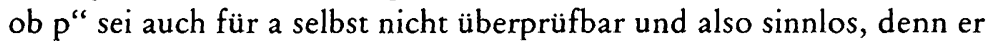
sei logisch verträglich mit dem Satz ,,a wird gleich in gutem Glauben sagen: „Mir erschien es nicht, als ob $p$ “" ". Hier wird jedoch erstens wieder die Erinnerung ins Spiel gebracht, und zweitens lassen sich SSätze selbstverständlich überprüfen: Den Satz „Es erscheint mir, als ob dies Blatt Papier weiß ist", prüfe ich so, daß ich mir das Blatt $\mathrm{Pa}$ pier ansehe; erscheint es mir dabei als weiß, so ist der Satz wahr, sonst falsch.

\subsection{Wirklichkeit und Existenz}

In erkenntnistheoretischen Kontexten ist oft von der ,Welt" oder „,der Wirklichkeit“ die Rede. Auch diese Wörter bedürfen einer Erläuterung.

„Die Welt ist alles, was der Fall ist“ beginnt Wittgensteins „Tractatus logico-philosophicus". Das heißt: die Welt ist der Inbegriff oder die Menge aller bestehenden Sachverhalte oder Tatsachen. Das ist eine zwar ungewöhnliche, aber sinnvolle Festlegung des Begriffes „Welt". Üblicher ist es, die Welt als konkretes, physikalisches Gebilde aufzufassen, als den Kosmos. Aber die wahren Aussagen über dieses Gebilde sind dann doch jene Aussagen, die Tatsachen ausdrücken; und die Menge aller Tatsachen kennzeichnet andererseits dieses Gebilde vollständig. Daher sind beide Redeweisen gleichberechtigt. Aussagen über die Welt sind ja nicht nur Sätze, in denen das Wort ,,die Welt" oder „,der Kosmos" vorkommt; auch die Aussage, daß Köln am Rhein liegt, ist eine Aussage über die Welt. Wir wollen im folgenden die Wittgensteinsche Redeweise zugrunde legen. ${ }^{11}$

Das Wort ,wirklich" wird sowohl als Adjektiv wie als Adverb verwendet. Als Adjektiv weist es, wie J. L. Austin hervorgehoben hat, ${ }^{12}$ die gleiche Kontextabhängigkeit auf wie die Wörter "schwer" oder

${ }^{11}$ Man kann natürlich das Wort "Welt" auch enger als die Menge synthetischer Tatsachen verstehen.

12 Vgl. Austin (62), Kap.VII. 
„groß“. Die Bedeutung dieser Wörter variiert mit dem Kontext, in dem sie stehen. Ein wirklicher Freund ist ein zuverlässiger Freund. Eine wirkliche Krankheit dagegen keine zuverlässige, sondern eine nicht eingebildete Krankheit. Eine wirkliche Freude ist weder eine zuverlässige noch eine nicht eingebildete, sondern eine große Freude. Ebenso ist ein kleiner Elefant größer als eine große Mücke. Ein großer Irrtum hingegen hat keinerlei räumliche Ausdehnung, ebensowenig wie ein schwerer Fehler sich wiegen läßt. Und ein leichter Lastwagen ist schwerer als ein schweres Motorrad. Eine allgemeine Unterscheidung von ,,wirklichen“" und ,,nicht wirklichen“ Gegenständen oder Eigenschaften, die man für erkenntnistheoretische Zwecke verwenden könnte, ist daher aufgrund des üblichen Sprachgebrauchs ohne weitere Festlegungen nicht erklärt.

Erkenntnistheoretisch spielt aber auch weniger die adjektivische Verwendung von ,,wirklich“ eine Rolle als die adverbiale. In den Sätzen „Fritz kam wirklich“, „Ich war wirklich krank", „, Was Emma sagte, ist wirklich wahr" etc. dient das Wort zunächst einmal zum Ausdruck der Bestätigung. Der Sprecher betont damit, daß es sich so und nicht anders verhält, daß der Inhalt des Satzes in vollem Umfang zutrifft, oder daß der behauptete Sachverhalt entgegen vorherigen Erwartungen oder Wahrscheinlichkeiten eintrat. Von dieser expressiven Bedeutungskomponente können wir hier aber absehen. Worin liegt also der deskriptive Gehalt des Wortes? ${ }^{13}$ Die Sätze lassen sich auf die Normalform bringen „Es ist wirklich so, daß $\mathrm{p}$ “. Was heißt das? Nun das heißt nicht mehr, als daß p eine Tatsache ist. Wie das Wort ,wahr" folgt also das Adverb ,,wirklich" in seinem deskriptiven Gehalt der Bedingung: Es ist wirklich so, daß p, genau dann, wenn $p$. „Wirklich" besagt also in Anwendung auf Propositionen nicht mehr als ,,wahr". Wenn p gilt, so ist es auch wirklich so, daß p - wirklicher als tatsächlich geht es nicht. Und wenn es wirklich so ist, daß p, so gilt auch p.

Wenn man nun die Bezeichnung ,die Wirklichkeit" als Menge der Tatsachen deutet, so ist die Wirklichkeit nichts anderes als die Welt. Die ,wirkliche Welt" ist die Welt, wie sie wirklich ist, d. h. die tatsächliche Welt, unsere Welt, im Gegensatz zu einer bloß möglichen Welt.

${ }^{13}$ Zur Unterscheidung expressiver und deskriptiver Bedeutungskomponenten vgl. z. B. Kutschera (75), 1.2 und 2.4.5. 
Von seinem deskriptiven adverbialen Sinn her können wir das Wort „,wirklich“ als Adjektiv in Anwendung auf Gegenstände für einen allgemeinen erkenntnistheoretischen Gebrauch so deuten: ,, a ist wirklich" heißt soviel wie „Es ist wirklich so, daß a existiert", d. h. einfach soviel wie ,,a existiert". Das führt uns auf das Problem der Existenzaussagen.

Die Ontologie ist in traditioneller Auffassung jene philosophische Disziplin, in der man sich mit Fragen der Form "Gibt es Dinge der und der Art?" oder pauschaler „Was existiert?", „Was gibt es?" befaßt. Nun treten solche Fragen auch in den Einzelwissenschaften auf. In der Mathematik fragt man $z$. B., ob es natürliche Zahlen $x, y, z$ und $\mathrm{n}>2$ gibt, für die gilt $\mathrm{x}^{\mathrm{n}}+\mathrm{y}^{\mathrm{n}}=\mathrm{z}^{\mathrm{n}}$ (das ist das Große Fermatsche Problem); in der Biologie fragt man, ob es vererbbare erworbene Eigenschaften gibt, usf. Der Sinn solcher Existenzfragen ist klar. Weniger klar ist hingegen der Sinn allgemeinerer Existenzfragen wie "Gibt es Zahlen?" oder "Gibt es Eigenschaften?" Insbesondere erfordert die allgemeinste Existenzfrage, die ontologische Frage „Was gibt es?" einige Erläuterungen. Man kann sie aber, wie wir sehen werden, mit Quine einfach so beantworten: „Alles!“"14

Existenzfragen treten in zwei Formen auf. Man kann fragen

1. „Existieren Dinge mit der Eigenschaft F?، und man kann fragen

2. „Existiert der Gegenstand a?"๘

Fragen der Form (2) sind insofern problematisch, als man oft Eigennamen nur dann als bedeutungsvoll ansieht, wenn es einen Gegenstand gibt, den sie bezeichnen, wenn sie also einen Bezug haben. Dann wäre aber jede sinnvolle Frage von Typ (2) trivialerweise zu bejahen. Denn entweder hat der Name ,a" keinen Bezug - dann ist er und mit ihm auch der ganze Fragesatz sinnlos -, oder ,,a" bezeichnet einen Gegenstand, und dann gibt es diesen Gegenstand. Steht in der Frage (2) statt ,,der Gegenstand a" ein Kennzeichnungsterm der Form „Derjenige Gegenstand, der die Eigenschaft F hat", so kann man diese Frage so formulieren „Gibt es genau ein Ding mit der Eigenschaft F?"; man kann sie dann also auf die Form (1) bringen. Wie aber ist es im Fall von Eigennamen wie „Pegasus“? Quine schlägt vor,

${ }^{14}$ Vgl. Quine (48). 
statt dieses Eigennamens ein Prädikat ,,pegasieren“ (oder „Pegasussein") einzuführen (das man dann natürlich nicht durch den Ausdruck ,,mit Pegasus identisch sein“ definieren kann, sondern als undefiniertes Prädikat anzusehen hat) und ,Pegasus“ durch den Kennzeichnungsterm ,dasjenige Ding, das pegasiert" zu ersetzen. Auf diese Weise könnte man alle Eigennamen eliminieren, so daß Existenzfragen nur mehr in der Form (1) auftreten. Überzeugender ist es, zwischen Bezug und Bedeutung von Eigennamen zu unterscheiden (zwischen meaning und reference, oder Konnotation und Denotation). ${ }^{15}$ Es gibt viele bedeutungsvolle, aber bezugslose Namen wie „Die kleinste reelle Zahl, die größer ist als O", „Die Tochter Freges“ oder „Odysseus". Unterscheidet man auch zwischen (in unserer Welt) existierenden und möglichen Objekten (die in irgendwelchen anderen Welten existieren), so kann man sagen, daß alle Eigennamen mögliche Objekte bezeichnen, einige jedoch in unserer Welt keinen Bezug haben, und kann so der Frage (2) einen nichttrivialen Sinn geben. ${ }^{16}$ Wir wollen jedoch diesen Gedanken hier nicht weiter verfolgen, denn für das folgende spielen nur Existenzfragen der Form (1) eine Rolle, bzw. Existenzfragen, die sich auf die Form (1) bringen lassen.

Jeder Sprache S liegt ein universe of discourse, eine (nichtleere) Menge U von Objekten zugrunde, über die man in S sprechen kann. ${ }^{17}$ Wie Carnap in (50a) betont hat, muß man interne und externe Existenzfragen unterscheiden. Eine interne Existenzfrage bzgl. $S$ ist eine Frage des Inhalts, ob es in $U$ Dinge mit einer gewissen Eigenschaft gibt. Solche Fragen kann man in der Sprache S nach dem Schema (1) formulieren.

Eine externe Existenzfrage bzgl. S ist dagegen eine Frage, ob es Objekte der Art, wie sie im universe of discourse U der Sprache S vorausgesetzt werden, überhaupt gibt. Solche Fragen treten z. B. im Universalienstreit auf, wo diskutiert wird, ob es neben den konkreten Dingen

15 Vgl. dazu Kutschera (75), 2.1.2.

${ }_{16} \mathrm{Vgl}$. dazu Kutschera (76), 1.7 und 2.9. Quines Kritik an möglichen Objekten in (48) ist bei diesem Vorgehen nicht gerechtfertigt, denn aus ,Es ist möglich, daß es ein Objekt mit der Eigenschaft $F$ gibt" folgt nicht „Es gibt ein mögliches Objekt mit der Eigenschaft $F^{\text {“c. }}$.

17 Das wird besonders deutlich beim Aufbau von Kunstsprachen. Vgl. dazu z. B. Kutschera (76), 1.2, 2.4 oder Kutschera und Breitkopf (71), 9.2. 
(Tischen, Menschen und Sternen) auch abstrakte Entitäten (Begriffe, Klassen, Funktionen, Zahlen) gibt, wie das der Platonismus annimmt, oder nicht, wie der Nominalismus glaubt. ${ }^{18}$ Solche bzgl. S externen Existenzfragen lassen sich nun in $\mathrm{S}$ nicht sinnvoll stellen, denn in $\mathrm{S}$ sind sie trivialerweise immer zu bejahen. Das gilt einfach deswegen, weil bei der Interpretation von S, speziell bei der Deutung des Ausdrucks „Es gibt“, genau die Objekte aus U vorausgesetzt werden, so $\mathrm{da}$ man sich mit den in S formulierten Behauptungen immer im Rahmen dieser ontologischen Voraussetzungen bewegt. Ohne solche Voraussetzungen hätte der Ausdruck „Es gibt“ in S keinen Sinn. Man kann solche bzgl. S externen Existenzfragen also sinnvollerweise nur in einer anderen Sprache S' diskutieren, in der sie sich als interne Existenzfragen darstellen. Da aber jede Sprache einen universe of discourse voraussetzt, und da man Existenzfragen und Antworten darauf immer nur in einer Sprache, d. h. in einem Rahmen ontologischer Voraussetzungen, formulieren kann, lassen sich ontologische Fragen nicht ohne ontologische Voraussetzungen diskutieren.

Aufgrund solcher Überlegungen kann man Carnap darin beipflichten, daß ontologische Probleme weniger theoretische als praktische Probleme sind. ${ }^{19}$ Man fragt statt: „Was gibt es?" oft besser: „Welche Sprache sollen wir wählen?"،, und diese Frage läßt sich allgemein nur so beantworten: „Wähle eine Sprache, die für die intendierten Zwecke hinreichend präzise und ausdrucksstark ist, aber nicht ausdrucksreicher (d. h. nicht ontologisch voraussetzungsreicher) als dafür nötig“. Die letztere Bedingung entspricht Occams Ökonomieprinzip entia non sunt multiplicanda praeter necessitatem. Dieses Prinzip ontologischer Ökonomie muß man freilich gegen das Prinzip der Einfachheit des Ausdrucks abwägen: ontologisch reichere Sprachen ermöglichen es oft, Sachverhalte einfacher auszudrücken.

Auf die Frage ,Welche Dinge gibt es?"“, formuliert in S, kann man also nur antworten: „Alle, die zum universe of discourse von $S$ gehö-

${ }^{18}$ Zum Universalienproblem vgl. Stegmüller (65).

19 W.V.Quine hat in (51a) Kritik an Carnaps Unterscheidung zwischen externen und internen Existenzfragen geübt. Diese Kritik trifft aber den Kern des Carnapschen Arguments nicht, daß jede Existenzfrage und jede Existenzaussage die ontologischen Voraussetzungen derjenigen Sprache übernimmt, in der sie formuliert ist. 
ren“, oder technisch ausgedrückt: „Alle, die zum Definitionsbereich der Variablen von S gehören". Das ist die Antwort Quines: "To be is to be the value of a bound variable". ${ }^{20}$ Es gibt also alles, worüber man sinnvoll reden kann.

\subsection{Reduktionen und Modelle}

Im Sinn der Carnapschen Ideen zum Verhältnis von Sprache und Ontologie kann man oft ontologische Aussagen mit Gewinn an Präzision in Aussagen über Sprachen übersetzen. Insbesondere lassen sich Behauptungen, daß es Objekte der und der Art nicht gibt, daß es überflüssig ist, solche Objekte anzunehmen, oder daß Objekte der Art A nichts anderes sind als Objekte der Art B, als Reduzierbarkeitsbehauptungen für Sprachen oder für Theorien formulieren.

Von einer Reduktion einer Sprache $S_{2}$ auf eine Sprache $S_{1}$ spricht man meist dann, wenn $S_{1}$ eine Teilsprache von $S_{2}$ ist. $S_{2}$ heißt in diesem Fall auf $S_{1}$ reduzierbar, wenn sich in $S_{1}$ explizite Definitionen für die in $S_{1}$ nicht vorkommenden Grundterme von $S_{2}$ angeben lassen, die ihnen die ursprünglichen Bedeutungen zuordnen. ${ }^{21}$ Ist $S_{2} z$. B. eine Sprache über Verwandtschaftsbeziehungen mit den Grundtermen „Bruder“", „Vater“, „Onkel mütterlicherseits“ etc., und ist $S_{1}$ jene Teilsprache von $S_{2}$, die als Grundterme nur ,männlich" und ,ist ein Elternteil von" enthält, so ist $S_{2}$ in diesem Sinn auf $S_{1}$ reduzierbar.

Generell wird man sagen:

D 3.3-1: Eine Sprache $S_{2}$ heißt reduzierbar auf eine Sprache $S_{1}$, wenn sich die Aussagen von $S_{2}$ in solche von $S_{1}$ übersetzen lassen.

${ }^{20}$ Quine hat sein Kriterium nicht für Sprachen, sondern für Theorien formuliert. Es lautet: „Die Existenz einer Entität (bzw. von Entitäten einer Art K) wird von einer Theorie $T$ genau dann vorausgesetzt, wenn sie zu den Werten gebundener Variablen gehören muß (bzw. müssen), falls die Behauptungen der Theorie wahr sein sollen" (vgl. z. B. Quine (64),103). Nun liegt jeder Theorie eine bestimmte Interpretation V der Sprache zugrunde, in der sie formuliert ist; im Sinn dieser Interpretation sind die Aussagen der Theorie zu verstehen. Dann gilt aber: ist $T$ nach $V$ falsch, so setzt $T$ beliebige Entitäten voraus. Darauf haben Cartwright in (54) sowie Scheffler und Chomsky 
Um den Begriff der Übersetzung näher zu erläutern, beziehen wir uns auf den einfachen Fall, daß die wohlgeformten Ausdrücke von $S_{1}$ und $\mathrm{S}_{2}$ (die Sätze, Prädikate und Eigennamen) nach denselben Formregeln gebildet werden, so daß sich beide Sprachen - syntaktisch gesehen - nur in ihrem Vokabular unterscheiden. Eine Zuordnung der Ausdrücke von $S_{2}$ zu Ausdrücken von $S_{1}$ wird angegeben durch eine Funktion $\varphi$, die jedem Grundterm $A$ von $S_{2}$ einen Term gleicher syntaktischer Kategorie $\varphi(A)$ von $S_{1}$ zuordnet. Ist $R\left(A_{1}, \ldots, A_{n}\right)$ ein mit der Formregel $R$ aus den Termen $A_{1}, \ldots, A_{n}$ gebildeter Ausdruck von $S_{2}$, so soll ihm der Ausdruck $\varphi\left(R\left(A_{1}, \ldots, A_{n}\right)\right)=R\left(\varphi\left(A_{1}\right), \ldots, \varphi\left(A_{n}\right)\right)$ von $S_{1}$ zugeordnet werden. Eine solche Zuordnung stellt eine Übersetzung von $S_{2}$ in $S_{1}$ dar, wenn für alle Ausdrücke $A$ von $S_{2}$ gilt, daß $A$ (nach der Interpretation von $S_{2}$ ) mit $\varphi(A)$ (nach der Interpretation von $S_{1}$ ) synonym ist, d.h. daß $\varphi(A)$ dieselbe Bedeutung hat wie A. ${ }^{22}$ Eine solche Übersetzung von $S_{2}$ in $S_{1}$ gibt es genau dann, wenn sich in $S_{1}$ Explizitdefinitionen für die in $S_{1}$ nicht vorkommenden Grundterme von $S_{2}$ angeben lassen, die ihnen (nach der Interpretation von $S_{1}$ ) dieselbe Bedeutung zuordnen, die sie nach der Interpretation von $S_{2}$ in $S_{2}$ haben. ${ }^{23}$

in (58) hingewiesen. Auf ihre Vorschläge zur Verbesserung des Quineschen Ontologiekriteriums wollen wir hier nicht eingehen, da auch sie wenig überzeugend sind. Bei unserem Ontologiekriterium für Sprachen stellt sich dieses Problem von vornherein nicht.

${ }^{21}$ Zur Definitionstheorie vgl. z. B. Kutschera (67), 6.3 und Kutschera und Breitkopf (71), Kap.14.

${ }^{22}$ Man könnte die Synonymitätsforderung auf Sätze beschränken. Denn im üblichen Sinn sind zwei Sätze $R\left(A_{1}, \ldots, A_{n}\right)$ und $R\left(\varphi\left(A_{1}\right), \ldots, \varphi\left(A_{n}\right)\right)$ nur dann synonym, wenn auch die entsprechenden Satzteile $A_{i}$ und $\varphi\left(A_{i}\right)$ $(i=1, \ldots, n)$ synonym sind. - Für Übersetzungen natürlicher Sprachen, z. B. von Englisch in Deutsch, wäre es wegen der Verschiedenheit ihrer Formregeln sehr viel schwieriger, Übersetzungskriterien anzugeben. Man könnte hier auch weder strenge Synonymität von Original und Übersetzung fordern, sondern nur eine für den Kontext hinreichende Bedeutungsähnlichkeit, noch eine Übersetzbarkeit Wort für Wort, sondern nur eine Übersetzung ganzer Sätze. Daher beziehen wir uns hier der Einfachheit halber auf Kunstsprachen.

${ }^{23}$ Lassen sich die Grundterme von $S_{2}$ in $S_{1}$ nur implizit definieren, so kann keine Synonymität behauptet werden. Dazu wäre mindestens erforderlich, $\mathrm{da} ß$ diesen Termen durch die Postulate der impliziten Definitionen im Rah- 
Eine Theorie im allgemeinen Sinn des Wortes ${ }^{24}$ ist eine Menge von Sätzen, wobei diese Menge entweder nur die Axiome der Theorie enthält, oder daneben auch alle Theoreme der Theorie, d.h. alle Sätze, die aus den Axiomen folgen. ${ }^{25}$ Nicht jede Menge $T$ von Sätzen, die bzgl. logischer Folgerungen geschlossen ist, stellt eine Theorie dar. T ist vielmehr nur dann eine Theorie, wenn es eine entscheidbare ( $z$. B. endliche) Teilmenge ' $T$ ' von $T$ gibt, aus deren Sätzen alle übrigen Sätze von $T$ folgen. Die Menge aller wahren Sätze einer Sprache ist z. B. in der Regel keine Theorie. Im folgenden verstehen wir unter "Theorie" die Menge der Axiome der Theorie.

Jede Theorie $T$ ist in einer Sprache $S$ formuliert. Als Sprache von $T$ - symbolisch $S(T)$ - bezeichnen wir jene Teilsprache von $S$, die nur die in den Axiomen von $T$ (wesentlich) vorkommenden (nicht-logischen und nicht-mathematischen) Grundterme enthält.

men der vorgegebenen Interpretation von $\mathrm{S}_{1}$ genau eine Bedeutung zugeordnet wird; dann ließen sich die Terme in $S_{1}$ aber auch explizit definieren. Vgl. dazu Kutschera (72),271 f. Anders sieht die Sache aus, wenn die Terme von $\mathrm{S}_{2}$ schon in dieser Sprache selbst nicht eindeutig interpretiert sind, und man nur von jenen Ausdrücken von $\mathrm{S}_{2}$ sagen kann, sie hätten eine bestimmte Bedeutung, denen alle Interpretationen einer Menge $V_{2}$ dieselbe Bedeutung zuordnen; wenn also $S_{2}$ mithilfe von super-valuations interpretiert ist. (Vgl. dazu van Fraassen (69) und Fine (75).) In diesem Fall kann man von einer Synonymität reden, wern diejenigen Erweiterungen der Interpretation von $S_{1}$, welche die Postulate der impliziten Definitionen erfüllen und auch für die Grundterme von $S_{2}$ definiert sind, diesen dieselben Werte zuordnen wie die Interpretationen aus $V_{2}$. Man kann die Grundterme $t_{i}$ von $S_{2}$ dann aber auch in $S_{1}$ explizit definieren, indem man sie in den impliziten Definitionen zunächst durch Terme $\tau_{i}$ ersetzt (um die $S_{1}$ also erweitert wird), und sie dann explizit durch diese $\tau_{i}$ definiert.

${ }^{24}$ Auf einen spezielleren Sinn des Wortes „Theorie“, der seinen Verwendungen in den Naturwissenschaften besser angepaßt ist, gehen wir im Abschnitt 9.5 ein.

${ }^{25}$ Wir beschränken uns hier der Einfachheit halber auf logische Folgen. Ein Satz $B$ einer Sprache $S$ folgt logisch aus Sätzen $A_{1}, \ldots, A_{n}$ dieser Sprache, wenn alle Interpretationen von $S$, welche die Sätze $A_{1}, \ldots, A_{n}$ erfüllen (d. h. wahr machen), auch den Satz $B$ erfüllen. Geht man von intensionalen Interpretationen aus, so besteht die logische Folgebeziehung, wenn für alle Interpretationen $\mathrm{V}$ von $\mathrm{S}$ und alle (bei $\mathrm{V}$ betrachteten) Welten $\mathrm{i}$ gilt: Wenn $\mathrm{V}$ in $\mathrm{i}$ die Sätze $A_{1}, \ldots, A_{n}$ erfüllt, so erfüllt $V$ in $i$ auch den Satz B. Vgl. dazu z. B. Kutschera (76), 1.2 und 2.4. Von solchen logischen Folgebeziehungen sind 
Man kann nun sagen:26

D 3.3-2: Eine Theorie $T_{2}$ ist auf eine Theorie $T_{1}$ reduzierbar, wenn es in $S\left(T_{1}\right)$ Definitionen aller in $S\left(T_{1}\right)$ nicht vorkommenden Grundterme von $S\left(T_{2}\right)$ gibt - die Konjunktion dieser Definitionsformeln sei $D_{1}-$, so daß alle Sätze von $T_{2}$ in logische Folgen von $T_{1}$ und $D_{1}$ (in der mit $D_{1}$ erweiterten Sprache $S_{1}$ ) übergehen.

Dabei wird nun nicht verlangt, daß diese Definitionen den Termen von $\mathrm{S}\left(\mathrm{T}_{2}\right)$ dieselbe Bedeutung zuordnen, die sie in $\mathrm{S}\left(\mathrm{T}_{2}\right)$ haben. Die Aussagen von $T_{2}$ müssen also nicht dasselbe bedeuten wie die ihnen nach $D_{1}$ entsprechenden Aussagen von $T_{1}$, die man aus den ersten durch Ersetzung der Grundterme durch die sie nach $\mathrm{D}_{1}$ definierenden Ausdrücke von $S\left(T_{1}\right)$ erhält. Anders ausgedrückt: die Sätze von $S\left(T_{2}\right)$ - als syntaktische Gebilde verstanden - können nach der Interpretation, die dieser Sprache zugrundeliegt, etwas anderes bedeuten als nach der Interpretation, die sie aufgrund von $D_{1}$ in $S_{1}\left(T_{1}\right)$ erhalten. Besteht Synonymie, so liegt eine Reduktion von $S\left(T_{2}\right)$ auf $S\left(T_{1}\right)$ vor, bei der $T_{2}$ in eine Folge von $T_{1}$ übergeht. Von größerer Bedeutung ist jedoch der allgemeine Fall einer Theorienreduktion. Beispiele dafür

analytische Folgebeziehungen zu unterscheiden, die sich jeweils auf eine bestimmte Interpretation $\mathrm{V}$ beziehen. Ist $\mathrm{V}$ eine extensionale Interpretation, so folgt $B$ aus $A_{1}, \ldots, A_{n}$ im Sinne von $V$ analytisch, wenn $V$ B erfüllt, falls $V$ die Sätze $A_{1}, \ldots, A_{n}$ erfüllt (falls also die Implikation $A_{1} \wedge \ldots \wedge A_{n} \supset B$ bei $V$ wahr ist). Bei extensionalen Interpretationen sind also analytische Folgebeziehungen insofern trivial, als sie mit materialen Implikationen zusammenfallen. Ist $V$ hingegen eine intensionale Interpretation, so folgt $B$ aus $A_{1}, \ldots, A_{n}$ im Sinne von $V$ analytisch, wenn für alle (bei $V$ betrachteten) Welten $i$ gilt: falls $V$ in i $A_{1}, \ldots, A_{n}$ erfüllt, erfüllt $V$ in $i$ auch $B$. Es wird oft die Meinung vertreten, analytische Folgebeziehungen ergäben sich aus logischen durch Verwendung von Definitionen, wie sich z. B. der Schluß „Alle Junggesellen sind unverheiratet" aus der logischen Folgebeziehung ,Alle unverheirateten Männer sind unverheiratet" mithilfe der Definition ,Junggesellen sind unverheiratete Männer" ergibt. Das ist jedoch nicht immer der Fall, wie das Beispiel "Wenn a Vater von b ist, so ist b nicht Vater von a“ zeigt.

${ }^{26}$ Zur Erörterung von Reduktionsbegriffen für Theorien vgl. a. J. Kemeny und P. Oppenheim (56), Stegmüller (70), 2.Halbbd. Kap. VIII, \9, Kutschera (72), 4.6. 
sind die Reduktionen der phänomenologischen Thermodynamik auf die statistische Mechanik, oder die Reduktion der Arithmetik auf die Mengenlehre nach den Definitionen von Frege oder von Neumann. Würde man keine explizite, sondern nur eine implizite Definierbarkeit der Terme aus $\mathrm{S}\left(\mathrm{T}_{2}\right)$ in $\mathrm{S}\left(\mathrm{T}_{1}\right)$ fordern, so erhielte man einen zu weiten Reduzierbarkeitsbegriff. Als Menge der Postulate einer impliziten Definition dieser Terme ließe sich ja $T_{2}$ selbst ansehen. Ist $T_{2}$ bzgl. $T_{1}$ nicht kreativ, so daß aus $T_{1}$ und $T_{2}$ keine Sätze von $S\left(T_{1}\right)$ folgen, die nicht auch aus $T_{1}$ alleine folgen, so ist $T_{2}$ ein System von bzgl. $T_{1} z u-$ lässigen impliziten Definitionen, und es gilt dann trivialerweise, daß $T_{2}$ aus $T_{1}$ und $T_{2}$ folgt. Ist dagegen $T_{2}$ bzgl. $T_{1}$ kreativ, so gibt es keine bzyl. $T_{1}$ zulässigen impliziten Definitionen der Terme aus $S\left(T_{2}\right)$ in $S\left(T_{1}\right)$, mit denen $T_{2}$ aus $T_{1}$ folgt. ${ }^{27}$ Reduzierbarkeit in diesem erweiterten Sinn besagt also nur, daß $T_{2}$ bzgl. $T_{1}$ nicht kreativ ist. ${ }^{28}$

Eine andere Trivialisierung ergibt sich aus folgendem Gedanken: In den Naturwissenschaften spricht man auch oft von einer Reduktion einer Theorie $T_{2}$ auf eine Theorie $T_{1}$, wenn sich $T_{2}$ mithilfe passender Definitionen aus $T_{1}$ nur unter Zuhilfenahme gewisser Zusatzbedingungen $Z$ als Sätzen von $S\left(T_{1}\right)$ ableiten läßt. So kann man die Keplersche Theorie der Planetenbewegung auf die Newtonsche Mechanik nur dann reduzieren, wenn man das Gravitationsgesetz hinzunimmt. Würde man jedoch definieren: „, $T_{2}$ ist auf $T_{1}$ reduzierbar, wenn es Definitionen $D_{1}$ der Terme aus $S\left(T_{2}\right)$ in $S\left(T_{1}\right)$ gibt und mit $T_{1}$ verträgliche Sätze $Z$ von $S\left(T_{1}\right)$, so daß $T_{2}$ analytisch aus $T_{1}, D_{1}$ und $Z$ folgt", so wäre jede Theorie $T_{2}$ auf jede andere reduzierbar. Denn für $Z$ könnte man die Übersetzung $T_{2}^{+}$von $T_{2}$ nach $D_{1}$ wählen. $D a$ aus $T_{1}$ und $D_{1} T_{2}^{+} \equiv T_{2}$ folgt, erhielte man so, daß $T_{2}$ aus $T_{1}, D_{1}$ und $T_{2}^{+}$ folgt. Es genügt auch nicht, die Bedingung hinzuzunehmen, daß $\mathrm{T}_{2}$ nicht aus $D_{1}$ und $Z$ folgen soll. Denn nun könnte man für $Z$ den Satz $\mathrm{T}_{1} \supset \mathrm{T}_{2}^{+}$wählen und erhielte so abermals eine Trivialisierung des Reduktionsbegriffs.

${ }^{27}$ Ist $P_{1}$ ein System impliziter Definitionen, das bzgl. $T_{1}$ nicht kreativ ist, so gilt für alle Sätze $A$ von $S\left(T_{1}\right)$ : Wenn $A$ aus $P_{1}$ und $T_{1}$ folgt, so auch aus $T_{1}$. Folgt also $T_{2}$ aus $T_{1}$ und $P_{1}$, so folgt aus $T_{1}$ und $T_{2}$ kein Satz $A$, der nicht aus $\mathrm{T}_{1}$ folgt, d. h. $\mathrm{T}_{2}$ ist dann nicht kreativ.

${ }^{28}$ Das ist für prädikatenlogische Sprachen mit Identität z. B. dann der Fall, wenn $S\left(T_{2}\right)$ keine Terme aus $S\left(T_{1}\right)$ enthält und wenn aus $T_{2}$ keine Anzahlaussagen folgen, die nicht auch aus $T_{1}$ folgen. 
Da keine Möglichkeit in Sicht ist, auf logischem Weg akzeptable von nicht akzeptablen Zusatzannahmen zu unterscheiden und insbesondere ad-hoc-Annahmen auszuschließen, läßt sich dieser Gedanke zur Definition eines erweiterten Reduktionsbegriffs nicht realisieren. Im obigen Beispiel ist das Gravitationsgesetz eine akzeptable Zusatzannahme, weil es noch zahllose andere Anwendungen hat. Daher bildet die Newtonsche Mechanik erweitert um das Gravitationsgesetz auch eine eigenständige Theorie, so daß man besser von einer Reduktion der Keplerschen Theorie auf die Gravitationstheorie als von einer solchen auf die Newtonsche Mechanik spricht.

Der Grundgedanke des Reduzierbarkeitsbegriffs nach D 3.3-2 hängt nicht davon $a b$, daß $T_{1}$ und $T_{2}$ Theorien sind. $T_{1}$ und $T_{2}$ könnten auch irgendwelche (konsistenten) Satzmengen sein. Für das folgende ist aber nur jene Erweiterung von D 3.3-2 von Interesse, bei der $T_{1}$ und $T_{2}$ die Mengen aller wahren Sätze von $S_{1}$, bzw. $S_{2}$ sind. Man kann dann weder von einer Reduktion von Theorien sprechen die Menge der wahren Sätze empirischer Sprachen ist in der Regel nicht axiomatisierbar - noch von einer Reduktion von Sprachen - es wird ja nicht, wie nach D 3.3-1, eine Reduktion der Ausdrucksmittel von $S_{2}$ auf die von $S_{1}$ gefordert. ${ }^{29}$ Wir wollen in Ermangelung eines besseren Ausdrucks von einer W-Reduktion (,W" für "Wahrheit") von $S_{2}$ auf $S_{1}$ sprechen. Wir definieren also:

D 3.3-3: Die Sprache $S_{2}$ ist auf die Sprache $S_{1} W$-reduzierbar, wenn es in $S_{1}$ explizite Definitionen aller in $S_{1}$ nicht vorkommende Grundterme von $S_{2}$ gibt - ihre Konjunktion sei $D_{1}$ - , so daß die Sätze von $S_{2}$, die aus $D_{1}$ und wahren Sätzen von $S_{1}$ logisch folgen, genau die wahren Sätze von $S_{2}$ sind.

Ist $A$ ein Satz von $S_{2}$ und ist $A^{+}$der Satz von $S_{1}$, der aus $A$ entsteht, indem man alle Grundterme in $A$, die in $S_{1}$ nicht vorkommen, durch die sie nach $D_{1}$ definierenden Ausdrücke ersetzt, so gilt $D_{1} \Rightarrow A \equiv A^{+}$. Dabei soll das Symbol " $\Rightarrow$ " die Beziehung logischer Folge ausdrükken. Nach D 3.3-3 ist $A$ in $S_{2}$ wahr genau dann, wenn es eine Menge $\boldsymbol{B}$ wahrer Sätze von $S_{1}$ gibt mit $D_{1}, \boldsymbol{B} \Rightarrow A$. Wegen $D_{1}, A \Rightarrow A^{+}$gilt also $D_{1}, \mathfrak{B} \Rightarrow A^{+}$, und da $A^{+}$und die Sätze aus $\mathcal{B}$ Sätze von $S_{1}$ sind, $2 B \Rightarrow$

${ }^{29}$ Wie viel schwächer die Bedingung der folgenden Definition D3.3 - 3 gegenüber der nach D 3.3-1 ist, hängt freilich vom Ausdrucksreichtum von $S_{2}$ ab. Darauf gehen wir unten ein. 
$A^{+}$. Wegen $D_{1}, A^{+} \Rightarrow A$ gilt also: $A$ ist in $S_{2}$ wahr genau dann, wenn $A^{+}$in $S_{1}$ wahr ist; nach $D_{1}$ also auch: $A$ ist in $S_{2}$ genau dann wahr, wenn $A$ in $S_{1}$ wahr ist. Aus alldem folgt nicht, daß A als Satz von $S_{2}$ und $\mathrm{A}+$ dieselbe Bedeutung haben. Der Satz $\mathrm{A}$ hat nur bei der Deutung nach $D_{1}$ in $S_{1}$ dieselbe Bedeutung wie $A^{+}$; der Satz $A$ (als syntaktisches Gebilde) kann aber in $S_{2}$ eine ganz andere Bedeutung haben als in $S_{1}$. Nach D 3.3-3 gilt lediglich, daß $A$ in $S_{2}$ und $A$ in (der durch $D_{1}$ erweiterten Sprache) $S_{1}$ dieselben Wahrheitswerte haben. Sind die Sprachen $S_{1}$ und $S_{2}$ über derselben Menge I von möglichen Welten interpretiert, folgt nicht, daß $A$ in $S_{1}$ und $A$ in $S_{2}$ in allen Welten aus I denselben Wahrheitswert haben. Und selbst wenn das gelten würde, würden $\mathrm{A}$ und $\mathrm{A}+\mathrm{zwar}$ dieselbe Intension, nicht aber dieselbe Bedeutung haben, denn $S_{1}$ und $S_{2}$ können verschiedene Objektberei-che zugrunde liegen. ${ }^{30}$

${ }^{30}$ Wird ein Term $t$ von $S_{2}$ in $S_{1}$ durch $t^{+}$definiert, so brauchen also insbesondere $\mathrm{t}$ und $\mathrm{t}^{+}$nicht dieselbe Extension zu haben. Dadurch unterscheidet sich W-Reduzierbarkeit von Kriterien extensionaler „Übersetzbarkeit". Lassen sich in $S_{1}$ implizite Definitionen der Grundterme $t_{i}$ von $S_{2}$ angeben, so auch explizite: Man ersetzt diese Terme in den Postulaten der impliziten Definitionen zuerst durch neu in $S_{1}$ eingeführte Terme $t_{i}$ und definiert dann die $t_{i}$ explizit durch diese Terme $t_{;}$N.Goodman hat in (51), Kap.I ein Kriterium „extensionaler Isomorphie" für Rekonstruktionen entwickelt, das im Effekt darauf hinausläuft, daß ein homomorphes Modell für einen Gegenstandbereich angegeben wird. Insofern fordert er - wie wir unten sehen werden mehr als eine W-Reduzierbarkeit. Seine Einwände gegen so etwas wie WReduzierbarkeit beruhen z. T. darauf, daß er sich nicht auf wohlbestimmte Sprachen bezieht. Wenn z. B. $S_{2}$ die normale Sprache der Geometrie ist und die Aussagen über Punkte in $S_{2}$ in $S_{1}$ in Aussagen über spezielle Mengen (wir nennen sie nach Goodman P-Mengen) von Volumina übersetzt werden, so darf natürlich in $S_{2}$ nicht die Aussage vorkommen: „Punkte sind keine Mengen von Volumina“ - die in $S_{1}$ wiedergegeben würde durch „P-Mengen von Volumina sind keine Mengen von Volumina“; ferner ist das Wort ,Volumen", das in $S_{2}$ und in $S_{1}$ vorkommt, in beiden Sprachen verschieden zu deuten, damit der Satz von $S_{2}$,Jedes Volumen enthält viele Punkte" nicht übersetzt wird in ,Jedes Volumen enthält viele P-Mengen von Volumina“. Interessant ist Goodmans Bemerkung, daß bei einer Darstellung von Individuen, von denen in $\mathrm{S}_{2}$ die Rede ist, und einer entsprechenden Darstellung einer Menge $C$ von Individuen durch eine Menge $\mathrm{C}^{+}$von Paarmengen in $\mathrm{S}_{1}$ der wahre Satz von $S_{2}(1)$,Die Elemente von $C$ enthalten keine Elemente“ 
Ist $S_{2}$ mithilfe der Definitionen $D_{1} W$-reduzierbar auf $S_{1}$, so gibt es zu jeder Theorie $T_{2}$, die sich in $S_{2}$ formulieren läßt, eine Theorie $T_{1}$ in $S_{1}$, auf die sich $T_{2}$ mit $D_{1}$ im Sinne von D 3.3-2 reduzieren läßt. Dazu braucht man die Axiome von $T_{2}$ nur nach $D_{1}$ in Sätze von $S_{1} z u$ übersetzen, die dann als Axiome von $T_{1}$ dienen; $T_{1}$ ist also $T_{2}{ }^{+}{ }^{31}$ In diesem Fall lassen sich mit $T_{1}$ auch deduktive Erklärungen von in $S_{2}$ beschriebenen Phänomenen mit $T_{2}$ nachzeichnen. Gilt im Sinne des deduktiv-nomologischen Erklärungsbegriffs von Hempel und Oppenheim, ${ }^{32}$, daß sich ein mit einem Satz $E$ von $S_{2}$ beschriebenes Phänomen mit $T_{2}$ und wahren Antezedensbedingungen $A_{1}, \ldots, A_{n}$ (als Sätzen von $S_{2}$ ) erklären läßt, gilt also $T_{2}, A_{1}, \ldots, A_{n} \Rightarrow E$, so gilt auch $T_{1}, D_{1}, A_{1}, \ldots, A_{n} \Rightarrow E$, und da die Sätze $A_{1}, \ldots, A_{n}$ in $S_{2}$ wahr sind, sind sie auch in $S_{1}$ wahr. Ist $T_{2}$ auf $T_{1}$ reduzierbar im Sinn von D 3.32, so kann man dagegen nicht so schließen. Aus $T_{2}, A_{1}, \ldots, A_{n} \Rightarrow E$ folgt zwar $T_{1}, D_{1}, A_{1}, \ldots, A_{n} \Rightarrow E$, aber aus der Wahrheit der Sätze $A_{1}, \ldots, A_{n}$ in $S_{2}$ folgt nicht, daß diese Sätze auch in $S_{1}$ wahr, also brauchbare Antezedensbedingungen für Erklärungen sind.

Ist $S_{2}$ auf $S_{1} W$-reduzierbar, so kann man nicht sagen, alle Phänomene, die sich in $S_{2}$ beschreiben und mit Gesetzesaussagen oder Theorien in $S_{2}$ erklären lassen, ließen sich auch in $S_{1}$ erklären. Denn der erklärte Satz bedeutet in $S_{1}$ nicht dasselbe wie in $S_{2}$. Man sagt freilich oft, Wärmephänomene, wie z. B. die Wärmeleitung, ließen sich in der statistischen Mechanik erklären; oder optische Phänomene seien ,nichts anderes“ als elektromagnetische Phänomene. Solche Behauptungen sind aber streng genommen falsch. Ereignisse, Sachverhalte, Zustände, Phänomene sind Bedeutungen von Sätzen. Nur bedeutungsgleiche Sätze drücken also dieselben Ereignisse, Sachver-

in den falschen Satz von $\mathrm{S}_{1}$,Die Elemente von $\mathrm{C}^{+}$enthalten keine Elemente" zu übersetzen wäre. Da der Ausdruck für die Elementschaftsrelation ein logisches Symbol ist, kann man ihn in $S_{1}$ und $S_{2}$ nicht wie den Ausdruck „Volumen“ im obigen Beispiel verschieden interpretieren. $S_{2}$ wäre daher bei einer W-Reduktion so zu bestimmen, daß (1) kein sinnvoller Satz von $S_{2}$ ist, d. h. daß für Individuen a die Aussagen ,bEa“" nicht erklärt sind. Dagegen sind (sinnvolle) Sätze von $S_{2}$ wie „C enthält mindestens zwei Elemente“ natürlich in $S_{1}$ durch den analogen $\mathrm{Satz}$ „C, $\mathrm{C}^{+}$enthält mindestens zwei Elemente" wiederzugeben.

${ }^{31}$ Gilt $T_{2} \Rightarrow A$, so wegen $T_{2}^{+}, D_{1} \Rightarrow T_{2}$ auch $T_{1}, D_{1} \Rightarrow A$.

${ }^{32} \mathrm{Vgl}$. dazu den Abschnitt 2.2. 
halte, Zustände und Phänomene aus, nicht hingegen Sätze mit demselben Wahrheitswert. Ebenso sind Attribute Bedeutungen von Prädikaten, so daß nur bedeutungsgleiche, nicht aber umfangsgleiche Prädikate dieselben Attribute ausdrücken.

Betrachten wir ein einfaches Beispiel. $\mathrm{S}_{2}$ sei eine Sprache der elementaren Prädikatenlogik mit den drei Gegenstandskonstanten a,b und $\mathrm{c}$ und der einstelligen Prädikatkonstanten F, der zweistelligen Prädikatkonstanten $\mathrm{G}$ und der dreistelligen Prädikatkonstanten $\mathrm{H}$. $\mathrm{S}_{2}$ sei interpretiert über der Menge der Orte München, Nürnberg und Garmisch. $F(x)$ bedeute, daß $x$ eine Landeshauptstadt ist; $G(x, y)$, daß $\mathrm{x}$ mehr Einwohner hat als $\mathrm{y}, \mathrm{H}(\mathrm{x}, \mathrm{y}, \mathrm{z}), \mathrm{da} ß \mathrm{x}$ zwischen $\mathrm{y}$ und $\mathrm{z}$ liegt. a bezeichne München, b Nürnberg und c Garmisch. $S_{1}$ sei eine Sprache der elementaren Prädikatenlogik mit den drei Gegenstandskonstanten d,e,f und den drei ein-, bzw. zwei- und dreistelligen Prädikatkonstanten $I_{2}, K, S_{1}$ sei interpretiert über der Menge der Himmelskörper Sonne, Erde und Mond. $\mathrm{I}(\mathrm{x})$ bedeute, daß $\mathrm{x}$ ein heißer Stern ist; $J(x, y)$, daß y sich auf einer Bahn um $x$ bewegt; $K(x, y, z)$, daß die Masse von $x$ größer ist als die Summe der Massen von y und $z$. $d$ bezeichne die Sonne, e die Erde, $f$ den Mond. Definiert man die Konstanten $a, b, c, F, G, H$ in dieser Reihenfolge durch $d, e, f, I, J, K$, so wird damit $S_{2}$ auf $S_{1} W$-reduziert. Es kann aber keine Rede davon sein, daß z. B. der Sachverhalt, daß München Landeshauptstadt ist, derselbe Sachverhalt wäre wie jener, daß die Sonne ein heißer Stern ist.

Es ist nun zweifellos richtig, daß die W-Reduzierbarkeit einer Sprache $S_{2}$ auf eine Sprache $S_{1}$ mit zunehmendem Ausdrucksreichtum von $S_{2}$ kein derart trivialer und uninteressanter Tatbestand ist wie in unserem Beispiel. Trotzdem rechtfertigt es auch dann eine bloße WReduzierbarkeit nicht, von einer Identität der Sachverhalte zu sprechen, wie sie durch denselben Satz in $S_{2}$ und in $S_{1}$ dargestellt werden, oder zu sagen, der durch einen Satz in $S_{2}$ ausgedrückte Sachverhalt sei ",nichts anderes" als der durch diesen Satz in $S_{1}$ ausgedrückte Sachverhalt.

Oft werden Identitätsbehauptungen für Sachverhalte im Fall einer W-Reduzierbarkeit oder einer Theorienreduzierbarkeit durch folgende Argumente verteidigt:

1. Der Sinn eines Satzes A wird durch die Wahrheitsbedingungen für $A$ angegeben. Ist $B$ eine hinreichende, bzw. notwendige Bedingung für $A$ in $S_{2}$, so ist aber bei W-Reduzierbarkeit $B$ auch eine hinreichende, bzw. notwendige Bedingung für $A$ in $S_{1}$. Für $A$ gelten also in 
$\mathrm{S}_{2}$ und $\mathrm{S}_{1}$ dieselben Wahrheitsbedingungen, der Satz hat also in beiden Sprachen denselben Sinn. - Dagegen ist zu sagen: Erstens drückt $B$ eben in $S_{1}$ und $S_{2}$ nicht dieselbe Bedingung aus, denselben Sachverhalt; daher hat $\mathrm{A}$ auch nicht dieselben Wahrheitsbedingungen in $S_{1}$ und $S_{2}$. Zweitens werden Wahrheitsbedingungen, die den Sinn von A charakterisieren, nicht durch materiale Implikationen oder logische Folgebeziehungen, sondern durch analytische Aussagen angegeben. Logische Folgebeziehungen zwischen zwei Sätzen $A$ und $B$ von $S_{2}$ gelten in $S_{1}$ wie $S_{2}$; und ist $A \supset B$ in $S_{2}$ wahr, so bei W-Reduzierbarkeit von $S_{2}$ auf $S_{1}$ auch in $S_{1}$. Analoges gilt aber nicht generell für analytische Aussagen: In $S_{2}$ analytisch wahre Sätze können in $S_{1}$ nur kontingent gelten, und umgekehrt.

2. Soweit sich Bedeutungen objektiv bestimmen lassen, müssen sie sich aus dem Gebrauch der Sprache ergeben. Mit W.V.Quine könnte man sagen: "Language is a social art. In acquiring it we have to depend entirely on intersubjectively available cues as what to say and when. Hence there is no justification for collating linguistic meanings, unless in terms of mens' dispositions to respond overtly to socially observable stimulations". ${ }^{33}$ Gebraucht eine Person $a_{2}$ die Sätze in dem Sinn, wie sie in $S_{2}$ verstanden werden, die Person $a_{1}$ so, wie sie nach $D_{1}$ in $S_{1}$ interpretiert werden, so läßt sich der Unterschied der Bedeutungen der Sätze nicht an ihrem Gebrauch ablesen, wenn sie bei beiden Interpretationen denselben Wahrheitswert haben, zumindest dann nicht, wenn die Sätze bei beiden Deutungen in denselben Situationen (unter denselben ,Reizbedingungen“ in der Terminologie Quines) gebraucht werden können. Die unterschiedlichen Bedeutungen sind dann objektiv nicht feststellbar, also bloß subjektive Phänomene. - Dazu ist aber zu sagen: ${ }^{34}$ Die Bedeutungen werden für den Gebrauch, für die Wahrheitswerte relevant bei der Verwendung nichtextensionaler Kontexte, wie z. B. „Die Person a glaubt, daß p“. Hier hängt, wie wir im Abschnitt 1.1 sahen, der Wahrheitswert des ganzen Satzes nicht nur vom Wahrheitswert des Teilsatzes „,p“ ab, sondern von dessen Intension, bzw. bei Verwendung eines deskriptiven Glaubensbegriffs sogar von dessen Bedeutung. Zu den nicht-extensionalen

${ }^{33}$ Quine (60), S.IX.

${ }^{34}$ Zur Diskussion des Quineschen Versuchs der Elimination von Bedeutungen und seiner These von der Indeterminiertheit der Übersetzungen vgl. a. Kutschera (75),2.3. 
Kontexten gehören z. B. auch Aussagen über die analytische Gültigkeit von Sachverhalten. Besagt NA in $S_{2}$, daß der Sachverhalt A analytisch gilt, so ist das Prädikat $\mathrm{N}$ in $\mathrm{S}_{1}$ durch ein Prädikat $N *$ von $S_{1}$ zu definieren, das auch die Eigenschaften einer analytischen Notwendigkeit haben muß, zumindest in Anwendung auf Sätze von $S_{2} . \mathrm{Da}$ aber in $S_{1}$ nach der definitorischen Erweiterung mit $D_{1}$ nicht zwischen Sätzen von $S_{2}$ und solchen von $S_{1}$ unterschieden wird, muß $N^{*}$ allgemein diese Eigenschaft haben. Gilt also z. B. $N^{*}(A \equiv B)$, so müssen $A$ und $B$ in allen (intensionalen) Kontexten salva veritate für einander substituierbar sein. $D a$ nun in $S_{2}$ gilt $N(A \equiv A)$, muß in $S_{1}$ gelten $\mathrm{N}^{*}(\mathrm{~A} \equiv \mathrm{A})$; also muß wegen $\mathrm{N}^{*}\left(\mathrm{~A}^{+} \equiv \mathrm{A}\right) \mathrm{A}^{+}$in $\mathrm{S}_{1}$ dieselbe Intension haben wie $A$. Nimmt man entsprechend in $S_{2}$ einen Ausdruck für Synonymität an, so fällt die W-Reduzierbarkeit von $S_{2}$ auf $S_{1}$ mit einer Reduzierbarkeit von $S_{2}$ auf $S_{1}$ im Sinne von D 3.3-1 zusammen. Ferner enthält der Begriff der W-Reduzierbarkeit nicht einmal die Quinesche Forderung, daß die Sätze von $S_{2}$ in beiden Deutungen in denselben Situationen verwendet werden können. In unserem obigen Beispiel ist das nicht der Fall: Sätze über Himmelskörper sind nicht in den gleichen Situationen passend wie Sätze über Städte. Auch wenn man eine Gebrauchstheorie der Bedeutung im Sinne Quines vertritt, kann man also nicht von einer Bedeutungsgleichheit bei W-Reduzierbarkeit sprechen.

Auch die Tatsache, daß für eine W-Reduzierbarkeit von $S_{2}$ auf $S_{1}$ nicht erforderlich ist, daß die universes of discourse dieser beiden Sprachen gemeinsame Elemente enthalten, daß also die einander zugeordneten Sätze $\mathrm{A}$ und $\mathrm{A}^{+}$über dieselben Objekte reden, zeigt, wie schwach dieser Reduzierbarkeitsbegriff ist. Wir werden ihn im folgenden auch nur so verwenden, daß wir zeigen: gewisse Sprachen sind nicht auf einander $W$-reduzierbar, also können erst recht keine anspruchsvolleren Reduzierbarkeitsbeziehungen zwischen diesen Sprachen bestehen. Wir beanspruchen also nicht, mit diesem Reduktionsbegriff eine Form inhaltlich sinnvoller Reduktion zu erfassen.

Ist eine Theorie $T_{2}$ auf die Theorie $T_{1}$ reduzierbar, so sagt man oft, es gebe $T_{2}$-Modelle von $T_{1}$, in den früheren Beispielen also: es gebe ein mechanisches Modell der Thermodynamik und ein mengentheoretisches Modell der Arithmetik. Wegen der Bedeutung des Modellbegriffs für erkenntnistheoretische Fragen wollen wir noch einige kurze Bemerkungen dazu anschließen. 
Man muß zunächst zwischen semantischen und algebraischen Modellen unterscheiden. Eine Interpretation einer (Kunst-)Sprache $S$ bezeichnet man oft als Modell dieser Sprache. Und ist A ein Satz, bzw. Aeine Menge von Sätzen von $S$, so bezeichnet man auch eine Interpretation von S, die den Satz A, bzw. alle Sätze aus A erfüllt (d. h. wahr macht), als Modell von A. In diesem Sinn spricht man auch von „Modellen von Theorien“. Beides sind semantische Modellbegriffe.

Was ein algebraisches Modell ist, wollen wir an einem einfachen Fall verdeutlichen. Ist $U$ eine Menge von Objekten und sind $F_{1}, \ldots, F_{m} m_{i^{-}}$ stellige Begriffe $(i=1, \ldots, m)$ und $f_{1}, \ldots, f_{n} n_{j}$-stellige Funktionen $(j=1, \ldots, n)$, die auf $U$ definiert sind, so bezeichnet man das $(\mathrm{m}+\mathrm{n}+1)-$ Tupel $\mathbb{A}=<\mathrm{U}, \mathrm{F}_{1}, \ldots, \mathrm{F}_{\mathrm{m}}, \mathrm{f}_{1}, \ldots, \mathrm{f}_{\mathrm{n}}>$ als Algebra. Es sei $\boldsymbol{A}^{\prime}=\left\langle U^{\prime}, \mathrm{F}_{1}^{\prime}, \ldots, \mathrm{F}_{\mathrm{m}}^{\prime}, \mathrm{f}_{1}^{\prime}, \ldots, \mathrm{f}_{\mathrm{n}}^{\prime}\right\rangle$ eine zweite Algebra vom gleichen $T y p$ wie $\boldsymbol{A}, \mathrm{d}$. h. mit gleichvielen Begriffen und Funktionen, wobei die sich in $\mathcal{A}$ und $A^{\prime}$ entsprechenden Begriffe $F_{i}^{\prime}$ und $F_{i}$ und Funktionen $f_{j}$ und $\mathrm{f}_{\mathrm{j}}^{\prime}$ dieselbe Stellenzahl $\mathrm{m}_{\mathrm{i}}$, bzw. $\mathrm{n}_{\mathrm{j}}$ haben. Man sagt dann:

D 3.3-4: A ist ein Modell von $\mathcal{A}^{\prime}$, wenn es eine Abbildung $\varphi$ von $U$ ' auf $U$ gibt, so daß für alle $i=1, \ldots, m, j=1, \ldots, n$ und alle $\mathrm{m}_{\mathrm{i}}-$, bzw. $\mathrm{n}_{\mathrm{j}}$-tupel von Objekten $\mathrm{a}_{1}, \ldots, \mathrm{a}_{\mathrm{m}_{\mathrm{i}}}$, bzw. $a_{1}, \ldots, a_{n}$ aus $U^{\prime}$ gilt:

a) $F_{i}\left(a_{1}, \ldots, a_{m_{i}}^{j}\right) \equiv F_{i}\left(\varphi\left(a_{1}\right), \ldots, \varphi\left(a_{m}.\right)\right)$ und

b) $\varphi\left(f_{j}\left(a_{1}, \ldots, a_{n_{j}}\right)\right)=f_{j}\left(\varphi\left(a_{1}\right), \ldots, \varphi\left(a_{n_{j}}\right)\right) \cdot{ }^{35}$

Es sei z. B. U' eine Menge von Stäben. F' sei eine zweistellige Relation, so daß F'(a,b) besagt, daß der Stab a höchstens so lang ist wie $\operatorname{der}$ Stab b. f' sei eine zweistellige Funktion, so daß f'(a,b) der Stab ist, der sich durch Aneinanderfügen von a und $b$ ergibt. $U$ sei eine Menge von Zahlen, F sei die Relation $\leq$ (kleiner oder gleich) und $f$ die Funktion + der Addition. $\varphi(a)$ sei die Länge des Stabes a in Metern. Dann ist, bei passender Wahl von $U,\langle U, \leq,+\rangle$ ein Modell von $<U^{\prime}, F^{\prime}, f^{\prime}>$ im Sinne von D 3.3 - 4. Allgemein besteht der Prozeß der Metrisierung von qualitativen Begriffen, die auf einem Gegenstandsbereich erklärt sind, in der Angabe numerischer Modelle dafür. ${ }^{36}$

${ }^{35}$ In diesem Fall spricht man auch von einem homomorphen Modell. Ist die Abbildung umkehrbar eindeutig, so liegt ein isomorphes Modell vor.

${ }^{36}$ Zur Theorie der Metrisierung vgl. Krantz, Luce, Suppes und Tversky (71); für eine Einführung Stegmüller (73), 1.Halbbd., Kap.I, oder Kutschera (72), Kap.1. 
Der algebraische Modellbegriff nach D 3.3-4 läßt sich in mannigfacher Weise verallgemeinern. Wir wollen darauf jedoch hier nicht eingehen, um uns nicht in technischen Details zu verlieren. Der einfache Fall genügt, um daran die methodologische Bedeutung des algebraischen Modellbegriffs deutlich zu machen. Nehmen wir an, daß sich die Phänomene einen Gegenstandsbereichs U' mithilfe von $\mathrm{Be}-$ griffen $F_{i}$ und Funktionen $f_{j}$ bestimmen lassen. Dann bedeutet die Angabe eines Modells $\boldsymbol{A}$ für $\boldsymbol{A}$ ', daß wir die Struktur dieser Phänomene, ihre Zusammenhänge und Gesetzmäßigkeiten darstellen können durch Aussagen mit den $F_{j}$ und $f_{j}$, die sich auf Objekte aus $U$ beziehen. Ist nun $\not$ e eine Struktur, die wir gut beherrschen, so haben wir mit dem Modell ein handliches Mittel zur Beschreibung der fraglichen Phänomene gewonnen. Die Aussagen über das Modell $\mathbb{A}$ sind zwar keine Aussagen über das Original A', denn die Gegenstände von U' können von ganz anderer Art sein als jene von $U$, und entsprechend können die Aussagen über $\mathbb{A}$ einen ganz anderen Sinn haben als jene über A', aber solange es uns nicht auf die Natur der Phänomene ankommt, sondern nur auf ihre Struktur, ihren Zusammenhang, also nicht auf das „Was“, sondern auf das „Wie“, enthält $\mathcal{A}$ (wo die Zuordnung $\varphi$ angegeben ist) alle relevanten Informationen über $\AA^{\prime}$.

Diese modellhafte Beschreibung ist für die moderne Naturwissenschaft, vor allem die Physik, charakteristisch. Insbesondere geht es dabei um die Angabe mathematischer Modelle für die Phänomene in der Natur. ${ }^{37}$ Physikalische Theorien sind nichts anderes als mathematische Modelle der physischen Wirklichkeit. Diese Wendung der modernen Naturwissenschaft von einer Analyse der Natur der Phänomene zu einer Analyse ihrer Struktur hat Descartes schon in den "Regulae ad directionem ingenii" vorgezeichnet. Descartes war der Begründer der analytischen Geometrie, in der geometrische Sachverhalte durch numerische Modelle dargestellt werden, geometrische Probleme also in algebraische transformiert werden. Diesen Gedanken hat Descartes dann verallgemeinert zu einem methodologischen Prinzip: Er forderte die Entwicklung einer abstrakten Algebra als einer allgemeinen Lehre von Größe und Ordnung, einer Mathesis universalis, in deren Rahmen sich für alle Erscheinungsbereiche Modelle

${ }^{37}$ Mathematische Modelle sind nicht immer numerische Strukturen; einen sehr viel weiteren Anwendungsbereich haben algebraische Strukturen. 
angeben lassen..$^{38}$ Sein Argument für die universale Anwendbarkeit dieser Mathesis universalis war folgendes: Erkennen heißt vergleichen. Vergleichen läßt sich nur, was ein Mehr-oder-Weniger zuläßt, d. h. Größen. Alle physikalischen Größen stehen aber in einem gesetzmäßigen Zusammenhang zur geometrischen Größe, wie z. B. die Temperatur zur Länge einer Quecksilbersäule, oder die Tonhöhe zur Länge einer Saite. Nun gibt es aber ein algebraisches Modell der Geometrie, also auch ein solches für alle Größen. ${ }^{39}$ Dieses Argument ist sicher schwach; an der Behauptung selbst, daß es zu jeder Struktur ein mathematisches Modell gibt, ist aber nicht zu zweifeln. Freilich gibt es nicht für alle Strukturen interessante mathematische Modelle, denn ein Modell soll ja eine durchsichtigere Darstellung der fraglichen Originalstruktur erlauben. Seit Pythagoras entdeckte, daß es interessante mathematische Modelle für physikalische Erscheinungen gibt, hat sich jedoch die Mathematisierung der Physik in immer weiteren Bereichen als fruchtbar erwiesen.

Ausschlaggebend für Descartes Programm war sicher auch sein Rationalismus, der sich in seiner schon in 1.5 zitierten Evidenzforderung für wissenschaftliche Sätze ausdrückt: Nur Sätze, die ebenso evident sind wie mathematische, stellen wissenschaftliche Erkenntnisse dar. Eine in diesem Sinn exakte Naturwissenschaft reicht nur so weit wie die Darstellbarkeit in mathematischen Modellen. Für Descartes war die physische Welt eine mathematisch beschreibbare Welt: "Apud me omnia fiunt mathematice in Natura", schreibt er an Mersenne. ${ }^{40}$

Ist eine Theorie $T_{2}$ im Sinne von $\mathrm{D} 3.3-2$ auf eine Theorie $T_{1}$ reduzierbar, so sind die semantischen Modelle von $T_{1}$ und $D_{1}$ algebraische Modelle von semantischen Modellen von $\mathrm{T}_{2}$. Daher bedeutet die Reduzierbarkeit, daß man die Phänomene, die $\mathrm{T}_{2}$ beschreibt, auch in Modellen darstellen kann, die sich auf solche Phänomene beziehen, wie sie $T_{1}$ beschreibt. Man kann aber nicht generell sagen, das inten-

${ }^{38} \mathrm{Vgl}$. ,Regulae“, Anhang zur Regel 4.

${ }^{39}$ Vgl. ,Regulae“, Regel 14, Abs. 4 f.

${ }^{40}$ Descartes (W) III,36. - Zum Programm der modernen Physik vgl. a. Helmholtz und „Über die Tatsachen in der Wahrnehmung“ (1878), abgedruckt in (21),S.109- 152 und H. Hertz (94), Einleitung. Vgl. dazu auch die Zitate im Abschnitt 4.3. 
dierte semantische Modell von $\mathrm{T}_{1}$ und $\mathrm{D}_{1}, \mathrm{~d}$. h. die Interpretation, die der Sprache $S\left(T_{1}\right)$ zugrunde liegt, sei ein algebraisches Modell des intendierten semantischen Modells von $\mathrm{T}_{2}$. Das gilt nur in speziellen Fällen, z. B. dann wenn die Theorie $\mathrm{T}_{2}$ kategorisch ist, $\mathrm{d}$. h. wenn alle ihre semantischen Modelle isomorph sind; wenn $\mathrm{T}_{2}$ also die Struktur ihrès Gegenstandsbereichs vollständig beschreibt. Daher kann man im Sinne unseres Modellbegriffs nicht allgemein behaupten, es gäbe ein $T_{1}$-Modell für die durch $T_{2}$ beschriebenen Phänomene, wenn $T_{2}$ auf $T_{1}$ reduzierbar ist. Auch wenn die Sprache $S_{2}$ im Sinne von D 3.3-3 auf die Sprache $S_{1} W$-reduzierbar ist, stellt die Interpretation von $S_{1}$ (oder eine Teilstruktur davon) nicht immer ein algebraisches Modell der Interpretation von $\mathrm{S}_{2}$ dar. W-Reduzierbarkeit bedeutet also nicht, $\mathrm{da} ß$ die Dinge, über die man in (einem Teil) der Sprache $S_{1}$ spricht, dieselbe Struktur haben wie die Dinge, über die man in $S_{2}$ spricht. Dagegen gilt die Umkehrung: Ist die Interpretation von $S_{1}$ ein Modell der Interpretation von $S_{2}$, so ist $S_{2}$ auf $S_{1}$ W-reduzierbar.

\subsection{Realismus}

Wir werden uns im nächsten Kapitel mit der Kontroverse zwischen Realismus und Idealismus befassen. Der Realismus stellt die normale Auffassung dar, die Ansicht des gesunden Menschenverstandes, oder wie man im Englischen treffender sagt: des common sense; eine Selbstverständlichkeit also für den Alltagsverstand. Der Idealismus ergibt sich aus kritischen Einwänden gegen diese Konzeption, und daher wollen wir zunächst auf sie eingehen, bevor wir uns mit idealistischen Thesen und Argumenten beschäftigen.

Zunächst sind zwei Formen des Realismus zu unterscheiden: der ontologische und der erkenntnistheoretische Realismus. Die These des ontologischen Realismus wird meist etwa so formuliert: Es gibt eine Realität, die in ibrer Existenz wie Beschaffenbeit unabbängig davon ist, $o b$ und wie sie von uns Menschen erfabren, gedeutet oder erkannt wird. ${ }^{41}$

${ }^{41}$ Als „ontologischen Realismus“ bezeichnet man auch die These des Platonismus von der von unserem Denken unabhängigen Existenz abstrakter Entitäten wie Begriffe, Propositionen, Zahlen, Funktionen oder Mengen, oder von der ,objektiven“ Geltung logischer und mathematischer Sätze. Die Erörterung dieses Begriffsrealismus ist aber Thema der Philosophie von Logik und Mathematik, nicht der Erkenntnistheorie. 
Im Sinne von 3.2 können wir unter ,Realität" eine Menge von Tatsachen verstehen. Es soll also Sachverhalte geben, deren Bestehen unabhängig davon ist, ob es Menschen gibt und was sie erfahren, glauben, wollen, fühlen etc. Solche Sachverhalte wollen wir hier kurz als ,,objektiv" bezeichnen; sie werden also ,subjektiven" Sachverhalten des Empfindens, Glaubens, Wollens, Fühlens etc. gegenübergestellt. Dann läßt sich die These auch so auch so ausdrücken:

\section{R 1: Es gibt objektive Tatsachen.}

Sie sagt noch nichts darüber aus, von welcher Art diese objektiven Tatsachen sind - ob z. B. physikalische Tatsachen objektiv sind in diesem Sinn - und auch nichts über die Erfahrbarkeit oder Erkennbarkeit der angenommenen objektiven Realität.

Die These des erkenntnistheoretischen Realismus besagt hingegen:

\section{R 2: Es gibt Erfabrungen von objektiven Sacbverbalten.}

Solche Erfahrungen sind im Sinne von 3.1 äußere Erfahrungen. Der erkenntnistheoretische Realismus setzt den ontologischen voraus. Der ontologische Realismus impliziert aber keinen erkenntnistheoretischen; er ist auch mit einem erkenntnistheoretischen Idealismus verträglich. So vertritt Kant einen ontologischen Realismus, aber einen erkenntnistheoretischen Idealismus. Die These R1 läßt sich aber wohl nur über $\mathrm{R} 2$ begründen, und daher hat auch schon Fichte Kant vorgeworfen, ein ontologischer Realismus sei nicht haltbar, wenn man einen erkenntnistheoretischen Idealismus annehme.

Das Argument des common sense für den Realismus im Sinn von $\mathrm{R} 1$ und R2 lautet etwa so: Es gibt Erfahrungen von Physischem. Ich sehe z. B., daß dieses Blatt Papier weiß ist; daß es schneit; daß in meinem Garten ein Baum steht. Solche synthetischen Aussagen über konkrete, wahrnehmbare, körperliche Dinge wie Papierblätter, Bäume, Tische, Sterne etc. und ihre wahrnehmbaren Eigenschaften wie Gestalt, Farbe, gegenseitige Lage, Härte, usw. - solche Aussagen der Dingsprache in der Terminologie Carnaps ${ }^{42}$ - drücken nun Sachverhalte aus, für deren Bestehen es gleichgültig ist, ob und wie wir sie erfahren, erfahren haben oder erfahren werden. Wenn das Blatt Papier

${ }^{42}$ Die Dingsprache ist Teil der normalen Sprache und ist von der Sprache der Physik zu unterscheiden, in der Fachausdrücke wie „Masse“ oder „Feldstärke" vorkommen. Vgl. dazu den Abschnitt 6.1. 
weiß ist, so ist es auch dann weiß, wenn ich es nicht betrachte oder wenn es mir bei der Betrachtung als gelb erschiene. Daraus, daß es mir so erscheint, bzw. nicht so erscheint, als läge vor mir ein weißes Blatt Papier, folgt weder, daß hier tatsächlich ein Blatt Papier liegt, noch daß es tatsächlich weiß ist - es könnte ja eine Sinnestäuschung vorliegen; das, was mir als Papier erscheint, könnte eine Plastikfolie sein; und aufgrund der Beleuchtungsverhältnisse könnte mir etwas als weiß erscheinen, was tatsächlich gelb ist. Und umgekehrt folgt daraus, daß hier tatsächlich ein weißes Blatt Papier liegt, auch nicht, daß es mir so scheint, als sei das der Fall; es kann ja sein, daß ich es nicht wahrnehme oder wiederum, daß ich einer Sinnestäuschung unterliege. Die physischen Sachverhalte, die wir erfahren, sind also objektiv. Es gibt daher äußere Erfahrungen als Erfahrungen von objektiven Sachverhalten.

Das ist eine prima facie höchst plausible Begründung des erkenntnistheoretischen Realismus im Sinn von R2, und damit zugleich eine Begründung für R1. Die physische, körperliche Welt ist danach eine objektive Realität. Diese Welt existiert, wie man heute annimmt, seit ca. 13 Milliarden Jahren. Die Erde, auf der wir leben, ist nur ca. 5 Milliarden Jahre alt, und Menschen gibt es - selbst bei großzügigen Kriterien für Menschlichkeit - erst seit etwa 1,5 Millionen Jahren. Die physische Natur hat also schon lange existiert, bevor es Menschen gab, und sie wird auch bestehen bleiben, wenn es keine Menschen mehr gibt. Angesichts der ungeheuren zeitlichen und räumlichen Dimensionen, in denen sich kosmisches Geschehen abspielt, und den vergleichweise engen Grenzen, auf die sich menschliches Leben beschränkt, erscheint es als absurd, Existenz und Beschaffenheit der physischen Natur als abhängig von der Existenz und Beschaffenheit menschlicher Erfahrung anzusehen. Aufgrund dieser Argumente wird man insbesondere physikalische Sachverhalte als objektiv ansehen und einen Realismus bzgl. der physischen Welt vertreten.

Aus der Erfahrbarkeit objektiver Sachverhalte im Sinn von R 2 folgt nicht unmittelbar die Erkennbarkeit solcher Sachverhalte, also die Behauptung

R 3: Es gibt eine Erkenntnis objektiver Tatsachen.

Verwirft man diese Behauptung, so vertritt man einen Skeptizismus bzgl. der äußeren Erfahrung, und wenn wir das Wort ,empirisch“ im 
Sinn äußerer Erfahrung verstehen, einen empirischen Skeptizismus. Dieser ist aber kaum weniger absurd als der universelle Erkenntnisskeptizismus, den wir in 1.7 diskutiert haben. Wir alle haben empirische Überzeugungen, die wir - als Überzeugungen - auch für richtig halten und an deren Richtigkeit wir keine Zweifel haben. Wenn man R3 für falsch erklärt, muß man die Behauptung vertreten, alle empirischen Sätze, die wir für wahr halten, gehalten haben und halten werden, seien tatsächlich falsch. Da diese Behauptung - bei der hier zugrunde gelegten engen Deutung des Wortes „empirisch“ - selbst kein empirischer Satz ist, führt sie, wenn sie als wahre Überzeugung ausgegeben wird, zwar nicht zu einem Selbstwiderspruch, aber jeder Versuch einer Widerlegung von R3 tut das. Um R3 zu widerlegen, müßte man ja für jeden empirischen Satz A, den jemand für wahr hält, zeigen, daß nicht-A gilt. $\mathrm{Da}$ nicht-A aber auch ein empirischer Satz ist, würde man sich damit in einen Widerspruch verwickeln.

Konkreter gesagt: Um zu zeigen, daß der Satz 1. Dieses Blatt Papier ist weiß, den wir für wahr halten, tatsächlich falsch ist, müßte man z. B. durch Messungen des von diesem Blatt reflektierten Lichtes zeigen, daß es nicht weiß, sondern etwa gelb ist. Dann würde man sich aber auf empirische Annahmen (auf Meßergebnisse und Naturgesetze) stützen, um eine andere empirische Annahme zu widerlegen. Es ist also nicht möglich, R3 zu widerlegen.

Tritt dieselbe Schwierigkeit aber nicht auch auf, wenn man versucht, R3 zu rechtfertigen? Erfordert eine Begründung der Annahme (1) nicht auch einen Rückgriff auf andere empirische Annahmen, z. B. wieder auf Messungen über das von diesem Blatt Papier reflektierte Licht? Dann würde der Versuch einer Widerlegung von R 3 auf einen Widerspruch, der Versuch einer Rechtfertigung von R 3 aber auf eine petitio principii hinauslaufen. Wir haben jedoch schon früher gesehen, daß nicht alle Annahmen einer Begründung fähig oder bedürftig sind. Im Falle des Satzes (1) genügt uns zunächst seine Evidenz. Damit ist uns aber auch die These R3 evident. Eine Begründung für (1) wird erst dann erforderlich, wenn Gründe angegeben werden, die gegen die Annahme (1) sprechen. Das sind dann aber, wie wir sahen, zugleich Gründe, welche die These R3 stützen. Der Opponent kann sich dagegen nicht auf Evidenz berufen. Denn erstens kann man eine so universelle und starke Behauptung wie die Negation von R 3 nicht als evident bezeichnen, und zweitens müßte eine solche universelle 
Evidenz z. B. die spezielle Evidenz einschließen, daß (1) falsch ist, d. h. die Evidenz, daß dieses Biatt Papier nicht weiß ist, und würde so R 3 wiederum stützen, da Evidenz immer Evidenz für Wahrheit, also ein Argument für Wissen ist. Man kann also auch bzgl. der empirischen Skepsis mit Wittgenstein sagen: „Wer an allem zweifeln wollte, der wird auch nicht bis zum Zweifel kommen. Das Spiel des Zweifelns setzt selbst schon die Gewißheit voraus." 43 Wie in 1.7 betont wurde, bezieht sich der Skeptizismus in der Regel nicht auf den Begriff des Wissens als wahrer Überzeugung, sondern auf einen Begriff perfekten Wissens. Perfektes empirisches Wissen gibt es aber nicht, wie wir dort gesehen haben. ${ }^{44}$ Daraus folgt jedoch nicht, daß es keine „echte“ empirische Erkenntnis gibt, sondern nur, daß die Forderung perfekten Wissens im empirischen Bereich nicht sinnvoll ist.

Die These R 3 setzt R 2 voraus, denn objektive Sachverhalte sind synthetisch, und synthetische Sachverhalte lassen sich nur aufgrund von Erfahrung erkennen. Wenn umgekehrt auch R3 keine unmittelbare Folge von R2 ist, so ist doch Erfahrung etwas, was Aufschluß über die Geltung des erfahrenen Sachverhalts gibt. Versteht man die Rede von „Erfahrungen“ im weiten Sinn, so daß auch der Satz „Es erscheint mir, als ob dies Papier weiß ist" über eine Erfahrung redet, so ist nicht jede Erfahrung eine Erkenntnis und nicht jede Erfahrung bewirkt, daß wir vom Bestehen des erfahrenen Sachverhalts überzeugt sind. Aber jede Erfahrung eines Sachverhalts $p$ bedingt doch zumindest eine prima-facie-Vermutung von der Geltung dieses Sachverhalts. Wenn es mir also erscheint, als ob ein ins Wasser gehaltener Stock an dem Punkt, an dem er ins Wasser eintaucht, einen Knick hätte, und ich das trotzdem nicht glaube, so nur deswegen, weil ich aufgrund anderer empirischer Überzeugungen diese Erfahrung als nicht zuverlässig ansehe. Es gibt also Erfahrungen, mit denen sich keine Überzeugungen von der Geltung des Erfahrenen verbinden, aber es kann nicht nur solche Erfahrungen geben. Es gibt sie nur, weil es andere Erfahrungen gibt, von deren Verläßlichkeit wir überzeugt sind. Daher kann man die These $R 2$ nicht vertreten, ohne auch $R 3$ zu akzeptieren.

${ }^{43}$ Wittgenstein (74), 115.

${ }^{44}$ Das ergibt sich auch schon aus der Bestimmung objektiver Sachverhalte. Vgl. dazu die folgenden Präzisierungsvorschläge für diesen Begriff. 
Als naiven Realismus bezeichnet man die These:

R 4: Die Dinge, die wir wabrnebmen, existieren an sich, und die Attribute, die wir an ibnen wabrnebmen, kommen ibnen selbst $z u$.

Man kann hier ,,wahrnehmen“ nicht durch ,erfahren“ (in dem weiten Sinn, den wir gerade charakterisiert haben) oder ,beobachten" ersetzen, denn bei Erfahrungen und Beobachtungen können wir uns irren. Ein solcher „naiver Realismus“ wäre sicher zu naiv.

Wenn ich wahrnehme, daß meine Tabaksdose rot ist, so ist es nach der Bestimmung des Begriffs ,wahrnehmen“ eine Tatsache, daß sie rot ist, und daraus folgt, daß es auch eine Tatsache ist, daß es eine rote Tabaksdose gibt. In welchem vernünftigen Sinn könnte man sagen, die Tabaksdose existierte ,, an sich" nicht oder sie sei ,,an sich“ nicht rot? Der Ausdruck ,,an sich“ kann sich nur auf die Objektivität der Tatsache beziehen, und im Sinn der obigen Argumente drückt der Satz ",Meine Tabaksdose ist rot" einen objektiven empirischen Sachverhalt aus. Da der Satz den Inhalt einer Wahrnehmung ausdrücken sollte, beschreibt er also eine objektive physische Tatsache. Akzeptiert man daher, daß die Aussagen der Dingsprache objektive Sachverhalte ausdrücken, so hat der naive Realismus unbestreitbar recht.

Der naive Realismus setzt $\mathrm{R} 3$ und damit auch $\mathrm{R} 2$ voraus, denn Wahrnehmungen objektiver Tatsachen sind Erkenntnisse und Erfahrungen solcher Tatsachen. Umgekehrt ergibt sich aber R 4 auch aus R3; denn eine Erkenntnis objektiver Tatsachen beruht auf Erfahrungserkenntnissen, also Wahrnehmungen, und nehme ich wahr, daß ein objektiver Sachverhalt besteht, so existieren, wie wir gesehen haben, auch die wahrgenommenen Objekte, und die an ihnen wahrgenommenen Attribute kommen ihnen selbst (objektiv) zu.

Einwände g̀egen den naiven Realismus werden nicht nur von idealistischen Positionen aus vorgebracht, über die wir im nächsten Kapitel reden wollen, sondern sie stützen sich insbesondere auch darauf, daß wir den Inhalt von Wahrnehmungen mit der normalen Sprache beschreiben, speziell mit der Dingsprache, mit Termen also, die in der Sprache der Physik nicht vorkommen. Wir erfahren Dinge als rot oder hart, die Physik verwendet diese Begriffe aber nicht, bzw. nur in einem vom normalen Sprachgebrauch abgehobenen Sinn; sie redet statt dessen von Quarks, schwarzen Löchern und Feldern - von Dingen also, die wir nicht wahrnehmen können - , und von Massen, Geschwindigkeiten und Wellenlängen, gebraucht also Begriffe, die kei- 
nen anschaulichen Eigenschaften entsprechen. Wenn wir nun annehmen, erst die Physik zeige uns, wie die physische Natur ,,in Wahrheit“ beschaffen ist, so entzieht sich die wahre Beschaffenheit der Natur unserer Wahrnehmung. Der Tisch, den wir sehen, besteht ,,in Wirklichkeit" aus unzähligen Molekülen, diese aus Atomen und Elementarteilchen, die Farben, die wir sehen, sind ,,in Wahrheit" elektromagnetische Schwingungen, die Töne, die wir hören, Schwingungen der Luft, usw.

Verschiedene Sprachen sind für verschiedene Zwecke verschieden gut geeignet. Die Sprache der Physik ist für physikalische Beschreibungen entwickelt worden, und sie ist dafür sicher sehr viel brauchbarer als die normale Sprache. Man kann aber nicht sagen, erst die physikalische Beschreibung der Phänomene zeige uns deren eigentliche Natur, erst die physikalische Theorie des Lichts ließe uns z. B. die eigentliche Natur der optischen Phänomene erkennen. Eine Beschreibung zeigt uns die „,eigentliche Natur" von Phänomenen, wenn sie diese so darstellt, wie sie wirklich sind. Sie sind wirklich so, wie sie sind; und wie sie sind, sagt uns jede Beschreibung mit wahren Sätzen. Der Vorteil der physikalischen Beschreibungen liegt allein darin, daß sie sehr präzise, allgemeine, fruchtbare und informative Systematisierungen erlauben. Insofern sind sie den Beschreibungen mit der normalen Sprache eindeutig überlegen. Daraus folgt aber nicht, daß sie wahrer wären als diese. Im übrigen haben wir im letzten Abschnitt betont, daß die physikalischen Theorien nur mathematische Modelle physischer Phänomene sind, die weder den Anspruch erheben können noch wollen, das „Wesen“ oder die „,wahre Natur" dieser Bereiche zu beschreiben. Endlich gibt es auch keine physikalische Erkenntnis ohne direkte, anschauliche Wahrnehmungen. Ohne Bezug auf die normale Dingsprache hätte die Sprache der Physik keinen empirischen Gehalt, und ohne schlichte Wahrnehmungen könnte der Physiker seine Instrumente nicht ablesen. Die normale Sprache - zumindest ein Teil davon - ist die unverzichtbare methodische Voraussetzung für den Aufbau der physikalischen Sprache, und schlichte Wahrnehmungen sind die unverzichtbare methodische Voraussetzung zur. Überprüfung physikalischer Aussagen.

Die Vorstellung, erst die Physik und allgemein die Naturwissenschaften zeigten uns die eigentliche Natur der Welt, ist heute weit verbreitet. Ein Beispiel mag für viele stehen: $H$. von Ditfurth - ein um die allgemeinverständliche Darstellung wissenschaftlicher Resultate 
im übrigen sehr verdienter Mann - erörtert in einem der in (74) gesammelten Aufsätze das Phänomen des Unanschaulichen, Abstrakten in der modernen Kunst und sieht darin einen Ausdruck der ins allgemeine Bewußtsein dringenden Erkenntnis, daß der naive Realismus falsch und daß die Welt an sich ganz anders sei, als uns das der Augenschein, die direkte sinnliche Wahrnehmung zeigt. Auf S. 88 schreibt er: „Wir sind so Zeugen der letzten, hoffnungslosen Schlacht des Augenscheins, der in seinen letzten Reservaten aufgespürt und ausgeräuchert wird. Wenn sie vorüber ist, wird nichts unmittelbar sinnlich Erlebtes mehr gelten, wird die Augenscheinlichkeit des leibhaftig Wahrgenommenen in allen ihren Formen endgültig als eine grandiose Illusion entlarvt sein." Was hier und in ähnlichen Ausführungen entlarvt wird, ist jedoch nichts anderes als ein Mangel an Unterscheidungsvermögen.

Wir haben bisher nur die üblichen Formulierungen und Argumente des Realismus referiert. Um sie genauer erörtern und das Gewicht idealistischer Einwände gegen sie abschätzen zu können, müssen wir den realistischen Thesen einen präziseren Sinn geben. Das kann nur dadurch geschehen, daß wir die Sachverhalte, die wir „objektiv“ genannt haben, genauer bestimmen. „Objektive“ Sachverhalte sollen von psychologischen Sachverhalten, von Überzeugungen, Beobachtungen, Empfindungen, Gefühlen, Präferenzen etc. abgegrenzt werden und von derartigen Phänomenen unabhängig sein. Nennen wir Sätze „objektiv“, die objektive Sachverhalte ausdrücken, so bieten sich drei Gedanken für eine Bestimmung dieses Begriffs an:

A) Objektivität als Unabbängigkeit des Wabrbeitswertes von Sätzen von den Wahrheitswerten von Sätzen über Psychisches,

B) Objektivität als Unabbängigkeit des Sinnes von Sätzen vom Sinn der Sätze über Psychisches,

C) Objektivität als Irreduzibilität von Sätzen auf Sätze über Psychisches.

$\mathrm{Da}$ alle Sachverhalte $\mathrm{p}$ des Glaubens, Wollens, Empfindens usw. einer Person a für sie selbst (im Zeitpunkt, auf den sie sich beziehen) problemlos sind, so daß analytisch gilt: ,,a glaubt, daß p, genau dann, wenn $\mathrm{P}^{\text {" }}, 45$ kann man sich beim Vorschlag (A) auf die Unabhängigkeit

${ }^{45}$ Vgl. dazu die Definition D 1.3-3. 
der Wahrheitswerte von den Wahrheitswerten der Sätze über Überzeugungen beschränken. Ist nun $\mathbb{B}$ die Menge aller Sätze, die nur von Überzeugungen (verschiedener Personen zu verschiedenen Zeiten) reden, so kann man einen (nicht analytischen) Satz A als „objektiv“ bezeichnen im Sinn des Vorschlags (A), wenn es keine konsistente Ménge von Sätzen aus $\mathbb{B}$ gibt, die mit $A$ oder nicht- $A$ unverträglich ist..6 Ist $\mathfrak{P}$ eine Menge von Sätzen, z. B. die Menge der Sätze der Dingsprache, so wird man $\mathfrak{P}_{\text {als }}$ unabhängig von $\mathbb{B}$ bezeichnen, wenn es keine konsistenten Satzmengen $\mathbb{A}$ von $\mathbb{B}$ und $\boldsymbol{B}$ von $\not{P}$ gibt, die mit einander unverträglich sind. ${ }^{47}$ Ist der Satz $\mathrm{A}$, bzw. die Satzmenge $\mathfrak{P}_{\text {in }}$ diesem Sinn objektiv, so wollen wir A, bzw. Pals objektiv im starken Sinn bezeichnen.

Im Sinn des Vorschlags (B) wird man einen Satz A - wir beschränken uns wieder auf nicht analytische Aussagen - „objektiv" nennen, wenn weder A noch nicht-A eine analytische Folge von Sätzen ist, die nur über Psychisches reden. Nach der obigen Bemerkung können wir uns dabei wieder auf Sätze über $\mathbb{G}$ beschränken. Dann gilt aber, daß Sätze, bzw. Mengen von Sätzen, im Sinne von (B) objektiv sind genau dann, wenn sie im Sinne von (A) objektiv sind; wenn sie also im starken Sinn objektiv sind.

$\mathrm{Zu}$ einem erheblich schwächeren Objektivitätsbegriff führt hingegen der Vorschlag (C). Im Sinn von D 3.3-1 kann man sagen, ein Satz A sei auf die Sprache über Psychisches reduzierbar, wenn er in diese Sprache übersetzbar ist. Dann gibt es aber einen Satz B über Psychisches, so daß die Äquivalenz $\mathrm{A} \equiv \mathrm{B}$ analytisch gilt und $\mathrm{A}$ ist mit nicht- $B$ unverträglich, also auch mit gewissen Sätzen aus $\mathbb{B}$. Ist also A übersetzbar in eine Aussage über Psychisches, so ist er auch nicht im

${ }^{46}$ Man kann ,,rein doxastische Sätze“ als Sätze, die nur von Überzeugungen reden, in Analogie zu den „,rein deontischen Sätzen“ definieren. Vgl. dazu Kutschera (77). Für eine prädikatenlogische Sprache sieht das so aus:

1. Sätze der Form $G_{t}(a, p)$ für beliebige Zeitpunkte $t$, Personen a und Sachverhalte $p$ sind rein doxastisch.

2. Ist der Satz A rein doxastisch, so auch $\neg \mathrm{A}$.

3. Sind die Sätze $A$ und $B$ rein doxastisch, so auch $A \supset B$.

4. Ist $A[a]$ ein rein doxastischer Satz, so auch $\wedge x A[x]$.

${ }^{47}$ Es gilt dann, daß jeder nicht analytische Satz von $\mathfrak{P}$ von $\mathbb{B}$ unabhängig ist. Die Umkehrung gilt, wenn man Kompaktheit annehmen kann, so daß jede inkonsistente Satzmenge eine endliche inkonsistente Teilmenge enthält. 
starken Sinn objektiv. Die Umkehrung gilt hingegen nicht: Aus dem Bestehen gewisser analytischer Beziehungen zwischen A und Sätzen von $\mathbb{B}$ folgt nicht, daß A mit einem dieser Sätze synonym ist. Wir wollen Sätze, die in diesem Sinn objektiv sind, als objektiv im schwachen Sinn bezeichnen. ${ }^{48} \mathrm{Je}$ nachdem, wie wir das Wort ,objektiv“ in den Thesen $R 1$ und $R 2$ deuten, ob im starken oder im schwachen Sinn, kommen wir nun zu ganz verschiedenen realistischen Thesen. Verstehen wir das Wort im starken Sinn, so definieren R 1 und R 2 einen starken oder, wie wir auch sagen wollen, einen absoluten Realismus. Deuten wir es hingegen im schwachen Sinn, so erhalten wir einen schwachen Realismus. Der schwache ontologische Realismus ist die Negation jenes ontologischen Idealismus, für den das Psychische die einzige Realität ist. ${ }^{49}$ Der starke ontologische Realismus behauptet dagegen weit mehr, als daß ein solcher Idealismus falsch ist, so daß nicht jeder, der diese Art von Idealismus ablehnt, damit auch schon einen absoluten Realismus vertreten müßte.

Den common-sense-Realismus wird man weder mit dem starken noch mit dem schwachen Realismus identifizieren können; dazu ist er zu wenig präzise. Meist wird er aber im Sinn des starken Realismus gedeutet. Bevor wir zu den realistischen Thesen in den verschiedenen Deutungen Stellung nehmen, wollen wir in den folgenden Kapiteln zunächst einmal die idealistischen Einwände kennenlernen. Auf den ersten Blick jedenfalls machen die common-sense-Argumente, die wir oben referiert haben, auch den starken Realismus recht plausibel, und wir werden zu prüfen haben, ob der Idealismus bessere Argumente vorzubringen hat.

${ }^{48}$ Andere Reduzierbarkeitsbegriffe als den nach D 3.3-1 wollen wir hier nicht betrachten. Ließen sich die Terme der Dingsprache z. B. im Sinn von D 3.3-3 durch psychologische so definieren, daß die Dingsätze genau dann wahr sind, wenn die ihnen aufgrund dieser Definitionen entsprechenden psychologischen Sätze wahr sind, so würde das noch nicht gegen eine Objektivität der Dingsätze sprechen.

${ }^{49}$ Vgl. dazu den Abschnitt 5.1. 


\section{Repräsentationstheorie und Idealismus}

\subsection{Repräsentationstheoretische Konzeptionen}

Die neuzeitliche Erkenntnistheorie setzt mit der Kritik am naiven Realismus ein und führt über die repräsentationstheoretischen Konzeptionen von Descartes und Locke zum Idealismus, der von Berkeley bis Fichte die Philosophie beherrschte. Wir wollen, hegelsch gesprochen, diesem Weg des Weltgeists durch die Geschichte folgen und uns den Zugang zu idealistischen Theorien über die Diskussion repräsentationstheoretischer Gedanken erschließen.

Den Ansatz zur Entwicklung solcher Ideen bildet die Unterscheidung primärer und sekundärer Qualitäten bei Descartes. Er geht bei dieser Unterscheidung davon aus, daß wir nur von klaren und distinkten Perzeptionen behaupten können, sie stellten die Dinge so dar, wie sie an sich sind. Was klare und distinkte Perzeptionen sind, wird nun zwar weder klar noch distinkt gesagt, man kann aber annehmen, daß es Beobachtungen sind, deren Inhalte sich mit präzisen $\mathrm{Be}-$ griffen beschreiben lassen. Nicht alle Beobachtungsinhalte sind nun begrifflich präzise bestimmbar. Das gilt nach Descartes nur für jene, die einer mathematischen Analyse zugänglich sind, und das sind allein geometrische Strukturen. Nur geometrische Begriffe sind für ihn also primär, d. h. nur sie lassen sich den Objekten selbst zuschreiben, und die Natur der physischen Dinge besteht für ihn in ihren geometrischen Eigenschaften. ${ }^{1}$ Andere Prädikate, mit denen wir den Inhalt von Beobachtungen beschreiben, wie Farbprädikate, Prädikate für Töne,

${ }^{1}$ Diese Identifikation von primären mit geometrischen Begriffen überrascht zunächst, da ja Descartes die physische Welt mit mechanischen Begriffen bestimmt, also auch mit Begriffen wie Zeit und Masse. Es ist aber zu beachten, daß für ihn alle metrischen Begriffe, alle Größenbegriffe insofern ,,geometrisch" sind, als sich die Metrisierung eines Begriffs nach der Bemerkung in 3.3 für Descartes durch Herstellung gesetzmäßiger Zusammenhänge zwischen ihm und geometrischen Begriffen vollzieht. 
Gerüche, Geschmackseigenschaften etc. drücken hingegen keine Attribute aus, die den Objekten selbst zukommen; diese Prädikate stehen also nicht für primäre, sondern für sekundäre Qualitäten.

In den „Meditationes de Prima Philosophia“ bezieht Descartes die realistischen Thesen in seinen radikalen $Z$ weifel ein, und sieht insbesondere auch den ontologischen Realismus als begründungsbedürftig an. Nach seiner Ansicht haben wir zwar eine starke Neigung, Beobachtungen so zu deuten, daß sie von Dingen einer Außenwelt hervorgerufen werden, aber diese Neigung allein rechtfertigt noch keine realistische Deutung unserer Erfahrung. Descartes Argument ist nun: Wäre diese Deutung falsch, so wäre Gott, der uns diese Neigung gegeben hat, ein Betrüger. $\mathrm{Da}$ Gott aber vollkommen ist, kann er kein Betrüger sein, also existiert eine Außenwelt. Es ist möglich, daß uns nicht alle Beobachtungen die Welt so zeigen, wie sie wirklich ist. Wir begreifen sie mit den Sinnen und sinnliche Erkenntnis ist oft unklar oder verworren. Wir sind aber jedenfalls sicher, daß all das existiert, was wir klar und deutlich erkennen, also das, was mathematisch erfaßbar ist: „Cum enim nullam plane facultatem mihi dederit ad hoc agnoscendum, sed contra, magnam propensionem ad credendum illas [ideas] a rebus corporeis emitti, non video qua ratione posset intelligi ipsum non esse fallacem, si aliunde quam a rebus corporeis emitterentur: Ac proinde res corporeae existunt. Non tamen forte omnes tales omnino existunt, quales illas sensu comprehendo; quoniam ista sensuum comprehensio in multis valde obscura est et confusa; sed saltem illa omnia in iis sunt, quae clare et distincte intelligo, id est omnia generaliter spectata quae in purae Matheseos objecto comprehenduntur." ${ }^{\text {"2 }}$

Auch die Beobachtung sekundärer Qualitäten wird von den beobachteten Dingen verursacht. Das ergibt sich für Descartes daraus, daß es nicht in unserer Macht liegt, die entsprechenden Empfindungen zu haben oder nicht zu haben. ${ }^{3}$ Wir können sie aus zwei Gründen aber nicht den Dingen selbst zusprechen: Erstens sind diese Begriffe nicht distinkt, nicht exakt definierbar, und zweitens ergäben sich Widersprüche, wenn man diese Qualitäten den Dingen selbst zuordnen würde. Descartes Beispiel ist die Härte: Wir empfinden ein Ding als

${ }^{2}$ Descartes (MP), 138 f. Vgl. dazu auch (PP), 31.

${ }^{3}$ Vgl. Descartes (PP), 31. 
hart, wenn es dem Druck unserer Hände Widerstand leistet. Würde es aber vor diesem Druck zurückweichen, würden wir es nicht als hart empfinden. Nun kann aber die Bewegung relativ zu unseren Händen nicht die objektive Natur der Dinge verändern, also können wir Härte nicht den Dingen selbst zuschreiben. ${ }^{4}$ Wir können nur sagen: den sekundären Qualitäten entspricht etwas in den Dingen, wir wissen aber nicht was. Es sind Eigenschaften der Objekte, kraft derer sie in den Nerven unserer Sinnesorgane gewisse Wirkungen hervorrufen, die wir als qualitative Bestimmungen der Dinge erleben. ${ }^{5}$ Dieser Konzeption entspricht das mechanistische Weltbild Descartes, nach dem sich alle Eigenschaften der Körper durch mechanische Eigenschaften erklären lassen, und die Annahme, bei Kenntnis der Physiologie unserer Wahrnehmungsorganisation - als einer ebenfalls mechanisch funktionierenden Maschine - ließen sich auch jene Eigenschaften der Dinge rein mechanisch beschreiben, die unsere Empfindungen bewirken und damit für das Auftreten sekundärer Qualitäten verantwortlich sind.

Die Objekte, die wir erfahren, sind also nach Descartes Dinge der Außenwelt. In der Erfahrung erscheinen sie uns aber mit Eigenschaften, die ihnen nur zum Teil selbst zukommen. Daher sind auch die erfahrenen Sachverhalte nicht sämtlich Sachverhalte der Außenwelt, und wir müssen zwischen erscheinenden oder, wie man auch sagt, phänomenalen Attributen und Sachverhalten und (außenweltlich) realen Attributen und Sachverhalten unterscheiden. Die phänomenalen Attribute und Sachverhalte repräsentieren dabei zwar reale; welche realen Attribute oder Sachverhalte ihnen entsprechen, ist uns aber nicht, oder doch nicht vollständig bekannt.

Das ist der Beginn einer Repräsentations-oder Abbildtbeorie der Erfahrung. Solche Theorien widersprechen dem naiven Realismus, stellen aber nicht immer erkenntnistheoretisch-idealistische Positionen dar. Der erkenntnistheoretische Idealismus ist die Gegenthese zum erkenntnistheoretischen Realismus, nach dem die Außenwelt selbst Gegenstand unserer Erfahrung ist. Er behauptet:

I1: Gegenstand unserer Erfabrung sind nur Phainomena.

${ }^{4}$ Vgl. Descartes (PP), $32 \mathrm{f}$.

${ }^{5}$ Vgl. Descartes (W) VII, 323. 
Statt „Phainomenon“ verwendet man auch Ausdrücke wie „Idee“ (z. B. Descartes, Locke, Berkeley), „Erscheinung“ (Kant) oder „,Sinnesdatum". Was darunter zu verstehen ist, werden wir im folgenden zu untersuchen haben. Zunächst ist nur klar, daß Phainomena mentale oder psychische Objekte sein sollen, die nur in unserem Kopf (in mente) existieren, nicht aber in der Außenwelt. Sie sind also von ganz anderer Art als die physischen Dinge der Außenwelt, so daß auch die Attribute, die für sie erklärt sind, nicht für die außenweltlichen Dinge, die Dinge ,an sich“ definiert sind. Pbänomenale Attribute sind dann Attribute, mit denen die Phainomena (die ,,phänomenalen Objekte") in unseren Beobachtungen erscheinen; also beobachtbare Attribute phänomenaler Objekte. Und ,phänomenale Sachverhalte“ sind Sachverhalte des Zutreffens oder Nichtzutreffens solcher Attribute auf Phainomena. Nach repräsentationstheoretischer Auffassung sind die Phainomena mentale Bilder von Dingen der Außenwelt.

Die repräsentationstheoretische Konzeption der Erfahrung können wir uns mithilfe des algebraischen Modellbegriffs verdeutlichen, von dem in 3.3 die Rede war. Ist die Algebra Aein Modell der Algebra A', so kann man sagen, $\mathbb{A}$ repräsentiere die Algebra $\boldsymbol{A}^{\prime}$, oder $\mathbb{A}$ sei ein Bild von $\mathcal{A}^{\prime}$. Die These der Abbildtheorien geht nun dahin, daß die phänomenalen Objekte und Attribute ein Modell der realen Objekte der Außenwelt und ihrer realen Attribute bilden. ${ }^{6}$ Sie setzt also immer einen ontologischen Realismus voraus, während das beim erkenntnistheoretischen Idealismus nicht allgemein der Fall ist. Die verschiedenen Abbildtheorien lassen sich durch ihre Annahmen über die Zuordnungen zwischen Modell (Phänomenen) und Original (Außenwelt) kennzeichnen. Eine Abbildtheorie stellt nur dann einen erkenntnistheoretischen Idealismus dar, wenn die Objekte, über denen das Modell erklärt ist, von den Objekten verschieden sind, über denen das Original definiert ist; wenn also kein Ding der Außenwelt Gegen-

- Dabei wird es sich natürlich nicht um ein homomorphes Modell im Sinne der Definition D 3.3-4 handeln. Die Phainomena werden vielmehr nur ein partielles Modell der Außenwelt bilden, denn nicht alle realen Objekte und Attribute sind beobachtbar. Ferner kann es sein, daß den phänomenalen Objekten nicht einzelne reale entsprechen sollen, sondern Aggregate von realen Objekten. Man hat also von einem sehr allgemeinen Modellbegriff auszugehen, den wir hier jedoch nicht charakterisieren wollen. Der zentrale Gedanke der Abbildtheorien und seine Problematik läßt sich auch so deutlich machen. 
stand der Erfahrung ist, so daß alle Gegenstände der Erfahrung bloße Phainomena sind. Descartes vertritt also keinen erkenntnistheoretischen Idealismus, denn die Objekte, die wir erfahren, sind für ihn reale Objekte, Dinge der Außenwelt. Die erfahrene Struktur der Welt bildet für ihn nur insofern ein Bild der Welt, als sich diese Struktur auch durch die sekundären Qualitäten definiert, die Struktur der Welt hingegen nur mit den primären.

Eine solche Repräsentationstheorie ist aber nicht haltbar: Auch die sekundären Qualitäten sind danach ja Attribute realer Objekte. Was sollen aber ,,reale Attribute" sein, wenn nicht Attribute, die für reale Objekte erklärt sind? Auch nach Descartes kommt eine sekundäre Qualität realen Dingen zu; denn sie kommt beobachteten Dingen zu, und diese sind nach seiner Theorie reale Objekte. $\mathrm{Da}$ wir rote Dinge in bestimmter Weise empfinden und daß wir sie bei anderer Wahrnehmungsorganisation anders empfinden würden, hat mit der Frage nichts zu tun, ob ,Rot' ein Dingattribut ist. Nur wenn wir mit dem Prädikat ,,rot“ Empfindungen oder Erfahrungsweisen charakterisierten, könnte man sagen, nicht Dinge der Außenwelt, sondern allein Psychisches sei rot. Wir sagen aber nicht ,Ich erfahre das Objekt b in roter Weise“, sondern „Ich erfahre das Objekt b als rot", „Mir erscheint es, als ob b rot ist", oder "Ich beobachte, daß b rot ist". Deswegen ist auch Descartes Argument, nach dem Härte eine sekundäre Qualität sein soll, nicht haltbar: $\mathrm{Daß}$ wir einen Gegenstand nicht als hart empfinden, impliziert nicht, daß er nicht hart ist. Wir unterscheiden sehr deutlich zwischen "Ich empfinde den Gegenstand b als hart" und ,b ist hart“. Es ist kein Widerspruch zu sagen „Der Gegenstand $\mathrm{b}$ ist hart, aber ich empfinde ihn nicht so" oder "Ich empfinde ihn als hart, aber er ist es nicht".

Entsprechendes gilt für Lockes bekanntes Argument, mit dem er den sekundären Charakter von Wärmebegriffen nachweisen wollte: Vor mir stehen drei Eimer mit Wasser. Der erste enthalte Wasser von $5^{\circ} \mathrm{C}$, der zweite Wasser von $25^{\circ} \mathrm{C}$, der dritte Wasser von $50^{\circ} \mathrm{C}$. Wenn ich nun einige Zeit die linke Hand in den ersten, die rechte in den dritten Eimer stecke und dann beide Hände in den zweiten, so empfinde ich das Wasser in diesem zweiten Eimer mit der linken Hand als warm, mit der rechten dagegen als kalt. Daraus schließt Locke: Da das Wasser im zweiten Eimer nicht zugleich warm und kalt sein kann, kommt dem Wasser ,,an sich“ keine der beiden Qualitäten zu; ,Warm‘ und ,Kalt‘ sind nicht primäre Qualitäten, nicht Eigen- 
schaften der Dinge selbst, sondern sekundäre Qualitäten.7 Wieder ist aber zwischen den Aussagen: „Ich empfinde b als warm" (oder ,Mir erscheint es so, als wäre b warm") und solchen der Gestalt ,b ist warm" zu unterscheiden. Im Lockeschen Beispiel werde ich nicht sagen „Das Wasser im zweiten Eimer ist warm und kalt zugleich“, sondern: „Ich empfinde das Wasser mit der linken Hand als warm, mit der rechten als kalt". Der letztere Satz enthält im Gegensatz zum ersten keinen Widerspruch. In beiden Redeweisen wird „warm" zum Ausdruck einer Eigenschaft verwendet, die wir den Dingen selbst zuschreiben. Wir charakterisieren unsere Empfindungsweisen oft durch gegenständliche Eigenschaften. Wärmeempfindungen können wir schlecht anders beschreiben als Empfindungen von der Art wie wir sie normalerweise haben, wenn es warm ist oder wenn wir warme Gegenstände berühren.

Als Rest der Thesen von Descartes bleibt dann aber nur die Reduzierbarkeitsbehauptung: Die Sprache, mit der wir unsere Beobachtungen physischer Dinge beschreiben - die Dingsprache - läßt sich auf die Sprache der Physik - für Descartes war das die Sprache der Mechanik - reduzieren, d.h. die sekundären Qualitäten lassen sich durch primäre definieren. Diese Behauptung hat aber mit einer Abbildtheorie nichts mehr zu tun.

Locke ist in seinen Aussagen zur Erkenntnistheorie recht unklar. Er schwankt zwischen einer Abbildtheorie im Sinne von Descartes und einer Abbildtheorie im Sinn des erkenntnistheoretischen Idealismus, nach der nicht reale Objekte die Gegenstände unserer Erfahrung bilden, sondern allein „Ideen“. In der Geschichte der Philosophie hat wohl selten ein einziges Wort soviel Verwirrung gestiftet wie das Wort „Idee“. Schon bei Descartes sind Ideen Objekte, Begriffe und Sachverhalte. Er unterscheidet aber immerhin noch zwischen Idee als einem Akt des Vorstellens, Denkens etc. (,idea“ als ,modus cogitandi") und Idee als etwas Vorgestelltem, Gedachtem etc. (,,idea" als ",res per istam operationem [cogitandi] repraesentata"). ${ }^{8}$ Bei Locke fehlt auch diese Unterscheidung. Das einzige Prinzip, das er beim Ge-

7 Vgl. Locke (E), S. 108.

${ }^{8}$ Vgl. Descartes (MP), 68. Andererseits sind für Descartes Ideen sowohl Vorstellungen, als auch die ihnen entsprechenden Gehirnzustände. Zur Bedeutungsvielfalt des Wortes ,Idee“ bei Descartes vgl. a. Kenny (68), $227 \mathrm{ff}$. 
brauch des Wortes „Idee“ konsequent verfolgt, ist das der Nichtunterscheidung des Verschiedenen, dessen Fruchtbarkeit Frege gerühmt hat. ${ }^{9}$ Als „Ideen“" werden insbesondere Sachverhalte des Beobachtens, Vorstellens, Wahrnehmens, Denkens bezeichnet, ebenso wie beobachtete, vorgestellte, wahrgenommene, gedachte Sachverhalte, Attribute oder Objekte. Daher begegnet eine Interpretation der Lockeschen Texte erheblichen Schwierigkeiten. Wenn man seine Abbildtheorie im Sinn eines erkenntnistheoretischen Idealismus deutet - und dafür spricht wohl die Mehrzahl seiner Aussagen - so sind die Gegenstände, die wir erfahren, phänomenale Objekte; mentale Bilder von Dingen der Außenwelt. Nach Locke entsprechen also allen phänomenalen Objekten reale Objekte oder Substanzen, aber nur den primären Ideen entsprechen objektive Attribute. ${ }^{10}$

Bei Locke begegnen wir nun einem Gedanken, der bei der Unterscheidung primärer und sekundärer Ideen, bzw. Qualitäten eine wichtige Rolle gespielt hat. Für ihn, wie für die gesamte traditionelle Logik, stellen mit Relationsbegriffen definierte Attribute, wie z. B. ,größer als 3 sein' oder, Vetter von Fritz sein' keine Eigenschaften dar. Man nahm an, alle Relationen ließen sich durch Eigenschaften der Relationsglieder definieren. Nach diesem Muster kann man z. B. die Relation , $a$ ist $b$ gleich bzgl. der Eigenschaft $F^{c}-$ symbolisch $a_{\bar{F}} b-$ definieren durch , $F$ trifft genau dann auf a $z u$, wenn $F$ auf $b$ zutrifft ${ }^{t}$ $(F(a) \equiv F(b))$. Nach traditioneller Auffassung ist dann $x_{\bar{F}} b$ (b gleich sein bzgl. der Eigenschaft F) keine Eigenschaft. Eigenschaften sind nur $F$ und nicht-F, und je nachdem, welche dieser Eigenschaften auf a und $b$ zutrifft, gilt $\mathrm{a} \overline{\mathrm{F}} \mathrm{b}$, oder es gilt nicht. Generell soll sich also das Bestehen oder Nichtbestehen von Relationen zwischen Objekten allein aus den Eigenschaften der Relationsglieder ergeben. Nun sind sekundäre Qualitäten für Locke Relationen der Dinge, die sie bzgl. un-

S Vgl. Frege (67), 241.

${ }^{10}$ Locke unterscheidet in (E) Buch II, Kap. VIII, S. $103 \mathrm{f}$. zwischen Ideen und Qualitäten. Ideen sind phänomenale Attribute, Qualitäten reale Dingeigenschaften. Als primäre, bzw. sekundäre Ideen bezeichnen wir hier die den primären, bzw. sekundären Qualitäten entsprechenden Ideen. Was bei dieser Unterscheidung freilich bei Locke sekundäre Qualitäten sein sollen, ist unklar. Seine Aussagen über sekundäre Qualitäten gehören zu jenen, die schlecht zur Annahme passen, er hätte einen erkenntnistheoretischen Idealismus vertreten. 
serer Wahrnehmungsorganisation haben. Was mir als Eigenschaft erscheint, z. B. das Rotsein eines Objekts b, ist in Wirklichkeit eine Relation $\mathrm{R}(\mathrm{a}, \mathrm{b})$ zwischen dem Objekt $\mathrm{b}$ und mir selbst, bzw. meiner Wahrnehmungsorganisation, a. Im Sinn der traditionellen Logik kann man nun nicht sagen, $b$ habe die Eigenschaft $R(a, x)$. Vielmehr kann man $b$ nur jene Eigenschaften zuschreiben, aus denen sich - zusammen mit den Eigenschaften von a - die Relation R(a,b) ergibt. Wir kennen aber die Definition der Relation $R$ nicht, und selbst wenn sie uns bekannt wäre, könnten wir, ohne die Eigenschaften von a zu kennen, nicht aus $R(a, b)$ auf bestimmte Eigenschaften von $b$ schließen. Im obigen Beispiel: Auch wenn ich weiß, daß $a_{\bar{F}} b$ gilt, kann ich daraus nicht auf $F(b)$ oder $\neg F(b)$ schließen, solange ich nicht weiß, ob $F(a)$ oder $\neg \mathrm{F}(\mathrm{a})$ gilt. Locke bringt ein anschauliches Beispiel für diesen Gedanken: Ein Schlüssel, b, hat eine bestimmte Form F. Ein Schloß, a, hat eine bestimmte Bauart G. Daß der Schlüssel b das Schloß a sperrt, $R(a, b)$, ist eine Relation zwischen a und $b$, die sich aus ihren Eigenschaften $F$ und $G$ ergibt. Es ist aber keine Eigenschaft des Schlüssels selbst, das Schloß zu sperren. Die Tatsache, daß er das Schloß sperrt, besagt noch nichts über seine Form, solange die Bauart des Schlosses nicht bekannt ist. Dem Schlüssel entspricht das Objekt, dem Schloß unsere Wahrnehmungsorganisation, dem Sperren die Produktion eines Erlebnisses in uns. ${ }^{11}$

Auch dieser Gedanke liefert jedoch kein brauchbares Argument für eine Abbildtheorie. Die Reduzierbarkeitsthese für Relationen ist falsch. Nicht alle Relationen lassen sich durch Eigenschaften definieren, und daher besteht auch kein Grund, mit Relationen definierte Eigenschaften wie $R(a, x)$ nicht als ,echte“ Eigenschaften anzusehen. Man kann also den sekundären Ideen ebenso wie den primären Dingeigenschaften zuordnen. Es ist eine Eigenschaft des Schlüssels, ein bestimmtes Schloß zu sperren, die ihn von anderen Schlüsseln unterscheidet. Und es ist eine Eigenschaft eines Objekts, daß es bei einer so und so gearteten Wahrnehmungsorganisation im Betrachter gewisse Empfindungen hervorruft.

${ }^{11}$ Locke folgt hier Ideen, die R. Boyle in "The origin of forms and qualities according to the corpuscular philosophy" und ,Excursion about the relative merits of physical qualities" (abgedruckt in den Werken, hrsg. von Th. Birch, 6 Bde. London 1772) entwickelt hatte. Vgl. dazu auch den Aufsatz von R. Jackson in Martin und Armstrong (74). 
Schon Berkeley hat Lockes Unterscheidung primärer und sekundärer İdeen angegriffen. Ihm ging es jedoch darum zu zeigen, daß alle Ideen sekundär sind, und so brachte er für die Subjektbezogenheit auch der von Locke als primär bezeichneten Ideen analoge Argumente vor, wie sie dieser für die von ihm als sekundär angesehenen Ideen formuliert hatte. ${ }^{12}$ So wies er darauf hin, daß die Dinge nicht dieselbe Größe haben, mit der sie uns erscheinen. Ein Gegenstand erscheint uns um so größer zu sein, je mehr wir uns ihm nähern, und die Sonne ist scheinbar so groß wie eine Tomate. Wir können also die beobachteten Größen nicht den Dingen selbst zuschreiben, ohne in Widersprüche zu geraten. Ferner betont Berkeley, daß man nicht manche Eigenschaften den Dingen selbst, manche aber nur ihren Abbildern zusprechen könne. Was ausgedehnt ist, ist z. B. auch farbig; man kann aber nicht sagen, ein Ding sei ausgedehnt, farbig sei hingegen nur seine Idee. Das letztere betrifft aber nur manche Aussagen von Locke, der, wie wir schon oben bemerkt haben, nicht immer klar zwischen Ideen und Dingen unterschieden hat. Es ist ein Argument für einen konsequenten erkenntnistheoretischen Idealismus, nach dem nur Phainomena Gegenstände der Erfahrung sind. Endlich betont Berkeley, Ideen seien immer Ideen eines Subjekts; sie bedürften eines Subjekts als Träger, und daher könne man sie nicht als Dingeigenschaften ansehen: "Let it be considered, the sensible qualities are colour, figure, motion, smell, taste, and such like, that is, the ideas perceived by sense. Now for an idea to exist in an unperceiving thing, is a manifest contradiction; for to have an idea is all one as to perceive: that therefore wherein colour, figure, and the like qualities exist, must perceive them; hence it is clear there can be no unthinking substance or substratum of those ideas". ${ }^{13}$ Für Berkeley sind also Ideen Arten von Perzeptionen; Perzeptionen haben aber nur Subjekte, nicht physikalische Objekte.

Hier wird nun schon der grundlegende Fehler des erkenntnistheoretischen Idealismus deutlich, auf den wir im nächsten Abschnitt noch ausführlich eingehen werden: Ideen sollen zugleich beobachtete Eigenschaften (die Eigenschaft, z. B., rot zu sein) und Attribute von Beobachtungen dieser Eigenschaften sein - also z. B. das Attribut,

\footnotetext{
${ }^{12}$ Auf Kants Argumente für die Idealität von Raum und Zeit gehen wir im Abschnitt 8.7 ein.
}

${ }^{13}$ Berkeley (T), 116. 
Wahrnehmung von etwas Rotem zu sein - und außerdem auch noch Attribute von Personen, die diese Beobachtungen machen - also z. B. das Attribut, etwas Rotes zu sehen. Beobachtete Dinge sind aber von Beobachtungen und von beobachtenden Personen zu unterscheiden, und ebenso die Attribute dieser verschiedenen Objekte. Die Tabaksdose, die ich beobachte, ist rot, aber weder meine Beobachtung ist rot noch ich als Beobachter. Es ist einfach absurd, die Eigenschaft Rot mit der Eigenschaft zu identifizieren, eine Rotwahrnehmung zu sein, oder etwas Rotes zu sehen. Auch die Eigenschaft, als rot beobachtet zu werden (d. h. beobachtet und dabei als rot empfunden zu werden), kann man nicht als Idee im Sinn Berkeleys ansehen, denn diese Eigenschaft kommt Dingen, nicht aber Subjekten zu.

Hume hat die Argumente von Berkeley übernommen, sie aber in einem Punkt noch radikalisiert: Nach seiner Ansicht gibt es auch keine direkte innere Wahrnehmung von uns selbst als beobachtendem, denkendem, fühlendem oder wollendem Subjekt. Auch in der inneren Erfahrung sind uns nur Ideen gegeben, Eindrücke, Empfindungen, nicht aber ein Subjekt, so wie es an sich ist. Wir haben keine Perzeptionen von unserer Seele, und hätten wir sie, so würden sie uns nur Bilder, Vorstellungen oder Ideen der Seele liefern, von denen wir nicht sagen könnten, wie weit ihnen etwas Reales entspricht. Während wir nach Berkeley jedenfalls von einer Substanz, nämlich uns selbst, direkte Kenntnis haben, beschränkt sich Erfahrung nach Hume generell nur auf Ideen, die wir zwar gewöhnlich als Beobachtungen eines Subjekts von realen Dingen deuten, ohne jedoch bei genauerem Zusehen diese Deutung rechtfertigen zu können. Sie beruht für Hume, wie für Descartes, auf einer angeborenen psychischen Neigung. Während aber Descartes den Begriff der Idee so verstand, daß Ideen immer Ideen eines Subjekts sind, und auf dem Weg über seinen Gottesbeweis die sachliche Angemessenheit unserer Neigung begründete, eine Außenwelt anzunehmen, ist für Hume die Existenz Gottes noch fragwürdiger als die der Seele und der Außenwelt, und daher vertritt er im Gegensatz zu Descartes einen radikalen Skeptizismus, nicht nur bzgl. der Außenwelt, sondern auch bzgl. der Seele als Träger der Ideen. Für ihn ist der Mensch „,... nothing but a bundle or collection of different perceptions, which succeed each other with an inconceivable rapidity, and are in a perceptual flux and movement" .14 ,"The mind is a

${ }^{14}$ Hume (T), Buch 1, S. 329. 
kind of theater, where several perceptions successively make their appearance; pass, glide away, and mingle in a infinite variety of postures and situations ... The comparison of the theater must not mislead us. They are the successive perceptions only, that constitute the mind; nor have we the most distant notion of the place where these scenes are represented, or of the materials of which it is composed ". 15

Diese Auffassung ist aber völlig unhaltbar: Ideen als Sachverhalte des Beobachtens oder als beobachtete Sachverhalte setzen ein Subjekt voraus, das beobachtet. Das impliziert natürlich nicht, daß es eine direkte Wahrnehmung unserer „Seele“, „,des Bewußtseins“ oder „des Ich" mit allen zugehörigen Eigenschaften gäbe. Innere Wahrnehmung ist nicht Wahrnehmung der Seele als eines Objekts, sondern Selbstbewußtsein, Bewußtsein unserer eigenen Empfindungen, Überzeugungen, Strebungen, unserer Akte des Denkens, Vorstellens, Erinnerns, usf. ${ }^{16}$ Man kann auch nicht sagen, Ideen seien nur Ideen einer Idee, denn das würde zu einem unendlichen Regress führen. Und alle erkenntnistheoretischen Aussagen Humes wie „Uns sind nur Ideen gegeben“, , Wir können nicht wissen, ob es eine Substanz als Träger der Ideen gibt", , Wir haben keine Perzeptionen der Seele“, , Wir können nicht begründen, daß es eine Außenwelt gibt", ,Wir haben eine Neigung, die Existenz eines Subjekts anzunehmen" setzen ja gerade das voraus, was er bezweifelt: ein Subjekt, das erfährt, dem Ideen gegeben sind, das zweifelt, das argumentiert. Hier ist sicher Descartes Aussage

${ }^{15}$ Hume (T), Buich 1, S. $239 \mathrm{f}$.

${ }^{16}$ Kant hat Humes Argumente übernommen: auch für ihn gibt uns Erfahrung keinen Aufschluß über Existenz und Beschaffenheit der Seele als einer Substanz. Vgl. (KrV), B 68. - Zur Kritik an der Humeschen These: ,Alles ist Vorstellung, auch das vorstellende Subjekt" vgl. auch Freges Ausführungen in (KS), S. $356 \mathrm{f}$. Dort schreibt er: ,Wenn alles Vorstellung ist, so gibt es keinen Träger der Vorstellungen. Und so erlebe ich wieder einen Umschlag ins Entgegengesetzte. Wenn es keinen Träger der Vorstellung gibt, so gibt es auch keine Vorstellungen; denn Vorstellungen bedürfen eines Trägers, ohne den sie nicht bestehen können... Was wäre dieses ganze Schauspiel ohne einen Zuschauer? Kann es nun Schmerz geben ohne jemanden, der ihn hat? Das Empfundenwerden gehört notwendig zum Schmerze, und zum Empfundenwerden gehört wieder jemand, der empfindet. Dann aber gibt es etwas, was nicht meine Vorstellung ist und doch Gegenstand meiner Betrachtung, meines Denkens sein kann, und ich bin von der Art". 
sehr viel richtiger, daß ich eins sicher nicht mehr sinnvoll bezweifeln kann: meine cogitationes, die Sachverhalte, daß ich denke, glaube, bezweifle, erfahre etc. und daß das eben meine Tätigkeiten sind, daß ich es bin, der sie vollzieht. ${ }^{17}$

Berkeley und Hume vertreten keine Abbildtheorie der Erfahrung; beide lehnen den ontologischen Realismus ab, der Voraussetzung dieser Theorie ist. Bevor wir auf ihre Argumente dafür eingehen, wollen wir zunächst noch auf weitere Formen der Abbildtheorie hinweisen und uns im nächsten Abschnitt ausführlich mit Argumenten für den erkenntnistheoretischen Idealismus befassen.

Erheblich loser als bei Locke wird die Zuordnungsrelation in der Repräsentationstheorie von $\mathrm{H}$. von Helmholtz angesetzt. Er schreibt: „Unsere Empfindungen sind Wirkungen, welche durch äußere Ursachen in unseren Organen hervorgebracht werden; und wie eine solche Wirkung sich äußert, hängt natürlich ganz wesentlich von der Art des Apparates ab, auf den gewirkt wird. Insofern die Qualität unserer Empfindung uns von der Eigentümlichkeit der äußeren Einwirkung, durch welche sie erregt ist, eine Nachricht gibt, kann sie als ein Zeichen derselben gelten, aber nicht als ein Abbild. Denn vom Bilde verlangt man irgendeine Art von Gleichheit mit dem abgebildeten Gegenstande, von einer Statue Gleichheit der Form, von einer Zeichnung Gleichheit der perspektivischen Projektion im Gesichtsfelde, von einem Gemälde auch noch Gleichheit der Farben. Ein Zeichen aber braucht gar keine Art der Ähnlichkeit mit dem zu haben, dessen Zeichen es ist. Die Beziehung zwischen beiden beschränkt sich darauf, daß das gleiche Objekt, unter gleichen Umständen zur Einwirkung kommend, das gleiche Zeichen hervorruft, und daß also ungleiche Zeichen immer ungleicher Einwirkung entsprechen. - Der populären Meinung gegenüber, welche auf Treu und Glauben die volle Wahrheit der Bilder annimmt, die uns unsere Sinne von den Dingen liefern, mag dieser Rest von Ähnlichkeit, den wir anerkennen, sehr geringfügig erscheinen. In Wahrheit ist er es nicht; denn mit ihm kann noch eine Sache von der allergrößten Tragweite geliefert werden, nämlich die Abbildung der Gesetzmäßigkeit in den Vorgängen der wirklichen Welt. Jedes Naturgesetz sagt aus, daß auf Vorbedingungen, die in gewisser Beziehung gleich sind, immer Folgen eintreten, die in

${ }^{17}$ Zur Problematik der Auffassung des Subjekts als Substanz vgl. 7.5. 
gewisser anderer Beziehung gleich sind. Da Gleiches in unserer Empfindungswelt durch gleiche Zeichen angezeigt wird, so wird der naturgesetzlichen Folge gleicher Wirkungen auf gleiche Ursachen, auch eine ebenso regelmäßige Folge im Gebiete unserer Empfindungen entsprechen. - Wenn Beeren einer gewissen Art beim Reifen zugleich rotes Pigment und Zucker ausbilden, so werden in unserer Empfindung Beeren dieser Form rote Farbe und süßer Geschmack sich immer zusammenfinden. - Wenn also unsere Sinnesempfindungen in ihrer Qualität auch nur Zeichen sind, deren besondere Art ganz von unserer Organisation abhängt, so sind sie doch nicht als leerer Schein zu verwerfen, sondern sie sind eben Zeichen von Etwas, sei es etwas Bestehendem oder Geschehendem; und, was das Wichtigste ist: das Gesetz dieses Geschehens können sie uns abbilden". 18

Die Art der Zuordnung zwischen realen Dingen und phänomenalen Objekten (Zeichen) bleibt hier völlig offen. Es wird nur angenommen, daß es eine solche Zuordnung gibt und eine Entsprechung zwischen phänomenalen (Zeichen-) Attributen und Attributen der Dinge, daß also die Zeichen ein Modell der Wirklichkeit bilden und daß daher die Gesetzmäßigkeiten der Außenwelt sich in gesetzmäßigen Zusammenhängen der Zeichen widerspiegeln. $\mathrm{Da} ß$ gewisse Attribute der Zeichen auch Attribute der bezeichneten Dinge sind, wird hingegen nicht mehr behauptet.

Ähnlich drückt sich H. Hertz, ein Schüler von Helmholtz aus: „Wir machen uns innere Scheinbilder oder Symbole der äußeren Gegenstände, und zwar machen wir sie von solcher Art, daß die denknotwendigen Folgen der Bilder stets wieder die Bilder seien von den naturnotwendigen Folgen der abgebildeten Gegenstände. Damit diese Forderung überhaupt erfüllbar sei, müssen gewisse Übereinstimmungen vorhanden sein zwischen der Natur und unserem Geiste. Die Erfahrung lehrt uns, daß die Forderung erfüllbar ist und daß also solche Übereinstimmungen in der Tat bestehen ... Die Bilder, von welchen wir reden, sind unsere Vorstellungen von den Dingen; sie haben mit den Dingen die eine wesentliche Übereinstimmung, welche in der Erfüllung der genannten Forderung liegt, aber es ist für ihren Zweck nicht nötig, daß sie irgend eine weitere Übereinstimmung mit den Dingen haben. In der Tat wissen wir auch nicht, und haben auch kein

${ }^{18}$ Helmholtz (21), S. $115 \mathrm{f}$. 
Mittel zu erfahren, ob unsere Vorstellungen von den Dingen mit jenen in irgend etwas anderem übereinstimmen, als allein in eben jener einen fundamentalen Beziehung ". ${ }^{19}$

In Kants Theorie der Erfahrung verbindet sich zwar ein ontologischer Realismus mit einem erkenntnistheoretischen Idealismus, aber er vertritt keine Abbildtheorie. Unsere Sinnesempfindungen werden durch Dinge der Außenwelt verursacht, aber über diese Ursache-Wirkungs-Beziehung läßt sich nichts aussagen und wir können so keine auch noch so schwache Korrespondenz zwischen der Welt unserer Erscheinungen und der Welt an sich behaupten. Insbesondere können wir auch aus den Gesetzmäßigkeiten im Bereich der Erscheinungen nicht auf analoge Gesetzmäßigkeiten im Bereich der Dinge an sich schließen, denn diese Gesetzmäßigkeiten (zumindest die fundamentalen Naturgesetze) legen wir selbst in die Erscheinungen hinein. Auch die Kategorien Ursache und Wirkung sind Verstandesbegriffe, die sich nur auf Gegenstände der Erfahrung anwenden lassen - die Rede von der „Verursachung“ unserer Empfindungen durch Dinge an sich ist nur metaphorisch gemeint, mit ihr läßt sich weder der normale noch ein anderer präziser Sinn verbinden.

\subsection{Argumente für den erkenntnistheoretischen Idealismus}

Wir haben uns im letzten Abschnitt schon überlegt, daß allenfalls Abbildtheorien haltbar sind, nach denen die Gegenstände der Erfahrung nie Dinge einer Außenwelt, sondern immer nur mentale Objekte: Phainomena, Erscheinungen, Sinnesdaten oder Ideen sind; Abbildtheorien also, die Formen des erkenntnistheoretischen Idealismus sind. Wir wollen nun in diesem Abschnitt Argumente für diesen Idealismus besprechen, wobei wir von den weiteren Thesen der Abbildtheorien über das Bestehen einer Entsprechung zwischen Phainomena und Dingen an sich absehen.

Die wichtigsten Argumente für den erkenntnistheoretischen Idealismus sind: ${ }^{20}$

${ }^{19}$ H. Hertz (94), Einleitung, S. $1 \mathrm{f}$. 


\section{Das Argument von der Subjektbezogenbeit des Beobachteten ${ }^{21}$}

Alle Objekte, Attribute und Sachverhalte, die wir beobachten, sind beobachtete Objekte, beobachtete Attribute und beobachtete Sachverhalte. Die Dinge, Attribute und Sachverhalte an sich, wie sie unabhängig von unserer Beobachtung sind, können wir nicht beobachten. $\mathrm{Da}$ ferner alles empirische Wissen deduktiv oder induktiv aus Beobachtungen abgeleitet ist, bezieht es sich insgesamt nur auf Beobachtungsphänomene.

Dieses Argument beruht - soweit es sich auf Sachverhalte bezieht - auf einer Verwechslung von beobachteten Sachverhalten, d. h. Sachverhalten $\mathrm{p}$, die von einer Person a beobachtet werden, für die also gilt $\mathrm{B}(\mathrm{a}, \mathrm{p})$, und Sachverhalten des Beobachtens, d. h. Sachverhalten $\mathrm{q}$, die sich darstellen lassen durch Sätze der Form ,,B(a,p)“. Daraus, daß $\mathrm{p}$ ein beobachteter Sachverhalt ist, folgt nicht, daß p auch ein Sachverhalt des Beobachtens ist oder umgekehrt: Weder folgt aus $B(a, p)$, $\mathrm{da} ß$ es einen Sachverhalt q gibt, so daß p mit $B(a, q)$ identisch ist, noch folgt $B(a, p)$ aus $p=B(a, q)$. Beobachtet jemand, daß der Mond rund ist, so folgt daraus weder, daß er diese Beobachtung beobachtet, noch daß er eine andere Tatsache des Beobachtens beobachtet. Nach den Ausführungen in 3.1 lassen sich vielmehr Sachverhalte des Beobachtens nicht beobachten; wir beobachten nicht, daß wir etwas beobachten, sondern wir glauben oder wissen nur, daß wir etwas beobachten. Auch aus diesem Grund sind Sachverhalte des Beobachtens nie beobachtete Sachverhalte.

Wenn ein „Beobachtungsphänomen“ nun ein beobachteter Sachverbalt sein soll, so gilt zwar trivialerweise, daß wir nur Sachverhalte beobachten können, die durch die Beobachtung zu Beobachtungsphänomenen werden, aber diese Tatsache besagt nichts gegen die realisti-

${ }^{20}$ Auf Argumente, die zunächst Gründe gegen den absoluten erkenntnistheoretischen Realismus vorbringen, um dann aus dessen Unhaltbarkeit auf die These I 1 des erkenntnistheoretischen Idealismus zu schließen, gehen wir hier nicht ein. Wir haben ja schon in 3.4 betont, daß ein solcher Schluß ungültig ist. Auch das in 5.2 erörterte Illusionsargument läßt sich als Argument für die These I 1 ansehen. Da es jedoch das zentrale Argument für den Phänomenalismus ist, behandeln wir es erst im Zusammenhang mit dieser speziellen Version des Idealismus.

21 Vgl. dazu z. B. Berkeley (T), S. $113 \mathrm{ff}$. 
sche These R2. Denn das Argument enthält dann keinen Hinweis darauf, wieso die Beobachtungsphänomene - hier also beobachtete Sachverhalte - nicht objektive Tatsachen im Sinn von 3.4 sein sollen. Man kann auch nicht behaupten, wir könnten nur Beobachtungsphänomene beobachten - ebensowenig wie man sagen kann, man könne nur verheiratete Frauen heiraten, da sie ja durch die Heirat zu verheirateten Frauen würden. Wenn ein „Beobachtungsphänomen“ hingegen ein Sachverbalt des Beobachtens sein soll, so sind zwar diese „Phänomene" keine objektiven Sachverhalte, aber das, was wir beobachten, sind dann keine Beobachtungsphänomene. Die Behauptung, wir könnten nur Beobachtungsphänomene beobachten, ist dann also wiederum falsch.

Statt des Prädikats „,beobachten“ werden in Argumenten dieses Typs auch oft Prädikate wie ,,vorstellen“, ,denken“, ,erscheinen“, „,bewußt sein“ etc. verwendet. In allen diesen Fällen beruht das Argument darauf, daß man nicht zwischen vorgestellten, gedachten, erscheinenden oder bewußten Sachverhalten und Sachverhalten des Vorstellens, Denkens, Erscheinens oder Bewußtseins unterscheidet.

\section{Das Argument vom unmittelbar Gegebenen}

Wir beobachten, daß hier ein Stück gelbes Papier liegt. Der Satz

a) Hier liegt ein Stück gelbes Papier

beschränkt sich nun nicht darauf, den Inhalt der schlichten und direkten Beobachtung wiederzugeben. Daß dieses gelbe Etwas ein Stück Papier ist, und nicht etwa eine Plastik- oder Metallfolie, ist eine Aussage, die nur durch weitere Beobachtungen zu verifizieren wäre, nicht aber schon durch bloßes Hinsehen gerechtfertigt ist. Es gehört also nicht zum Inhalt der einfachen Beobachtung, von der wir ausgehen, daß dieses Etwas aus Papier ist. Diese Beobachtung erlaubt es vielmehr nur zu sagen „Hier liegt etwas Gelbes, das aussieht, als ob es aus Papier wäre“. Aber auch diese Aussage ist noch zu stark, denn wir verwenden das Wort ,gelb" so, daß wir nur solche Dinge als gelb bezeichnen, die unter physikalisch weißem Licht (bei normaler Beleuchtung) in jedem Betrachter (mit normaler visueller Organisation) den Farbeindruck Gelb hervorrufen, bzw. Licht aus einem Wellenlängenbereich von ca. 0,6 $\mu \mathrm{m}$ reflektieren. Ob aber das Licht, das jetzt auf dieses Etwas fällt, physikalisch weiß ist, und welche Wellenlänge das reflektierte Licht hat, können nur komplizierte physikalische Tests klären; das gehört also nicht zum Inhalt der schlichten Beobachtung, und ebensowenig, daß dieses Etwas in allen normalen Betrachtern den 
Farbeindruck ,gelb“ hervorruft. Wir können also aufgrund der schlichten Beobachtung nur sagen „Hier liegt etwas, das so aussieht wie ein Stück gelbes Papier". Aber auch das ist noch zuviel! Denn die Aussage „Hier liegt etwas...", d. h. „Hier liegt ein Ding ..." beinhaltet $j a, d a ß$ tatsächlich ein Ding vorhanden ist, daß auch andere es sehèn können, daß ich beim Hingreifen gewisse Tasteindrücke haben werde, usw. Unsere Beobachtung allein rechtfertigt also nur den Satz „Es erscheint mir, als ob hier ein Stück gelbes Papier liegt". Nur dieser Satz gibt den tatsächlichen Inhalt der Beobachtung wieder. Dieses Beispiel zeigt, daß Aussagen über das in der Erfahrung unmittelbar Gegebene nur Sätze der Form $S(a, p)$ sind, also Aussagen über Phainomena als Sachverhalte des Erscheinens-als-ob. Da sich nun unser gesamtes empirisches Wissen aus dem in der Erfahrung unmittelbar Gegebenen herleitet, und da aus Sätzen S(a,p) die Sätze p weder deduktiv noch induktiv folgen, beschränkt sich unser empirisches Wissen bei genauerem Zusehen auf Sachverhalte des Erscheinens-als-ob, auf Phainomena. ${ }^{22}$

Dieses Argument enthält zwei Fehler: Erstens ist der Gegenstand einer Beobachtung $B(a, p)$ der Sachverhalt $p$, aber weder der Sachverhalt $B(a, p)$ selbst, noch der Sachverhalt $S(a, p)$ (der daraus nach dem Prinzip S2 aus $3.1 \mathrm{folgt}$ ), und erst recht nicht das Subjekt a der Beobachtung. Und das gilt unabhängig davon, ob die Person a, der es er-

22 Ähnliche Argumente für den erkenntnistheoretischen Idealismus findet man bei Fichte in der „Bestimmung des Menschen“, (W) II. Daraus einige Zitate: „In aller Wahrnehmung nimmst du zunächst nur dich selbst, und deinen eigenen Zustand wahr; und was nicht in dieser Wahrnehmung liegt, wird überhaupt nicht wahrgenommen“ (201). „Kannst du sagen: ich bin mir äußerer Gegenstände bewußt? . . Keineswegs, wenn ich es genau nehme: denn das Sehen und Fühlen usw., womit ich die Dinge umfasse, ist ... dasjenige, dessen ich mir am ersten und unmittelbarsten bewußt bin. Der Strenge nach könnte ich nur sagen: ich bin mir meines Sehens oder Fühlens der Dinge bewußt“ (202). „Das Bewußtsein des Gegenstandes ist nur ein nicht dafür erkanntes Bewußtsein meiner Erzeugung einer Vorstellung vom Gegenstande“ (221). „Du siehst, sonach ein, daß alles Wissen lediglich ein Wissen von dir selbst ist, daß dein Bewußtsein nie über dich selbst hinausgeht, und daß dasjenige, was du für ein Bewußtsein des Gegenstandes hältst, nichts ist als ein Bewußtsein deines Setzens eines Gegenstandes, welches du nach einem inneren Gesetze deines Denkens mit der Empfindung zugleich notwendig vollziehst?" (221 f). 
scheint, als ob $\mathrm{p}$, vom Bestehen von $\mathrm{p}$ überzeugt ist und ob p tatsächlich besteht. Empirische Erkenntnis, die durch äußere Erfahrung vermittelt wird, ist nie ein Wissen von Tatsachen des Erscheinens-als-ob, sondern nur von erscheinenden Tatsachen. Das Argument könnte also höchstens zeigen, daß es keine empirische Erkenntnis in diesem Sinn gibt, daß wir ein Wissen immer nur für subjektive (problemlose) Sachverhalte beanspruchen können. Es wäre dann aber kein Argument gegen den erkenntnistheoretischen Realismus. Auch in diesem Sinn ist es aber unhaltbar, und damit kommen wir zum zweiten Fehler.

Wir haben schon in den Abschnitten 1.7 und 3.4 betont, daß die Annahme einer prinzipiellen Fehlbarkeit unserer Beobachtungen noch keinen vernünftigen Zweifel an der Korrektheit bestimmter Beobachtungen begründet. Erscheint es der Person a, als ob der Sachverhalt $\mathrm{p}$ bestünde, so ist sie in der Regel auch davon überzeugt; es gilt also nicht nur $\mathrm{S}(\mathrm{a}, \mathrm{p})$, sondern auch $\mathrm{B}(\mathrm{a}, \mathrm{p})$. So urteilen wir im angegebenen Beispiel mit großer Sicherheit, weil wir die Dinge bei verschiedenen Beleuchtungen farbkonstant sehen, weil wir zumeist gute Gründe haben, vom normalen Funktionieren unserer Sinne überzeugt zu sein, und weil sich Papier in der Regel schon rein optisch von Plastik oder Metall unterscheidet. Gilt aber B(a,p), so ist a davon überzeugt, daß p besteht, und hat a damit recht, so weiß a, daß p gilt. Nach dem Prinzip W9* aus 1.2 gilt allgemein $B(a, p) \supset G(a, W(a, p))$ - beobachtet $a$, daß p, so glaubt a auch zu wissen, daß p gilt. Es gibt also eine Fülle von Sätzen über die Außenwelt, die wir zu wissen glauben, und das Argument enthält keinen Gedanken, der eine empirische Skepsis begründen könnte; aus dem sich also ergäbe, daß alle diese Überzeugungen falsch sind. Gibt es aber ein Wissen von der Geltung beobachteter Sachverhalte, so lassen sich damit auch Aussagen über nicht-beobachtete oder nicht beobachtbare Sachverhalte begründen.

Der Versuch, das, was in einer Beobachtung ,,unmittelbar gegeben“ ist, von hypothetischen Elementen seiner Beschreibung abzugrenzen, ist im Argument mißlungen, weil das ,unmittelbar Gegebene“ in einem Sachverhalt des Erscheinens-als-ob gesucht wird. Wir haben schon oben betont, daß solche Sachverhalte nichts in der Beobachtung Gegebenes sind, keine Gegenstände von Beobachtungen. Das, was in einer Beobachtung ,gegeben“ ist, ist im üblichen Sinn nicht die Tatsache, des Beobachtens oder des Erscheinens-als-ob, sondern der beobachtete Sachverhalt. Es ist aber auch nie gelungen, in sinnvoller 
und exakter Weise einen Teil des beobachteten Sachverhalts als ,,unmittelbar gegeben" abzugrenzen. Auf die Schwierigkeiten, denen ein solcher Versuch begegnet, hat insbesondere N. Goodman hingewiesen. ${ }^{23}$ Wollte man $\mathrm{z}$. B. sagen: ,,a ist mir unmittelbar als ein $\mathrm{F}$ gegeben genau dann, wenn ich im Augenblick der Wahrnehmung von a weiß, $\mathrm{da} ß$ a die Eigenschaft $F$ hat", so wäre mir dieses Blatt Papier jetzt unmittelbar als 150 Millionen $\mathrm{km}$ von der Sonne entfernt gegeben, da ich jetzt weiß, daß die Erde diese Entfernung von der Sonne hat. Und wenn man im Hinblick darauf, daß eine Aussage über das unmittelbar Gegebene möglichst wenig Hypothetisches enthalten soll, sagen würde: „Ein Gegenstand a ist mir unmittelbarer als ein $F$ denn als ein $G$ gegeben genau dann, wenn alle G's F's sind, aber nicht umgekehrt, so wäre mir dies Blatt Papier am unmittelbarsten als Kuh-oder-NichtKuh gegeben. Die Suche nach allgemeinen Kriterien für das, was in einer Beobachtung unmittelbar gegeben ist, sind auch aus folgenden Gründen verfehlt: Erstens enthalten auch die einfachsten sprachlichen oder begrifflichen Bestimmungen beobachteter Sachverhalte hypothetische Elemente, wie wir im 9. Kapitel sehen werden. Und zweitens soll das „,unmittelbar Gegebene“ das sein, was dem Beobachter aufgrund seiner Beobachtung evident ist. Verschiedenen Leuten ist aber oft unter gleichen Beobachtungsbedingungen Verschiedenes evident; nicht weil sie Verschiedenes sehen, sondern weil sie Verschiedenes glauben. Was wir glauben und was wir als problematisch ansehen, hängt auch von unseren vergangenen Erfahrungen ab, von Vorurteilen oder vom Untersuchungskontext - bei wissenschaftlichen Untersuchungen sind wir z. B. meist vorsichtiger in unseren beobachtungsmäßigen Feststellungen als im Alltag.

Weitere Argumente für den erkenntnistheoretischen Idealismus stützen sich auf die Annahme, unsere Erfahrungen seien Produkte der Wechselwirkung zwischen realen Zuständen, Ereignissen oder Gegenständen und subjektiven Faktoren wie unserer Wahrnehmungsorganisation, unserer Verstandesorganisation oder unserer Sprache.

${ }^{23} \mathrm{Vgl}$. Goodman (51), S. $138 \mathrm{ff}$. - Auch J. L. Austin betont in (62), S. 15 ff., daß die Redeweise vom ,direkt Beobachteten“ nicht dem normalen Sprachgebrauch entspricht und keinen präzisen Sinn hat. 
3. Das Argument vom Einfluß unserer Wabrnehmungsorganisation auf die Erfabrung ${ }^{24}$

Unsere Erfahrung wird von der Natur der menschlichen Wahrnehmungsorganisation geprägt. Unsere Sinne sprechen nur auf einen Teil der Umweltreize an, z. B. nur auf gewisse Schall- und Lichtfrequenzen. Es gibt eine Fülle von Phänomenen aus der Wahrnehmungspsychologie, die zeigen, daß die physikalischen Reize durch die sinnliche Wahrnehmung erheblich umorganisiert werden, daß weglassen, hinzugefügt und verändert wird. Die Sinnesqualitäten, wie $z$. B. die Farben, entsprechen nicht direkt physikalischen Merkmalen: Das Kontinuum der Lichtfrequenzen von Rot bis Violett wird qualitativ in Farbklassen aufgespaltet, und die lineare Ordnung der Frequenzen wird in den geschlossenen Farbkreis umgeordnet, in dem Rot dem Violett benachbart ist. Zusätzlich wird das sinnliche Erleben unter dem Einfluß von Stimmungen und Strebungen modifiziert und ausgestaltet. Darauf beruhen z. B. die projektiven Tests der Psychologie. Unsere Erfahrungen sind also das Ergebnis einer Transformation physikalischer Einwirkungen der Umwelt auf unsere Sinne durch physiologische und psychologische Mechanismen. Sie stellen daher die Wirklichkeit nicht so dar, wie sie an sich ist, sondern sie liefern uns nur Bilder der Außenwelt, die sich von den Originalen erheblich unterscheiden.

$\mathrm{Zu}$ diesem Argument ist folgendes zu bemerken:

a) Es ist richtig, daß die Art und Weise, wie wir Dinge und Ereignisse der Außenwelt erleben, von unserer Wahrnehmungsorganisation und von psychischen Faktoren abhängig ist. Daraus folgt aber nicht, daß die beobachteten Dinge und Ereignisse subjektabhängig sind, daß sie etwas Psychisches sind, mentale Bilder von etwas draußen in der Welt. Im Argument werden wieder beobachtete Sachverhalte nicht von Sachverhalten des Beobachtens unterschieden. Es werden aber auch zwei Verwendungen des Wortes „Bild“ verwechselt, die normale und die übertragene. Bilder im normalen Sinn sind Abbildungen; ein Bild des Herrn Meier ist z. B. eine Fotografie von ihm. Im übertragenen Sinn des Wortes sagen wir, daß wir uns ,ein Bild“ von Herrn Meier machen, z. B. aufgrund unserer Begegnungen und Erlebnissen mit ihm. Und das heißt dann nicht, daß wir eine Fotografie von ihm anfertigen, sondern daß wir zu gewissen Annahmen über ihn gelangen.

${ }^{24}$ Vgl. dazu z. B. N. Goodman (75). 
Diese Annahmen können subjektiv gefärbt sein, etwa durch die besonderen Umstände, unter denen wir ihm begegnet sind, durch Gefühle der $\mathrm{Zu}$ - oder Abneigung. Erfahrungen, aufgrund derer wir uns ein Bild von Herrn Meier machen, sind aber nicht Erfahrungen von einem Bild des Herrn Meier. Wenn man also sagt, unser „Bild“ der Welt sei durch die Besonderheiten unserer Erfahrung bestimmt, so ist das richtig im übertragenen Sinn des Wortes. Unsere Erfahrungen der Außenwelt sind deswegen aber nicht Erfahrungen von (mentalen) Bildern dieser Welt. Wenn jemand Spinnen in besonderer Weise erlebt, so folgt daraus nicht, daß er besondere Spinnen erlebt, subjektive oder gar mentale Spinnen.

b) Wie wir schon bei der Diskussion des naiven Realismus im Abschnitt 3.4 sahen, kann man nicht behaupten, die Sätze der normalen Sprache, mit denen wir die Inhalte von Beobachtungen ausdrücken also die einfachen Sätze der Dingsprache - , beschrieben im Gegensatz zu denen der Physik die Welt nicht so, wie sie an sich ist. Die Sätze der Physik sind in mathematischer Sprache formulierte Aussagen, deren Bezug zur Realität nur durch Sätze der Dingsprache hergestellt wird. Würden die Aussagen der der Dingsprache nicht von der physischen Realität reden, sondern allein von mentalen Bildern, so könnte man mit ihnen die Sprache der Physik nicht so interpretieren, daß sich mit ihr etwas über die Außenwelt aussagen ließe. Mit Aussagen über mentale Bilder von Meßordnungen, Instrumente und deren Anzeigen ließen sich keine Aussagen über die gemessenen realen physikalischen Größen begründen.

c) Die Behauptung, die Art und Weise, wie wir die Außenwelt erfahren, hänge von der physiologischen und psychologischen Faktoren ab, stützt sich auf Annahmen darüber, wie die Welt an sich beschaffen ist. Da Beobachtungen die Grundlage unserer Annahmen über die Welt sind, kann man nicht zugleich behaupten, sie zeigten uns allesamt die Welt nicht so, wie sie an sich ist. Man kann auch nicht sagen, Beschreibungen der Welt mit der Sprache der Physik seien korrekter als solche mit der Dingsprache. Sie sind, wie schon in 3.4 betont wurde, sicher präziser und für wissenschaftliche Zwecke geeigneter, deswegen aber nicht wahrer als wahre Sätze der Dingsprache. Zu beachten ist auch, daß oft Terme der normalen Sprache wissenschaftlich in einem anderen Sinn gebraucht werden. So sind z. B. phänomenologische Aussagen über Farben von physikalischen Aussagen über Farben zu unterscheiden. Die phänomenologische Aussage, daß Rot Vio- 
lett benachbart ist, besagt nicht, daß die Wellenlänge roten Lichts kontinuierlich in jene violetten Lichts übergehen; sie widerspricht also nicht der physikalischen Aussage, Rot und Violett bildeten die beiden Enden des sichtbaren Spektrums.

\section{Das Argument vom Einfluß von Vernunftorganisation und Sprache auf die Erfabrung}

Nach Kant verursachen die Dinge an sich in uns gewisse Empfindungen. Empfindungen stellen aber nicht schon Erfahrungen dar, sondern Erfahrung entsteht daraus erst durch die Organisation der Empfindungen nach den Anschauungsformen Raum und Zeit und durch begriffliche Verarbeitung. Die Kategorien begrifflicher Auffassung sind nach Kant in unserer Vernunftorganisation begründet, so daß man sagen kann, daß wir den Erscheinungen eine begriffliche Struktur aufprägen; daß wir mit den Aussagen, die wir über Erfahrungen machen, das Erfahrene in einer Weise strukturieren - z. B. mit der Subjekt-Prädikat-Struktur der Sätze in Substanz und Akzidens -, die den Sachverhalten an sich vielleicht nicht angemessen ist. Wir können nicht behaupten, daß die Gegenstände der Welt an sich unter jene Kategorien fallen, unter die wir die Gegenstände der Erfahrung aufgrund unserer Vernunftorganisation subsumieren.

Wir wollen dieses Argument im Sinne der modernen Wendung der Vernunftkritik in eine Sprachkritik als Argument von der Sprachbezogenheit unserer Erfahrungen formulieren. Thesen vom Anteil der Sprache an der Erfahrung, wie sie vor allem W. v. Humboldt, E. Sapir und B. Whorf formuliert haben ${ }^{25}$, verbinden sich nun keineswegs immer mit idealistischen Positionen. Um damit einen erkenntnistheoretischen Idealismus zu begründen, müßte man etwa so argumentieren: Erkenntnis vollzieht sich immer im Medium der Sprache. Jede Erkenntnis wird ausgedrückt durch einen wahren Satz. Der Gegenstandsbereich der Erkenntnis beschränkt sich also auf Propositionen als Bedeutungen von Sätzen. In 3.2 wurde die die Welt als Menge der Tatsachen bestimmt. Tatsachen sind aber nichts anderes als Bedeutungen wahrer Sätze. Daher ist die Welt immer nur relativ zu einer Sprache $S$ bestimmt als Menge der durch die wahren Sätze von $S$ ausgedrückten Sachverhalte. Je nach den Ausdrucksmöglichkeiten von $\mathrm{S}$ wird die bzgl. S definierte Welt anders aussehen. Daher ergibt sich

${ }^{25}$ Vgl. dazu Kutschera (75), Kap. 4 und das Argument von der Pluralität der Welten in 9.7. 
eine Relativität des Begriffes, Welt' bzgl. der Sprache, und da Sprachen von Menschen geschaffene konzeptuelle Instrumente sind, eine Abhängigkeit der Welt unserer Erfahrungen vom Erkenntnissubjekt. „Die Grenzen meiner Sprache bedeuten die Grenzen meiner Welt" könnte man mit Wittgenstein sagen. ${ }^{26}$ Und man könnte, wie in 3.2, auch darauf hinweisen, daß jede Sprache eine Ontologie voraussetzt, die in dieser Sprache sinnvoll nicht mehr diskutiert werden kann. Wenn aber unsere Erfahrung auch von der Sprache geprägt ist, wenn Sprache nicht nur ein Beschreibungsmittel für sprachunabhängig vorgegebene Tatsachen ist, sondern die erfahrenen Sachverhalte mit bestimmt, dann sind die Gegenstände der Erfahrung nicht Tatsachen an sich, sondern begriffliche Konstrukte oder Ideen.

Zur Kritik eines solchen Arguments ist zunächst anzumerken: Die Definition der Tatsachen als Bedeutungen wahrer Sätze ist nicht ganz adäquat. Wir können auch ohne, oder ohne explizite sprachliche Formulierungen Feststellungen machen, und was wir dabei feststellen, wird man auch zu den Tatsachen rechnen. Könnten wir nicht auch ohne Sprache Tatsachen erkennen und unterscheiden, so wären wir nicht in der Lage, eine Sprache zu erlernen; denn um einen Satz erlernen zu können, muß man nicht nur ihn selbst von anderen unterscheiden können, sondern auch den Sachverhalt, auf den er sich bezieht, von anderen Sachverhalten. Zudem wissen wir auch komplexe Gestalten, spezifische Farben, Gerüche oder Gefühle zu unterscheiden, die sich sprachlich nur unvollkommen beschreiben lassen. Die Behauptung, Sachverhalte seien Bedeutungen von Aussagesätzen, ist also nur bei Voraussetzung von Sprachen mit unbeschränktem Ausdrucksreichtum korrekt. Für eine natürliche Sprache, wie das Deutsche, gilt das nur in einer praktisch recht guten Annäherung. Auch in dieser Beschränkung ergibt sich aus der Behauptung aber kein idealistisches Argument. Man muß inzwischen zwei Deutungen der These von den Sprachgrenzen als Grenzen unserer Welt unterscheiden: Die erste besagt einfach, daß die Grenzen unserer Sprache die Grenzen dessen darstellen, worüber wir sinnvoll reden können. In dieser trivialen Deutung ist die These aber mit dem erkenntnistheoretischen Realismus verträglich, denn aus ihr folgt dann nicht, daß die Sachverhalte,

${ }^{26}$ Wittgenstein (22), 5.6. Bei Wittgenstein verbindet sich mit diesem Satz kein Idealismus. 
die wir sprachlich ausdrücken, sich auf sprachliche oder begriffliche Gegenstände beziehen. Der Satz „Dieses Pferd hat vier Beine“ redet nicht über sprachliche oder begriffliche Konstrukte, er handelt weder von dem Begriff ,Pferd' noch von dem Wort „Pferd“. Obwohl ,Pferd“ ein Begriff ist, sind Pferde keine begrifflichen Konstrukte oder Ideen. Deutet man die These hingegen so, daß wir nur über begriffliche Konstrukte sprechen können, so ist sie schlicht falsch. Wir können nicht nur über Ausdrücke und ihre Bedeutungen sprechen, wir können mit der Sprache nicht nur Aussagen über sprachliche Ausdrücke und Begriffe machen, sondern normalerweise verwenden wir die Sprache, um über Nichtsprachliches zu reden, und Begriffe, um konkrete Dinge zu charakterisieren. Mit dem allgemeinen Hinweis auf den Einfluß der Sprache auf die Erfahrung läßt sich also kein erkenntnistheoretischer Idealismus begründen.

Mit unserer Kritik an den Argumenten (3) und (4) wollen wir natürlich nicht behaupten, Untersuchungen der Funktionsweisen unserer Sinne oder den Abhängigkeiten unseres Erlebens von physiologischen, psychologischen und sprachlichen Faktoren seien nicht wichtig. Es ging hier nur darum zu zeigen, daß solche Abhängigkeiten keine Stütze für einen erkenntnistheoretischen Idealismus bilden.

\section{Das Argument von Leibniz}

Leibniz hat darauf hingewiesen, daß zwischen einem Ereignis und seiner Wahrnehmung eine gewisse Zeit verstreicht. Die Zeit, die das Licht z. B. braucht, um von einem Gegenstand zu unserer Retina zu gelangen, ist für nahe Objekte zwar gering, aber wir sehen im Teleskop Sterne, die schon vor langer Zeit explodiert sind. Es kann also sein, daß der beobachtete Gegenstand im Moment $t$ der Beobachtung gar nicht mehr existiert, und es kann auch sein, daß er die Eigenschaft, die wir in $t$ an ihm beobachten, in $t$ nicht mehr hat. Wir nehmen daher in einem Zeitpunkt t, so meint Leibniz, nicht Ereignisse oder Zustände der Außenwelt in $t$ wahr, sondern nur Eindrücke, die wir in $t$ haben. ${ }^{27}$

Aus dem Phänomen der Zeitverschiebung zwischen einem Ereignis und seiner Wahrnehmung, ergibt sich aber nur, daß wir in Fällen, in denen ein Zustand $\mathrm{p}$ im Zeitpunkt $\mathrm{t}$ seiner Wahrnehmung nicht mehr

${ }^{27}$ Vgl. Leibniz (NE), $114 \mathrm{f}$. 
besteht, nicht sagen können, wir nähmen in $t$ wahr, daß $\mathrm{p}$ in $t$ besteht. Es erscheint uns dann nur so, als ob $\mathrm{p}$ in $t$ bestünde, während $\mathrm{p}$ tatsächlich nur in einem früheren Zeitpunkt t' bestand. Ist es eine objektive Tatsache, daß $\mathrm{p}$ in $\mathrm{t}$ nicht besteht, so ist auch der Sachverhalt, daß $\mathrm{p}$ in $\mathrm{t}$ besteht, als objektiver Sachverhalt anzusehen, wenn nicht ,objektive Sachverhalte“ immer nur Tatsachen sein sollen. Hört ein objektiver Zustand auf zu bestehen, so wird er dadurch nicht zu einem ,,subjektiven" Zustand, insbesondere nicht zu einem Zustand des Empfindens, Beobachtens etc. Versteht man hingegen unter ,objektiven Sachverhalten“ Tatsachen und unter ,,subjektiven Sachverhalten“ vermeintliche Tatsachen, also Sachverhalte, die jemand fälschlich als bestehend ansieht, so kann man nicht behaupten, daß wir immer nur „,subjektive Sachverhalte“ beobachten; denn das würde auf die skeptische These führen, daß alle Beobachtungen falsch sind. Diese These ist aber nicht haltbar, wie wir in 3.4 gesehen haben. Auch Leibniz hat sie nicht vertreten.

\subsection{Der ontologische Idealismus}

Die Argumente für Abbildtheorien und den erkenntnistheoretischen Idealismus beruhen, wie wir gesehen haben, darauf, daß man nicht z.wischen beobachtenden Sachverhalten und Sachverhalten des Beobachtens unterscheidet. Tut man das, so ist diesen Konzeptionen ihre Grundlage entzogen. Die historische Entwicklung hat aber einen anderen Weg genommen: Die Kritik an Lockes Repräsentationstheorie richtete sich darauf, daß sie nicht konsequent genug sei und führte so zum erkenntnistheoretischen Idealismus, und von diesem dann und darauf wollen wir hier eingehen - zum ontologischen Idealismus.

Berkeley, der Begründer des ontologischen Idealismus, stimmt mit Locke in der Aussage überein: "Since the mind, in all its thoughts and reasonings, hath no other immediate object but its own ideas, which it alone does or can contemplate, it is evident that our knowledge is only conservant about them. Knowledge then seems to me to be nothing but the perception of the connexion and agreement, or disagreement and repugnancy, of any of our ideas. In this alone it consists" ${ }^{28}$

${ }^{28}$ Berkeley (T) 2, 133. 
Er setzt sich aber von der Repräsentationstheorie Lockes ab, indem er aus diesem erkenntnistheoretischen Idealismus folgende Konsequenzen zieht:

1. Wir können über die Natur der Abbildungsrelation, der Zuordnung zwischen Bildern und Originalen nichts aussagen.

$\mathrm{Daß}$ eine bestimmte Zuordnung zwischen Ideen und ihren Originalen besteht, ließe sich nur dann behaupten, wenn man die Bilder mit den Originalen vergleichen, wenn man also Aussagen über die Originale selbst machen könnte. Da uns aber in der Erfahrung immer nur Ideen gegeben sind und wir ohne eine Zuordnung zwischen ihnen und ihren Originalen schon vorauszusetzen, aus ihnen nichts über die Originale erschließen können, läßt sich nichts über die Originale, und damit auch nichts über die Abbildrelation aussagen. ${ }^{29}$

2. Wir können nicht einmal behaupten, daß es Originale zu unseren Ideen gibt.

Wir können die Existenz von Originalen weder empirisch erkennen - denn empirisch sind uns nur Ideen gegeben - noch analytisch. Aus der Existenz von Ideen überhaupt oder bestimmten Ideen können wir nicht darauf schließen, daß es Originale gibt; denn es ist denkbar, d. h. logisch möglich, daß jemand Ideen hat, ohne daß ihnen irgendwelche äußeren Gegenstände entsprechen. Auf diesem Gedanken beruhte Descartes Traumargument: Es ist nicht logisch auszuschließen, daß wir, was wir zu erfahren glauben, nur träumen; daß unseren $\mathrm{Er}$ lebnissen keine realen Tatsachen entsprechen. Berkeley sagt: "But though it were possible that solid, figured, moveable substances may exist without the mind, corresponding to the ideas we have of bodies, yet how is it possible for us to know this? either we must know it by sense, or by reason. As for our senses, by them we have the knowledge only of our sensations, ideas, or those things that are immediately perceived by sense, call them what you will: but they do not inform us that things exist without the mind, or unperceived, like to those which are perceived. This the materialists themselves acknowledge. It remains therefore that if we have any knowledge at all of ex-

${ }^{29}$ Berkeley argumentiert speziell gegen die Annahme einer Äbnlichkeit von Ideen und ihren Originalen: Eine Idee als ein Bewußtseinsmodus kann nicht einem materiellen Objekt ähnlich sein. Der beständig variierende Fluß meiner Ideen kann nicht konstanten und festen Objekten gleichen. Vgl. dazu (D), 239 und (T), $116 \mathrm{f}$. 
ternal things, it must be by reason, inferring their existence from what is immediately perceived by sense. But (I do not see) what reason can induce us to believe the existence of bodies without the mind, from what we perceive, since the very patrons of matter themselves do not pretend, there is any necessary connexion between them and our ideas. I say, it is granted on all hands (and what happens in dreams, frenzies, and the like, puts it beyond dispute) that it is possible we might be affected with all the ideas we have now, though no bodies existed without, resembling them. Hence it is evident the supposition of external bodies is not necessary for ... producing our ideas: since it is granted they are produced sometimes, and might possibly be produced always, in the same order we see them in at present, without their concurrence". 30

Man kann nach Berkeley nicht einmal sagen, daß die Annahme einer Außenwelt unsere Erfahrungen erklärt, daß sie also, wenn sie schon nicht beweisbar ist, jedenfalls einen Erklärungswert besitzt. Denn es bleibt unklar, wie körperliche Substanzen mit seelischen in Wechselwirkung treten sollten. Das Leib-Seele-Problem, das diese Hypothese aufwirft, ist nicht weniger schwierig zu lösen als die Annahme von Ideen, die nicht durch die Außenwelt verursacht werden: "The existence of external bodies affords no explication of the manner in which our ideas are produced. - But though we might possibly have all our sensations without them, yet perhaps it may be thought easier to conceive and explain the manner of their production, by supposing external bodies in their likeness rather than otherwise; and so it might be at least probable there are such things as bodies that excite their ideas in our minds. But neither can this be said; for though we give the materialists their external bodies, they, by their own confession, are never the nearer knowing how our ideas are produced: since they own themselves unable to comprehend in what manner body can act upon spirit, or how it is possible it should imprint any idea in the mind". ${ }^{31}$

Die Konsequenzen dieser Argumente ist zunächst die totale Skepsis bzgl. der Erkenntnis der Außenwelt, wie sie Hume vertrat: Wir können keine begründeten Aussagen über die Natur der Außenwelt machen, ja nicht einmal über deren Existenz. Akzeptiert man die These

\footnotetext{
${ }^{30}$ Berkeley (T), $121 \mathrm{f}$.

${ }^{31}$ Berkeley ('T), 122.
} 
des erkenntnistheoretischen Idealismus, nach der allein Ideen oder Sinnesdaten Gegenstand der Erfahrung sind, so ist diese Folgerung nur konsequent. Wenn man das Bewußtsein als ,Geist in der Maschine" ansieht, wie Ryle sagt, und seine Gegenstände als Bilder auf einer Projektionswand in einer dunklen Kammer dieser Maschine, so wird es weder feststellen können, ob diese Maschine (der Körper) selbst die Bilder erzeugt, oder ob sie Abbilder äußerer Objekte sind. Und wenn es letzteres annimmt, so wird es doch nicht feststellen können, von welcher Art der Abbildungsmechanismus ist und wie die abgebildeten Objekte an sich beschaffen sind.

Hume war der Ansicht, man könne die Existenz einer Außenwelt zwar nicht beweisen, sie andererseits aber auch nicht widerlegen. Denn auch eine Widerlegung müßte von Ideen ausgehen. Ideen aber stellen sich uns dar als Ideen von Originalen, als Empfindungen, Eindrücke oder Erfahrungen von etwas; sie haben, wie wir heute sagen würden, intentionale Struktur. ${ }^{32}$ Nimmt man also keine Außenwelt an, so sind auch die Ideen - die einzige Basis unserer Behauptungen für oder gegen die Existenz einer Außenwelt - trügerisch. ${ }^{33}$ Demgegenüber hat Berkeley die Ansicht vertreten, die Annahme einer Außenwelt sei nicht nur nicht begründbar und überflüssig, sondern sogar widerlegbar. Für ihn ist der Begriff einer körperlichen, materiellen Substanz ein Unbegriff: "Let us examine a little the description that is here given us of matter. It neither acts, nor perceives, nor is perceived: for this is all that is meant by saying it is an inert, senseless, unknown substance; which is a definition entirely made up of negatives, excepting only the relative notion of its standing under or supporting: but then it must be observed, that it supports nothing at all; and how nearly this comes to the description of a nonentity, I desire may be considered". ${ }^{34}$

${ }^{32}$ Das betont auch Kant, für den jede Erscheinung Erscheinung eines Dings an sich ist. Vgl. dazu auch den Abschnitt 8.7.

${ }^{33} \mathrm{Vgl}$. Hume (T), $182 \mathrm{ff}$. Es ist freilich zu beachten, daß Hume viele Thesen seines "Treatise“ später nicht mehr aufrecht erhalten hat. Vgl. dazu das „Advertisement“ zur Ausgabe der „Enquiries concerning Human Understanding" von 1777 .

${ }^{34}$ Berkeley (T), 147 (vgl. dazu auch (D), 230 f.). - Daß materielle Substanzen nicht wahrgenommen werden, ergibt sich aus der These des erkenntnistheoretischen Idealismus. Insbesondere sind alle Qualitäten nach Berkeley se- 
Alles, was wir wahrnehmen können, sind Ideen. Aber auch alles, was denkbar ist, sind Ideen, denn ich kann nicht ungedachte Objekte denken, gedachte Objekte sind aber Ideen. ${ }^{35}$ Es ist also nichts denkbar als Ideen, und wenn reale Objekte gerade so bestimmt werden, $\mathrm{daß}$ sie keine Ideen sind, so ist es nicht denkbar, daß es solche Objekte gibt. - Hier wird wieder die Verwechslung von Wahrnehmung und Denken mit Wahrgenommenem und Gedachtem deutlich. ${ }^{36}$

kundär, so daß wir materiellen Substanzen keine einzige Eigenschaft zuschreiben können.

${ }^{35}$ Vgl. das Argument von der Subjektbezogenheit des Beobachteten in 4.2. Dazu Berkeley (T), 124.

${ }^{36}$ Auf diese Unterscheidung geht Berkeley in (D), $226 \mathrm{ff}$. ein. Er argumentiert dort so: Was wir wahrnehmen, sind nur Qualitäten, nicht Substanzen als Träger von Qualitäten. Substanzen sind nur Bündel von Qualitäten - oder Qualia, wie man im Blick auf Berkeleys Nominalismus besser sagt. (Er bezieht sich dabei auf Lockes Kritik am traditionellen Substanzbegriff in (E), II, 23.) Während Locke noch sagte: Substanz ist ein Bündel von Eigenschaften, verbunden mit einer nicht mehr begreifbaren, aber unverzichtbaren Vorstellung eines Trägers (eines Individuums, dem diese Eigenschaften zukommen), lehnt Berkeley - wie auch Leibniz - die Vorstellung eines Trägers, eines Substrats der Eigenschaften ab. Nach Leibniz lassen sich Individuen nur durch ihre Eigenschaften bestimmen und unterscheiden. Wenn man also von den Eigenschaften absieht, so bleibt nichts mehr übrig, von dem man sinnvoll reden könnte. Vgl. (NE), 225.) Bei Qualitäten kann man aber nicht Wahrgenommenes und Wahrnehmung unterscheiden: Eine Wahrnehmung ist ein Akt des Verstandes, der von unserem Willen abhängt, die Wahrnehmung von Qualitäten ist aber nicht von unserem Willen abhängig (wir können nicht die Eigenschaften sehen, die wir sehen wollen, nicht etwas Rotes, wo etwas Blaues ist). Daher kann man bzgl. Qualitäten Wahrnehmungsakte nicht vom Wahrgenommenen unterscheiden. Gegenstand und Wahrnehmungsakt fallen zusammen; Qualitäten sind Wahrnehmungsweisen, und Wahrnehmungsweisen kommen nur dem Subjekt, nicht ,unthinking objects" zu; Qualitäten sind ,sensations". - All das ist aber völlig unhaltbar: Die Unterscheidung zwischen einem Sachverhalt $B(a, F(b))$ und dem Sachverhalt $F(b)$ hängt nicht davon $a b$, ob das Bestehen des Sachverhalts $B(a$, $F(b))$ in der freien Verfügung der Person a liegt. Rot als Körperfarbe ist ferner von der Art und Weise, in der ich rote Dinge empfinde oder erlebe zu unterscheiden. Endlich ist die Auffassung von Objekten als Mengen von Eigenschaften nicht korrekt, denn selbst wenn man die Objekte eindeutig den 
Für Berkeley ist die Annahme einer Außenwelt, von materiellen Substanzen eine unhaltbare Fiktion: "It is indeed an opinion strangely prevailing amongst men, that houses, mountains, rivers, and in a word all sensible objects have an existence natural or real, distinct from their being perceived by the understanding. But with how great an assurance and acquiescence soever this principle may be entertained in the world; yet whoever shall find in his heart to call it in question, may, if I mistake not, perceive it to involve a manifest contradiction. For what are forementioned objects but the things we perceive by sense, and what do we perceive besides our own ideas or sensations; and is it not plainly repugnant that any one of these or any combination of them should exist unperceived?"37 - "Some truths there are so near and obvious to the mind, that a man need only open his eyes to see them. Such I take this important one to be, to wit, that all the choir of heaven and furniture of the earth, in a word all those bodies which compose the mighty frame of the world, have not any subsistence without a mind, that their being (esse) is to be perceived or known; that consequently so long as they are not actually perceived by me, or do not exist in my mind or that of any other created spirit, they must either have no existence at all, or else subsist in the mind of some eternal spirit: it being perfectly unintelligible and involving all the absurdity of abstraction, to attribute to any single part of them an existence independet of a spirit". ${ }^{38}$

Damit ergibt sich die These des ontologischen Idealismus:

12: Es gibt keine objektiven Sachverhalte. Die Wirklichkeit besteht nur aus Subjekten und ibren Ideen - esse est percipi - oder besser: esse est percipi vel percipere.

Es stellt sich dann natürlich die Frage, wie es im Rahmen dieses Wirklichkeitsverständnisses die normalen Redeweisen zu rekonstruieren sind, nach denen wir $z$. B. auch von nicht wahrgenommenen Objekten sprechen und einen Unterschied zwischen realen und nur vorgestell-

Mengen ihrer Eigenschaften zuordnen kann, so sind doch Eigenschaften immer Eigenschaften von Objekten; man kann also nicht den Begriff der Eigenschaft als primär gegenüber dem des Objekts ansehen. Vgl. dazu auch Kutschera (79).

${ }^{37}$ Berkeley (T), $114 \mathrm{f}$.

${ }^{38}$ Berkeley (T), $115 \mathrm{f}$. 
ten Dingen machen. Es lag Berkeley fern, diese Unterscheidungen einfach als unbegründet zu verwerfen, denn er meinte merkwürdigerweise, gerade seine Theorie entspreche der common-sense-Auffassung besonders gut. Er stand also vor der Aufgabe, die Sätze der normalen Sprache in die Sprache über Ideen zu übersetzen. Darauf wollen wir jedoch erst im nächsten Kapitel im Zusammenhang mit dem Phänomenalismus eingehen.

Wir haben in 3.4 zwei Vorschläge zur Explikation des Wortes „objektiv" gemacht. Im Sinn der Berkeleyschen Argumente ist die These I2 im Sinn des schwachen Begriffs der Objektivität zu verstehen, denn alle Aussagen über die Welt lassen sich nach seiner Ansicht in Aussagen über Subjekte und ihre Ideen übersetzen. Eine Verschärfung des ontologischen Idealismus stellt die These des Solipsismus dar:

I2*: Die Wirklichkeit besteht nur aus mir selbst und meinen Ideen.

Der erkenntnistheoretische Idealismus besagt naturgemäß, daß die Gegenstände der Erfahrung jeder Person nur ibre eigenen Ideen sind - Ideen anderer kann ich nicht erfahren. Es ist dann aber im Sinn der Argumente des ontologischen Idealismus auch nur konsequent, anstelle von I2 auch I2* zu behaupten. Wenn der Gegenstand meiner Erfahrung nur meine Ideen sind, so habe ich ebenso wenig Grund, die Existenz anderer Personen und ihrer Ideen anzunehmen wie die einer Außenwelt; ich habe dann immer nur eigene Ideen von anderen Personen. Die These des Solipsismus ist aber schon auf den ersten Blick absurd. Für das Pronomen ,mir" ist ja jeweils ein Name einzusetzen. Gustav Meier muß also behaupten ,Die Wirklichkeit besteht nur aus Gustav Meier und seinen Ideen", und das ist für alle anderen Personen sicher völlig unakzeptabel. Die Thesen I2*, bezogen auf verschiedene Personen, sind mit einander unverträglich. Wer von uns sollte aber die privilegierte Stellung des allein Existierenden erhalten und wieso gerade er?

I1 ist - bei beiden Deutungen des Wortes „objektiv“ - die Gegenthese zum erkenntnistheoretischen Realismus nach R2. I2 ist die Gegenthese zum ontologischen Realismus nach R1. I2* entspräche eine realistische These des Inhalts: „Es gibt für mich objektive Tatsachen". Dabei wäre ein Sachverhalt p für die Person a objektiv, wenn $p$ mit beliebigen Überzeugungen von a verträglich ist, bzw. nicht mit solchen Sachverhalten äquivalent ist. Darauf gehen wir hier jedoch nicht ein, da ein solcher Realismus ohne tieferes Interesse ist. Die ein- 
ander entsprechenden Thesen sind jeweils konträr, aber nicht kontradiktorisch: I1 ist eine sehr viel stärkere Behauptung als die Verneinung von R2, und Analoges gilt in den übrigen Fällen.

Aus den besprochenen Argumenten für Repräsentationstheorien und Idealismen haben sich, so können wir abschließend feststellen, keine brauchbaren Einwände gegen die realistischen Thesen aus 3.4 ergeben. Die Argumente beruhen sämtlich auf der Verwechslung von beobachteten Sachverhalten und Sachverhalten des Beobachtens. Daß eine derart elementare begriffliche Konfusion so weitreichende Folgen haben konnte, daß sie von den bedeutendsten Philosophen von Descartes bis Hegel nicht bemerkt wurde und ihren Erkenntnistheorien schon vom Ansatz her eine falsche Richtung gegen konnte, läßt sich wohl nur geistesgeschichtlich erklären. ${ }^{39}$ Es gibt Auffassungen, die den Zielen und Intentionen einer Epoche so sehr entgegenkommen, daß sie gegen Kritik praktisch immun sind.

Mit der Reduplikation der Erkenntnisgegenstände in den Abbildtheorien - zwischen das erkennende Subjekt und die Außenwelt schieben sich Ideen als unmittelbare Objekte der Erfahrung - entstand die an Platons Höhlengleichnis erinnernde Metapher vom Geist in einer camera obscura. So sagt Locke: "For, methinks, the understandings is not much unlike a closet wholly shut from light, with only some little opening left, to let in external visible resemblances, or ideas of things without; would the pictures coming into such a dark room but stay there, and lie so orderly as to be found upon occasion, it would very much resemble the understanding of a man in reference to all objects of sight and the ideas of them". ${ }^{40}$ Dieses Bild menschlicher Erkenntnis ist ein stehender Topos der philosophischen Literatur der Zeit, den

${ }^{39}$ Auch diejenigen, die den Idealismus ablehnten, standen ihm ohnmächtig und ohne Argumente gegenüber. Typisch ist die Reaktion von S. Johnson, von der Boswell berichtet: "After we came out of church we stood talking for some time together of Bishop Berkeley's ingenious sophistry to prove the non-existence of matter, and that everything in the universe is merely ideal. I observed, that though we are satisfied his doctrine is not true, it is impossible to refute it. I never shall forget the alacrity with which Johnson answered, striking his foot with mighty force against a large stone, till he rebounded from it, "I refute it thus!"” (Von Lindsay zitiert in der Einleitung zu Berkeley (L), S. IX.)

${ }^{40}$ Locke (E), 129. 
z. B. auch Leibniz übernimmt. ${ }^{41}$ Wir haben im Abschnitt 1.7 über das verfehlte Streben nach perfekter Erkenntnis gesprochen. Gerade dieses Streben führte paradoxerweise in einen Zustand, den man kaum anders bezeichnen kann als eine Umnachtung der Vernunft. Daß diese Bezeichnung nicht zu stark ist, belegt schon das obige Bild von der camera obscura. Descartes beginnt seine Bemühung um Erkenntnis der Außenwelt mit den Worten: „Claudum nunc oculos, aures obturabo, avocabo omnes sensus, imagines etiam rerum corporalium omnes vel ex cogitatione mea delebo, vel certe, quia hoc fieri vix potest, illas ut inanes et falsas nihili pendam, meque solum alloquendo, et penitius inspiciendo, meipsum paulatim mihi magis notum et familiarem reddere conabor" ${ }^{42}$ Leibniz spricht davon, daß wir unsere Sinne gebrauchen wie ein Blinder seinen Stock, ${ }^{43}$ und für Hume ist die Konsequenz seines Idealismus ein theoretisch unausweichlicher, praktisch aber nicht realisierbarer, totaler Skeptizismus.

${ }^{41} \mathrm{Vgl}$. Leibniz -(NE), 126.

${ }^{42}$ Descartes (MP), 58. „Ich werde jetzt die Augen schließen, die Ohren verstopfen, alle Sinne abberufen und auch alle Bilder körperlicher Dinge entweder aus meinem Denken tilgen, oder sie doch wenigstens - weil das kaum möglich ist - als eitel und falsch für nichts achten, und werde versuchen, allein mich ansprechend und tiefer in mich blickend, mich mit mir selbst ein wenig bekannter und vertrauter zu machen". In diesem Ansatz ist schon der idealistische Versuch vorgezeichnet, durch eine Art innere Nabelschau die Welt zu erkennen.

${ }^{43}$ Vgl. Leibniz (WP) VI, $499 \mathrm{f}$. 



\section{Der Phänomenalismus}

\subsection{Formen und Thesen des Phänomenalismus}

Der Phänomenalismus ist die heute am meisten diskutierte idealistische Theorie. An seinem Beispiel läßt sich auch die Behauptung am besten diskutieren, die Aussagen über die Welt, wie wir sie normalerweise im Alltag und in den Wissenschaften machen, ließen sich im Rahmen einer idealistischen Ontologie rekonstruieren. Mit der Erörterung dieser Reduzierbarkeitsthese kommen wir auf das Verhältnis von Physischem und Psychischem zu sprechen, auf das Leib-SeeleProblem (im weiteren Sinn dieser Bezeichnung), und das ist das zentrale Thema dieses und der folgenden Kapitel.

Wie beim Idealismus insgesamt, kann man auch beim Phänomenalismus eine erkenntnistheoretische und eine ontologische These unterscheiden. Nach dem erkenntnistheoretischen Phänomenalismus bilden allein Sinnesdaten (Sinneseindrücke oder -empfindungen) die Gegenstände äußerer Erfahrung. Von dieser Annahme gelangt man dann aufgrund der schon in 4.3 erörterten Argumente zu einem ontologischen Phänomenalismus, nach dem die Sinnesdaten - neben anderen psychischen Phänomenen und den Subjekten als Trägern seelischen Erlebens, Denkens etc. - die gesamte Wirklichkeit darstellen. Alle Aussagen über eine Außenwelt, über Atome, Tische und Sterne, die wir im Alltag oder in den Wissenschaften machen, sind danach nur insoweit sinnvoll und lassen sich nur insoweit begründen, als sie sich als komplexe Aussagen über Sinnesdaten interpretieren lassen, und es ist die Überzeugung der Phänomenalisten, daß man sie so verstehen kann. Da nur meine eigenen Sinnesdaten den Gegenstand meiner Erfahrung bilden, ist der Phänomenalismus zumindest ein methodischer Solipsismus. Auch Aussagen über die Existenz anderer Personen und über ihre Empfindungen sind danach nur insoweit sinnvoll und begründbar, als sie sich deuten lassen als Aussagen über eigene Sinnesdaten.

Wir haben schon in 3.2 gesehen, daß Formulierungen wie „Nur Sinnesdaten und Subjekte sind wirklich" unbrauchbar sind. Im nor- 
malen Sinn des Wortes ,wirklich“ ist diese Behauptung sinnlos oder falsch. Daher konnte S. Johnson den Phänomenalismus ,,widerlegen“, indem er mit dem Fuß gegen einen Stein stieß;1 ein ,, wirklicher" Stein ist im Gegensatz zu einem bloß vorgestellten Stein oder einem Komplex von Sinnesdaten eben ein solcher, gegen den man stoßen kann. Und G. E. Moore widerlegte die Behauptung, es gäbe nur Sinnesdaten, damit, daß er beide Hände hochhob und sagte: „Hier ist eine Hand und hier eine andere. Also gibt es zumindestens zwei Dinge, die keine Sinnesdaten sind". ${ }^{2}$ Ontologische Fragen, so hatten wir in 3.2 gesehen, sind externe Existenzfragen, und die Wahl einer Ontologie rechtfertigt sich durch den Wert (den Ausdrucksreichtum, die Einfachheit, die ontologische Sparsamkeit) einer Sprache, der diese Ontologie zugrunde liegt. Daher liegt es nahe, die ontologische These des Phänomenalismus in eine Übersetzbarkeitsthese zu transformieren, um so den Phänomenalismus einer rationalen Diskussion zugänglich zu machen.

Dem Phänomenalismus geht es speziell um die Reduzierbarkeit des Physischen auf Psychisches, auf Sinnesdaten. Ist $\Psi$ eine phänomenalistische Sprache, deren deskriptive Grundterme für Sinnesdaten einer Person und ihre Attribute stehen, und ist $\Phi$ die Sprache über Physisches, d.h. unsere normale Dingsprache, erweitert um die Sprache der Physik - wir bezeichnen $\Phi$ auch als physikalische Sprache -, dann lautet diese phänomenalistische Kernthese als Übersetzbarkeitsthese so:

PN: Die Sprache $\Phi$ läßt sich in die Sprache $\Psi$ übersetzen.

Besteht eine solche Übersetzbarkeit, so lassen sich alle Aussagen über Dinge, über Steine und Hände, als Aussagen über Sinnesdaten deuten, d. h. PN gibt die wesentliche Intention des Phänomenalismus wieder. Diese These besagt allerdings nichts über die Reduzierbarkeit von Fremdseelischem auf Eigenseelisches im Sinn des Solipsismus. Ist $\Psi *$ eine Sprache über eigenseelische Zustände des Solipsisten, die insbesondere auch die Sprache $\Psi$ über seine eigenen Sinnesdaten enthalten soll, und ist $\Omega$ die normale Sprache, erweitert um die Wissenschaftssprachen - so daß $\Omega$ auch $\Phi$ enthält - , so würde die stärkere These des Phänomenalismus lauten:

${ }^{1}$ Vgl. dazu die Anmerkung 39 zu S. 220.

${ }^{2}$ Vgl. dazu G. E. Moore (39). 


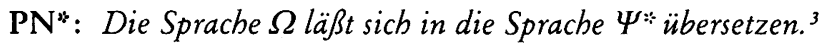

$\mathrm{PN}$ * besagt, daß man für alle Zwecke mit der von der Sprache $\Psi *$ vorausgesetzten Ontologie auskommt, enthält allerdings nicht mehr den Ausschließlichkeitsanspruch des ontologischen Idealismus, nur eine solche Ontologie sei akzeptabel und nur eine solche Sprache wie $\Psi *$ sei sinnvoll. PN* ist vielmehr z. B. durchaus auch mit der These des Physikalismus verträglich, alle Sätze von $\Omega$ ließen sich in Sätze der physikalischen Sprache $\Phi$ übersetzen. Wir wollen uns im folgenden vor allem für die schwächere These PN interessieren. Alle Argumente gegen sie, die wir im folgenden vorbringen werden, sind auch Argumente gegen die stärkere These $\mathrm{PN}$ *

Wie soll nun die Sprache $\Psi$ aussehen? $\Psi$ soll eine Sprache über die Sinnesdaten einer bestimmten Bezugsperson sein - des Autors z. B., bzw. des Lesers. Was aber Sinnesdaten genauer sind, und wie Aussagen darüber aussehen sollen, ist zunächst unklar. In der phänomenalistischen Literatur wird eine solche Sprache - mit vereinzelten Ausnahmen wie Carnap (28) und Goodman (51) - nicht angegeben. Man begnügt sich meist mit vagen Andeutungen und Beispielen phänomenalistischer Sätze, die sich bei näherem Zusehen aber meist entweder als unsinnig oder als nicht streng phänomenalistisch erweisen. ${ }^{4}$ Dabei zeigt sich, daß $\Psi$, im Gegensatz z. B. zur physikalischen Sprache, weder ein Teil der normalen Sprache noch einer üblichen Wissenschaftssprache ist, so daß es ein um so dringenderes Erfordernis wäre, diese Sprache durch Angabe einer präzisen Syntax und Semantik zu bestimmen. Daß solche Bestimmungen fehlen, stellt den ersten grundsätzlichen Einwand gegen den Phänomenalismus dar. Solange $\Psi$ nicht angegeben wird, läßt sich mit der These PN kein genauer Sinn verbinden. Wir wollen jedoch diesen Einwand zunächst zurückstellen und sehen, ob sich nicht aus den Argumenten der Phänomenalisten ein Aufschluß über den Begriff des Sinnesdatums gewinnen läßt und damit eine genauere Vorstellung über die Sprache $\Psi$.

${ }^{3} \mathrm{Da}$ sich schon nicht alle Aussagen über seelische Zustände des Solipsisten Aussagen z. B. über seine Überzeugungen, Präferenzen oder Stimmungen in Aussagen über seine Sinnesdaten übersetzen lassen, ließe sich $\Omega$ sicher nicht in die Sprache $\Psi$ übersetzen.

${ }^{4}$ Diesen Mangel betont auch Black in (52). 
Schwächere Reduzierbarkeitsbehauptungen als PN sind im Fall des Phänomenalismus ohne Interesse. Eine W-Reduzierbarkeit von $\Phi$ auf $\Psi$ entspräche, wie wir in 3.3 gesehen haben, nicht dem phänomenalistischen Gedanken, daß physische Objekte nichts anderes sind als Komplexe von Sinnesdaten. Im übrigen werden wir im Abschnitt 5.4 zeigen, daß auch eine solche Reduzierbarkeit nicht angenommen werden kann. Eine Reduzierbarkeitsbehauptung des Inhalts, daß sich physikalische Theorien auf Theorien über Sinnesdaten reduzieren lassen, die in der Sprache $\Psi$ formuliert sind, ist schon deswegen ohne Interesse, weil es keine Theorien über gesetzmäßige Zusammenhänge zwischen dem Auftreten von Sinnesdaten gibt.

\subsection{Argumente des Phänomenalismus}

Wir gehen zunächst auf die Hauptargumente des Phänomenalismus ein. Sie beziehen sich nicht direkt auf die Übersetzungsthese PN, sondern auf die erkenntnistheoretische und die ontologische These des Phänomenalismus, von denen wir im letzten Abschnitt ausgegangen sind. Neben den schon in 4.2 erörterten Argumenten werden vor allem zwei Begründungen für den Phänomenalismus immer wieder vorgetragen: ${ }^{5}$

\section{Das Illusionsargument}

Bei unseren sinnlichen Beobachtungen können wir uns täuschen, und zwar sowohl bzgl. der Beschaffenheit der wahrgenommenen Objekte wie auch bzgl. ihrer Existenz: Der ins Wasser gehaltene Stock erscheint uns als geknickt, obwohl er tatsächlich nicht geknickt ist; der Betrunkene glaubt zwei Weinflaschen zu sehen, wo tatsächlich nur eine steht; und im Traum erscheinen uns Dinge und Ereignisse, die tatsächlich nicht existieren, bzw. stattfinden. Solche Illusionen, wie wir sie zusammenfassend bezeichnen wollen, beweisen, daß wir die Dinge und Sachverhalte, wie sie uns erscheinen, nicht mit den Dingen und Sachverhalten, wie sie wirklich sind, identifizieren können. Wir können das aber nicht so ausdrücken, daß wir sagen, bei einer Illusion

${ }^{5}$ Für den ontologischen Phänomenalisten kommen nur die Argumente (1) und (2) aus 4.2 infrage, da er ja die Existenz einer Außenwelt leugnet. 
würden reale Dinge und Sachverhalte falsch aufgefaßt, denn sie brauchen keine realen Entsprechungen zu haben, wie wir gerade sahen. Welche realen Dinge und Ereignisse erscheinen mir denn z. B. falsch, wenn ich träume, daß ich von einem Einhorn verfolgt werde? Wir müssen also sagen, daß wir bei solchen trügerischen Erfahrungen nur phänomenale Objekte oder Sinnesdaten wahrnehmen. Tun wir das, so können wir dann aber nicht behaupten, in einer korrekten Beobachtung erführen wir reale Entitäten, in einer trügerischen aber phänomenale. Denn wegen des fundamentalen Unterschieds zwischen realen und phänomenalen Objekten müßten sich sonst korrekte und trügerische Erfahrungen qualitativ wesentlich voneinander unterscheiden, so daß wir also bei Beachtung dieses qualitativen Unterschieds keinen Täuschungen mehr unterliegen würden. Es wäre also eine Konsequenz dieser Analyse von Illusionen, daß es sie nicht geben kann. Ferner gibt es kontinuierliche Übergänge zwischen Illusionen und korrekten Erfahrungen. Wenn z. B. ein weißes Blatt Papier unter Licht betrachtet wird, das kontinuierlich von Rot in Weiß übergeht, so geht die Illusion, das Papier sei rot, ebenso kontinuierlich in die korrekte Beobachtung über, daß es weiß ist. Das widerspricht aber der Annahme ganz verschiedenartiger Objekte trügerischer und korrekter Beobachtungen. ${ }^{6}$

Wir müssen also sagen, daß wir generell nur phänomenale Entitäten erfahren, und daß reale Entitäten nicht direkt erfahren, sondern allenfalls aus phänomenalen Gegebenheiten erschlossen werden können.

\section{Das Argument der Evidenz}

In den empirischen Wissenschaften zielen wir auf die Gewinnung möglichst sicherer Aussagen ab. Manche naturwissenschaftlichen Sätze, wie z. B. allgemeine Gesetzesaussagen, sind in hohem Maße problematisch, andere weniger. Alle irgendwie problematischen Aussagen müssen aber überprüft werden und diese Überprüfung muß letztlich auf Sätze zurückgreifen, die nicht mehr problematisch sind, sondern als durch Beobachtungen definitiv verifiziert gelten können. Solche Sätze dürfen in ihrem Inhalt nicht über das in der Beobachtung unmittelbar Gegebene hinausgehen, es sind also nach dem Argument des unmittelbar Gegebenen in 4.2 Sätze der Form „Es erscheint

'Vgl. dazu auch Ayer (40), Kap. 1. 
mir, als ob p“. Solche Sätze sind introspektiv definitiv entscheidbar: Der Satz „Mir erscheint es (jetzt), als ob hier ein Stück gelbes Papier liegt“ ist, im Gegensatz zur Behauptung „Hier liegt ein Stück gelbes Papier", (jetzt) für mich problemlos; nur er ist von hypothetischen Elementen frei und nur ihn kann man vernünftigerweise nicht mehr bezweifeln. Die Basissätze der empirischen Erkenntnis als Sätze, die durch einfache Beobachtungen definitiv entscheidbar sind, müssen also phänomenalistische Sätze sein, und damit ergibt sich, wie im Argument des unmittelbar Gegebenen, daß alle empirischen Sätze phänomenalistische Sätze sein müssen.

\section{Das Illusionsargument läßt sich nun so rekonstruieren}

a) Es erscheint mir, als ob p.

b) Also gibt es einen Sachverhalt p, von dem es mir erscheint, als ob er bestehe.

c) Ist nun $p$, wie bei einer Illusion, kein realer Sachverhalt, so gilt also: Es gibt einen phänomenalen Sachverhalt $\mathrm{p}$, von dem es mir erscheint, als ob er besteht.

d) Wegen der qualitativ gleichen Erscheinungsweise realer und phänomenaler Sachverhalte gilt dann auch: Wenn es mir erscheint, als ob p, so ist $\mathrm{p}$ ein phänomenaler Sachverhalt.

Wenn in diesem Argument reale und phänomenale Sachverhalte einander gegenübergestelli werden, so ist ein ,,phänomeraler Sachverhalt" entweder ein Sachverhalt, von dem es mir fälschlich so erscheint, als ob er bestünde - dann ist (c) richtig, (d) aber falsch (denn nicht alle Sachverhalte, von denen es mir so erscheint, als ob sie bestehen, sind falsch) - oder ein ,phänomenaler Sachverhalt“ ist ein Sachverhalt des Erscheinens, d. h. ein Sachverhalt, der durch einer Satz der Gestalt „Es erscheint mir, als ob q“ ausgedrückt wird. Dann ist aber der Schritt von (b) zu (c) nicht korrekt, und ebensowenig (d). Es liegt dann ein analoger Fehler vor wie im Argument von der Subjektbezogenheit des Beobachteten, d. h. die Annahme, ein erscheinender (beobachteter) Sachverhalt sei ein Sachverhalt des Erscheinens (Beobachtens).

Um die Kritik am Illusionsargument noch deutlicher $\mathrm{zu}$ machen, und um die mysteriösen Sinnesdaten ins Spiel zu bringen, wollen wir das Argument noch in einer leicht modifizierten Form wiederholen: a') Es erscheint mir, als ob ein Objekt $b$ die Eigenschaft $F$ habe. 
b') Also gibt es einen Gegenstand b und eine Eigenschaft F, so daß es mir erscheint, als ob b die Eigenschaft $F$ habe.

c') Existiert nun, wie in einem Traum, kein realer Gegenstand b und keine reale Eigenschaft $F$, so daß man sagen könnte, sie erschienen mir, so gilt: Es gibt ein phänomenales Objekt $b$ und eine phänomenale Eigenschaft $F$, so daß es mir erscheint, als ob b die Eigenschaft $F$ habe.

d') Wegen der qualitativ gleichen Erscheinungsweise von phänomenalen und realen Objekten und Attributen gilt dann generell: Wenn es mir erscheint, als ob $b$ die Eigenschaft $F$ habe, ist $b$ ein phänomenales Objekt und $\mathrm{F}$ eine phänomenale Eigenschaft.

Hier ist die Verwirrung perfekt. Der erste Fehler ist bereits der Übergang von (a') zu (b'), denn da ,Es erscheint mir, als ob a die Eigenschaft $F$ hat" - symbolisch $S(a, F(b))$, wo ,,a" ein Name für mich sei - ein intensionaler Kontext ist, gelten die Partikularisierungsregein $\mathrm{S}(\mathrm{a}, \mathrm{F}(\mathrm{b})) \supset \mathrm{VxS}(\mathrm{a}, \mathrm{F}(\mathrm{x}))$ und $\mathrm{S}(\mathrm{a}, \mathrm{F}(\mathrm{b})) \supset \mathrm{VfS}(\mathrm{a}, \mathrm{f}(\mathrm{b}))$ nicht allgemein. ${ }^{7}$ Ferner ist wieder unklar, was phänomenale Objekte und Attribute sein sollen. Wenn es mir z. B. im Traum erscheint, als unterhielte ich mich mit Popper, so wird Popper dadurch nicht weniger real als er es vorher war. Auch wenn es mir im Traum so erscheint, als lebte Odysseus, so ist Odysseus nur in dem Sinn kein reales Objekt, als er (in unserer Welt) nicht existiert. Das macht ihn aber noch nicht zu einem ,phänomenalen“ Objekt. Was soll ferner heißen, die Eigenschaft, lebendig zu sein, sei nicht real? Man kann nur sagen, daß es neben den realen Objekten auch Vorstellungen von Objekten gibt die man als „phänomenale Objekte“ bezeichnen könnte - und daß auf diese Vorstellungen von Objekten andere Attribute zutreffen die man dann ,,phänomenal" nennen kann - als auf die Objekte selbst. So kann ein Mensch hungrig oder satt sein; die Vorstellung von einem Menschen ist aber nicht hungrig oder satt, sondern z. B. klar oder verschwommen. Bei dieser Deutung ist (c') jedoch falsch und es liegt wieder der frühere Fehler vor: Es gibt nicht eine Vorstellung und eine Eigenschaft von Vorstellungen, so daß es mir erscheint, als ob diese Vorstellung diese Eigenschaft habe - Vorstellungen sind nicht Gegenstände von Sinneswahrnehmungen. ${ }^{8}$ Dem Illusionsargument

7 Vgl. dazu z. B. Kutschera (76), $43 \mathrm{ff}$. und $95 \mathrm{ff}$.

${ }^{8}$ Vgl. 3.1. Zur Kritik des Illusionsarguments vgl. auch Austin (62), Kap. III und S. $50 \mathrm{f}$. 
liegt also wieder die Verwechslung von Sachverhalten des Erscheinens mit erscheinenden Sachverhalten zugrunde.

Wenn man fragt: „Was erscheint mir denn, wenn es mir erscheint, als ob p, p aber nicht gilt?" , so enthält diese Frage schon eine falsche Suggestion, die Suggestion nämlich, daß ,erscheinen“ ein zweistelliges Prädikat sei, dessen Argumentstellen nur von Namen für ,,existierende" Entitäten ausgefüllt werden können, wobei ein Sachverhalt nur dann existiert, wenn er besteht. Wenn es mir erscheint, als ob p, so besagt das aber nicht mehr, als daß ich irgendwelche nicht näher spezifizierten Eindrücke habe, die ich in der Regel nur dann habe, wenn p gilt. Eine Illusion ist eine Ausnahme von dieser Regel. Der phänomenalistische Schluß von der Existenz von Erscheinungen (als Sachverhalten des Erscheinens-als-ob) auf die Existenz von Erscheinendem, von der erscheinenden Welt auf eine Welt der Erscheinungen hält einer genaueren Analyse nicht stand.

Auch das Argument der Evidenz beruht auf demselben Fehler. Wir haben schon bei der Kritik des Arguments von unmittelbar Gegebenen in 4.2 betont: Erscheint es der Person a, als ob der Sachverhalt $p$ bestünde - gilt also $S(a, p)$ - , so glaubt sie in der Regel auch, daß p besteht -- also $\mathrm{B}(\mathrm{a}, \mathrm{p})$. Damit weiß a aber nicht nur von ihrer Beobachtung, sondern a weiß auch, daß p gilt, falls das der Fall ist. Die Erfahrungserkenntnis von a beschränkt sich also nicht nur auf Sachverhalte der Art S(a,p), sondern es gibt auch Erkenntnis erfahrener Sachverhalte. Ferner begründe ich, wenn ich glaube, daß $\mathrm{p}$ gilt, andere Annahmen damit nicht so, daß ich sie aus $G(a, p)$ ableite, sondern damit, daß ich sie aus $p$ ableite. Die Behauptung, unsere durch Beobachtung begründeten Annahmen seien immer Sätze der Form G(a,p) und mit ihnen ließen sich wieder nur Annahmen dieser Gestalt begründen, ist also falsch.

Das Argument der Evidenz orientiert sich wieder an einem Begriff perfekter Evidenz. Sachverhalte des Glaubens, Beobachtens oder Erscheinens sind zwar in diesem ausgezeichneten Sinn evident, aber die Problemlosigkeit von Aussagen über solche Sachverhalte beruht, wie wir in 1.7 sahen, darauf, daß sie keine Informationen über die Außenwelt enthalten. Was man mit dem Rückzug auf solche Sätze an Sicherheit gewinnt, verliert man also an empirischem Gehalt. 


\subsection{Sinnesdaten}

Im Abschnitt 4.1 war offengeblieben, was ,,Sinnesdaten“ sind. Wir wollen nun versuchen, uns eine Übersicht über die verschiedenen Deutungen dieses Ausdrucks zu verschaffen. Allgemeiner reden wir zunächst wieder von „Phainomena".9 Phainomena können sowohl Sachverhalte als auch Objekte und Attribute sein. Der Kürze wegen sprechen wir im folgenden aber meist nur von Sachverhalten.

a) Phainomena als Sachverbalte des Beobachtens, Vorstellens, Erlebens oder Erscheinens-als-ob.

Phainomena in diesem Sinne kommen in allen Argumenten vor, die wir hier erörtert haben. Von solchen Sachverhalten kann man jedoch, wie schon mehrfach betont wurde, nicht sagen, sie seien Gegenstände oder Inhalte von Beobachtungen, Vorstellungen oder von Erscheinungen oder Erlebnissen. Da solche Phainomena durch Sätze der Form $\mathrm{B}(\mathrm{a}, \mathrm{A})$ oder $\mathrm{S}(\mathrm{a}, \mathrm{A})$ dargestellt werden, wobei die Teilsätze A auch Sätze von $\Phi$ sein können, enthielte eine phänomenalistische Sprache $\Psi$ als Sprache über solche Phainomena ferner die Sprache $\Phi$ als Teilsprache, so daß die These PN trivial würde.

b) Phainomena als beobachtete, vorgestellte oder erscheinende Sachverbalte

Solche Phainomena sind Gegenstände von Beobachtungen, Vorstellungen etc., aber es sind Sachverhalte im normalen Sinn, so daß ihre Bezeichnung als „Phainomena" überflüssig und nichtssagend ist. ${ }^{10} \mathrm{An}$ ihrem ontologischen Status ändert sich auch dadurch nichts, daß sie beobachtet oder vorgestellt werden. Eine Sprache $\Psi$ über solche Phainomena enthält ebenfalls die Sprache $\Phi$, ist also für die phänomenalistische Übersetzbarkeitsthese unbrauchbar.

c) Phainomena als Sachverhalte, die uns in der Beobachtung unmittelbar gegeben sind

"Der Ausdruck "Sinnesdaten" (engl. sense-data) ist vor allem von G. E. Moore und B. Russell eingeführt worden.

${ }^{10}$ Eine solche Auffassung der Sinnesdaten vertritt z. B. Ayer in (63), S. $131 \mathrm{ff}$. Und C. D. Broad sagt in (54): “. . I shall give the name of 'sensing' to the act or process of being immediately aware of a particular as having certain qualities... I shall call any particular which is capable of being sensed a 'sensible' ... I can now define the word 'sense-datum'. I shall say that a sensible is a 'sense-datum' for a certain person on a certain occasion, if and only if he is sensing that sensible on that occasion." 
Was uns in einer Beobachtung gegeben ist - unmittelbar oder mittelbar - ist Inhalt der Beobachtung. Solche Phainomena müssen also spezielle Sachverhalte unter den Phainomena nach (b) sein. Dann gilt auch das dort Gesagte. Ferner haben wir in 4.2 bei der Diskussion des zweiten Arguments gesehen, daß eine brauchbare Abgrenzung unmittelbar beobachteter von nur mittelbar beobachteten Sachverhalten nicht in Sicht ist.

d) Phainomena als empirischẻ Sachverbalte, bzgl. deren Bestehen oder Nichtbestehen wir uns nicht täuschen können

Das sind im Sinne der oben referierten Argumente Phainomena im Sinne von (a), wobei noch einmal zu betonen ist, daß ich mich zwar jetzt bzgl. der Geltung eines Satzes wie "Ich beobachte (jetzt), daß p“ nicht täuschen kann, wohl aber früher und später. Solche Phainomena kommen im Illusionsargument vor, und man bestimmt Sinnesdaten unter Bezugnahme auf die zweite oben angegebene Version dieses Arguments oft als Objekte, die genau dann existieren, wenn sie mir erscheinen und die immer so beschaffen sind, wie sie mir erscheinen; als Objekte also, bzgl. derer es keine Illusionen gibt. Solche Sinnesdaten sollen daher eine Art Erscheinungsbilder sein. Phänomenale Attribute sind danach Attribute solcher Sinnesdaten, mit denen sie erscheinen, und phanomenale Sachverinalte sind Sachverhalte c'es Zutreffens oder Nichtzutreffens phänomenaler Attribute auf Sinnesdaten.

Solche Sinnesdaten sind aber merkwürdige Gebilde, denn sie können verborgene Eigenschaften haben. Darauf haben $\mathrm{H}$. H. Price und R. Chisholm hingewiesen: ${ }^{11}$ Ein Tiger kann als gestreift wahrgenommen werden, ohne daß wahrgenommen wird, wieviele Streifen er hat. In phänomenalistischer Sprechweise: Das Erscheinungsbild des Tigers ist gestreift, aber es gibt keine Zahl n, so daß es n Streifen hat. Der „,phänomenale“ Tiger hat also eine verborgene Eigenschaft, nämlich eine bestimmte Anzahl von Streifen. Das widerspricht aber der obigen Bestimmung phänomenaler Entitäten und ihrer Attribute. Ein weiteres Argument gegen die Annahme solcher Sinnesdaten ist folgendes: Wenn Sinnesdaten oder phänomenale Attribute als identisch erscheinen, so sind sie es auch. Nun kommt es aber vor, daß wir drei Farb-

${ }^{11}$ Vgl. Price (41) und Chisholm (42). - Ayers Einwand gegen dieses Argument in (63), S. $92 \mathrm{ff}$. ist unbrauchbar. 
töne $F_{1}, F_{2}, F_{3}$ beobachten, so daß $F_{1}$ und $F_{2}$ sowie $F_{2}$ und $F_{3}$ jeweils ununterscheidbar sind, während wir $F_{1}$ und $F_{3}$ als verschieden ansehen. Für die phänomenalen Farben würde also gelten $F_{1}=F_{2}$ und $F_{2}=F_{3}$, aber nicht $F_{1}=F_{3}$, im Widerspruch zur Transitivität der Identität.

Dieser ganze Unsinn ergibt sich aus den Fehlern im Illusionsargument. Aus $(\alpha)$,,Es erscheint mir, als ob der Tiger gestreift sei“ folgt $(\beta)$,Es erscheint mir, als gäbe es eine Zahl n, so daß der Tiger $n$ Streifen hat", aber nicht $(\gamma)$,Es gibt eine Zahl n, so daß es mir erscheint, als hätte der Tiger $\mathrm{n}$ Streifen". Wenn ich dagegen von $(\alpha)$ übergehe zu $(\beta)$,,Der phänomenale Tiger ist gestreift", so folgt daraus $\left(\gamma^{\prime}\right)$,Es gibt eine Zahl n, so daß der phänomenale Tiger $n$ Streifen hat" - im Widerspruch zur Falschheit von $(\gamma)$. Ebenso folgt aus $(\delta)$, Es erscheint mir, als ob $F_{1}=F_{2}$ sei, und es erscheint mir, als ob $F_{2}=F_{3}$ sei“ nicht $(\varepsilon)$,Es erscheint mir, als ob $F_{1}=F_{3}$ sei." man jedoch von $(\delta)$ übergeht zu $\left(\varepsilon^{\prime}\right)$,,Die phänomenalen Farben $F_{1}$ und $F_{2}$ sowie $F_{2}$ und $F_{3}$ sind identisch", so folgt daraus im Widerspruch zur Falschheit von $(\varepsilon)$,Die phänomenalen Farben $F_{1}$ und $F_{3}$ sind identisch."

\section{e) Pbainomena als Aspekte}

Nach dieser Bestimmung erscheint mir, wenn es mir erscheint, als ob ein (an sich runder) Tisch elliptische Form habe, nicht der runde Tisch, sondern ein elliptischer Aspekt des runden Tisches. Bei einer Illusion, und daher nach den Argumenten der Gleichartigkeit von und der Kontinuität zwischen korrekten und trügerischen Erfahrungen auch generell, nehme ich nicht Dinge, sondern nur Dingaspekte wahr und diese werden als Sinnesdaten aufgefaßt. ${ }^{13}$

Man kann aber den Satz „Es erscheint mir, als ob b die Eigenschaft $F$ hat" nicht deuten als ,Mir erscheint ein F-Aspekt von b“. Aspekte sind Eigenschaften, die Dinge für einen Beobachter zu haben scheinen, nicht aber Entitäten, die uns erscheinen. Definiert man: Die Eigenschaft $\mathrm{F}$ ist (für die Person a) ein Aspekt von b genau dann, wenn

${ }^{12}$ Aus ,Ich glaube, daß $F_{1}=F_{2}$ ist, und ich glaube, daß $F_{2}=F_{3}$ ist" folgt nach den G-Axiomen in 1.1 ,Ich glaube, daß $F_{1}=F_{3}$ ist". Ein entsprechendes Gesetz gilt für Sachverhalte des Erscheinens-als-ob aber nicht.

${ }^{13}$ Eine solche Auffassung scheint z. B. G. E. Moore in (42), S. $630 \mathrm{ff}$. zu vertreten. 
gilt $\mathrm{S}(\mathrm{a}, \mathrm{F}(\mathrm{b}))$, so sind solche ",Aspekte“ normale Dingeigenschaften. ${ }^{14}$ Die Rede von ,Sinnesdaten“" ist also wieder sinnlos oder irreführend.

f) Phainomena als Empfindungen

Mit dieser Auffassung verbindet sich meist ein Atomismus, nach dem es einfachste sinnliche Empfindungen (Farb-, Ton-, Tastempfindungen etc.) gibt, aus denen sich alle Empfindungen und Erfahrungen zusammensetzen. Ein solcher Empfindungsatomismus widerspricht aber den Ergebnissen der Wahrnehmungspsychologie, nach der Wahrnehmungen nicht durch eine Synthese einfacher Empfindungen entstehen, sondern solche Empfindungen umgekehrt durch einen Prozeß der Analyse und Abstraktion aus gestalthaft-komplexen Wahrnehmungen gewonnen werden, so daß es - da sich dieser Prozeß prinzipiell unbeschränkt fortsetzen läßt - keine atomaren Empfindungen gibt. Da das phänomenalistische Reduktionsprogramm aber nicht die Behauptung impliziert, komplexe Empfindungen ließen sich auf elementare zurückführen, sehen wir hier von diesem Atomismus ab. Wir stehen dann wieder vor der alten Frage: Ist eine Empfindung der Sachverhalt, daß ich etwas empfinde - also ein Phainomenon im Sinne von (a) - oder das, was ich empfinde, also ein Phainomenon im Sinne von (b)? In beiden Fällen enthielte eine Sprache über solche Empfindungen dann wieder die physikalische Sprache $\Phi$, so daß das phänomenalistische Übersetzungsprogramm trivialisiert würde. Es gibt aber Aussagen über Empfindungen im ersteren Sinn wie „Ich empfinde Schmerzen" oder "Ich habe eine Rotempfindung",15 die nicht nach dem Schema $S(a, p)$ konstruiert sind, in denen also nicht von physischen Dingen und ihren Attributen die Rede ist. Der Aufbau einer phänomenalistischen Sprache mit solchen Sätzen würde jedoch erhebliche Schwierigkeiten machen, denn unsere Sprache über Empfindungen ist aus der Sprache über Physisches abgeleitet. In diesem Sinn sagt W.V. Quine: "Talk of subjective sense qualities comes

${ }^{14}$ Bezeichnet man $F$ als ,Aspekt" von $b$ für $a$, wenn gilt $\vee x(x=b \wedge$ $\mathrm{S}(\mathrm{a}, \mathrm{F}(\mathrm{x})))$, so gilt im Gegensatz zur Definition im Text, daß a $\mathrm{F}$ für eine Eigenschaft des Objekts hält, das tatsächlich b ist, wenn $F$ ein Aspekt von b für $a$ ist. (Vgl. dazu Kutschera (76), 95 ff.) Auch nach dieser Definition sind jedoch ,Aspekte“ normale Dingeigenschaften.

15 Eine Rotempfindung kann auch durch einen Druck auf das geschlossene Auge erzeugt werden, läßt sich also nicht immer durch einen Satz der Form „Es erscheint mir, als ob hier etwas Rotes wäre" beschreiben. 
mainly as a derivative idiom. When one tries to describe a particular sensory quality, he typically resorts to reference to public things -describing a color as orange or heliotrope, a smell as like that of rotten eggs. In this there is little cause for wonder. Each of us learns his language from other people, through the observable mouthing of words under conspicuously intersubjective circumstances. Linguistically, and hence conceptually, the things in sharpest focus are the things that are public enough to be talked of publicly, common and conspicuous enough to be talked of often, and near enough to sense to be quickly identified and learned by name; it is to these that words apply first and foremost." Und zum Begriff des Sinnesdatums: "Impressed with the fact that we know external things only mediately through our senses, philosophers from Berkeley onward have undertaken to strip away the physicalistic conjectures and bare the sense data. Yet even as we try to recapture the data in all their innocence of interpretation, we find ourselves depending upon sidelong glances into natural science. We may hold with Berkeley, that the momentary data of vision consist of colors disposed in a spatial manifold of two dimensions; but we come to this conclusion by reasoning from the bidimensionality of the ocular surface or by noting the illusions which can be engendered by twodimensional artifacts such as paintings and mirrors or, more abstractly, simply by noting that the interception of light in space must necessarily take place along a surface. Again we may hold that the momentary data of audition are clusters of components each of which is a function of just two variables, pitch and loudness; but not without knowledge of the physical variables of frequency and amplitude in a stimulating string."'16

Diese Hinweise Quines, daß ,man seine Sinnesdaten am besten identifiziert, indem man sie an äußeren Dingen spiegelt ${ }^{\text {" }},{ }^{17}$ implizieren zwar nicht, daß es unmöglich wäre, eine phänomenalistische Sprache als Sprache über Empfindungen aufzubauen, die $\Phi$ nicht enthält, sie zeigen aber die Schwierigkeiten, denen ein solcher Versuch begegnen würde. Eine derartige Sprache über Sachverhalte des Empfindens, die nicht Phainomena im Sinn von (a) sind, wäre die einzige,

\footnotetext{
${ }^{16}$ Quine (60), S. 1 f. - Vgl. dazu auch Ryle (49), S. 20 ff., 203, 236 f.

${ }^{17}$ Quine (60), S. 1.
} 
die als Sprache $\Psi$ der phänomenalistischen These $P N$ infrage käme. ${ }^{18}$ Solche Empfindungen sind aber nicht etwas, das in einer Beobachtung (,unmittelbar") gegeben wäre, oder etwas, das Gegenstand von Beobachtungen, Empfindungen oder Vorstellungen wäre. Der erkenntnistheoretische Phänomenalismus, nach dem die Gegenstände unserer Erfahrung Sinnesdaten sind, ist also bei dieser Deutung nicht haltbar. Empfindungen hat man; man erfährt sie nicht.

\subsection{Kritik der Reduzierbarkeitsthese}

Für das folgende setzen wir voraus, $\Psi$ sei eine wohldefinierte Sprache über Empfindungen, die keine Ausdrücke für Physisches enthält. Wir wollen zeigen, daß das phänomenalistische Übersetzungsprogramm nach $\mathrm{PN}$ sich auch bei dieser großzügigen Vorgabe nicht durchführen läßt. ${ }^{19}$

a) Ein Satz wie (1), ,Zum Zeitpunkt t gibt es am Ort $x$ ein Ding der Beschaffenheit F“ kann nicht übersetzt werden durch einen Satz ,Zur Zeit $t$ habe ich bei $x$ Empfindungen der Art G", bzw. allgemeiner (2) „Es gibt eine Person, die zur Zeit $t$ bei $x$ eine Empfindung der Art G hat", da (1) richtig, (2) aber falsch sein kann, wenn sich zur Zeit $t$ bei $x$ niemand befindet. ${ }^{20}$

${ }^{18}$ Der phänomenalistischen Sprache in Carnap (28) liegen „Elementarerlebnisse" zugrunde, die als vollständige momentane Querschnitte durch den Erlebnisstrom eines Subjekts bestimmt werden. Es wird nicht klar, ob diese Elementarerlebnisse sich ohne Verwendung der physikalischen Sprache $\Phi$ beschreiben lassen. Die Grundobjekte der phänomenalistischen Sprache von Goodman in (51) sind nicht Empfindungen, sondern Qualia, Attribute' von Empfindungen. Diese Festlegung der Qualia ist allerdings bei Goodman nicht eindeutig, da er auch Eigenschaften von Dingen, die diese zu haben scheinen, als Qualia bezeichnet. Auch hier also wieder die Verwechslung der Phainomena im Sinne von (a) und von (b)!

${ }^{19}$ Vgl. dazu auch Stegmüller (58).

${ }^{20}$ Ersetzt man (2) durch ,Alle Personen, die sich zur Zeit $t$ bei $x$ befinden, haben eine Empfindung der Art G", so existiert überall dort, wo keine Beobachter sind, alles. Daher wären entsprechende Modifikationen dieser Bedingung vorzunehmen, wie sie im folgenden für (2) besprochen werden, und es ergäben sich dabei entsprechende Probleme. 
Auf diese Schwierigkeit der Identifikation von Dingen mit aktuellen Emptindungen (esse est percipi), daß danach nicht wahrgenommene Dinge nicht existieren, war schon Berkeley gestoßen. Er schreibt: "It will be objected, that from the foregoing principles it follows, things are every moment annihilated and created anew. The objects of sense exist only when they are perceived: the trees therefore are in the garden, or the chairs in the parlour, no longer than while there is somebody by to perceive them. Upon shutting my eyes, all the furniture in the room is reduced to nothing, and barely upon opening them it is again created." 21

Berkeley macht dann den Vorschlag, Existenzsätze für Dinge nach dem Muster (3) „,Wenn sich zum Zeitpunkt $t$ jemand bei $\mathrm{x}$ befindet, so hat er Empfindungen der Art G", so zu übersetzen: "The table I write on, I say, exists, that is, I see and feel it; and if I were out of my study I should say it existed, meaning thereby that if I was in my study I might perceive it, or that some other spirit actually does perceive it." 22

Berkeley hat jedoch dieses Übersetzungsschema, das heute meist von Phänomenalisten verwendet wird, nicht weiter verfolgt. Für ihn waren Dinge Bündel von Ideen, und da Ideen nur als aktuell ,,wahrgenommene" Entitäten im Bewußtsein existieren, gilt das auch für Dinge. Daher sagt er: Ideen, und damit Dinge, werden immer wahrgenommen, wenn nicht von Menschen, so von Gott. Er bleibt also bei der Formel ,esse est percipi“". J. St. Mill führte mögliche Empfindungen ein, so daß auch Dinge existieren, denen nur mögliche, nicht aber tatsächliche Empfindungen entsprechen. Man könnte in diesem Sinn sagen, (1) sei äquivalent mit (4) ,Es ist möglich, daß es eine Person gibt, die zur Zeit $\mathrm{t}$ bei $\mathrm{S}$ eine Empfindung der Art $\mathrm{G}$ hat." Aber welcher Möglichkeitsbegriff soll hier zugrunde gelegt werden? Der Begriff der logischen Möglichkeit scheidet aus, denn sonst wäre die $\mathrm{Ne}$ gation von (1) äquivalent mit dem Satz. „Es ist logisch notwendig, daß (2) nicht gilt, d. h. ein kontingenter Satz wäre mit einem logisch determinierten Satz analytisch äquivalent - die Äquivalenz zwischen (1) und (4) soll ja nach PN analytisch gelten. Also müßte man einen naturwissenschaftlichen Möglichkeitsbegriff verwenden. Naturwis-

\footnotetext{
${ }^{21}$ Berkeley (T), 134.

${ }^{22}$ Berkeley (T), 114. Vgl. a. (T), 136, 141.
} 
senschaftlich ist es aber nicht möglich, daß sich z. B. im Innern der Sonne ein Beobachter befindet, der Empfindungen vom Sonnenkern hat, so daß also der Satz ,Der Sonnenkern hat eine Temperatur von 20 Millionen Grad" nach dem angegebenen Übersetzungsschema falsch wäre. ${ }^{23}$ Entsprechendes gilt, wenn man (2) durch den Konditionalsatz (3) ersetzt. ${ }^{24}$ Es würde eher gelten ,, Wenn eine Person sich im Sonneninnern befände und dort Beobachtungen anstellen könnte, so wäre dort die Temperatur wesentlich niedriger als 20 Millionen Grad", als daß diese Person dann Wärmeempfindungen hätte, die einer Temperatur von 20 Millionen Grad entsprechen.

b) Selbst wenn man von der Schwierigkeit (a) absieht, steht man vor einem weiteren Problem: Die Wahrheit von (2) ist keine hinreichende Bedingung für die Wahrheit von (1), denn (1) läßt sich erst dann behaupten, wenn noch weitere Bedingungen erfüllt sind, nach denen sich von anderen, benachbarten Beobachtungsstandpunkten gewisse andere Sinnesdaten ergeben, beim Berühren des beobachteten Objekts gewisse Tastempfindungen usw. Wir erhielten also anstelle von (2) eine evtl. unendliche Konjunktion von Sätzen der Form (2) als hinreichende Bedingung für (1). Diese Bedingung wird aber für das Bestehen von (1) nicht notwendig sein, denn derselben physikalischen 'Tatsache kann eine Vielzahl von Enpfindungen entsprechen. Die Gestalt eines Körpers läßt sich z. B. ebenso rein optisch wie allein durch Tasten feststellen. Man müßte also eine evtl. wieder unendliche Disjunktion - nennen wir sie (5) - solcher Konjunktionen bilden, um die faktische Äquivalenz mit (1) zu sichern. Das bedeutet aber, daß sich evtl. keine endlichen phänomenalistischen Sätze als Übersetzungen von Dingsätzen angeben lassen..$^{25}$

${ }^{23}$ Vgl. zu diesen Überlegungen auch das Dr. Crippen-Beispiel von Ayer in (63), S. $150 \mathrm{ff}$. Price nimmt zur Überwindung der Schwierigkeit des esse est percipi sogar unbeobachtete Sinnesdaten an. Da das dem Begriff des Sinnesdatums als einer Empfindung widerspricht, wollen wir darauf nicht näher eingehen. Auch Russell hat in (18) für seine ,,sensibilia“ eine ähnliche Position vertreten, sie jedoch später aufgegeben.

${ }^{24} \mathrm{Zu}$ den Wahrheitsbedingungen für Konditionalsätze vgl. wieder Kutschera (76), Kap. 3.

${ }^{25}$ Stegmüller meint in (58), S. 78 f., daß ein Existenzsatz der Dingsprache wie (1) abermals eine evtl. unendliche Disjunktion von Konjunktionen erfordere, die den einzelnen konkreten Dingen mit der Eigenschaft $F$ entspre- 
c) Sieht man auch von dem Problem (b) ab, so muß man, um Sinnestäuschungen auszuschließsen, doch fordern, daß die fraglichen Beobachtungen unter Normalbedingungen erfolgen, daß also keine Illusionen vorliegen. (2) wäre also zu ersetzen durch den Satz (6) „Es gibt einen Beobachter a, der zur Zeit $t$ bei $x$ ist, dessen Beobachtung unter normalen Bedingungen stattfindet und der eine Empfindung der Art $G$ hat. “ Der Satz ,Die Beobachtung von a erfolgt unter normalen Bedingungen" ist nun aber kein Satz der Sprache $\Psi$; er wäre also selbst in einen phänomenalistischen Satz zu übersetzen, der besagt, daß sich zur Zeit $t$ bei $S$ ein zweiter Beobachter, $b$, befindet, der vom ersten Beobachter, a, und den Umständen von dessen Beobachtung gewisse Empfindungen hat. Aber auch für $b$ ist dann wieder die Normalbedingung zu fordern, usw.

d) Wenn man nun auch von dieser Schwierigkeit absehen wollte, so bliebe doch noch die Aufgabe, die Bedingung ,, a befindet sich zur Zeit $\mathrm{t}$ am Ort x" in (2) in die phänomenalistische Sprache zu übersetzen. Denn die Orts- und Zeitangaben nehmen ja auf physikalische Orts- und Zeitbestimmungen Bezug. I. Lewis hat vorgeschlagen, ${ }^{26}$ diese Bedingung zu ersetzen durch ,a glaubt sich zur Zeit $\mathrm{t}$ bei $\mathrm{x}$ zu befinden." Aber erstens ist das kein Satz der phänomenalistischen Sprache, da in ihm der physikalische Teilsatz ,, a befindet sich zur Zeit $\mathrm{t}$ bei $\mathrm{x}$ " vorkommt, und zweitens wäre auch hier wieder zu fordern, $\mathrm{da}$ a seine Überzeugung unter Normalbedingungen gewinnt, und das führt dann wieder auf das Problem (c) zurück. H. H. Price schlägt hingegen vor, ${ }^{27}$ die physikalischen Orts- und Zeitbestimmung so durch eine phänomenalistische zu ersetzen, daß der Zeitpunkt $t$, ausgehend vom gegenwärtigen Zeitpunkt durch den dazwischen liegenden Erlebnisstrom charakterisiert wird, und daß der Ort x, ausgehend vom gegenwärtigen Ort, bestimmt wird durch die Erlebnisse

chen, weil diese verschiedenen Dinge kraft ihrer, in (1) nicht erwähnten verschiedenen zusätzlichen Eigenschaften ganz verschiedene Sinnesdaten hervorrufen könnten. Zur Feststellung der Eigenschaft $G$ müssen die entsprechenden Sinnesdaten aber nicht in allen Details festliegen. Von anderen Schwierigkeiten einmal abgesehen ließe sich z. B. der Satz ,Zur Zeit t gibt es in $x$ ein blaues Ding“" wiedergeben durch „Es gibt einen Beobachter, der zur Zeit $t$ bei $x$ ist und einen Sinneseindruck ,Blau' hat."

${ }^{26} \mathrm{Vgl}$. I. Lewis (45), S. $179 \mathrm{ff}$.

${ }^{27}$ Vgl. Price (40), S. $183-188$. 
(die kinästhetischen Empfindungen und dergl.), die man hätte, wenn man sich nach $x$ begeben würde. Wie aber A. J. Ayer hervorgehoben hat, enthielte eine solche phänomenalistische Orts- und Zeitangabe eine Fülle faktischer Informationen, die über (1) hinausgehen, so daß von einer Synonymität der physikalischen und der phänomenalistischen Orts- und Zeitangaben nicht die Rede sein könnte. Außerdem wären für phänomenalistische Orts- und Zeitangaben auch immer wieder die Normalbedingungen zu fordern. Das gilt auch für den Vorschlag Ayers, die Orts- und Zeitbestimmungen durch Empfindungen zu geben, die sich auf Uhrablesungen und Orientierungen beziehen. ${ }^{28}$

e) Ferner bliebe auch noch folgende Schwierigkeit: Für mich ist eine Aussage der Gestalt ,, a hat einen Sinneseindruck der Art G“ nur dann eine phänomenalistische Aussage, wenn ich mit a identisch bin. Insofern wäre (2) zu ersetzen durch, ,Wenn ich mich zur Zeit $t$ in $x$ befinde, habe ich einen Sinneseindurck der Art G." Dieser Satz muß aber nach (c) durch einen Satz ersetzt werden, in dem auch von anderen Beobachtern die Rede ist. So ein Satz ist aber für mich kein phänomenalistischer Satz mehr.

f) Für Sätze der Dingsprache gilt die zweiwertige Logik, d. h. alle (Behauptungs-)Sätze dieser Sprache sind wahr oder faisch. Wenn man aber den Satz (1) durch (3) übersetzt, so ergibt sich eine dreiwertige Logik: (1) ist wahr, wenn für alle Personen a gilt: wenn sich a in t bei $\mathrm{x}$ befindet, so hat a Empfindungen der Art G“; (1) ist falsch, wenn für alle Personen a gilt: wenn sich a in $t$ bei $x$ befindet, so hat a keine Empfindungen der Art G; und (1) ist indeterminiert, wenn einige Personen in $t$ bei $x$ G-Empfindungen haben, einige aber nicht. Selbst wenn alle Leute (unter Normalbedingungen) an denselben RaumZeit-Stellen dieselben Sinnesempfindungen hätten - was kaum anzunehmen ist - so wäre die Zweiwertigkeit der Sätze der physikalischen Sprache nach PN dann nur eine empirische Tatsache; diese Sätze hätten also nicht denselben Sinn wie in $\Phi$.

g) Endlich wäre nach PN jeder Satz der normalen Sprache ein Satz über die Empfindungen des Sprechers. Es gäbe dann aber keine gemeinsame intersubjektive Sprache. Wenn a sagt ,Der Mond ist rund“, so sagt er etwas über seine Empfindungen, und wenn b sagt ,,Der Mond ist nicht rund“, so sagt er etwas über seine. Beide Sätze wider-

${ }^{28} \mathrm{Vgl}$. Ayer (63), S. $156 \mathrm{ff}$. 
sprechen einander also nicht, und da jeder seine Empfindungen am besten beurteilen kann, wäre eine Diskussion über die Wahrheit von Sätzen sinnlos. Frege sagt: ,, Wenn jeder mit dem Namen ,Mond“ etwas Anderes bezeichnete, nämlich eine seiner Vorstellungen, etwa so, wie er mit dem Ausrufe, au!' seinen Schmerz äußerte, so wäre freilich die psychologische Betrachtungsweise gerechtfertigt; aber ein Streit über die Eigenschaften des Mondes wäre gegenstandslos; der Eine könnte von seinem Monde ganz gut das Gegenteil von dem behaupten, was der Andere mit demselben Rechte von seinem sagte. Wenn wir nichts erfassen könnten, als was in uns selbst ist, so wäre ein Widerstreit der Meinungen, eine gegenseitige Verständigung unmöglich, weil ein gemeinsamer Boden fehlte, und ein solcher kann keine Vorstellung im Sinne der Psychologie sein."29

Diese Argumente zeigen schon, daß selbst von einer W-Reduzierbarkeit der physikalischen Sprache $\Phi$ auf die phänomenalistische $\Psi$ im Sinne von D 3.3-3 nicht die Rede sein kann. Erst recht ist also die These PN nicht haltbar. Selbst wenn sich die Schwierigkeiten (a) bis (d) irgendwie überwinden ließen, so hätte doch die Diskussion gezeigt, daß zwischen (1) und den vorgeschlagenen Übersetzungen keine analytische Äquivalenz besteht. Die naturgesetzlichen Aussagen, auf die in (a) Bezug genommen wird, die notwendigen und hinreichenden Bedingungen nach (b), die Normalbedingungen nach (c) und die phänomenalistischen Orts- und Zeitbestimmungen nach (d) sind rein empirische Bedingungen für die Adäquatheit der Übersetzungen; sie können also die These PN von der Bedeurungsgleichheit zwischen (1) und einer phänomenalistischen Übersetzung nicht rechtfertigen. Zusätzlich bleiben auch gegen PN die Argumente (e) bis (g) bestehen. Wir können also feststellen, daß die These PN und damit der Phänomenalismus nicht haltbar ist. ${ }^{30}$

${ }^{29}$ Frege (GGA) I, XVIIIf.

${ }^{30}$ Für weitere, allerdings nicht immer stichhaltige oder schwerwiegende Einwände gegen den Phänomenalismus vgl. Stegmüller (58) und Ayer (63), $125 \mathrm{ff}$. Solche Einwände sind z. B.: 1. Physikalische Sätze sind nicht subjektiv entscheidbar, wohl aber phänomenalistische Sätze. Also lassen sich physikalische Sätze nicht analytisch in phänomenalistische übersetzen (Ayer). Die Behauptung, alle phänomenalistischen Sätze seien problemlos, ist viel zu stark, das gilt nur für solche Sätze, die sich auf gegenwärtige Empfindungen beziehen. 2. Ein beobachteter physikalischer Vorgang, dem tatsächliche Empfindungen entsprechen, kann eine unbeobachtete Ursache haben, der 
Im Dickicht der phänomenalistischen Literatur gibt es nur zwei Arbeiten, die sich durch Präzision und Ausführlichkeit auszeichnen: R. Carnaps „Der logische Aufbau der Welt" (1928) und N. Goodmans ,,The Structure of Appearance“ (1951). Auf beide Arbeiten können wir hier nicht näher eingehen. Wir beschränken uns auf folgende Hinweise:

1. Carnaps „Aufbau“ stellt nur eine Skizze dar, in der lediglich die Begriffe der ersten Stufe exakt definiert werden, während die Begriffe der höheren Stufen nur angedeutet sind. Nun geht es aber auf der ersten Stufe lediglich darum, das phänomenalistische Vokabular auf ein einziges Grundprädikat zu reduzieren - ein Bestreben, das für die Begründung der Übersetzbarkeitsthese PN nichts beiträgt. Von einer Definition von Termen von $\Phi$ durch solche von $\Psi$ kann auf den höheren Stufen des Carnapschen Begriffssystems nicht die Rede sein. Vielmehr werden Sätzen von $\Phi$ Sätze von $\Psi$ zugeordnet, die aufgrund teilweise fragwürdiger - empirischer Annahmen denselben Wahrheitswert haben. So kann man aber die These PN nicht begründen. ${ }^{31}$

2. Goodman unternimmt in (51) nicht den Versuch, die Übersetzbarkeitsthese zu beweisen. Er entwickelt im 1. Kapitel seines Buches einen sehr liberalen Reduktionsbegriff, diskutiert jedoch auch im Sinn dieses Begriffs nicht die Frage der Reauzieriarkeit physikalisciner Aussagen auf phänomenalistische. Gerade die präzisen Definitionen und Axiome Goodmans zeigen, wie viele Voraussetzungen dafür noch fehlen. Diese Präszision beschränkt sich aber auf die formale Ebene. Es wird keine inhaltlich klare Interpretation der phänomenalistischen Sprache angegeben; die Natur der Grundobjekte, der Qualia, bleibt weitgehend im Dunkeln.

Auch diese beiden Arbeiten tragen also letztlich nichts zur Begründung des Phänomenalismus bei.

also nur mögliche Empfindungen entsprechen. Mögliche Empfindungen können aber nicht aktuelle verursachen. (Vgl. Stout (38), Hardie (45) und Ayer (63), 143 ff.) - Wenn aber der Satz A die Ursache, der Satz B die Wirkung ausdrückt und A' und B' die zugeordneten phänomenalistischen Sätze sind, so sind nach (a) sowohl A' wie B' Sätze über mögliche Empfindungen, und zwischen $A^{\prime}$ und $B^{\prime}$ kann ein entsprechendes Verhältnis gesetzmäßiger Folge bestehen, wie zwischen A und B. Richtig ist jedoch, daß eine Geltung von $\mathrm{PN}$ implizieren würde, daß allen physikalischen Gesetzen Gesetze im Bereich der Empfindungen entsprächen, während solche Gesetze nirgends in Sicht sind. 


\subsection{Die Intentionalität des Erlebens}

Man könnte nun fragen: Ist es denn nicht unbestreitbar, daß all unsere Aussagen über die Welt sich letztlich auf sinnliche Wahrnehmungen gründen, d. h. auf Sinnesempfindungen? Und ist es dann nicht eine unabweisbare Aufgabe einer Theorie empirischer Erkenntnis zu zeigen, wie wir von Aussagen über Sinnesempfindungen zu Aussagen über die Dinge der Außenwelt kommen? Und wie soll diese Aufgabe anders als durch das phänomenalistische Reduktionsprogramm lösbar sein? In diesen Fragen drückt sich aber schon die Konfusion aus, die dem Phänomenalismus zugrunde liegt. Die sinnliche Wahrnehmung läßt sich eben nicht so beschreiben, daß wir gewisse Sinnesempfindungen haben, Farb-, Ton-, Tastempfindungen, etc. Sinnliche Wahrnehmungen haben, wie das gegenüber der Empfindungspsychologie vor allem F. Brentano und $\mathrm{E}$. Husserl betont haben, intentionale Struktur, d. h. sie sind Wahrnehmung von etwas, sie haben einen Gegenstand. ${ }^{32}$ Wahrnehmungen werden durch Sätze beschrieben wie „Ich sehe eine Katze, die auf der Fensterbank sitzt“, „Ich höre, daß im Nebenzimmer jemand "Yesterday" singt", oder, wenn wir im Sinne von 3.1 von den durch solche Erfolgsverben implizierten Tatsachenbehauptungen absehen, durch Sätze wie „Ich beobachte, daß eine Katze auf der Fensterbank sitzt", bzw. „,Es erscheint mir, als ob eine Katze auf der Fensterbank sitzt". Wahrnehmungen oder Beobachtungen werden hingegen nicht adäquat durch Aussagen wiedergegeben wie ,Ich habe graue und braune Farbempfindungen, die sich in meinem Gesichtsfeld so und so verteilen" oder "Ich habe eine Folge gedämpfter Tonempfindungen d-c-c.." ". Tatsache ist, daß bei Beobachtungen Sinnesempfindungen in aller Regel gar keine Rolle spielen. Ich kann die Katze am Fenster sitzen sehen, ohne irgendwelche Empfindungen angeben zu können, die ich dabei habe. In der Wahrnehmung sind also primär Gegenstände oder Sachverhalte gegeben, nicht aber Empfindungen.

${ }^{31}$ Zur Kritik am ,Aufbau“ vgl. Carnaps Vorwort zur 2. Auflage sowie Goodman (51).

${ }^{32}$ Es war das Verdienst Fichtes, zuerst klar und nachdrücklich auf die intentionale Struktur der Erfahrung hingewiesen zu haben. Bei ihm ist sie die Grundstruktur alles (,empirischen“) Bewußtseins. Vgl. dazu auch den Abschnitt 8.7. 
Wenn wir ein sinnliches Erleben in der Form ausdrücken ,,a sieht, daß p“ oder ,,a hört, daß p“ etc., so stellen wir es als eine Beziehung zwischen einem erlebenden Subjekt a und einem erlebten Gegenstand oder Inhalt - hier dem Sachverhalt p - dar. Ein solches Erleben ist also gekennzeichnet 1. durch seinen Gegenstand (seinen Inhalt), 2. durch das erlebende Subjekt und 3. durch die Art und Weise des Erlebens, die spezielle Natur der Relation. Im Erleben sind wir normalerweise auf den Gegenstand gerichtet. Brentano spricht dann von einer intentio recta. Wir selbst als Subjekt, die Tatsache, daß wir es sind, die den Gegenstand erleben, und die Art des Erlebens sind in dieser gegenständlichen Einstellung nicht Themen des Erlebens. In dieser Einstellung ist es zwar mitgegeben, daß ich es bin, der den Gegenstand erlebt, und wie ich ihn erlebe, aber nur mehr oder minder undeutlich. Wenn ich darauf reflektiere, mache ich das erlebende Subjekt oder die Art und Weise des Erlebens zum Thema meiner Aufmerksamkeit; Brentano spricht hier von einer intentio obliqua. Empfindungen kommen erst ins Spiel, wenn von der Art und Weise des Erlebens die Rede ist. Empfindungen im normalen Sinn des Wortes sind nicht Erlebnisinhalte, sondern Erlebnisweisen. Empfindungen erleben wir nicht, sondern wir haben sie. Mit Empfindungswörtern charakterisieren wir primär die Art und Weise eines Erlebens, nicht seinen Gegenstand. Und wenn es sich nicht nur um gefühlsmäßige Reaktionen auf Beobachtetes handelt, wie in dem Satz „Entsetzt sah Fritz, wie sich der Mauerhaken löste", sondern um Empfindungen, welche die Beobachtung selbst kennzeichnen wie in „Fritz hatte den Eindruck, als schlüge in der Ferne ein Hund an", so charakterisieren wir diese Empfindung meist vom Inhalt der Beobachtung her. Daher erscheint es als verfehlt, die Sprache über Gegenstände des Erlebens aus der Sprache über Empfindungen ableiten zu wollen. Darauf haben wir schon in 5.3 unter Bezugnahme auf Aussagen von Quine hingewiesen.

Nicht alles Erleben, Fühlen, Streben etc. ist intentional, d. h. auf einen Gegenstand gerichtet. ${ }^{33}$ Ein nichtintentionales Erleben drücken wir mit Sätzen aus wie ,Mir ist unwohl", ,Mir ist warm", ,Ich bin müde“, „Ich bin zufrieden“, ,Ich habe Schmerzen“, ,Ich bin froh"

${ }^{33}$ „Erleben“, ,Streben“, „Erfahren“ bezeichnen allerdings meist intentionale Akte oder Dispositionen. Insofern ist die Rede vom ,, nichtintentionalen Erleben" schief, aber es gibt hier keine feste Terminologie. 
etc. In solchen Fällen vor allem reden wir von Empfindungen. Dabei haben Empfindungen wiederum nicht, oder nicht direkt, mit äußerer Erfahrung zu tun.

Zwischen beobachtbaren Eigenschaften und Empfindungsweisen gibt es natürlich Beziehungen und Verwandtschaften. Farben kommen z. B. nicht nur als Körperfarben an der Oberfläche von Körpern vor, sondern auch als freie Farben, als Raum- oder Lichtfarben, wie z. B. das Blau des Himmels. Wenn ich in den Himmel schaue, kann ich nicht behaupten, ich nähme etwas wahr (,,den Himmel"), und dies etwas sei blau. In diesem Fall könnte man sagen, man habe eine BlauEmpfindung. Und da das Blau des Himmels mit der blauen Farbe eines Balls Verwandtschaft hat, so liegt es nahe, zu behaupten, auch bei der Wahrnehmung dieses Balls hätten wir eine Blauempfindung. Körperfarben werden aber, wie z. B. das Phänomen der Farbkonstanz zeigt, anders wahrgenommen als Raum- oder Lichtfarben. Unser Gehirn verrechnet die Oberflächenfarben aus einfallendem und reflektiertem Licht, so daß uns z. B. ein Stück weißes Papier unter einem roten Sonnenschirm immer noch als weiß erscheint, obwohl es rotes Licht reflektiert. Wenn dagegen das Auge weder Lichtquellen noch andere Körper wahrnehmen kann, aus denen sich die Farbe des einfallenden Lichts ergibt, erscheint uns das Papier als rot. Die Rede von Farbempfindungen läßt sich also aus dem Erleben freier Farben, wo sie am Platz ist, nicht auf die Wahrnehmung von Körperfarben übertragen. Farben werden in beiden Fällen verschieden wahrgenommen.

Erlebnisweisen hängen ferner mit Erlebnisinhalten zusammen. Man kann daher den Erlebnisinhalt teilweise auch durch Angabe der Erlebnisweise charakterisieren, wie man das, was jemand sagt, zum Teil auch durch die Weise charakterisieren kann, wie er es sagt. Und umgekehrt erleben wir natürlich wahrnehmbare Dingeigenschaften in bestimmter Weise, wir erleben rote Dinge anders als blaue, usw. $\mathrm{Da}$ her werden Erlebnisweisen und -inhalte in natürlichen Sprachen manchmal durch dieselben Wörter charakterisiert. So verwenden wir „,warm“ z.ur Beschreibung von Empfindungen wie von Dingen (,Mir ist warm", „Das Wasser ist warm“) und ebenso ,schwer" (im Sinn von ,,anstrengend“ oder von ,,gewichtig“). Wir bezeichnen Schnee als „,blendend weiß“ und reden von einer ,,verwirrenden Vielfalt von Formen". Auf diese Verwendung derselben Wörter für Empfindungen wie für Dingeigenschaften stützen sich, wie wir im 3. Kapitel sahen, manche Argumente für den Idealismus. Wir haben dort aber auch be- 
tont, daß wir in der normalen Sprache durchaus zwischen beiden Verwendungstypen unterscheiden, selbst wenn wir dabei dieselben Wörter verwenden.

Diese Verwandtschaften machen den Versuch des Phänomenalismus verständlich, den Inhalt intentionaler Erfahrungen vom Empfindungen her zu charakterisieren. Im übrigen beruht der Phänomenalismus aber auf einer veralteten Wahrnehmungstheorie. Nach dieser Theorie erzeugen verschiedene, isolierbare physikalische Reize, die auf unsere Sinnesorgane einwirken, verschiedene isolierbare Empfindungen, so daß in jedem Moment die Struktur unserer Empfindungen die Struktur der Reize im Bewußtsein widerspiegelt. Aus diesen Empfindungen bauen wir uns dann das Bild einer gegenständlichen Welt auf. Diese Theorie ist aber nicht haltbar. Wie die Gestalt- und Konstanzphänomene der Wahrnehmung zeigen, entsprechen nicht einzelnen Reizen einzelne Empfindungen, sondern Erlebnisse entsprechen Klassen von Reizkonfigurationen. Wir hören dieselbe Melodie und nehmen oft nicht wahr, in welcher Tonart sie gespielt wird oder von welchen Instrumenten. Die Reize sind also verschieden, nur ihre Struktur, die Relation der Tonhöhen ist konstant, und diese Strukturen nehmen wir wahr. Wir sehen eine runde Tischplatte aus verschiedenen Blickwirkelin als runa' und nicht als oval; wir seinen sie bei verschiedenen Beleuchtungen in derselben Farbe und aus verschiedener Entfernung in derselben Größe. Wir fassen, wie z. B. die Rubinsche Becherfigur zeigt, dieselbe Figur entweder als zwei Profile auf oder als Becher, und wir haben Schwierigkeiten, von einer Deutung zur anderen überzugehen. ${ }^{34}$ Unsere Wahrnehmungsorganisation selbst leistet also schon das, was der Phänomenalist einer bewußten Interpretation zumutet: die Konstitution einer gegenständlichen Welt aus den Reizen. Diese Leistung vollzieht sich unbewußt; den Reizen selbst entsprechen - im Normalfall - keine Bewußtseinsinhalte, keine Empfindungen, sondern erst dem fertigen Produkt. Dabei geht in die Konstitution, die Reizverarbeitung, auch eine Fülle von Erfahrungen ein, ebenso wie Strebungen und Wertungen, und auch sprachlich-begriffliche Systematisierungen spielen dabei eine Rolle. All diese Faktoren wirken zusammen und geben unserer Erfahrung von Anfang an intentionale Struktur: die Struktur einer Erfahrung von Ge-

${ }^{34} \mathrm{Zu}$ den Konstanz- und Gestaltphänomenen der Wahrnehmung vgl. z. B. Ph. Lersch (54), 324 ff. oder D. Katz (48). 
genständlichem. Die Differenzierung von subjektiven und objektiven Momenten, von Erlebnisweise und Erlebnisgegenstand ist also in der Erfahrung bereits angelegt. Sie ist freilich in verschiedenen Typen des Erlebens verschieden stark ausgeprägt, und auch bewußte Deutungen haben einen Einfluß darauf. ${ }^{35}$

- Nicht Empfindungen bilden also die Grundlage der Erfahrung, sondern intentionales, bereits gegenständlich strukturiertes Erleben. Daher ist auch die Sprache über Gegenstände ein zentraler und unverzichtbarer Bestandteil unserer Sprache über Beobachtungen. Erfahrung stellt sich immer schon dar als Erfassen einer gegenständlichen Weit. Frege sagt: „Wenn wir überhaupt aus dem Subjektiven herauskommen wollen, so müssen wir das Erkennen auffassen als eine Tätigkeit, die das Erkannte nicht erzeugt, sondern das schon Vorhandene ergreift. Das Bild des Ergreifens ist recht geeignet, die Sache zu erläutern. Wenn ich einen Bleistift ergreife, so geht dabei in meinem Leibe mancherlei vor: Nervenerregungen, Veränderungen der Spannung und des Druckes von Muskeln, Sehnen und Knochen, Veränderungen der Blutbewegung. Aber die Gesamtheit dieser Vorgänge ist weder der Bleistift, noch erzeugt sie ihn. Dieser besteht unabhängig von diesen Vorgängen. Und. es ist wesentlich für das Ergreifen, daß etwas da ist, was ergriffen wird; die innern Veränderungen allein sind das Ergreifen nicht. So besteht auch das, was wir geistig erfassen, unabhängig von dieser Tätigkeit, von den Vorstellungen und deren Veränderungen, die zu diesem Erfassen gehören oder es begleiten; ist weder die Gesamtheit dieser Vorgänge, noch wird es durch sie als Teil unseres seelischen Lebens erzeugt." ${ }^{\text {"36 }}$

${ }^{35}$ Ausführlicher gehen wir darauf im 8. Kapitel ein.

${ }^{36}$ Frege (GGA) I, XXIV. 



\section{Der Physikalismus}

\subsection{Formen und Thesen des Physikalismus}

Der Physikalismus ist die moderne Form des Materialismus. ${ }^{1}$ Als materialistisch bezeichnet man eine Auffassung, nach der die Welt nur aus materiellen Objekten besteht, z. B. aus Elementarteilchen und deren Komposita, und alle Phänomene materieller Natur sind. Die allumfassende Realwissenschaft ist demnach die Physik. Lebendige Organismen, wie sie die Biologie untersucht, sind nichts anderes als besonders komplexe physikalische Gebilde; ihre Eigenschaften und ihr Verhalten lassen sich prinzipiell rein physikalisch erklären. Seelische Vorgänge sind nichts anderes als physikalische Prozesse, die im $\mathrm{Zu}$ sammenhang mit komplexen Organismen auftreten. Und kulturelle und geistige Phänomene endlich lassen sich mit biologischen und psychologischen, d. h. letztlich aber wieder mit physikalischen Mitteln beschreiben und erklären.

Der Materialismus ist fast so alt wie die Philosophie selbst. Einen Atomismus hat schon Demokrit vertreten, einen generellen Materialismus Epikur. Während der Materialismus von Demokrit bis hin zur Aufklärung eine metaphysische, mit apriorischen Argumenten begründete und stark ideologie-kritisch orientierte Position war, versteht er sich seit dem 19. Jahrhundert als eine Verallgemeinerung naturwissenschaftlicher Resultate. Durch die Entwicklung der Naturwissenschaften seit dem 17. Jahrhundert wurden dem Materialismus zunehmend konkrete wissenschaftliche Argumente zur Verfügung gestellt. Mit der Entwicklung der Physiologie im 17. Jahrhundert, insbesondere der Entdeckung des Blutkreislaufs durch W. Harvey, wurden

${ }^{1}$ Dieser theoretische Materialismus ist vom praktischen Materialismus zu unterscheiden, nach dem sich moralische Unterscheidungen allein an den materiellen Lebensbedürfnissen des Menschen orientieren, sowie vom dialektischen oder bistorischen Materialismus, der sich primär auf die gesellschaftliche Wirklichkeit bezieht. 
die Vorgänge im menschlichen Körper zum Gegenstand physikalischer, und mit der Entdeckung der chemischen Vorgänge bei der Atmung von J. Priestley und A. L. Lavoisier im 18. Jahrhundert auch zum Gegenstand chemischer Untersuchungen. Die Arbeiten von Charles Darwin über die Evolution der biologischen Arten stützten zwei der Grundthesen des Materialismus, daß auch der Mensch ein Teil der Natur ist und daß sich die Entwicklung biologischer Organismen nicht-teleologisch erklären läßt. Durch die Fortschritte der Biologie, Biochemie und Physiologie wurde die Grenze zwischen Anorganischem und Organischem immer mehr verwischt, so daß heute wohl nur mehr eine kleine Minderheit von Biologen eine grundsätzliche ontologische Verschiedenheit zwischen beiden Bereichen und damit eine Irreduzibilität organischer auf anorganische Phänomene annimmt. So steht gegenwärtig im wesentlichen nur mehr die Frage der Reduzierbarkeit psychischer auf physikalische Phänomene zur Diskussion. Aber auch hier sind durch die Entwicklung auf dem Gebiet der Neurophysiologie, die weitgehende Abhängigkeiten zwischen psychischen und physischen Vorgängen aufgedeckt hat, dem Materialismus viele neue Argumente geliefert worden. Die Psychologie hat mit den Arbeiten von Sigmund Freud die Grenzen der alten Bewußtseinspsychologie gesprengt und entwickelt sich unter dem Einfluß der methodologischen Kritik des Behaviorismus zu einer empirischen Naturwissenschaft, die sich nicht wesentlich von der biologischen Verhaltensforschung unterscheidet. Die meisten Psychologen würden heute wohl der Ansicht Freuds zustimmen, daß es einst möglich sein werde, das menschliche Verhalten rein physiologisch zu erklären.

Ontologische Formulierungen der materialistischen These erweisen sich nun aber aus analogen Gründen als unbrauchbar wie die ontologischen Formulierungen des Phänomenalismus. ${ }^{2}$ Die Behauptung ,Es gibt nur Materielles" ist als Satz der normalen Sprache falsch. Denn nach den Regeln dieser Sprache verwenden wir den Ausdruck ,,materiell“ so, daß Sätze wie „Der Wert dieser Expedition ist nicht materiell, sondern rein wissenschaftlich“ oder „Für seine Entscheidung waren ideelle, nicht materielle Gründe ausschlaggebend" oft richtig sind. Aus ihnen folgt aber, daß es Nichtmaterielles gibt. Ferner gehört

${ }^{2}$ Vgl. dazu den Abschnitt 5.1. 
die Sprache der Mathematik zur physikalischen Sprache; Zahlen, Mengen und Funktionen sind aber sicher keine materiellen Objekte. Endlich ist die Rede von "materiellen Dingen“ nicht nur in der normalen Sprache vage ${ }^{3}$ - sind Töne, Regenbogen oder Spiegelbilder materiell? - , sondern sie kommt auch in der heutigen Physik kaum mehr vor, die von Feldern, Wellenpaketen, masselosen Elementarteilchen, einer Umwandlung von Masse in Energie etc. spricht. Und endlich haben wir im Abschnitt 3.2 gesehen, daß sich so generelle Existenzfragen wie „Gibt es nichtmaterielle Objekte?" auf Fragen nach der Ausdrucksfähigkeit von Sprachen reduzieren. Daher wird man auch die materialistische These besser als eine These des Inhalts formulieren, daß man alle empirischen Phänomene in einer physikalischen Sprache beschreiben kann. Damit entfällt auch der Bezug auf Materie und materielle Objekte, welcher der modernen Physik nicht mehr entspricht, für die der Begriff des Feldes grundlegender ist als jener der Materie. Man spricht in diesem Sinn besser von einem Physikalismus.

$\Omega$ sei wie in 5.1 unsere normale Sprache, erweitert um die zusätzlichen Ausdrücke der Wissenschaftssprachen, der Mathematik, Physik, Biologie, Psychologie etc. $\Phi$ sei wieder die physikalische Sprache. Dazu gehört zunächst die Sprache der Physik, in der von "Massen“, „Ladungsdichten“, „Feldstärken“, „Neutrinos“ etc. die Rede ist. Die Einführung solcher quantitativer und theoretischer Terme vollzieht sich auf der Basis einer Sprache, in der von konkreten Dingen und ihren Attributen die Rede ist, die wir beobachten können; in der also Sätze vorkommen wie „Dieser Stein ist schwerer als jener", „Diese Fläche ist rot" oder „Der Zeiger des Meßinstruments schlägt aus“. Diese Sprache haben wir in 3.4 als Dingsprache bezeichnet. Sie ist, systematisch gesehen, Grundlage der physikalischen Sprache, und wir sehen sie daher als Teil der physikalischen Sprache $\Phi$ an. Wie die physikalische Sprache $\Phi$ im einzelnen aussieht, welche Ausdrücke ihr Vokabular umfaßt, ist für die folgende Diskussion unwichtig. Im Fortschreiten der Physik werden ohnehin laufend neue Terme eingeführt. Man könnte daher auch sagen: zur physikalischen Sprache gehören alle theoretischen Terme, die sich auf der Basis der Dingsprache als

${ }^{3}$ Zur Kritik an der Verwendung des Ausdrucks „,materielles Ding“ vgl. Austin (62), S. $7 \mathrm{f}$. 
Beobachtungssprache (mithilfe entsprechender logischer und mathematischer Mittel) einführen lassen. ${ }^{4}$

Die physikalische Übersetzbarkeitsthese lautet nun so:

PK: Jeder Satz von $\Omega$ läßt sich in einen Satz von $\Phi$ übersetzen.

Gilt diese These, so kann man über alles, über das man in $\Omega$ reden kann, auch in $\Phi$ reden; man kommt dann mit der Sprache $\Phi$ und ihrer Ontologie aus; der Physikalismus ist also in diesem Sinn gerechtfertigt. Dagegen enthält PK nicht den Absolutheitsanspruch der ontologischen These des Materialismus; PK ist insbesondere auch mit der phänomenalistischen These $\mathrm{PN}$ verträglich.

Im Gegensatz zum Phänomenalismus hat der Physikalismus nicht das Problem, daß seine Grundsprache $\Phi$ keine existierende Sprache ist, sondern erst definiert werden müßte: $\Phi$ ist ein Teil von $\Omega$ selbst, die übliche Sprache über Physisches, kein philosophisches Konstrukt.

Neben der These PK spielen in der Diskussion des Physikalismus auch schwächere Thesen eine wichtige Rolle. Von Interesse ist dabei vor allem die Behauptung:

PS: Die Sprache $\Omega$ ist $W$-reduzierbar auf die Sprache $\Phi$.

${ }^{4}$ Man könnte in diesem Sinn auch $\Phi$ mit der Dingsprache identifizieren. Andererseits lassen sich für die Dingterme physikalische Äquivalente angeben (z. B. $x$ ist grün genau dann, wenn $x$ unter physikalisch weißem Licht elektromagnetische Strahlung im Wellenlängenbereich von ca. 0,53 $\mathrm{m} \mu$ reflektiert), so daß sich auch die Terme der Dingsprache durch physikalische Terme erklären lassen, wenn man diese als in ihrer Bedeutung gegeben voraussetzt. Daher scheint es, als könne man $\Phi$ auch mit der Sprache der Physik identifizieren. Da deren Terme aber tatsächlich mithilfe von Dingtermen erklärt worden sind, würde eine solche Reduktion der Dingsprache auf die Sprache der Physik die faktischen Verhältnisse auf den Kopf stellen. Vgl. dazu auch den Abschnitt 8.3. - Carnap hat in (32) und (32a) betont, daß man die quantitativen physikalischen Terme unter Bezugnahme auf die Dingterme, die für Objekte und Qualitäten eines beliebigen Sinnesgebiets (z. B. für visuell Wahrnehmbares) stehen, einführen kann, und daß sich auch da noch eine begrenzende Auswahl treffen läßt. Es genügt also prinzipiell, der physikalischen Sprache einen kleinen Teil der Dingsprache zugrundezulegen. Solche möglichen Reduktionen der Sprache $\Phi$ sind für das folgende aber ohne Interesse. 
Die schwächere These einer Reduzierbarkeit aller in $\Omega$ formulierbaren Theorien auf physikalische Theorien im Sinne von D 3.3..2 ist ohne tieferes Interesse, da der Physikalismus in jeder Version behauptet, alle Phänomene ließen sich physikalisch erklären. Das läuft aber, wie wir in 3.3 sahen, auf PS hinaus, denn allen wahren Antezedensbedingungen von Erklärungen in $\Omega$ müssen dann wahre Sätze von $\Phi$ entsprechen. Im folgenden werden wir zeigen, daß PS nicht haltbar ist. Damit ist aber zugleich gezeigt, daß auch alle stärkeren Thesen des Physikalismus nicht haltbar sind, insbesondere die nach den Ausführungen in 3.3 stärkere These, daß es ein physikalisches Modell der Gesamtwirklichkeit gibt. Es genügt also, wenn wir im folgenden die Thesen PK und PS diskutieren. Ja, es würde schon eine Kritik von PS genügen, da auch PK stärker ist als PS. Wegen der Verschiedenartigkeiten der Argumente für beide Thesen und im Blick auf die Entwicklung des Physikalismus gehen wir aber zunächst auf PK ein.

Im Sinn der Ausführungen in 3.3 ist zu betonen, daß PS eigentlich keine physikalistische These im engeren Sinn dieser Bezeichnung ausdrückt. Der Anspruch des Physikalismus geht auch dort, wo er nicht behauptet, psychologische Vorgänge seien ,,nichts anderes als" physikalische Prozesse -- womit im Effekt die These PK vertreten wird -, dadurch über PS hinaus, daß sich erstens im Sinne der Reduktion die zueinander zugeordneten Sätze von $\Omega$ und von $\Phi$ in irgendeinem Sinn auf dasselbe beziehen sollen, daß also z. B. eine Aussage über die Stimmungslage eines Menschen in physikalischer Rekonstruktion eine Aussage über neurophysiologische Zustände dieses Menschen zur gleichen Zeit sein soll. Und zweitens wird behauptet, daß es einen naturgesetzlichen Zusammenhang zwischen den korrelierten Sätzen, bzw. Satzformen gibt, so daß die Sätze unter allen naturgesetzlich möglichen und nicht nur unter den tatsächlich gegebenen Umständen dieselben Wahrheitswerte haben. Die These PS wird uns also hier nur dazu dienen, mit ihr auch solche stärkeren, im einzelnen aber sehr viel schwieriger $\mathrm{zu}$ formulierenden Thesen zu widerlegen. ${ }^{5}$

5 Eine Sammlung neuerer Aufsätze zum Physikalismus hat J. O'Connor in (69) herausgegeben. Für eine physikalistische Theorie des Seelischen vgl. z. B. Armstrong (68). 


\subsection{Die Übersetzbarkeitsthese}

Die These PK könnte man so begründen, daß man zu jedem deskriptiven Term von $\Omega$ einen synonymen Term von $\Phi$ angibt. Ein solches direktes Begründungsverfahren ist aber aussichtslos, da wir heute noch nicht einmal in der Lage sind, extensionale Entsprechungen für die meisten Terme von $\Omega, z$. B. für psychologische Terme anzugeben. Daher laufen alle Begründungen für PK darauf hinaus, daß man apriorische Gründe für die Möglichkeit einer Übersetzung anführt. In diesem Sinn spricht man auch von einem logischen Physikalismus. Solche ,logischen“ Argumente z. B. sind folgende:

1. Nach dem Verifikationskriterium der Bedeutung - einer frühen Form des empiristischen Sinnkriteriums - ist ein Satz A nur dann sinnvoll, wenn er aus Beobachtungssätzen $B_{1}, B_{2}, \ldots$ logisch folgt. ${ }^{6}$ Als Beobachtungssätze im wissenschaftlichen Sinn kann man nur Sätze der Dingsprache ansehen, denn nur sie beschreiben Sachverhalte, die für alle in gleicher Weise überprüfbar sind. Sätze über Empfindungen oder Erlebnisse sind hingegen intersubjektiv nicht direkt überprüfbar, kommen also als wissenschaftliche Beobachtungssätze nicht in Betracht. Die Dingsprache ist aber Teil von $\Phi$. Da aus Sätzen von $\Phi$ nur Sätze von $\Phi$ folgen oder Sätze, die mit solchen Sätzen synonym sind, sind daher alle sinnvollen Sätze in $\Phi$ übersetzbar.?

Entsprechende Argumente ergeben sich auch aus anderen empiristischen Sinnkriterien. So ist nach dem Bestärigungskriterium - einer späteren Version des empiristischen Sinnkriteriums - ein Satz empirisch sinnvoll genau dann, wenn er (prinzipiell) durch Beobachtungen

${ }^{6}$ Zum Begriff des Beobachtungssatzes und der Beobachtungssprache vgl. den Abschnitt 9.1.

7 Dieses und das folgende Argument finden sich in Carnap (32), (32a) und (36), sowie in Hempel (35). Zu den empiristischen Sinnkriterien vgl. z. B. Hempel (65), Kap. X, Stegmüller (70), 1. Halbbd. Kap. III oder Kutschera (72), 3.4. - Da aus einem Satz A der Dingsprache auch ein Satz A $\vee$ B folgt, wo $B$ nicht der Dingsprache angehört, müßte man im Sinn des Arguments genauer sagen: (synthetische) Sätze, die keine Terme der Dingsprache enthalten, folgen nicht aus Sätzen der Dingsprache; sie sind also sinnlos. Ist aber der Satz B sinnlos, so auch A V B, so daß alle sinnvollen Sätze, die aus solchen der Dingsprache folgen, in sie übersetzbar sind. Analoges gilt für das folgende Argument mit dem Bestätigungskriterium. 
(positiv oder negativ) bestätigt werden kann. Beobachtungssätze sind aber aus den genannten Gründen, Sätze von $\Phi$. Eine Relation deduktiver oder induktiver Bestätigung eines Satzes A durch Sätze $B_{1}, \ldots, B_{n}$ besteht wieder nur dann, wenn in $A$ nur solche Terme vorkommen, die auch in $B_{1}, \ldots, B_{n}$ vorkommen. Ein Satz von $\Omega$, der sich nícht in einen Satz von $\Phi$ übersetzen läßt, ist also nicht empirisch sinnvoll. ${ }^{8}$

2. $\Omega$ ist eine intersubjektive Sprache, nicht eine Privatsprache im Sinne Wittgensteins. ${ }^{9}$ Für die Wörter von $\Omega$ muß es also Gebrauchsregeln geben, die sich auf intersubjektiv kontrollierbare Anwendungskriterien beziehen. Insbesondere muß es auch für Wörter der Sprache über Psychisches wie "Schmerzen haben" andere als private, introspektive Gebrauchskriterien geben. Intersubjektiv kontrollierbare Kriterien lassen sich aber in der Dingsprache formulieren. Sie ergeben sich insbesondere aus Verhaltensweisen oder -dispositionen, die für die fraglichen seelischen Zustände typisch sind. Damit lassen sich operative Definitionen solcher Zustände angeben. Für alle Terme von $\Omega$, die nicht selbst schon Terme von $\Phi$ sind, gibt es also in $\Phi$ formulierbare Anwendungskriterien, und diese bestimmen auch die intersubjektive Bedeutung dieser Terme.

3. In Schillp (63), S. 882-886 gibt Carnap folgendes Argument für den Physikalismus an: Eine Aussage ist empirisch sinnvoll, wenn sie zumindest prinzipiell subjektiv bestätigungsfähig ist. Es gilt nun:

a) Es gibt andere Subjekte, mit denen ich mich sprachlich verständigen kann. b) Ich bin imstande, anderen meine Beobachtungen, auch introspektiver Art, mitzuteilen, und soweit das nicht möglich ist, können andere meine Beobachtungen aus meinen, für sie beobachtbaren körperlichen Zuständen erschließen. c) Daher ist alles, was ich empirisch feststellen kann, einschließlich dessen, was ich durch Introspektion weiß, prinzipiell für andere bestätigungsfähig auf der Basis ihrer Beobachtungen. d) Daher ist es möglich (und für praktische Zwecke sinnvoll), den Aufbau der Sprache mit Grundtermen zu beginnen, die sich auf intersubjektiv Beobachtbares beziehen. Terme für nur subjektiv Beobachtbares wie Empfindungen, lassen sich dann auf dieser $\mathrm{Ba}$

${ }^{8}$ Dieses Argument findet sich ebenfalls bei Carnap in (36). Zum Bestätigungsbegriff vgl. Kutschera (72), Kap. 5 und Lenzen (73).

${ }^{9}$ Vgl. dazu Kutschera (75), 2.4.6. 
sis implizit definieren. Daher formuliert Carnap die These des Physikalismus nun als Konjunktion von (a) bis (c) ${ }^{10}$ oder, äquivalent, als These e) Was subjektiv bestätigungsfähig ist, ist auch intersubjektiv bestätigungsfähig. Oder $f$ ) als Vorschlag, eine Sprache zu verwenden, in der alle Aussagen intersubjektiv bestätigungsfähig sind, zusammen mit der Behauptung, daß sich in dieser Sprache alle sinnvollen Aussagen formulieren lassen.

Das Argument Carnaps beruht also auf dem Gedanken, daß alles, was subjektiv bestätigungsfähig ist, auch intersubjektiv bestätigungsfähig sei, und schließt sich dann dem 1. Argument an.

Diese Argumente illustrieren die Entwicklung des Physikalismus bei Carnap, seinem prominentesten Vertreter in der analytischen Philosophie. $\mathrm{Zu}$ ihnen ist nun folgendes zu sagen:

$\mathrm{Zu}$ (1): Erstens haben sich alle empiristischen Sinnkriterien als unhaltbar erwiesen. ${ }^{11}$ Das Verifikationskriterium ist unhaltbar, da danach eindeutig empirisch sinnvolle Sätze als bedeutungslos anzusehen wären, wie z. B. alle generellen Hypothesen, die sich durch endlich viele Beobachtungen nicht verifizieren lassen. Das Bestätigungskriterium bleibt ohne präzisen Inhalt, solange man nicht den verwendeten Bestätigungsbegriff angibt. Nach den üblichen deduktiven oder induktiven Bestätigungstegrifíen gibt es aber immer empirische Säize, auch Sätze von $\Phi$ selbst, die danach jeweils als ,,nichtempirisch“ erklärt werden. ${ }^{12}$ Ein Grund dafür liegt darin, daß wir in der Regel nicht einzelne Hypothesen durch Beobachtungen bestätigen können, sondern nur Systeme von Hypothesen. So kann man aus einer physikalischen Hypothese $\mathrm{H}$ nicht direkt den Wert einer Größe ableiten, der sich bei einer Messung ergeben wird, sondern man benötigt dazu Randbedingungen, eine Theorie, in deren Rahmen diese Größe implizit definiert wird, Annahmen über die Funktionsweise des Meßinstruments, etc. Für einen Bestätigungsbegriff, der die Bestätigung, die $\mathrm{H}$ durch einen Beobachtungssatz B erfährt, auf gewisse Annahmen A re

${ }^{10}$ Man braucht eigentlich nur (c) oder aber die Sätze (a), (b), aus denen ja (c) folgen soll.

${ }^{11} \mathrm{Vgl}$. dazu die oben angegebene Literatur.

${ }^{12}$ Bei induktiven Kriterien hängt das, was danach bestätigungsfähig ist, auch wesentlich von der apriori-Wahrscheinlichkeit ab, von der man ausgeht. Vgl. dazu Kutschera (78), $203 \mathrm{f}$. 
lativiert, gilt jedoch die Behauptung nicht mehr, daß B H nur dann bestätigen kann, wenn $\mathrm{H}$ keine Terme enthält, die in $\mathrm{B}$ nicht vorkommen: solche Terme können ja in A enthalten sein.

Zweitens ist die These, intersubjektiv kontrollierbare Beobachtungssätze seien immer Sätze von $\Phi$, nicht haltbar. Es gibt viele Aussagen, die Beobachtungssätze im intersubjektiven Sinn darstellen, aber nicht der Sprache $\Phi$ angehören, wie z. B. ,Fritz sagt, daß es regnet“, „Hans kann den Stein nicht heben“ oder „,Der Baum ist abgestorben“. Angesichts solcher Sätze kann man entweder behaupten, diese Sätze seien zwar als (wissenschaftliche) Beobachtungssätze zulässig, sie seien aber in Sätze von $\Phi$ übersetzbar - dann liegt aber, sofern die Übersetzungen nicht tatsächlich angegeben werden, eine petitio principii vor, denn die Übersetzbarkeit sollte ja gerade durch das Argument bewiesen werden und kann daher in ihm nicht vorausgesetzt werden - oder man behauptet, diese Sätze seien keine (wissenschaftlichen) Beobachtungssätze. Da sie aber in vielen Kontexten als Beobachtungssätze verwendet werden, ist das eine dogmatische These, die auf einer Einengung des Begriffs ,empirisch“ durch die definitorische Festsetzung: ,Empirisch heißt ein Beobachtungssatz nur dann, wenn er ein Satz von $\Phi$ ist" beruht. Eine solche Einengung entspräche aber nicht dem üblichen Sprachgebrauch, wäre daher inadäquat und würde im übrigen das Argument trivialisieren.

$Z u$ (2): Die Behauptung ist sicher richtig, $\Omega$ sei eine intersubjektive Sprache und für die Wörter einer intersubjektiven Sprache müsse es Gebrauchsregeln geben, die sich auf intersubjektiv kontrollierbare Kriterien stützen. Ein Ausdruck wie „Schmerzen haben“ kann, wie Wittgenstein betont, ${ }^{13}$ nur deswegen der intersubjektiven Sprache angehören, weil er nicht allein nach introspektiven, privaten Kriterien verwendet wird, sondern weil es auch für andere beobachtbare Anzeichen dafür gibt, daß jemand Schmerzen hat, z. B. verengte Pupillen, Stöhnen, oder andere typische Verhaltensweisen. Daraus folgt aber nicht, daß „Schmerzen haben“ in der intersubjektiven Sprache soviel bedeutet wie verengte Pupillen haben, stöhnen etc. Vielmehr muß man analytische und empirische Kriterien unterscheiden: Die Bedeutung von "Schmerzen haben" wird im wesentlichen durch introspektive Kriterien bestimmt; „Fritz hat Schmerzen“ heißt soviel wie „Fritz

${ }^{13}$ Vgl. Wittgenstein (53), 384. Vgl. dazu auch Ryle (49), S. 15, 21, 52, sowie die Diskussion in Kutschera (75), 2.4.6. 
empfindet dasselbe wie ich, wenn ich Schmerzen habe". Verengte Pupillen etc. sind dagegen Schmerzsymptome, empirische Kriterien dafür, daß jemand Schmerzen hat. Wir haben schon in 2.2 bei der Diskussion von Dispositionsprädikaten gesehen, daß man nicht alle $\mathrm{Ge}$ brauchsprinzipien für einen Ausdruck als bedeutungsbestimmend, d. h. als analytisch ansehen kann, da sonst empirische Sätze, und zwar auch solche, die den fraglichen Ausdruck nicht enthalten, zu analytischen Sätzen würden. Man kann z. B. nicht beide Sätze ,Wenn X stöhnt, so hat X Schmerzen" und ,Wenn X Schmerzen hat, so verengen sich seine Pupillen" als analytisch ansehen, denn sonst wäre auch der Satz ,Wenn X stöhnt, so verengen sich seine Pupillen“ analytisch. Notwendige und hinreichende Symptome für psychische Zustände hat im übrigen noch niemand angeben können.

C. S. Chihara und J. A. Fodor haben in (65) Wittgensteins Argument so dargestellt: Es sei $\Sigma$ eine intersubjektive Sprache über Psychisches. Damit man einen $\Sigma$-Term erlernen kann, muß es intersubjektiv kontrollierbare Kriterien für seine Anwendung geben. Diese Kriterien können empirisch oder analytisch sein. Es muß aber zumindest einige analytische Kriterien geben, denn eine empirische Korrelation von psychischen Zuständen und physischen Symptomen muß ja wieder intersubjektiv überprüft weróen, d. h. man braucht dazu wieder andere Kriterien für psychische Zustände. Das würde aber zu einem infiniten Regress führen, wenn es keine analytischen Kriterien gäbe. ${ }^{14}$

Dazu ist zu sagen: Eine Person $X$ kann zunächst Korrelationen $z$ wischen seinen eigenen seelischen Zuständen und seinem Verhalten oder körperlichen Symptomen als empirische Korrelationen überprüfen. Bei anderen kann X eine solche Korrelation ohne die Voraussetzung genereller Verhaltenskriterien nicht überprüfen. Es ist aber für $\mathrm{X}$ eine naheliegende Hypothese, daß die Korrelation für andere Personen auch gilt, und diese Hypothese leistet sehr gute Dienste für die Erklärung des Verhaltens anderer. Diese Hypothesen sind aber nicht

${ }^{14}$ Die Autoren modifizieren die übliche behsprache $\Phi$ übersetzen lassen. Sie sind vielmehr der Ansicht, daß die $\Sigma$-Terme ein System bilden, das nur als Ganzes analytisch mit Termen von $\Phi$ korreliert werden kann, so daß die $\Sigma$ Terme ein System theoretischer Terme auf der Grundlage der Sprache $\Phi$ als Beobachtungssprache bilden. 
analytisch. Man kann jedoch auch ohne weiteres analytische Sätze annehmen, in denen $\Sigma$-Terme und Verhaltensterme vorkommen. Tatsächlich bestimmen auch unsere grundlegenden Annahmen über die Korrelationen zwischen psychischen und physischen Vorgängen und Zuständen die Bedeutung der $\Sigma$-Terme mit, so daß wir geneigt sind, solchen Annahmen analytische Geltung zuzusprechen. Aus der Existenz solcher analytischer Sätze folgt aber nicht, daß sich die $\Sigma$-Terme durch andere Terme explizit oder auch implizit definieren lassen. Dazu müßten diese analytischen Korrelationen viel stärker sein als sie es tatsächlich sind.15 Die Existenz von analytischen Korrelationen zwischen $\Sigma$-Termen und Verhaltenstermen erklärt auch, daß wir, wie Wittgenstein hervorhebt, Aussagen über die seelischen Zustände anderer mit großer Sicherheit machen können; daß wir z. B. sagen können „Ich weiß, daß X Schmerzen hat". ${ }^{16}$

Wittgenstein vergleicht nur introspektiv erfaßbare Empfindungen mit Käfern in Schachteln: „Angenommen, es hätte jeder eine Schachtel, darin wäre etwas, was wir „Käfer“ nennen. Niemand kann je in die Schachtel des Anderen schauen ... Aber wenn nun das Wort „Käfer" dieser Leute doch einen Gebrauch hätte? - So wäre er nicht der der Bezeichnung eines Dings. Das Ding in der Schachtel gehört überhaupt nicht zum Sprachspiel ... durch dieses Ding in der Schachtel kann ,gekürzt werden'; es hebt sich weg, was immer es ist" ". ${ }^{17}$

Wittgensteins Beispiel beruht darauf, daß die Käfer in den Schachteln isolierte Phänomene sind; es macht für die „öffentlichen“ Sachverhalte keinen Unterschied, wie sie beschaffen sind. Das gilt aber nicht für Empfindungen, Erlebnisse oder Gedanken, die mit unserem gesamten Verhalten und Leben eng verwoben sind. Daher, meint Wittgenstein, sind sie auch intersubjektiv beobachtbar. Man kann seinen Gedanken so formulieren: Entweder entsprechen allen Unterschieden im seelischen Leben und Erleben einer Person unter geeigne-

${ }^{15}$ Die $\Sigma$-Terme spielen in diesen analytischen Sätzen nicht die Rolle von theoretischen Termen, denn ihre Anwendung folgt nicht nur Verhaltenskriterien, sondern im Fall von Aussagen über Eigenpsychisches auch introspektiven Kriterien, und der Sinn dieser Terme ist wesentlich auch durch ihre Anwendung auf eigenpsychische $\mathrm{Phänomene} \mathrm{bestimmt.}$

${ }_{16}$ Wittgenstein (53), 246. Vgl. dazu auch Austin (46), S. 78.

17 Wittgenstein (53), 293. 
ten Umständen Unterschiede in ihrem Verhalten - dann hat der Physikalismus recht -, oder es gibt Unterschiede des Erlebens, die sich nicht in verschiedenem Verhalten widerspiegeln - dann sind diese Unterschiede nicht in einer intersubjektiven Sprache beschreibbar, also auch nicht intersubjektiv erkennbar; ja nach Wittgensteins Argumenten gegen Privatsprachen sind sie nicht einmal subjektiv erkennbar. Im Bereich erkennbaren und unterscheidbaren Erlebens gilt also der Physikalismus. Sehen wir einmal von dem letzten Teil des Arguments ab, der sich auf nicht hinreichend begründete Einwände gegen Privatsprachen stützt, ${ }^{18}$ so setzt dieses Argument voraus, daß sich das intersubjektiv Beobachtbare mit dem Physischen deckt. Dagegen hatten wir aber schon oben Bedenken angemeldet. Im normalen Sinn des Wortes ,beobachten“ ist auch nicht rein Physisches beobachtbar. In vielen Fällen können wir $z$. B. beobachten, daß sich jemand freut, daß er etwas sagt (Sätze mit einem bestimmten Sinn äußert), oder daß er etwas absichtlich tut. Man kann auch nicht behaupten, direkt beobachtbar sei nur Physisches, z. B. die Miene einer Person, die Bewegungen ihres Körpers, die Laute, die sie von sich gibt, Aussagen über seelische Einstellungen oder Absichten, die sich damit verbinden, beruhten hingegen auf hypothetischen Deutungen des direkt Beobachteten. Wir haben schon im Abschnitt 4.2 gesehen, daß eine Abgrenzung des beobachtungsmäßig unmittelbar Gegebenen von seinen Deutungen nicht in Sicht ist, und wir werden später, im 9. Kapitel, sehen, daß alle Beobachtungssätze hypothetische Elemente enthalten. Im normalen Sinn von ,beobachten“" könnte man durchaus sagen, $\mathrm{daß}$ sich alle Unterschiede im seelischen Leben und Erleben einer Person unter geeigneten Umständen in Unterschieden ihres beobachtbaren Verhaltens widerspiegeln, aber das impliziert dann keinen Physikalismus. Die normale Sprache ist sicher intersubjektiv, in ihr kann man aber nicht nur über Physisches reden und über das, was sich mit physikalischen Begriffen definieren läßt. Unsere Sprache enthält eine Unmenge psychologischer Ausdrücke, die wir sinnvoll zu gebrauchen wissen, ohne für einen einzigen von ihnen rein physikalische Anwendungskriterien zu kennen. Die Sprache über Psychisches ist, wie in 5.1 betont wurde, eng auf die Sprache über Physisches bezogen. Sie ist aber ebenso wenig allein aus dieser abgeleitet, wie die Sprache über Physisches aus jener über Psychisches. Im übrigen folgt aus der Tatsa-

${ }^{18}$ Vgl. dazu Kutschera (75), 2.4.6. 
che, daß sich alle Unterschiede seelischen Lebens und Erlebens unter geeıgneten Umständen in Unterschieden des Verhaltens widerspiegeln, nicht, daß die Beschreibungen der psychischen Zustände mit jenen des entsprechenden Verhaltens synonym wären.

Die These, alle Aussagen über Seelisches seien in Aussagen über Verhalten übersetzbar, kennzeichnet den Behaviorismus. Der „logische Behaviorismus" sucht, ebenso wie der „logische" Physikalismus, diese These apriorisch zu rechtfertigen. Der Behaviorismus ist als Gegenbewegung gegen den Subjektivismus in der Psychologie entstanden und seine Überbewertung der introspektiven Methode zur Überprüfung psychologischer Gesetze. Demgegenüber betont er zurecht, daß eine wissenschaftliche Psychologie nur Aussagen machen darf, die intersubjektiv kontrollierbar sind. Er geht aber über diese Forderung noch einen wesentlichen Schritt hinaus: Intersubjektiv beobachtbar ist für ihn allein das menschliche Verhalten, und daher soll auch nur das Verhalten Gegenstand der wissenschaftlichen Psychologie sein, und Aussagen über psychische Zustände sollen in ihr nur insoweit Platz haben, als sie sich in Verhaltensaussagen übersetzen lassen. Es ist nun die These des Behaviorismus, daß eine solche Übersetzung immer möglich ist. Ist $\Sigma$ eine Sprache über Psychisches, $\Phi^{*}$ die physikalische Sprache, erweitert um ein Vokabular zur Beschreibung von Verhaltensformen, so lautet diese These in Analogie zu PK: „Alle Sätze von $\Sigma$ lassen sich in Sätze von $\Phi^{*}$ übersetzen ". Diese These ist schon deshalb schwächer als PK, da $\Sigma$ nur eine Teilsprache von $\Omega$ ist. Je nachdem, wie Verhaltensbegriffe verstanden werden, ob man sie, wie z. B. James B. Watson, ${ }^{19}$ als spezielle physikalische Begriffe ansieht (so daß $\Phi^{*}$ mit $\Phi$ zusammenfällt), als biologische Begriffe, oder ob man darüber hinaus auch speziell auf menschliches Verhalten bezogene Begriffe zuläßt, wie das z. B. G. Ryle in (49) tut, ergeben sich ferner ganz verschiedene Versionen des Behaviorismus. Es ist einer der problematischen Punkte des Behaviorismus, daß der Verhaltensbegriff, auf den er sich stützt, kaum je genauer expliziert wird; ebenso

19 Watson nimmt gelegentlich eine Übersetzbarkeit von psychologischen in neurophysiologische Aussagen an. Er vertritt damit dann - zumindest bzgl. der Sprache $\Sigma$ - eher einen Physikalismus als einen Behaviorismus. Verhaltensbegriffe sind, auch bei physikalischer Deutung, Begriffe, die die äußeren Makrozustände und -zustandsänderungen eines Organismus beschreiben, nicht seine inneren Mikrozustände. 
wenig der damit eng verwandte Begriff der Handlung. Die Wörter „sich verhalten" und "handeln" werden in der normalen Sprache häufig nur dann angewendet, wenn eine absichtliche und bewußte Tätigkeit vorliegt. ,Absichtlich“ und ,,bewußt" sind aber Wörter jener Art, die der Behaviorismus vermeiden will. Für eine klare Bestimmung seines Programms ist es also entscheidend, eine Definition des Verhaltensbegriffs anzugeben, die solche Konnotationen ausschließt.

Die „logische“ Begründung der behavioristischen These im Sinne des Arguments (2) für den Physikalismus wurde schon besprochen. Ein zusätzliches Argument von Carnap lautet so: ${ }^{20}$ Wenn man sprachliches Verhalten mit in Betracht zieht, kann man trivialerweise jede psychologische Aussage in eine Verhaltensaussage übersetzen. So läßt sich z. B. die Aussage „X ist zornig“ übersetzen in „Auf die Frage: ,Bist du zornig?" antwortet $\mathrm{X}$ mit $\mathrm{Ja}$ “" . - Es ist nun aber - von der Synonymität dieser Sätze einmal ganz abgesehen - für ihre empirische Äquivalenz erforderlich, daß man die Bedingung hinzufügt, daß $\mathrm{X}$ nicht lügt, daß X die Frage versteht und daß X seine Antwort im Sinne der Bejahung meint. Diese Bedingungen sind aber nicht Sätze der Verhaltenssprache, vielmehr sind die Wörter ,lügen“ (,,etwas sagen, was man nicht glaubt, in der Absicht, den Hörer zu täuschen"), „verstehen" und „meinen" typische $\Sigma$-Prädikate. Wolle man auf diese Bedingungen wiederum die Carnapsche Übersetzungsregel anwenden, so müßte man abermals $\Sigma$-Bedingungen hinzufügen, und so ad infinitum.

G. Ryle hat in (49) die behavioristische These durch Einzelanalysen zu belegen versucht. Er legt dabei einen Verhaltensbegriff zugrunde, der auf spezifisch menschliches Verhalten zugeschnitten ist, und verwendet die Wörter „Verhalten“ und „Handlung“ vorwiegend im Sinn von ,,bewußtes“, ,,absichtliches“, „planvolles“ oder ,,intelligentes Verhalten".21 Diese Adjektive sind wieder typische $\Sigma$-Terme. Wenn man sie nicht explizit als Grundterme verwenden, sondern nur zur vorläufigen Erläuterung der Grundterme des Verhaltens gebrauchen würde, um sie dann hinterher durch diese Grundterme zu definieren, so wäre dagegen nichts einzuwenden. Es zeigt sich aber, daß Ryle bei seinen Analysen immer wieder zwischen intelligentem und nichtintel-

${ }^{20} \mathrm{Vgl}$. Carnap (32a).

${ }^{21}$ Vgl. Ryle (49), S. 327 f. 
ligentem (zufälligen, planlosen, instinkthaftem) Verhalten unterscheiden muß, daß er den $\Sigma$-Term ,,intelligent" also explizit verwendet, ohne ihn mit Verhaltenstermen definieren zu können. Hinzukommt, $\mathrm{da}$ der Verhaltensbegriff Ryles sich im Laufe seiner Reduktionsbemühungen zusehends auflöst. Wenn zuerst noch von ,äußerem Verhalten" (overt bebavior) die Rede ist, so taucht später unvermittelt ein „,stilles Verhalten“ (silent behavior) auf, ${ }^{22}$ womit sowohl Denkvorgänge charakterisiert werden als auch Gefühle und Empfindungen; ${ }^{23}$ und auch das Sprachverhalten, auf dessen Problematik im Zusammenhang eines behavioristischen Programms wir gerade hingewiesen haben, wird im Notfall von Ryle bemüht. ${ }^{24}$

Ryle zeigt für verschiedene psychologische Terme, daß sie sich als Verhaltensterme auffassen lassen - das sind aber psychologische Terme, die auch sonst vielfach als Leistungs-, Dispositions- und Verhaltensbegriffe eingestuft werden. Daneben finden sich auch falsche Bedeutungsanalysen. ${ }^{25}$ Die Stärke des Buches von Ryle liegt nicht darin, daß es etwas zur Begründung der behavioristischen These beitrüge, sondern in seinen Argumenten gegen den Dualismus, sowie gegen falsche Interpretationen von Aussagen über Psychisches. Darauf gehen wir im nächsten Kapitel ein.

$\mathrm{Zu}$ (3): Zu diesem Argument Carnaps ist zu sagen, daß (c), (e) und (f) keine physikalistischen Thesen sind. Auch ein Phänomenalist kann (c) und (e) akzeptieren, und eine Sprache, wie sie in (f) vorgeschlagen

${ }^{22}$ Vgl. Ryle (49), S. 169.

${ }^{23}$ Es wird auch einmal das Problem der Empfindungen kurzerhand aus den Diskussionen ausgeklammert mit der Begründung, daß Empfindungen nicht zum mind (im Sinne von "Verstand") gehörten. Bis dahin hatte aber Ryle mit ,mind" nicht nur den Verstand bezeichnet, sondern allgemein das Bewußtsein.

${ }^{24}$ Vgl. Ryle (49), S. 61, 115, $181 \mathrm{ff}$.

${ }^{25}$ Vgl. dazu z. B. die Analyse von voluntary, (49), S. 69. Ryle analysiert dieses Wort im Sinne von ,absichtlich“ nur in Verbindungen mit Handlungen, die falsch oder schlecht sind. Oder die Analyse von believe, (49), $44 \mathrm{f}$. Ryle faßt dort ,glauben, daß p“ auf als Disposition, gewisse Handlungen zu vollziehen oder zu unterlassen. Nach den Entscheidungsprinzipien hängen unsere Handlungen aber nicht nur von unseren Annahmen, sondern auch von unseren Präferenzen ab, und auch das gilt nur für rationale Handlungen. 
wird, kann auch Sätze über Erlebnisse enthalten, da sie nach (e) intersubjektiv bestätigungsfähig sind. Auch der Satz (d) stellt keine physikalistische These dar, sofern man hier die Sätze über ,intersubjektiv Beobachtbares" nicht wieder stillschweigend als Sätze von $\Phi$ ansieht. Dann wären aber (b), (c) und (d) nicht ausreichend begründet.

Die angeführten Argumente für die These PK sind also nicht stichhaltig. Überblickt man die Entwicklung des Physikalismus, so kann man feststellen, daß sich die Argumente für die These PK dabei zusehends, wenn auch uneingestandenermaßen, so abschwächen, daß sie nur mehr die These PS zu stützen vermögen. Das wird besonders in der Wandlung der Auffassungen von Carnap deutlich, der in (32) und (32a) noch eine explizite Definierbarkeit aller Terme von $\Omega$ (die nicht ohnehin $\Phi$ angehören) durch Terme von $\Phi$ behauptet, diese Behauptung dann in (36) so abschwächt, daß nur mehr die Existenz operativer Definitionen (sogenannter „Reduktionssätze") für solche Terme von $\Omega$ mithilfe von Termen von $\Phi$ angenommen wird, die zudem nicht mehr analytisch, sondern empirisch adäquat sind, um dann in (63) hauptsächlich so zu argumentieren, daß der Fortschritt der Wissenschaften immer tiefere Entsprechungen zwischen nicht-physikalischen und physikalischen Phänomenen aufweise.

Um dieser Schwierigkeit zu entgehen, daß man eigentlich eine Übersetzungsthese begründen will, aber doch nur Argumente für eine empirische Äquivalenz zwischen psychologischen und physikalischen Aussagen findet, hat $\mathrm{H}$. Feigl eine Identitätsthese entwickelt, die seitdem in einer Vielzahl von Veröffentlichungen diskutiert worden ist. ${ }^{26}$ Sie besagt:

IT: Jeder mentale Zustand, in dem sich eine Person a befindet, ist identisch mit einem gleichzeitigen Gebirnzustand dieser Person. ${ }^{27}$

${ }^{26} \mathrm{Vgl}$. Feigl (58), S. 438 - 465, Feigl (63) und die Aufsätze in O’Connor (69). 27 Manche Autoren sprechen auch von einer Identität mentaler Zustände mit solchen des gesamten Nervensystems, aber darauf kommt es für die folgenden Argumente nicht an. Andere Autoren beschränken den Bereich der mentalen Zustände, für die eine solche Identität gelten soll. Aber wenn sie nicht - unmittelbar oder mittelbar - für alle Zustände des Erlebens, Wahrnehmens, Fühlens, Denkens, Glaubens und Wollens gelten soll, so begründet die These IT keinen Physikalismus. 
Besagt der Satz , $M(a, t) “$, daß sich die Person a zur Zeit $t$ im mentalen Zustand $M$ befindet, und drückt der Satz ,B(a,t) “ den Sachverhalt aus, daß sich a's Gehirn zur Zeit $t$ im physiologischen Zustand B befindet, so soll es also für jedes $M$ ein $B$ geben, so daß beide Sätze denselben Zustand ausdrücken.

Weder die Aussage

a) $\wedge \mathrm{xt}(\mathrm{M}(\mathrm{x}, \mathrm{t}) \equiv \mathrm{B}(\mathrm{x}, \mathrm{t}))$ - Alle Personen befinden sich immer genau dann im Zustand $M$, wenn sie sich im Zustand B befinden noch die Aussage

b) (a) gilt mit naturgesetzlicher Notwendigkeit impliziert jedoch eine Identität der Sachverhalte $M(a, t)$ und $B(a, t)$. Aus dem physikalischen Gesetz, daß genau die mathematischen Pendel mit der Länge 1 eine Schwingungsdauer $t=2 \pi \sqrt{1 / g}$ haben, folgt nicht, daß für solche Pendel eine Länge $l$ zu haben und eine entsprechende Schwingungsdauer $t \mathrm{zu}$ haben dasselbe wäre. Nur wenn gilt c) $N \wedge x t(M(x, t) \equiv B(x, t))$ - es ist analytisch notwendig (d. h. analytisch wahr), daß (a) gilt - , kann man behaupten, die $M$ - und die B-Zustände seien identisch. Sie treten dann nicht nur, wie nach (a), in unserer Welt, und auch nicht nur, wie nach (b), in allen Welten, in denen dieselben Naturgesetze gelten wie in unserer Welt, zusammen auf, sondern sie treten in allen möglichen Welten, d. h. unter beliebigen Bedingungen zusammen auf, wie z. B. die Sachverhalte, daß a Elternteil von $b$ und daß b Kind von $a$ ist. Nun stimmen alle Vertreter der Identitätsthese darin überein, daß die behauptete Identität von $\mathrm{M}$ - und $\mathrm{B}-$ Zuständen nicht implizieren soll, daß (c) gilt. Sonst hätten ja die Sätze , $M(a, t)$ “ und , $B(a, t)$ “ dieselbe Bedeutung, und wir könnten die Erforschung der Zusammenhänge zwischen mentalen Zuständen und Gehirnzuständen einem Linguisten überlassen, der die Probleme, mit denen sich heute ungezählte Gehirnspezialisten in mühevoller empirischer Kleinarbeit befassen, aufgrund seiner Sprachkenntnis mit geschlossenen Augen im Lehnstuhl lösen könnte.

Die Identität, so wird gesagt, ist nur eine kontingente Identität, wie die zwischen Morgenstern und Abendstern, zwischen Wasser und $\mathrm{H}_{2} \mathrm{O}$, zwischen Molekularbewegung und Temperatur. Die Feststellung solcher kontingenten Identitäten ist aber Sache der Erfahrung. Selbst wenn die Feststellung einer empirischen Korrelation von Mund B-Zuständen im Sinn von (a) oder (b) die Identität dieser Zustände nicht impliziert, könne man sie doch bei Geltung solcher Kor- 
relationen aus Gründen der Einfachheit oder der Begriffsökonomie identifizieren. ${ }^{28}$ Es gibt aber keine Rechtfertigung, verschiedene Dinge zu identifizieren. Die sparsamste Ontologie wäre sicher die von Parmenides, nach dem alles Eins ist, aber die Nichtunterscheidung des Verschiedenen ist sicher der falsche Weg zur Einfachheit. Die Frage ist also: Sind die Zustände identisch oder sind sie es nicht. Sind sie es nicht, so kann man sie auch nicht identifizieren, sondern allenfalls - im Sinn einer Theorienreduktion oder einer W-Reduktion aufeinander reduzieren.

Die Rede von ,kontingenten Identitäten“ ist aber zumindest schief: Es gibt keine kontingenten Identitäten, sondern nur kontingente Identitätsaussagen. Ähnliches hat schon S. Kripke in (71) und (72) gesagt. $\mathrm{Da}$ er sich dabei aber auf spezielle Voraussetzungen über die Funktion von Namen stützt und auf einige zentrale Argumente der Verfechter der IT nicht eingeht, soll diese Behauptung hier noch einmal ausführlicher begründet werden.

Betrachten wir zunächst das Beispiel vom Morgenstern und Abendstern. Beide Ausdrücke stellen Kennzeichnungen dar: Der Morgenstern ist der Stern, der am Morgenhimmel am hellsten leuchtet, der Abendstern jener, der am Abendhimmel am hellsten leuchtet. Diese Kennzeichnungsterme bezeichnen in unserer Weit denselben Stern, die Venus. Daß nun die Venus mit sich selbst identisch ist, ist kein kontingenter Sachverhalt, wohl aber, daß der Stern, der am Morgenhimmel am hellsten leuchtet, das auch am Abendhimmel tut. Im Rahmen der intensionalen Semantik, in dem man solche Probleme exakt diskutieren kann, geht man aus von einer Menge I möglicher Welten und von einem Gegenstandsbereich $U$ - demselben für jede mögliche Welt bei dem heute gebräuchlichsten Ansatz. ${ }^{29}$ Dabei wird der Begriff der Identität von Objekten als Grundbegriff angesehen. Es gilt dann 1. $\wedge \mathrm{xy}(\mathrm{x}=\mathrm{y} \supset \mathrm{N}(\mathrm{x}=\mathrm{y}))$ - identische Objekte sind notwendig (d. h. in allen Welten) identisch - , und

2. $\wedge x y(x \neq y \supset N(x \neq y))$ - nicht identische Objekte sind notwendigerweise nicht identisch.

\footnotetext{
${ }^{28}$ So äußert sich z. B. J. Smart in (62).

${ }^{29} \mathrm{Vgl}$. dazu Kutschera (76). Man kann U als Menge möglicher Objekte bezeichnen und für jede Welt $i$ eine Teilmenge $E_{i}$ von $U$ als Menge von in i existierenden Objekten betrachten, so daß sich die $E_{i}$ für verschiedene Welten $i$ unterscheiden können.
} 
Die Identität und Verschiedenheit der Objekte aus U wird bei der Interpretation einer Sprache über $U$ vorausgesetzt, nicht aber erklärt, sie hat nichts mit den möglichen Welten zu tun. Definiert wird nur das Zeichen ",=", das in der Sprache diese Identität ausdrücken soll, und es muß so interpretiert werden, daß es die vorgegebenen Identitäten und Verschiedenheiten wiedergibt. Da diese nicht von den Welten abhängt, ist also das Zeichen ,,=" so zu erklären, daß zwei Objekte entweder in allen Welten oder aber in keiner Welt verschieden sind. Es gibt daher keine kontingenten Identitäten, keine Objekte, die in manchen Welten identisch, in anderen aber verschieden wären. ${ }^{30}$

Es gibt nun Eigennamen, die in allen Welten dasselbe Objekt bezeichnen. Man bezeichnet sie als "Standardnamen“ oder rigid designators. Sind a,b Standardnamen, so gilt also auch

3. $a=b \supset N(a=b)$.

Daneben gibt es aber auch andere Eigennamen (non-rigid designators) wie z. B. Kennzeichnungen, die in verschiedenen Welten verschiedene Objekte bezeichnen. Für sie gilt (3) nicht. Wenn z. B. in unserer Welt der Stern, der am Morgenhimmel am hellsten leuchtet, mit dem Stern identisch ist, der am Abendhimmel am hellsten leuchtet, so folgt daraus nicht, daß das in allen Welten so ist. Es gilt hingegen: Das Objekt (der Planet Venus), das die Ausdrücke „Morgenstern“ und „Abendstern" in unserer Welt bezeichnen, ist in allen Welten mit sich selbst identisch, also, wo s,t für „Morgenstern" und „Abendstern“ stehen: 4. $s=\mathrm{t} \supset \wedge \mathrm{xy}(\mathrm{x}=s \wedge \mathrm{y}=\mathrm{t} \supset \mathrm{N}(\mathrm{x}=\mathrm{y})) \cdot{ }^{31}$

Wir können das so lesen: „,Wenn der Morgenstern mit dem Abendstern identisch ist, so gilt vom Morgenstern (d. h. von dem Objekt, das in unserer Welt der Morgenstern ist) und vom Abendstern (von dem Objekt, das in unserer Welt der Abendstern ist), daß sie notwendigerweise identisch sind“. Den Satz ,N(s=t)“ kann man nicht als

${ }^{30}$ Es gibt Semantiken, in denen man verschiedenen möglichen Welten $i$ und $k$ verschiedene Objektmengen $U_{i}$ und $U_{k}$ zuordnet und anstelle einer QuerWelten-Identität eine Relation der Entsprechung, eine counterpart-Relation verwendet, wie z. B. D. Lewis in (68). Ist $R$ eine solche Relation, so besagt also $R(x, y)$, daß das Objekt $x$ aus $U_{i}$ dem Objekt $y$ aus $U_{k}$ entspricht. Es kann dann zwei verschiedenen Objekten $x$ und $x^{\prime}$ aus $U_{i}$ auch dasselbe $O b-$ jekt $y$ aus $U_{k}$ entsprechen. Aber $R$ ist dann eben keine Identitätsrelation, und daher kann man nicht behaupten, $x$ und $x^{\prime}$ seien in $k$ identisch.

${ }^{31}$ Vgl. dazu Kutschera (76), S. 43 ff. 
eine Aussage über Objekte lesen, denn es gibt keine festen Objekte, die durch die Terme ,,$"$ " und , $t$ " in allen Welten bezeichnet würden. Er besagt also nur: Die Aussage , $s=t^{\prime \prime}$ gilt notwendigerweise, und kann auch dann gelten, wenn die Terme ,, $s$ und , $t$ " keine Standardnamen sind, falls sie nämlich - welche Objekte sie auch immer in verschiedenen Welten bezeichnen - in jeder Welt dasselbe Objekt bezeichnen. Es gibt also keine kontingenten Identitäten von Objekten, sondern nur kontingente Aussagen über die Identität von Objekten. Läßt sich „N $\mathrm{N}(s=\mathrm{t})$ “ als Aussage über Objekte verstehen - ist im Sinne Quines der Bezug von , $s$ " und ,t" in dieser Aussage transparent, so daß man $z$. B. aus ihr auf die Sätze $\operatorname{VxN}(\mathrm{x}=\mathrm{t})$ und $\mathrm{VyN}(\mathrm{s}=\mathrm{y})$ schließen kann - , so sind die ,s" und „t" Standardnamen für Objekte, und es gilt dann auch $\mathrm{N}(s=\mathrm{t})$ oder $\mathrm{N}(s \neq \mathrm{t}){ }^{32}$

Wie steht es nun mit kontingenten Identitäten von Attributen und Zuständen? Wenn man einen Zustand als eine Proposition einer gewissen Art auffaßt, im Rahmen der intensionalen Semantik also als Menge von Welten (in denen er besteht), so drücken die Sätze , $M(a, t)$ “ und , $B(a, t)$ “ genau dann denselben Zustand aus, wenn ihre Äquivalenz analytisch gilt, und , $M(x, t)$ “ und , $B(x, t)$ “ drücken genau dann dieselbe Zustandsart aus, wenn (c) gilt. Daher muß sich die Ider,titätschese auf einen anderen Eegriff des Zustands stützer. Da man mit Quine sagen kann: „No entity without identity“, sind dann zunächst Identitätskriterien für Zustände anzugeben. Es genügt hier, elementare Zustände zu betrachten, wie sie durch einfache Sätze der Form ,,$F\left(a_{1}, \ldots, a_{m}\right)$ “ und , $G\left(b_{1}, \ldots, b_{n}\right)$ “ ausgedrückt werden. Man hat vorgeschlagen, diese beiden Sätze sollten genau dann denselben Zustand ausdrücken, wenn „,F“ und „G“ für dasselbe Attribut stehen - dann muß $n=m$ sein - und wenn (für $r=1, \ldots, n$ ),, $a_{r}$ " und , , $b_{r}{ }^{\prime \prime}$ dasselbe Objekt bezeichnen. Eine kontingente Identität der beiden $\mathrm{Zu}$ stände läge danach also genau dann vor, wenn entweder für ein $\mathrm{r}$ die Objekte $a_{r}$ und $b_{r}$ nur kontingent identisch sind, oder wenn die Attribute $F$ und $G$ nur kontingent identisch sind. Daß es eine kontingente Identität von Objekten nicht gibt, haben wir bereits gesehen. Die Frage einer kontingenten Identität von Zuständen reduziert sich damit auf die Frage, ob es eine kontingente Identität von Attributen

${ }^{32}$ Vgl. dazu Kutschera (76), S. 95 ff. Nach der traditionellen Unterscheidung ist $\mathrm{N}(s=\mathrm{t})$ eine Modalaussage de dicto (über Sachverhalte), $\Lambda \mathrm{xy}(\mathrm{x}=\mathrm{s} \wedge$ $\mathrm{y}=\mathrm{t} \supset \mathrm{N}(\mathrm{x}=\mathrm{y})$ ) hingegen eine Modalaussage de re (über Objekte). 
gibt. Wann stehen , $\mathrm{F}^{\prime \prime}$ und ,G“ für dasselbe Attribut? Gilt das nur im Fall von $N \wedge x_{1} . . x_{n}\left(F\left(x_{1}, \ldots, x_{n}\right) \equiv G\left(x_{1}, \ldots, x_{n}\right)\right)$, so werden wir wieder auf (c) geführt. Die Identitätsthese muß sich also auf eine schwächere, kontingente Identität von Attributen stützen. Fälle kontingenter Identität von Attributen, so sagen z. B. Kim in (66) oder Putnam in (66), sind

d) Blau ist die Farbe des Himmels

e) Gerechtigkeit ist die von Platon am höchsten geschätzte charakterliche Eigenschaft.

Das sind sicher kontingente Identitätsaussagen. Es ist nicht analytisch notwendig, daß der Himmel blau ist, noch daß Platon Gerechtigkeit am höchsten schätzt. Aber daraus folgt ebensowenig, daß im Fall (d) die Eigenschaften ,Blau' und ,die Farbe des Himmels' kontingent, d. h. nur in unserer Welt, identisch wären, wie das im Fall der Eigennamen „Morgenstern“ und „Abendstern“ folgte. In der intensionalen Semantik sind Eigenschaften Funktionen g, die jeder Welt i aus I eine Funktion g(i) zuordnet, die ihrerseits den Gegenständen aus U Wahrheitswerte w (,,wahr") und $f($,falsch") zuordnet, je nachdem ob ein Objekt in $i$ die Eigenschaft hat oder nicht. Ist $A^{B}$ die Menge der Funktionen, welche die Menge $B$ in die Menge $A$ abbilden, so ist $g(i)$ eine Funktion aus $\{w, f\} \cup$ und $g$ eine Funktion aus $(\{w, f\} U)$. Wie wir Objekte aus $U$ und Individualbegriffe - Funktionen aus $U^{l}$, die jeder Welt i ein Objekt aus $U$ zuordnen (die Extension in i des Namens, der diesen Individualbegriff ausdrückt; solche Individualbegriffe entsprechen dem, was man als Sinn eines Eigennamens bezeichnet) - unterscheiden müssen, so müssen wir auch die Funktionen aus $\{\mathrm{w}, \mathrm{f}\} \mathrm{U}$ und jene aus $(\{\mathrm{w}, \mathrm{f}\} \mathrm{U})^{\mathrm{I}}$ unterscheiden. Die letzteren bezeichnet man normalerweise als „Eigenschaften“, die ersten sind so etwas wie ,extensionale Eigenschaften", die eindeutig Begriffsumfängen entsprechen, d. h. Mengen von Objekten. Entsprechendes gilt für mehrstellige Attribute. Extensionale wie intensionale Eigenschaften sind identisch oder sie sind nicht identisch, und ihre Identität hat nichts mit möglichen Welten zu tun. Sind sie also identisch, so sind sie notwendig identisch, und sind sie verschieden, so sind sie notwendigerweise verschieden.

Die Aussagen (d) und (e) haben die Gestalt:

f) $F=\imath f G(f)$.

Dabei ist nun die Frage: Sollen „,F“ und , $\mathrm{LGG}(\mathrm{f})$ “ Namen für extensionale oder Namen für intensionale Eigenschaften sein? Im ersten 
Fall haben wir eine genaue Analogie zu Identitätsaussagen über Objekte. Wie ein Eigenname ,"s in jeder Welt i ein bestimmtes Objekt bezeichnet, und einen Individualbegriff ausdrückt, (also die Funktion, die jeder Welt $\mathrm{i}$ aus I das Objekt zuordnet, das , $s$ “ in i bezeichnet), so bezeichnet ein Name wie , $F^{\prime \prime}$ in jeder Welt $i$ eine extensionale Eigenschaft, und er drückt eine intensionale Eigenschaft aus. In den Beispielen (d) und (e) bezeichnet der Name , , $\mathrm{fG}(\mathrm{f}$ ) " in verschiedenen Welten verschiedene extensionale Eigenschaften. Aus ( $f$ ) folgt also nicht $\mathrm{N}(\mathrm{F}=\mathrm{lfG}(\mathrm{f}))$, sondern nur $\wedge \mathrm{gh}(\mathrm{g}=\mathrm{F} \wedge \mathrm{h}=\mathrm{lFG}(\mathrm{f}) \supset$ $\mathrm{N}(\mathrm{g}=\mathrm{h})) \cdot{ }^{33} \mathrm{~N}(\mathrm{~F}=\mathrm{tfG}(\mathrm{f}))$ ist keine Aussage über extensionale Eigenschaften, weil $\mathrm{ifG}(\mathrm{f})$ kein Standardname für solche Eigenschaften ist. Daher kann man die (richtige) Aussage, daß der Satz $F=\mathrm{lfG}(\mathrm{f})$ ) kontingent ist, nicht als Aussage über eine Kontingenz der Identität von zwei extensionalen Eigenschaften verstehen.

Auch im zweiten Fall, in dem „,F“ und „,fG(f) “ Namen für intensionale Eigenschaften sein sollen, kann , $\mathrm{lfG}(\mathrm{f})$ “ in verschiedenen Welten Verschiedenes bezeichnen, so daß auch hier der Satz $N(F=t f G(f))$ nicht gilt. Auch bei dieser Deutung ist die Aussage dann aber keine Aussage über die Identität zweier intensionaler Eigenschaften, sondern es gilt nur: Die beiden Eigenschaften, die ,, $F^{“}$ und ,, $\mathrm{fG}(\mathrm{f})$ “ in unserer Welt beztichmen, sind notwendigerweise iclentisch, d.h. $\wedge \mathrm{gh}(\mathrm{g}=\mathrm{F} \wedge \mathrm{h}=\mathrm{lfG}(\mathrm{f}) \supset \mathrm{N}(\mathrm{g}=\mathrm{h}))$.

Wir können also auch für Attribute - und damit für die nach dem obigen Vorschlag definierten Zustände - sagen: Es gibt keine kontingenten Identitäten, sondern nur kontingente Identitätsaussagen.

${ }^{33}$ Die Quantoren über Eigenschaften sind dabei analog zu definieren wie jene über Objekte. Vgl. dazu Kutschera (76), 2.4. Setzt man nicht voraus, daß die Gegenstandskonstanten a,b,c, ... als Standardnamen gedeutet werden, so soll die Interpretation $\Phi$ den Satz $\wedge \mathrm{xA}(\mathrm{x})$ in der Welt i wahr machen genau dann, wenn alle Interpretationen $\Phi^{\prime}$, die sich von $\Phi$ höchstens bzgl. der Deutung einer Gegenstandskonstanten a unterscheidet, die im Satz $\wedge \mathrm{xA}(\mathrm{x})$ nicht vorkommt, und die a als Standardnamen deuten, den Satz $\mathrm{A}(\mathrm{a})$ in $\mathrm{i}$ wahr machen - symbolisch

$\Phi_{\mathrm{i}}(\Lambda \mathrm{xA}(\mathrm{x}))=$ w genau dann, wenn $\Lambda \Phi^{\prime}\left(\Phi^{\prime} \overline{\mathrm{a}} \Phi \wedge \Lambda \mathrm{k}\left(\Phi_{\mathrm{k}}(\mathrm{a})=\Phi_{\mathrm{i}}(\mathrm{a})\right) \supset\right.$ $\left.\Phi_{1}^{\prime}(A(a))=w\right)$. Analog setztman

$\Phi_{\mathrm{i}}(\Lambda \mathrm{f} A(\mathrm{f}))=$ w genaudann, wenn $\Lambda \Phi^{\prime}\left(\Phi_{\overline{\mathrm{F}}}^{\prime} \Phi \wedge \Lambda \mathrm{k}\left(\Phi_{\mathrm{k}}(\mathrm{F})=\Phi_{\mathrm{i}}^{-}(\mathrm{F})\right) \supset\right.$

$\Phi(\mathrm{A}(\mathrm{F}))=\mathrm{w})$, wobei die Prädikatkonstante $F$ nicht in $\Lambda \mathrm{fA}(\mathrm{f})$ vorkommen soll. 
Gibt es aber keine kontingenten Identitäten, so ist die Identitätsthese II' nicht haltbar.

Unsere Argumentation stützt sich auf die intensionale Semantik. Aber das ist der einzige Rahmen, in dem man gegenwärtig solche Fragen exakt diskutieren kann. Wollte man behaupten, in einer anderen Semantik könne für so etwas wie kontingente Identitäten Platz sein, so müßte man eine solche Semantik angeben; dazu ist aber kein geeigneter Ansatz in Sicht.

Der Materialismus ist ein Proteus: wenn man ihn zu fassen sucht, ändert er seine Gestalt. Nach Behaviorismus und Identitätsthese ist gegenwärtig der ,Funktionalismus“ en vogue, wobei diese Bezeichnung aber für recht verschiedenartige Konzeptionen verwendet wird. (Eine Sammlung einschlägiger Arbeiten ist Block [80]. Dort finden sich auch ausführliche Literaturhinweise.) Die beiden einflußreichsten Versionen sind die von H. Putnam und D. Lewis. Putnam hat in (60) und späteren Aufsätzen die Ansicht vertreten, der Mensch (oder der menschliche Geist) sei eine Maschine - dieselbe Idee also, die schon im 18. Jahrhundert Leute wie J. Lamettrie vertreten hatten. Neu ist nur, daß nun von Maschinen in einem sehr allgemeinen Sinn die Rede ist, von Turing-Maschinen oder probabilistischen Automaten. So eine Maschine S läßt sich in ihrer Funktionsweise ohne Bezug auf ihre physikalische Realisierung (z. B. mit Transistoren und Schaltkreisen) durch die Maschinentafel charakterisieren, in der die möglichen Eingaben, Ausgaben und Maschinenzustände angegeben werden und spezifiziert wird, von welchen Zuständen (bei welchen Eingaben) $S$ (mit welchen Wahrscheinlichkeiten) zu anderen Zuständen (und Ausgaben) übergeht. Verschiedene physikalische Systeme können dieselbe Maschine darstellen, d. h. sich in gleicher Weise funktional beschreiben lassen. Solche Systeme bezeichnet man (bzgl. dieser Beschreibung) als „funktional isomorph". Psychische Zustände sollen nun nach Putnam solche funktionalen Zustände einer Maschine sein. Sie werden also nicht im Sinn der Identitätsthese mit physikalischen $\mathrm{Zu}$ ständen identifiziert. Das menschliche Gehirn ist nur eine physikalische Realisierung so einer Maschine neben anderen. Für Putnam sind funktionale Zustände kategorial von den physikalischen Zuständen verschieden, die sie realisieren. Daher ist für ihn die Frage sinnlos, ob mentale Zustände mit gewissen physikalischen identisch sind. $\mathrm{Da}$ es aber nach seiner Ansicht nur physikalische Realisierungen von Automaten gibt - diese materialistische Prämisse begründet er nicht wei- 
ter -, spricht das nicht gegen einen Materialismus. Den Fortschritt gegenüber der Identitätsthese sieht er darin, daß man nach dieser Konzeption Lebewesen mit ganz verschiedenen Gehirnstrukturen also z. B. Eidechsen ebenso wie Menschen - dieselben psychischen Zustände (wie etwa Schmerzempfindungen) zuschreiben kann. Die Behauptung ist also: Das System aus Sinnesreizen (als Eingaben), psychischen Zuständen und dem Verhalten (als Ausgabe) bildet einen Automaten, der physikalisch realisiert ist im zentralen Nervensystem (oder dem gesamten menschlichen Organismus).

Das Ganze ist aber nicht mehr als ein Programm wie alle anderen Versionen des Materialismus auch. Es wäre zunächst zu zeigen, daß sich das Psychische in seiner Abhängigkeit von Umweltreizen und seinen Wirkungen auf unser Verhalten als Automat darstellen läßt. Es genügt nicht $\mathrm{zu}$ zeigen, daß sich gewisse psychische Phänomene so verstehen lassen. In hinreichend grober und unvollständiger Weise läßt sich alles funktional beschreiben. Es gibt z. B., wie W. Kalke in (69) betont, eine funktionale Beschreibung der Katze, bei der sie funktional isomorph ist mit einer Mausefalle; daraus folgt aber nicht, daß Katzen Mausefallen sind oder sich vollständig wie diese beschreiben lassen. Es wäre also nachzuweisen, daß sich das Psychische vollständig als Automat charakterisieren läßt. Dafür bringt aber Putnam kein einziges Argument. Die funktionale Isomorphie zweier Systeme impliziert ferner nicht ihre Identität. Selbst wenn es also gelänge, das Psychische vollständig als Automat zu beschreiben und nachzuweisen, $\mathrm{da} ß$ es eine Beschreibung der Gehirnvorgänge gibt, bei der diese mit dem Psychischen funktional isomorph sind, würde daraus nicht die zentrale These des Materialismus folgen, das Psychische sei etwas Materielles. Putnam setzt vielmehr, wie schon betont wurde, den Materialismus voraus, wenn er annimmt, es gebe nur physikalische Realisierungen von Automaten. Programme sind keine Argumente, und daher macht auch der Putnamsche Funktionalismus den Materialismus nicht plausibel.

D. Lewis hat in (70) die Auffassung vertreten, theoretische Terme ließen sich durch nicht-theoretische definieren. Als ,theoretisch“ bzgl. einer Theorie $T$ bezeichnet er jene Terme in $T$, die keine von $T$ unabhängige Bedeutung haben, sondern erst im Kontext von $\mathrm{T}$ gedeutet werden. Seine Ansicht, sie ließen sich definieren, stützt sich darauf, daß er eine Theorie $T$ mit den theoretischen Termen $t_{1}, \ldots, t_{n}$ nur dann als wahr ansieht, wenn es genau ein $n$-tupel von Objekten 
gibt, das T erfüllt. (Da Lewis Prädikate als Namen für Klassen deutet, sind auch Begriffsumfänge solche Objekte.) Diese Voraussetzung ist aber im Blick auf die Terme, die man üblicherweise als ,theoretisch“ bezeichnet, völlig unplausibel, und Lewis gibt auch kein Argument an, das diese Voraussetzung als sinnvoll erscheinen ließe. In (72) geht er nun davon aus, daß eine psychologische Theorie $T$ vorliegt, in der die psychologischen Terme als theoretische Terme fungieren. Sie lassen sich dann durch die in $T$ vorkommenden nicht-psychologischen Terme, also durch Verhaltensterme definieren. Gelingt es außerdem zu zeigen, daß die Theorie auch erfüllt ist bei Ersetzung der psychologischen Terme durch gewisse neuro-physiologische, so folgt daraus unmittelbar, daß diese dasselbe bezeichnen wie jene. Da Lewis es für wahrscheinlich hält, daß so etwas gelingt, vertritt er die Identitätsthese.

Dazu ist - neben dem bereits erwähnten Einwand - zu sagen: Erstens sind psychologische Terme - auch im Sinn von Lewis - keine theoretischen Terme; sie haben auch außerhalb einer (vollständigen) psychologischen Theorie eine Bedeutung. Tatsächlich verwenden wir sie, ohne über eine solche Theorie zu verfügen. Zweitens gibt es keine scharfe Grenze zwischen psychologischen und nichtpsychologischen Termen, z. B. Verhaltenstermen, wie wir im Abschnitt 7.4 sehen werden. Drittens besagt eine Identität der Extensionen zweier Prädikate nicht, daß sie dieselben Eigenschaften, Beziehungen oder Zustände ausdrücken. Haben genau die Lebewesen ein Herz, die eine Niere haben, so folgt daraus nicht, daß ein Herz zu haben nichts anderes ist, als eine Niere zu haben. Viertens ist der Lewische Funktionalismus nur eine Variante der Identitätsthese, also allen Einwänden ausgesetzt, denen diese begegnet. Schließlich scheitern beide Versionen des Funktionalismus, die von Lewis wie die von Putnam, daran, daß die psychologische Sprache ausdrucksreicher ist als die physikalische. Darauf gehen wir im nächsten Abschnitt ein. Auch der Funktionalismus ist also nur eine andere Einkleidung des Materialismus, die nichts zu seiner Begründung beiträgt: des Kaisers neue Kleider.

Bisher haben wir nur die Argumente für die These PK einer Kritik unterzogen. Aus deren negativem Resultat folgt nicht, daß die These selbst falsch ist. Gegen diese These gibt es aber einen ebenso einfachen wie schlagenden Einwand, der oben schon angeklungen ist: Wäre PK wahr, so wären die Äquivalenzen zwischen biologischen oder psychologischen Zuständen einerseits und physikalischen $\mathrm{Zu}$ - 
ständen andererseits Bedeutungswahrheiten. Die Biologen, Psychologen und Neurophysiologen, die sich auf empirischem Wege bemühen, solche Äquivalenzen zu entdecken, könnten ihre mühsame Arbeit dann einfach den Linguisten überlassen, die solche Probleme allein aufgrund ihrer Sprachkompetenz lösen würden. Eine solche Vorstellung ist aber doch zu phantastisch. Es ist einfach falsch, daß wir mit einem Satz wie ,Eva ist fröhlich“ meinen, daß sich Eva so und so verhält oder daß ihr zentrales Nervensystem sich in dem und dem $\mathrm{Zu}$ stand befindet. Wir wissen, was wir mit diesem Satz meinen, können aber trotzdem - im Gegensatz zur These PK - kein notwendiges und hinreichendes Verhaltens- oder neurophysiologisches Kriterium für Fröhlichkeit angeben, und das liegt nicht daran, daß wir der deutschen Sprache und der naturwissenschaftlichen Fachsprachen nicht mächtig wären. ${ }^{34}$

\subsection{Die Reduzierbarkeitsthese}

Wenden wir uns nun der These PS zu. Die beiden Hauptargumente für diese These können wir nach den vorausgegebenen Erörterungen kurz so zusammenfassen:

1. Für alle Terme einer intersubjektiven Sprache wie $\Omega$ muß es intersubjektiv kontrollierbare Gebrauchskriterien geben. Solche Kriterien werden aber durch Sätze von $\Phi$ dargestellt.

${ }^{34}$ Man kann auch so argumentieren: Ist $\mathrm{S}$ eine Sprache über physikalische Sachverhalte, die über einer Menge I möglicher Welten interpretiert ist, und $S$ eine Sprache über das, was eine Person a bzgl. dieser Sachverhalte glaubt, so ist $S$ ' über einer Menge I' möglicher Welten zu interpretieren, die von höherer Mächtigkeit ist als I. Denn für jeden möglichen physikalischen Sachverhalt, $d$. h. für jede nichtleere Teilmenge $X$ von $I$ ist es möglich, daß a genau von den Sachverhalten überzeugt ist, die aus $X$ folgen. (Im Sinn der Definition D 4.2-2 in Kutschera (76), S. 91 muß es also für jede solche Menge $X$ eine Welt $i$ aus I' geben mit $S_{i}=X$.) I' muß also dieselbe Mächtigkeit haben wie die Potenzmenge von I, abzüglich der leeren Menge. Da es nicht analytisch notwendig ist, daß a nur bestimmte unter den möglichen physikalischen Sachverhalten glauben kann, muß es also viele Welten geben, die sich physikalisch nicht unterscheiden, wohl aber bzgl. dessen, was die Person a in ihnen glaubt. Daher ist es nicht möglich, die Sprache $S^{\prime}$ in die Sprache $S$ zu übersetzen. 
2. Die Entwicklung der Wissenschaften deckt immer engere und weiter reichende Beziehungen zwischen organischen und psychischen Phänomenen einerseits und physikalischen Phänomen andererseits auf. Es ist also wahrscheinlich, daß man einmal die These PS in allen Details wissenschaftlich belegen kann.

$\mathrm{Zu}$ (1) ist wie oben zu sagen: Intersubjektive Gebrauchskriterien sind nicht immer nur physikalische Kriterien; aber auch die Existenz solcher Kriterien stellt noch nicht sicher, daß sich alle Terme von $\Omega$, die nicht schon selbst Terme von $\Phi$ sind, so in $\Phi$ definieren lassen, $\mathrm{da} ß$ dadurch alle wahren Sätze von $\Omega$ in wahre Sätze von $\Phi$ übergehen.

(2) ist eine Prognose über künftige wissenschaftliche Entwicklungen, bei der man zunächst darauf hinweisen muß, daß die heute bekannten Korrelationen nichtphysikalischer und physikalischer Phänomene bei weitem nicht hinreichen, um der These PS eine hohe Wahrscheinlichkeit zu sichern. Die bekannten Korrelationen sind ferner so komplex, daß man aus ihnen auch nicht Definitionen der Terme aus $\Omega$ im Sinne von PS gewinnen kann. Die Annahme einer Korrespondenz im Sinne von PS geht sehr viel weiter als die Annahme starker gegenseitiger Abhängigkeiten der Phänomene.

Sinnvoller als Spekulationen über künftige Entwicklungen der Wissenschaften anzustellen wäre es auch, bereits erzielte Resultate zur Kenntnis zu nehmen und sich klar zu machen, daß der Pbysikalismus durch die Entwicklung der Physik seit gut 50 Jabren überbolt ist. Im Sinn des Physikalismus sind Beobachter physikalische Systeme und Beobachtungen physikalische Wechselwirkungen zwischen ,beobachtenden“ und „,beobachteten“ Systemen. In der Quantenmechanik ist es aber nicht möglich, Beobachtungen so zu deuten. Darauf gehen wir im Abschnitt 8.4 näher ein. Hier wollen wir ein einfaches logisches Argument gegen die These PS angeben, das, kurz gesagt, so lautet: Die normale Sprache, und damit $\Omega$, ist reich an nichtextensionalen Prädikaten. Das gilt insbesondere für die Sprache über Psychisches. Prädikate wie „Glauben“, ,Wollen“, „Beabsichtigen“, „Vorziehen“, „Wünschen“, ,Hoffen“, ,Sagen“, Bezweifeln" usw. sind nichtextensionale Prädikate. Wir haben z. B. schon in 1.1 gesehen, daß die Sätze „,a glaubt, daß $A$ “ und ,,a glaubt, daß $B^{\prime \prime}$ nicht immer denselben Wahrheitswert haben, wenn das für A und B gilt. Die physikalische Sprache ist hingegen eine rein extensionale Sprache. Nichtextensionale Prädikate sind aber nicht mit extensionalen äquivalent, und da- 
her lassen sich nichtextensionale Sprachen nicht auf extensionale Teilsprachen W-reduzieren.

Überlegen wir uns das genauer. Nehmen wir z. B. an, daß das Prädikat ,,a glaubt, daß $\mathrm{p}^{\text {" }}$ - symbolisch $G(\mathrm{a}, \mathrm{p})$ - in $\Phi$ durch ein Prädikat $G^{+}(a, p)$ definiert wird. $G^{+}$ist nur dann ein physikalisches Prädikat, wenn es extensional ist. Haben also zwei Sätze $A$ und $B$ von $\Phi$ denselben Wahrheitswert, so gilt $G^{+}(a, A) \equiv G^{+}(a, B) . \Omega$ enthält nun $\Phi$ als Teilsprache, d. h. es sind auch $G(a, A)$ und $G(a, B)$ Sätze von $\Omega$, und ihre Entsprechungen in $\Phi$ lauten $G+(a, A)$ und $G+(a, B)$. Es müßte also bei W-Reduzierbarkeit von $\Omega$ auf $\Phi$ auch allgemein gelten $(A \equiv B) \supset(G(a, A) \equiv G(a, B))$, für alle Sätze $A, B$ von $\Phi$. Aus PS folgt nun zwar nicht, daß diese Implikationen analytisch gelten, $d$. h. unabhängig davon, daß sie in unserer Welt gelten. Es muß aber jedenfalls für alle in unserer Welt wahren Sätze A und B von $\Phi G(a, A)$ genau dann wahr sein, wenn $G(a, B)$ wahr ist. Ist also a von der Geltung eines einzigen wahren physikalischen Satzes überzeugt, so von der Geltung aller solchen Sätze. Weiß also a von einem physikalischen Sachverhalt, daß er besteht, so von allen; $d$. h. a hat keinerlei physikalische Kenntnisse oder a ist allwissend auf physikalischem Gebiet. Das ist offensichtlich absurd. Ist ferner $A^{+}$die Entsprechung eines Satzes $A$ von $\Omega$ in $\Phi$, so kann gelten $G(a, A)$, aber nicht $G\left(a, A^{+}\right)$- es kann der Person a ja unbekannt sein, daß $\mathrm{A}$ mit $\mathrm{A}+$ äquivalent ist - wie uns tatsächlich ja fast alle der in PS geforderten Äquivalenzen unbekannt sind. Die Entsprechung dieser Annahme in $\Phi: G^{+}\left(a, A^{+}\right) \wedge$ $\neg \mathrm{G}^{+}\left(\mathrm{a}, \mathrm{A}^{+}\right)$ist aber kontradiktorisch.

Gegen diese Überlegung kann man folgendes einwenden:

a) Es sind nicht Prädikate wie $G(a, p)$ in $\Phi$ zu definieren, sondern Vollausdrücke solcher Prädikate, d. h. den Sätzen $G\left(a, A_{1}\right), G\left(a, A_{2}\right)$, usf. sind äquivalente Sätze $G_{1}^{+}(a), G_{2}^{+}(a)$, usf. von $\Phi$ zuzuordnen. So könnten z. B. den Sachverhalten, daß die Person a glaubt, daß sie $75 \mathrm{~kg}$ wiegt, und daß sie glaubt, daß die Mumie von Ramses II im Ägyptischen Museum von Kairo ausgestellt ist, nicht nur verschiedene, sondern verschiedenartige neurophysiologische Zustände entsprechen. - Solche Entsprechungen wären aber erstens nicht ausreichend, um eine W-Reduzierbarkeit zu begründen. Im Sinn von D3.3 - 3 läge eine $W$-Reduktion von $\Omega$ auf $\Phi$ nur dann vor, wenn sich das Glaubensprädikat $G$ explizit in $\Phi$ definieren ließe. Das ist aber nicht möglich, wie wir gesehen haben. Zweitens müßte man unendlich viele Definitionen angeben - zu jedem Satz A eine für das Prädikat 
$\mathrm{G}(\mathrm{a}, \mathrm{A})$. Drittens ließen sich dann in $\Phi$ keine Entsprechungen zu generellen Aussagen in $\Omega$ wie $\wedge p(G(a, p) \supset \neg G(a, \neg p))$ angeben. Und viertens würde damit die Behauptung der W-Reduzierbarkeit von $\Omega$ auf $\Phi$ durch eine völlig triviale These ersetzt, die Behauptung nämlich, daß es eine Zuordnung von physikalischen Sätzen $A^{+} z u$ den Sätzen $\mathrm{A}$ von $\Omega$ gibt, bei der $\mathrm{A}$ und $\mathrm{A}^{+}$denselben Wahrheitswert haben. Eine solche Zuordnung ist aber ohne jedes Interesse, denn man kann ja z. B. allen wahren Sätzen von $\Omega$ (abzüglich der Teilsprache $\Phi$ ) den Satz „Die elektrische Ladung eines Elektrons beträgt 1,602. 10-19 Coulomb" von $\Phi$ zuordnen, und allen falschen Sätzen von $\Omega$ den Satž ,Die Ladung eines Elektrons beträgt 15 Coulomb“.

b) Man könnte in $\Phi$ einen theoretischen Term $G^{+}$einführen, mit dem sich das Glaubensprädikat $G$ definieren läßt. Es ist also nicht notwendig, $G$ durch die üblichen physikalischen Terme explizit zu definieren, sondern es genügt, hinreichend starke physikalische Bedingungen für eine implizite Definition von $G$ anzugeben. - Nun gilt aber für alle Personen a und alle Sachverhalte $p$ immer $G(a, p)$ oder $\neg \mathrm{G}(\mathrm{a}, \mathrm{p})$. Daher müßten die Postulate der impliziten Definition von $G^{+}$so stark sein, daß sie allen $G^{+}-$Sätzen einen bestimmten Wahrheitswert zuordnen. Dann läßt sich $G^{+}-$und damit auch $G$ selbst in $\Phi$ aber explizit definieren. ${ }^{35} \mathrm{Im}$ übrigen wären die Bedingungen für $\mathrm{G}^{+}$extensional; auf diesem Weg kann man daher unserem Argument nicht entgehen.

c) Intensionale Sprachen lassen sich in extensionale übersetzen: Die Semantik solcher Sprachen wird ja in einer Sprache formuliert, in der nur von Extensionen: von Objekten, Mengen und Funktionen die Rede ist. - Die Semantiksprache ist aber keine physikalische Sprache, und man kann sie auch nicht in die Sprache $\Phi$ übersetzen. Da die Sprache über das, was Personen glauben, die Sprache $\Phi$ enthält ihre Annahmen beziehen sich ja insbesondere auch auf die physische Welt - , enthielte sonst $\Phi$ seine eigene Metasprache. Dann ließen sich aber in $\Phi$ semantische Antinomien konstruieren. ${ }^{36}$

Der Physikalismus nimmt in all seinen Versionen an, daß der physikalische Zustand der Welt in jedem Zeitpunkt $t$ die psychischen Zustände aller Menschen in $t$ eindeutig bestimmt. Er nimmt spezieller

${ }^{35} \mathrm{Vgl}$. dazu Kutschera (72), $271 \mathrm{ff}$.

${ }^{36}$ Vgl. dazu z. B. Tarski (44). 
an, daß die physikalischen Zustände des Zentralnervensystems jeder Person $\mathrm{x}$ in $\mathrm{t}$ ihre psychischen Zustände in $\mathrm{t}$ bestimmen. Es muß also, wenn schon keine W-Reduzierbarkeit besteht, doch insbesondere eine Funktion $\varphi$ geben, die jeder Person $x$, jedem Zeitpunkt $t$ und jedem Sachverhalt $\mathrm{p}$ einen Gehirnzustand $\varphi(\mathrm{x}, \mathrm{t}, \mathrm{p})$ von $\mathrm{x}$ in $\mathrm{t}$ zuordnet, der genau dann besteht, wenn $\mathrm{x}$ in $\mathrm{t}$ glaubt, $\mathrm{daß} \mathrm{p}-$ symbolisch

1. $\wedge \operatorname{xtp}\left(\varphi(x, t, p) \equiv G_{t}(x, p)\right)$.

Noch liberaler läßt sich eine physikalische These sicher nicht formulieren. Eine Person a erkennt im Zeitpunkt t', daß diese physikalische These richtig ist, wenn neben (1) gilt: a glaubt in t', daß (1) wahr ist, also

2. $G_{t},\left(a, \wedge x t p\left(\varphi(x, t, p) \equiv G_{t}(x, p)\right)\right)$.

Nun gilt aber, wie wir im 1. Kapitel gesehen haben, trivialerweise, daß jedermann das für wahr hält, wovon er überzeugt ist. Es muß also im Sinn des Axioms $G 4$ aus 1.1 auch gelten

3. $G_{t},\left(a, \wedge p\left(G_{t},(a, p) \supset p\right)\right)$.

Daraus ergibt sich mit (2) aber

4. $G_{t},\left(a, \wedge p\left(\varphi\left(a, t^{\prime}, p\right) \supset p\right)\right)$

d. h.: $a$ ist in ' $t^{\prime}$ davon überzeugt, daß sich sein Gehirn nur dann in dem Zustand $\varphi\left(a, t^{\prime}, p\right)$ befindet (der einem Glauben, daß p, entspricht), weenn der Sachverinalt tátsächlich besieht. à müßte also, um Physikalist zu sein, annehmen, daß seine Gehirnzustände die gesamte Außenwelt insofern abbilden, als für alle Tatsachen $p$ sein Gehirn sich nicht im Zustand $\varphi\left(a, t^{\prime}, \neg p\right)$ befinden kann. Der Physikalist a muß also in ' $t^{\prime}$ überzeugt sein, daß $z$. B. ein räumlich und zeitlich weit von ihm entferntes Ereignis $\mathrm{p}$ - etwa ein mikrophysikalisches Ereignis in einem fernen Sternsystem in einigen Jahrmilliarden - bedingt, daß sein Gehirn jetzt (in $\left.\mathrm{t}^{\prime}\right)$ nicht im Zustand $\varphi\left(a, t^{\prime}, \neg p\right)$ ist. Wenn a (in $t^{\prime}$ ) das Bestehen von (1) als naturgesetzliche Tatsache ansieht, wie das jeder Physikalist tut, so muß a annehmen, daß beliebige Ereignisse in der Zukunft seine gegenwärtigen Gehirnzustände determinieren. Das wäre aber eine allzu phantastische Vorstellung. Macht man sie sich jedoch nicht zu eigen, so ist man kein konsequenter Physikalist - auch im liberalsten Sinn dieses Wortes. 


\subsection{Determinismus}

An die Bemerkungen zum Physikalismus wollen wir in diesem Abschnitt noch einige Überlegungen zum Determinismusproblem anschließen.

Als ,deterministisch“ kann man Gesetze, Theorien und Systeme bezeichnen. ${ }^{37}$ Was deterministische Gesetze und was Kausalgesetze sind, haben wir schon im Abschnitt 2.2 erläutert. Eine Theorie heißt deterministisch, wenn ihre Grundgesetze sämtlich deterministisch sind, genauer: deterministisch bzgl. aller Zustandsgrößen oder -arten, von denen in diesen Gesetzen die Rede ist. Wie Stegmüller in (69), S. 487 betont, ist dabei die Bezugnahme auf die Grundgesetze der Theorie insofern wesentlich, als sich aus deterministischen Gesetzen - wie z. B. in der Mechanik - auch statistische Gesetze ableiten lassen und umgekehrt. Enthält eine Theorie auch statistische Grundgesetze, so nennen wir sie indeterministisch. Eine Theorie, deren Grundgesetze Kausalgesetze sind, nennen wir eine kausale Theorie. Ein System endlich heißt deterministisch, bzw. kausal, wenn es sich durch eine deterministische, bzw. kausale Theorie vollständig beschreiben läßt. So ist z. B. unser Planetensystem (in seinen Bewegungszuständen) im Rahmen der deterministischen Gravitationstheorie vollständig beschreibbar.

Bei der Diskussion des (universellen) Determinismus sind zwei Thesen zu unterscheiden. Die starke Determinismusthese besagt:

$\mathrm{D}^{*}$ : Unsere Welt ist ein deterministisches System.

Die schwächere These besagt:

D: Alle Ereignisse lassen sich deterministisch erklären.

Erklärungen sollen dabei DN-Erklärungen im Sinn von Hempel und Oppenheim sein, wie sie in 2.2 charakterisiert worden sind. Gilt daher $\mathrm{D} \%$, so auch D, da man dann Erklärungen von Ereignissen immer mit der fraglichen deterministischen Theorie vornehmen kann. Die Umkehrung gilt aber nicht. Gibt es zu jedem Ereignis eine Erklärung mit Gesetzen und passenden Antezedensbedingungen, so ist nicht gesagt, $\mathrm{da}$ all diese Gesetze eine Theorie bilden, daß man eine entscheidbare

${ }^{37}$ Vgl. dazu, wie zum ganzen Determinismusproblem, die ausführlichen Erörterungen von Stegmüller in (69), Kap. VII. 
Menge von Axiomen angeben kann, aus denen all diese Gesetze folgen.

Die entsprechenden Kausalitätsprinzipien lauten:

$\mathrm{K}^{*}$ : Die Welt ist ein kausal determiniertes System.

$\mathrm{K}$ : Alle Ereignisse lassen sich kausal erklären.

Eine klassische Formulierung der ersten These findet sich bei P. S. Laplace am Anfang seines ,Essai philosophique sur les probabilités" von 1812. Für ihn war die Newtonsche Mechanik die Theorie, mit der sich die gesamte Wirklichkeit beschreiben läßt. Wäre es möglich, den Zustand der Welt (die Orte und Impulse aller Teilchen) in einem Zeitpunkt vollständig und exakt zu ermitteln und die nötigen Kalkulationen durchzuführen, so könnte man den Zustand der Welt zu jedem anderen Zeitpunkt in allen Details bestimmen. Als ,Kausalitätsprinzip" bezeichnet man meist die These K oder den Satz ,Jedes Ereignis hat eine Ursache“. Nennt man ein Ereignis A „Ursache" eines Ergebnisses B, wenn es eine kausale DN-Erklärung von B mit A als Antezedenzbedingung gibt, so besagt dieser Satz dasselbe wie K. Wir haben aber in 2.2 betont, daß diese Bestimmung des Wortes ,Ursache" nicht dem normalen Sprachgebrauch entspricht. Nach diesem gibt es Ursachen vielmehr nur dann, wenn nicht ailes Gescheinen determiniert ist, wenn also die schwache Determinismusthese $\mathrm{D}$ und damit auch $\mathrm{K}$ (bei dem von uns hier vorausgesetzten Erklärungsbegriff) nicht gilt.

Wenn von „Determinismus“ die Rede ist, so meint man damit meist Auffassungen, wie sie durch $\mathrm{K}$, bzw. $\mathrm{K}^{*}$ ausgedrückt werden. Insbesondere gilt das, wenn Determinismus und Freiheit einander gegenüber gestellt werden. Denn deterministische Gesetze liegen auch dann vor, wenn es möglich ist, aus der Gegenwart auf die Vergangenheit zu schließen, aber nicht umgekehrt. Gibt es Handlungsfreiheit, so besagt das aber nicht, daß man die Entscheidung eines Menschen nicht aus auf sie folgenden Zuständen (z. B. aus seinen Handlungen selbst oder ihren Folgen) bestimmen könnte, sondern daß man sie nicht aus vorhergehenden Zuständen ableiten kann. Daher versteht man auch oft unter deterministischen Thesen die entsprechenden Kausalitätsprinzipien.

Bisher haben wir uns nur mit Sinnfragen beschäftigt, die sich mit den Determinismus- und Kausalitätsprinzipien verbinden. Wir wollen nun zu den Geltungsfragen übergehen. Eine Geltungsfrage stellt sich 
nicht, wenn die Prinzipien nicht als Behauptungen gedeutet werden. So gibt es Auffassungen des Kausalprinzips $K$, nach denen es eine Voraussetzung oder ein Programm wissenschaftlicher Arbeit darstellt. ${ }^{38}$ Als Voraussetzung würde es besagen: Da die (natur-)wissenschaftliche Arbeit wesentlich in der Suche nach allgemeinen Gesetzen besteht, unter die sich die Erscheinungen subsumieren lassen, ist sie nur dann sinnvoll, wenn es immer solche Gesetze gibt. Die Suche nach Kausalgesetzen ist jedoch auch dann sinnvoll, wenn sich manches nicht kausal erklären läßt. Da ferner auch statistische Gesetze nützlich sind und ihre Entdeckung einen Erkenntnisfortschritt darstellt, ist die wissenschaftliche Arbeit selbst dann nicht sinnlos, wenn man - wie z. B. in der Quantenphysik - annimmt, daß alle Grundgesetze statistischen Charakter haben. Als Programm würde K besagen: Die Wissenschaft soll bemüht sein, die Erscheinungen möglichst vollständig durch Kausalgesetze zu beschreiben. Aber auch dieses Programm ist angesichts der Quantenphysik zumindest problematisch geworden. Auf solche Deutungen wollen wir hier nicht näher eingehen; uns geht es um die Diskussion der Thesen als Behauptungen, und als Behauptungen haben wir sie formuliert.

Wir wollen zunächst zeigen, daß das starke Determinismusprinzip D*, und damit auch das starke Kausalitätsprinzip $\mathrm{K}^{*}$, nicht haltbar ist. Dazu zwei Argumente:

1. Es gibt keine Theorie, deterministisch oder statistisch, mit der sich alle empirischen Tatsachen erklären lassen, da es keine Sprache gibt, in der sich alle diese Tatsachen ausdrücken lassen, also auch keine Sprache, in der sich eine solche Theorie formulieren ließe. Es gibt also keine vollständige Theorie der Welt als Menge aller empirischer Tatsachen. $\mathrm{Zu}$ den empirischen Sachverhalten sind dabei im Sinn des universellen Determinismus nicht nur solche zu zählen, die sich auf die Außenwelt beziehen, sondern auch psychologische Sachverhalte.

Ist $S$ eine beliebige Sprache und MS eine Metasprache zu S, in der man über die Ausdrücke von $S$ reden und ihre Bedeutungen charakterisieren kann, so muß MS immer eine ausdrucksreichere Sprache sein als $\mathrm{S}$; andernfalls lassen sich in S semantische Antinomien konstruieren, d. h. Sätze angeben, die zugleich wahr und falsch sind. S wäre dann aber keine konsistent interpretierte Sprache, und jede vollstän-

${ }^{38}$ Vgl. dazu Stegmüller (69), Kap. VII, 7. 
dige Theorie in S (in der sich alle wahren Sätze von $\mathrm{S}$ beweisen lassen) wäre inkonsistent. Es gibt also zu jeder eindeutig interpretierten Sprache $S$ metasprachliche Aussagen, die sich nicht in $S$ übersetzen lassen. Das brauchen nun zwar keine empirischen Aussagen sein, aber zu jeder solchen Aussage A ist $G(a, A)$ ein empirischer Satz, wo a eine bestimmte Person ist. ${ }^{39}$ Mit $G(a, A)$ ist aber auch $\neg \mathrm{G}(\mathrm{a}, \mathrm{A})$ ein empirischer Satz, der nicht in $S$ formulierbar ist. Einer dieser Sätze drückt aber eine empirische Tatsache aus. Also gibt es für jede eindeutig interpretierte Sprache empirische Tatsachen, die sich nicht in ihr ausdrücken lassen. ${ }^{40}$

2. Wie K. Gödel in (31) bewiesen hat, gibt es zu jeder widerspruchsfreien Theorie $T$, die in einer Sprache $S$ formuliert ist, in der sich arithmetische Sachverhalte ausdrücken lassen - die Sprache der Physik ist so eine Sprache -, wahre Sätze von S, die in T nicht beweisbar sind. ${ }^{41}$ Wie die Konstruktion solcher Sätze zeigt, lassen sie sich als wahr erkennen. Die wahre Aussage, daß eine Person a weiß, daß ein solcher Satz A gilt, läßt sich also mit $T$ nicht beweisen, da daraus der

${ }^{39}$ Das gilt zumindest dann allgemein, wenn wir den deskriptiven Glaubensbegriff verwenden (vgl. dazu 1.1).

${ }^{40}$ In jeder Sprache $S$ lassen sich nur abzählbar viele Sachverhalte ausdrücken, da sich aus den endlich vielen Grundzeichen von S nur abzählbar viele Ausdrücke von $S$ (als endliche Folgen solcher Grundzeichen), also auch nur abzählbar viele Sätze von S bilden lassen. Da es überabzählbar viele reelle Zahlen gibt, gibt es z. B. keine Sprache, in der sich für jeden Wert, den eine Größe wie Masse oder Länge annehmen kann, die Aussage formulieren läßt, diese Größse habe diesen spezielien Wert. Nun lassen sich zwar nicht Namen für alle reellen Zahlen zugleich in eine Sprache $\mathrm{S}$ einführen, wohl aber $\mathrm{Na}$ men für jede beliebige reelle Zahl, und es lassen sich in S Aussagen formulieren, aus denen folgt, daß eine Größe einen bestimmten Wert hat oder nicht hat, wenn sich diese Folgerung auch nicht in S formulieren läßt. Unser Argument stützt sich nicht auf diese Begrenzung der Ausdrucksfähigkeit von Sprachen, sondern auf die Tatsache, daß es zu jeder Sprache Aussagen über sie gibt, die sich nicht in sie übersetzen lassen, ohne daß ein Widerspruch entsteht. Jede semantische Antinomie - wie die des Lügners, die von K. Grelling, J. Richard oder J. König - ergibt eine solche Aussage.

${ }^{41}$ Rosser hat in (36) gezeigt, daß die von Gödel in seinem Beweis vorausgesetzte $\omega$-Konsistenz von T entbehrlich ist. 
Satz A selbst folgen würde. ${ }^{42}$ Folgt $A$ nicht aus $T$, so auch nicht aus $T$ und Beobachtungssätzen, da A ein rein arithmetischer Satz ist; er ist also mit $T$ nur dann erklärbar, wenn er aus $T$ folgt.

Der Gödelsche Beweis für die Unvollständigeit jeder formalen Theorie der Arithmetik und verwandte metamathematische Resultate sind oft für Argumente gegen den Physikalismus bemüht worden speziell gegen die These, es ließen sich Komputer konstruieren, die dasselbe leisten wie der menschliche Verstand, es spräche also nichts gegen ein materialistisches Modell geistiger Phänomene. Solche Argumente, wie z. B. die von J. Lucas in (61), sind aber oft schlecht begründet. Das Gleiche gilt allerdings auch für die Gründe, die gegen eine Relevanz metamathematischer Resultate für das Verhältnis von Psychischem und Physischem vorgebracht wurden ${ }^{43}$ Einwände gegen den Physikalismus beinhalten oft, der menschliche Verstand könne Leistungen vollbringen, zu denen Maschinen prinzipiell nicht imstande sind. Man beruft sich dabei entweder darauf, daß es für jede Maschine Probleme gibt, die nicht sie, wohl aber menschliche Intelligenz entscheiden kann. ${ }^{44}$ Oder man behauptet, es gebe Probleme, die keine Maschine, wohl aber menschliche Intelligenz entscheiden kann. Das erste ist richtig; daraus folgt aber nicht die Behauptung, denn es kann andere Maschinen geben, die das fragliche Problem entscheiden können. Es kann also für alle Probleme, die wir entscheiden können, Maschinen geben, die diese Probleme ebenfalls lösen können. Die zweite Behauptung würde für den gewünschten Nachweis ausreichen, sie wird aber nicht hinlänglich begründet. Keine Maschine kann z. B. die Frage der Zugehörigkeit zu einer nicht-rekursiven Menge, etwa der Menge der Theoreme der Prädikatenlogik, generell entscheiden. $\mathrm{Daß}$ das ein Mensch tun könne, ist aber nicht nur unbewiesen, son-

${ }^{42}$ Der Grundgedanke des Beweises von Gödel ist derselbe wie bei der Konstruktion einer semantischen Antinomie. Der „Trick“ von Gödel bestand darin, daß er ein Verfahren angab, mit dem sich metasprachliche Aussagen über die Beweisbarkeit in $T$ in arithmetische Aussagen von $S$ übersetzen lassen.

4j Vgl. z. B. Webb (68) oder Benaceraff (68).

${ }^{44}$ Das entspricht der Behauptung, daß es in jeder formalen Theorie, welche die Arithmetik enthält, unentscheidbare Sätze gibt. Beide Thesen gehen von einer Festlegung des Begriffs der Maschine, bzw. des Begriffs des formalen Systems aus, die wir hier als unproblematisch ansehen können. 
dern unbeweisbar. Denn nach der gut begründeten These von A. Church fällt Entscheidbarkeit mit Rekursivität zusammen, und wenn man diese These aufgibt, kann man auch nicht mehr behaupten, keine Maschine könnte nicht rekursive Mengen entscheiden, da dann der Begriff der Maschine wieder unklar würde. Geht man von der Churchschen These aus, so kann man diesen Begriff z. B. mit Hilfe von Turingmaschinen erfassen, die so definiert sind, daß Rekursivität mit Turingberechenbarkeit zusammenfällt. ${ }^{45}$ Korrekt wäre nur das Argument: Es läßt sich nicht beweisen, daß es eine Maschine gibt, die all das leistet, was menschliche Intelligenz leisten kann; denn zu jeder Maschine, die man zum Zweck eines Beweises angibt, gibt es Probleme, die sie nicht lösen kann, wohl aber der Adressat des Beweises, der die Funktionsweise dieser Maschine kennt. Immer, wenn jemand behauptet, eine bestimmte, von ihm beschriebene Maschine, könne all die Probleme lösen, die ich zu lösen vermag, kann ich ihn also widerlegen.

Einwände gegen die erkenntnistheoretische Relevanz metamathematischer Resultate behaupten, solche Resultate besagten nichts über das menschliche Erkenntnisvermögen. ${ }^{46}$ So pauschal ist das aber sicher falsch: Die Metamathematik ist Teil der Philosophie der Mathematik und damit auch Teil der Erkenntnistheorie in dem umfassenden Sinn des Wortes, von dem wir in der Einleitung gesprochen haben. Es ist auch nicht einzusehen, wieso Einsichten über die Grenzen der Ausdrucksfähigkeit von Sprachen und der Leistungsfähigkeit von Theorien erkenntnistheoretisch nicht relevant sein sollten.

Die These D* ist also nicht haltbar. ${ }^{47}$ Daraus folgt freilich nicht, daß sich unsere Welt, gewissermaßen von außen betrachtet, nicht als

${ }^{45} \mathrm{Zu}$ diesen Begriffen und Resultaten vgl. z. B. Hermes (61).

${ }^{46} \mathrm{So} \mathrm{z}$. B. Webb in (68) und Lorenzen in (62), S. $132 \mathrm{f}$.

${ }^{47}$ Für eine Widerlegung von $D *$ genügt es nicht, darauf hinzuweisen, daß Theorien durch Beobachtungen nie verifizierbar sind, also immer hypothetischen Charakter haben. Auch eine Hypothese kann ja richtig sein, und man kann im Sinne unseres Wissensbegriffs auch sagen, man wisse, daß sie richtig ist. - K. Popper hat in (50) ein Argument formuliert, nach dem auch dann, wenn es eine umfassende Theorie $T$ gäbe, mit der sich im Prinzip alles erklären läßt, es dem Menschen als endlichem Teilsystem der Welt doch unmöglich wäre, all die Informationen über die Antezedensbedingungen zu gewinnen, die erforderlich wären, um mit T z. B. sein eigenes Verhalten und seine eigenen Annahmen vorauszusagen. Unser Argument ist dagegen: Es gibt keine solche Theorie. 
ein deterministisches oder kausales System darstellen könnte. Unser Beweis sagt nur, daß sich die Welt für einen Betrachter in ihr, dessen Überzeugungen Sachverhalte dieser Welt sind, nicht so darstellen kann. Wir haben deterministische Systeme so definiert, daß es Theorien gibt, mit denen sie sich vollständig beschreiben lassen. Solche Theorien sind aber in Sprachen zu formulieren, die wir beherrschen, über die wir verfügen, und beide Argumente beruhen darauf, daß es zu jeder Sprache $S$ eine Metasprache MS gibt, in der man über $S$ reden kann, und daß es in MS Sätze gibt, die sich nicht in $S$ ausdrücken lassen. Für ein absolutes Wesen, das unsere Welt und uns selbst von außen betrachten könnte, würde eine solche Restriktion nicht gelten; $\mathrm{da}$ dieses Wesen kein Teil unserer Welt wäre, müßten sich Aussagen über seine Überzeugungen nicht in jene Sprache übersetzen lassen, in der es unsere Welt beschreibt, denn diese Überzeugungen wären nicht Teil dieser Welt. „An sich“ mag also alles Geschehen deterministisch ablaufen, aber erkennen läßt sich das für uns nicht. Es mag eine Menge von Propositionen geben, die deterministische Gesetze darstellen, mit denen sich alles erklären läßt. Aber es gibt keine Sprache, in der sich all diese Gesetze formulieren ließen und keine Theorie, die uns sagte, welche Gesetze zu diesen Mengen gehören. Wenn wir mit Leibniz Sachverhalte, die für uns erkennbar determiniert sind, von solchen unterscheiden, die ,, an sich" determiniert sind - Leibniz unterscheidet ,unendlich analytische" von ,endlich analytischen" oder ,notwendigen“ Aussagen - , so muß nicht alles, was an sich determiniert ist, auch erkennbar determiniert sein. Im Gegensatz zu Leibniz behaupten wir aber, daß sich eine durchgängige Determination alles Geschehens ,,an sich“ nicht beweisen läßt; daß wir für empirische Ereignisse $\mathrm{p}$ nicht nur nicht wissen, ob $\mathrm{p}$ oder ob nicht-p notwendigerweise eintreten muß, sondern daß wir auch nicht berechtigt sind anzunehmen, einer dieser Sachverhalte müsse notwendigerweise eintreten.

Aus der Unhaltbarkeit der These $D^{*}$ folgt auch nicht, daß D oder $\mathrm{K}$ falsch wäre. Die Behauptung K besagt: Für jedes Ereignis gibt es Kausalgesetze und Antezedensbedingungen, mit denen es sich (im Sinn einer DN-Erklärung) erklären läßt. Das ist eine Behauptung der Struktur ,Für alle F's gibt es ein G, so daß . . “", und solche Sätze lassen sich durch Beobachtungen weder verifizieren noch falsifizieren. $\mathrm{Da}$ es unendlich viele Ereignisse gibt, können wir nicht für alle Erklärungen angeben, und da es unendlich viele Gesetze und Antezedens- 
bedingungen gibt, können wir nie definititiv sagen, ein Ereignis lasse sich nicht kausal erklären. Gegen $\mathrm{K}$ spricht aber erstens die alltägliche Erfahrung, daß wir in vielen Fällen frei sind, so oder so zu handeln, daß wir etwas tun, aber auch unterlassen können - es hat sich aber auch schon manches Verhalten als biologisch oder psychologisch erklärbar erwiesen, von dem wir das zunächst nicht annahmen. Gegen $\mathrm{K}$ spricht zweitens auch die Tatsache, daß sich in der Quantenphysik nicht alle Ereignisse kausal erklären lassen - aber es läßt sich nicht ausschließen, daß diese Theorie eines Tages durch eine kausal-deterministische ersetzt wird.

Nach dem gegenwärtigen Erkenntnisstand kann man also nicht behaupten, alles Geschehen sei kausal determiniert. $\mathrm{Da}$ in der These $\mathrm{K}$ aber nicht nur von den gegenwärtig bekannten Kausalgesetzen die Rede ist und von den gegenwärtig bekannten empirischen Tatsachen, die als Antezedensbedingungen für Erklärungen mit diesen Gesetzen infrage kommen, läßt sich die These so nicht widerlegen. Die Frage, ob K gilt, stellt vielmehr ein Grenzproblem im Sinn von 1.7 dar. Sie ist nicht entscheidbar, denn ein Beweis für $\mathrm{K}$ liefe auf einen Beweis für $\mathrm{K}^{*}$ hinaus, den es nach unseren Argumenten nicht geben kann, und eine Widerlegung wäre nur dann möglich, wenn alle Kausalgesetze und alle für Erklärungen mit ihnen passenden Antezedensbedingungen bekannt wären und wir uns eine Übersicht über sie verschaffen könnten. Dazu müßten wir aber eine Sprache angeben, in der sich alle solche Gesetze und Bedingungen ausdrücken lassen. Wegen des beschränkten Ausdrucksreichtums aller Sprachen ist das aber nicht möglich, wie wir gesehen haben. Die Frage, ob $\mathrm{K}$ gilt, ist im Zusammenhang mit dem Problem der Handlungsfreiheit von eminenter praktischer Bedeutung. ${ }^{48}$ Freie Handlungen, Handlungen im Sinn von 2.4, gibt es nur dann, wenn nicht alles Geschehen kausal determiniert ist. (Ist $\mathrm{K}$ falsch, so folgt daraus freilich noch nicht, daß nicht alles Verbalten kausal determiniert ist.) $\mathrm{Da}$ wir uns in der Praxis rationalerweise nach dem richten, was wir wissen, bzw. nach dem, was wir für wahrscheinlich halten, werden wir uns so verhalten müssen, als ob $\mathrm{K}$ nicht gelten würde, da gegenwärtig alles gegen diese These spricht. Entsprechendes gilt für die These D.

${ }^{48}$ Zum Freiheitsproblem vgl. Kutschera (81), Kap. 6. 
Kant hat in der ,Kritik der reinen Vernunft" versucht, die These $\mathrm{K}$ (für den Bereich der Erscheinungen) apriorisch zu begründen. ${ }^{49}$ Sein Grundgedanke ist dabei folgender: Ein Ereignis ist nur dann objektiv, wenn es mit anderen in einem gesetzmäßigen Zusammenhang steht. Gesetzmäßigkeit ist ein Kriterium für objektive Ereignisse im Gegensàtz zu subjektiven Vorstellungen. In ähnlichem Sinn hat man oft darauf hingewiesen, daß wir als Phänomen in den Naturwissenschaften nur einen unter gleichen Bedingungen regelmäßig auftretenden Effekt ansehen. So sagt K. Popper: „Nur dort, wo gewisse Vorgänge (Experimente) auf Grund von Gesetzmäßigkeiten sich wiederholen, bzw. reproduziert werden können, nur dort können Beobachtungen, die wir gemacht haben, grundsätzlich von jedermann nachgeprüft werden. Sogar unsere eigenen Beobachtungen pflegen wir wissenschaftlich nicht ernst zu nehmen, bevor wie sie nicht selbst durch wiederholte Beobachtungen oder Versuche nachgeprüft und uns davon überzeugt haben, daß es sich nicht nur um ein einmaliges ,,zufälliges Zusammentreffen" handelt, sondern um Zusammenhänge, die durch ihr gesetzmäßiges Eintreffen, durch ihre Reproduzierbarkeit grundsätzlich intersubjektiv nachprüfbar sind. So hat wohl schon jeder Experimentalphysiker überraschende, unerklärliche „Effekte“ beobachtet, die sich vielleicht sogar einige Male reproduzieren ließen, um schließlich spurlos zu verschwinden; aber er spricht in solchen Fällen noch nicht von einer wissenschaftlichen Entdeckung (obwohl er sich vielleicht bemühen wird, Reproduktionsanordnungen für den Vorgang aufzufinden). Der wissenschaftlich belangvolle physikalische Effekt kann ja geradezu dadurch definiert werden, daß er sich regelmäßig und von jedem reproduzieren läßt, der die Versuchsanordnung nach Vorschrift aufbaut. Kein ernster Physiker wird jene ,okkulten Effekte", zu deren Reproduktionen er keine Anweisung geben kann, der wissenschaftlichen Öffentlichkeit als Entdeckung unterbreiten, denn nur zu bald würde man auf Grund des negativen Resultats der Nachprüfungen die „Entdeckung“" als ein Hirngespinst ablehnen. " 50

${ }^{49}$ Auf die Argumente von Leibniz haben wir schon in 1.4 hingewiesen. Die beiden wesentlichen Argumente stützen sich darauf, daß er Wahrheit mit analytischer Wahrheit gleichsetzt, und daß er Objekte als Mengen ihrer Eigenschaften auffaßt. Vgl. dazu Kutschera (79).

so Popper (66), S. 19. Popper bezieht sich an dieser Stelle auf Kant. 
Sähe man aber die Subsumierbarkeit unter Kausalgesetze als Bedingung der „,Objektivität" von Ereignissen an, so würde $\mathrm{K}$ zu der trivialen und damit uninteressanten These ,Alles was sich kausal erklären läßt, läßt sich kausal erklären“". Im normalen Sinn sind jedenfalls auch mikrophysikalische Ereignisse ,objektiv“; solche Ereignisse (wie z. B. daß ein einzelnes Photon nach dem Durchgang durch eine Blende auf einen bestimmten Punkt einer Photoplatte auftrifft) lassen sich aber nicht beliebig reproduzieren. 


\section{Der Dualismus}

\subsection{Grundgedanken}

Physikalismus und Phänomenalismus sind monistische Thesen. Sie behaupten, alles Wirkliche sei von gleicher Natur, und sehen die Einheit der Realität in ihrem durchgängig physischen, bzw. psychischen Charakter. Daneben gibt es noch eine dritte monistische These, nach der Physisches wie Psychisches Phänomene derselben einen Realität sind. Damit diese These nicht trivial wird - denn es gibt tatsächlich im normalen Sinn des Wortes nur eine Realität, die alles, insbesondere auch Physisches und Psychisches umfaßt - , ist sie als Reduzierbarkeitsthese etwa so zu formulieren:

M: Es gibt eine Sprache M, die weder physikalische noch psychologische Grundterme enthält, so daß man jeden Satz von $\Omega$ in einen Satz von $M$ übersetzen kann.

Dabei sei $\Omega$ wieder die normale Sprache, erweitert um die Wissenschaftssprachen.

Diese Auffassung bezeichnet man als neutralen Monismus. Eine spezielle Version des neutralen Monismus ist die Zweiaspektenlebre, nach der Physisches und Psychisches zwei Aspekte sind, unter denen sich die eine Realität darstellt. Sie ist in der neuzeitlichen Philosophie zuerst von Spinoza vertreten worden. Um wieder mehr zu sagen als eine bloße Trivialität, muß man hier annehmen, daß sich die eine Realität vollständig sowohl physisch als auch psychisch darstellen läßt. Das läuft dann auf die Zusatzthese hinaus:

$\mathbf{M}^{+}$: Die Sprache M läßt sich sowohl in die physikalische Sprache $\Phi$ wie in eine psychologische Sprache $\sum$ übersetzen.

Daraus ergibt sich mit der Transitivität der Übersetzbarkeitsrelation, daß $\Phi$ in $\Sigma$ und $\Sigma$ in $\Phi$ übersetzbar wäre; wir haben aber in den letzten Kapiteln gesehen, daß beides nicht möglich ist.

Der grundsätzliche Mangel des neutralen Monismus ist darin zu sehen, daß die neutrale Realität nicht genauer charakterisiert wird, 
bzw. daß die Sprache $M$ überhaupt nicht angegeben wird. Unsere Umgangssprache enthält sowohl physikalische wie psychologische Terme, aber keine Terme für neutrale Entitäten in dem hier intendierten Sinn, und es dürfte daher außerordentlich schwierig sein, eine „,neutrale“ Sprache M aufzubauen. Da der neutrale Monismus kaum näher präzisiert worden ist, lohnt es sich nicht, ihn weiter zu diskutieren. Verschiedene Vertreter der These $M$ haben die ,,neutrale“ Natur der Realität in ganz verschiedener, immer aber in sehr vager Weise beschrieben. So war z. B. für Spinoza die eigentliche Realität Gott oder die Natur, für William James war es die „reine Erfahrung“ und für Bertrand Russell waren es in seiner frühen Phase die ,,sensibilia“. ${ }^{1}$

Wenn sich nun die Monismen bezüglich des Verhältnisses von Physischem und Psychischem als undurchführbar erweisen, so scheint nur eine Position übrig zu bleiben: die des psycho-physischen Dualismus, nach der das Psychische und das Physische zwei verschiedenartige, weder aufeinander noch auf eine gemeinsame Wurzel reduzierbare Seinsbereiche sind. Der Dualismus beschränkt sich aber nicht auf diese negative Behauptung. Für ihn sind darüber hinaus zwei Aussagen charakteristisch:

1. Alles Reale ist physischer oder psycniscner Natur.

2. Die Natur des Physischen ist von der des Psychischen totaliter verschieden.

Wir wollen nun versuchen, diese Aussagen zu präzisieren, und ersetzen sie zu diesem $Z$ weck wieder durch Aussagen über die Sprachen $\Phi$, $\Sigma$ und $\Omega$. Dabei genügt es nicht, $\Sigma$ als Sprache über Psychisches zu kennzeichnen. Zum Psychischen gehören im normalen Sinn des Wortes

a) Gedanken, Erinnerungen, Vorstellungen, Empfindungen, doxastische und voluntative Einstellungen (insbesondere Präferenzen), daneben aber auch

b) charakterliche Dispositionen und Fähigkeiten, wie Intelligenz, Kreativität, Sensibilität, Kontaktfähigkeit etc., sowie

${ }^{1}$ Vgl. z. B. Russell (18) und (26). - Die neutralen Entitäten werden auch oft als eine Art von Empfindungen aufgefaßt, die vom Subjekt teils als psychische, teils als physikalische Phänomene interpretiert werden. Daher fällt es oft schwer, zwischen phänomenalistischen und neutral-monistischen Standpunkten zu unterscheiden. 
c) das Verhalten, Verhaltensdispositionen und -fähigkeiten, wie manuelle Geschicklichkeit, körperliche Leistungsfähigkeit, Ausdauer, etc.

Nur die Phänomene der ersten Gruppe bilden den Bereich, den der Dualismus dem Physischen gegenüberstellt - wir wollen es zur Abhebung vom Psychischen im normalen Sinn hier als rein Psychisches bezeichnen. ${ }^{2}$ Wir können die für eine Person a rein psychischen Sachverhalte wie in 3.4 als jene Sachverhalte bestimmen, die für a (im Zeitpunkt, auf den sie sich beziehen) problemlos sind im Sinne von D1.3-3. Wenn ich denke, von etwas überzeugt bin, etwas einer anderen Sache vorziehe, mir etwas vorstelle, mich an etwas erinnere, etwas empfinde, so bin ich davon überzeugt, daß es so ist, und umgekehrt. Ich kann immer durch „Introspektion“ entscheiden, ob solche Sachverhalte bestehen; sie stellen die Gegenstände meiner inneren Erfabrung dar. Was für eine Person nicht rein psychisch ist - z. B. die Überzeugungen einer anderen Person - kann für diese rein psychisch sein. Wenn wir von rein psychischen Sachverhalten reden wollen, ohne auf bestimmte Personen Bezug zu nehmen, so müssen wir also sagen: Ein Sachverhalt $\mathrm{p}$ ist rein psychisch, wenn er aus Komponenten besteht, deren jede für eine Person rein psychisch ist. ${ }^{3}$ Die rein psychischen Phänomene sind die cogitationes im Sinne von Descartes. ${ }^{4}$ Sie bilden den Bereich des Psychischen, den der Dualismus dem Physischen gegenüberstellt. Alles Verhalten hingegen hat auch äußere, physische Aspekte: die körperlichen Bewegungen, die der Handelnde dabei ausführt, wie ihre physischen Wirkungen lassen sich physika-

${ }^{2}$ Die Bezeichnung dieser Phänomene als ,geistig“ wäre ebenso schief wie die als ,psychisch“, denn erstens zählen zum Bereich des Geistigen im üblichen Sinn auch überindividuelle Phänomene wie Sprache und Wissenschaft, und zweitens gehören zum individuell Geistigen Denken und Wollen, aber z. B. nicht Empfindungen.

${ }^{3} \mathrm{Da}$ man auch einen Sachverhalt wie $\mathrm{G}(\mathrm{a}, \mathrm{p}) \wedge \mathrm{G}(\mathrm{b}, \mathrm{q})$ als rein psychisch ansprechen wird, dieser Sachverhalt aber weder für a noch für $b$ rein psychisch ist, kann man nicht sagen, ein Sachverhalt sei rein psychisch, wenn es eine Person gibt, für die er rein psychisch ist. - Die Definitionen des rein Psychischen lassen sich bei Bezugnahme auf exakte Sprachen nach dem Muster von Anmerkung 46 zu S. 187 präzisieren.

${ }^{4}$ Descartes nimmt Empfindungen aus, aber das ist hier unwesentlich. 
lisch beschreiben. ${ }^{5}$ Es gibt also für das Vorliegen von Handlungen und Verhaltensweisen auch physikalische Kriterien. Verhaltensaussagen sind nicht unabhängig von physikalischen Aussagen. Daher sind sie im Sinne des Dualismus in Komponenten zu analysieren, die entweder nur rein Psychisches beschreiben (wie z. B. die Absichten und Überzeugungen des Handelnden) oder rein Physisches (wie z. B. die körperlichen Bewegungen). Entsprechendes gilt aber auch für die Sachverhalte der zweiten Gruppe (b). Charakterliche Dispositionen sind immer auch Verhaltensdispositionen, und daher gibt es für das Vorliegen solcher Dispositionen auch Verhaltenskriterien, also auch physikalische Kriterien.

Im Sinn einiger dualistischer Theorien, insbesondere der von Descartes, ist die Bestimmung des rein Psychischen noch enger zu fassen. Für Descartes ergibt sich die These (2) daraus, daß rein psychische Aussagen von anderen Substanzen handeln als rein physische Aussagen. Er unterscheidet zwei Arten von Substanzen: res extensae, d. h. Körper, und res cogitantes, d. h. Träger von cogitationes, die Subjekte, die solche cogitationes vollziehen. Während im normalen Verständnis Personen Subjekte solcher cogitationes sind und wir Personen als leibseelische Einheiten auffassen und ebenso sagen „Hans glaubt, daß $2+2=4$ ist" wie „Hans ist 1,78 groß“, sind nach Descartes Denken und Ausgedehntsein Attribute zweier verschiedener Substanzen. Der Mensch Hans besteht also aus einer Seele, die Subjekt seiner cogitationes ist, und einem Körper, so daß man genauer sagen müßte: „Die Seele von Hans glaubt, daß $2+2=4$ ist“ und „Der Körper von Hans ist 1,78 groß". Mit dem ersteren Satz läßt sich aber nicht ohne weiteres ein vernünftiger $\operatorname{Sinn}$ verbinden. Uns ist die Rede von seelischen Vorgängen, Phänomenen etc. vertraut, aber was eine Seele ist, ist alles andere als klar. Wir formulieren Aussagen über Seelisches nicht als Aussagen über Seelen, sondern als spezielle Aussagen über Personen. Die Identität und Verschiedenheit von Personen beurteilen wir aber auch nach physikalischen Kriterien.

Je nachdem, welche Version des Dualismus vorliegt, ist also $\Sigma$ entweder als Sprache über rein Psychisches im oben charakterisierten Sinn zu bestimmen oder es ist darüber hinaus zu fordern, daß die in

\footnotetext{
${ }^{5}$ Viele Handlungsverben sind, wie schon in 2.4 betont wurde, Erfolgsverben, d. h. sie sind nur dann anwendbar, wenn die Handlung gewisse Wirkungen zeitigt.
} 
den Sätzen dieser Sprache verwendeten Subjektnamen nur seelische Substanzen oder kurz. Seelen bezeichnen.

Die erste These des Dualismus läßt sich dann, wo $\Phi+\Sigma$ die Verbindung der beiden Sprachen $\Phi$ und $\Sigma$ ist $^{6}$, als Übersetzbarkeitsthese so formulieren:

D 1: Alle Sätze von $\Omega$ lassen sich in $\Phi+\Sigma$ übersetzen. ${ }^{7}$

Ist $\Sigma$ eine Sprache über Seelen, so braucht man in $\Phi+\Sigma$ freilich ein Prädikat wie „Das physische Objekt $\mathrm{x}$ ist Körper der Seele y“, das weder zu $\Phi$ noch zu $\Sigma$ gehört. Man kann Sätze wie „Hans glaubt, daß p“, ,Hans turnt", ,Harıs sieht Fritz", ,Hans sagt, daß es 12 Uhr sei“ etc. nicht sinnvoll in Aussagen über Körper und Seelen übersetzen, wenn man nicht zumindest ausdrücken kann, daß sich gewisse seelische Vorgänge bei einer Person a (Vorgänge in ,,der Seele“ von a) mit gewissen körperlichen Vorgängen bei a (Vorgängen im oder am Körper von a) verbinden. Es ist aber denkbar, daß man ein solches Prädikat in $\Phi+\Sigma$ definieren kann, etwa so, daß man sagt, x sei Körper von $\mathrm{y}$, wenn gewissen physischen Einwirkungen auf $\mathrm{x}$ immer gewisse Empfindungen von y entsprechen. ${ }^{8}$ Auf dieses, für die cartesische Version des Dualismus allerdings zentrale Problem wollen wir hier jedoch nicht eingehen. Wir werden im folgenden so vorgehen, daß wir zunächst die nicht-cartesische, also die schwächere Form des Dualismus einer Kritik unterziehen. Daraus ergeben sich dann auch Einwände gegen die stärkere, cartesische Version. Auf deren Hauptproblem, das Problem seelischer Substanzen, gehen wir dann im Abschnitt 7.5 ein.

Erheblich schwieriger ist eine Präzisierung der zweiten These des Dualismus, der Behauptung, rein Psychisches und Physisches seien von gänzlich verschiedenartiger Natur. Bei Descartes ergibt sie sich daraus, daß Seelen und Körper verschiedene Substanzen sind, die

- Die Verbindung ist so zu denken, daß alle einfachen Sätze von $\Phi+\Sigma$ entweder $\Phi$ oder $\Sigma$ angehören, während alle logischen Verbindungen solcher Sätze zu $\Phi+\Sigma$ gehören. Ist also z. B. A ein Satz von $\Phi$ und B ein Satz von $\Sigma$, so ist $\mathrm{A} \wedge \mathrm{B}$ ein Satz von $\Phi+\Sigma$.

'Schwächere Reduzierbarkeitsthesen sind, soweit ich sehe, nicht formuliert worden.

${ }^{8}$ Einen solchen Definitionsvorschlag hat Locke gemacht. 
keine gemeinsamen essentiellen Eigenschaften haben. Man wird die Rede von der ",totalen Verschiedenartigkeit" in (2) aber auch so zu deuten haben, daß sie nicht einmal gemeinsame akzidentelle Eigenschaften haben. Akzidentelle Eigenschaften von Körpern sind z. B. solche, die sich auf ihre Lage oder Geschwindigkeit beziehen. Akzidentelle Eigenschaften von Seelen sind z. B. , sich an das und das erinnern', ,das und das wollen' oder, dies oder jenes glauben'. Attribute von Körpern lassen sich, wie diese Beispiele zeigen, nicht sinnvoll auf Seelen anwenden, und umgekehrt. Enthalten aber die Sprachen $\Phi$ und $\Sigma$ keine gemeinsamen Vokabeln, so sind die Sätze von $\Phi$ von jenen von $\Sigma$ unabhängig.

Auch bei der nicht-cartesischen Version des Dualismus wird man (2) so deuten müssen, daß zwischen den Sätzen von $\Phi$ und jenen von $\Sigma$ keine analytischen Beziehungen bestehen. Empirische Beziehungen müssen offenbar bestehen, da ja z. B. unser sinnliches Erleben nicht völlig unabhängig von allem physischem Geschehen sein soll. Will man also von ,eigenständigen" Seinsbereichen sprechen, so darf es jedenfalls keine analytischen Beziehungen geben. Analytisch zumindest muß es möglich sein, daß alle rein psychischen Tatsachen auch dann bestünden, wenn die physische Welt ganz anders beschaffen wäre oder wenn $\in$ üiberhaupt keine pinysische Außenwelt gäbe', und $\mathrm{da}$ umgekehrt die physischen Tatsachen bestehen blieben, wenn die rein psychische Wirklichkeit ganz anders aussähe oder wenn es überhaupt keine psychische Welt gäbe. Alle tatsächlich bestehenden Beziehungen zwischen rein Psychischem und Physischem sind also kontingent, und beide Bereiche lassen sich isoliert von einander betrachten. Eine solche Eigenständigkeit nimmt man heute noch meist für das Physische an; man versteht physikalische Aussagen so, daß sie ihrem Sinn - und also auch ihrer Geltung nach - von Psychischem unabhängig sind. Diese Annahme ist nur dann sinnvoll, wenn man psychische Sachverhalte nicht als spezielle physikalische ansieht, wenn man also keinen Physikalismus vertritt. Dann impliziert sie aber auch den Dualismus, also auch eine Unabhängigkeit des Psychischen vom Phy-

\footnotetext{
9 Deshalb stellt sich für eine dualistische Konzeption das Problem der Existenz einer Außenwelt und der Zuverlässigkeit unseres sinnlichen Erlebens in besonderer Schärfe. Descartes konnte es nur lösen durch einen Rückgriff auf Gott.
} 
sischen. Denn ,unabhängig' ist eine symmetrische Relation, d. h. wenn $A$ von $B$ unabhängig ist, so auch $B$ von $A$.

In diesem Sinn können wir die These (2) etwa so formulieren:

D 2: Zwischen den Sätzen von $\Phi$ und denen von $\Sigma$ bestehen keine analytischen Beziehungen. ${ }^{10}$

Daraus ergibt sich unmittelbar, daß sich der Dualismus mit einem absoluten ontologischen Realismus im Sinn von 3.4 verbindet.

Es ist zu betonen, daß die Thesen des Dualismus, speziell die These (2), in der Literatur nie genauer präzisiert worden sind. Die genauesten Aussagen sind die von Descartes, aber sie führen, wie wir sahen, zu einer sehr starken und damit auch besonders problematischen Version dieser Thesen. Darin liegt sicher ein grundlegender Einwand gegen den Dualismus. Wenn man es aber nicht dabei bewenden lassen will - was uns in der Sache nicht weiterbrächte -, muß man versuchen, seine Thesen so zu interpretieren, daß jedenfalls eine an das sachliche Kernproblem heranführende Diskussion möglich wird.

Ideengeschichtlich hat der psycho-physische Dualismus eine sehr alte Tradition. Ein Dualismus von Geist und Materie, von Seelischem und Körperlichem findet sich in vielen Religionen und er begegnet uns schon in der antiken Philosophie, z. B. bei Platon. Entscheidend für seinen Einfluß in der neueren Philosophie war seine Formulierung durch Descartes, insbesondere in den ,Meditationes de prima philosophia". Von Descartes reicht die dualistische Tradition bis in die Gegenwart hinein, so daß sie G. Ryle noch in (49) als ,offizielle Doktrin" bezeichnete. Als ,,offizielle Doktrin“ wird man aber gegenwärtig wohl eher den Physikalismus ansehen müssen. Daß der Dualismus jedoch auch heute noch nicht tot ist, zeigen Veröffentlichungen wie Popper und Eccles (77) oder Wigner (61).

${ }^{10}$ Das verstehen wir nicht nur so, daß für alle Sätze $A$ von $\Phi$ und $B$ von $\Sigma$ weder $\mathrm{A}$ aus $\mathrm{B}$, noch $\mathrm{B}$ aus $\mathrm{A}$ folgt, sondern stärker so, daß jede konsistente Satzmenge von $\Phi$ mit jeder konsistenten Satzmenge von $\Sigma$ verträglich ist. In diesem Sinn sagen wir auch, zwischen den beiden Sprachen bestünden keine analytischen Zusammenhänge. 


\subsection{Argumente des Dualismus}

Die Diskussion des Dualismus wie seine eigenen Argumente konzentrieren sich ganz auf die These D2. Für die These D 1 sind kaum Argumente oder Gegenargumente angeführt worden. Dabei ist auch diese These alles andere als unproblematisch. Es ist z. B. völlig unklar, welche Sätze von $\Phi+\sum$ Aussagen entsprechen sollen wie

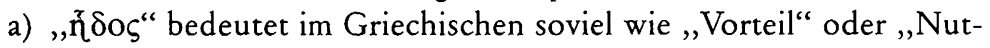
zen".

b) Hans ist bestrebt, sein Examen möglichst früh abzulegen.

c) Es ist (moralisch oder gesetzlich) verboten, einen anderen zu töten, es sei denn in Notwehr.

d) Die Bundesversicherungsanstalt für Angestellte ist eine Selbstverwaltungskörperschaft.

Aussagen über sprachliche Bedeutungen sind weder Aussagen über rein Psychisches noch über rein Körperliches, und es ist durchaus nicht klar, ob ihnen Komplexe solcher Aussagen entsprechen und ggf. welche. Man könnte versuchen, etwa im Sinne von P. Grice, Aussagen uber Bedeutungen als Aussagen über Intentionen zu deuten, welche die Angehörigen der Sprachgemeinschaft mit der Verwendung von Ausdrücken verbinden. Aber diese Theorie ist erstens bisher noch nicht ausführlich entwickelt worden, und zweitens nimmt eine solche Rekonstruktion des Bedeutungsbegriffs nicht nur auf Physisches und rein Psychisches Bezug, sondern auch auf Verhaltensweisen; es wäre also zu zeigen, daß sich Verhaltensaussagen im Sinn von D 1 in die Sprache $\Phi+\Sigma$ übersetzen lassen. (b) wäre zu übersetzen in eine psychologische Aussage über eine Absicht von Hans. „Bestrebt sein“ heißt aber aktiv bestrebt sein, so daß Hans auch etwas tun muß (z. B. Vorlesungen besuchen, Bücher lesen, Anmeldefristen einhalten etc.), damit der Satz (b) richtig ist. Welche körperlichen Vorgänge das aber sind, ist in (b) nicht spezifiziert. Man könnte also nun sagen: Der Körper von Hans führt gewisse Bewegungen aus, die Hans geeignet erscheinen (das wäre wieder eine psychologische Aussage), sein Ziel zu erreichen. Offenbar wäre aber ein solcher Satz nicht synonym mit (b); vielmehr wäre hinzuzufügen, daß diese körperlichen Bewegungen von Hans willentlich verursacht werden. Aber dann sieht man sich mit dem Problem der psycho-physischen Wechselwirkung konfrontiert, auf das wir im Abschnitt 6.3 eingehen. Für (c) und (d) endlich sind keine auch nur einigermaßen plausiblen Übersetzungen in Sicht. 
Das zentrale Problem des Dualismus ist aber die These D 2 von der völligen Verschiedenartigkeit von Physischem und rein Psychischem, und dieses Problem allein ist auch im Zusammenhang mit dem LeibSeele-Problem von Interesse, mit dem wir uns seit dem 5 . Kapitel befassen.

Descartes Argument für die Verschiedenartigkeit von res extensae und res cogitantes in der 6 . Meditation ist unklar. ${ }^{11}$ Zunächst gilt sicher: Als res cogitans könnte ich nur mit einer res extensa, einer körperlichen Substanz, identisch sein: mit meinem Körper. Um aber die Verschiedenheit der res cogitans von ihrem Körper zu beweisen, vermischt Descartes zwei Prinzipien:

I) Wenn ich die Natur eines Gegenstandes b vollständig, klar und deutlich erkennen kann, ohne dabei zu erkennen, ob b auch die Eigenschaft $F$ hat, so ist $F$ kein essentielles Merkmal von b.

Das ist richtig. Daraus kann man aber nicht folgern: $\mathrm{Da}$ ich erkennen kann, daß ich ein denkendes Etwas - eine Seele - bin, ohne zu erkennen, ob ich ausgedehnt bin (das sei Descartes einmal zugestanden), ist Ausdehnung keins meiner essentiellen Eigenschaften; da aber Ausgedehntheit ein essentielles Merkmal von Körpern ist, bin ich als res cogitans von meinem Körper verschieden. Denn hier wird vorausgesetzt, was zu beweisen war, daß ich nämlich meine Natur vollständig erkennen kann, ohne zu erkennen, ob ich ein ausgedehntes Etwas bin.

II) Wenn ich deutlich und klar erkenne, daß ein Gegenstand $b$ eine Eigenschaft $F$ hat, ohne dabei zu erkennen, ob b auch die Eigenschaft $G$ hat, so ist es möglich, daß b die Eigenschaft $G$ nicht hat.

11 Descartes sagt: "Et primo quoniam scio omnia quae clare et distincte intelligo, talia a Deo fieri posse qualia illa intelligo, satis est quod possim unam rem absque altera clare et distincte intelligere, ut certus sim unam ab altera esse diversam ... ac proinde, ex hoc ipso quod sciam me existere, quodque interim nihil plane aliud ad naturam, sive essentiam meam pertinere animadvertam, praeter hoc solum quod sim res cogitans, recte concludo, meam essentiam in hoc uno consistere, quod sim res cogitans. Et quamvis fortasse (vel potius ut postmodum dicam, pro certo) habeam corpus, quod mihi valde arcte conjunctum est, quia tamen ex una parte claram et distinctam habeo ideam mei ipsius quatenus sum tantum res cogitans, non extensa; et ex alia parte distinctam ideam corporis, quatenus est tantum res extensa, non cogitans, certum est me a corpore meo revera esse distinctum, et absque illo posse existere". (MP, S. 136) 
Daraus könnte man folgern: Da ich erkennen kann, daß ich denke, ohne zu erkennen, ob ich ausgedehnt bin, ist Ausgedehntsein kein essentielles Merkmal von mir; da aber Ausgedehntsein ein essentielles Merkmal von Körpern ist, bin ich kein körperliches Ding. Hier ist jedoch das Prinzip falsch, und so haben schon Caterus, Arnauld, Hobbes und Gassendi in ihren Einwänden an der Beweisführung von Descartes Kritik geübt. Ich kann erkennen, daß ein Dreieck drei Seiten hat, ohne zu erkennen, ob seine Winkelsumme $180^{\circ}$ beträgt. Daraus folgt aber nicht, daß eine Winkelsumme von $180^{\circ} \mathrm{zu}$ haben kein essentielles Merkmal von Dreiecken wäre.

Heute sind es vor allem zwei Argumente, die zur Stützung der These D 2 vorgebracht werden:

1. Entweder hat der Monismus recht und das Psychische läßt sich auf das Physische reduzieren (Physikalismus) oder das Physische auf das Psychische (Phänomenalismus) oder beide auf ein Drittes (Neutraler Monismus) - oder aber der Monismus ist falsch; dann muß der Dualismus richtig sein, nach dem Psychisches und Physisches eigenständige Seinsbereiche sind. Nun sind aber, wie die obigen Argumente zeigen, die drei möglichen Formen des Monismus nicht haltbar, also muß die dualistische These gelten. In diesem Sinne verteidigen viele Autoren ihre dualistische Position einfach dadurch, daß sie Gründe gegen die monistischen Positionen anführen.

Dieses Argument ist jedoch nicht haltbar. Es wäre nur dann korrekt, wenn der Dualismus aus der Negation der Monismen folgen würde. Wir haben aber schon betont, daß das nicht der Fall ist. Man kann nicht sagen: Entweder handeln Physik und Psychologie nur von ein und derselben Realität oder von zwei verschiedenartigen Realitäten; im letzteren Fall gilt aber der Dualismus. Denn aus einer Verschiedenartigkeit der Realitäten folgt nicht ihre totale Verschiedenartigkeit im Sinne der These D 2.

2. Das zweite Hauptargument bilden Versuche, eine grundsätzliche Verschiedenheit von Psychischem und Physischem direkt aufzuweisen. Sie wird in folgenden Punkten gesehen:

a) Physisches ist räumlich lokalisiert und ausgedebnt, Psychisches nicht.

Das ist die cartesische Unterscheidung von res extensa und res cogitans. Dazu ist zu sagen: Räumlich lokalisiert sind nur Dinge, nicht aber deren Attribute und Zustände. Ein Magnet hat zu einer bestimmten Zeit einen bestimmten Ort und eine bestimmte Ausdehnung, nicht aber die 
Eigenschaft magnetisch zu sein. Ebenso wie es unsinnig ist, nach dem Ort der Eiger,schaft Magnetisch zu fragen, ist es auch unsinnig, nach dem Ort eines seelischen Zustands zu fragen und zu sagen: ,, a ist ärgerlich - wo ist sein Ärger? Er läßt sich nicht lokalisieren, also ist er nichts Physisches“. Ebenso könnte man sagen: ,a wiegt $80 \mathrm{~kg}-$ Wo ist der Zustand, $80 \mathrm{~kg}$ zu wiegen? Er läßt sich nicht lokalisieren, also ist er nichts Physisches". Psychische Objekte wie Seelen oder auch Sinnesdaten und dergleichen wären aber erst einmal aufzuweisen, bevor man die Frage sinnvoll stellen könnte, ob sie sich räumlich lokalisieren lassen. Diese Unterscheidung leistet also nichts.

Der entsprechende Einwand wäre auch gegen die Unterscheidung zu machen, nach der Physisches materiell, Psychisches aber immateriell ist. ${ }^{12}$

b) Physisches ist objektiv, Psychisches subjektiv.

Es ist zwar richtig, daß Schmerzen immer Schmerzen von jemand sind, eine Wahrnehmung immer Wahrnehmung von jemand. Das besagt aber nur, daß solche psychologischen Prädikate immer nur auf Subjekte angewendet werden können, daß also der Definitionsbereich (einer) ihrer Variablen aus Subjekten (Personen) besteht. Das zeichnet sie aber nicht vor Prädikaten wie „turnen“ oder „krank sein“ aus, die nicht als psychologisch gelten können.

c) Physisches ist quantitativ bestimmbar, Psychisches nur qualitativ.

Diese Unterscheidung ist völlig unhaltbar, da sich alle qualitativen Begriffe metrisieren lassen. Im übrigen sind subjektive Wahrscheinlichkeiten und Nutzensfunktionen quantitative Begriffe. Auch in der Psychologie macht man quantitative Aussagen, z. B. Aussagen über Intelligenz mithilfe des Intelligenz-Quotienten.

d) Psychisches ist zielgerichtet, teleologisch bestimmt, Physisches ist mechanisch, kausal bestimmt.

Selbst wenn man davon absieht, daß die Charakterisierung des Physischen als "mechanisch" viel zu eng ist - es gibt $\mathrm{z}$. B. keine mechanische Theorie elektromagnetischer Erscheinungen --, so ist diese Unterscheidung ganz ungenau: Eine Dampfturbine, also etwas Physisches, funktioniert „mechanisch“, „kausal“, dient aber z. B. dem Zweck, ein Schiff anzutreiben. Manche physikalischen Gesetze lassen sich auch so formulieren, daß ein Prozeß immer in der Weise abläuft, $\mathrm{daß}$ eine bestimmte Größe einen minimalen oder maximalen Wert hat;

${ }^{12}$ Vgl. dazu den Abschnitt 6.1. 
man könnte sie also „teleologisch“ deuten, und Leibniz hat sie auch so gedeutet. ${ }^{13}$ Endlich gibt es auch Kausalzusammenhänge im psychischen Geschehen.

e) Psychisches ist mnemisch bestimmt, Physisches nicht.

Unsere gegenwärtigen Handlungen sind durch frühere, evtl. weit zurückliegende Ereignisse mitbestimmt, an die wir uns erinnern. Physikalische Vorgänge hingegen hängen nur von den unmittelbar vorhergehenden und den mit ihnen koexistierenden Zuständen ab.

Es ist aber nicht so, daß die vergangenen Ereignisse unsere gegenwärtigen Handlungen bestimmen, sondern die gegenwärtigen oder unmittelbar vorausgegangenen Erinnerungen an sie oder gegenwärtige, durch vergangene Erfahrungen erworbene Verhaltensdispositionen. Außerdem gibt es auch bei Maschinen so etwas wie bedingte Reflexe oder Lernvorgänge, in denen frühere Zustände der Maschine ihr späteres Verhalten beeinflussen. Endlich kann man auch sagen, daß die vergangene Magnetisierung eines Eisenstabes über das Phänomen der Hysterese den gegenwärtigen Zustand, das gegenwärtige Verhalten bei Ummagnetisierung bestimmt. - Auch diese Unterscheidung ist also unklar und leistet nichts.

f) Psychisches ist ganzheitlich, Physisches atomistisch.

Parallel zu dieser Unterscheidung läuft die Charakterisierung des Psychischen als emergent und das Physischen als kompositionell. Dabei besagt die Emergenz, daß sich die Eigenschaften psychischer Komplexe nicht aus den Eigenschaften der einfachen Teile dieser Komplexe ableiten lassen.

Die Gestaltpsychologie hat den Gestaltcharakter von Erlebnissen betont, die moderne Biologie den Systemcharakter von Organismen. Aber Strukturen spielen auch in der Physik eine Rolle. So hängen z. B. chemische Reaktionen zwischen Stoffen von deren molekularer Struktur ab, nicht von der Summe der atomaren Bestandteile. Und eine Maschine ist nicht bloß ein Haufen von Einzelteilen, ihre Wirkungsweise hängt nicht nur von den Eigenschaften der Einzelteile ab, sondern auch davon, wie sie zusammengesetzt sind.

g) Psychische Akte sind intentional, physische Vorgänge nicht.

Wahrnehmungen sind Wahrnehmungen von etwas, Strebungen sind Strebungen nach etwas, etc. Bewußtseinsakte sind in diesem Sinn intentional, d. h. auf Gegenstände oder Sachverhalte gerichtet. ${ }^{14}$

${ }^{13}$ Vgl. dazu den Abschnitt 2.3.

${ }^{14}$ Vgl. dazu den Abschnitt 5.5. 
Erstens ist aber nicht alles Psychische intentional: Schmerzempfindungen sirid es z. B. nichc. Wenn man zweitens intentionale Sachverhalte nicht näher eingrenzt als durch die Forderung, daß sie durch Sätze der Gestalt „F $(\mathrm{a}, \mathrm{p})$ “ ausgedrückt werden, in denen , $\mathrm{a}$ “ eine Person bezeichnet und „,p“ einen Satz, und die bzgl. des zweiten Argưments nichtextensional sind, so gilt zwar, daß solche Sätze in der Physik als einer extensionalen Sprache nicht vorkommen; es gibt aber derartige Sätze über Personen, wie z. B. „,Hans sagt, daß es 12 Uhr ist", die man nicht als Sätze über rein Psychisches ansprechen kann.

h) Psychisches ist privat, Pbysisches öffentlich. ${ }^{15}$

Physische Dinge und Sachverhalte sind intersubjektiv beobachtbar, psychische Zustände sind aber nur für ihre Träger introspektiv oder „privat" direkt beobachtbar.

Auch dieses Begriffspaar eignet sich ohne nähere Spezifizierung nicht dazu, Psychisches und Physisches zu unterscheiden. Erstens ist nicht alles Psychische introspektiv beobachtbar; man denke z. B. an verdrängte Triebe. Zweitens kann ein Komputer ,introspektiv“ auf in ihm gespeicherte Daten zurückgreifen, die von außen nur mittelbar zugänglich sind.

Zusammenfassend können wir sagen: Keines dieser Begriffspaare eignet sich ohne genauere Präzisierungen für eine Charakterisierung des Unterschieds zwischen Physischem und Psychischem. Selbst wenn aber ein Begriff aufgewiesen wird, der auf alle psychischen, nicht aber auf physische Phänomene zutrifft oder umgekehrt, so folgt daraus nicht die Behauptung D 2 oder eine ,totale Verschiedenartigkeit" von Psychischem und Physischem. Kühe lassen sich von Pferden unterscheiden, aber deshalb sind sie nicht ,totaliter verschiedenartig“, denn beides sind Säugetiere. Auch aus unserer Bestimmung rein psychischer Sachverhalte ergibt sich nicht, daß rein psychische Sachverhalte von gänzlich anderer Art sind als physikalische Sachverhalte. Es folgt daraus auch nicht die These D2, wie wir im Abschnitt 7.4 sehen werden.

Einen engagierten Angriff auf den Dualismus hat Gilbert Ryle in seinem Buch „The Concept of Mind“ (49) unternommen. Die falsche Grundthese des Dualismus, des "Cartesianischen Mythos" oder des „Dogmas vom Gespenst in der Maschine“, wie er ihn polemisch nennt, sieht Ryle darin, daß neben der physischen Realität eine ei-

${ }^{15}$ Vgl. dazu z. B. Ayer (63a), $52 \mathrm{ff}$. 
gene, auf jene nicht reduzierbare psychische Realität angenommen wird. Ryle vertritt, wie wir im Abschnitt 6.2 gesehen haben, eine behavioristische Position, wobei er als Verhaltenssprache freilich nicht die physikalische Sprache $\Phi$ wählt, sondern eine Sprache, in der die normalen Verhaltensterme vorkommen. Die dualistische These entspringt nach Ryle einem Fehler in der logischen Analyse psychologischer Aussagen, einem Kategoriefehler. Ryle unterscheidet, wie üblich, zwischen manifesten und dispositionellen Attributen. ${ }^{16}$ Ryles Theorie der Dispositionen, die er im 5. Kapitel seines Buchs entwickelt, ist nun in vielen Punkten unklar oder fragwürdig. ${ }^{17}$ Auf diese Punkte brauchen wir hier jedoch nicht einzugehen. Wichtig ist nur, daß Ryle in der Konsequenz dieser Unterscheidung manifester und dispositioneller Begriffe drei Typen von Aussagen unterscheidet:

1. kategorische Aussagen als Aussagen über momentane Zustände und Vorgänge (,Episoden“) (wie z. B. „Hans bremst vor der Ampel ab“),

2. hypothetische Aussagen als Aussagen über Dispositionen (wie z. B. „Hans ist ein vorsichtiger Fahrer"), und

3. misch-kategorische Aussagen (mongrel-categorials), Mischformen der beiden ersten Typen (wie z. B. „Hans fährt vorsichtig“).

Nach Ryle besteht nun der Kategoriefehler des Dualismus darin, daß hypothetische, bzw. misch-kategorische Alissagen als kategorische Aussagen aufgefaßt werden, und daß dann, da diesen Aussagen keine manifesten physischen Eigenschaften und Vorgänge entsprechen, eine psychische Realität als Träger solcher manifester Eigenschaften oder Vorgänge fingiert wird. Wenn man z. B. sagt, ,a weiß,

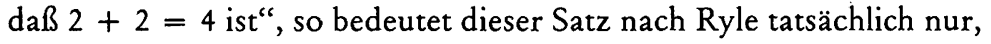
$\mathrm{da} ß$ a die Frage, wieviel $2+2$ ist, richtig beantworten kann; er drückt also eine Fähigkeit, eine Disposition von a aus. Faßt man aber das Wissen als manifesten (episodischen) Zustand auf, so muß man, da

${ }^{16}$ Vgl. dazu den Abschnitt 2.2

${ }^{17}$ So bezeichnet Ryle z. B. auch Sätze der kategorischen Form ,,a ist ein F“ als „hypothetisch“, wenn F ein Dispositionsprädikat ist. Ferner werden Dispositionsbegriffe nicht als "reale" Attribute angesehen, und hypothetische Sätze werden als ,,inference-tickets" aufgefaßt - ohne daß mit diesen Redeweisen ein präziser oder fruchtbarer Sinn verbunden würde. Vgl. dazu (49), S. $119 \mathrm{ff}$. - Wir haben freilich in 2.2 gesehen, daß die Unterscheidung dispositioneller und manifester Eigenschaften nicht nur bei Ryle unscharf ist. 
kein entsprechender physischer Zustand von a in Sicht ist, einen seelischen Zustand des Wissens fingieren, auf den sich diese Aussage bezieht. Oder wenn man sagt ,a will Arzt werden“, so bedeutet das nach Ryle tatsächlich nur, daß a in gewissen Situationen jene Handlungen vollzieht, die für diese Berufswahl zweckmäßig sind, daß also a gewisse Verhaltensdispositionen hat. Wenn man diese Aussage dagegen als kategorischen Satz versteht, so muß man in Ermangelung entsprechender aktueller physischer Vorgänge einen seelischen Vorgang postulieren, einen Willensakt, auf den sich diese Aussage beziehen soll. Führt man aber die Unterscheidung zwischen kategorischen und hypothetischen, bzw. misch-kategorischen Sätzen korrekt durch, so wird man nach Ryle der Notwendigkeit enthoben, eine eigenständige psychische Realität anzunehmen..$^{18}$

Wenn ein Satz hypothetisch im Sinne Ryles ist, folgt daraus jedoch nicht schon, daß dieser Satz ein Satz über Verhaltensweisen ist. Dafür ist vielmehr allein entscheidend, ob das fragliche Dispositionsprädikat $\mathrm{D}$ durch Bedingungen der Art ,Eine Person $\mathrm{x}$ hat die Eigenschaft D genau dann, wenn sie die Reaktion $R$ unter der Testbedingung $T$ zeigt" erklärt ist, in denen $\mathrm{R}$ und $\mathrm{T}$ Verhaltensterme sind. ${ }^{19}$ Für die interessanten psychologischen Prädikate bleibt aber Ryle, wie wir schon früher betont haben, den Nachweis schuldig, daß sie sich im Sinne von Verhaltensdispositionen deuten lassen.

\subsection{Das Problem der psycho-physischen Wechsel- wirkung}

In ernste Schwierigkeiten gerät der Dualismus mit dem Problem der psycho-physischen Wechselwirkung. Wie kann er erklären, daß physikalische Ereignisse psychische Wirkungen und psychische Vorgänge physikalische Wirkungen haben? Zwischen physischen und psychischen Ereignissen bestehen ja tatsächlich sehr enge wechselseitige Beziehungen: Unsere Wahrnehmungen hängen von physikalischen Reizen ab, die auf unsere Sinnesorgane einwirken und von deren Funktionsweise. Krankheiten und Drogen können unser Gefühlsleben, ja

${ }^{18}$ Vgl. dazu z. B. Ryle (49), S. 50, 51, 117.

${ }^{19}$ Vgl. dazu den Abschnitt 2.2, S. $105 \mathrm{f}$. 
den Charakter, die Persönlichkeitsstruktur verändern. Gehirnverletzungen führen zu Ausfällen im Denk-, Sprach-und Urteilsvermögen. Umgekehrt wirken sich unsere Strebungen und Entschlüsse in Handlungen aus, mit denen wir auf die Umwelt einwirken. Das Faktum der Wechselwirkung bedarf also keiner weiteren Diskussion. Wenn man aber versucht, diese Wechselwirkungen zu erklären und dazu z. B. die Kette der Wirkungen einer physikalischen Ursache verfolgt, so bleibt man immer im physikalischen Bereich: Licht, das von einer roten Fläche reflektiert wird, trifft auf die Netzhaut auf. Dort wird der optische Reiz auf komplizierten chemischen Wegen in elektrische Impulse verwandelt, die über die Nervenbahnen ins Gehirn.gelangen und dort gewisse physiologische Wirkungen hervorrufen, die man teilweise beschreiben kann und hofft, einst vollständig beschreiben zu können. Wie kommt es aber zu einer Rotempfindung und zu der bewußten Wahrnehmung der roten Fläche? Hier scheint ein unbegreiflicher Sprung vorzuliegen. ${ }^{20}$ Oder was geschieht, wenn wir uns entschließen, das Fenster zu öffnen? Da ist zunächst etwas Psychisches, der Willensentschluß. Wie ergibt sich daraus aber ein physiologischer Vorgang im Gehirn? Wenn dieser Vorgang im Gehirn gegeben ist, so läßt sich alles folgende, der Übergang von Strömen in den Nerven zur Betatigung von Muskeln und zu körperlichen Bewegungen - wenn auch gegenwärtig nur äußerst skizzenhaft - physikalisch erklären: aber zwischen diesem Vorgang und dem Willensentschluß scheint wieder ein unerklärlicher Sprung zu bestehen.

Im Rahmen des Dualismus bleiben solche Wechselwirkungen in der Tat unverständlich. Nimmt man an, Psychisches und Physisches bildeten verschiedene Welten, so ist nicht einzusehen, wie physikalische Ereignisse psychische Ursachen und physikalische Ursachen psychi-

${ }^{20}$ So schreibt z. B. K. Lorenz in (73), S. 227: ,,Der Leib-Seele-Hiatus dagegen ist unüberbrückbar, vielleicht, wie Nicolai Hartmann gesagt hat, ,nur für uns', d. h. für den Erkenntnisapparat, mit dem wir ausgerüstet sind. Ich glaube, daß diese Kluft nicht etwa nur für den heutigen Stand unseres Wissens unüberbrückbar ist. Selbst eine utopische Zunahme unserer Kenntnisse würde uns der Lösung des Leib-Seele-Problems nicht näherbringen. Die Eigengesetzlichkeiten des Erlebens können grundsätzlich nicht aus chemischphysikalischen Gesetzen und aus der wenn auch noch so komplexen Struktur der neurophysiologischen Organisation erklärt werden." 
sche Wirkungen haben sollen. Geht man von derjenigen Konzeption der Kausalbeziehung aus, die Descartes vertrat und die bis hin zu Hume herrschend blieb, so wären Wechselwirkungen sogar unmöglich. Denn dann sind Kausalgesetze Wesensgesetze und gelten mit Notwendigkeit. Und ist eine Ursache eines Ereignisses eine Bedingung, mit der es sich kausal erklären läßt, ${ }^{21}$ so gilt auch die Beziehung zwischen Ursache und Wirkung mit Notwendigkeit. Nun kann aber zwischen zwei Ereignissen keine Beziehung einer notwendigen Folge bestehen, wenn das eine rein psychisch ist, also durch einen Satz von $\Sigma$ ausgedrückt wird, das andere hingegen rein physisch, so daß ihm eine Aussage von $\Phi$ entspricht. Denn nach D 2 sollen zwischen Sätzen von $\Sigma$ und solchen von $\Phi$ keine analytischen, d. h. notwendigen Beziehungen bestehen. ${ }^{22}$ Descartes nahm trotzdem psycho-physische Wechselwirkungen an. Für ihn besteht der Mensch und er allein Tiere sind für Descartes seelenlose Automaten, Gott aber ist ein körperloser Geist - aus materieller und seelischer Substanz und beide sind in ihm so eng verbunden, $\mathrm{da} ß$ sie in Wechselwirkung stehen. Die Nervenströme, die Descartes als Ströme sehr feiner Partikel - der esprits animaux - ansah, bewirken, daß in der Zirbeldrüse gewisse Strömungszustände herrschen. Diese kann die Seele irgendwie ablesen, und sie kann sie auch willentlich beeinflussen - wie bleibt offen. Wie A. Kenny betont hat, ist es erstaunlich, daß Descartes das Wechselwirkungsproblem in der Beschränkung auf die Zirbeldrüse für einfacher lösbar hielt als im allgemeinen. ${ }^{23}$ Gelegentlich äußert sich Descartes freilich auch so, daß die Natur dieser Wechselwirkung prinzi-

${ }^{21}$ Vgl. dazu den Abschnitt 2.2.

${ }^{22}$ Bei Descartes findet sich (vgl. (MP) III, 14) die Aussage, es könne nichts in der Wirkung sein, was nicht auch in der Ursache ist, sonst entstünde es ,,aus nichts". Danach kann z. B. eine gestoßene Kugel durch eine stoßende nur deshalb in Bewegung gesetzt werden, weil diese ihr ihren Bewegungsimpuls mitteilt. Ein physisches Ereignis kann danach kein rein psychisches verursachen, weil in ihm nichts Psychisches ist, und umgekehrt.

${ }^{23}$ Vgl. Kenny (68), S. 225. - Descartes nahm einen Satz von der Erhaltung der Impulsbeträge an, sah also in einer Richtungsänderung der Gehirnströme durch die Seele keinen Eingriff in das physikalische System. Demgegenüber hat Leibniz betont (vgl. (T), $134 \mathrm{ff}$.), daß auch die Richtung des Gesamtimpulses in einem System erhalten bleibt, und daß generell das, was auf physikalisches Geschehen einwirkt, immer eine physikalische Ursache darstellt und physikalisch beschrieben werden kann. 
piell unerkennbar sei. Man kann aber nicht eine Theorie entwickeln, die ein so alltägliches Phänomen wie die psycho-physischen Wechselwirkungen unerklärlich macht, und dann sagen, die Theorie zeige, $\mathrm{daß}$ es prinzipiell unerklärlich sei. Tatsache ist wohl einfach, daß Descartes diesem Problem nicht genügend Aufmerksamkeit geschenkt hat. Einem Mann wie ihm wären sonst die Implikationen dieses Problems für seine Philosophie kaum verborgen geblieben.

Die Aussagen von Descartes hat man von Anfang an als unbefriedigend empfunden, und die Dualisten haben es in seiner Nachfolge für konsequenter gehalten, den Gedanken einer psycho-physischen Wechselwirkung ganz aufzugeben und statt dessen einen psycho-physischen Parallelismus anzunehmen: Physikalische Ereignisse sollen danach immer nur physikalische Ursachen haben und psychische Ereignisse nur psychische. Es besteht dann aber eine erhebliche Schwierigkeit, die psychologischen Ursachen von psychologischen Vorgängen anzugeben, die wir normalerweise als durch Physisches verursacht ansehen, und umgekehrt. Und es stellt sich die Frage, wie die Parallelität physikalischer und psychischer Ereignisse zu erklären ist, die man sonst durch eine Wechselwirkung begreiflich macht. Im Okkasionalismus, wie er von Arnold Geulincx und Nicole Malebranche begründet wurde, wird Gotr als a'exis ex maclivia benüht: Er soll anläßlich eines menschlichen Willensentschlusses zu einer Handlung, also anläßlich eines psychischen Ereignisses, das entsprechende physikalische Ereignis der Körperbewegung bewirken, und er soll andererseits anläßlich eines physikalischen Vorgangs den entsprechenden psychischen Vorgang der Empfindung oder des Erlebens hervorrufen. Wenn aber schon die Wechselwirkung von Psychischem und Physischem fragwürdig erschien, so muß erst recht diese göttliche Verursachung dunkel bleiben. So schreibt A. Pap mit Recht: „, One wonders why the action of the divine mind upon matter should have seemed to these philosophers any more intelligible than the action of the human (or animal) mind upon matter". ${ }^{24}$

Angesichts der Schwierigkeiten der okkasionalistischen Vorstellungen suchte Leibniz den durch pausenloses Verursachen zu sehr beschäftigten göttlichen Geist durch die in seiner Lehre von der prästabilierten Harmonie formulierte Annahme zu entlasten, Gott habe bei der Erschaffung der Welt und der einzelnen Subjekte den Ablauf ihrer

${ }^{24}$ Pap (49), S. 277. 
Zustände so eingerichtet, daß er parallel läuft - so wie zwei Uhren, einrnál gleichgesceilt und aufgezogen, immer die gleiche Zeit anzeigen, ohne daß man sagen könnte, der Zeigerstand der einen, verursache den der anderen. ${ }^{25}$

Als eine weitere These zum Leib-Seele-Problem ist der Epiphänomenaiismus zu erwähnen. Danach soll Psychisches nicht auf Physisches einwirken, so daß das Physische sich vollständig aus sich selbst erklären läßt, also ein in sich geschlossenes System bildet, während das Psychische nicht - oder doch nicht vollständig - aus sich selbst verständlich ist, also kein in sich geschlossenes System darstellt. Manche Epiphänomenalisten nehmen eine Einwirkung von Physischem auf Psychisches an - dann liegt also so etwas wie eine einseitige Wechselwirkungstheorie vor, die nicht plausibler ist als die normale, zweiseitige Wechselwirkungstheorie. Auch sie bleibt eine Antwort auf

${ }^{25}$, ,Nam Deus ab initio ita condidit animam pariter et corpus tanta sapientia et tanto artificio, ut ex ipsa cujusque prima constitutione notioneve omnia quae in uno fiunt per se perfecte respondeant omnibus quae in altero fiunt, perinde ac si ex uno in alterum transiissent. Quam ego Hypothesin concomitantiae appello". Zitiert in Couturat (02), S. 5. Gegen die Okkasionalisten sagt Leibniz: „Et je n'ay pas cru qu'on put écouter icy des philosophes, tres habiles d'ailleurs, qui font venir un Dieu comme dans une machine de theatre, pour faire le denouement de la piece, en soutenant que Dieu s'employe tout expres pour remuer les corps comme l'ame le veut, et pour donner des perceptions à l'ame comme le corps le demande; d'autant que ce Systeme, qu'on appelle celuy des causes occasionnelles (parce qu'il enseigne que Dieu agit sur le corps à l'occasion de l'ame, et vice versa) outre qu'il introduit des miracles perpetuels pour faire le commerce de ces deux substances, ne sauve pas le derangement de loix naturelles, établies dans chacune de ces mêmes substances, que leur influence mutuelle causeroit dans l'opinion commune". ((WP) VI, 136; (T), 135f.) Sein berühntes Uhrengleichnis findet sich in (WP) IV, S. $500 \mathrm{ff}$. Auf die Lehre von der prästabilierten Harmonie können wir hier nicht näher eingehen, da sie in der Leibnizschen Metaphysik tief verankert ist. Das Problem der psycho-physischen Wechselwirkung war für Leibniz ein Anlaß zur Entwicklung seiner Metaphysik, es bildet aber keine systematische Stütze für seine Philosophie. Die Lehre von der prästabilierten Harmonie bezieht sich auch nicht allein auf das Verhältnis von Körperlichem und Seelischem. Alle Beziehungen zwischen Monaden werden so internalisiert, daß sie sich ohne kausale Wechselwirkungen allein aus den Programmen der einzelnen Monaden ergeben. 
die Frage schuldig, wie Physisches auf so Verschiedenartiges wie das Psychische einwirken kann. In einer anderen Version stellt sich der Epiphänomenalismus als eine Art Parallelismus dar: Psychische Ereignisse sollen so etwas wie „Begleitphänomene“ gewisser physikalischer Ereignisse sein. So sagt T. H. Huxley in (98), S. 240, das Bewußtsein sei ein Nebenprodukt der Vorgänge im Körper und habe auf diese ebensowenig Einfluß wie das Pfeifen einer Dampflokomotive auf deren Bewegung. Auch dieser Parallelismus ist aber nicht verständlicher als der normale, und bildliche Vergleiche helfen hier wenig.

Sieht man nun von der traditionellen Konzeption der Kausalgesetze ab und versteht sie im Sinn des Abschnitts 2.2, so scheint die Annahme kausaler Beziehungen zwischen beiden Bereichen keine Schwierigkeiten mehr zu machen. Aus der Verschiedenheit physikalischer und psychischer Ereignisse kann man dann nicht darauf schließen, daß sie nicht in einer Kausalbeziehung stehen können. Auch mechanische Vorgänge können elektromagnetische Wirkungen haben, und umgekehrt, obwohl mechanische und elektromagnetische Vorgänge verschiedenartig sind. Wenn man von einem Kausalgesetz nicht mehr fordert, als daß es ein deterministisches Sukzessionsgesetz ist, so gibt es offenbar psycho-physische Wechselwirkungen. Eine solche Wechseiwirkung beschreibi z. B. das Weber-Fechreische Gesetz, nach dem die Empfindungsstärke dem Logarithmus der Reizstärke entspricht. Für den Gehörsinn gilt danach: Die vom Ohr empfundene Lautstärke ist proportional dem Logarithmus der (physikalisch gemessenen) Schallintensität. ${ }^{26}$

Die Probleme, die der Dualismus mit der Annahme psycho-physischer Wechselwirkung hat, entstehen jedoch nicht nur aus ihrer Verbindung mit dem traditionellen Kausalitätsbegriff. Wenn man von einer Einwirkung psychischer Faktoren auf ein physikalisches Geschehen redet, so meint man damit, daß dieses Geschehen nicht so abläuft, wie es ohne die fragliche Einwirkung ablaufen würde. Sieht man nun diese Faktoren im Sinn des Dualismus als nicht-physikalisch an, so läßt sich das Geschehen, wie es tatsächlich verläuft, nicht allein mit physikalischen Gesetzen aus physikalischen Antezedensbedingun-

${ }^{26}$ Das Weber-Fechnersche Gesetz ist kein Sukzessionsgesetz, sondern ein Koexistenzgesetz. Es läßt sich aber in ein Sukzessionsgesetz umformen, indem man es so formuliert: Die Veränderung der Empfindungsstärke ist proportional der Veränderung der Schallintensität J, dividiert durch J. 
gen erklären; das Physische bildet also kein in sich geschlossenes, autonomes System. Nach dualistischer Konzeption wie nach Auffassung der klassischen Physik ist aber das Physische ein in sich geschlossenes System. Mit anderen Worten: Gibt es Einwirkungen von Psychischem auf Physisches, so gilt entweder der Physikalismus (oder ein anderer Monismus) - dann ist aber der Dualismus falsch - oder das Physische bildet kein geschlossenes System, d. h. nicht alle physikalischen Ereignisse lassen sich rein physikalisch erklären. Auch das widerspricht aber vielen dualistischen Konzeptionen; für sie kann es also keine Einwirkung von Psychischem auf Physisches geben. Und für andere Dualismen gilt jedenfalls: Solche Einwirkungen sind mit der Annahme der Geschlossenheit der Physik nicht verträglich, welche die seit Descartes herrschende Konzeption des Physischen bestimmt. Nach dieser Konzeption ist ein Dualismus nur mit einem Parallelismus verträglich, der aber völlig unverständlich bleibt.

Mit der Einwirkung physikalischer Vorgänge auf Psychisches steht es nicht anders: Es gibt in der Physik keine Wirkungen physikalischer Vorgänge, die nicht einen Energieaufwand erfordern. Gibt aber ein System Energie nach außen ab, so ist es nicht geschlossen. Selbst das Pfeifen einer Dampflokomotive, von dem Huxley sprach, oder ihr Geräusch, benötigt einen Aufwand an Energie. Der Kern der Schwierigkeiten des Dualismus mit der Wechselwirkungstheorie liegt also nicht darin, daß dieser ein überholter Ursachenbegriff zugrundegelegt wurde, sondern er liegt in der Annahme, das Physische bilde ein in sich geschlossenes System; er besteht darin, daß wir alle Ursachen physikalischer Ereignisse als physikalisch ansehen und ebenso ihre Wirkungen. Die Annahme, die Grundgesetze der Physik seien statistisch, ändert an diesem Problem nichts. Denn dann folgen doch die Wahrscheinlichkeiten der Ereignisse deterministischen Gesetzen, und das System ist genau dann geschlossen, wenn sich die Wahrscheinlichkeiten späterer Zustände des Systems nach diesen Gesetzen aus den beobachteten Verteilungen in früheren Zuständen ergeben. Ist das aber der Fall, so kann von einer Einwirkung von außen nicht die Rede sein. Ist die (objektive) Wahrscheinlichkeit, mit einem Würfel die Augenzahlen 1 bis $6 \mathrm{zu}$ werfen, jeweils $1 / 6$, so ist die Behauptung eines Spielers absurd, er könne die Ergebnisse der einzelnen Würfe bestimmen, nicht aber deren Wahrscheinlichkeiten. Eine Einwirkung auf die Resultate läßt sich nur feststellen, wenn sich die Wahrscheinlichkeiten der Ergebnisse ändern, die relativen Häufigkeiten auf lange Sicht. 
Der indeterministische Charakter physikalischer Gesetze eröffnet also keine Möglichkeit, die Annahme der Geschlossenheit der Physik mit der Annahme einer psychophysischen Wechselwirkung zu vereinbaren, ebensowenig wie Descartes Annahme, in der Mechanik gelte nur ein Gesetz über die Erhaltung der Impulsbeträge.

H. Feigl hat den Unterschied zwischen Wechselwirkungstheorie und Parallelismus so bestimmt ${ }^{27}$ : Der Parallelismus behauptet, daß die Zuordnung von psychologischen Sachverhalten, die sich auf eine Person a beziehen, zu physikalischen Sachverhalten, die sich auf das zentrale Nervensystem von a beziehen, eine ein-ein-deutige oder einmehr-deutige Relation sei, während die Wechselwirkungslehre behauptet, diese Zuordnung sei mehr-ein-deutig oder mehr-mehr-deutig. Feigls Gedanke ist dabei folgender: Im Fall der Parallelität kann man alle psychischen Phänomene physikalisch erklären; man kann dann aber auch jene physikalischen Ereignisse, die wir normalerweise als durch Psychisches bewirkt ansehen, rein physikalisch erklären. Denn jedem physikalischen Zustand des zentralen Nervensystems von a entspricht dann nur ein seelischer Zustand von a. Im Fall der Wechselwirkung läßt sich dagegen das Auftreten eines bestimmten unter denjenigen psychischen Zuständen von a, die ein und demselben physikalischen Zustand von a entsprechen, physikalisch nicht mehr erklären, und falls nur mehr-mehr-deutige Zuordnungen bestehen, lassen sich auch nicht alle physikalischen Phänomene physikalisch erklären. Denn ein bestimmter, physikalisch nicht erklärbarer psychischer $\mathrm{Zu}$ stand kann dann eine physikalische Wirkung haben, die sich physikalisch nicht erklären läßt. Der Physikalismus ist so nur mit dem Parallelismus vereinbar. Feigl hält die Frage ,Wechselwirkung oder Parallelität?“ für prinzipiell experimentell entscheidbar. Es sei denkbar, daß einmal ein Auto-Cerebroskop entwickelt wird, eine Art Gehirnspiegel, der es erlaubt, die neurophysiologischen Vorgänge im eigenen Gehirn zu beobachten und sie mit den introspektiv erfahrenen psychischen Zuständen zu vergleichen. Dieser Gedanke ist aber viel zu naiv: Wir könnten mit diesem Instrument nur die Gehirnzustände beobachten, die psychischen Zuständen entsprechen, in denen wir unter anderem eben Beobachtungen über unsere Gehirnzustände machen; wir könnten also nicht feststellen, welche Zustände des Nervensystems psychischen Zuständen ohne gleichzeitige autocerebroskopische Be-

${ }^{27}$ Vgl. Feigl (58), $376-387$. 
obachtungen entsprechen. Im übrigen stimmen Feigls Begriffsbestimmungen nicht mii den üblichen überein: Er versteht unter Paralielismus einen Epiphänomenalismus, da er nicht fordert, daß sich alles Psychische rein psychisch erklären läßt. Und eine Wechselwirkung in seinem Sinn kann auch dann bestehen, wenn sich alles Physische rein physikalisch erklären läßt. Beim Leib-Seele-Problem geht es aber nicht nur um Fragen der Korrespondenz psychologischer und physikalischer Ereignisse, sondern um die Frage, ob Psychisches und Physisches in sich geschlossene, autonome Systeme bilden. Parallelismus und Wechselwirkungstheorie sind zudem Theorien, die sich im Rahmen des Dualismus bewegen; sie schließen also beide einen Physikalismus aus.

Das Wechselwirkungsproblem stellt sich sicher nicht nur für den Dualismus. Es stellt sich aber für jede nicht monistische Position, insbesondere also auch für den Dualismus, und für ihn wird es besonders dringlich, weil er auf einer "totalen Verschiedenartigkeit" von Physischem und Psychischem beharrt. Wir kommen auf dieses Problem im Abschnitt 8.5 zurück.

\subsection{Die wechselseitige Bezogenheit von Psychi- schem und Physischem}

In diesem Abschnitt wollen wir zeigen, daß die dualistische These D 2 nicht haltbar ist, daß es also analytische Beziehungen zwischen Sätzen über Physisches und solchen über rein Psychisches gibt.

Zunächst kann man sagen, daß die Sprache über rein Psychisches die Sprache über Physisches voraussetzt und mit ihr eng verknüpft ist. Dazu können wir auf frühere Ausführungen zurückgreifen, denn wir haben schon im 5 . Kapitel betont, daß die Sprache über intentionale Akte und Einstellungen, also z. B. jene über doxastische Einstellungen, über das, was Personen glauben oder für wahrscheinlich halten, die Sprache über Physisches als Teilsprache enthält. Dasselbe gilt auch für Aussagen über Präferenzen. Die Sachverhalte, die geglaubt oder bewertet werden, sind selten, beobachtete Sachverhalte aber nie rein psychischer Natur. Im 6. Kapitel haben wir auch gesehen, daß sich die Sprache über Physisches nicht in eine Sprache über Psychisches übersetzen läßt. 
Im Abschnitt 5.3 haben wir im Anschluß an Hinweise von Quine dargelegt, daß auch die Sprache über nichtintentionale Sinnesempfindungen zum großen Teil aus der Sprache über Physisches abgeleitet ist. Entsprechendes gilt für andere Empfindungen, für Gefühle und Stimmungen. Wir charakterisieren unsere Empfindungen häufig durch die physischen Sachverhalte, die sie normalerweise verursachen. Wir reden z. B. von ,stechenden“ oder „brennenden“ Schmerzen; wir sagen, jemand sei ,niedergeschlagen“, ,, wie vor den Kopf geschlagen" oder ,,gebrochen". Wir benützen also die vielfältigen $\mathrm{Zu}$ sammenhänge zwischen äußeren Vorgängen und Empfindungen oder Gefühlen, um diese näher zu bestimmen. Typisch für dieses Phänomen sind die physischen, speziell räumlichen Metaphern oder Vergleiche für Seelisches. ${ }^{28}$ So sprechen wir von ,tiefer Trauer", „, hohen Zielen“, wir ,begreifen“ etwas, machen es uns ,,klar", sind ,,verblendet", fühlen uns ,,in die Enge getrieben“. Endlich wird Seelisches häufig durch typische Verhaltensweisen charakterisiert. Wir sagen, daß jemand ,hochgeht", wenn er wütend reagiert, bezeichnen Dünkel als „Hochnäsigkeit", usf.

Im Abschnitt 6.2 haben wir ferner gesehen, daß es nahe liegt, Bedeutungsbeziehungen zwischen Ausdrücken für Psychisches und solchen über Verhaltensweisen anzunehmen. Wir erlernen die Wörter, mit denen Psychisches bezeichnet wird, anhand von intersubjektiv beobachtbaren Verhaltenskriterien. Selbst wenn Verhaltensaussagen, wie wir betont haben, sich nicht nur als Aussagen über Physisches verstehen lassen, so enthalten sie doch Komponenten, die P'nysisches bezeichnen. Überzeugungen, Präferenzen, Empfindungen, Gefühle usw. lassen sich nicht allein durch ihre Rolle im seelischen Leben charakterisieren, sondern ihre Eigenart bestimmt sich auch aus der Rolle, die sie für unser Verhalten, für unser ganzes Leben spielen. Ein Satz wie „Wenn eine Person will, daß ein Ereignis p eintritt, glaubt, daß p genau dann eintritt, wenn sie $f$ tut, und $f$ tun kann, so wird sie in der Regel $f$ tun" ist kein bloß empirischer Satz, sondern ein Satz, der auch die Bedeutung der in ihm vorkommenden rein psychologischen Terme wie ,glauben" und ,wollen“ mitbestimmt. Entspräche unser tatsächliches Verhalten nur gelegentlich dem, was wir glauben und wollen, so hätten die Wörter ,glauben“ und ,wollen“ nicht die Bedeutung, die sie haben. Es wäre dann nicht mehr möglich, aus dem

${ }^{28}$ Vgl. dazu auch Snell (55), Kap. XI. 
Verhalten einer Person auf ihre Präferenzen zurückzuschließen, und Praferenzen würden zu jenen Käfern in seelischen Schacnteln, von denen Wittgenstein spricht. Phänomene wie Schmerz, Wut, Enttäuschung, Fröhlichkeit drücken sich im Verhalten, in der Miene und in körperlichen Haltungen aus und werden darin sinnfällig. Und dieser leíbliche Ausdruck ist nicht etwas, was dem Seelischen äußerlich wäre, sondern darin realisiert und bestimmt es sich. Daher kann man es auch von diesem Ausdruck her charakterisieren.

Nun haben wir früher betont, daß es nicht möglich ist, einzelne Aussagen über rein Psychisches in Aussagen über Verhaltensweisen, geschweige denn in rein physikalische Aussagen zu übersetzen. Die dualistische These D2 ist aber schon falsch, wenn nicht jede konsistente Menge von Sätzen über rein Psychisches mit jeder konsistenten Menge von Sätzen über Physisches verträglich ist. Wäre das der Fall, so wären aber all unsere Beobachtungen über Physisches damit verträglich, daß die physische Welt völlig anders beschaffen ist, als wir das aufgrund unserer Beobachtungen annehmen; daß sie sich plötzlich völlig ändert oder gar aufhört zu existieren, ohne daß wir das bemerken. Diese Vorstellung ist aber doch zu abwegig, und wir könnten dann auch den Anspruch nicht begründen, unsere Erfahrung liefere uns Informationen über die Außenwelt. Die These D 2 hat also eine nicht mehr auflösbare Skepsis bzgl. Beschaffenheit und Existenz der Außenwelt zur Folge, eine Skepsis, auf deren Unhaltbarkeit wir schon im Abschnitt 3.4 hingewiesen haben. Die Welt, die wir meinen und über die wir allein sinnvoll reden können, ist eben die Welt, die wir erfahren, und die erfahrene Welt kann nicht völlig anders beschaffen sein, als wir sie erfahren.

Diese Überlegung ist zugleich ein Argument gegen den absoluten ontologischen Realismus, der sich einerseits aus der These D 2 ergibt, und der andererseits normalerweise einen Dualismus darstellt. Selbst wenn er das nicht tut, wenn also die von ihm angenommene objektive Realität nicht physikalischer Natur ist, impliziert er aber eine analoge These wie D $2 .^{29}$

Endlich haben wir im Abschnitt 7.1 auch betont, daß Personennamen, die in allen rein psychologischen Aussagen vorkommen, nicht „Seelen“ bezeichnen, sondern Menschen als leib-seelische Einhei-

${ }^{29} \mathrm{Vgl}$. dazu die ausführlichere Argumentation in 8.6. 
ten. ${ }^{30}$ Auch aus diesem Grund können Aussagen über rein Psychisches nicht völlig unabhängig von Aussagen über Physisches sein: Wenn jemand nicht mehr lebt - und dafür gibt es rein physiologische Kriterien - so ist es nicht nur empirisch, sondern analytisch falsch zu behaupten, er habe gewisse Empfindungen.

Daß Sätze über Psychisches sich ihrer Bedeutung nach auch auf Physisches beziehen, erscheint uns nicht ungewöhnlich. Weniger geläufig und den heute verbreiteten Vorstellungen sehr viel weniger gemäß ist die Umkehrung dieser Behauptung, daß auch die Sprache über Physisches subjektive, auf Psychisches bezogene Bedeutungskomponenten enthält. Das ergibt sich zwar schon aus dem obigen Argument gegen D 2 - denn Unabhängigkeit ist ein symmetrischer Begriff, wie wir schon betont haben -, aber wir wollen uns das doch genauer überlegen.

Daß wir die Terme der physikalischen Sprache in bestimmter Weise verwenden, daß wir mit ihnen bestimmte Bedingungen verbinden, das hat sicher etwas mit Psychischem zu tun. Die Bedeutung des Satzes A einer Sprache S ist im Sinne von P. Grice das, was die (kompetenten) Sprecher von $S$ dem jeweiligen Hörer mit einer Äußerung von A anzuzeizen versuchen; Bećeutungen haben also etwas mit Absichten zu tun, und Absichten sind etwas Psychisches. Die Sprache über Physisches hätte daher auch dann etwas mit menschlichem Verhalten und dessen subjektiven Faktoren zu tun, wenn das Physische selbst nichts damit zu tun hätte. Der Sachverhalt, daß dieses Blatt Papier weiß ist, ist jedoch von dem Sachverhalt zu unterscheiden, daß alle kompetenten Sprecher des Deutschen mit der Äußerung des Satzes „Dieses Blatt Papier ist weiß" dem Hörer diesen Sachverhalt anzuzeigen versuchen. Daher kann man so nicht begründen, daß es analytische $\mathrm{Zu}$ sammenhänge zwischen Sätzen der physikalischen Sprache und solchen der psychologischen gibt.

Wir betrachten ein einfaches Beispiel: Das Prädikat „,rot“" ist ein Wort der Dingsprache, also der Sprache $\Phi$; es drückt eine Eigenschaft physischer Gegenstände aus. Der Ausdruck „,Die Person a empfindet den Gegenstand b als rot" ist hingegen ein Prädikat der Sprache $\Sigma$ über rein Psychisches. Es gilt nun nicht allgemein, daß rote Dinge von

${ }^{30} \mathrm{Vgl}$. dazu auch den Abschnitt 7.5. 
jedermann als rot empfunden werden - rote Dinge werden nicht immer beobachtet, und sie können bei Beobachtungen unter grünem Licht auch als schwarz empfunden werden. Aus dem letzten Grund gilt auch nicht allgemein, daß von jemand als rot empfundene Dinge tatsächlich rot sind. In der Regel und unter normalen Beobachtungsbedingungen gilt aber: Wenn a den Gegenstand b betrachtet, so ist $b$ genau dann rot, wenn $a b$ als rot empfindet.

„Rot“ ist ein Prädikat unserer gemeinsamen Sprache. Das kann es nur sein, wenn es eine breite Übereinstimmung im Gebrauch dieses Prädikats gibt, wenn es also Kriterien für die Anwendung von ,,rot“ gibt, die weitgehend invariant sind gegenüber Differenzen im subjektiven Erleben der verschiedenen Sprecher und gegenüber Unterschieden ihrer Beobachtungen. Wenn mehrere Personen ein Ding wahrnehmen, so werden sie es in der Regel unter verschiedenen Blickwinkeln, aus verschiedenen Entfernungen und vor verschiedenem Hintergrund sehen. All das kann einen gewissen Einfluß auf die spezifische Weise haben, in der sie die Farbe dieses Objektes empfinden. Die Beobachter werden sich daher leichter über eine farbliche Bestimmung des Objekts verständigen können, die mit all diesen Erşcheinungsweisen verträglich ist, als über etwas, das individual spezifischen Erlebnisformen entspricht. Dieser Zug zur intersubjektiven Vereinheitlichung wirkt dem Zug zur subjektiv einfachsten, d. h. direkten Assoziation von Dingeigenschaft und Empfindungsweise entgegen - in unserem Beispiel also der Bestimmung: Ein Ding ist rot, wenn ich es bei der Betrachtung als rot empfinde. W. V. Quine spricht von einem objektiven Zug (objective pull), der dem subjektiven Zug zur direkten Assoziation mit bestimmten Empfindungen entgegenwirkt, und sagt, die Anwendungskriterien für einfache Prädikate stellten einen Kompromiß zwischen beiden Faktoren dar: „In general, if a term is to be learned by induction from observed instances where it is applied, the instances have to resemble one another in two ways: they have to be enough alike from the learner's point of view from occasion to occasion to afford him a basis of similarity to generalize upon, and they have to be enough alike from simultaneous distinct points of view to enable the teacher and learner to share the appropriate occasions" ${ }^{31}$

Die für die Ausdrücke einer intersubjektiven Sprache nötige Übereinstimmung im Gebrauch bewirkt also, daß der Gebrauch von Ding-

${ }^{31}$ Quine (60), S. 7. 
Prädikaten wie ,,rot“ nicht nur Kriterien der Empfindungsweise folgt. Hinzu kommt, daß wir die Unterscheidung von Farben, die Klassifikation der Vielfalt von Farbnuancen, erst mit der Sprache erlernen. Verschiedene Sprachen stellen dazu verschiedene Wortfelder bereit. Mit der Sprache erwerben wir also nicht nur Bezeichnungen für uns bereits geläufige Unterscheidungen, sondern auch die Fähigkeit, diese Unterscheidungen zu machen. Wittgenstein sagt: „Wie erkenne ich, daß diese Farbe Rot ist?“ - Eine Antwort wäre: „Ich habe Deutsch gelernt ${ }^{\text {"32 }}$. Wir erlernen also nicht gemeinsame Benennungen, nachdem uns die ihnen entsprechenden Aufgliederungen der Phänomenfelder schon geläufig sind, sondern wir lernen, die Phänomene zu unterscheiden, indem wir sprechen lernen - jedenfalls in einem weiten Bereich, denn über gewisse Unterscheidungen müssen wir natürlich schon verfügen, um eine Sprache überhaupt erlernen zu können. Wir lernen also von vornherein, die Welt in einer gemeinsamen Weise zu sehen, in einer für unsere Kultur spezifischen Weise, die sich nicht schon aus angeborenen, artspezifischen Unterscheidungen ergibt. Das zeigt sich schon bei der Klassifikation der Farben, wird aber natürlich besonders deutlich bei den verschiedenen Wortfeldern zur Beschreibung von Psychischem und Geistigem.

Der objektive Zug in cier Verwendung des Piädikats ,rot“ ergibt sich endlich auch daraus, daß es eine Reihe von generellen Verknüpfungen dieser Eigenschaft mit anderen Dingattributen gibt wie z. B. „,Hummer wird beim Kochen rot“ oder „Eisen ist bei einer Temperatur von $550^{\circ} \mathrm{C}$ rotglühend". Solche Sätze liefern weitere objektive Kriterien für die Verwendung von ,rot", und diese Kriterien sind so vielfältig, daß auch ein Farbenblinder die Verwendung von „,rot“ recht gut erlernen kann, indem er sich z. B. merkt, daß unreife Tomaten grün, reife aber rot sind, daß Gras grün ist, das oberste Licht an der Verkehrsampel rot, usf. Die Gesamtheit dieser Sätze charakterisiert auch die Bedeutung des Prädikats ,rot“. Unsere Ansichten, welche Dinge rot sind, könnten sich nicht völlig ändern, ohne daß sich auch die Bedeutung dieses Prädikates ändern würde. ${ }^{33}$ Seine Bedeutung wird also sicher nicht allein durch Kriterien des Farberlebens be-. stimmt.

32 Wittgenstein (53), 381; vgl. dazu auch Kutschera (75), 2.4.7.

${ }^{33}$ Vgl. dazu auch Kutschera (75), 2.3.2 und 2.4.4. 
Neben diesen objektiven Kriterien für den Gebrauch von Dingprädikaten sind aber subjektive, d. h. Erlebniskriterien unentbehrlich. Wir erlernen den Gebrauch eines Dingprädikats wie ,,rot“ aus Beispielen und Gegenbeispielen seiner Verwendung. Man beherrscht den Gebrauch von ,,rot" jedoch noch nicht, wenn man eine Klasse K von Dingen kennt, auf die man das Prädikat anwenden darf, und eine Klasse $\mathrm{K}^{\prime}$ von Dingen, auf die man es nicht anwenden darf, sondern erst dann, wenn man über ein allgemeines Kriterium verfügt - das natürlich nicht explizit formulierbar sein muß - , nach dem man das Prädikat verwendet und das so geartet ist, daß es auf alle Dinge aus $\mathrm{K}$, aber auf kein Ding aus $\mathrm{K}^{\prime}$ zutrifft; wenn man also das Prädikat über die Klasse der Beispiele und Gegenbeispiele hinaus richtig anwenden kann. Als ein solches Kriterium kommt erstens die Übereinstimmung mit anderen nicht infrage, denn wir halten nicht vor jeder Anwendung des Prädikats auf ein neues Ding eine Abstimmung darüber ab, ob wir es als ,,rot" oder als ,nicht rot" bezeichnen wollen. „Rot" würde sonst nicht mehr bedeuten als ,,wird übereinstimmend ,rot' genannt". Man kann aber nicht alle Prädikate F im Sinne von ,wird übereinstimmend $\mathrm{F}$ genannt" verstehen, weil der Ausdruck "wird übereinstimmend so und so genannt" selbst ein Prädikat ist, so daß man auf diese Weise in einen unendlichen Regreß geriete: , $a$ ist ein $F^{\prime \prime}$ wäre zu deuten als ,, a wird übereinstimmend , $F^{\star}$ genannt", das als ,a wird übereinstimmend, ,übereinstimmend , $F^{6}$ genannt" genannt", usf. Ferner würden dann Aussagen mit solchen Prädikaten nichts über die Dinge sagen, auf die wir sie anwenden, sondern nur etwas über unseren Sprachgebrauch. Anwendungskriterien können zweitens aber auch nicht immer nur objektive Kriterien sein. Um z. B. mithilfe des Kriteriums „Eine Tomate ist rot, wenn sie reif ist“ bestimmen zu können, ob ein Ding rot ist oder nicht, muß ich zunächst den Gebrauch der Prädikate "Tomate" und ,reif“, also anderer Dingprädikate beherrschen. Die Annahme, alle Dingprädikate würden allein nach objektiven Kriterien verwendet, führt also auf einen Zirkel oder einen unendlichen Regreß. ${ }^{34}$

${ }^{34}$ Dem entspricht, daß eine Festlegung, welche Sätze einer Sprache wahr und welche falsch sind, die Interpretation dieser Sprache noch nicht eindeutig bestimmt, sondern, wie in der Logik gezeigt wird, mit den verschiedensten Annahmen über ihren universe of discourse verträglich ist. Vgl. dazu z. B. Kutschera (67), 2.2.2. 
Subjektive Anwendungskriterien für Dingprädikate sind daher unverzichtbar. Wir verwenden das Wort „rot“ für beobachtete Dinge normalerweise nach Maßgabe der Art und Weise, wie wir es erleben, also nach Empfindungs- oder Erlebniskriterien, im Sinn seines anschaulichen, erlebnismäßigen Gebalts. ${ }^{35}$ Diese Kriterien werden im Sinn der vorstehenden Bemerkungen durch die Forderung einer intersubjektiven Übereinstimmung im Gebrauch und durch objektive Kriterien überlagert und ergänzt, bleiben aber eine unverzichtbare Grundlage unserer Sprache über Dinge. Die Korrespondenz zwischen Erlebnisweise und Dingeigenschaft ist im Fall des Prädikats ,,rot" recht eng, in anderen Fällen, wie z. B. bei ,reif“ erheblich lockerer. Es gibt sicher keine eineindeutige Beziehung zwischen Differenzen in unserem Erleben und Unterschieden im Bereich der Dinge. Diese gewinnen durch ihre Verflechtungen untereinander eine relative Selbständigkeit, aber eben nur eine relative Selbständigkeit. Ohne eine Verknüpfung mit unserem Erleben, ohne anschaulichen Gehalt gäbe es keine Verwendungskriterien für die Terme der Dingsprache. Ihr anschaulicher Gehalt macht einen Teil ihrer Bedeutung aus. „Rot sein“ heißt eben auch soviel wie ,normalerweise in gewisser Weise erlebt werden".

Sätze wie

1) Betrachtet a das Objekt b, so gilt in der Regel: b ist rot genau dann, wenn es a erscheint, als ob b rot ist

lassen sich nicht alle als empirische Sätze auffassen. (1) läßt sich ja nur dann empirisch rechtfertigen, wenn wir ohne Rekurs darauf, wie $\mathrm{b}$ der Person a erscheint, feststellen können, ob b rot ist. Das macht. nun zwar keine Schwierigkeiten im Einzelfall. Wir können ja z. B. prüfen, ob b bei Bestrahlung mit physikalisch weißem Licht vorwiegend Licht im Wellenlängenbereich von ca. 0,7 $\mu \mathrm{m}$ reflektiert. Das Ergebnis der Ablesung, daß z. B. der Zeiger des Instruments auf einer bestimmten Stelle der Meßskala steht, wird aber wieder durch einen Satz der Dingsprache ausgedrückt, und wir verlassen uns bei der Ablesung darauf, daß der Zeiger dort steht, wo er uns zu stehen scheint, setzen also einen Satz der Art (1) voraus. Auch das können wir in-

${ }^{35}$ Kant spricht von einem anschaulichen „Schema“, das Begriffen zugeordnet ist und mit dessen Hilfe wir Dinge unter Begriffe subsumieren. Vgl. dazu den Abschnitt „Von dem Schematismus der reinen Verstandesbegriffe “ in der „Kritik der reinen Vernunft". 
frage stellen, aber wir müssen letztlich bei allen Überprüfungen empirischer Sätze Aussagen der Art (1) voraussetzen. Das heißt natürlich nicht, daß es bestimmte solche Sätze gibt, die in jedem Untersuchungskontext vorauszusetzen wären, aber wir setzen immer Sätze dieser Art voraus. Ohne solche Voraussetzungen kämen wir nicht zu Dingaussagen.

Wie sind nun solche Voraussetzungen zu rechtfertigen? Wir können nicht einfach sagen, in der Regel seien wir eben davon überzeugt oder es sei uns evident, daß b rot ist, wenn es uns so erscheint; wir hätten in den meisten Fällen allen Grund anzunehmen, daß b rot ist, und keinen Grund, das zu bezweifeln, wenn es uns erscheint, als sei es so. Unsere Frage ist nicht, ob wir tatsächlich in der Regel glauben, $\mathrm{daß} \mathrm{b}$ rot ist, falls es uns so erscheint, sondern mit welchem Recht wir das tun. Und diese Frage läßt sich wohl nur so beantworten, daß wir sagen, „Rot sein“ bedeute unter anderem auch ,,bei Betrachtung in der Regel als rot erscheinen“. Das Dingprädikat „,rot“ hat dann aber eine subjektive, auf unser Erleben bezogene Bedeutungskomponente.

Ebenso erleben wir räumliche Relationen optisch, haptisch und bei Bewegungen in gewisser Weise. Die Art, wie wir räumliche Verhältnisse physikalisch beschreiben, entspricht nicht genau der Weise, wie wir sie erleben, aber ohne ein Raumerleben könnten wir die physikalisch-geometrischen Begriffe nicht anwenden. Wie weit sich die Theorien von der Struktur des physikalischen Raums - z. B. in der allgemeinen Relativitätstheorie - auch immer von unserem Raumerleben entfernen mögen, so bleibt dieses doch Grundlage unserer physikalischen Feststellungen über Räumliches. ${ }^{36}$ Diese Korrespondenz zwischen Erleben und erlebter Welt ist also die Basis unserer Aussagen über diese, die Grundlage unserer Sprache über Physisches.

Gehört nun aber der erlebnismäßige Gehalt der Terme der Dingsprache, gehören die subjektiven Bedeutungskomponenten $\mathrm{zu}$ den sprachlichen Bedeutungen dieser Terme? Zählen diese Komponenten zur intersubjektiven Bedeutung der Terme, oder sind sie nur Konnotationen oder Assoziationen, die der einzelne mit diesen Termen verbindet?

Peirce, Wittgenstein und Quine, um nur einige Namen zu nennen, vertreten die Auffassung, sprachliche Bedeutungen seien durch den

${ }^{36}$ Vgl. dazu den Abschnitt 8.3. 
allgemeinen Sprachgebrauch festgelegt, so daß Terme sich in ihren sprachlichen Bedeutungen nur dann unterscheiden, wenn die Terme in verschiedener Weise verwendet werden. Peirce sagt: „, . there is no distinction of meaning so fine as to consist in anything but a possible difference of practice“ ${ }^{37}$ Nach Wittgenstein kann man ,für eine große Klasse von Fällen der Benutzung des Wortes „Bedeutung“ wenn auch nicht für alle Fälle seiner Benutzung - dieses Wort so erklären: Die Bedeutung eines Wortes ist sein Gebrauch in der Sprache" ${ }^{38}$ Und Quine meint: ,... there is no justification in collating linguistic meanings unless in terms of mens' dispositions to respond overtly to socially observable stimulation". ${ }^{39}$ Dieser These kann man im Großen und Ganzen durchaus zustimmen, wenn man das Wort "Gebrauch" hinreichend weit versteht und es nicht im Sinne des Behaviorismus nur auf physikalisch beschreibbares Verhalten beschränkt. Sprachliche Äußerungen sind Handlungen, und Handlungen sind, wie schon mehrfach betont wurde, nicht einfach physische Vorgänge, sondern sie werden auch durch Absichten, Ziele, Überzeugungen, Wertungen des Handelnden charakterisiert, d. h. durch rein Psychisches. Bestimmt man also Bedeutungen durch Gebrauchsweisen, so eliminiert man damit keineswegs die Bezüge auf rein Psychisches, sondern betont sie eher. ${ }^{40}$

Wir haben in 6.2 gesehen, daß wir aufgrund des Verhaltens anderer Aussagen über ihr Erleben und ihre Empfindungen machen können. Solche Aussagen sind hypothetisch, sie können sich als falsch erweisen, aber das gilt auch für generelle Aussagen über den Gebrauch von Wörtern in einer Sprachgemeinschaft, wie eng man die Rede vom "Gebrauch" auch immer versteht. Wenn nun unsere Verwendung sprachlicher Ausdrücke anschaulichen oder empfindungsmäßigen Kriterien folgt und wir feststellen, daß andere diese Ausdrücke in gleicher Weise verwenden, so liegt die Annahme nahe, daß sie in etwa den gleichen erlebnismäßigen Kriterien folgen. Würde ein anderer

\footnotetext{
${ }^{37}$ Peirce (65), V, S. 257.

38 Wittgenstein (53), 43.

${ }^{39}$ Quine (60), S. IX. - Zu den Gebrauchstheorien der Bedeutung vgl. Kutschera (75), Kap. 2.

${ }^{40} \mathrm{Vgl}$. dazu wieder die Ansätze zu einer handlungstheoretischen Bedeutungstheorie bei P. Grice und D. Lewis, auf die wir oben und in 2.3 hingewiesen haben.
} 
rote Dinge ganz anders erleben als ich, so käme das in seiner Verwendung des Wortes ,rot" oder in seinem sonstigen Verhalten vermutlich irgendwie zum Ausdruck, wäre also für mich prinzipiell feststellbar. Wir können z. B. aus dem Verhalten eines Farbblinden erschließen, daß er Rot und Grün nicht direkt unterscheiden kann, daß er keine direkten erlebnismäßigen Unterscheidungskriterien hat, und daraus entnehmen wir, daß er rote Dinge anders erlebt als wir. Wir haben also keinen Grund anzunehmen, daß andere, die das Wort ,,rot" ebenso verwenden wie wir und Feststellungen darüber, ob etwas rot ist, ebenso direkt machen, rote Dinge anders erleben als wir, und daher besteht auch kein Grund, unsere Erlebniskriterien für das Wort „rot" als eine bloß individuelle Assoziation anzusehen. Ohne die Annahme, der Gebrauch der Wörter sei im Erleben intersubjektiv etwa in gleicher Weise verankert, könnten wir nach den obigen Bemerkungen auch nicht behaupten, daß wir mit denselben Sätzen über dasselbe reden. Die Behauptung, erlebnismäßige Konnotationen könnten grundsätzlich nie Teil der sprachlichen Bedeutung sein, wäre völlig unbegründet. Es gibt, wie im Fall des Wortes „Nigger“, auch emotive Konnotationen, die sich für jeden Sprecher mit diesem Wort verbinden und daher zur Bedeutung dieses Wortes zu rechnen sind. Bedeutung ist nicht immer nur deskriptive Bedeutung, und deskriptive Bedeutung ist nicht immer physikalische Bedeutung.

Versteht man die Rede vom Sprachgebrauch wie Quine so, daß zwei Sprecher die Sprache in gleicher Weise verwenden, wenn für sie die Sätze bezogen auf die gleichen Komplexe von Sinnesreizen dieselben Wahrheitswerte haben, so beruhen Feststellungen über diesen Gebrauch ebenfalls auf Annahmen über Fremdpsychisches. Daß jemand einen bestimmten Komplex von Sinnesempfindungen hat, ist nicht direkt ,sozial beobachtbar" und ebensowenig, daß er seine Äußerungen im Sinne aufrichtiger Behauptungen macht. Auch solche Gebrauchsbeschreibungen schließen also rein Subjektives nicht aus dem Feld sprachlicher Bedeutungen aus. Quine hat eine These von der Indeterminiertheit der Übersetzungen aufgestellt. Sie lautet: „The infinite totality of sentences of any given speaker's language can be so permuted or mapped into itself, that (a) the totality of the speaker's dispositions to verbal behavior remains invariant, and yet (b) the mapping is no mere correlation of sentences with equivalent sentences, in any plausible sense however loose“. ${ }^{41}$ Dabei ist mit ,equivalence“ eine Bedeutungsgleichheit im üblichen Sinn gemeint. Die Begründung dieser 
These, die das, was wir normalerweise als Bedeutungen bezeichnen, aus dem Bereich der "linguistic meanings" ausschließen und in das Feld bloß subjektiver, individueller Assoziationen und Vorstellungen verweisen soll, beruht darauf, daß Quine Annahmen über Fremdpsychisches, wie sie in Aussagen über den Sprachgebrauch in seinem Sinn stecken, in anderen Fällen nicht zuläßt. Wie wir aber Handlungen anderer nur aufgrund von Annahmen über ihren Absichten, Präferenzen und Überzeugungen verstehen, so verstehen wir auch ihre sprachlichen Äußerungen nur aufgrund solcher Annahmen. Läßt man solche Annahmen nicht zu, so kommt man natürlich zu einer Auffassung von Sprache, nach der wir uns mit ihr nicht mehr verständigen können, nach der wir nicht mehr verstehen, was uns andere mitteilen wollen. Die Konsequenz, daß Sprache - das Verständigungsmittel schlechthin - zur Verständigung nicht ausreicht, führt aber die Prämissen ad absurdum, aus denen sie sich ergibt.

Es gibt also Wörter der Dingsprache, die subjektive, d. h. auf Erlebnisweisen bezogene Bedeutungskomponenten enthalten. Ohne hinreichend verläßliche subjektive Anwendungskriterien könnte man den allgemeinen Gebrauch solcher Wörter aus Beispielen ihrer Verwendung nicht erlernen. Diese Kriterien müssen bei den verschiedenen Sprechern hinreichend' genau übereinstimmen, damic sich ein cinheitlicher intersubjektiver Gebrauch der Wörter ergibt. Obwohl die spezielle Weise, in der jemand die Farbe Rot empfindet, z. B. die Art und Intensität von Gefühlswerten, die er damit assozïert, nicht zur Bedeutung des Wortes ,,rot" gehört, charakterisieren doch die intersubjektiv in etwa gleichen Merkmale dieser Empfindungsweise die Bedeutung des Wortes, da sie seinen allgemeinen Gebrauch bestimmen. Diese Kriterien gelten analytisch, weil das Wort so verstanden wird, daß es nach diesen Kriterien gebraucht werden kann. Die „Ob-

${ }^{41}$ Quine (60), S. 27. Daß mit demselben Gebrauch - derselben äußeren Gesamterscheinung - verschiedene individuelle Deutungen der Sprache verträglich sind, illustriert Quine durch folgenden Vergleich: „Different persons growing up in the same language are like different bushes trimmed and trained to take the shape of identical elephants. The anatomical details of twigs and branches will fulfill the elephantine form differently from bush to bush, but the overall outward results are alike" ((60), S. 8). Zur Diskussion dieser These vgl. Kutschera (75), 2.3.3. 
jektivität" der Dingsprache wird damit nicht infrage gestellt. Ihre Prädikate charakterisieren Dinge - Physisches, nicht Psychisches. Und zur sprachlichen Bedeutung gehören auch nicht alle individuellen, sondern nur die allgemeinen, interpersonell gleichen Assoziationen mit Erlebnisweisen. Im normalen Sinn des Wortes ist aber das objektiiv, was nicht von individuellen Meinungen, Assoziationen, etc. abhängt.

Das Wort „,rot" war ein Beispiel für Wörter der physikalischen Sprache, für deren Anwendung es relativ direkte und zuverlässige subjektive (Erlebnis-)Kriterien gibt. Andere Wörter dieser Art sind „,heil", „laut", ,hart", ,schwer", ,,warm“, ,,rund“ etc., d. h. diejenigen Prädikate, die als Standardbeispiele von Beobachtungsprädikaten angeführt werden und von denen man sagen kann, es sei durch einfache und direkte Beobachtungen mit einer meist ausreichenden Sicherheit entscheidbar, ob sie auf einen Gegenstand zutreffen. Auf der Basis solcher Prädikate kann man dann weitere Terme der physikalischen Sprache, insbesondere auch theoretische Terme einführen. Für sie gibt es in der Regel keine direkten subjektiven Anwendungskriterien mehr, sondern nur indirekte, die sich aus dem Zusammenhang dieser Terme mit Beobachtungstermen aufgrund der Bedeutungspostulate und den subjektiven Kriterien für die Beobachtungsterme ergeben. Sie enthalten also praktisch keine spezifischen subjektiven Bedeutungskomponenten, haben keinen anschaulichen Gehalt. Das gilt insbesondere für die Terme der Sprache der Physik wie ,,magnetisch“ oder „Neutron“. Die Sprache der theoretischen Physik ist zunächst eine rein mathematische Sprache. Als solche ist sie aber keine Sprache über Empirisches. Sie wird dazu erst dadurch, daß wir die Größen wie Kraft, Masse, Feldstärke, etc., von denen sie redet, in Beziehung zu Beobachtbarem setzen. Nur so lassen sich physikalische Theorien auf empirische Phänomene anwenden. Dazu müssen aber die Ausdrücke für mathematische Funktionen als theoretische Terme durch Bedeutungspostulate mit Beobachtungstermen verknüpft werden. Und für diese Beobachtungsterme gelten unsere Aussagen über subjektive $\mathrm{Be}-$ deutungskomponenten, und sie gelten damit im Prinzip auch für die gesamte physikalische Sprache. ${ }^{42}$ Entscheidend für die Erlernbarkeit wie den Gebrauch dieser Sprache ist, daß sie Wörter wie „,rot" enthält, für die es relativ direkte Erlebniskriterien gibt.

${ }^{42} \mathrm{Vgl}$. dazu die Abschnitte 8.3 und 9.2. 
Die Sprache über Physisches ist also nicht unabhängig von jener über Psychisches. Wir haben oben betont, daß auch die Umkehrung gilt. Die Sprachen sind also wechselseitig aufeinander bezogen. Der Satz (1) charakterisiert nicht nur die Dingeigenschaft Rot durch Bezugnahme auf Rotempfindungen, sondern es gilt auch das Umgekehrte: Rotempfindungen charakterisieren wir als Empfindungen von $\operatorname{der}$ Art, wie wir sie normalerweise haben, wenn wir rote Dinge wahrnehmen. Man kann also weder behaupten, die Sprache über Physisches sei aus der Sprache über Psychisches abgeleitet oder überhaupt ableitbar, noch kann man das Umgekehrte sagen. Vielmehr stellt sich ihr Zusammenhang so dar: Die Gesamtsprache bildet sich - gewissermaßen onto- wie phylogenetisch - als eine Einheit, in der sich die Sprachen über Physisches und über Psychisches zunehmend von einander differenzieren. ${ }^{43}$ Beide Teilsprachen bleiben aber miteinander verbunden und auf einander bezogen: Wir charakterisieren Psychisches durch Physisches und umgekehrt. Physikalische wie psychologische Unterscheidungen bestimmen sich wechselseitig, wenn natürlich auch nicht jeder physikalischen Unterscheidung eine psychologische entspricht oder umgekehrt. Man kann nicht sagen, Unterscheidungen im Bereich des Physischen könnten nicht von Unterscheidungen im Bereich des I'sycnischen abhängen, weil diese wiederum von jenen abhängen. Man muß von einem Denken nach dem Schema einer Priorität - zuerst dies, dann damit jenes - abkommen und sehen, daß sich beide Sprachen simultan und im Zusammenhang miteinander entwikkeln; man muß den Prozeß als Entwicklung einer Sprache begreifen, die sich in nur relativ eigenständige Teilsprachen differenziert.

Wir können abschließend feststellen:

1. Die Thesen des Dualismus, die Behauptungen (1) und (2) des Abschnitts 7.1, lassen sich ohne nähere Präzisierung nicht sinnvoll diskutieren. Solche Präzisierungen fehlen in der Literatur aber weitgehend. 2. Bei einer Deutung dieser Thesen im Sinne von D 1 und D 2 sind beide unhaltbar: Es gibt keine ausreichenden Argumente für eine Übersetzbarkeit aller empirischen Aussagen in die Verbindung der Sprachen $\Phi$ und $\Sigma$, und die Behauptung, zwischen $\Sigma$ und $\Phi$ gebe es keine analytischen Beziehungen, ist falsch.

${ }^{43}$ Vgl. dazu den Abschnitt 8.2. 
3. Unsere Argumente widerlegen nicht nur die These D2, also die Bchauptung, jede konsistente Menge von $\Phi$-Säizen sei mit jeder konsistenten Menge von $\Sigma$-Sätzen verträglich, sondern auch die sehr viel schwächere These

D 2+: Es gibt kontingente $\Phi$-Sätze, die mit jeder konsistenten Menge von $\Sigma$-Sätzen verträglich sind, oder kontingente $\Sigma$ Sätze, die mit jeder konsistenten Menge von $\Phi$-Sätzen verträglich sind.

Für keine einzige physikalische Aussage läßt sich sagen, sie gelte völlig unabhängig davon, wie wir die Welt erleben, und für keine einzige psychologische Aussage, ihre Geltung sei ganz unabhängig davon, wie die physische Welt beschaffen ist. Das letztere ergibt sich daraus, daß man zwar nicht jedem psychischen Zustand einer Person ein bestimmtes Verhalten oder einen bestimmten körperlichen Zustand zuordnen kann, daß er aber jedenfalls nicht mit beliebigen Verhaltensweisen und körperlichen Zuständen zusammen auftreten kann. Das war für uns nicht eine empirische, sondern eine analytische Behauptung. Das erstere ergibt sich daraus, daß alle physikalischen Aussagen, sei es unmittelbar oder mittelbar, ihrem Sinn nach auf unser sinnliches Erleben Bezug nehmen; daß sie, wie wir gesagt haben, subjektive Bedeutungskomponenten enthalten. Auch diese sind nicht so direkt, daß wir jeder physikalischen Aussage A eine rein psychologische zuordnen könnten, die aus analytischen Gründen immer dann gelten muß, wenn A gilt; aber A ist jedenfalls nicht mit beliebigen psychologischen Gegebenheiten analytisch verträglich.

\subsection{Seele und Ich}

Wir haben im Abschnitt 7.1 von einer cartesischen Version des Du-alismus gesprochen, die insofern über die beiden dort formulierten Thesen D1 und D2 hinausgeht, als sie seelische Substanzen oder ",Seelen“ annimmt und physikalische und psychologische Aussagen als Aussagen über verschiedene Arten von Substanzen deutet. Da diese Konzeption die Behauptungen D1 und D2 impliziert, unterliegt sie der Kritik, die wir an diesen Thesen geübt haben. Wegen der philosophiegeschichtlichen Bedeutung dieser Konzeption wollen wir aber doch noch einmal auf ihre besondere Problematik zurückkommen. 
Wenn von „seelischen Substanzen“ die Rede ist, so stellt sich die Frage, ob - und wenn ja: wie - sich Körper und Seele als verschiedene Substanzen charakterisieren lassen. Wir haben nun schon in 7.2 gesehen, daß Descartes Argument für die Annahme eigenständiger seelischer Substanzen nicht haltbar ist. Für eine Unterscheidung Seele-Körper kann man nur vom Unterschied seelischer und körperlicher Eigenschaften von Personen ausgehen. Dieser Unterschied bietet aber keine Grundlage für eine Definition der Seelen durch Abstraktion. Man kann nicht sagen, die Seele einer Person sei die Menge ihrer seelischen Eigenschaften. Denn eine Menge von Eigenschaften ist keine Substanz. Ferner soll die Seele Träger der seelischen Eigenschaften sein; die Elemente einer Menge von Eigenschaften sind aber nicht Eigenschaften dieser Menge. Eine Menge von Eigenschaften kann nicht denken, fühlen oder etwas wollen, wie das für Seelen der Fall sein soll.

Man könnte versuchen, Seele und Körper implizit zu definieren, und sagen:

1. Ist F eine seelische Eigenschaft, so soll die Seele der Person a die Eigenschaft $\mathrm{F}$ genau dann haben, wenn a sie hat. Nicht-seelische Eigenschaften sollen für Seelen nicht erklärt sein. ${ }^{44}$

2. Ist F eine körperliche (physische) Eigenschaft, so soll der Köroer von a diese Eigenschaft genau dann haben, wenn a sie hat. Nichtkörperliche Eigenschaften sollen für Körper nicht definiert sein.

Man kann dann zwar sinnvolle Aussagen über Seelen und Körper machen, aber die Zahl der Entitäten hat man damit nicht vergrößert: Man kann nicht behaupten, die Seele der Person a sei von ihrem Körper verschieden, wie das der cartesische Dualismus annimmt. Nach dem Leibnizprinzip ist das Objekt b vom Objekt c genau dann verschieden, wenn es eine Eigenschaft F gibt, die b, aber nicht $c$ (im selben Zeitpunkt) zukommt, oder umgekehrt. ${ }^{45}$ Nun gibt es aber nach

${ }^{44}$ Man kann nicht sagen, alle Sätze, die Seelen nicht-seelische Eigenschaften zuschreiben, seien falsch, denn damit wäre das Prinzip vom ausgeschlossenen Widerspruch verletzt: $\mathrm{Da}$ eine Seele z. B. weder die Eigenschafi hat, weniger als $80 \mathrm{~kg} \mathrm{zu}$ wiegen, noch die Eigenschaft, mindestens $80 \mathrm{~kg}$ zu wiegen, hätte sie dann zugleich mindestens und höchstens dieses Gewicht.

${ }^{45}$ Dieses Prinzip kann natürlich nur dann als Identitätskriterium verwendet werden, wenn unter den Eigenschaften, von denen man ausgeht, nicht schon die Identität mit einem anderen Objekt enthalten ist. Leibniz ging wie Locke 
(1) und (2) keine Eigenschaften, die zugleich für Körper und Seelen defiritert sind, die man also der Seele von a zusprechen, dem Körper von a hingegen absprechen könnte, oder umgekehrt. Daher sind Identität und Verschiedenheit von Seelen und Körpern nicht erklärt. Würde man den Identitätsbegriff so erweitern, daß auch Objekte verschieden sind, für die nicht dieselben Eigenschaften erklärt sind, und daß Objekte genau dann identisch sind, wenn alle Eigenschaften für beide erklärt oder für beide nicht erklärt sind und sie in den erklärten Eigenschaften übereinstimmen, so könnten verschiedene Personen dieselbe Seele oder denselben Körper haben - wenn sie nämlich trotz körperlicher Verschiedenheit dieselben seelischen Eigenschaften haben, bzw. trotz seelischer Verschiedenheit dieselben körperlichen Merkmale. Im übrigen könnte man auf diese Weise beliebige Substanzen in mehrere andere zerlegen: Körper z. B. in Form- und in Materiesubstanzen, wobei die „Formsubstanz“ eines Körpers genau dessen Form- und seine „Materiesubstanz" genau seine stofflichen Eigenschaften hat. Man könnte insbesondere auch mehrere seelische Substanzen ansetzen, z. B. eine glaubende, eine wollende, und eine empfindende Seele. All das zeigt, daß der ganze Ansatz nur eine unnötige, unfruchtbare und irreführende Komplizierung der Aussagen über Personen als leib-seelische Einheiten bewirkt.

Locke geht in seiner Theorie der personalen Identitä $t^{46}$ nicht von der Identität als Übereinstimmung in allen Eigenschaften (zu allen Zeiten) aus, sondern von einer Gleichbeit bzgl. gewisser Eigenschaften, der Eigenschaften einer Menge $\mathcal{A}$, und legt fest, daß zwei Objekte bzgl. der Eigenschaften aus A gleich sind, wenn sie (zu allen Zeiten) in all diesen Eigenschaften übereinstimmen. Man kann z. B. sagen: ,Als Ehemänner sind sich Hans und Fritz gleich“, d. h. sie stimmen in allen für Ehemänner charakteristischen Eigenschaften (insbesondere in ihrem Verhalten zu ihren Frauen) überein. Man kann in diesem Sinn auch sagen: „Körperlich sind sich Hans und Fritz gleich, aber nicht geistig“. Daraus folgt natürlich nicht, daß der Körper von Fritz mit dem Körper von Hans identisch wäre, ihre Seelen aber ver-

(vgl. dazu 4.1) davon aus, daß alle Relationen durch Eigenschaften der Relationsglieder definierbar seien. Daher sind bei ihm Aussagen über alle Eigenschaften immer als Aussagen über alle undefinierten - insbesondere nicht mit Relationsbegriffen erklärten - Eigenschaften zu verstehen.

${ }^{46} \mathrm{Vgl}$. Locke (E), II, Kap. 27. 
schieden. Aus der Tatsache, daß a mit b bzgl. der Eigenschaften aus Agleich, bzgl. der Eigenschaften aus $2 B$ hingegen verschieden ist, folgt nicht, daß a und $b$ aus zwei Substanzen $a_{1}, a_{2}$, bzw. $b_{1}, b_{2}$ zusammengesetzt sind, so daß gilt: $a_{1}$ ist (im normalen $\operatorname{Sin} n$ ) mit $b_{1}$ identisch, und $a_{2}$ ist nicht identisch mit $b_{2}$. Locke unterscheidet Gleichheit als Mensch im Sinne körperlicher Gleichheit und Gleichheit als Person (als Selbst), die er durch die Gleichheit der Bewußtseinsinhalte bestimmt. Er betont aber auch, daß wir mit dem Wort „Ich“ - wir können auch sagen: mit Eigennamen oder Pronomina für Personen - gewöhnlich Körperliches wie Geistiges meinen, und daß personale Identität in seinem Sinn keine substantielle Identität beinhaltet. ${ }^{47}$ Locke sagt: „,To find wherein personal identity consists, we must consider what person stands for; which, I think, is a thinking intelligent being that has reason and reflection and can consider itself as itself, the same thinking thing in different times and places; which it does only by that consciousness which is inseparable from thinking and, as it seems to me, essential to it; it being impossible for anyone to perceive without perceiving that he does perceive... As far as this consciousness can be extended backwards to any past action or thought, so far reaches the identity of that person". ${ }^{48}$ Die Rede vom ,thinking thing" ist dabei allerdings - auch im Sirın Lockes - höchst miffverständiich, denn sie legt eine Deutung der Person als selbständige Substanz mit jenen Eigenschaften nahe, die für personale Identität erheblich sind.

Auch Lockes Bestimmung personaler Identität ist also kein geeigneter Ansatz zur Einführung von Seelen. Aber muß denn, wenn man schon nicht von "Seelen“" sprechen will, jedenfalls das erkennende, wollende, empfindende Subjekt als Träger psychischer Eigenschaften, Dispositionen und Akte nicht etwas Psychisches sein? Und muß ich als dieses Subjekt nicht von meinem Körper verschieden sein, da ich mir, nicht aber meinem Körper seelische Eigenschaften zuschreiben kann? Die Antwort ist: Das Subjekt muß sicher in dem Sinn etwas Psychisches sein, als es psychische Eigenschaften hat. Daraus folgt aber nicht, daß es etwas rein Psychisches wäre. Eine Eisenkugel hat

${ }^{47} \mathrm{Vgl}$. Locké(E), 287.

${ }^{48}$ Locke (E), 281. Am Lockeschen Kriterium personaler Identität haben schon J. Butler und Leibniz Kritik geübt. Vgl. dazu Leibniz (NE), Kap. 27, und Butlers ,Dissertation I: On Personal Identity“, (W)I. 
geometrische Eigenschaften; daraus folgt aber nicht, daß sie etwas rein Geometrisches ist - sie besteht auch aus Eisen, hat ein bestimmtes Gewicht, etc. Die Kugel ist ferner von dem Stoff verschieden, aus dem sie besteht, von ihrer Form wie ihrer Farbe. Aus Eisen zu bestehen, kugelförmig zu sein und schwarz zu sein sind Eigenschaften, die nätürlich ebenso untereinander wie von dem Gegenstand verschieden sind, auf den sie zutreffen. Aber das heißt nicht, daß dieser Gegenstand sich aus seinen Eigenschaften wie aus Teilen zusammensetzt. Eigenschaften sind nicht im gleichen Sinn Objekte wie die Eisenkugel. ${ }^{49}$

Es ist also keine Bestimmung von Seelen in Sicht, nach der man sie als von Körpern verschiedene Substanzen ansehen könnte. Im Abschnitt 7.2 sind wir auf Ryles Kritik am Dualismus eingegangen. Wir haben dort seine Erklärung seelischer Eigenschaften als körperliche Dispositionen abgelehnt. Zur Kritik am Dualismus - wenn auch nicht zur Begründung eines Behaviorismus - genügt es im Sinn unserer Ausführungen zu sagen: Aus der Tatsache, daß es seelische Eigenschaften und Dispositionen gibt, die sich von körperlichen unterscheiden lassen, folgt nicht, daß es eine vom Körper verschiedene Seele als Träger dieser Eigenschaften und Dispositionen gibt. Auch damit erweist sich die von Ryle angegriffene dualistische Vorstellung vom „Geist in der Maschine" als unhaltbar. ${ }^{50}$

${ }^{49} \mathrm{Als}$ „Objekt“ im weitesten Sinn dieses Wortes bezeichnet man alles, worüber man reden kann, also ebenso Gegenstände (Individuen) wie Attribute (Begriffe) und Sachverhalte (Propositionen). Statt von „Objekten“ in diesem umfassendsten Sinn reden wir meist von „Entitäten“. Gegenstände sind konkrete Dinge wie Tische ebenso wie Lebewesen, Personen und abstrakte Dinge wie Zahlen. Als „Objekte“ bezeichnet man oft auch Gegenstände. Will man aber Objekte von Subjekten abgrenzen, so wird das Wort in einem noch engeren Sinn verwendet; Objekte sind dann Gegenstände, die keine Subjekte sind. Das Wort „Objekt" hat also keine feste Bedeutung. Wir wollen hier auch nicht versuchen, es auf eine dieser Bedeutungen festzulegen. Was damit gemeint ist, wird jeweils gesagt, wo es sich nicht aus dem Kontext ergibt. Entsprechendes gilt für die Ausdrücke „Gegenstand“ und „Ding“. Werden Objekte Gegenständen, bzw. Entitäten gegenübergestellt, so sind diese Bezeichnungen im oben skizzierten Sinn zu verstehen.

${ }^{50} \mathrm{Zu}$ betonen ist aber, daß nicht nur der Geist in der Maschine, die körperlose Seele eine Fiktion ist, sondern auch die Maschine, der seelenlose Körper. Aussagen über den Körper eines Menschen sind nicht Aussagen über ein von 
Das Problem seelischer Substanzen ist nur ein Aspekt der Frage, wie sich der Begriff des Erkenntnissubjekts bestimmen läßt. Die Schwierigkeiten ergeben sich dabei erstens aus dem in der dualistischen und idealistischen Tradition immer wieder unternommenen Versuch, dieses Subjekt als etwas rein Psychisches zu bestimmen, und zweitens aus der merkwürdigen Verwandlung in einen Gegenstand, in eine Substanz, die das Subjekt im Verlaufe dieser Bestimmung erfährt. Über den ersten Punkt haben wir schon gesprochen. Den zweiten wollen wir am Beispiel des „Ich“ und des „Bewußtseins“ verdeutlichen, die vor allem von Hume bis Hegel eine so große Rolle in der Philosophie gespielt haben.

Eine Quelle der Vergegenständlichung von Subjekten zu Substanzen ist die unkritische Verwendung von Nominalisierungen. Im Deutschen können wir nicht-substantivische Wörter und Phrasen ebenso freizügig zu Substantiven machen wie im Griechischen. Wir können z. B. aus den Adjektiven ,rot", ,schön“ oder ,,wahr" die Substantive „die Röte“, „die Schönheit (das Schöne)", ,die Wahrheit (das Wahre)" bilden. Die Verwendung solcher Nomina ist jedoch nicht für alle Kontexte erklärt. Nicht jeder Satz, der grammatikalisch (syntaktisch) korrekt gebildet ist, ist auch sinnvoll. So ist z. B. der Satz „Prinzahlen lachen“ syntakuisch ikcrrekt, aber sinnlos. Säize wie „Das Rot dieser Rose ist blasser als das dieser Tulpe“ oder ,Es ist geboten, die Wahrheit zu sagen" haben einen hinreichend klaren Sinn. Für den Satz „,Die Wahrheit ist erkennbar" gilt das jedoch ohne nähere Erläuterungen nicht. Was wir erkennen, sind Sachverhalte. „,Die Wahrheit" als Menge aller wahren (bestehenden) Sachverhalte, oder als die Eigenschaft, wahr zu sein, ist kein Sachverhalt, also nicht erkennbar. Damit der Satz bedeutungsvoll wird, ist er also entweder im Sinn der generellen Aussage „Alle 'Wahrheiten', d. h. alle Tatsachen sind erkennbar" oder im Sinn der Existenzaussage „Einige Wahrhei-

diesem Menschen verschiedenes Objekt, sondern Aussagen über körperliche Eigenschaften dieses Menschen. Anerkennt man gegenüber dem Physikalismus eine Eigenständigkeit des Seelischen, bestreitet aber die Existenz von Seelen, so kann man auch nicht behaupten, Körper seien eigene Gegenstände. Denn da wir seelische Eigenschaften haben, diese aber nicht Eigenschaften des Körpers sind, wäre dann ein Träger dieser seelischen Eigenschaften anzunehmen, und da unsere körperlichen Eigenschaften als Eigenschaften des Körpers angesehen werden, wäre dieser Träger dann so etwas wie eine körperlose Seele. 
ten (Tatsachen) sind erkennbar" zu interpretieren, oder - wo auf eine bestimmte Frage Bezug genommen wird - im Sinn von , Es läßt sich erkennen, wie die richtige Antwort auf diese Frage lautet". Völlig sinnlos wäre eine Aussage wie „Die Röte ist die Wahrheit der Rose“.

Ein Satz, in dem die Nominalisierung eines Ausdrucks vorkommt, ist'sicher nicht nur dann sinnvoll, wenn er die Paraphrase eines Satzes darstellt, der diesen Ausdruck nur nichtnominalisiert enthält. Andernfalls müßte man auch die Sprachen höherer Logiksysteme als sinnlos erklären, in denen Attribute von Objekten wie Objekte höherer Stufe behandelt werden. Es ist aber jeweils zu prüfen, ob die Verwendung einer Nominalisierung im gegebenen Kontext erklärt ist. $\mathrm{Daß}$ die $\mathrm{Ob}-$ jektivierung von Eigenschaften auch in der Logik nicht unproblematisch ist, zeigen die logischen Antinomien. ${ }^{51}$

In philosophischen Texten kommen nun auch Nominalisierungen vor, die nicht nur in der normalen Sprache völlig ungebräuchlich und daher ohne neue semantische Festlegungen sinnlos sind, sondern mit deren Sinn und Funktion eine Nominalisierung gar nicht verträglich ist. Beispiele dafür sind „,das Nichts" und „das Ich“. Wenn mein Portemonnaie leer ist, so kann ich sagen „In meinem Portemonnaie ist nichts"; ich kann aber nicht sagen ,In meinem Portemonnaie ist das Nichts“. Denn der Ausdruck „,das Nichts“ würde nach den Regeln unserer Sprache einen Gegenstand bezeichnen, in meinem Portemonnaie befindet sich aber kein Gegenstand. „Nichts“ hat, logisch gesehen, nicht die Funktion eines Eigennamens, sondern die eines Quantors, mit dem ich im Beispiel ausdrücke, daß der Begriff ,in meinem

${ }^{51}$ Vgl. dazu z. B. Tarski's Antinomie der Erfüllungsrelation in (44), S. 82, Anm. 19. - Andere Beispiele falschen philosophischen Sprachgebrauchs sind manche Aussagen über den Willen und den Verstand. Die Rede vom Willen und vom Verstand ist in vielen Kontexten sicherlich sinnvoll. Statt „Fritz will Eva heiraten“ kann man auch sagen „Es ist der Wille von Fritz, Eva zu heiraten". Man kann den Willen insbesondere als Vermögen zu wollen oder zu wählen ansehen. Aber wenn in der philosophischen Literatur dann vom ,freien Willen“ die Rede ist, wobei der Wille selbst als frei angesehen wird, oder wenn gesagt wird, der Wille wolle etwas, so ist das unsinnig. Nicht mein Wille ist frei, sondern ich bin frei; nicht mein Wille will etwas, sondern ich selbst. Ebenso ist der Verstand das Vermögen zu denken, denkt aber nicht. Ein Magnèt hat das Vermögen, Eisenfeilspäne anzuziehen, aber nicht dieses Vermögen zieht sie an, sondern der Magnet. 
Portemonnaie befindlicher Gegenstand' auf kein Objekt zutrifft. ${ }^{52}$ Dieser logischen Funktion des Wortes ,nichts“ widerspricht seine Nominalisierung, die es zu einem Gegenstandsnamen macht. Entsprechend verhält es sich mit ,,dem Ich“. „Ich“ ist ein Personalpronomen, mit dem der jeweilige Sprecher sich selbst bezeichnet. Bei Aussagen über sich selbst verwendet der Sprecher das Pronomen ,,ich“, ebenso wie er für alle Aussagen über den Adressaten seiner Äußerung das Pronomen ,du“, bzw. die Höflichkeitsform „Sie“ gebraucht. Personennamen und Kennzeichnungen wie „Der so und so" werden nur für Dritte verwendet. ${ }^{53}$ Rede ich von ,,dem Ich“, so steht dieser Ausdruck nach den sprachlichen Regeln also für etwas vom Sprecher Verschiedenes. Sage ich statt „Ich lese“ „Das Ich liest“ oder „Mein Ich liest", so impliziere ich damit, es gäbe ein von mir (als Sprecher) verschiedenes Etwas, „mein Ich“, das liest. Dieser Satz ist daher unsinnig. Bei dieser Nominalisierung geht der Bezug des Wortes ,,ich“ auf den Sprecher verloren, und er erscheint somit gewissermaßen als verdoppelt. Im Blick auf die Funktion des Wortes ,,ich“ ist also die Nominalisierung ,,das Ich“ widersinnig. Gibt es ,,das Ich“, so auch ,die Iche“ („,mein Ich“, ,dein Ich“, ,,sein Ich“, usf.) - normalsprachlich unsinnige Bildungen.

Auch aus eirnem zweiterı Gruna' ist eine Norninalisierung des Fronomens ,,ich“ nicht sinnvoll. „Ich“ ist ein Indexausdruck, dessen Bezug vom Äußerungskontext abhängt und das den jeweiligen Sprecher bezeichnet. Andere Indexausdrücke sind ,,dieses“, ,,hier" und ,,jetzt". All diese Indexausdrücke lassen sich nicht sinnvoll nominalisieren, da „,das Ich“, ,,das Hier“, usw. nach den sprachlichen Regeln Namen für bestimmte Objekte sein müßten. Welche Folgen die Mißachtung dieser einfachen grammatikalischen Tatsache haben kann, zeigen Hegels Aussagen in der „Phänomenologie des Geistes“. Da er diese Indexausdrücke nominalisiert und sie dann auch prompt als Eigennamen mit einem festen, vom Äußerungskontext unabhängigen Bezug ansieht, stellt sich ihm die Frage: „Was ist das Dieses? Das Hier? Das Jetzt?", und er steht vor dem Problem, daß jeweils andere Dinge hier

52 Darauf hat schon Frege hingewiesen.

53 Weiß der Sprecher, daß der Hörer ihm ein Buch weggenommen hat und sagt zu ihm ,Ich werde den Dieb anzeigen, wenn er mir das Buch nicht bis morgen zurückgibt", so verbirgt er ihm dieses Wissen, indem er über den Dieb wie über einen Dritten redet. 
und jetzt vorhanden sind, daß also „das Hier" und ,das Jetzt“ Dinge von höchst beachtlicher Wandlungsfähigkeit sind: „Das Hier ist z. B. der Baum. Ich wende mich um, so ist diese Wahrheit verschwunden und hat sich in die entgegengesetzte verkehrt: Das Hier ist nicht ein Baum, sondern vielmehr ein Haus. Das Hier selbst verschwindet nicht, sondern es ist bleibend im Verschwinden des Hauses, Baumes usf. und gleichgültig, Haus, Baum zu sein“. ((W)III,85). So ergeben sich dann im weiteren Verlauf Aussagen wie „,Das Dieses ist also gesetzt als nicht dieses oder als aufgehoben, und damit nicht Nichts, sondern ein bestimmtes Nichts oder ein Nichts von einem Inhalte, nämlich dem Diesen“. ((W)III,94). „Auch die Tiere sind nicht von dieser Weisheit ausgeschlossen, sondern erweisen sich vielmehr, am tiefsten in sie eingeweiht zu sein; denn sie bleiben nicht vor den sinnlichen Dingen als an sich seienden stehen, sondern verzweifelnd an dieser Realität und in der völligen Gewißheit ihrer Nichtigkeit langen sie ohne weiteres zu und zehren sie auf; und die ganze Natur feiert wie sie diese offenbaren Mysterien, welche es lehren, was die Wahrheit der sinnlichen Dinge ist" ((W)III,91). Wenn irgendwelche Texte die Notwendigkeit sprachkritischer Untersuchungen belegen, so sind es die des deutschen Idealismus, dessen Systeme weithin Produkte eines völligen Mangels an logischem und grammatikalischem Unterscheidungsvermögen sind.

Die Rede von ,,dem Ich“ suggeriert nun eine Auffassung, nach der Subjekte Gegenstände sind, die wir ebenso - wenn auch mit anderen Prädikaten - beschreiben können wie physische Dinge. Schon Kant hat aber betont, daß das "Ich denke“ alle meine Vorstellungen begleiten können muß. ${ }^{54}$ Man kann das auch so ausdrücken: Alle Aussagen, die ich mache, sind nur dann korrekt, wenn sie mit der Tatsache verträglich sind, daß ich sie mache. Ich kann mich also z. B. nicht vollständig beschreiben, weil unter den Eigenschaften, die ich mir in einer Beschreibung zuspreche, jene fehlt, daß ich mir all diese Eigenschaften zuspreche. Dieser Gedanke, daß das urteilende Subjekt sich in seinen Urteilen nicht vollständig erfassen kann, sich selbst daher nicht in gleicher Weise zum Gegenstand werden kann wie z. B. physische Dinge, daß es sich nicht gewissermaßen von einem Standpunkt über sich betrachten kann, kam auch in unseren Argumenten gegen den Physikalismus und gegen den starken Determinismus im 6. Kapitel

${ }^{54} \mathrm{Vgl}$. Kant (KrV) B,131f. 
zum Ausdruck. Wenn jemand etwas über sich selbst aussagt, z. B. in dem Satz ,Ich schreibe", so ist durch das Pronomen ,,ich" die Identität der Person, über die er etwas aussagt, mit ihm selbst als Sprecher deutlich. Es ist z. B. klar, daß er nicht nur schreibt, sondern auch darüber spricht. Wenn man aber von ,dem Ich" redet, so geht dieser Rückbezug verloren, und damit wird der Annahme Vorschub geleistet, man könne über sich wie über andere Gegenstände sprechen. Darin liegt auch die Problematik der Annahme seelischer Substanzen.

Entsprechendes wie für „,das Ich“ gilt für die Bezeichnung eines Subjekts als „Bewußtsein“. Auch der Ausdruck ,das Bewußtsein“ ist eine Nominalisierung. Wir unterscheiden Tatsachen, die jemand bewußt sind, von solchen, die ihm nicht bewußt sind, und sagen, jemand sei sich seiner Schuld bewußt, oder es sei ihm nicht bewußt, in welcher Gefahr er sich befinde. Aussagen über das, was jemand bewußt ist, haben die Gestalt „Es ist der Person a bewußt, daß p“, wo p eine Tatsache ist, und wir verwenden solche Sätze im Sinn von ,a weiß, daß $\mathrm{p}$ ". Das Bewußtsein einer Person ist dann ihr Wissen, also die Menge der Sachverhalte, die sie weiß. Neben dieser prädikativen Verwendung von ,,bewußt“ gibt es auch adverbiale Verwendungen. Wir sagen z. B., jemand habe bewußt die Unwahrheit gesagt, er erlebe etwas bexußt ocier er formuliere eine Aussage bewußt. „Berwißi" heißt hier soviel wie ,,absichtlich“, ,,deutlich“, ,,überlegt“" oder ,,konzentriert". In diesem Sinn meinen wir mit „Bewußtsein" nicht die Tatsachen, die uns bewußt sind, sondern den Zustand, in dem wir selbst uns befinden, wenn uns etwas bewußt ist, den besonderen Grad der Bewußtheit, oder die Fähigkeit des Erlebens oder Erkennens, des absichtlichen Handelns etc. Fehlt jemand diese Fähigkeit, so sagen wir auch, er sei nicht bei (vollem) Bewußtsein. In beiden Bedeutungen dieses Wortes kann man aber nicht sagen, jemand sei ein (oder mein) Bewußtsein. Ich bin offenbar weder die Menge der mir bekannten Tatsachen, noch die (oder meine) Fähigkeit zu erleben oder zu handeln. Daher ist es auch sinnlos, jemand als „Bewußtsein“ zu bezeichnen. Ich babe Bewußtsein, aber ich bin es nicht; nicht das Bewußtsein erlebt und handelt, sondern ich erlebe und handle bewußt.

Der erste, der sich gegen eine Auffassung der Seele als Substanz wandte, war Kant. Nach ihm ist ,Substanz' eine Verstandeskategorie und kann daher nur auf Gegenstände der Erfahrung, nicht aber auf „,das Ich“ als Noumenon angewendet werden. Radikaler war die Kri- 
tik Fichtes, der die Fähigkeit des Menschen zur Selbstbestimmung betonte, durch die er sich von allen Substanzen unterscheidet. Und Hegel hat gesehen, daß die Substanzvorstellung der Seele oder ",des Ich“ der Grundfehler von Dualismus und Materialismus ist. Bei allen drei Autoren treten jedoch neue Konfusionen an die Stelle der alten. Die Abschnitte der „,Kritik der reinen Vernunft“, in denen Kant auf „,das Ich“, oder besser die verschiedenen ,Iche“ eingeht ${ }^{55}$ - er unterscheidet deren nicht weniger als vier: die reine Apperzeption, das empirische Ich, das Ich als Noumenon und das Gemüt als Träger der verschiedenen Vermögen, wobei das Verhältnis dieser Iche weitgehend unklar bleibt - , zählen nicht nur zu den schwierigsten Teilen dieses Buches, wie man meist sagt, sondern - abgesehen von dem oben erwähnten Hinweis auf die Rolle des „Ich denke“ (der reinen Apperzeption) - auch zu den schwächsten. Die Umarbeitung dieser Partien in der 2. Auflage zeigen deutlich, daß auch Kant selbst damit nicht zufrieden war. Nach Fichte können wir uns durch die Reflexion auf uns selbst zur Idee des ,,reinen Ich“ erheben. Wir können uns unserer selbst bewußt werden. Wenn wir dabei nun von all dem abstrahieren, was nicht zu diesem Selbstbewußtsein gehört, von unseren sonstigen Eigenschaften und Tätigkeiten, so ergibt sich das ,,reine Ich", dessen Wesen und Sein allein darin besteht, sich selbst Gegenstand zu sein, sich selbst zu ,setzen“, wie Fichte sagt. Diese Abstraktion liefert aber kein „Ich", sondern einen Begriff. Wenn ich von den besonderen Eigenschaften eines Pferdes (seiner Farbe, seinem Alter, etc.) abstrahiere, so erhalte ich nicht ein ,,reines Pferd“, sondern den Begriff ,Pferd', und dieser Begriff ist kein Pferd, denn er hat nicht vier Beine. Ebenso erhalte ich durch Abstraktion aus dem Sachverhalt, $\mathrm{da}$ jemand sich seiner selbst bewußt ist, nicht ein reines Ich, sondern den Begriff, sich seiner selbst bewußt sein'. Und dieser Begriff ist kein Ich, weil Begriffe weder denken noch handeln können und sich ihrer selbst nicht bewußt sind. ${ }^{56}$ Wenn also Fichte beklagt, daß die ,,derzeiti-

${ }^{55}$ Vgl. die "Transzendentale Deduktion der reinen Verstandesbegriffe" und die „Paralogismen der reinen Vernunft".

56 Letzteres erkannte auch Fichte an. Vgl. zu dieser Einführung des ,reinen Ich“ z. B. (W)II,364f. - Wie wir oben gesehen haben, drückt ,sich bewußt sein" wie ,wissen" eine Beziehung zwischen Personen und Sachverhalten aus. Ich bin mir also nicht irgendwelcher Objekte bewußt, z. B. der Wand vor mir, sondern nur der Tatsache, daß da eine Wand ist, daß sie weiß ist, 
gen Philosophen" dieses reine Ich ,unendlich spaßhaft gefunden" hätten, so ist das nur allzu verständlich. ${ }^{57}$

Mit dem Begriff des Erkenntnissubjekts verbinden sich also eine Fülle von Konfusionen. Wir vermeiden sie, indem wir unter ,,Subjekten" einfach Personen, Menschen im normalen Sinn dieses Wortes verstehen, nicht irgendwelche rein seelischen oder geistigen Entitäten. ${ }^{58}$ Subjekte haben also seelische wie körperliche Eigenschaften, bestehen aber nicht aus Seele und Körper wie aus Teilen. Subjekte sind auch nichts anderes als wir selbst, und daher sind Aussagen über sie am besten als Aussagen über uns, als Sätze mit Pronomina der 1. Person zu formulieren.

oder ähnliches. Daher ist auch ein Selbstbewußtsein durch Aussagen auszudrücken wie ,,Die Person a ist sich bewußt, daß sie das und das tut (die oder jene Eigenschaft hat)". Die Abstraktion ergibt also sogar einen zweistelligen Begriff, $\mathrm{x}$ ist sich bewußt, das $\mathrm{x}$ die Eigenschaft $\mathrm{F}$ hat'. Nach Fichte kann man jedoch Objekte denken oder wissen. Er fordert den Leser auf „Wisse die Wand! Nun wisse dich selbst!“ (vgl. (WLO1),93).

${ }^{57}$ Fichte (W)II,365.

${ }^{58}$ Ist von ,,dem Subjekt" die Rede, so bezeichnet das Wort nicht ein ,,allgemeines Subjekt", sondern der Artikel ist im Sinn einer Generalisierung zu verstehen, wie ,der Löwe“ in dem Satz „Der Löwe ist ein Säugetier“. 


\section{Subjekt und Objekt}

\subsection{Das polare Verhältnis von Psychischem und Physischem}

In den Kapiteln 4 bis 7 haben wir die wichtigsten Theorien über das Verhältnis von Physischem und Psychischem besprochen: Idealismus (insbesondere Phänomenalismus), Physikalismus und Dualismus. Wir sind dabei zu dem Ergebnis gekommen, daß alle drei Positionen nicht haltbar sind, stehen also nun vor der Aufgabe, eine eigene Alternative zu entwickeln. Durch unsere Kritik an den drei genannten Theorien ist diese Alternative aber schon vorgezeichnet, so daß wir sie im folgenden nur mehr zusammenfassen und ergänzen müssen.

Die wichtigsten Ergebnisse unserer Kritik waren:

I) Psychische Phänomene lassen sich weder auf physische reduzieren, noch physische auf psychische.

Bei der Diskussion des Phänomenalismus haben wir gezeigt, daß sich die physikalische Sprache nicht in eine Sprache über Psychisches übersetzen läßt, und bei der Diskussion des Physikalismus haben wir gesehen, daß auch eine umgekehrte Übersetzung nicht möglich ist. Psychisches und Physisches bilden also eigenständige Bereiche.

II) Psychisches und Pbysisches sind keine von einander unabbängigen Bereiche.

Bei der Diskussion des Dualismus haben wir gezeigt, daß es Bedeutungszusammenhänge zwischen Aussagen über Psychisches und solchen über Physisches gibt, daß sich der Sinn von Termen für rein Psychisches nicht vollständig ohne Bezugnahme auf Physisches charakterisieren läßt und umgekehrt. Aus diesen analytischen Beziehungen ergab sich, daß nicht beliebige psychologische Tatsachen mit beliebigen physikalischen koexistieren können. Wenn man also nach I von einer Eigenständigkeit beider Bereiche reden kann, so nach II nur von einer relativen Eigenständigkeit. Wir haben das im Abschnitt 7.4 so ausgedrückt: Die Sprache über Physisches und die über Psychisches bilden relativ selbständige, auf einander nicht reduzierbare Teilsprachen der Gesamtsprache, zwischen denen aber vielfältige Bedeutungs- 
beziehungen bestehen. Dem entspricht die ontologische Aussage: Es gibt nur eine Realität; Psychisches und Physisches sind Teilbereiche dieser Realität, die aufeinander bezogen und miteinander verbunden, also nicht voneinander isolierbar sind.

Im Sinn des alltäglichen, vorwissenschaftlichen Sprachgebrauchs bilden die Begriffe ,Physisch' und ,Psychisch“ ein Gegensatzpaar: Die Bezeichnung eines Phänomens als ,psychisch“ besagt, daß wir es nicht als physisches Phänomen ansehen, und umgekehrt. Beide Begriffe sind nicht rein klassifikatorisch. Die Phänomene zerfallen nicht eindeutig in physische und psychische, sondern sie haben vielfach sowohl physische als auch psychische Aspekte. Beide Begriffe werden auch komparativ verwendet. So sind Überzeugungen etwas in einem engeren Sinn Psychisches als charakterliche Dispositionen; diese sind wieder in einem engeren Sinn psychisch als Handlungen, usf. Wenn wir etwas nicht als ", in einem engeren Sinn" oder "typischer" psychisch als etwas anderes bezeichnen, sondern als "psychisch“ schlechthin, so ist damit gemeint: eher psychisch als physisch. Es handelt sich also um gegensätzliche Typenbegriffe. Für Typenbegriffe ist es charakteristisch, daß sie sowohl klassifikatorisch wie komparativ verwendet werden. ${ }^{1}$ Typenbegriffe sind auch dann sinnvoll, wenn es reine Typen nicht gibt. So sind z. B. die Körperbautypen des Pyinnikers und des Leptosomen ebenso wie die von E. Kretschmer damit in Zusammenhang gebrachten Charaktertypen des Zyklothymen und des Schizothymen ${ }^{2}$ auch dann brauchbar, wenn man zugesteht, daß es den reinen Pykniker oder Leptosomen, bzw. den rein Zyklothymen oder Schizothymen nicht gibt, sondern daß jeder Pykniker, bzw. Zyklothyme auch Züge des Leptosomen, bzw. Schizothymen aufweist, und umgekehrt, so daß man nur von mehr oder weniger typischen Fällen von Pyknikern etc. sprechen kann.

Ein so verstandenes Verhältnis von Physischem und Psychischem kann man polar nennen. Unter „Polarität“ versteht man allgemein ein „,Verhältnis der Gegensätzlichkeit zwischen zwei voneinander abhängigen, sich gegenseitig bedingenden Momenten oder Prinzipien" ". In

${ }^{1}$ Vgl. dazu Kutschera (75), 2.4.7.

${ }^{2}$ Vgl. dazu Kretschmer (44).

${ }^{3}$ So der „Große Brockhaus“, Bd. 15, Wiesbaden 1972. - Ein oft gebrauchtes Beispiel für ein polares Verhältnis ist das von Licht und Finsternis. Helligkeit und Dunkelheit bilden ein Gegensatzpaar, bei dem sich jedes Glied durch die 
einem polaren Verhältnis stehen auch die Begriffe ,subjektiv' und ,objektiv". Als ,subjektiv" bezeichnet man alles, was in eirem Subjekt (einer Person als Individuum) begründet ist, seine Eigenart charakterisiert oder was von individuellen Faktoren (Meinungen, Entschlüsse, Persönlichkeit) abhängig ist, ${ }^{4}$ als , objektiv“ hingegen alles, was nicht subjektiv ist. Auch diese Begriffe bilden ein Gegensatzpaar von Typenbegriffen; sie lassen komparative Abstufungen zu und sind aufeinander bezogen. ${ }^{5}$

Wir haben bisher meist nur über das Verhältnis von rein Psychischem (im Sinn von 7.1) und rein Physischem gesprochen, und diese Begriffe kann man als rein klassifikatorisch ansehen. Im 7. Kapitel haben wir aber nicht nur an der dualistischen These von der absoluten

Gegenüberstellung zum anderen bestimmt und in dem alle Zustände $\mathrm{Zu}$ stände mehr oder minder großer Helligkeit wie Dunkelheit sind. Dieses Bild gebraucht Fichte, um das Verhältnis von Subjekt (Ich) und Objekt (NichtIch) zu charakterisieren: „Licht und Finsternis sind überhaupt nicht entgegengesetzt, sondern nur den Graden nach unterschieden. Finsternis ist bloß eine sehr geringe Quantität Licht. - Gerade so verhält es sich zwischen dem Ich und dem Nicht-Ich". ((W)I,301.)

${ }^{4} \mathrm{Im}$ abwertenden Sinn heißt dagegen „subjektiv“ soviel wie ,,voreingenommen", ,,von rein persönlichen Wertungen oder Meinungen abhängig", ,willkürlich".

${ }^{5}$ Hier lassen sich auch verschiedene Aspekte unterscheiden, unter denen man etwas (mehr oder minder) ,subjektiv“, bzw. „,objektiv“ nennen kann. Es gibt verschiedene subjektive Faktoren, z. B. Überzeugungen, charakterliche Dispositionen, Präferenzen, von denen etwas abhängen kann, und verschiedene Weisen, wie es davon abhängen kann. Empfindungen sind z. B. subjektiv als Zustände des Subjekts, von denen es introspektiv entscheiden kann, ob sie bestehen oder nicht. Empfindungen stehen aber nicht in der freien Verfügung des Subjekts; es kann nicht bestimmen, ob es z. B. Schmerzen hat oder nicht. Doxastisch gesehen sind also Empfindungen subjektiver als Handlungen wir haben ja schon früher betont, daß Handlungsweisen oft durch Erfolgsverben ausgedrückt werden; über den Erfolg unserer Handlungen können wir uns aber täuschen. Unter dem Aspekt der Determinierbarkeit sind dagegen Handlungen, die in unserer Kontrolle stehen, subjektiver als Empfindungen. Verschiedene solche Aspekte können in der Bewertung von Phänomenen zusammenkommen. Ebenso wie Farbähnlichkeit ein mehrdimensionaler komparativer Begriff ist, da wir Ähnlichkeiten nach Farbton, Sättigung und Helligkeit unterscheiden können, sind also ,subjektiv' und ,objektiv' mehrdimensionale Begriffe. 
Eigenständigkeit dieser beiden Bereiche Kritik geübt, sondern auch an der These, daß sich alle empirischen Phänomene in rein psychische und rein physikalische Komponenten zerlegen lassen. ${ }^{6}$ Hat man sich einmal von der wechselseitigen Bezogenheit von Psychischem und Physischem überzeugt, so sieht man leichter, daß sich in vielen Erscheinungen beides unauflöslich verbindet. Wir haben schon betont, daß Personen als körperlich-seelische Einheiten zu verstehen sind. Charakterliche Dispositionen, Geschicklichkeit, Willenskraft und Persönlichkeit lassen sich weder rein psychologisch noch rein physikalisch beschreiben. Sie zerfallen auch nicht in rein psychologische und rein physikalische Komponenten, deren gewissermaßen zufälliges Zusammentreffen die fraglichen Phänomene ergibt. Willenskraft z. B. - etwas, wie wir sagen würden, Psychisches - realisiert sich nur in einem konsequenten Verfolgen gesetzter Ziele, das auch äußere Widerstände überwindet, und drückt sich in diesem Verhalten aus. Es ist sinnlos, von einem starken Willen zu reden, der sich nicht in der Praxis zeigt. Willenskraft besteht so nicht in einer rein psychischen Eigenschaft, die sich mit gewissen Verhaltensweisen verbindet; es gibt keine solche Eigenschaft, die auch ohne die Verhaltenskomponente bestehen könnte. Willenskraft ist vom Begriff her auch eine Verhaltensdisposition. Das Psychische läßt sich hier nur unter Einschluß der Verhaltensseite fassen. Umgekehrt läßt sich aber auch die Verhaltensweise nur unter Einschluß von Psychischem zureichend bestimmen: ohne Kenntnis der Ziele und Überzeugungen des Handelnden kann man eine Handlung nicht als zielstrebig bezeichnen. Zur Persönlichkeit gehört ebenso das Seelische wie das Körperliche und beides ist nicht unabhängig voneinander so, wie es ist, sondern der seelische Charakter drückt sich im Körperlichen aus, in Haltung, Bewegung, Miene, Gestik, Sprache, Verhaltensweise und realisiert sich darin. Und umgekehrt reflektieren sich körperliche Eigenart und körperliche Vorgänge in seelischen Zuständen und im Erleben. Der menschliche Leib ist nicht etwas bloß Physisches, sondern auch Ausdruck des Seelischen. Er ist, wie Hegel sagt, ,,Organ der Seele“, d. h. Seelisches realisiert und bestimmt sich im körperlichen Verhalten und Habitus. Seelische Haltungen und Vorgänge werden darin nicht nur anschaulich und erfahrbar, sondern sie vollziehen sich zugleich leiblich. Im

${ }^{6}$ Vgl. dazu die These D 1 in 7.1 
Sprechen wird nicht ein Gedanke in Töne übersetzt, sondern er formiert sich sprachlich. Im Handeln wird nicht ein Willensentschluß durch eine körperliche Bewegung ausgeführt, sondern er vollzieht sich im Handeln - wenn er nicht ein Plan für spätere Handlungen ist.' Beim Sehen werden nicht physikalische Reize in Empfindungen übersetzt, sondern der Vorgang ist von Anfang an eine zielstrebige Aktivität. Eine Gefühlsregung wie Zorn findet nicht nur in ,,der Seele" statt, sondern auch im Körper: der Blutdruck steigt, Miene und Haltung ändern sich, usw. Und viele körperliche Vorgänge finden auch mehr oder minder bewußt in ,der Seele“ statt. Wenn man angesichts dieser Verbindungen von einem „Ausdruck“ des Seelischen im Körperlichen und einer „Internalisierung" des Körperlichen im Seelischen spricht, so sind das freilich nur sehr ungenaue Redeweisen. ${ }^{8}$ Nicht alles Seelische drückt sich direkt in Körperlichem aus. Nicht in allen Handlungen zeigt sich die sie leitende Absicht; Absichten können auch verborgen werden, wir können uns verstellen. Es lassen sich auch nicht alle körperlichen Vorgänge, die sich mit seelischen verbinden, als "Ausdruck" des Seelischen bezeichnen. So sind z. B. physiologische Vorgänge im Gehirn kein „Ausdruck“" seelischer Vorgänge. Ebenso wird nicht alles körperliche Geschehen erlebnismäßig internalisiert. Eine genauere Analyse der Zusammenhänge zwischen körperlichen und seelischen Vorgängen ist Aufgabe der Empirie, und es ist nicht sehr sinnvoll, eine Terminologie zur Beschreibung von Phänomenen einzuführen, bevor man diese im einzelnen übersieht.

7 Nach dem Reafferenzprinzip steuern wir unser Handeln aufgrund der Rückmeldungen, die wir im Verlauf des Handelns erhalten. Wie wir z. B. einen Gegenstand richtig ergreifen, zeigt sich erst, wenn wir ihn anfassen, seine Konsistenz, seinen Schwerpunkt fühlen, usf.

${ }^{8}$ Hegel verwendet statt „Internalisierung" die Bezeichnung ,Symbolisierung“" (vgl. (W)V,108f). Er geht dabei davon aus, daß Körperliches im Erleben für das Subjekt bedeutungsvoll wird, und nennt den Prozeß der Bedeutungsverleihung allgemein „Symbolisierung“. Aber auch dieses Wort hat einen sehr unspezifischen Sinn, und zudem ist es hier insofern schief, als nicht das Erleben Symbol des Körperlichen ist, sondern Körperliches Symbol für Geistiges. Es ist aber eine der wichtigsten Leistungen Hegels, daß er die Einheit von Physischem und Psychischem in ihrer wechselseitigen Bezogenheit gegen den Dualismus geltend gemacht und damit viele Phänomene einem besseren Verständnis erschlossen hat. 
Hier ging es nur darum, plausibel zu machen, daß man Phänomene der Art, wie wir sie oben besprochen haben, besser versteht, wenn man von der Einheit von Seelischem und Körperlichem ausgeht nicht von einer Einheit natürlich, innerhalb derer sich nichts mehr unterscheiden läßt, sondern von einer in sich differenzierten Einheit - , als wenn man im Sinn des Dualismus getrennte Bereiche des Seelischen und Körperlichen voraussetzt. Dem entspricht auch die übliche Bestimmung des Psychischen, die, wie wir in 7.1 gesehen haben, nicht nur das rein Psychische umfaßt.

Damit wird noch einmal das Ergebnis unterstrichen, das wir schon im Abschnitt 7.2 gewonnen hatten, daß sich nämlich die Gesamtsprache $\Omega$ nicht in die Verbindung der dualistischen Teilsprachen $\Phi$ und $\Sigma$ übersetzen läßt. Wir halten also fest:

III) Die empirischen Phänomene lassen sich nicht so analysieren, daß sie sämtlich aus rein psychologischen und rein physikalischen Komponenten besteben.

Damit stellt sich auch für uns das Verhältnis von Physischem und Psychischem als ein polares Verhältnis dar. Es gibt nicht nur das rein Physische und das rein Psychische und außerdem Phänomene, die sich in solchen Komponenten analysieren lassen, sondern es gibt Übergänge, d. h. Erscheinungen, in denen sich Physisches unauflöslich mit Psychischem verbindet.

Den aus der Kritik an Phänomenalismus, Physikalismus und Dualismus gewonnenen Aussagen I bis III woller, wir noch eine vierte hinzufügen, die bereits im Abschnitt 7.4 angeklungen ist:

IV) Die Unterscheidung physischer und psychischer Phänomene ist nicht allein durch die Natur der Sache vorgegeben, sondern auch ein Produkt der Interpretation der Erfabrung und damit diachronischen Veränderungen unterworfen.

$\mathrm{Daß}$ die alltägliche, vorwissenschaftliche Unterscheidung PhysischPsychisch in verschiedenen Theorien unterschiedlich rekonstruiert wird, ist nichts Neues - Beispiele dafür haben wir ja schon kennengelernt. Weniger geläufig ist hingegen, daß auch diese alltägliche Unterscheidung Produkt einer historischen Entwicklung ist, in der sich eine zunehmende Differenzierung beider Bereiche vollzogen hat. Da diese Tatsache unsere Behauptung stützt, Physisches und Psychisches seien keine autonomen Bereiche, sondern eng aufeinander bezogen, soll sie im nächsten Abschnitt durch einige ethnologische und ent- 
wicklungspsychologische Hinweise belegt werden. Auch ein Blick auf die Geschichte der Sprache zeigt, daß diese sich als Einhieit entwickelt hat und daß sich dabei die Sprache über Psychisches und jene über Physisches erst allmählich als relativ selbständige Teilsprachen herausdifferenziert haben. Darauf haben wir im Abschnitt 7.4 hingewiesen.

Mit den Aussagen I bis IV ist nun die Konzeption vom Verhältnis von Physischem und Psychischem umrissen, die wir hier vertreten und im folgenden näher erläutern und begründen.

\subsection{Die Differenzierung von Physischem und Psy- chischem}

Ein Zugang zum besseren Verstärıdnis des polaren Verhältnisses von Psychischem und Physischem eröffnet sich mit der Einsicht, daß unsere vom Dualismus beeinflußte Deutung dieses Verhältnisses nicht so selbstverständlich oder naturgegeben ist, wie sie uns aufgrund langer Gewöhnung erscheint. Das wollen wir durch einige kurze Hinweise auf Ergebnisse der Ethnologie und der Sprachgeschichte deutlich machen. Diese Hinweise können systematische Argumente natürlich nicht ersetzen. Unsere zentrale Frage ist, welches Verhältnis zwischen Psychischem und Physischem besteht, und für diese Frage ist es unerheblich, wie die heute gängige Unterscheidung entstanden ist.

Wenn im folgenden von ,primitiven“ Vorstellungen und Kulturen die Rede ist, so soll damit nicht, wie besonders in älteren ethnologischen Arbeiten, z. B. denen von L. Levy-Bruhl, vorausgesetzt werden, $\mathrm{da}$ ß diese Kulturen überwundene Entwicklungsstufen auf dem Weg zu unseren eigenen seien. Unter diesem Aspekt bekäme man kaum mehr zu Gesicht als eine Sammlung kurioser Vorstellungen und Verhaitensweisen. Das Interesse der Ethnologie besteht vielmehr vor allem darin, daß sie die Eigenart anderer Vorstellungswelten im Sinn von Alternativen zu unserer eigenen aufzeigt. „Primitiv“ versteht sich also einfach als kurze Bezeichnung für das, was entwicklungsmäßig früher ist. Neben Ergebnissen der Ethnologie verwenden wir im folgenden auch solche der Entwicklungspsychologie in dem weiteren Sinn, in dem sie sich nicht nur mit der Entwicklung der einzelnen Persönlichkeit befaßt, sondern auch mit stammesgeschichtlichen Entwicklungen, wie das eine Vielzahl paralleler Tatbestände auf diesen 
Gebieten nahelegt. Eine gute Darstellung der Grundzüge der Entwicklungspsychologie in diesem Sinn hat $\mathrm{H}$. Werner in (59) gegeben.

Für primitive Stufen des Erlebens und der Erfahrung sind unter anderem folgende Bestimmungen charakteristisch:

1. Die Polarisierung des Erlebens ist wenig ausgeprägt

Das Erleben stellt sich viel weniger als für uns dar als Erleben eines Subjekts von Gegenständlichem, von Dingen oder anderen Personen, sondern mehr als eine umgreifende und nach subjektiven und objektiven Momenten wenig differenzierte Zuständlichkeit. Gefühle und Strebungen des Subjekts werden von gegenständlichen Sachverhalten nicht scharf unterschieden. „Der Gegensatz“, sagt Werner, ,,zwischen dem Ich und der Welt der Gegenstände ist umso weniger scharf ausgeprägt, in je primitivere Zonen der Naturmenschheit man vordringt." Und ,... gerade darin besteht die besondere Eigenart primitiver Organismen, daß sich das seelische Leben in Gesamtverhaltungsweisen vollzieht, die eine verhältnismäßig geringe Differenziertheit von Objekt und Subjekt, von Gegenstand und Zustand, von Wahrnehmung, Gefühl und handelnder Bewegung bekunden."9

Die Grenzen zwischen Subjektivem und Objektivem sind fließend: Wahrnehmung und Vorstellung, Gefühl und Realität, Traum und Wirklichkeit verfließen ineinander. Das in der Wahrnehmung Gegebene wird unter dem Einfluß affektiver Strebungen bestimmt: „Der Naturmensch bedarf, besonders unter dem Einfluß affektiver Strebungen, nicht der objektiv-vollständigen Merkmale einer sachlich auf̈gebauten Gegenständlichkeit, ihm genügen oft Fragmente des AuBenweltlichen, um daraus eine illusionäre Ganzheit zu formen." Das Gegenständliche wird ,durch affektive Bewertung gewissermaßen ausgestaltet; man kann hier direkt von einer affektiven Perspektive sprechen". ${ }^{10}$ In diesem Zusammenhang ist das Phänomen der Visionen interessant. Werner betont, ,daß der Naturmensch in anscheinend viel weitergehenden $\mathrm{Maße}$ als der Kulturmensch dem $Z$ wange von Visionen, die entweder frei steigen, oder an undeutlich gesehene Gegenstände, wie etwa Baumstämme, Felsen usw. geknüpft sind, ausgesetzt ist. Sicher spielen hier affektive Momente eine sehr wesentliche Rolle. Aber diese Visionen wären in ihrem sinnhaften Charakter

${ }^{9}$ Werner (59), 317 und 38.

10 Werner (59), 105 und 107. Ein ähnlicher Tatbestand bildet die Grundlage des projektiven Tests. 
unmöglich, würde nicht die objektive, die wahrnehmungsmäßige, und die subjektive, die Vorstellungswelt eine solche geringe Differenziertheit besitzen, daß sie eben ein visionäres Anschauungsbild ermöglichten. Wir finden überall, daß visionäre Erscheinungen in primitiven Gesellschaften nicht nur als objektiv gewertet werden, sondern daß ihnen sogar eine höhere Wirklichkeit zugeschrieben wird".i1 Entsprechendes gilt für die Durchdringung von Traum- und Wirklichkeitssphäre, von Spiel und Realität. So schreibt G. W. Allport: „It is only in the most highly developed individuals that one finds a sharpening of the polarity between subject and world. Between the primitive or the child and his environment there exists a raw, syncretic relation illustrated by fusions between dreams and imagery and perceptions of outer reality".12 Auch die Vorstellungen von der magischen Kraft von Bildern, Wörtern und Handlungen lassen sich aufgrund dieser engen Verknüpfung zwischen subjektiver und gegenständlicher Sphäre besser verstehen. ${ }^{13}$

Die Bedeutungen, welche die Dinge für den Menschen haben, werden ihnen als Eigenschaften oder Aktionen zugeschrieben. Sie werden emotional und affektiv ausgestaltet und sind so bedrohlich, heiter, traurig, usw. Sie werden physiognomisch erlebt, nicht als Sachen, sondern als beseelt. „In physiognomic perception which has often been described as typical of a primitive mentality, there is no differentiation between the objective qualities of the objects and the emotion they arouse in the person. Objects appear not as large or small, having this or that color or shape, but as powerful, weak, harmonious, threatening etc. "14

\section{Psychisches und Körperliches sind nur wenig differenziert.}

Seelisches und Körperliches werden als Einheit gesehen, seelische und körperliche Eigenschaften nicht unterschieden. Mut und Kraft, Trauer und Müdigkeit bilden ein Phänomen. Man kann sich von Schuld durch Waschungen oder Schwitzbäder reinigen, und diese Handlungen haben dabei nicht nur symbolische Bedeutung, sondern eine direkte moralische Wirkung. Geistige Eigenschaften lassen sich

\footnotetext{
11 Werner (59), 103.

12 Im Vorwort zu Werner (48), S. XI.

${ }^{13} \mathrm{Vgl}$. dazu z. B. Lévi-Strauss (58), $183 \mathrm{ff}$.

${ }^{14} \mathrm{E}$. Hanfmann und J. Kasanin: Conceptual thinking in schizophrenia, Nerv. Ment. Dis. Monogr. 1942,67. Vgl. dazu auch Werner (59), 271.
} 
durch Berührung übertragen. ,Durch die Berührung fließt nach dem Glauben, auf dem dieser Zauber beruht, die Eigenschaften sozusagen über: Der Papua reibt sich Rücken und Glieder an einem Felsen, um stark und widerstandsfähig wie dieser Felsen zu werden ".15 Gefühle und Empfindungen sind sehr viel stärker an Verhaltensweisen geknüpft als bei uns. ${ }^{16}$ Das Seelische wird auch nicht als autonomer Bereich verstanden. Seelisches wie Körperliches erscheinen als in ein kosmisches Geschehen einbezogen: Vorstellungen, Gedanken, Träume und Willensentschlüsse werden erlebt als von außen bewirkt, und andererseits auch direkt nach außen hin wirksam, wie z. B. Wünsche, Haßgefühle etc. Das zeigt sich noch deutlich bei Homer, wo Entschlüsse oder Einsichten nicht aus einem autonomen Subjekt hervorgehen, wo Gefühle wie Mut oder Angst nicht aus dem Innern der Person entstehen, sondern von den Göttern den Menschen eingegeben werden. ${ }^{17}$ Auf das Phänomen der psychischen Beeinflussung weist auch Dodds hin. ${ }^{18}$ Die Verblendung (ä $\left.\eta\right)$ durch die Götter als zeitweilige Trübung des normalen Bewußtseins, ebenso wie plötzliche Erkenntnis oder Erinnerung und das Eingießen von Kraft in den $\vartheta u$ ós sind Beeinflussungen des Psychischen von außen. Das Seelische steht im Spannungsfeld äußerer Kräfte und erscheint noch nicht als etwas in sich Geschlossenes. Wille, Denken, Erinnern, Vorstellen stehen nicht in der Verfügung des Subjekts; es gibt das Subjekt in unserem Sinn noch nicht. Im Menschen wirken viele Kräfte, er ist ein Konglo-

15 Werner (59), 294.

${ }^{16}$ Noch bei Homer erscheint Angst als ein körperlicher Vorgang, bei dem die Glieder erschlaffen, die Knie sich lösen. Die Knie werden dabei als Sitz der

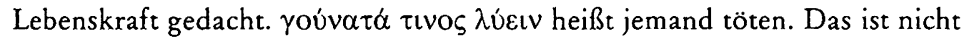
nur eine dichterisch bildhafte Wendung, sondern seelischer und körperlicher Vorgang werden als eins gesehen.

${ }^{17}$ Vgl. dazu B. Snell (55), Kap. II. Snell bezieht sich auf die Szene am Anfang der Ilias (I,188-222), wo Athene mäßigend auf Achill einwirkt. Er weist auch auf die merkwürdige Verwandtschaft dieser Vorstellungen mit der Rolle hin, welche die assistentia dei, das Einwirken Gottes auf die menschliche Seele im Okkasionalismus spielt, und, so kann man ergänzen, bei Berkeley. Es ist in der Tat ein Symptom für die Überzeichnung der Eigenständigkeit des Subjekts in Dualismus und Idealismus, daß diese Autonomie sich in ihr Gegenteil verkehrt: Das von der physischen Welt abgetrennte Subjekt wird zum Objekt der Einwirkung Gottes.

${ }^{18}$ Vgl. Dodds (66), Kap. 1. 
merat verschiedener Fähigkeiten, Organe, Kräfte, die eine relative Selbständigkeit haben. Es besteht so eine gewisse psychische Labilität. Antriebe stehen nicht in der Kontrolle des Subjekts. Man blickt erstaunt oder entsetzt zurück auf das, was man eben getan hat, und das eigene Verhalten kann dabei fremd erscheinen. Alle Abweichungen von normalem menschlichem Verhalten, deren Gründe nicht einsichtig sind, werden übernatürlichen Kräften zugeschrieben, ebenso wie Abweichungen von normalen Verläufen in der Natur. Das bewirkt ein Gefühl beständiger Abhängigkeit alles Geschehens von Übernatürlichem.

In primitiven Kulturen ist auch die Differenzierung zwischen Individuen und Gruppe weniger ausgeprägt. Es besteht eine unmittelbare Verbundenheit im Erleben, Fühlen und Handeln. Mit einem Familienmitglied werden auch die anderen schuldig; der Vater kann das Medikament für sein erkranktes Kind schlucken, usf. ${ }^{19}$

3. Die Dinge baben Funktionscharakter.

Im primitiven Erleben erscheinen die Dinge nicht als Substanzen, als etwas, das im Wechsel der Erscheinungen und im Wandel der Situationen beharrt und konstante Wesensmerkmale aufweist, sondern eher als Bestandteile des jeweiligen Erlebens- und Verhaltenszusammenhangs. Man kann in diesem Sinn von „Aktions-“ oder „Signaldingen" reden. Entsprechendes hat J. von Uexküll für die Umwelt von Tieren nachgewiesen. Dinge werden oft nur dann und insoweit wahrgenommen, als sie eine bestimmte Valenz oder Bedeutung für den Wahrnehmenden haben; als Objekte oder Hindernisse von Strebungen oder als Instrumente. Funktionsgleiche Dinge werden trotz objektiver Verschiedenheiten als gleichartig angesehen. Umgekehrt kann das gleiche Ding in verschiedenen Handlungskontexten und affektiven Situationen wegen seiner verschiedenen Funktionen und Bedeutungen als verschieden erscheinen. Wirklich ist, was im Lebenszusammenhang eine Rolle spielt. ${ }^{20}$ Eine objektive Welt, die unabhängig vom Subjekt da ist und an sich gewisse Eigenschaften hat, gibt es für das primitive Erleben nicht im selben Maße wir für uns. Aus dem Funk-

${ }^{19}$ Vgl. dazu z. B. Werner (59),317ff.

${ }^{20}$ Experimente mit Spinnen zeigen z. B., daß sie nicht auf Mücken reagieren, sondern nur auf die komplexe Einheit „Mücke im Netz“ - eine Mücke ist für sie eben nicht als solche bedeutsam, sondern erst als Beute. 
tionscharakter und der emotiven Ausgestaltung der Dinge ergibt sich ihre Labilität im primitiven Erleben. Indem sie sich aus der jeweiligen Erlebnissituation bestimmen, aus dem Handlungskontext, affektiv und physiognomisch erfaßt werden, wandeln sie sich mit der Situation. In verschiedenen Aktionszusammenhängen kann ein und dasselbe Ding seinen Charakter völlig ändern. Im primitiven Erleben bleiben nicht die Dinge konstant und ändern nur ihre akzidentellen Eigenschaften, sondern ihre Situationsbezogenheit bewirkt, daß sich mit einzelnen Eigenschaften, einzelnen Teilen und mit der Situation das Ganze ändert, daß verschiedene Erscheinungsweisen des gleichen Dings als Erscheinungen verschiedener Dinge aufgefaßt werden. Das drückt sich auch sprachlich in der Vielfalt der Bezeichnungsweisen für ein und dasselbe Ding aus: Wenn z. B. für die aufgehende Sonne, die Mittagssonne und für die untergehende Sonne verschiedene $\mathrm{Be}$ zeichnungen ohne sprachlich gleiche Bestandteile existieren, so ist das ein Symptom dafür, daß die verschiedenen Erscheinungsweisen der Sonne als Erscheinungen verschiedener Gegenstände aufgefaßt werden. ${ }^{21}$

Ethnologie und Entwicklungspsychologie belegen also, daß im primitiven Erleben subjektive und objektive Momente viel stärker miteinander verwoben sind und viel weniger differenziert werden; daß also die relative Eigenständigkeit von Subjektivem und Objektivem man könnte von einer Pol-Distanz sprechen - sehr viel geringer ist als für uns.

Mit dem Entstehen menschlicher Kultur tritt an die Stelle der Entwicklungsgeschichte der menschlichen Natur die Geistesgeschichte. Auch die Differenzierung von Psychischem und Physischem ist das Produkt von Interpretationen der Erfahrung und ihrer Erprobung im Leben, also ein Thema der Geistesgeschichte. Den Wandel des Weltund des Menschenbildes unter diesem Aspekt zu untersuchen, wäre für unser Thema sicher von besonderem Interesse, liegt aber weit jenseits der Zielsetzungen dieser Arbeit. Wir beschränken uns hier auf einige kurze Hinweise auf die Sprachgeschichte. In ihr spiegelt sich die Entwicklung der Begriffssysteme am unmittelbarsten, weil unbeeinflußt von einzelnen theoretischen Entwürfen. $\mathrm{Zu}$ den Arbeiten über Sprachgeschichte als Geistesgeschichte, die für unsere Fragestellung

${ }^{21} \mathrm{Vgl}$. dazu Werner (59), 227. 
einschlägiges Material enthalten, gehört insbesondere das Buch von B. Snell „Diє Entdeckung des Geistes" (55), in dem die Entwicklung der griechischen Konzeption von Mensch und Welt und ihrem Verhältnis in archaischer und klassischer Zeit im Spiegel der Sprache untersucht wird. Wir geben einige sprachgeschichtliche Parallelen zu den drei oben genannten Punkten an.

Zurgeringeren Differenzierung zwischen Subjektivem und Objektivem Das Feld der Verben, die mit „,sehen“ und ,blicken“ sinnverwandt sind, enthält bei Homer verschiedene Wörter, die mit dem Vorgang der optischen Wahrnehmung zugleich gewisse Gefühle oder Einstellungen ausdrücken, mit denen jemand auf das reagiert, was er sieht. Es fehlen dagegen Wörter, die ein neutrales Sehen in unserem Sinn bezeichnen. Das ist ein Indiz dafür, daß Gegenstände und Ereignisse der Außenwelt sehr viel stärker unter subjektiven Aspekten, unter Gesichtspunkten ihrer Bedeutung für den Menschen erfahren wurden; $\mathrm{da}$ ßeniger zwischen Erlebtem und Erlebnisweise differenziert wurde. Charakteristischerweise verschwinden solche Wörter, wie $\lambda \varepsilon v ́ \sigma \sigma \varepsilon \imath v$ (etwas Glänzendes sehen, im Doppelsinn, der auch in diesem deutschen Wort steckt: etwas Leuchtendes, Helles, wie z. B. blinkende Waffen, mit Stolz oder Freude sehen) oder ö $\sigma \sigma \varepsilon \sigma \vartheta \alpha$ l (etwas Drohendes vor Augen haben und dabei Schlimmes ahnen) im klassischen Griechisch und werden durch Wörter wie $\beta \lambda \varepsilon \dot{\tau} \pi \tau \varepsilon \iota v$ und $\checkmark \varepsilon \omega \rho \varepsilon \tau v$ ersetzt, die keine solchen Gefühlskonnotationen mehr enthalten. ${ }^{22}$

Wie E. Heitsch in (74), S. 90ff hervorgehoben hat, wird im Griechischen sprachlich nicht zwischen Wahrheit und Evidenz unterschieden. $\dot{\alpha} \lambda \hat{\eta} \vartheta \varepsilon \iota \alpha$ bedeutet etymologisch etwa soviel wie „Unverborgenheit“", d. h. für Sachverhalte soviel wie Offensichtlichkeit, Klarheit, Gegenwärtigkeit. Evidenz als subjektives Wahrheitskriterium und Wahrheit als objektive Eigenschaft von Sachverhalten, bzw. Sätzen werden also mit demselben Wort bezeichnet, und das heißt: zwischen ihnen wird meist nicht unterschieden. ${ }^{23}$

Verben drücken (im Aktiv) ursprünglich sehr viel eindeutiger als nach heutigem Verständnis Handlungen eines Subjekts aus. Von Handlungen kann man aber nach unserer Auffassung im engeren Sinn

${ }^{22}$ Vgl. dazu Snell (55), S. $13 \mathrm{ff}$.

${ }^{23}$ Für analoge Verbindungen subjektiver und objektiver Bedeutungskomponenten im moralischen Vokabular vgl. Kutschera (81), Kap. 6. 
nur bei Menschen, im weiteren Sinn allenfalls bei Tieren reden. Wenn wir diese Bedeutung der Verben beachten - in unserem Sprachverständnis ist sie in vielen Fällen ganz in den Hintergrund getreten -, erscheinen Aussagen wie „Der Wind bläst“, „Das Wasser läuft“, „Die Strömung reißt den Ast fort" usw. als Anthropomorphismen oder als bloße Metaphern. Snell erwähnt in (55) neben solchen Beispielen einen Vergleich Homers (Il.15,615): Die Schlachtreihe hält sich gegen den feindlichen Ansturm ,,so wie ein Fels im Meer, der Wind und Wellen zum Trotz verharrt", und sagt dazu, „daß der Fels ein menschliches Verhalten deutlich macht, also ein toter Gegenstand anthropomorph gesehen wird: das unbewegliche Stehen der Klippe in der Brandung wird gedeutet als Ausharren, so wie der Mensch ausharrt in einer bedrohten Situation. Der Gegenstand wird also tauglich, im Gleichnis etwas zu veranschaulichen, dadurch, daß in diesen Gegenstand das hineingesehen wird, was er dann seinerseits illustriert. Dies eigentümliche Verhältnis, daß menschliches Verhalten deutbar wird durch etwas, das selbst erst nach diesem menschlichen Verhalten gedeutet ist, gilt auch für alle anderen homerischen Gleichnisse, ja, es gilt weit darüber hinaus bei den echten Metaphern und überhaupt überall dort, wo der Mensch etwas ,,versteht". Es ist also schon bedenklıch, wenn wir sagen, der Fels würde ",anthropomorph" gesehen - man müßte dann hinzufügen, daß der Mensch den Felsen nur dadurch anthropomorph sehen kann, daß er sich selbst zugleich petromorph sieht, daß er nur dadurch, daß er den Felsen von sich aus interpretiert, ein eigenes Verhalten gewahr wird und den treffenden Ausdruck dafür findet. Daß der Mensch sich selbst nur so im Echo hören und verstehen kann, ist grundlegend für das Verständnis der Gleichnisse". ${ }^{24}$

Der Mensch begreift sich also im Spiegel seiner Umwelt und entnimmt aus ihr Beschreibungen für sich selbst, seine Handlungen, seine Eigenschaften, seinen Charakter, speziell auch für Geistiges und Seelisches, wie räumliche und optische Metaphern zeigen. Das Erken-

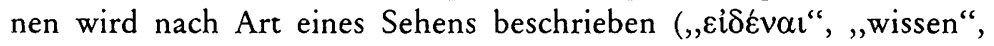
heißt etymologisch soviel wie ,gesehen haben"); wenn etwa erkannt wird, so wird es ,klar", ,,durchsichtig“, ,,hell" (dasselbe Phänomen läßt sich also auch im Deutschen beobachten); das Unverständliche ist dunkel, das Rätselhafte tief. Menschliche Charaktere werden durch

${ }^{24}$ Snell (55),S. 185. 
Vergleiche mit Tieren beschrieben; ${ }^{25}$ die Dinge der Außenwelt erscheinen als von denselben elementaren Kräften bewegt wie der Mensch. Umgekehrt ist die Natur ein Spiegel des Menschlichen. Die, wie wir sagen würden, „,metaphorische" Übertragbarkeit - tatsäch" lich ist es eine Sinnverwandtschaft - von Termen für Dinge der AuBenwelt auf menschliche Phänomene, speziell auf Psychisches, ist charakteristisch für die frühe Sprache und belegt unsere Behauptung, daß sich die Gesamtsprache als eine Einheit entwickelt hat, innerhalb der sich die Sprache für Physisches und die für Psychisches langsam differenziert haben, wobei erst in späteren Stadien keine direkte Sinnverwandrschaft mehr besteht, so daß man dann von „Metaphern“ reden kann.

\section{Zur geringen Differenzierung von Psychischem und Physischem}

Die zunehmende Differenzierung von Seelischem und Körperli-

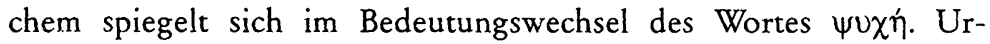
sprünglich bedeutet es soviel wie „Lebensodem“, „Leben“, daneben die abgeschiedene Seele des Toten. Der Gegensatz $\psi v \chi \dot{\eta}-\sigma \tilde{\omega} \mu \alpha$ (Seele - Körper) nimmt seinen Ausgang von der Unterscheidung Leichnam - Totenseele, und erst bei Heraklit nimmt $\psi v \chi \eta \dot{~ d i e ~ B e-~}$ deutung von Seele als Inbegriff geistigen und emotionalen Lebens an, werden Seelisches und Körperliches als verschiedene Bereiche gesehen. ${ }^{26}$

Bei Homer gibt es weder eine einheitliche Bezeichnung für Seelisch-Geistiges, noch für Physisches, ja nicht einmal für den menschlichen Körper. Es ist nur von einzelnen Gliedern die Rede, und es kommen Ausdrücke für verschiedene seelische Vermögen oder Kräfte vor

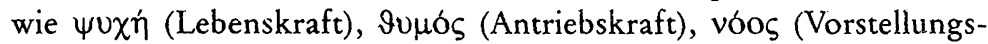
kraft), die aber von körperlichen Vermögen, vom Leben, daß sich im Atmen und in der Bewegung darstellt, von den Regungen der Glieder und von der Erfassung der Gegenstände mit den Sinnen nicht deutlich abgesetzt werden, so daß man hier von einer in sich wenig differenzierten Einheit von Seelischem und Körperlichem sprechen kann.

${ }^{25}$ Vgl. dazu den „Weiberjambus“ des Semonides (Fr. 7), den Snell in (55), S. 191 zitiert.

${ }^{26}$ Vgl. dazu Snell (55),S. $18 \mathrm{ff}$. 
J. Warden hat in (69) auch darauf hingewiesen, daß bei Homer terminologisch wie sachlich nicht zwischen Kraft und Tapferkeit, zwischen Schwäche und Feigheit unterschieden wird, also nicht zwischen körperlichen Fähigkeiten und charakterlichen Haltungen.

Die Tatsache, daß es keine einheitliche Bezeichnung für Seelisches gibt, ist auch ein Symptom dafür, daß das Psychische nicht als etwas in sich Geschlossenes aufgefaßt wird, sondern sich eher wie ein Aggregat von Kräften, Fähigkeiten und Organen nach Art körperlicher Organe darstellt, die relativ selbständig agieren können, so daß sich eine gewisse psychische Labilität der Person ergibt. ${ }^{27}$

\section{Zum Funktionscharakter der Dinge}

Dem konkreten Charakter primitiver Erfahrungen entspricht auf der sprachlichen Seite die Plastizität und Vieldeutigkeit der Wörter. In verschiedenen sprachlichen Kontexten und Situationen können sie ganz Verschiedenes bedeuten. Die Satzbedeutung bestimmt sich nicht nur aus den Wortbedeutungen, sondern es findet - stärker als in unserer Sprache - auch eine Determination der Wortbedeutungen durch das Satzganze und durch den Sprach- und Situationskontext statt. Bezeichnend für den Funktionscharakter ist auch die Häufigkeit der pars-pro-toto-Ausdrücke, in denen der Teil für das Ganze stehen kann, und derselbe Teil (wie wir sagen würden) in verschiedenen Kontexten für verschiedene Ganzheiten. Die Objektivierung der Aktionsdinge zu Dingen an sich zeigt sich sprachgeschichtlich besonders deutlich an Bezeichnungen für Geistiges und Psychisches. vóos, der

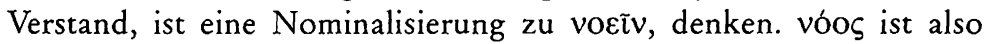
das, was denkt (vorstellt, einsieht). Da aber das Wort zugleich für „Gedanke“ und „Vorstellung" steht, bezeichnet es noch nicht das Vermögen des Verstehens, sondern das, was präsent ist, wenn jemand etwas denkt oder versteht oder sich verständig zeigt. Erst wenn vóos vom vó $\mu \alpha$, dem Gedachten unterschieden wird, kann man ihn als Vermögen des Denkens auffassen, das auch dann vorhanden ist, wenn es nicht betätigt wird.

Man kann also sagen, daß die zunehmende Differenzierung zwischen Subjektivem und Objektivem, Psychischem und Physischem ei-

${ }^{27}$ Vgl. dazu Snell (55), Kap. 1 und Dodds (66), Kap. 1. 
nen geschichtlichen Prozeß darstellt, und daß erst im Stadium einer relativ starken Differenzierung so etwas wie die dualistische Konzeption entstehen konnte, nach der Psychisches und Physisches selbständige und voneinander unabhängige Bereiche sind. Von dieser Konzeption her läßt sich aber diese Entwicklung nicht mehr verstehen. Frühere Vorstellungswelten erscheinen dann nur als Formen „falschen Bewußtseins"; die Entwicklung stellt sich dar als Fortschreiten des Unterscheidungsvermögens, nicht als Folge verschiedener möglicher Interpretationen der Erfahrung, verschiedener Alternativen zu unserer eigenen Deutung. Wegen ihrer größeren Allgemeinheit ermöglicht die polare Konzeption dagegen einen sinnvolleren Vergleich solcher Interpretationen, ist also für geistesgeschichtliche Untersuchungen brauchbarer. So, wie wir sie skizziert haben, bildet sie freilich nur einen Ansatz zum Verständnis solcher Phänomene, der erst in sehr viel detaillierteren Ausgestaltungen und Untersuchungen seine Fruchtbarkeit erweisen kann.

\subsection{Raum und Zeit}

Wir wollen uns nun jenem Punkt unserer Bestimmung des Verhältnisses von Physischem und Psychischem zuwenden, der deswegen besonders problematisch ist, weil er am weitesten von der normalen Konzeption abweicht: der Aussage, Physisches sei auf Psychisches bezogen. Die Bezogenheit psychischer Phänomene auf Physisches ist demgegenüber sehr viel unproblematischer. Wir haben schon früher in den Abschnitten 5.3 und 7.4 gesehen, daß die Sprache über Psychisches die Sprache über Physisches voraussetzt, und mit ihr eng verknüpft ist. Ferner gibt es eine Vielzahl von Abhängigkeiten unseres Erlebens von physischen Vorgängen. Es gibt also weder eine Sprache über Psychisches, die unabhängig von der physikalischen Sprache wäre, noch lassen sich psychische Phänomene ganz ohne Bezugnahme auf physische erklären. Die physikalische Natur sehen wir dagegen normalerweise in ihrer Existenz wie Beschaffenheit als völlig unabhängig von subjektiven Faktoren wie Erlebnissen und Erlebensweisen, Überzeugungen und Präferenzen an, ja als unabhängig von der Existenz von Menschen, die sie erleben und erkennen. Die Geltung von Aussagen wie „Dieses Blatt ist grün“ oder "Quecksilber schmilzt bei $-39^{\circ} \mathrm{C}$ " hängt nach üblichem Verständnis nicht von ir- 
gendwelchen psychologischen Umständen ab. Die Sprache der Physik nimmt nicht explizit auf Psychisches Bezug, und daher liegt es nahe zu sagen, der Bereich des Physischen und die einzelnen physikalischen Phänomene ließen sich ohne Rekurs auf Psychisches charakterisieren.

Wir haben zwar in 7.4 Argumente angeführt, nach denen die physikalischen Terme einen mehr oder minder ausgeprägten erlebnismäßigen, anschaulichen Gehalt haben, diese Argumente genügen aber noch nicht, um eine Konzeption überzeugend zu verteidigen, die üblichen Vorstellungen so radikal widerspricht. Daher wollen wir in diesem und dem folgenden Abschnitt sehen, ob sie sich mit dem Naturbegriff der modernen Physik vereinbaren läßt. Was ,das Physische“ ist, wird man wohl am besten von physikalischen Theorien her bestimmen, und es wird sich zeigen, daß der modernen Physik ein ganz anderes Naturverständnis entspricht als der klassischen, auf die sich die üblichen Vorstellungen vom Physischen beziehen.

Wir haben in 7.4 den anschaulichen Gehalt einfacher Terme der Dingsprache wie ,rot", „hart" etc. aufgewiesen. Diese Terme drükken Qualitäten aus, die Descartes und Locke als ,,sekundäre Qualitäten" bezeichnen, d. h. Qualitäten, die nicht den Dingen selbst zukommen, sondern nur die Weise charakterisieren, wie wir sie erleben. Der Realismus Descartes und Lockes gründet sich darauf, daß es neben solchen sekundären Qualitäten auch primäre gibt, z. B. räumliche und zeitliche Eigenschaften und Beziehungen, die sich den Dingen selbst zuschreiben lassen. ${ }^{28}$ Berkeley versuchte dagegen nachzuweisen, daß auch raum-zeitliche Eigenschaften nur sekundäre Qualitäten darstellen, um daraus zu schließen: Wenn uns Beobachtungen immer nur zeigen, wie uns die Dinge erscheinen, aber nicht, wie sie an sich beschaffen sind, so gibt es keine Erkenntnis und keine Erfahrung von Dingen an sich; denn es gibt keine Erkenntnis von Objekten ohne Erkenntnis ihrer Eigenschaften. Kant ist in seiner transzendentalen Ästhetik Berkeley in diesem Gedanken gefolgt und sein transzendentaler Idealismus ist ein Ergebnis dieser Ästhetik, in der er nachzuweisen sucht, daß Raum und Zeit Anschauungsformen sind; daß sich räumliche und zeitliche Bestimmungen daher nicht den Dingen an sich zusprechen lassen, sondern nur ihren Erscheinungen. ${ }^{29}$ Wir wollen hier

${ }^{28}$ Vgl. dazu den Abschnitt 4.1.

${ }^{29}$ Vgl. dazu den Abschnitt 8.7. 
anhand der Gedanken, die der speziellen Relativitätstheorie zugrunde liegen, ebenfalls zeigen, daß raum-zeitliche Bestimmungen nicht Dinge an sich charakterisieren, sondern nur die Natur, so wie sie sich uns in der Erfahrung zeigt; daß diese Bestimmungen einen nicht eliminierbaren Bezug auf Beobachtungen haben. Daraus ergibt sich freilich kein Idealismus, sondern nur eine Kritik am absoluten Realismus, wie er der klassischen Physik zugrundelag und der heute noch verbreiteten Auffassung der Physik als Wissenschaft von einer Natur, für die es unwesentlich ist, daß sie Gegenstand menschlicher Erfahrung ist.

Die klassische Physik gehr von einem dreidimensionalen euklidischen Raum und einer eindimensionalen Zeit aus. Newton spricht in den "Philosophiae naturalis principia mathematica" von 1687 von einem ,,absoluten, unveränderlichen, unbeweglichen Raum", der unabhängig von dem Vorhandensein von Körpern in ihm existiert, und einer ,,absoluten und wahren Zeit", die ebenfalls unabhängig von physikalischen Ereignissen in ihr besteht. Raum und Zeit sind also gewissermaßen Behälter, deren Struktur unabhängig von ihrem Inhalt ist. Dem Raum ist eine Metrik aufgeprägt, d.h. es ist für alle Raumpunkte $x$ und y eine reelle $Z$ ahl $d(x, y) \geq 0$ als Entfernung bestimmt, die eindeutig ist bis auf die Wahl einer Einheit, die also festliegt, wenn wir z. B. dem Urmeter in Paris die Länge 1 zuordnen. Die Wahl dieser Einheit ist Sache der Konvention. Es ist sinnlos zu fragen, ob das Urmeter ,,in Wahrheit" die Länge 1 habe. Nicht konventionell ist jedoch die euklidische Metrik, nach der

$$
\mathrm{d}(\mathrm{x}, \mathrm{y})=\sqrt{\left(\mathrm{x}_{1}-\mathrm{y}_{1}\right)^{2}+\left(\mathrm{x}_{2}-\mathrm{y}_{2}\right)^{2}+\left(\mathrm{x}_{3}-\mathrm{y}_{3}\right)^{2}}
$$

ist, wo die $x_{i}(i=1,2,3)$ die Koordinanten von $x$ und die $y_{i}$ jene von $y$ in einem cartesischen Koordinatensystem sind. Sie bestimmt die Struktur des Raums. Ebenso ist für alle Zeitpunkte $t$ und $t^{\prime}$ eine zeitliche Entfernung bis auf die Wahl einer Einheit festgelegt, z. B. der Sekunde als des 86400 . Teiles eines mittleren Sonnentages. Raum und Zeit sind zwei voneinander unabhängige Ordnungsschemata.

Nach der Newtonschen Mechanik gibt es keine physikalischen Kriterien, mit denen man feststellen könnte, ob man sich gegenüber dem absoluten Raum in Ruhe oder im Zustand einer gleichförmig geradlinigen Bewegung befindet. In einem gleichförmig geradlinig fahrenden Zug z. B. laufen die mechanischen Prozesse in derselben Weise ab wie auf dem Bahndamm, so daß ein Fahrgast im Zug, der nicht nach draußen sehen kann, nicht festzustellen vermag, ob der Zug steht 
oder fährt. Die Gesetze der Mechanik sind also invariant gegenüber Formulierungen in gegeneinander geradlinig gleichförmig bewegten Bezugs- oder Koordinatensystemen, d. h. gegenüber Transformationen, die solche Koordinatensysteme ineinander überführen. Diese Tatsache bezeichnet man als Mechanisches Relativitätsprinzip. Es drückt sich im ersten Newtonschen Gesetz aus, nach dem ein Körper im Zustand der Ruhe oder gleichförmig geradlinigen Bewegung verharrt, wenn keine Kräfte auf ihn wirken. Die Aussage, ein Körper habe die und die absolute Geschwindigkeit, hat also keine physikalische Bedeutung: die Größe der Geschwindigkeit hängt von der Wahl des Bezugssystems ab, ebenso wie Ortsangaben. Kräfte hingegen sind objektive physikalische Realitäten. Sie werden nach dem zweiten Newtonschen Gesetz, der Bewegungsgleichung, in Relation zur Beschleunigung gesetzt: Kraft ist Masse mal Beschleunigung. Beschleunigungen sind physikalisch beobachtbar über die Wirkung der Kräfte, die dabei auftreten. So kann man im Zug feststellen, ob er seine Fahrt beschleunigt oder abbremst oder ob er eine Kurve durchfährt. In gegeneinander beschleunigten Bezugssystemen gelten also nicht dieselben physikalischen Gesetze. Die Gesetze der Newtonschen Mechanik zeichnen daher eine Klasse von Bezugssystemen aus, in denen sie gelten: die Inertialsysteme. Ein solches Inertialsystem ist das, in dem die Fixsterne ruhen. Inertialsysteme sind solche, die sich gegenüber dem absoluten Raum in einem Zustand der Ruhe oder gleichförmig geradlinigen Bewegung befinden. Da wir zwischen diesen beiden Zuständen physikalisch nicht unterscheiden können, können wir auch nicht feststellen, ob ein Inertialsystem absolut ruht oder nicht.

Der Übergang von einem raum-zeitlichen (cartesischen) Koordinatensystem $\mathrm{K} \mathrm{zu}$ einem anderen $\mathrm{K}^{\prime}$ wird dadurch bestimmt, daß räumliche und zeitliche Entfernungen dabei konstant bleiben. Sind also $\mathrm{x}_{1}, \mathrm{y}_{1}, \mathrm{z}_{1}$ und $\mathrm{x}_{2}, \mathrm{y}_{2}, \mathrm{z}_{2}$ die räumlichen Koordinaten zweier Punkte in $\mathrm{K}$, $\mathrm{x}_{\hat{1}}, \mathrm{y}_{\mathfrak{i}}, \mathrm{z}_{\overline{1}}$ und $\mathrm{x}_{\hat{2}}, \mathrm{y}_{\overline{2}}, \mathrm{z}_{\hat{2}}$ die Koordinaten derselben Punkte in $\mathrm{K}^{\prime}$, so muß gelten

1a) $\begin{aligned} & \left(x_{1}-x_{2}\right)^{2}+\left(y_{1}-y_{2}\right)^{2}+\left(z_{1}-z_{2}\right)^{2}= \\ & \left(x_{1}-x_{2}\right)^{2}+\left(y_{1}-y_{2}\right)^{2}-\left(z_{i}-z_{2}\right)^{2} .\end{aligned}$

Und sind $t_{1}$ und $t_{2}$ die zeitlichen Koordinaten zweier

Ereignisse in $K, t_{1}$ und $t_{2}$ jene in $K^{\prime}$, so muß gelten

1b) $\left(t_{1}-t_{2}\right)^{2}=\left(t_{1}-t_{2}\right)^{2}$.

Die Konstanz der räumlichen und zeitlichen Distanzen ist die Konsequenz der voneinander unabhängigen Metriken von Raum und Zeit; 
die Darstellung dieser Konstanz in der Form (1) ist eine Folge der euklidischen Metrik des Raums. Daraus ergibt sich irisbesondere das klassische Additionstheorem der Geschwindigkeiten:

2) Sind die Achsen von $K^{\prime} z u$ jenen von $K$ parallel, fallen die $x$-Achsen von $\mathrm{K}$ und $\mathrm{K}^{\prime}$ zusammen und bewegt sich $\mathrm{K}^{\prime}$ gegenüber $\mathrm{K}$ in

- Richtung der x-Achse mit der Geschwindigkeit v, so hat ein Körper, der sich in $\mathrm{K}$ in derselben Richtung mit der Geschwindigkeit w bewegt, von $\mathrm{K}^{\text {' }}$ aus gesehen die Geschwindigkeit w-v.

Zwei cartesische Koordinatensysteme $\mathrm{K}$ und $\mathrm{K}^{\prime}$ gehen nach (1) auseinander hervor durch eine Drehung und eine Translation, die beide von $\mathrm{t}$ abhängen könner. Ist nun $\mathrm{K}^{\prime}$ gegenüber $\mathrm{K}$ gleichförmig geradlinig bewegt, so darf die Drehung nicht zeitabhängig sein und die Translation muß mit konstanter Geschwindigkeit erfolgen. Aus (1) und dem Mechanischen Relativitätsprinzip ergibt sich so die Gestalt der Galilei-Transformationen, bzgl. derer die Gesetze der Newtonschen Mechanik invariant sind. In dem einfachen Fall, der in (2) vorausgesetzt wurde, lauten sie

3) $x^{6}=x-v \cdot t$

$y^{6}=y$

$\mathrm{z}^{\mathrm{c}}=\mathrm{z}$

$\mathrm{t}^{\mathrm{t}}=\mathrm{t}$.

Die Gesetze der Elektrodynamik unterscheiden sich dadurch wesentlich von jenen der Mechanik, daß in ihnen die Konstante $c$ für die Lichtgeschwindigkeit (im Vakuum) vorkommt. Hängt der Wert dieser Konstanten nicht vom Bezugssystem ab, so sind diese Gesetze nicht invariant bzgl. Galilei-Transformationen. Das mechanische Relativitätsprinzip kann dann nicht gelten, denn nach dem Additionsgesetz (2) müßte das Licht in bewegten Systemen eine andere Geschwindigkeit haben. Versuche, wie die von A. A. Michelson und E. W. Morley (1881 bis 1887), die den Einfluß der Geschwindigkeit des Bezugssystems auf die Lichtausbreitung nachweisen sollten, schlugen jedoch fehl. Man stand damit vor der Wahl, entweder eine Abhängigkeit der Länge von Stäben und der Geschwindigkeiten physikalischer Prozesse vom Bewegungszustand des Bezugssystems anzunehmen, so daß die mit Maßstäben und Uhren im normalen Sinn gemessene Lichtgeschwindigkeit in bewegten Systemen nicht die wahre Lichtgeschwindigkeit ergibt, ${ }^{30}$ oder aber die Konstanz der Lichtgeschwindigkeit im

${ }^{30}$ Die Annahme einer bloßen Längenkontraktion wie sie H. A. Lorentz vor- 
Vakuum in allen gegeneinander gleichförmig geradlinig bewegten $\mathrm{Be}$ zugssystemen anzunehmen. Nach dem ersten Vorschlag konnte man die Newtonsche Raum-Zeit-Lehre (kurz NRZL) beibehalten, mußte aber das mechanische Relativitätsprinzip preisgeben, da nun in Abweichung von der Mechanik deformierende Kräfte anzunehmen waren, die von der Geschwindigkeit abhingen. Beim zweiten Weg mußte man hingegen die NRZL aufgeben und durch eine andere ersetzen. Diesen Weg ist Einstein mit seiner speziellen Relativitätstheorie gegangen. ${ }^{31} \mathrm{Daß}$ dieser Weg aber überhaupt gangbar war, mußte er durch methodologische und erkenntnistheoretische Überlegungen zeigen.

Wenn die Konstanz der Lichtgeschwindigkeit gelten soll, muß für verschiedene (zulässige) Koordinatensysteme $\mathrm{K}$ und $\mathrm{K}^{\prime}$ gelten

4) $\left(x_{1}-x_{2}\right)^{2}+\left(y_{1}-y_{2}\right)^{2}+\left(z_{1}-z_{2}\right)^{2}-c^{2}\left(t_{1}-t_{2}\right)^{2}=0$ genau dann, wenn $\left(x_{1}-x_{2}\right)^{2}+\left(y_{i}-y_{2}\right)^{2}++\left(z_{1}-z_{2}\right)^{2}-c^{2}\left(t_{1}-t_{2}\right)^{2}=0$.

Denn die erste Gleichung beschreibt eine Lichtwellenfront, die in $t_{1}$ vom Punkt $\left(\mathrm{x}_{1}, \mathrm{y}_{1}, \mathrm{z}_{1}\right)$ ausgeht. Breitet sich nun die Lichtwelle in $\mathrm{K}^{\mathrm{c}}$ mit derselben Geschwindigkeit in allen Richtungen aus wie in $\mathrm{K}$, so erscheint sie auch von $\mathrm{K}^{\mathrm{c}}$ aus gesehen als Kugelwelle um den Punkt $\left(\mathrm{x}_{1}, \mathrm{y}_{1}, \mathrm{z}_{1}\right)$. Die Lichtwellenfront befindet sich also in $\mathrm{K}^{\mathrm{c}}$ in den entsprechenden Zeiten an den entsprechenden Punkten. Ebenso gilt die Umkehrung. (4) ist nun nicht mit der Forderung (1) verträglich. Insbesondere ist nach (4) das Additionstheorem (2) für Geschwindigkeiten verletzt. Danach müßte sich ja in $\mathrm{K}^{\prime}$ bei dem in (2) angenommenen Fall das Licht in der Richtung der $\mathrm{x}^{\prime}$-Achse mit der Geschwindigkeit $\mathrm{c}-\mathrm{v}$ fortpflanzen, in der Gegenrichtung mit der Geschwindigkeit $c+v$. Nimmt man das Mechanische Relativitätsprinzip hinzu - und

schlug, erwies sich als nicht zureichend angesichts anderer Experimente, insbesondere der von R. J. Kennedy und E. M. Thorndike. Vgl. dazu Grünbaum (63), S. 388ff.

${ }^{31}$ Diese stark vereinfachende Darstellung soll natürlich keine wissenschaftsgeschichtlich getreue Schilderung der Situation der Physik vor 1905 sein - in diesem Jahr erschien Einsteins grundlegende Arbeit zur speziellen Relativitätstheorie „Elektrodynamik bewegter Körper". So ist insbesondere die Zielsetzung des Michelson-Morley-Versuchs ohne Erläuterung der Äthertheorie und des ,,Mitführungskoeffizienten“ nicht adäquat zu schildern, auf die wir hier jedoch nicht eingehen wollen. Dieser spezielle Versuch hat ferner wohl bei der Entwicklung der Relativitätstheorie durch Einstein keine entscheidende Rolle gespielt. Vgl. dazu Grünbaum (63),Kap. 12. 
die Möglichkeit, es beizubehalten war für Einstein ein wesentlicher Grund sich für die Aufgabe der NRZL zu entschließen - , so erhält man aus (4) in dem einfachen Fall, von dem wir bei (2) und (3) ausgingen, anstelle der angegebenen Galilei-Transformationen die Lorentz-Transformationen:

5) $\begin{aligned} x^{\prime} & =\frac{x-v t}{\sqrt{1-\beta^{2}}} \\ y^{\prime} & =y \\ z^{\prime} & =z\end{aligned}$

$t^{\prime}=\frac{t-\frac{v}{c^{2}} x}{\sqrt{1-\beta^{2}}}$, wo $\beta=\frac{v}{c}$ ist.

Die Invarianz gegenüber Lorentz-Transformationen tritt also in der speziellen Relativitätstheorie an die Stelle der Galilei-Invarianz in der klassischen Mechanik. Die Lorentz-Transformationen beschreiben nun den Übergang von einem Inertialsystem zum anderen. Und sie machen das noch deutlicher, was schon in (4) steckt, daß nämlich die Unabhängigkeit von Raum und Zeit nun aufgegeben wird: in die Transformationsgleichung der Zeitkoordinate geht die Ortskoordinate ein, und aus diesen Gleichungen ergibt sich, daß vom ruhenden Bezugssystem $\mathrm{K}$ aus gesehen die Länge von mit $\mathrm{K}^{\prime}$ bewegten Stäben, die in der $x$-Achse von $K^{\prime}$ liegen, verkürzt erscheint um den Faktor $\sqrt{1-\beta^{2}}$, während die Zeitstrecken in $K^{\prime}$, von $K$ aus gesehen, um den Faktor $1 / \sqrt{1-\beta^{2}}$ länger sind als in $K$, so daß also $z$. B. die Uhren in

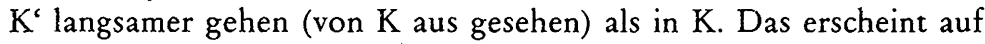
den ersten Blick als eine höchst merkwürdige Modifikation der NRZL und es stellt sich die Frage, inwiefern eine solche Änderung dieser RZL überhaupt sinnvoll und begründbar ist. ${ }^{32}$

${ }^{32} \mathrm{Da}$ wir im folgenden nur kurz auf die Philosophie von Raum und Zeit eingehen können, sei auf die einschlägige Literatur verwiesen. Das klassische Werk dazu ist Reichenbach (28); Teile davon hatte Reichenbach in einer mehr formalen Weise schon in (24) dargestellt. Ein wichtiges neueres Werk ist Grünbaum (63); dort finden sich auch ausführliche Literaturangaben. Besonders gut lesbar ist auch Einstein (20). Ein Standardwerk, das freilich mehr auf mathematische und physikalische Probleme ausgerichtet ist, ist noch immer Weyl (23). Mit der Raum-Zeitlehre befassen sich auch die ersten beiden Kapitel von Mittelstaedt (66). 
Newton stellte Geometrie und Chronometrie, also seine RaumZeit-Lehre, der Physik als einer empirischen Disziplin voraus. Aussagen darüber waren für ihn nicht empirisch, sondern apriorisch. Das erklärt sich vor allem aus der Tatsache, daß bis hin zu Gauß, Riemann und den Begründern der nicht-euklidischen Geometrie Bolyai und Lobatschewsky nur eine mathematische Geometrie, die euklidische, bekannt war, die auch in einer recht guten Näherung unserer Raumanschauung entspricht. Es gilt aber nun:

I) Die Verwendung raum-zeitlicher Beschreibungsmittel in der Physik als einer empirischen Disziplin ist nur dann sinnvoll, wenn man für räumliche und zeitliche Größen Meßverfabren angeben kann.

Andernfalls könnte man weder die Daten ermitteln, die man braucht, um mit den physikalischen Gesetzen Voraussagen zu machen, noch könnte man diese Gesetze empirisch überprüfen. Schon Newton hat betont, daß sich Raum und Zeit als solche, d. h. der leere Raum und die leere Zeit, nicht empirisch erfassen lassen. Was wir beobachten können, sind immer nur Relationen zwischen Körpern oder Ereignissen. Um diese empirischen Relationen von jenen im absoluten Raum oder der absoluten Zeit zu unterscheiden, wollen wir die ersteren als E-Relationen (als E-geometrische oder E-chronometrische Relationen) bezeichnen, die letzteren als A-Relationen. Wenn wir räumliche Entfernungen zwischen Körpern messen, benutzen wir Maßstäbe, d.h. andere Körper, ermitteln also E-geometrische Relationen. Mit welchem Recht können wir nun behaupten, daß wir mit realen Maßstäben, also durch E-geometrische Relationen, die wahren räumlichen Entfernungen, also A-geometrische Relationen erfassen? Das einzige Kriterium, was wir dafür haben, besteht darin, daß die mit realen Maßstäben empirisch ermittelte E-Geometrie dieselbe (metrische) Struktur hat wie die vorausgesetzte A-Geometrie - im Fall der klassischen Physik also: daß die E-Geometrie eine dreidimensionale euklidische Geometrie ist. Die Annahme einer A-Geometrie besagt, in der Natur sei eine bestimmte von vielen möglichen mathematischen Geometrien realisert. Diese Behauptung kann man nicht anders begründen als damit, daß es eine E-Geometrie gleicher mathematischer Struktur gibt. Da aber die Wahl verschiedener Meßverfahren verschiedene E-Geometrien ergibt und man nicht behaupten kann, es gebe mehrere wahre Geometrien (in der Natur seien mehrere verschiedene Raumstrukturen verwirklicht), muß man die Rede von dem ,wahren“ Raum aufgeben und sagen: 
II) Von dem (realen, physikalischen) Raum kann man nur sprechen bzgl. der Wabl gewisser Meßverfahren. Er ist der Kaum, der sich mit diesen Verfabren ergibt.

Die Rede von einem absoluten, wahren Raum ist also sinnlos, genauso sinnlos wie die Frage nach der wahren oder absoluten Länge dès Urmeters. Wie die Wahl der Längeneinheit eine Sache der Konvention ist und man erst bzgl. einer bestimmten Konvention von der Größe einer Entfernung reden kann, so ist auch die Wahl geometrischer Meßverfahren eine Sache der Konvention, und man kann erst bzgl. bestimmter Meßverfahren von dem Raum reden.

Man kann nun entweder gewisse Meßverfahren festlegen, mit denen man dann kraft Konvention die räumlichen Verhältnisse (d.h. eben: die durch diese Verfahren definierten E-geometrischen Relationen) korrekt erfaßt. Welche Raumstruktur sich dabei ergibt, ist eine Frage der Erfahrung. Bestimmen wir z. B. Lichtstrahlen als Gerade, so ergibt sich daraus noch nicht, welche Winkelsumme ein Dreieck hat; das ist vielmehr nachzumessen. Gauß hat als erster die Frage nach der Struktur des physikalischen Raums als eine empirische Frage gesehen und hat 1831 die Winkelsumme des Dreiecks Inselberg Brocken - Hoher Hagen mit Lichtstrahlen vermessen, fand aber innerhalb der Fehlergrenzen keine Abweichung von $180^{\circ}$. Die Wahl der Meßverfahren kann sich dabei nach deren Einfachheit richten, aber auch nach der Einfachheit der sich damit ergebenden Raumstruktur.

Man kann also zweitens auch die Meßverfahren so wählen, daß sich damit eine bestimmte Raumstruktur ergibt, z. B. die euklidische. Dann gelten die Aussagen über die Raumstruktur kraft Festsetzung und insofern apriorisch, hingegen ist es nun eine empirische Hypothese, daß bestimmte Meßverfahren korrekt sind bzgl. dieser Wahl, d. h. daß sie tatsächlich eine E-Geometrie mit dieser Struktur definieren. Und ebenso sind dann alle mit diesem Verfahren gewonnenen Meßergebnisse hypothetisch, da sie unter dem Vorbehalt stehen, daß die Meßverfahren korrekt sind. Auch dieses Vorgehen ist methodisch sauber und darf nicht mit der Idee verwechselt werden, eine mathematische Raumstruktur ließe sich ohne Bezugnahme auf Meßverfahren als real auszeichnen. Hier wird nicht ,die reale“, sondern eine bestimmte mathematische Geometrie ausgezeichnet, und ihre „Realität" besteht lediglich darin, daß es in ihrem Sinn korrekte Meßverfahren gibt. Solche Meßverfahren gibt es aber aus mathematischen Gründen immer. 
Dazu zwei Beispiele: Hätte sich im Experiment von Gauß eine andere Winkelsumme als $180^{\circ}$ ergeben, so hätte man auch ein solches Ergebnis mit der Annahme eines euklidischen Raums vereinbaren können durch die Hypothese, daß die bei der Messung verwendeten Lichtstrahlen nicht gerade Linien bilden, sondern durch Kräfte abgelenkt werden. Einstein hat die Ausmessung einer Tischplatte mit kleinen Stäben diskutiert. Man kann entweder diese Stäbe als starr ansehen und damit die geometrische Struktur der Fläche bestimmen. Wenn sie sich z. B. nicht in ein Quadratnetz anordnen lassen, so ist die Platte keine euklidische Ebene, sondern gekrümmt. Man kann aber auch festlegen, daß die Fläche euklidisch ist und aufgrund dieser Annahme die Objekte bestimmen, die als starre Meßstäbe infrage kommen, d. h. nur solche Stäbchen als Meßstäbe zulassen, mit denen sich ein Quadratnetz legen läßt. Für andere Stäbe muß man dann deformierende Kräfte annehmen, z. B. eine Temperaturabhängigkeit ihrer Länge und eine entsprechende Temperaturverteilung auf der Tischplatte - wenn eine solche Annahme mit den übrigen Beobachtungen und Gesetzen verträglich ist.

In der klassischen Physik hat man sich - abgesehen von einzelnen Ausnahmen wie vor allem $\mathrm{H}$. Helmholtz ${ }^{33}$ - nur wenig Gedanken über die Messung von Raum und Zeit gemacht. Man kann die Begründung der NRZL aber etwa so rekonstruieren: ${ }^{34}$ Für die Messung räumlicher Entfernungen werden reale Stäbe bestimmter Art als Einheitsstäbe ausgewählt, die wir auf den zu messenden Strecken abtragen. Diese Stäbe haben per Definition an allen Stellen und in allen Richtungen dieselbe Länge, sie sind also kraft Festsetzung starr, d. h. ihre Länge unterliegt nicht dem Einfluß physikalischer Kräfte. Um Messungen mit solchen Stäben erklären und auf diese Weise den Begriff der räumlichen Entfernung einführen zu können, müssen wir gewisse intuitive, anschaulich erklärte Begriffe voraussetzen. Wir müssen nicht nur die Stäbe und die mit ihnen auszuführenden Operationen beschreiben können, sondern wir brauchen z. B. auch den Begriff

${ }^{33} \mathrm{Vgl}$. z. B. Helmholtz (83) und (96).

${ }^{34}$ Eine ausführliche Diskussion von Problemen der Messung würde zu weit aus dem Kontext herausführen, in dem dieser Abschnitt steht. Wir müssen daher von verschiedenen Fragen der Raum-Zeit-Lehre absehen, und gehen nur auf die in unserem Zusammenhang wichtigsten Punkte ein. 
der räumlichen Koinzidenz zweier Punkte (zweier kleiner Körper, zweier Markierungen auf Körpern, etc.).

Es gilt also

III) Meßverfabren lassen sich nur mithilfe anschaulicher Begriffe erklären.

Durch Meß- oder Beobachtungsverfahren erklärte Begriffe sind in einem hinreichend weiten Sinn - operativ definierte Begriffe. Die Grundlage solcher Definitionen bilden Begriffe der Dingsprache, für deren Anwendung es anschauliche Kriterien gibt.

Die durch Meßverfahren eingeführten Begriffe müssen gewissen Forderungen genügen, die erstens ihre eindeutige Anwendbarkeit garantiereri und die zweitens sicher stellen, daß sie die mathematischen Eigenschaften der entsprechenden geometrischen Beziehungen haben. So sind z. B. Längenmessungen so zu erklären, daß die damit ermittelten Abstände die mathematischen Axiome für Abstandsfunktionen erfüllen. Daß die Messungen solche Abstände ergeben, ist eine empirische Feststellung. Daher gilt auch

IV) Die Adäquatheit der durch Meßverfabren festgelegten Begriffe hängt von empirischen Bedingungen ab.

Die Eindeutigkeit der Längenmessung mit unseren Einheitsstäben ist z. B. nur dann gegeben, wenn die Stäbe an jedem Ort und in jeder Richtung zur Deckung gebracht werden können. Das kann man empirisch prüfen. Dagegen kann man nicht empirisch prüfen, ob zwei Einheitsstäbe in verschiedenen Lagen dieselbe Länge haben. Das wird vielmehr durch die Konvention festgelegt, mit der wir die Einheitsstäbe als starr erklären.

Analog geht man bei der Zeitmessung vor, wobei hier Uhren als Maßstäbe verwendet werden. Die Verbindung von Raum und Zeit wirft nun neue Probleme auf. Wir wollen das an dem Problem der Gleichzeitigkeit von Ereignissen erläutern, die an verschiedenen Punkten stattfinden. Zunächst ist nur die Messung zeitlicher Distanzen von Ereignissen erklärt, die am selben Ort stattfinden, nicht aber die von Ereignissen, die an verschiedenen Orten stattfinden. Die Gleichzeitigkeit von Ereignissen im selben Punkt, ihre zeitliche Koinzidenz, ist ein Grundbegriff, der bei der Zeitmessung ebenso vorausgesetzt wird, wie jener der räumlichen Koinzidenz zweier Punkte (zur selben Zeit) bei räumlichen Messungen. Ohne eine solche zeitliche Koinzidenz feststellen zu können, könnten wir keine Zeitmessung mit Uhren vornehmen, da sie darauf beruht, daß wir den Ereignissen gleichzeitige Stellungen des Uhrzeigers zuordnen können. Um die Gleich- 
zeitigkeit von Ereignissen festzustellen, die an verschiedenen Punkten stattfinden, genügen anschauliche Kriterien jedoch nicht. Es ist z. B. nicht ,anschaulich“ evident, daß ein Ereignis in Peking im selben Moment stattfindet wie ein Ereignis in München. Im Sinne der NRZL würde man nun sagen: Wir versammeln Uhren einer bestimmten Bauart in einem Punkt, stellen sie gleich, und nachdem wir geprüft haben, $\mathrm{da} ß$ sie dort gleichlaufen, transportieren wir sie an die verschiedenen Stellen des Raums. Dann ist ein Ereignis E im Punkt $\mathbf{x}$ gleichzeitig mit einem Ereignis $\mathrm{F}$ im Punkt $\mathrm{y}$, wenn die Uhr in $\mathrm{x}$ für $\mathrm{E}$ dieselbe Zeit anzeigt wie die Uhr in y für F. Es ist wichtig zu sehen, daß hier eine Festlegung vorliegt und eine empirische Voraussetzung. Diese besteht darin, daß zwei Uhren im selben Punkt, egal wie wir sie dorthin transportieren, immer dieselbe Zeit anzeigen. Das können wir prüfen. Wir können dagegen nicht prüfen, ob Uhren in verschiedenen Punkten dieselbe Zeit anzeigen. „Dieselbe Zeit“ ist für verschiedene Punkte vielmehr erst zu definieren, und das tun wir mithilfe dieser Uhren. Daß sie in verschiedenen Punkten dieselbe Zeit anzeigen, also „starr" sind, ist eine Folge unserer Festlegung.

Gäbe es unendlich schnelle Signale, d. h. Wirkungen, die sich im Raum unendlich schnell fortpflanzen, so wäre die Gleichzeitigkeitsrelation eindeutig festgelegt: ${ }^{35}$ Das Ereignis $\mathrm{E}$ in $\mathrm{x}$ wäre genau dann gleichzeitig mit dem Ereignis $F$ in $y$, wenn ein von $x$ zugleich mit $E$ ausgesandtes Signal in y zugleich mit $\mathrm{F}$ ankommt. Nun zeigt die Erfahrung, daß es keine unendlich schnellen Signale gibt. Man kann aber auch mit Signalen endlicher Geschwindigkeit, z. B. mit Lichtsignalen, die Gleichzeitigkeit so bestimmen: $E$ ist gleichzeitig mit $F$ genau dann, wenn die zugleich mit $E$ und $F$ von $x$, bzw. y ausgehenden Lichtsignale simultan am Mittelpunkt $\mathrm{z}$ der Strecke von $\mathrm{x}$ nach y ein-

${ }^{35}$ Die Geschwindigkeit v eines Signals von $\mathrm{x}$ nach y läßt sich erst bestimmen, wenn bereits eine Gleichzeitigkeitsdefinition vorliegt. Denn v ist der Quotient der Entfernung von $\mathbf{x}$ und $\mathrm{y}$ und der zeitlichen Distanz zwischen Aussendung in $x$ und Ankunft in $y$. Diese Zeitdifferenz kann ich aber erst ermitteln, wenn ich sagen kann, zu weicher Zeit, von y aus gesehen, das Signal in $x$ abging. Man kann hingegen die Zeit messen, die ein von $x$ ausgehendes, in $\mathrm{y}$ reflektiertes und wieder nach $\mathrm{x}$ zurückkehrendes Signal für den gesamten Weg benötigt, ohne eine Definition der Gleichzeitigkeit für verschiedene Punkte vorauszusetzen. Trifft das Signal in $x$ zur selben Zeit ein, in der es von $\mathrm{x}$ ausgesandt wird, so hat das Signal unendliche Geschwindigkeit. 
treffen. Auch das ist eine korrekte Definition der Gleichzeitigkeit, nach der die Gleichzeitigkeit unter gewissen empirischen Voraussetzungen eine Äquivalenzrelation ist. ${ }^{36}$ Wichtig ist dabei, daß es keine schnelleren Signale gibt als die gewählten. Denn sonst könnte es sein, daß das Eintreffen des schnelleren Signals von $\mathrm{x}$ in $\mathrm{z}$ als später erklärt würde als der Zeitpunkt, zu dem es nach $\mathrm{x}$ zurückkehrt.

Diese Definition der Gleichzeitigkeit hat nun Einstein gewählt. Aus ihr folgt aber unmittelbar die Konstanz der Lichtgeschwindigkeit in allen Bezugssystemen, also die Forderung (4). Damit ergibt sich aus ihr auch eine ganz andere Struktur von Raum und Zeit - insbesondere die Abhängigkeit von Raum und Zeit - als in der NRZL. Es war die Leistung Einsteins, daß er durch methodologische Überlegungen von der Art, wie wir sie hier angestellt haben, gezeigt hat, daß in der NRZL mit der Festlegung der Gleichzeitigkeit entfernter Ereignisse eine Konvention steckt, die als solche nicht ,richtiger" ist als die Einsteinsche. Man kann es also auch so machen, und wenn man es so macht, ergibt sich der Ansatz der relativistischen RZL. Daß man es so machen sollte und nicht nach dem klassischen Uhrenverfahren, kann man nur im Blick auf physikalische Tatsachen begründen. Die erste ist die, daß die Beobachtungen die Annahme einer Lichtgeschwindigkeit nahelegen, die unabhängig vom Bezugssystem ist, wie das die Ergebnisse von Michelson und Morley taten. Andernfalls wäre die Definition zwar nicht falsch, würde aber eine tiefgreifende Umgestaltung der Physik erfordern. Die zweite ist, daß die Lichtgeschwindigkeit die höchste (im Vakuum) erreichbare Geschwindigkeit ist; denn dazu wird sie durch die Lorentz-Transformationen erklärt, die sich mit (4) und dem Mechanischen Relativitätsprinzip ergeben. Auch dafür gab es experimentelle Hinweise. Endlich ist sicherzustellen, daß die empirischen Voraussetzungen der Adäquatheit dieser Definition erfüllt sind. Das ist aber nach den bekannten Tatsachen der Fall. (Hingegen ergibt sich aus der Relativitätstheorie, daß die empirischen Voraussetzungen der klassischen Definition der Gleichzeitigkeit, die Unabhängigkeit der Uhranzeigen vom Transportweg, nicht erfüllt sind.) Durch diese Definition der Gleichzeitigkeit wird nun auch die Form der Lorentz-Transformationen verständlich, insbesondere die Tatsache, daß die transformierten Zeitkoordinaten $t^{\prime}$ nach (5) nicht nur von $t$, sondern auch von $\mathrm{x}$ abhängen.

${ }^{36}$ Vgl. dazu z. B. Reichenbach (24), $\$ 7$ und (28), $\$ 27$. 
Wenn man, wie wir sagten, Meßvorschriften, d. h. die operativen Definitionen raum-zeitlicher Begriffe, im Blick auf physikalische Tatsachen oder Hypothesen auswählt, so wirft das folgendes Problem auf: Physikalische Tatsachen kann man erst dann feststellen, wenn man die raum-zeitlichen Begriffe bereits erklärt hat. Denn alle Aussagen der physikalischen Sprache werden unter Bezugnahme auf raum-zeitliche Größen formuliert; sie sind also ohne deren Definition gar nicht erklärt. Daß wir tatsächlich Meßvorschriften für raum-zeitliche Größen im Blick auf physikalische Tatsachen auswählen, ist klar. Wir verwenden z. B. nicht leicht deformierbare Gummistäbe für Längenmessungen, sondern Stäbe, von denen wir wissen, daß sie relativ starr sind, also z. B. Stäbe aus Metall; andernfalls ergäbe sich eine höchst komplexe Physik. Ebenso verwenden wir als Uhren regelmäßige periodische Prozesse, Prozesse also, die ihre Zustände immer in derselben Zeit durchlaufen und deren Periode weitgehend unabhängig ist von äußeren Einwirkungen. Ich könnte die Zeit auch mit einem unregelmäßigen periodischen Vorgang messen, z. B. mit meinem Pulsschlag, also den Abstand zweier Pulsschläge als Zeiteinheit wählen. ${ }^{37}$ Damit hätte ich dann per definitionem einen ganz regelmäßigen Pulsschlag, die Physik würde aber recht kompliziert, weil nun die physikalischen Prozesse im Universum, z. B. die Bewegung der Planeten um die Sonne, langsamer abliefen, wenn ich mich aufrege, Fieber habe oder Sport treibe.

All das besagt aber nur, daß ich die Meßvorschriften für die raumzeitlichen Größen so festlege, daß sich eine einfache Physik ergibt. $\mathrm{Daß}$ wir Konventionen im Blick auf gewisse Tatsachen so und nich: anders treffen, bedeutet nicht, daß sie zu Behauptungen würden, die man mit diesen Tatsachen begründen kann. Eine Physik ohne Geometrie und Chronometrie gibt es nicht, und daher kann man diese auch nicht physikalisch begründen. Das Verhältnis zwischen der Feststellung physikalischer Tatsachen und der Festlegung physikalischer Begriffe durch Meßverfahren ist also nicht von der Art ,erst das eine, dann auf dieser Grundlage das andere", sondern jedes Moment bedingt das andere: physikalische Tatsachen bestimmen unsere Auswahl von Meßverfahren, und diese die Begriffe, mit denen wir physikalische Tatsachen beschreiben. Das Ziel ist eine einfache Physik, und es

${ }^{37}$ Das Beispiel gibt Carnap in (66), S. $83 \mathrm{ff}$ an. 
wird durch schrittweise Anpassung von Begriffsbildung und Erkenntnisfortschritt erreicht. ${ }^{38}$

Die erkenntnistheoretisch wichtigsten Einsichten zur Raum-ZeitLehre, die sich aus der Diskussion der Relativitätstheorie ergeben haben, wurden oben in den Sätzen I bis IV festgehalten. Inwiefern stützen nun diese Aussagen unsere These von der Bezogenheit des Physischen auf Psychisches? Beobachtungsverfahren sind im normalen Sinn des Wortes Handlungsweisen, die zu Erkenntnissen des Bestehens von Sachverhalten führen, also Überzeugungen vermitteln. Insofern sind Aussagen über Beobachtungen sicher Aussagen über Psychisches. Wir müssen aber zwischen Aussagen über Beobachtungen (als Sachverhalten des Beobachtens) und Aussagen über Beobachtungsresultate (als beobachteten Sachverhalten) unterscheiden. Die ersteren sind immer Aussagen über Psychisches, nicht aber die letzteren. Die Gleichzeitigkeit von zwei Ereignissen E und F, die an den Orten $\mathbf{x}$ und y stattfinden, wird, wie wir sahen, so erklärt, daß ein in der Mitte $\mathrm{z} z$ wischen $\mathrm{x}$ und $\mathrm{y}$ befindlicher Beobachter zwei simultan mit $\mathrm{E}$, bz.w. $\mathrm{F}$ von $\mathrm{x}$, bzw. y ausgehende Lichtsignale gleichzeitig wahrnimmt. Daraus folgt natürlich nicht, daß eine Aussage über die Gleichzeitigkeit von $\mathrm{E}$ und $\mathrm{F}$ eine Aussage über Beobachtungen ist; sie ist nur eine Aussage darüber, was jemand in $z$ beobachten würde, $d$. h. über das Resultat seiner Beobachtung, über die Koinzidenz des Eintreffens der Lichtsignale in z. Das ist aber ein physikalischer Sachverhalt. Wir versuchen hier nicht, physikalische Aussagen als psychologische zu deuten - das wäre offenbar absurd -, sondern wir argumentieren gegen die dualistische These $\mathrm{D}^{+}$aus 7.4. Und in unserem Fall sieht dieses Argument so aus: Wir bezeichnen Sachverhalte p nur dann als mögliche Resultate von Beobachtungen, wenn die Tatsache, daß jemand beobachtet, daß p, in der Regel (d.h. wenn nicht außergewöhnliche Umstände vorliegen) ein hinreichendes Kriterium für das Bestehen von $p$ ist. In unserem Beispiel ist die operative Definition der Gleichzeitigkeit von Ereignissen an verschiedenen Orten nur dann brauchbar, wenn die Feststellung der zeitlichen Koinzidenz der Signale in z unproblematisch ist, wenn der einzelne Beobachter darüber mit Si-

${ }^{38}$ Auf den Zusammenhang zwischen physikalischer Sprache und Theorie gehen wir im Kap. 9 ausführlicher ein. 
cherheit urteilen kann und wenn verschiedene Beobachter in ihren Urteilen übereinstimmen. Man kann also nicht behaupten, die Geltung physikalischer Aussagen über Gleichzeitigkeit sei völlig unabhängig von unseren Beobachtungen, denn ihre Geltung läßt sich allein durch Beobachtungen feststellen. Die Ereignisse $E$ und $F$ sind gleichzeitig genau dann, wenn die Signale in z simultan eintreffen. Sie treffen aber in $z$ in der Regel genau dann simultan ein, wenn es uns so erscheint, und das gilt, wie wir in 7.4 sahen, analytisch. Die Aussage ,,E und F sind gleichzeitig" ist also z. B. nicht verträglich mit der Aussage „Niemand erscheint es so, als ob die Signale in z koinzidieren." Die Bestimmung der Gleichzeitigkeit ist nur sinnvoll, wenn Beobachtungen über zeitliche Koinzidenzen in einem Punkt in der Regel zuverlässig sind. Wie wir in 7.4 gesehen haben, ist diese Zuverlässigkeit in Einzelfällen empirisch überprüfbar, aber nicht allgemein: gewisse Beobachtungen müssen wir als zuverlässig voraussetzen, um überhaupt zu empirischen Aussagen über die Welt zu kommen. In unserem Beispiel könnten wir, wenn wir der direkten Beobachtung zeitlicher Koinzidenzen nicht trauen, z. B. ein automatisch arbeitendes Instrument verwenden, das von $\mathrm{x}$ in $\mathrm{z}$ eintreffende Lichtsignale durch Striche auf der linken, von y eintreffende durch Striche auf der rechten Seite eines durchlaufenden Papierstreifens markiert, so daß gleichzeitig in $\mathrm{z}$ ankommende Signale durch koinzidierende Striche erfaßt werden. Dieser Registrierungsvorgang ist dann ein rein physikalischer Prozeß. Als Meßinstrument ist der Apparat aber nur dann brauchbar, wenn er erstens korrekt funktioniert, und wenn zweitens - und darauf kommt es uns hier an - seine Anzeigen sich unproblematisch ablesen lassen. Die Verbindung zwischen Beobachtungen und physikalischen Sachverhalten ist damit nicht eliminiert, sondern nur verschoben.

Physikalische Aussagen können wir nur deswegen begründen, weil es einfache physikalische Sätze gibt, die sich ihrem Sinn nach durch Beobachtungen entscheiden lassen. Und sie haben ihren empirischen Sinn nur aufgrund der Definitionen, welche die Kette der physikalischen Terme mit Beobachtungsbegriffen verbinden, die einen anschaulichen Gehalt haben. Wir haben das am Beispiel der Aussagen über Gleichzeitigkeit illustriert, im Sinn der Feststellung I gilt es aber für alle Aussagen der Physik.

Es ist oft behauptet worden, aus der Relativitätstheorie und den mit ihr verknüpften erkenntnistheoretischen Ideen zur Raum-Zeit-Lehre ergäben sich keine Einwände gegen die klassische Konzeption einer 
von Beobachtungen und Erfahrungsweisen unabhängigen Natur. ${ }^{39}$ Wenn die Rolle der Konventionen in der RZL und allgemein in der relativistischen Physik gegenüber der klassischen besonders hervorgehoben werden, so bedeute das nur, daß man nicht von der wahren Struktur von Raum und Zeit reden kann, sondern daß Aussagen über Raum und Zeit ihre Geltung relativ zu gewissen Meßvorschriften haben. Diese Meßvorschriften sind Bedeutungspostulate, die den physikalischen Aussagen erst ihren Sinn geben. Daß aber der Sinn und damit die Wahrheitsbedingungen von Aussagen von semantischen Konventionen abhängen, sei eine bloße Trivialität. Daraus folge nicht, daß die Aussagen irgendwelche subjektiven Bedeutungskomponenten enthalten.

Unsere Erörterungen haben jedoch keineswegs nur diese triviale Tatsache der Konventionalität sprachlicher Bedeutungen zutage gefördert. Der zentrale Gedanke war nicht: „Aussagen über die Natur sind erst möglich, wenn wir den physikalischen Termen (per Konvention) Bedeutungen zuordnen“, sondern: „Aussagen über die Natur sind erst möglich, wenn wir den physikalischen Termen durch $M e \beta$ vorschriften Bedeutungen zuordnen" ${ }^{40}$ Die Aussagen einer physikalischen Theorie erhalten erst dann einen empirischen Sinn, wenn wir sie mithilfe von Beobachtungsprädikaten deuten, die einen anschaulichen Sinn haben und deren Anwendungen aufgrund dieses Sinnes in der Regel unproblematisch sind. Da Aussagen mit solchen Prädikaten

${ }^{39}$ Vgl. dazu z. B. Reichenbach (49) und Grünbaum (63), S. $367 f$.

${ }^{40}$ Auch Grünbaum betont in (63), Kap. 4, daß die Konventionen der RaumZeit-Lehre nicht nur semantische sind. Er begründet das aber durch die Behauptung, die hier auftretende Konventionalität sei gewissermaßen der Geometrie als solcher immanent, da der Raum zunächst ein bloßes Kontinuum sei, dem man verschiedene metrische Strukturen aufprägen kann. Als ,Kontinuum" bezeichnet man aber in der Topologie spezielle metrische Räume, d. h. Räume, denen bereits eine Metrik aufgeprägt ist. Bei Grünbaum ist also zumindest die Ausdrucksweise irreführend. Man kann auch nicht sagen, der (reale) Raum sei nur (qualitativ) topologisch bestimmt; er habe nur eine bestimmte topologische Struktur, die mit verschiedenen Metriken verträglich sei. Die Auffassung, Konventionen spielten erst für die Feststellung der metrischen Struktur des Raums eine Rolle, nicht aber für die Festlegung seiner topologischen Struktur, wäre völlig falsch. Auch von ,,der Topologie des realen Raums" kann man erst bzgl. operativer Definitionen der topologischen Begriffe sprechen. 
nicht mit belicbigen Alissagen über unsere Erfahrungen verträglich sind, gilt das für alle physikalischen Aussagen. Die Natur, von der die Physik spricht, ist also nicht in der Weise unabhängig von allem Psychischen, wie das der absolute Realismus behauptet. Wie in 7.4 ist aber zu betonen, daß die Abhängigkeit physikalischer Aussagen von psychologischen dadurch verschleiert wird, daß die Verbindung zwischen Erfahrungsweisen und objektiven Tatbeständen recht komplex ist. Wir können z. B. nicht immer sagen, zwei Punkte koinzidierten, wenn es uns so erscheint, sondern das gilt nur unter der Voraussetzung, daß die Beobachtungen unter Normalbedingungen erfolgen; Normalbedingungen sind aber teilweise wieder in physikalischer Sprache zu formulieren. Ferner gibt es für jede physikalische Größe nicht nur ein Meßverfahren, sondern mehrere, die verschiedenartigen Beobachtungen entsprechen. Der Sinn der physikalischen Terme wird nicht nurdurch operative Definitionen festgelegt, sondern auch durch die Gesetze, die einen Zusammenhang zwischen den verschiedenen physikalischen Größen herstellen; aber er muß immer auch durch operative Definitionen festgelegt werden.

Aus all dem ergibt sich natürlich keine Subjektivierung der Physik. Die Aussagen der Physik gelten objektiv, d. h. sie sind unabhängig von individuellen Meinungen, Erfahrungen und Erfahrungsweisen. Die Beobachtungssprache ist eine intersubjektive Sprache. Zum Sinn ihrer Terme gehören nur solche Momente anschaulichen Gehalts, die intersubjektiv hinreichend übereinstimmen. Und nur jene Beobachtungen werden als zuverlässig erachtet, die von anderen bestätigt werden.

Die Sprache der Physik ist so trotz ihrer Unanschaulichkeit nicht eine Sprache über eine von unserer Erfahrung und unseren Erlebnisweisen unabhängige Welt, sondern eine Sprache über die Natur, wie sie sich uns in unserer Erfahrung zeigt. Und obwohl die Physik Aussagen über Phänomene macht, die sich unserer Anschauung und direkter Erfahrung entziehen, stützen sich alle ihre Behauptungen ihrem Sinn wie ihrer Geltung nach auf das, was wir erfahren können. Über eine Natur, die völlig unabhängig von menschlicher Erfahrung wäre, könnten wir weder begründete Aussagen machen, noch überhaupt sinnvoll reden. Das ist der einfache Kern der methodologischen Aussagen zur Raum-Zeit-Lehre. 


\subsection{Grenzen der Objektivierbarkeit physikalischer Aussagen}

Am Beispiel von Aussagen über Raum und Zeit haben wir im letzten Abschnitt gesehen, daß Physik nicht Wissenschaft von der Natur an sich ist, sondern von der Natur, wie sie sich zeigt, wenn sie mit bestimmten Beobachtungsverfahren untersucht wird. ${ }^{41}$ Die zweite für die moderne Physik grundlegende Theorie, die Quantenmechanik, stützt diese Aussage in einem noch sehr viel stärkeren Maß. ${ }^{42}$

Die Quantenmechanik ist die Mechanik mikro-physikalischer Objekte, die Mechanik der Elementarteilchen wie Photonen, Elektronen, Protonen, usw. Sie verwendet dieselben Begriffe oder Größen, dieselben Observablen zur Kennzeichnung ihrer Objekte wie die klassische Mechanik, also z. B. die Größen Ort und Impuls. Der entscheidende Unterschied zur klassischen Mechanik liegt darin, daß sie nicht den Ablauf der Zustände eines Systems solcher Objekte beschreibt - weder in Form von deterministischen noch in Form von statistischen $\mathrm{Ge}$ setzen - , sondern nur angibt, welche Wahrscheinlichkeiten für künftige Meßergebnisse sich aus vergangenen Meßresultaten ergeben. Aus jeder für Prognosen brauchbaren empirischen Theorie müssen sich solche Wahrscheinlichkeiten ergeben. In der klassischen Physik erhält man sie aber aus der mehr oder minder genauen Kenntnis vergangener Zustände des Systems mithilfe von Gesetzen, die den Ablauf der Zustände des Systems selbst beschreiben. Die klassische Physik stellt

${ }^{41}$ Eine ähnliche Formulierung verwendet Mittelstaedt in (66), 161.

${ }^{42}$ Die Quantenmechanik hat sich in der Erfahrung bestens bewährt und ihre mathematische Formulierung genügt allen Anforderungen an Exaktheit. Ihre Interpretation hingegen ist bis heute, und gerade heute wieder, heftig umstritten, in einem unvergleichlich stärkeren $\mathrm{Maß}$ als etwa jene der Relativitätstheorie. Die Standardinterpretation der Quantenmechanik ist aber immer noch die vor allem von Bohr, Heisenberg und Born entwickelte Kopenbagener Deutung. Sie hat sich in den Diskussionen behaupten können, wenn auch manche Aussagen ihrer Väter präzisierungsbedürftig sind. Mit anderen Deutungsversuchen können wir uns hier nicht auseinandersetzen; wir müssen uns dazu auf einzelne kurze Hinweise beschränken. - Für die Kopenhagener Deutung der Quantenmechanik vgl. z. B. Heisenberg (30) und v. Neumann (32). Bibliographien zu Grundlagenproblemen der Quantenmechanik finden sich in Scheibe (67) und Nilson (76). 
also Theorien über das Verhalten von Objekten auf und leitet daraus Prognosen für künftige Beobachtungen ab. In der Quantenmechanik ist es hingegen nicht möglich, deterministische oder auch nur statistische Gesetze für die Zustände des Systems selbst anzugeben. Die Quantenmechanik ist schon als Theorie über den Zusammenhang von Beobachtungen formuliert und läßt sich nicht objektivieren zu einer Theorie über den Zusammenhang objektiver Ereignisse.

Diese Eigenart der Quantenmechanik läßt sich aus der Tatsache verstehen, daß sie es mit Mikroobjekten zu tun hat. Man kann Makroobjekte als Objekte charakterisieren, die sich im Prinzip störungsfrei beobachten lassen. Bei jeder Beobachtung oder Messung tritt das beobachtete System $S$ in Wechselwirkung mit einem beobachtenden System B, denn die Eigenschaften von S werden aus den Änderungen erschlossen, die B im Prozeß der Messung erfährt, und diese Änderungen geben nur dann Aufschluß über $S$, wenn sie von $S$ bewirkt werden; jeder Wirkung entspricht aber eine Gegenwirkung. Eine Messung an S kann nur dann als annähernd störungsfrei angesehen werden, wenn die Änderung, die S durch die Messung erleidet, sehr klein ist. Die Annahme einer im Prinzip störungsfreien Beobachtbarkeit besagt also, daß sich die Meßmethoden im Prinzip so verfeinern lassen, daß ihre Einwirkung auf das System S, an dem die Messung vorgenommen wird, beliebig klein wird. Das muß sich darin ausdrükken, daß das Resulat einer Messung am System S unabhängig davon ist, ob und ggf. welche anderen Messungen vorher am System durchgeführt worden sind. Nur dann, wenn eine Messung einer Eigenschaft störungsfrei ist, kann man die Eigenschaft dem System S selbst zusprechen. Ist die Messung dagegen nicht störungsfrei, so gibt das Meßresultat keine Auskunft über das System S selbst, sondern besagt nur etwas über das Produkt der Wechselwirkung zwischen B und S. Makroobjekte sind also nach der obigen Charakterisierung jene, denen wir die beobachteten Eigenschaften zuschreiben können. Wenn wir z. B. Objekte wie ein Tintenfaß oder einen Stern mit bloßem Auge oder einem Fernrohr beobachten, so ist der Einfluß der Messung auf sie (die Wirkung des auf sie auftreffenden und von ihnen reflektierten Lichts) so minimal, daß wir ihn vernachlässigen können, und daher können wir die beobachteten Eigenschaften diesem Objekt selbst zuschreiben. Mikroobjekte und -ereignisse lassen sich nur mithilfe makroskopischer Meßinstrumente beobachten. Wir erkennen sie nur aufgrund ihrer makroskopischen, für uns beobachtbaren Wirkungen, 
insbesondere mithilfe makroskopischer Meßinstrumente, die ihre Wirkungen registrieren. Bei Mikroobjekten können wir nun keine störungsfreie Beobachtbarkeit annehmen. Eine störungsfreie Messung ist nur dann gegeben, wenn die Kräfte, die nötig sind, um Anzeigen des Meßinstruments hervorzurufen, klein sind gegenüber den Kräften, die im System wirken; wenn also z. B. die Lichtstrahlen, die uns Anzeigen in einem Mikroskop liefern, den Bewegungszustand der Objekte, von denen sie reflektiert werden, nicht verändern. Da nun der Auslösemechanismus jedes Meßinstruments aus Mikroobjekten besteht, kann im Bereich der Mikroobjekte nicht allgemein eine störungsfreie Beobachtung angenommen werden. Gibt es aber keine störungsfreie Beobachtbarkeit, so kann man die beobachteten Eigenschaften oder Werte von Größen auch nicht objektivieren; man kann sie nicht den Objekten selbst zuschreiben, sondern nur sagen, daß S die beobachtete Eigenschaft bei Messung mit $B$ zeigt. ${ }^{43}$ Man könnte aus dem Produkt der Wechselwirkung von S und B (aus der Anzeige des Meßinstruments) nur dann auf den Zustand von $S$ vor der Messung zurückschließen, wenn erstens die mikrophysikalischen Gesetze und zweitens der Zustand von B vor der Messung genau bekannt wären. Nun kann man aber die mikrophysikalischen Gesetze nur mithilfe von Beobachtungen ermitteln. Man müßte also voraussetzen, daß es gewisse störungsfreie Beobachtungen gibt, die uns direkt etwas über die beobachteten Systeme selbst sagen. Die Annahme einer störungsfreien Beobachtbarkeit ist aber im Rahmen der Mikrophysik, wie gesagt, nicht sinnvoll. $\mathrm{Da}$ es für den Schluß von der Anzeige des Meßinstruments auf $S$ ferner auf die mikroskopischen Eigenschaften von B ankommt, bräuchte man für die Ermittlung des Zustands von B vor der Messung ein anderes Meßinstrument $B^{c}$, mit dem wir den $\mathrm{Zu}$ stand von $B$ vor der Messung ermitteln; die Messung von B mit B würde dann aber B wieder stören, usf. Es ist also letztlich Sache der Konvention - freilich einer Konvention, die sich dadurch bewähren

${ }^{43}$ Damit man überhaupt von einer Beobachtung von $S$ reden kann, wird man jedenfalls fordern, daß eine direkt nachfolgende Beobachtung derselben $\mathrm{Ei}$ genschaft dasselbe Resultat ergibt. Daß eine nachfolgende Beobachtung anderer Eigenschaften dasselbe Resultat ergibt, egal ob die erste durchgeführt wurde, kann man hingegen wegen des Mangels an Störungsfreiheit nicht annehmen. 
muß, daß sich mit ihr eine kohärente Beschreibung von Beobachtungsresultaten ergibt -, daß wir die Anzeige von B als Auskunft über eine Eigenschaft von $S$ ansehen. Diese Eigenschaft läßt sich aber $S$ nur bei Messung mit $B$ zuordnen. Die Eigenschaften von $S$ sind also solche, die sich bei Messungen zeigen, und die sich $S$ nicht unabhängig von Messungen zuschreiben lassen.

Die Quantenmechanik ordnet nun jedem System S ein System von Vektoren zu (einen Hilbert-Raum), die Wahrscheinlichkeiten für die Resultate künftiger Beobachtungen angeben. Wenn z. B. das System S nur aus einem Elektron besteht, so geben die Vektoren Wahrscheinlichkeiten für seine möglichen Orts- und Impulskoordinaten bei Orts-, bzw. Impulsmessungen an. Diese Wahrscheinlichkeiten lassen sich nicht als Wahrscheinlichkeiten dafür deuten, daß das Elektron sich unabhängig von Messungen an gewissen Orten befindet, bzw. einen bestimmten Impuls hat. Nach den obigen Erörterungen kann man erstens die gemessene Größe wegen der Störung des Systems nicht objektivieren, und zweitens ergäbe sich damit auch ein Widerspruch: Die Quantenmechanik verwendet den klassischen Wahrscheinlichkeitsbegriff, und danach existiert zu den Wahrscheinlichkeitsverteilungen für die Werte zweiter Größen $A$ und $C$ immer auch die gemeinsame Wahrscheinlichkeitsverteilung für die Kombination von Werten beider Größen. Sind aber die Größen inkommensurabel, d. h. ergibt eine Messung von $A$ mit nachfolgender Messung von $C$ einen anderen Wert von $C$, als wenn man zuerst $C$ und dann $A$ mißt, stört also eine A-Messung die C-Messung und umgekehrt, so gibt es keine solche gemeinsame Verteilung: Ist die Wahrscheinlichkeit, einen Wert $a_{i}$ von $A$ zu messen, gleich $r$, und die Wahrscheinlichkeit, einen bestimmten Wert $c_{k}$ von $C z u$ messen, gleich $q$, so errechnet sich die Wahrscheinlichkeit, zugleich $a_{i}$ und $c_{k} z u$ messen, nicht aus $r$ und $q$, da eine Messung von $a_{i}$ bewirkt, daß sich die Wahrscheinlichkeiten für die Resultate einer nachfolgenden Messung von $C$ ändern. Die quantenmechanischen Wahrscheinlichkeiten für Eigenschaften und Größenwerte, wie sie sich aus den Vektoren ergeben, sind also nicht absolute, sondern in dem Sinn bedingte Wahrscheinlichkeiten, daß sie Wahrscheinlichkeiten für diese Ergebnisse bei entsprechenden Messungen sind. Diese Nichtobjektivierbarkeit der Eigenschaften des Systems wird oft übersehen, und man sucht dann nach einer „Quantenlogik" als einer Logik von Aussagen, wie sie der Quantenmechanik angepaßt ist, in der also die Konjunktion zweier sinnvoller Aussagen 
nicht immer sinnvoll ist. ${ }^{44}$ Über dieser Logik wären dann nicht-klassische Wahrscheirlichkeitsbewertungen zu definieren. Abgesehen davon, daß nach den obigen Ausführungen die Zielsetzung einer solchen Quantenlogik als von Anfang an verfehlt erscheint, wäre ihre Bezeichnung als „,Logik“ insofern nicht gerechtfertigt, als jede Logik ja generell anwendbar sein soll. Diese "Logik" bezieht sich aber nur auf die quantenmechanischen Ereignisse, während man in der gesamten Mathematik der Theorie weiterhin die klassische Logik verwendet. $^{45}$

Auch von Objekten kann man in der Quantenmechanik grundsätzlich nur relativ zu Messungen sprechen; auch Gegenstände sind also nicht objektivierbar, und daher paßt der Substanzbegriff nicht zur Quantenmechanik. Wenn man von Prozessen absieht, bei denen Elementarteilchen zerfallen oder sich neu bilden, wenn also das System z. B. im gesamten Verlauf der Untersuchung aus $n$ Elektronen besteht, so kann man zwar sagen, daß bei den Messungen gewisse Eigenschaften der Elektronen erhalten bleiben, z. B. ihre Ruhmasse, ihre elektrische Ladung, ihr Spin. Befinden sie sich aber in Wechselwirkung, so kann man sie nicht unterscheiden; man kann nicht sagen, welches Elektron sich an welcher Stelle befindet. Zudem ist der $\mathrm{Zu}$ standsraum, in dem dieses System zu beschreiben ist, als Produkt-

${ }^{44}$ Vgl. dazu z. B. Birkhoff und v. Neumann (36), v. Weizsäcker (55), Reichenbach (44), Kochen und Specker (65), Mittelstaedt (59), (61), (66), Suppes (63), (66), Suppes und Zanotti (76), um nur einige Arbeiten aus der Vielzahl solcher Versuche zu erwähnen.

${ }^{45} \mathrm{~J}$. Sneed hat in (70) zwar betont, daß man die quantenmechanischen Wahrscheinlichkeitsaussagen jedenfalls in einigen Fällen als Aussagen über bedingte klassische Wahrscheinlichkeiten auffassen könne, er deutet aber diese bedingten Wahrscheinlichkeiten im Sinne der Wahrscheinlichkeitstheorie, d. h. so, daß die Wahrscheinlichkeit, bei Messung der Größe A das Resultat $a_{i}$ zu erhalten, die Wahrscheinlichkeit des Ereignisses 'A wird gemessen und der Wert $a_{i}$ ergibt sich' geteilt durch die Wahrscheinlichkeit von 'A wird gemessen' ist (falls diese nicht $O$ ist). Das ist aber insofern fragwürdig, als die Quantenmechanik ja dem Ereignis, daß gewisse Größen gemessen werden, keine Wahrscheinlichkeit zuordnet. Die Rede von ,,bedingten Wahrscheinlichkeiten" ist also so zu verstehen, daß die Vektoren des Hilbertraums für jede Größe A eine klassische Wahrscheinlichkeitsbewertung für die möglichen Werte von A bei Messung von $A$ angibt, aber nicht Wahrscheinlichkeiten für Werte, die A unabhängig von Messungen hat. 
raum der Zustandsräume der einzelnen Elektronen anzusetzen. In diesem Zustandsraum gibt es meßbare Eigenschaften, die mit allen Eigenschaften der Teilräume inkommensurabel sind, die sich also nicht mit Eigenschaften der einzelnen Elektronen definieren lassen. ${ }^{46}$

$\mathrm{H}$. Reichenbach hat in (44) betont, daß Aussagen über unbeobachtete Objekte nicht nur in der Quantenmechanik, sondern grundsätzlich auch im Bereich von Makroobjekten ein erkenntnistheoretisches Problem bilden. Welchen Sinn haben die Aussagen über Existenz und Beschaffenheit unbeobachteter Objekte, die wir im Alltag wie in der klassischen Physik machen, und wie lassen sie sich begründen? Reichenbach schildert das Problem an einem einfachen Beispiel. Wir wollen ,annehmen, daß wir einen Baum ansehen und uns dann umdrehen. Wie können wir wissen, daß der Baum an seiner Stelle bleibt, wenn wir nicht hinsehen? Die Antwort, daß wir uns einfach wieder umwenden und so verifizieren können, daß der Baum nicht verschwunden ist, würde uns nicht helfen. Was wir auf diese Weise verifizieren, ist nur, daß der Baum immer $\mathrm{da}$ ist, wenn wir hinsehen; aber das schließt die Möglichkeit nicht aus, daß er immer verschwindet, wenn wir wegsehen, solange er nur wieder erscheint, wenn wir uns ihm wieder zuwenden. Solch eine Annahme wäre widerspruchslos durchführbar. Wir könnten nämlich annehmen, daß die Beobachtung eine gewisse Veränderung des Objekts verursache, obgleich es scheint, daß keine Veränderung vor sich gegangen ist. Wir haben kein Mittel, das Gegenteil dieser Annahme zu beweisen. Um den Einwand auszuschalten, daß eine andere Person den Baum beobachten könne, während wir nicht hinsehen, und so die Aussage bestätigen würde, daß der Baum nicht verschwindet, beschränken wir unsere Annahme auf Fälle, wo niemand den Baum ansieht; damit schreiben wir die Kraft, den Baum zu reproduzieren, der Beobachtung eines jeglichen Menschen zu. Wir müssen noch die Möglichkeit ausschalten, daß wir die Existenz des Baumes aus gewissen Wirkungen erschließen, die man beobachten kann, selbst wenn man den Baum nicht ansieht, wie $z$. B. aus dem Schatten des Baumes. Zu diesem Zwecke nehmen wir an, daß die Gesetze der Optik sich immer dann ändern, wenn das Objekt nicht beobachtet wird, und daß z. B. ein Schatten da sein kann, ohne $\mathrm{da}$ ein Baum vorhanden ist. Das Argument beweist daher nur, daß eine Annahme, die die Existenz oder das Verschwinden unbeobachte-

${ }^{46}$ G. Ludwig bezeichnet solche Eigenschaften in (54), 172 als ,,ganzheitlich“. 
ter Objekte betrifft, mit einer Annahme über das Fortbestehen oder eine Änderung der Naturgesetze verbunden sein muß. Es wäre falsch, zu behaupten, daß es für die Annahme, der Baum verschwinde nicht, wenn wir nicht hinsehen, einen induktiven Beweis gebe oder daß diese Annahme sehr wahrscheinlich sei. Es gibt keinen solchen induktiven Béweis. Wir können nicht sagen: Wir haben den unbeobachteten Baum so oft unverändert vorgefunden, daß wir annehmen, es werde immer so bleiben.' Die für diesen induktiven Beweis benutzte Voraussetzung ist nicht wahr, denn tatsächlich haben wir nie einen unbeobachteten Baum gesehen. Wir haben oft gesehen, daß der Baum da war, wenn wir hinsahen; aus dieser Tatsache können wir induktiv schließen, daß der Baum immer wieder da sein wird, wenn wir hinsehen - aber es gibt keinen induktiven Beweis, der von dieser Tatsache zu Behauptungen über den unbeobachteten Baum führt. Darum können wir nicht einmal sagen, daß die unveränderte Existenz des unbeobachteten Objekts wenigstens wahrscheinlich sei“".47

Die Frage läßt sich verschärfen: Wieso können wir sagen, der jetzt beobachtete Baum sei mit jenem identisch, den wir früher beobachtet haben oder später beobachten werden? Welche Kriterien haben wir für die Identität von zu verschiedenen Zeiten beobachteten Objekten? Diese Frage läßt sich am besten diskutieren, wenn wir von Momentanobjekten ausgehen. Jedes Momentanobjekt a ist einem bestimmten Zeitpunkt $\mathrm{z}$ (a) zugeordnet, nur in diesem Zeitpunkt existiert es. Ist $z(a)=t$, so deuten wir das auch durch die Schreibweise $a^{t}$ an. Für Momentanobjekte $a^{t}$ und $b^{t^{t}}$ gilt, daß sie nur dann identisch sind, wenn sie im selben Zeitpunkt existieren; es gilt also $a^{t}=b^{t} \supset t=t^{t}$. Ausgehend von solchen Momentanobjekten gelangt man zu Objekten im normalen Sinn durch Einführung einer Relation $G$ der Genidentität zwischen Momentanobjekten. $G$ ist eine Äquivalenzrelation, und ein Objekt im normalen Sinn ist eine Äquivalenzklasse zu dieser Relation, d. h. eine Klasse von Momentanobjekten, die untereinander in der Relation $G$ stehen. ${ }^{48}$ Unser Problem besteht nun darin, wie eine sol-

47 Reichenbach (44), S. $29 f$.

${ }^{48}$ Ist $\mathrm{T}$ eine Menge von Zeitpunkten $t, t$, $\ldots$, auf der eine Ordnung $\leq$ (nicht später als) definiert ist, so soll für alle Momentanobjekte a,b gelten:

1. $G(a, b) \wedge z(a)=z(b) \supset a=b$

2. $G(a, b) \supset \wedge t(z(a)<t<z(b) \supset V x(G(a, x) \wedge z(x)=t))$

Betrachten wir nur die Genidentität von beobacbteten Momentanobjekten, so 
che Relation der Genidentität definiert werden soll - sie ergibt sich offenbar nicht aus der Natur der Sache - und welchen Sinn wir damit den Aussagen über Objekte geben wollen. Ziel der Einführung von Objekten ist es, Gesetze über den Zusammenhang von Beobachtungsergebnissen oder Naturgesetze einfach formulieren zu können. Erst die Einführung einer Genidentität erlaubt es, aussagekräftige Gesetze zu formulieren, ${ }^{49}$ und durch eine geschickte Festlegung dieser Relation kann man die Gesetze erheblich vereinfachen. Relationen der Genidentität werden also zusammen mit Gesetzen eingeführt, und die Gesetze liefern dann auch Kriterien für die Identität von Objekten, bzw. für die Genidentität von Momentanobjekten.

Die Einführung einer Genidentität auch für unbeobachtete Objekte setzt nicht nur eine zeitliche Ordnung von Beobachtungen, sondern eine objektive Zeit voraus. Es ist also eine Menge $T$ von Zeitpunkten einzuführen und eine Ordnungsrelation $\leq$ (nicht später als) auf $T$. Jeder Beobachtung $B$ wird ein Zeitpunkt $t(B) \varepsilon T$ zugeordnet, und es muß gelten: $t(B) \leq t\left(B^{c}\right)$ genau dann, wenn $B \leq . B^{c}$, wo $\leq$. eine vorausgesetzte zeitliche Ordnung für Beobachtungen sei. ( $\leq$. ist also die „,subjektive“, $\leq$ eine „objektive“ Zeitordnung.) Im übrigen ist man bei der Einführung der objektiven Zeitordnung frei. Man wird sie so festlegen, daß sich einfache Gesetze für die Objekte ergeben. $\mathrm{Da} \mathrm{Ge}-$ setze mit stetigen Veränderungen von Größen besonders einfach sind, kann man z. B. T so wählen, daß sich stetige Gesetze ergeben. Aussagen über Existenz und Beschaffenheit von Objekten setzen also eine Relation der Genidentität und die Einführung einer objektiven Zeit voraus, und beides wird man umgekehrt so einführen, daß einfache

gilt (2) nicht, auch nicht, wenn $T$ die Menge aller Zeitpunkte ist, in denen Beobachtungen stattfinden; denn nicht jedesmal, wenn etwas beobachtet wird, wird dasselbe Objekt beobachtet.

${ }^{49}$ Das kann man sich an einem einfachen Beispiel verdeutlichen. Wir gehen davon aus, daß sich der Inhalt einzelner Beobachtungen durch Molekularsätze einer prädikatenlogischen Sprache mit Identität formulieren läßt, deren Eigennamen Momentanobjekte bezeichnen. Aussagen, die sich auf verschiedene Zeitpunkte beziehen, enthalten dann keine gemeinsamen Eigennamen. Man verfügt also nur über eine aussagenlogische Sprache, in der sich keine Gesetze ausdrücken lassen. Nach Einführung einer Genidentität hat man dagegen die sehr viel stärkere Sprache der Prädikatenlogik zur Verfügung, in der sich auch Gesetze formulieren lassen. 
Aussagen über die Welt der Gegenstände resultieren. Theorie und $\mathrm{Be}-$ griffsbildung hängen hier aiso ebenso eng zusammen wie im Fall der Relativitätstheorie.

Eine entsprechende Position vertritt auch Kant: Die Gegenstände der Erkenntnis werden nicht in der Anschauung (wir sprachen von Béobachtungen) vorgefunden, sondern durch Verstandestätigkeit (wir sprachen von der Einführung von Begriffen) konstituiert. Zum Begriff des Gegenstandes gehört nach Kant, daß etwas (die Substanz) in der Zeit beharrt, daß es Gesetze gibt für die Veränderung der Gegenstände und eine objektive Zeitordnung. Diese Zeitordnung wird bei Kant definiert mithilfe der Ursache-Wirkungsrelarion, also unter Be-zugnahme auf Gesetze. Die Konstitution von Objekten hängt daher auch bei Kant unlösbar zusammen mit dem, was wir als Festlegung einer Genidentität bezeichnet haben, mit der Einführung einer Zeitordnung und der Formulierung von Gesetzen für die Objekte. Bei Kant findet sich so eine wesentlich tiefere Analyse von Aussagen über $\mathrm{Ob}$ jekte als im Phänomenalismus.

Die Festlegung einer Genidentität auch für unbeobachtete Objekte ist nur dann möglich und sinnvoll, wenn es Gesetze gibt, aufgrund deren wir auch den unbeobachteten Objekten Eigenschaften zuordnen und sie damit unterscheiden können. Das ist im makroskopischen Bereich möglich, wo es störungsfreie Beobachtbarkeit gibt, aber nicht, wie wir sahen, im Mikrobereich. Die Grenze zwischen Makro- und Mikrobereich ist also die Grenze einer Objektivierbarkeit unserer Aussagen über die Welt, die Grenze für die Möglichkeit, sinnvolle Aussagen über unbeobachtete Objekte zu machen. ${ }^{50}$ Wenn also Einstein sagt, die Kopenhagener Interpretation der Quantenmechanik verstehe die Realität im Sinne des Berkeleyschen esse est percipisi, so ist das richtig. Wenn er aber meint, eine solche Beschreibung der Mikrophänomene könne nicht das letzte Wort sein, die Physik habe es auch hier mit einer objektiven, von Beobachtungen unabhängigen Realität zu tun, so ist das eine unbegründete und, wie wir zu zeigen suchten, auch verfehlte Position.

${ }^{50}$ Diese Grenze ist natürlich nicht scharf: Von der Quantenmechanik her kann man nur sagen, daß Objekte oder Phänomene in einer mehr oder minder guten Näherung als Makroobjekte angesehen werden können.

${ }^{51}$ Vgl. Schilpp (49), Bd. 2, 669. 
Die für uns zentrale Frage ist die nach der Rolle des Subjekts, des Beobachters in der Quantenmechanik. Wir haben schon gesehen, daß die Aussagen, welche die Quantenmechanik über ein System macht, Aussagen über die Wahrscheinlichkeit von Resultaten von Beobachtungen am System sind, und daß sich beobachtete Eigenschaften und Objekte nicht objektivieren lassen, daß also aus den Aussagen über Becbachtungsresultate keine Aussagen über das unbeobachtete System folgen. All das spricht prima facie dafür, daß die Aussagen der Quantenphysik ihrem Gehalt nach wesentlich auf menschliche Beobachtungen und Beobachter bezogen sind. Diese Subjektbezogenheit der Quantenphysik wird jedoch vielfach bestritten. Beobachtungen, so wird oft behauptet, haben in der Quantenphysik keine andere Funktion als in der klassischen Physik: Um Feststellungen über die Natur zu machen, Naturgesetze zu entdecken und Hypothesen zu prüfen, müssen wir natürlich Beobachtungen machen, aber diese Beobachtungen kommen in den Aussagen der Quantenmechanik nicht vor, bzw. sie lassen sich eliminieren. Beobachtungen von mikrophysikalischen Ereignissen werden mit Meßinstrumenten vorgenommen. Diese Meßinstrumente sind physikalische Objekte, die sich quantenmechanisch beschreiben lassen. Insbesondere läßt sich auch die Wechselwirkung des Objektsystems $S$ mit dem Instrument I beschreiben. Sie bewirkt, daß sich am Instrument eine gewisse Anzeige einstellt, und da I nur dann als Meßinstrument brauchbar ist, wenn seine Anzeige ein makroskopischer, für uns beobachtbarer Zustand ist, ist die Anzeige auch ein objektives Phänomen, das unabhängig davon besteht oder nicht besteht, ob wir es beobachten, d. h. ob wir die Anzeige ablesen. Die menschliche Beobachtung vollzieht sich also im Makrobereich, während der eigentliche Meßvorgang völlig unabhängig vom Beobachter abläuft. ${ }^{52}$

Diese Behauptung ist nun zu prüfen. Wir haben gesehen, daß die Quantenmechanik aufgrund der Informationen, die wir aus vergangenen Messungen am System gewonnen haben, Aussagen über die Wahrscheinlichkeiten von Resultaten künftiger Messungen macht. Den Inbegriff dieser Information über den Ausgang der verschiedenen möglichen Messungen am System, der (im reinen Fall) durch einen Vektor im Hilbertraum dargestellt wird, bezeichnet man etwas

52 Vgl. dazu z. B. Ludwig (53),(54a). 
unglücklich als „Zustand des Objektsystems“ - besser würde man von einer Zustandsangabe über das System reden. Dieser Zustand verändert sich in zweifacher Weise in der Zeit: Er verändert sich in stetiger Weise (nach der Schrödingergleichung), wenn keine Messungen vorgenommen werden - das bezeichnet man auch als Eigenbeweguing des Systems - und er verändert sich in unstetiger Weise bei einer Messung - das bezeichnet man als Zustandsreduktion. Dabei geht das System in den Zustand (den ,Eigenvektor") $\mathfrak{a}_{i}$ über, der dem ermittelten (Eigen-)Wert $a_{i}$ der gemessenen Observablen A entspricht. Wenn nun das obige Argument korrekt ist, so muß sich die Zustandsreduktion in zwei Prozesse aufspalten lassen: Beim ersten Prozeß, den G. Süßmann in seiner Analyse des Meßprozesses in (58) als Aufnabme bezeichnet, geht $\mathrm{S}$ aus dem Zustand $\mathbf{r}$ vor der Messung (vor der Wechselwirkung mit dem Instrument I) in ein Gemisch aus den Eigenvektoren $\boldsymbol{a}_{\mathrm{i}}$ der gemessenen Observablen A über, $d . h$. in einen Zustand, der einer Angabe entspricht, daß sich S mit den Wahrscheinlichkeiten $\mathrm{w}_{\mathrm{i}}$ in den Zuständen $\mathfrak{a}_{\mathrm{i}}$ befindet. Und I geht aus seinem $\mathrm{Zu}$ stand $\boldsymbol{y}$ vor der Messung in ein Gemisch aus den Zuständen $\boldsymbol{A}_{i}$ über, in denen es die Werte $a_{i}$ anzeigt. Da I, wie gesagt, nur dann als Meßinstrument für die Observable A brauchbar ist, wenn die Objektzustände $\mathfrak{a}_{\mathrm{i}}$ den Anzeigezuständen $\boldsymbol{A}_{\mathrm{i}}$ eineindeutig entsprechen, müssen dabei die Wahrscheinlichkeiten $\operatorname{der} \boldsymbol{A}_{\mathrm{i}}$ mit jenen der $\mathfrak{a}_{\mathrm{i}}$ gleich sein. Der zweite Prozeß ist dann die Ablesung, bei der ein Beobachter den wahren Wert von I und damit den wahren Zustand von S feststeilt. Dabei ist die Funktion des Beobachters dieselbe wie in der klassischen Physik, z. B. in der statistischen Mechanik, wo die Theorie nur statistische Aussagen macht und man durch Beobachtung feststellt, welcher der mit Wahrscheinlichkeit vorausgesagten Fälle tatsächlich vorliegt. Nach dem Argument muß dabei, und das ist der entscheidende Punkt, der Prozeß der Aufnahme quantenmechanisch ohne Benutzung des Projektionspostulats, d.h. des Gesetzes über die Zustandsreduktion zu beschreiben sein. D. h. die Aufnahme muß sich als Eigenbewegung von $\mathrm{S}+\mathrm{I}$ beschreiben lassen. Die Unstetigkeit soll allein durch die Ablesung hereinkommen.

Das gilt aber nicht: Solange man Prozesse betrachtet, die sich allein mit der Schrödingergleichung beschreiben lassen, kann kein Informationsverlust eintreten. Die Aufnahme, d. h. der Übergang von dem reinen Fall $\mathbf{r}$, der auch Aussagen über die Resultate der Messungen von mit A inkommensurablen Observablen erlaubt, zu dem Gemisch 
bedeutet jedoch einen Informationsverlust bzgl. solcher Aussagen. ${ }^{53}$ Dieser Informationsverlust resultiert daraus, daß im Gemisch die Interferenzterme zwischen den Objektzuständen $\boldsymbol{a}_{\mathrm{i}}$ fehlen; sind sie vorhanden, so kann man nicht sagen, daß sich S, bzw. I in einem der $\mathrm{Zu}$ stände $\mathfrak{a}_{\mathrm{i}}$ bzw. $\boldsymbol{A}_{\mathrm{i}}$ befindet. Der Wegfall dieser Terme ist Voraussetzung für das Resultat der Aufnahme. Er ergibt sich spätestens bei der Ablesung, ist aber bei der Trenrung von Aufnahme und Ablesung eigens zu begründen. Dafür gibt es in der Quantenmechanik jedoch kein Prinzip, wenn man das Projektionspostulat ausklammert. Vielmehr macht man dann eine Zusatzannahme, die nur damit zu rechtfertigen ist, daß sich I als Makroobjekt schon vor der Ablesung in einem der Zustände $\boldsymbol{A}_{\mathrm{i}}$ befinden muß. ${ }^{54}$ Wegen der eineindeutigen Korrespondenz zwischen den Zuständen von $S$ und jenen von I, die wir bei einem Meßinstrument voraussetzen, ist das aber nicht besser gerechtfertigt als die Annahme, $S$ befinde sich nach der Wechselwirkung unabhängig von einer Beobachtung in einem der Zustände $\mathfrak{a}_{\mathfrak{i}}$. Das Argument läßt sich also auch umkehren: Es gibt Zustände makroskopischer Objekte, wie z. B. der Meßinstrumente, die man nach der Quantenmechanik wegen der Kopplung mit Mikrozuständen nicht objektivieren kann.

Der Meßprozeß läßt sich daher nicht zerlegen in die Ablesung und einen Prozeß, der im System S+I selbst ohne einen Beobachter abläuft. Wenn man das Meßinstrument I als Teil des ursprünglich beobachtenden Systems B zum beobachteten System rechnet, das dann aus $\mathrm{S}+\mathrm{I}$ besteht, muß man ein System B' einführen, mit dem man S+I beobachten kann. Die Überführung von Zuständen von $S+I$ in solche Zustände, die eine bestimmte Anzeige von I (und damit einen bestimmten Zustand $\boldsymbol{a}_{\mathrm{i}}$ von $S$ ) anzeigen, ist dann quantenmechanisch mit einer Anwendung des Projektionspostulats auf diese Messung mit B'

${ }^{53}$ Das gilt auch für den Fall, daß sich $\mathrm{S}$ schon vor der Messung in einem Gemisch befindet. Vgl. dazu Süßmann (58).

${ }^{54}$ Das hat Schrödinger in (35) als Einwand gegen die Quantenmechanik formuliert in einem als "Schrödingersche Katze" berühmt gewordenen Beispiel: $\mathrm{Da}$ die Anzeigen des Meßinstruments makroskopische Phänomene sind und also von Beobachtungen unabhängig, kann die quantenmechanische Beschreibung nicht vollständig sein, da sich aus ihr diese Beobachtungsunabhängigkeit nicht ergibt. Ein ähnliches Argument verwendet auch Einstein in Schilpp (49), Bd. II, $667-671$. 
zurückzuführen. Man kann also den „Schnitt“ zwischen beobachtendem und beobachtetem System verschieben, man kommt aber nicht ohne ein beobachtendes System aus, und zu diesem gehört immer ein Beobachter. ${ }^{55}$ Anders ausgedrückt: Man kommt nicht ohne das Projektionspostulat aus, und dieses Postulat charakterisiert nicht nur den Prozeß des Ablesens von Objektzuständen, sondern die Herstellung einer Situation, in der man überhaupt von abzulesenden Objektzuständen reden kann.

Wir können also das Ergebnis dieser Diskussion so zusammenfassen: Die Quantenphysik ist die Physik des Mikrobereichs. Dieser Bereich ist uns nur zugänglich durch die Effekte, die mikrophysikalische Ereignisse auf makrophysikalische Ereignisse haben, also insbesondere durch Messungen mit makroskopischen Meßinstrumenten. Die Beobachtungsverfahren und die physikalischen Systeme, auf die sie angewendet werden, sind in der Sprache für Makroobjekte, in der Sprache der klassischen Physik zu beschreiben. Diese ist also eine methodische Voraussetzung der Quantenphysik; sie ergibt sich aber umgekehrt aus der letzteren für den Grenzfall hoher Quantenzahlen oder vernachlässigbarer Unschärfen. Im Bereich der Mikro-Physik erweist sich eine Objektivierung der physikalischen Aussagen als unmöglich. ${ }^{56}$ Diese sind daher zu deuten als Aussagen über die Natur, so

${ }^{55}$ Die Verschiebbarkeit des Schnitts zwischen Subjekt und Objekt läßı sich durch eine Analogie mit der Sprache illustrieren. Hier unterscheiden wir zwischen dem Objektbereich, über den wir mit einer Sprache S reden, und der Sprache $S$ selbst. Man kann nun auch die Sprache $S$ zum Gegenstand der Untersuchung machen. Dazu benötigt man aber eine Metasprache MS, in der man über $S$ reden kann. Die semantischen Antinomien zeigen, daß man die Sprache $S$ nicht in ihr selbst vollständig beschreiben kann. Man kann also Sprache nicht vollständig objektivieren.

${ }^{56}$ Das setzt voraus, daß es keine ,,verborgenen Parameter" gibt, d. h. kein System von Größen, mit denen sich die Mikroobjekte objektiv beschreiben lassen, so daß die Aussagen der Quantenmechanik sich in einem ähnlichen Sinn als statistische Aussagen ansehen ließen wie die Aussagen der statistischen Thermodynamik, die man nach klassischer Auffassung im Prinzip durch deterministische mechanische Aussagen über die einzelnen Partikel z. B. eines Gases ersetzen könnte. Schon J.v. Neumann hat aber in (32), Kap. IV.2 gezeigt, daß die normalen Observablen sich nicht durch solche verborgenen Parameter definieren lassen, weil man auch sie sonst objektivieren könnte; das ist aber unmöglich, wenn die Quantentheorie richtig ist. Ab- 
wie sie sich bei Beobachtungen zeigt; als Aussagen über die Wahrscheinlichkeit von Beobachtungsergebnissen. ${ }^{57}$ Die Zustandsbeschreibungen des Systems sind Beschreibungen von Informationen über das System; es sind also nicht Aussagen über Objekte, sondern über Informationen, und enthalten damit ihrem Sinn, ihrer empirischen Interpretation nach - wenn auch nicht in ihrer mathematischen Formulierung - einen unverzichtbaren Bezug auf die Subjekte dieser Informationen. Der Vorgang der Beobachtung durch ein Subjekt spielt in der Quantenmechanik eine ganz andere Rolle als in der klassischen Physik; er wird durch das Projektionspostulat ausgedrückt. Darin ist zwar wiederum nicht explizit von Personen die Rede, die Anwendung eines Operators, der einer Observablen entspricht, ist aber physikalisch als Anwendung eines Meßverfahrens auf das System zu interpretieren, und nur bei dieser Interpretation ist die Zustandsreduktion als Reduktion von Informationszuständen sinnvoll. Daß das Subjekt in der Quantenmechanik nicht eliminierbar ist, daß es sich nicht vollständig als Teil des Objektsystems ansehen läßt, entspricht unseren Aussagen im 6. Kapitel und im Abschnitt 7.5.

\subsection{Das Leib-Seele-Problem}

Das Leib-Seele-Problem im weiteren Sinn ist das Problem des Verhältnisses von Psychischem und Physischem. Der Kern dieses Problems ist die Frage nach der psycho-physischen Wechselwirkung, die wir im Abschnitt 7.3 im Zusammenhang mit dem Dualismus diskutiert haben. Wir wollen uns nun überlegen, wie sich diese Frage von unserer Konzeption eines polaren Verhältnisses von Psychischem und Physischem her darstellt.

gesehen davon, daß sich solche Parameter nicht messen lassen, ist ihre Einführung in dem Sinn fiktiv, daß sie neben den normalen Observablen stünden und zur Voraussage von Beobachtungen nichts beitrügen.

${ }^{57}$ Man kann aber nicht behaupten, die Quantenphysik sei eine Theorie von Beobachtungen, denn sie kann natürlich nicht vorhersagen, wann welche Messungen durchgeführt werden. Ihre Voraussagen haben die Form: ,,Wenn am System S im Zeitpunkt t eine Messung der Observablen A vorgenommen wird, so wird sich dabei mit der und der Wahrscheinlichkeit dies und jenes Resultat ergeben". 
Vorweg ist noch einmal zu betonen, daß dieses Problem nicht in der Frage besteht, ob es psycho-physische Wechselwirkungen gibt das ist (so, wie wir die Begriffe ,Ursache' und,Wirkung' heute verwenden) unbestritten. Es besteht auch nicht in der Frage, welche Zusammenhänge konkret zwischen Physischem und Psychischem gelten. Das herauszufinden ist Sache empirischer Forschung, und die Philosophie kann dazu nichts beitragen. ${ }^{58}$ Aufgabe der Philosophie ist es lediglich, grundsätzliche Schwierigkeiten aus dem Weg zu räumen, denen ein Verständnis solcher Beziehungen begegnet. Diese Schwierigkeiten sind nicht allein durch die Philosophie selbst, insbesondere durch den philosophischen Dualismus geschaffen worden, sondern sie ergeben sich auch aus der üblichen Auffassung der physischen $\mathrm{Na}$ tur. Wird kein Physikalismus vertreten - für den sich das Leib-SeeleProblem von vornherein nicht stellt - , so wird meist angenommen, das Physische sei ein in sich geschlossener Bereich, in dem sich alle Phänomene ohne Bezugnahme auf Psychisches beschreiben und erklären lassen. Dann kann es zumindest keine physikalischen Wirkungen psychischer Vorgänge geben. Wie wir in 7.3 gesehen haben, ergibt sich damit aber auch schon eine dualistische Konzeption. Nun wurde in den letzten beiden Abschnitten betont, daß diese Konzeption des Physischen von der modernen Physik selbst aufgegeben worden ist. Wir haben insbesondere gesehen, daß die Quantenmechanik nur Aussagen über beobachtbare Größen macht, daß die Beobachtung im mikrophysikalischen Bereich einen Eingriff in das beobachtete System bedeutet, daß aber die Quantenmechanik nichts darüber sagt und sagen kann, wann und wo welche Beobachtungen stattfinden. Sie begreift also die physische Natur nicht als ein in sich geschlossenes System, sondern als System, das durch Beobachtungen, d. h. durch menschliches Handeln beeinflußt wird. Der Schnitt zwischen beobachteter Natur und Beobachter kann, wie wir sahen, verschoben, aber er kann nicht so angesetzt werden, daß der physikalische Effekt der Beobachtung rein physikalisch erklärbar wäre.

Von der modernen Physik her ergeben sich somit zumindest keine grundsätzlichen Schwierigkeiten, die Existenz psycho-physischer Wechselwirkungen anzunehmen. Das gilt nur dann, wenn man von ei-

${ }^{58}$ So etwas wie eine vollständige Theorie psycho-physischer Wechselwirkung ist nach den Überlegungen in 6.4 allerdings nicht zu erwarten, nach denen es keine vollständige Theorie des Psychischen geben kann. 
ner Konzeption ausgeht, nach der Physisches und Psychisches eigenständige, voneinander unabhängige Realitätsbereiche bilden. Dann entsteht in der Tat eine Aporie: Einerseits gibt es offenbar Zusammenhänge zwischen beiden Bereichen, andererseits kann es sie nach einer solchen dualistischen Konzeption nicht geben. Diese Probleme entfallen für monistische Theorien, physikalistische, idealistische oder neutral-monistische. Zwischer ${ }_{1}$ Dualismus und diesen Monismen steht die in 8.1 umrissene Konzeption; in eine Aporie jedenfalls führt das LeibSeele-Problem auch hier nicht, da eine Unabhängigeit beider Bereiche von vornherein nicht angenommen wird.

Seelische Eigenschaften und Zustände sind, wie wir in 7.5 betont haben, nicht Eigenschaften oder Zustände seelischer Substanzen, sondern von Personen, die auch körperliche Eigenschaften haben. Derselbe seelische Zustand einer Person kann mit verschiedenen körperlichen Zuständen zusammen auftreten; er bestimmt diese also nicht eindeutig, aber er ist nicht mit allen körperlichen Zuständen verträglich. Es gibt in diesem Sinn keine „,bloß" psychischen Zustände, dic nicht auch körperliche Aspekte hätten. Wir haben in 7.4 gesehen, daß auch die dualistische These $\mathrm{D}+$ falsch ist, daß also psychologische Aussagen nicht mit beliebigen physikalischen Zuständen der Welt verträglich sind. In der Logik bezeichnet man die Menge der Zustandsbeschreibungen der Welt, in denen ein Satz A gilt, als logischen Spielraum von A. ${ }^{59}$ Der logische Spielraum eines Satzes ist danach ein Maß für seinen Gehalt: Je kleiner der Spielraum, desto größer der Informationsgehalt; desto mehr sagt er über die Welt aus, denn desto geringer ist die Anzahl der Weltzustände, in denen er gilt. Analog kann man die Menge der Beschreibungen der physikalischen Zustände, mit denen ein Satz verträglich ist, als physikalischen Spielraum dieses Satzes bezeichnen. Der physikalische Spielraum ist dann ein Maß für den physikalischen Gehalt eines Satzes, ${ }^{60}$ und unsere Behauptung war, daß alle kontingenten psychologischen Sätze einen nichtleeren physi-

${ }^{59}$ Auf semantischer Ebene entspricht dem Spielraum eines Satzes die Menge der möglichen Welten, in denen er gilt.

${ }^{60}$ Unter ,Verträglichkeit" ist hier nicht eine rein logische Verträglichkeit zu verstehen, sondern eine analytische Verträglichkeit, d. h. eine Verträglichkeit bei der zugrundegelegten Interpretation, der Sprache. Zwei Sätze (Satzmengen) sind in diesern Sinn verträglich, wenn es eine Welt gibt, in der sie beide gelten. 
kalischen Gehalt haben. Man kann diese Aussage so verschärfen: Jede psychologische Aussage über eine Person a ist nur mit gewissen, nicht aber mit allen physikalischen Aussagen über a (über den Körper von a und a's körperliches Verhalten) verträglich, und daher besagt sie auch etwas über die körperlichen Zustände von a.

Auch Handlungen haben sowohl seelische als auch körperliche Aspekte. Eine Beobachtung z. B. ist eine Handlung, bei der der Beobachter zu gewissen Überzeugungen gelangt, und zugleich ein körperliches Verhalten: er richtet seine Augen auf ein Objekt, hantiert mit Meßinstrumenten usw. Welche Feststellungen man bei einer Beobachtung macht, hängt auch von dem ab, was man dabei tut. Wir können also psychische und physische Momente an Handlungen unterscheiden, aber diese Momente sind nicht voneinander unabhängig. Wie Personen nicht aus einer seelischen und einer körperlichen Substanz bestehen, so setzt sich auch eine Handlung nicht aus einem psychischen und einem physischen Vorgang zusammen. Sie ist ein Vorgang, und psychologische wie physikalische Beschreibungen sind partielle Beschreibungen desselben Vorgangs, und sie erfassen nicht voneinander unabhängige Eigenschaften dieses Vorgangs. Die psychologische Beschreibung charakterisiert auch mehr oder minder deutlich den physischen Ablauf, und umgekehrt.

Der Anschein des Merkwürdigen, Unbegreiflichen psycho-physischer Wechselwirkungen, auf den wir in 7.3 hingewiesen haben, beruht darauf, daß man z. B. bei einer Wahrnehmung die physiologischen Prozesse als etwas rein Physikalisches ansieht, bei denen Seelisches noch gar nicht im Spiel ist, und dann natürlich den Sprung von rein physiologischen Vorgängen im Gehirn zu bloß psychischen Erlebnissen nicht verstehen kann; oder daß man den Entschluß zu einer Handlung als etwas bloß Seelisches ansieht, das noch gar nichts mit körperlichen Vorgängen zu tun hat, und dann die physiologischen Prozesse im Gehirn, den Nerven und Muskeln damit nicht in Verbindung bringen kann. Wittgenstein spricht vom „,Gefühl einer unüberbrückbaren Kluft zwischen Bewußtsein und Gehirnvorgang" und sagt: „Diese Idee dieser Artverschiedenheit ist mit einem leisen Schwindel verbunden, - der auftritt, wenn wir logische Kunststücke ausführen «. ${ }^{61}$ Dieses „,Kunststück“ ist eben die Annahme einer Unabhängigkeit beider Vorgänge. Die physiologische Beschreibung von

61 Wittgenstein (53), $\$ 412$. 
Wahrnehmungsprozessen charakterisiert denselben Vorgang, der von Anfang an auch ein psychologischer Vorgang ist, z. B. ein Fixieren eines Gegenstandes; und der Entschluß, etwas zu tun, vollzieht sich nicht nur auf seelischer Ebene, sondern er ist von Anfang an auch ein physiologischer Prozeß.

So verstanden ist die Rede von einer psycho-physischen „Wechselwirkung" oft schief. Bei einer Wahrnehmung bewirken nicht die physiologischen Prozesse im Gehirn ein Erleben, denn sie gehen dem Erleben nicht vorher, sondern sind schon mit Erleben verbunden, und bei einer Handlung bewirkt nicht der Entschluß Vorgänge im Gehirn, sondern er findet gleichzeitig mit ihnen statt: Psychologische und physiologische Aussagen charakterisieren verschiedene Aspekte desselben Vorgangs. Psycho-physische Zusammenhänge stellen sich also oft als Koexistenz-, nicht aber als Sukzessionsgesetze dar, so daß man nicht einen Zustand als Ursache des anderen bezeichnen kann, sondern ein Verhältnis wechselseitiger Bestimmung anzunehmen hat.

Auch in Fällen, in denen man von physikalischen Ursachen psychologischer Vorgänge sprechen kann, ist nicht immer ein Kausalgesetz anzunehmen, nach dem auf physikalische Zustände gewisse psychologische folgen. Und dasselbe gilt für psychologische Ursachen von Physischem. Ursache eines Ereignisses $\mathrm{p}$ im normalen Sprachgebrauch ist nicht die Antezedensbedingung einer kausalen $D N$-Erklärung von $\mathrm{p}$, sondern ein Ereignis $\mathrm{q}$, das $\mathrm{p}$ vorausgeht und für das der Satz ,p, weil q" gilt. ${ }^{62}$ Viele Behauptungen über physische Ursachen von Psychischem oder psychische Ursachen von Physischem sind in diesem Sinn zu verstehen. Wenn wir z. B. sagen, daß einem Beobachter ein Blatt Papier als weiß erscheint, weil es weiß ist, so wollen wir damit nicht behaupten, daß allgemein gilt, daß uns weiße Dinge als weiß erscheinen. Das ist vielmehr nur unter normalen Bedingungen der Fall: bei normalen Beleuchtungsverhältnissen und einem normalen Funktionieren der Farbwahrnehmung. Während also in der einfachen Aussage „Das Blatt Papier erscheint dem Beobachter als weiß, weil es weiß ist", ein psychisches Ereignis mit einem physikalischen erklärt wird, würde in einer kausalen DN-Erklärung das psychische Ereignis nicht nur mit physikalischen Prämissen begründet. Die Behauptung, die Farbwahrnehmung einer Person funktioniere normal, besagt ja, $\mathrm{daß}$ sie - normale Beleuchtungsverhältnisse vorausgesetzt - die

${ }^{62} \mathrm{Vgl}$. dazu den Absschnitt 2.2. 
Farben richtig sieht, daß sie ihr so erscheinen, wie sie sind, und in dieser Aussage wird auf das Erleben der Person Bezug genommen. Entsprechendes gilt für die Aussage „Hans läuft zum Bahnhof, weil er den $8^{15}$-Zug erreichen will und glaubt, daß es schon $8^{10}$ ist", in der etwas Physisches - die körperliche Bewegung von Hans - mit etwas Psychischem - einem Wunsch und einer Überzeugung von Hans erklärt wird. Es gibt kein Gesetz des Inhalts, daß alle Leute laufen, die einen Zug erreichen wollen und glauben, daß seine Abfahrt unmittelbar bevorsteht. Man kann nur sagen: Wenn eine Person a, die F tun kann und das auch weiß, will, daß ein Zustand $p$ eintritt, und glaubt, daß p genau dann eintritt, wenn sie $F$ tut, so wird sie $F$ tun, falls sie rational handelt. ${ }^{63}$ „Rational handeln" heißt aber, tun, was man im Sinne seiner Überzeugungen und Präferenzen für das Beste hält. Daß jemand rational handelt, ist daher keine rein psychologische Aussage. Auch bei Erklärungen des Verhaitens mit Gründen (Überzeugungen und Präferenzen) wird also nicht eine DN-Erklärung von Physischem mit rein psychologischen Antezedensbedingungen angegeben. Auch hier besteht keine gesetzmäßige Abhängigkeit von Physischem von bloß Psychischem. Nicht immer, wenn es so aussieht, als würde etwas Physisches allein mit psychologischen Bedingungen erklärt, liegt tatsächlich eine kausalgesetzliche Abhängigkeit zwischen beiden Bereichen vor.

In unseren beiden Beispielen stellt das Bestehen psycho-physischer Abhängigkeiten kein Problem dar. Daß einem Beobachter bei normalen Beleuchtungsverhältnissen ein weißes Stück Papier als weiß erscheint, wenn seine Farbwahrnehmung normal funktioniert, ist nicht merkwürdig, weil ein normales Funktionieren der Farbwahrnehmung eben besagt, daß bei normalen Beleuchtungsverhältnissen Farben richtig wahrgenommen werden. Und daß wir, wenn wir rational handeln und tun können, was wir wollen, das tun, was im Sinn unserer Präferenzen und Überzeugungen das Beste ist, ist nicht erstaunlich, weil rational handeln eben heißt, das tun, was im Sinne unserer Präferenzen und Überzeugungen das Beste ist. Diese trivialen Fälle sind also ohne tieferes Interesse. Interessant sind vor allem empirische Zusammenhänge zwischen Psychischem und Physischem, insbesondere die Zusammenhänge zwischen physiologischen Vorgängen im Gehirn und dem Erleben einer Person, ihrem Denken, Vorstellen,

${ }^{63}$ Vgl. dazu den Abschnitt 2.4. 
Erinnern, ihren Präferenzen, Annahmen usw. Dabei sind aber zwei Dinge zu beachten: Erstens sind hier, wie schon betont wurde, in der Regel nicht Sukzessionsgesetze, sondern Koexistenzgesetze anzunehmen. Zweitens ist das, was sich im Sinn des naturwissenschaftlichen Beobachtungsbegriffs feststellen läßt, ein Zusammenhang zwischen Gehirnzuständen und dem äußeren Verhalten der Person. Aus diesem Verhalten können wir nur dann auf das Erleben der Person zurückschließen, wenn wir voraussetzen, daß sie sich ihrem Erleben gemäß normal (z. B. rational) verhält. Ohne schon bestimmte Voraussetzungen über Zusammenhänge zwischen Psychischem und Physischem zu machen, kann man also auch hier solche Zusammenhänge nicht feststellen und Psychisches mit Physischem erklären, oder umgekehrt.

Die Schwierigkeiten, sich Wechselbeziehungen zwischen Psychischem und Körperlichem verständlich zu machen, beruhen z. T. auch darauf, daß man das Psychische mit dem Bewußten identifiziert. Für den Dualismus fällt das Seelische mit dem rein Psychischen im Sinne von 7.1 zusammen, mit dem Bereich dessen, was uns im Selbstbewußtsein problemlos ist, dem Bereich der cartesischen cogitationes. Diese Konzeption des Seelischen liegt der Bewußtseinspsychologie zugrunde, die bis zum Anfang unseres Jahrhunderts herrschend war. Unser bewußtes Erleben, Fühlen, Streben und Verhalten ist aber mit Vorgängen verbunden und wird von Faktoren beeinflußt, die unbewußt sind - von unbewußten, teilweise aus dem Bewußtsein verdrängten Trieben, von nicht bewußtem Erleben. Unser Verhalten geht nur zu einem 'geringen Teil aus Überlegungen hervor; daneben gibt es instinkthaftes und automatisches Verhalten sowie andere Formen unmittelbarer Reaktionen auf Erlebtes. Selbst das Denken, das Paradigma bewußter Aktivität, vollzieht sich nicht als Folge bewußter Vorgänge. Gedankenverbindungen werden nicht nur bewußt vollzogen, sondern drängen sich uns auf; sie werden oft von Assoziationsmechanismen produziert, die uns nicht einsichtig sind. Das Bewußt-Seelische ist also nicht aus sich erklärbar. Wie nur ein Zehntel eines Eisberges über die Wasseroberfläche ragt, so ragt nur ein kleiner Teil des seelischen Lebens ins Bewußtsein. Man kann nicht alles Unbewußte als ,,nicht seelisch“ bezeichnen, denn erstens sind die Grenzen zwischen Unbewußtem und Bewußtem nicht scharf, sondern es gibt kontinuierliche Übergänge - schon Leibniz spricht von Graden der Bewußtheit von Perzeptionen und nimmt unbewußte Perzeptionen an. Es kann jemand z. B. bewußt werden, daß er früher etwas gesehen 
hat, obwohl es ihm damals nicht bewußt wurde. Man kann also von einem unbewußten Erleben sprechen. Unser Erleben ist auch nicht entweder bewußt oder unbewußt, sondern zwischen klar bewußtem und unbewußtem Erleben gibt es alle Abstufungen eines mehr oder minder bewußten Erlebens. ,Bewußt' und ,unbewußt' sind also Typenbegriffe. Zweitens gibt es viele Verwandtschaften und Wechselwirkungen zwischen Bewußtem und Unbewußtem. Es gibt bewußtes wie unbewußtes Erleben, bewußte wie unbewußte Antriebe, und unbewußte Triebe und unbewußte Erfahrungen können unser bewußtes Verhalten ebenso steuern wie bewußte. Bewußte Erfahrungen und Formen überlegten Handelns können ins Unbewußte absinken und z. B. zu automatischen Verhaltensweisen werden oder unmittelbare Reaktionen auslösen. Die Grenzen seelischen Lebens sind also sehr viel weiter zu ziehen als jene des Bewußtseins. Wie das Bewußte kontinuierlich in das Unbewußte übergeht, so dieses in das Körperliche. Es gibt z. B. angeborene Verhaltensweisen, d. h. ein in unserer Erbsubstanz vorprogrammiertes Verhalten. Biologen sprechen ferner von einem ratiomorphen Apparat, der jene im Zentralnervensystem angelegten und im Verlauf der stammesgeschichtlichen Entwicklung entstandenen kognitiven Mechanismen umfaßt, auf welchen die rationale Vernunft beruht. Das vernünftige Denken umfaßt nur die uns bewußten und in der Selbstreflexion zugänglichen Leistungen, die unbewußt ablaufenden kognitiven Leistungen werden diesem ratiomorphen Apparat zugeschrieben. Auf diesen Leistungen beruht unsere (bewußte) Erkenntnis ebenso wie das instinktive, unreflektierte Verhalten. ${ }^{64}$ Man kann nun angeborene und im Zentralnervensystem angelegte Verhaltens- und Denkstrukturen nicht scharf von unbewußten Strukturen abgrenzen. Beides funktioniert unbewußt, und ererbte Verhaltensdispositionen wirken nicht unbeeinflußt von Bewußtem und Unbewußtem. Daher gibt es auch keine scharfen Grenzen zwischen Unbewußtem und Körperlichem, also auch nicht zwischen Bewußtem und Körperlichem. ${ }^{65}$

${ }^{64}$ Vgl. dazu Lorenz (73) und Riedl (79). Auf das Problem ,,angeborener“ Begriffe und Erwartungen gehen wir im Kap. 9 ein.

${ }^{65}$ Das Unbewußt-Seelische, das, was auch dann, wenn es zu Bewußtsein kommt, nicht als Eigenes erinnert wird, erscheint typischerweise als nichtindividuell. S. Freud spricht vom „Es“. Als Träger des Unbewußten wird „das Leben“ (ein nicht-individuelles, sondern artspezifisches Leben) angese- 
Heute bildet nicht nur das rein Psychische den Gegenstand der Psychologie, sondern daneben auch das Unbewußte, das Verhalten, die körperlich-seelischen Beziehungen. Psychologie ist eine Wissenschaft vom ganzen Menschen, die zwar primär auf das seelische Leben abzielt, sich aber wegen dessen Verflechtung mit Körperlichem mit Physiologie, Biologie und Medizin überschneidet. Wie sich die moderne Physik von der Konzeption der Natur als einer von menschlicher Erfahrung unabhängiger Realität abgewendet hat, so hat sich die Psychologie von der Auffassung des Seelischen als eines gegenüber der physischen Natur unabhängigen Bereichs befreit.

Das Unbewußte wurde in der Romantik entdeckt (oder wie man im Blick auf Leibniz besser sagt: wiederentdeckt), und schon Hegel hat in seiner Kritik am Dualismus die Grenzen des Seelischen sehr viel weiter gezogen als die des Bewußtseins. Er hat auf die enge Verbindung zwischen Seelischem und Körperlichem hingewiesen, auf den Einfluß körperlicher Vorgänge auf seelisches Geschehen und umgekehrt. ${ }^{66}$ Erst mit den Arbeiten von S. Freud ist jedoch das Unbewußte zum anerkannten Gegenstandsbereich der Psychologie geworden. Dieser Wandel im Verständnis des Seelischen konnte nicht ohne Rückwirkung auf das menschliche Selbstverständnis bleiben. Nach dualistischer Auffassung ist das Subjekt die Seele, die sich im Selbstbewußtsein erkennen und kraft ihres freien Willens selbst bestimmen kann. Sind wir auch in unserer leiblichen Existenz Naturgesetzen unterworfen und von äußeren Umständen abhängig, die sich unserer Kontrolle entziehen, so sind wir doch jedenfalls in unseren cogitationes frei, im Denken und Wollen. Reicht aber seelisches Leben weiter

hen. Bei C. G. Jung gibt es ein „Gattungsgedächtnis“, eine überindividuelle Seele, die im einzelnen lebendig ist, bei Freud ein „Überich“, als internalisierte gesellschaftliche Norm, die Erlebnisse oder Triebe aus dem individuellen Bewußtsein verdrängen kann. Auch das ist ein Hinweis auf die Nähe des Unbewußten zu den artspezifischen Anlagen, von denen die Biologie redet. Dem einzelnen Subjekt rechnen wir nur jenes seelische Geschehen zu, das ihm bewußt ist und als eigenes Erleben und Tun von uns erinnert werden kann. Die Einheit dieses Bezugs macht, wie schon Kant betont hat, die Einheit des Subjekts aus. Die Grenzen dieser Einheit verfließen freilich wie die Grenzen des Bewußtseins.

${ }^{66} \mathrm{Vgl}$. dazu Hegels Aussagen über den „subjektiven Geist“ in (W)X, sowie den Abschnitt 8.1. 
als unser Bewußtsein, ist es mit Körperlichem unlösbar verbunden und wird unser Verhalten nicht nur von bewußten Entscheidungen gesteuert, sondern auch von unbewußten Antrieben, die sich unserer Kontrolle entziehen, so läßt sich diese Vorstellung eines autonomen Subjekts nicht mehr halten. F. Nietzsche sagt: „Was weiß der Mensch eig̈entlich von sich selbst!. . . Verschweigt die Natur ihm nicht das Allermeiste, selbst über seinen Körper, um ihn, abseits von den Windungen der Gedärme, dem raschen Fluß der Blutströme, den verwickelten Fasererzitterungen, in ein stolzes, gauklerisches Bewußtsein zu bannen und einzuschließen!. . U Und wehe der verhängnisvollen Neubegier, die durch eine Spalte einmal aus dem Bewußtseinszimmer heraus und hinab zu sehen vermöchte, und die jetzt ahnte, daß auf dem Erbarmungslosen, dem Gierigen, dem Unersättlichen, dem Mörderischen der Mensch ruht in der Gleichgültigkeit seines Nichtwissens, und gleichsam auf dem Rücken eines 'Tigers in 'Träumen hängend « ${ }^{67}$

Die Einsicht, daß unser Denken, Erleben und Wollen in vielfacher Weise mit unbewußtem und körperlichem Geschehen verknüpft ist, hat einen Umschlag der dualistisch-idealistischen Idee eines autonomen Subjekts ins andere Extrem bewirkt: Der Mensch erscheint nun ähnlich wie im Materialismus als Teil der Natur. Er bestimmt nicht über sich, sondern er wird bestimmt; er ist Produkt seiner körperlichen und seelischen Anlagen, wie der Umwelteinflüsse. Beide Extreme sind aber nicht haltbar. Ebenso, wie bewußt-seelische Vorgänge vom Unbewußten beeinflußt werden, gilt die Umkehrung: der therapeutische Effekt der Psychoanalyse beruht z. B. gerade darauf, daß Unbewußtes dem Patienten zu Bewußtsein gebracht wird und damit von ihm beeinflußt und aufgearbeitet werden kann. Und Biologen übersehen oft, daß der Mensch ,,von Natur ein Kulturwesen“ ist, wie A. Gehlen sagt. Unser kognitiver „Apparat“ ist sicher zum Teil Produkt einer biologischen Evolution, zu einem sehr wesentlichen Teil ist er aber Produkt kreativer geistiger Leistungen. Unser Gehirn ist kein vorprogrammierter Komputer, sondern die Vernetzung der Neuronen durch Synopsen bildet sich erst mit der Erfahrung aus, ${ }^{68}$ und Erfahrungen werden auch durch die Sprache geprägt und die Weltsicht, die wir mit ihr übernehmen. Der ratiomorphe Apparat bestimmt also nicht nur unser Denken, sondern es gilt auch das Umgekehrte.

${ }^{67}$ Nietzsche (W)X, 191.

${ }^{68} \mathrm{Vgl}$. dazu z. B. Popper und Eccles (77). 
Die Differenzierung von Subjektivem und Objektivem, die Ausbildung von Selbstbewußtsein und Weltverständnis stellen einen Entwicklungsprozeß dar, in dem wir uns selbst als Subjekt wie die Außenwelt, die Natur, in Abgrenzung voneinander bestimmen. Dieser Prozeß vollzieht sich in der Deutung und begrifflichen Verarbeitung unseres Erlebens wie im bewußten Handeln, in der praktischen Auseinandersetzung mit der Umwelt. Darin stellt sich ein erkennendes, handelndes und leidendes Subjekt einer erkannten, gestalteten und wirkenden Natur gegenüber. ,Subjektiv - Objektiv', ,Bewußt - Unbewußt', ,Frei - Determiniert', ,Aktiv - Passiv' sind dabei als gegensätzliche Typenbegriffe zu verstehen, die komparative Abstufungen erlauben. Wir begreifen uns dabei weder als autonome seelische Subjekte, noch als Teil der Natur, sondern als konkrete Personen, die Bewußtsein haben und freier Handlungen fähig sind, und diese $\mathrm{Be}$ stimmung ist auch dann brauchbar, wenn man zugesteht, daß unser bewußtes Erleben, Denken und unser absichtliches Tun auch Bedingungen unterliegt, die sich unserer Kontrolle entziehen. Unsere früheren Überlegungen haben gezeigt, daß man weder das Psychische auf Physisches reduzieren kann, noch die Behauptung einer durchgängigen Determiniertheit unseres Verhaltens beweisen kann. Das Subjekt geht nicht in der Natur auf, denn „,Kein Subjekt - kein Objekt" ", wie Fichte sagt: Das Subjekt ist ebensowenig im Sinn des Physikalismus eliminierbar wie die Außenwelt im Sinn des Idealismus.

Für unsere polare Konzeption des psycho-physischen Verhältnisses wirft also das Leib-Seele-Problem keine prinzipiellen Verständnisschwierigkeiten auf. Es reduziert sich auf das empirische Problem, welche speziellen Verbindungen und wechselseitigen Abhängigkeiten zwischen Physischem und Psychischem bestehen. Historisch läßt sich eine zunehmende Differenzierung und Verselbständigung der beiden Pole der Erfahrung feststellen, des Subjekts der Erfahrung und der Welt seiner Gegenstände, des Psychischen und des Physischen. Erst bei einer starken Differenzierung konnte die Auffassung von der Eigenständigkeit und Unabhängigkeit beider Bereiche entstehen, der Dualismus. Während er die Wechselwirkungen nicht erklären kann und damit die Monismen auf den Plan ruft, werden diese der offensichtlichen Verschiedenheit der beiden Bereiche nicht gerecht, können also den Dualismus nicht aus dem Felde schlagen. Aus diesen Verwirrungen führt wohl nur der Weg heraus, den wir hier vorgeschlagen 
haben; eine Eigenständigkeit, aber nur eine relative Eigenständigkeit des Fhysischen und des Psychischen in einer unaufhebbaren wechselseitigen Bezogenheit anzunehmen. Dieser Vorschlag ist, um das abschließend noch einmal zu betonen, keine Theorie, denn es fehlen die konkreten Details, die nur die Einzelwissenschaften liefern können. Er ist auch nicht neu, denn ähnliche Gedanken finden sich schon im Deutschen Idealismus und bei W. Dilthey.

Mit diesem Abschnitt beenden wir die Diskussion des Leib-SeeleProblems und wenden uns im folgenden der erkenntnistheoretischen Frage nach dem Gegenstand der Erfahrung zu, von dem wir im Abschnitt 3.4 ausgegangen sind.

\subsection{Objektivität und Realismus}

Vom dritten Kapitel an haben wir uns mit Erfahrungserkenntnis befaßt. Zwei Fragen standen im Mittelpunkt unserer Überlegungen. Die erste war die nach dem Gegenstand der Erfahrung, mit der die Realismus-Idealismus-Kontroverse angesprochen ist. Die zweite war die nach dem Verhältnis von Physischem und Psychischem, das LeibSeele-Problem im weiteren Sinn. Oft wird nur die erste als erkenntnistheoretische Frage bezeichnet, während die zweite als ontologisches Problem der Metyphysik zugeordnet wird. Beide Fragen hängen aber eng zusammen, und daher gehen wir in dieser Arbeit auch auf beide ein. Jede Antwort auf die ontologische Frage muß der erkenntnistheoretischen Kritik standhalten; sie darf keine Annahmen über Existenz und Beschaffenheit einer Außenwirklichkeit machen, die über das hinausgeht, was sich durch Erfahrungen begründen läßt. Daher hängen unsere Annahmen über die Natur der Außenwelt von unseren Ansichten über die Gegenstände der Erfahrung ab. Umgekehrt enthält jede Antwort auf die erkenntnistheoretische Frage nach dem Gegenstand der Erfahrung auch eine ontologische Behauptung über die Existenz solcher Objekte, über die Existenz des Realitätsbereichs, der in der äußeren Erfahrung zur Gegebenheit kommt. In diesem Sinn setzt z. B. ein erkenntnistheoretischer Realismus einen ontologischen voraus, und ein erkenntnistheoretischer Idealismus legt einen ontologischen zumindest nahe. Setzt aber die Beantwortung einer Frage die der anderen voraus, so lassen sich diese Fragen nur zusammen sinnvoll diskutieren. 
Der Zusammenhang beider Probleme ist auch in unseren Erörterungen deutlich geworden: Wir sind im 4. Kapitel von der erkenntnistheoretischen Frage ausgegangen. Die Erörterung des erkenntnistheoretischen Idealismus hat uns im 5. Kapitel zur Diskussion des ontologischen Idealismus geführt, zur Frage nach der Reduzierbarkeit des Physischen auf das Psychische. Damit sind wir in die Behandlung der ontologischen Frage eingetreten, die wir mit der Erörterung des Physikalismus und Dualismus weiter verfolgt und im letzten Abschnitt abgeschlossen haben. Wir wollen nun auf die Frage nach dem Gegenstand der Erfahrung zurück kommen und die erkenntnistheoretische Seite unserer Konzeption vom Verhältnis von Psychischem und Physischem herausarbeiten.

Wir haben im Abschnitt 3.4 zwei Vorschläge zur Präzisierung des erkenntnistheoretischen und ontologischen Realismus gemacht, die zu einem starken (absoluten) und einem schwachen Realismus führen. Den schwachen Realismus haben wir im 5. Kapitel mit der Widerlegung der phänomenalistischen Übersetzungsthese gerechtfertigt. Am starken Realismus, sofern er sich auf die physische Natur bezieht, also die physikalischen Sachverhalte als objektiv im starken Sinn ansieht, haben wir in den Abschnitten 7.4, 8.3 und 8.4 Kritik geübt. Der Kernpunkt dieser Kritik läßt sich so formulieren: Die These des starken Realismus lautet, auf physikalische Sachverhalte bezogen:

I) Die Aussagen der physikalischen Sprache sind ibrem Sinn nach - und also erst recht ibrer Geltung nach - nicht auf Beobachtungen oder Beobachtungsverfabren bezogen. Sie beschreiben eine von Beobachtungen unabbängige Welt.

Daraus folgt, daß die wahren Sätze der physikalischen Sprache eine im starken Sinn objektive Realität beschreiben, also ein starker ontologischer Realismus. Aus der These ergibt sich nun, daß immer nur Erfahrungen und nicht apriorische Einsichten Aufschluß über die Welt geben. Schon Kant hat betont, daß wir über eine Welt, deren Existenz wie Beschaffenheit unabhängig von Beobachtungen ist, keine apriorischen Aussagen machen können. Apriorische Aussagen über die Gegenstände der Erfahrung ergeben sich nach Kant nur aus dem, was wir selbst in die Erfahrung hineinlegen, d. h. aus der Struktur, welche die Erfahrung durch unsere Wahrnehmungs- und Vernunftorganisation erhält. Über eine von Erfahrung im Sinne von I unabhängige Wirklichkeit können wir also apriorisch nichts erkennen:69 
II) Physikalische Aussagen lassen sich nur durch Erfabrungen überprüfen. Würden uns nun auch Beobachtungen keinen Aufschluß über die Geitung von Sätzen der physikalischen Sprache geben, so gäbe es keine Erkenntnis des Physischen, d.h. die Aussagen der physikalischen Sprache wären sämtlich weder begründbar noch widerlegbar oder bestätigungsfähig. Vertritt man keine so radikal skeptische Position, so muß also gelten:

III) Physikalische Aussagen lassen sich - zumindest teilweise - durch Beobachtungen überprüfen. Erfabrung gibt uns - zumindest eine partielle - Auskunft darüber, wie die physische Natur beschaffen ist. Aus I folgt nun, daß III nur dann gelten kann, wenn auch gilt:

IV) Es gibt Beobachtungsverfabren, von denen wir wissen, daß sie uns die Natur so zeigen, wie sie („an sich") ist.

Solche Beobachtungsverfahren wollen wir unproblematisch nennen. ${ }^{70}$ Als „Beobachtungsverfahren“ bezeichnen wir dabei nicht nur Verfahren schlichter, direkter sinnlicher Feststellung, sondern auch komplizierte Test- oder Meßverfahren. Es genügt, wenn es Beobachtungsverfahren gibt, aus deren Ergebnissen man die wahren Zustände der Natur nach bestimmten Regeln ermitteln kann. Diese Verfahren, verbunden mit einer Anwendung der Regeln, kann man dann auch als unproblematische Beobachtungsverfahren in unserem weiten Sinn des Wortes ansehen. Daß es gewisse Beobachtungen gibt, die uns tatsächlich die Natur so zeigen, wie sie ist, genügt nicht, um eine Erkennbarkeit der Natur zu begründen. Wir müssen auch davon überzeugt sein, daß sie uns die Welt so zeigen, wie sie ist. Daher muß es unproblematische Beobachtungsverfahren geben, so daß wir immer dann, wenn wir diese Verfahren anwenden, sicher sein können, daß die dabei gewonnenen Überzeugungen über die Natur richtig sind.

Diese Konsequenz zeigt aber nun, daß die These I nicht haltbar ist, denn ob uns eine Beobachtung die Natur so zeigt, wie sie (,,an sich") ist, läßt sich nach I nicht entscheiden. Wir müßten dazu ja unabhängig von dieser Beobachtung feststellen können, wie die Natur be-

${ }^{69} \mathrm{Vgl}$. Kant (KrV), 19 f. und (W) V, 146.

70 In 1.3 haben wir Sachverhalte als , unproblematisch" bezeichnet, für die gilt: Wenn jemand glaubt, daß sie bestehen, so gilt das. Beobachte ich, daß der Sachverhalt $p$ besteht, so glaube ich das im Sinne der Bestimmungen in 3.1 auch. 
schaffen ist. Nach II können wir aber über die Welt nur aufgrund von Beobachtungen etwas aussagen. Für die Frage, ob uns eine Beobachtung ein korrektes Bild physikalischer Tatsachen liefert, müssen wir also auf andere Beobachtungen rekurrieren, und für diese stellt sich dann dasselbe Problem, so daß wir in einen unendlichen Regreß geraten. Diese These I führt also dazu, daß sich die Behauptung III nicht begründen, also eine Skepsis bzgl. physikalischer Erkenntnis nicht widerlegen läßt. Tatsächlich sind wir aber doch davon überzeugt, daß es eine Erkenntnis des Physischen gibt. Das kann man auch so ausdrükken: Akzeptiert man - wie z. B. Kant - die These III nicht, so ergibt sich die Unerkennbarkeit der Welt ,an sich", die in I angenommen wird. Dann ist es aber nur konsequent, diese Annahme aufzugeben; denn über eine nicht erkennbare Welt kann man nicht sinnvoll reden und es läßt sich nicht einmal begründen, daß es eine solche Welt gibt. Sie ist also eine unbegründete Fiktion.

Der Ausweg aus dieser Aporie wird uns durch die Einsicht gewiesen, daß gewisse Beobachtungsverfahren per Konvention unproblematisch sind: Solche Konventionen sind nur in der Form möglich, daß man den Sinn der physikalischen Terme durch Rückgriff auf Beobachtungsverfahren festlegt. Wir haben schon in 7.4 gesehen, daß es anschauliche Kriterien für den Gebrauch solcher Terme geben muß, und daß sie deren Sinn mit bestimmen. Obwohl der Sachverhalt, daß ein Ding rot ist, keine analytische Folge des Sachverhalts ist, daß es mir als rot erscheint, so ist es doch eine Bedeutungswahrheit, daß das in der Regel gilt. Daher kann ich, sofern ich nicht Gründe habe, eine nicht-normale Beobachtungssituation anzunehmen, davon ausgeher, daß meine Beobachtung zuverlässig ist. Schlichte Beobachtungen von Farben sind also nicht generell, wohl aber in der Regel unproblematisch, und das genügt für eine Begründung von Aussagen über die Farbeigenschaften von Dingen.

Erklären wir den Sinn physikalischer Terme durch Rückgriff auf Beobachtungsverfahren, so verfügen wir über unproblematische Beobachtungsverfahren, und die Behauptung, es gebe physikalische Erkenntnis, läßt sich begründen. Dann muß man aber die These I aufgeben; physikalische Aussagen sind dann ihrem Sinn nach auf Beobachtungsverfahren bezogen.

V) Die Aussagen der physikalischen Sprache sind Aussagen über die $\mathrm{Na}$ tur wie sie sich bei Beobachtungen zeigt. Sie sind ibrem Sinn nach auf Beobachtungsverfabren bezogen. 
Der Übergang von I zu V kennzeichnet, wie wir in den Abschnitten 8.3 und 8.4 sahen, auch den Wandel der erkenntnis-theoretischen Konzeption, der sich mit der Entwicklung der modernen Physik vollzogen hat. Das erkenntnistheoretische Selbstverständnis der klassischen Physik - der Newtonschen Mechanik und ihrer Erweiterungen̉ (der Kontinuumsmechanik wie der statistischen Mechanik) sowie der Maxwellschen Elektrodynamik - ist realistisch im Sinn der These I. Es läßt sich in Entsprechung zu den Thesen I bis III so kennzeichnen:

I') Physik ist Wissenschaft einer von Beobachtungen unabbängigen Natur. Die Aussagen der Physik nebmen ibren Sinn (und also auch ibrer Geltung) nach nicht auf Beobachtungen oder Beobachtungsverfabren Bezug.

II') Die Aussagen der Physik lassen sich nur durch Experimente überprüfen.

III') Experimente geben uns zumindest partielle Aufschlüsse darüber, wie die Natur an sich beschaffen ist.

III' kann man wegen I' nur dann behaupten, wenn gilt:

IV') Es gibt Meßverfabren für die physikalischen Größen, von denen wir wissen, daß sie uns die wabren Werte dieser Größen mit binreichender Genauigkeit liefern.

Die Diskussion der Raum- und Zeitbegriffe in der Relativitätstheorie hat nun gezeigt, daß die Verwendung dieser Begriffe in der Physik nur dann sinnvoll ist, wenn sie durch Beobachtungsverfahren erklärt werden; daß die Verwendung von Observablen nur dann gerechtfertigt ist, wenn man sie mithilfe von Meßverfahren erklärt. Damit ist deutlich geworden, daß physikalische Aussagen ihrem Sinn nach auf Beobachtungsverfahren bezogen sind. In der Quantenphysik hat sich darüber hinaus gezeigt, daß die Annahme einer störungsfreien Beobachtbarkeit im Sinne der These IV' nur im Makrobereich haltbar ist, aber nicht in der Physik der Mikrophänomene, und daß sich die aus Beobachtungen gewonnenen Aussagen über Mikroobjekte nicht objektivieren lassen; Aussagen über Mikroobjekte sind Aussagen über Beobachtungsresultate. Daraus ergibt sich in Analogie zur Aussage V:

V') Die Aussagen der Physik sind Aussagen über die Natur wie sie sich bei Beobachtungen zeigt. Sie sind ibrem Sinn nach auf Beobachtungsverfabren bezogen und in der Mikrophysik in ibrer Geltung auf beobachtete Objekte beschränkt.

Die Relevanz der modernen Physik für das erkenntnistheoretische 
Problem des Realismus ergibt sich daraus, daß sie erstens die oben formulierten philosophischen Einwände gegen den starken Realismus im Sinn der These I und die Argumente für die These $V$ nicht nur stützt, sondern dadurch verstärkt, daß man von konkreten physikalischen Problemen her dazu gekommen ist. Ohne den Anstoß von seiten der Physik, nur aufgrund abstrakt-allgemeiner philosophischer Argumerıte, wäre ınan kaum tereit, eine dem gesunderı Menschenverstand so naheliegende, durch lange Gewöhnung so selbstverständlich gewordene und in der klassischen Physik so gut bewährte Konzeption preiszugeben wie den starken Realismus. Zweitens hat die Physik die Grenze aufgezeigt, innerhalb derer sich die realistische Fiktion praktisch - wenn auch nicht prinzipiell - und in einer hinreichend guten Näherung aufrechterhalten läßt. Damit kann sie auch die Brauchbarkeit der Fiktion in der alltäglichen Erfahrung erklären, bzgl. derer wir unsere empirischen Begriffe bilden. Drittens ist eine detaillierte Analyse der Einführung physikalischer Terme unter Bezugnahme auf Beobachtungsverfahren nur in einer hinreichend entwickelten Naturwissenschaft, nicht aber bzgl. der Alltagssprache möglich, ebenso wie eine exakte Diskussion des Einflusses von Beobachtungen auf das beobachtete System.

Mit dem starken ontologischen Realismus ist auch der starke erkenntnistheoretische Realismus aufzugeben, zumindest bzgl. physikalischer Sachverhalte. Ein Realismus läßt sich aber wohl überhaupt nicht vertreten, wenn nicht bzgl. der physikalischen Natur; physikalische Sachverhalte sind für uns ja die exemplarischen Fälle objektiver Sachverhalte. Aus der Unhaltbarkeit des starken Realismus folgt nicht, daß physische Sachverhalte nicht objektiv wären, sondern vielmehr, daß die starke Deutung des Wortes ,objektiv“ inadäquat ist. Im normalen Sinn sind Aussagen über Physisches der Standardfall objektiver Aussagen. Jede Erklärung des Wortes „objektiv“, nach der Aussagen über Physisches nicht objektiv sind, ist also unangemessen. Nun kann man natürlich in der Philosophie Termini einführen, die vom normalen Sprachgebrauch abweichen. Es muß dann aber gezeigt werden, daß diese Abweichung sinnvoll und systematisch fruchtbar ist. Wir haben jedoch gerade zu zeigen versucht, daß die Bestimmung des Objektiven im Sinne des starken Begriffs systematisch unfruchtbar, weil leer ist.

Wir müssen also den schwachen Realismus akzeptieren, den starken verwerfen. Diese Auskunft ist aber unbefriedigend, da der schwa- 
che Begriff der Objektivität zu schwach ist, um jenen Realismus zu definieren, an dem man festhalten möchte. Ein Satz wie „Hans weiß, daß München an der Isar liegt", der nicht in einen Satz über Psychisches übersetzbar ist, gilt im Sinne dieses Begriffs schon als ,objektiv", obwohl der Satz auch über rein Psychisches spricht, da ja ,Wisseñ ",Überzeugtsein“ impliziert. Es stellt sich also die Frage, ob man den Begriff der Objektivität nicht doch enger umreißen kann, ob sich nicht zwischen schwacher und starker Objektivität ein sinnvolles Drittes ansiedeln läßt. Nach den Bemerkungen in 3.4 bietet sich dafür kein zugleich präzises und generelles Kriterium an. Lassen sich aber nicht der Praxis der Unterscheidung von Subjektivem und Objektivem verallgemeinerungsfähige Kriterien entnehmen?

Eine Unterscheidung von Subjektivem und Objektivem vollzieht sich in jeder einzelnen (äußeren) Erfahrung. Erfahrungen haben, wie wir in 5.5 sahen, intentionale Struktur. Sie sind Erfahrungen von etwas, und das, was erfahren wird, ist der Gegenstand, das Objekt der Erfahrung, das wir uns gegenüberstellen und von unserer Erlebnisweise abgrenzen. In jeder Erfahrung legen wir also einen Schnitt zwischen das Erfahrene als etwas bzgl. dieser Erfahrung Objektives und das erfahrende Subjekt. Diese Struktur der Erfahrung ist ein Grundphänomen. Es gehört zum Begriff der Erfahrung, daß sich darin etwas zeigt, daß uns etwas gegeben ist, daß wir mit etwas konfrontiert werden, daß uns etwas begegnet, auf das wir reagieren, sei es gefühlsmäßig (z. B. mit Befriedigung oder Ablehnung), intellektuell (mit Interesse oder Aufmerksamkeit) oder praktisch im Handeln. Und das, was uns begegnet, stellt sich für uns als etwas Objektives dar. Wenn ich darauf reflektiere, daß ich etwas fühle, will oder glaube, so mache ich mir auch diese Tatsachen zum Gegenstand. Aber hier fehlt dieses Phänomen, daß mir etwas begegnet, das Reaktionen herausfordert; es handelt sich um ein Bewußtmachen eigener seelischer Zustände, Akte oder Dispositionen, eine Erhellung dessen, was implizit schon gegeben war, nicht um Erfahrung von etwas mir Entgegentretendem. Dieses Grundphänomen äußerer Erfahrung wollen wir - mangels eines besseren Ausdrucks - als Gegenständigkeit des Erfahrens bezeichnen.

Eine Differenzierung von subjektiven und objektiven Momenten im Erleben ist schon in unserer neuro-sensorischen Organisation angelegt. Besonders aufschlußreich dafür sind die Konstanzphänomene in der Farb-, Gestalt- und Größenwahrnehmung. Unsere Wahrneh- 
mungsorganisation ist so strukturiert, daß sie den Einfluß gewisser Beobachtungsbedingungen auf den Wahrnehmungsinhalt ausschaltet; daß sie uns etwas im Wechsel der Erscheinungsweisen relativ Konstantes zeigt. Wenn wir z. B. ein Blatt Papier betrachten, so sehen wir es unter variierenden Beleuchtungsverhältnissen immer als weiß, obwohl es jeweils Licht anderer Frequenzen reflektiert. Das wird dadurch erreicht, daß das reflektierte Licht mit dem Beleuchtungslicht von unserem physiologischen Wahrnehmungsapparat verrechnet wird. Dieser Mechanismus versagt - wie z. B. in der Dunkelkammer nur dann, wenn die Vergleichsmöglichkeiten fehlen. Entsprechend wird in der Wahrnehmung einer Gestalt, z. B. eines Rades, der Blickwinkel in Rechnung gestellt, unter dem wir es sehen, so daß wir auch bei einer Änderung des Blickwinkels eine konstante runde Form wahrnehmen. Und in der Wahrnehmung der Größe eines Gegenstandes wird auch seine Entfernung einkalkuliert, so daß wir ein entferntes Haus als größer sehen als einen nahen Tisch. ${ }^{71}$ Aufgrund solcher Phänomene kann man sagen, daß unsere Erfahrung schon durch die physiologische Organisation des Wahrnehmungsapparats als Erfahrung von Gegenständlichem strukturiert ist. Die Wahrnehmungsorganisation legt den Schnitt freilich nicht eindeutig fest, den wir in einer Erfahrung zwischen den Gegenstand und das erfahrende Subjekt legen. Wir haben in 8.2 darauf hingewiesen, daß der konkrete Charakter des primitiven Erlebens bewirkt, daß Gegenstände gesehen werden, die ,eigentlich“, d. h. nach heutigem Verständnis, gar nicht vorhanden sind; daß das Sichtbare unter dem Einfluß von Assoziationen und Strebungen ausgestaltet wird. Ferner wurde schon im letzten Abschnitt betont, daß der physiologische Verrechnungsmechanismus durch unsere Erfahrung und deren sprachliche Interpretation beeinfluß wird - auch die Sprache ist ein Erfahrungsorgan. Es gibt experimentelle Befunde, nach denen die Fähigkeit wahrnehmungsmäßiger Unterscheidungen von den sprachlichen Unterscheidungsmitteln abhängt, über die wir verfügen. ${ }^{72}$ Wir können nicht streng unterscheiden zwischen dem rein wahrnehmungsmäßig Gegebenem und seiner begrifflichen Interpretationen. Wir sehen z. B. einen Stuhl, nicht nur etwas, von dem wir aufgrund früherer Erfahrungen vermuten, daß man darauf sitzen kann. Die Wahrnehmungskonstanzen unterliegen einer

${ }^{11} \mathrm{Vgl}$. dazu z. B. Lorenz (73), $157 \mathrm{ff}$.

72 Vgl. dazu Kutschera (75), 4.2. 
onto- wie phylogenetischen Entwicklung. ${ }^{73}$ Das Kind merkt z. B. auf seinem Weg durch eine Straße, daß es nur so aussieht, als ob sie in der Ferne schmäler würde, und diese Erfahrung prägt dann künftige Wahrnehmungen. Wir gestalten also das Wahrgenommene aus und modifizieren es, indem wir es in den Horizont unserer sonstigen Erfahrungen und Annahmen einordnen. Ohne eine gewisse gegenständliche Vorstrukturierung der Erfahrung wäre es aber kaum verständlich, wieso es überhaupt zu intentionalen Erfahrungen kommt. Wir haben ja bei der Erörterung phänomenalistischer Reduktionstheorien im 5. Kapitel gesehen, daß eine Rekonstruktion der Aussagen über physische Dinge auf der Basis nicht-intentionaler Empfindungen nicht möglich ist.

Diese zunächst nur für einzelne Erfahrungen und auch für sie nicht eindeutige Differenzierung von Subjektivem und Objektivem wird dadurch verfestigt, daß wir Zusammenhänge zwischen den erfahrenen Sachverhalten feststellen und den Bereich objektiver Phänomene auch als einen Bereich bestimmen, in dem eine gesetzmäßige Ordnung herrscht, als eine in sich relativ geschlossene Welt. ${ }^{74}$ Wie wir in 7.4 sahen, erhalten Aussagen über Physisches aufgrund solcher $\mathrm{Zu}$ sammenhänge eine Bedeutung, nach der sie nur mehr oder minder lose mit Aussagen über Erlebnisweisen zusammenhängen. Wenn ich das Wort „,rot" nicht allein nach Empfindungskriterien verwende, wenn ich nicht genau die Dinge ,,rot" nenne, die mir als rot erscheinen, erhält das Wort einen nicht mehr allein auf Empfindungsweisen bezogenen Sinn. Die Kohärenz der objektiven Erscheinungen bewirkt also, daß es Sachkriterien und nicht nur Erlebniskriterien für ihr Bestehen gibt, und daß Aussagen über sie einen gegenüber Psychischem relativ eigenständigen Sinn haben. Diese Kohärenz wird nicht nur vorgefunden, sondern wir bestimmen das Objektive auch so, daß es einen in sich möglichst kohärenten Bereich darstellt.

${ }^{73} \mathrm{Vgl}$. dazu z. B. Werner (59), $98 \mathrm{ff}$., $152 \mathrm{ff}$.

${ }^{74}$ Wir haben schon darauf hingewiesen, daß für Kant das Bestehen kausaler Zusammenhänge zwischen den Phänomenen ein Kriterium dafür ist, daß sie einer objektiven Natur zugehören. Wenn wir hier von „Zusammenhängen“ reden, so sind hingegen nicht nur kausale Gesetze gemeint, und es wird auch nicht gefordert, daß sich alle objektiven Phänomene mit Gesetzen erklären lassen. 
Das Objektive ist für uns endlich auch das, was intersubjektiv gilt. Auch das wurde schon in 7.4 angesprochen: Intersubjektive Gebrauchskriterien für die Sprache bewirken einen ,objektiven Zug“, wie wir das im Anschluß an Quine genannt haben, eine Abstraktion des Sinns der Terme von individuellen Erlebnisaspekten. Nur wenn wir in den Anwendungen der Terme übereinstimmen, wenn sich die individuellen Gebrauchskriterien hinreichend gut entsprechen, sind sie Ausdrücke einer intersubjektiven Sprache. Solche Ausdrücke haben einen objektiven, nicht nur individuellen und privaten Sinn. Bereiche, in denen sich eine intersubjektive Übereinstimmung in den Urteilen erzielen läßt, sehen wir als objektiv an. Übereinstimmung ist uns ein Indiz dafür, daß wir über etwas Objektives sprechen, über etwas, was z. B. auch andere wahrnehmen können, was sich ihnen ebenso zeigt wie uns selbst. Wenn wir bzgl. einer einfachen empirischen Aussage keine Übereinstimmung mit anderen erzielen können, so werden wir das zunächst auf Beobachtungsfehler oder Unterschiede in den Kenntnissen zurückführen. Haben wir aber keinen Grund so etwas anzunehmen, dann werden wir am objektiven Charakter des Sachverhalts zweifeln. Wir werden uns dann fragen, ob die unterschiedlichen Beurteilungen sich nicht dadurch erklären lassen, daß sie nicht oder nicht nur eine objektive Tatsache betreffen, sondern (auch) unsere verschiedenartigen Einstellungen dazu oder unsere Erlebnisweisen. Wenn einer beim Baden sagt: „Das Wasser ist warm", der andere „Es ist kalt", so läßt sich das am einfachsten so erklären, daß es dem einen als warm, dem anderen als kalt erscheint und daß beide ihre Erlebnisweisen fälschlich objektivieren. ${ }^{75}$

Die Unterscheidung Subjektiv - Objektiv folgt also verschiedenen Kriterien, von denen nicht von vornherein gesagt ist, daß sie immer zusammenstimmen, und die einzeln wie zusammen auch keine klare und eindeutige Grenze definieren. Wir haben in 8.1 auch gesehen, daß die Begriffe ,Psychisch' und ,Physisch' ebenso wie ,Subjektiv' und ,Objektiv' keine klassifikatorischen Begriffe sind, sondern Typenbegriffe. Wir haben dort ferner betont, daß die Unterscheidung Subjektiv - Objektiv nicht eindeutig ist. Unter verschiedenen Aspekten wird man sie verschieden ansetzen. Da sie eine Interpretation der Erfahrung darstellt, muß sie sich bei der Systematisierung der Erfahrungen

${ }^{75}$ Vgl. dazu auch Berger und Luckmann (66), Kap. III, 1 c. 
bewähren. Verschiedene Abgrenzungen können sich aber unter verschiedenen Aspekten bewähren, und man kann nicht von vornherein annehmen, daß überall dieselbe Unterscheidung brauchbar ist. In der Physik bewährt sich eine sehr enge Begrenzung des Objektiven, obwohl man, wie wir sahen, auch hier nicht mit dem starken Objektivitäts'begriff arbeiten kann. In anderen Bereichen wird man die Grenze anders ziehen müssen. Wenn man z. B. eine Werterfahrung annimmt als eine Erfahrung von objektiven Gegebenheiten, so wird man auch das gefühlsmäßige Erleben in den Bereich der Erfahrung von Objektivem hereinnehmen müssen, da Werterfahrung eng mit gefühlsmäßigem Erleben zusammenhängt. ${ }^{76}$ Auch hier wird man sicher nicht alles Erleben als zuverlässig ansehen; man wird zwischen subjektiven und objektiven Sachverhalten, zwischen subjektiven Bewertungen und objektiven Werten unterscheiden, aber die Unterscheidung Subjektiv Objektiv ist dann doch deutlich anders als im Fall der Physik zu ziehen. Endlich haben wir in 8.2 gesehen, daß die Differenzierung zwischen objektiven und subjektiven Phänomenen einem historischen Wandel unterliegt. In der Abgrenzung drückt sich, wie gesagt, eine Interpretation der Erfahrung aus, eine Konzeption der Welt und des Verhältnisses von Mensch und Welt, und in verschiedenen Kulturen finden sich ganz unterschiedliche Konzeptionen. Daher kann man keine allgemeingültige Abgrenzung des Objektiven angeben und keinen Realismus definieren, der allgemeiner Auffassung entspräche.

Selbst wenn wir vom historischen Wandel des Wirklichkeitsverständnisses absehen, bleibt als Fazit dieser Überlegung: Wir können die unbefriedigende Unterscheidung Subjektiv - Objektiv mit dem schwachen Objektivitätsbegriff nicht durch eine generelle, für alle Zwecke angemessene und präzise Definition ersetzen. Trotzdem ist die Abgrenzung von Physischem und Psychischem, von subjektiven Phänomenen des Erlebens, Denkens, Wollens, Fühlens, Glaubens und von physischen Phänomenen natürlich in vielen Fällen hinreichend klar. Wir behaupten nicht, alle solchen Unterscheidungen seien unbrauchbar, sondern wenden uns nur dagegen, sie absolut zu setzen, als präzise anzusehen, was relativ unscharf ist, als eindeutig, was unter verschiedenen Aspekten verschieden zu beurteilen ist, und als voneinander unabhängig, was nur relativ eigenständig ist.

${ }^{16}$ Vgl. dazu Kutschera (81), Kap. 6. 
Ein Argument für den Realismus des ,,gesunden Menschenverstandes" war, die Existenz der physikalischen Natur könne nicht von menschlicher Erfahrung abhängen, da die Naturwissenschaften uns sagen, daß das Universum schon lange existiert hat, bevor es Menschen gab. Wie verträgt sich das mit unserer Behauptung von den Abhängigkeiten zwischen Physischem und Psychischem? Auch wenn man solche Abhängigkeiten annimmt, kann man behaupten, daß die Welt schon lange bestand, bevor auf unserem Planeten die ersten Menschen auftraten, und daß die Fortdauer der physischen Natur nicht vom Überleben unserer Gattung abhängt. Erfahrungen sind zwar die Basis unserer Aussagen über die Natur. Nur in der Sprache, die von erfahrungsmäßigen Unterscheidungen ausgeht, können wir sinnvoll über die Natur reden. Von einer anderen Welt als jener, die sich uns in Beobachtungen zeigt, haben wir nicht den mindesten Begriff. Aber mit dieser Sprache und auf der Grundlage unserer Erfahrungen lassen sich Aussagen über nicht beobachtete Ereignisse und Dinge machen - auch Aussagen über Ereignisse und Dinge, die nie ein Mensch beobachtet hat oder beobachten wird. Mit Begriffen, die durch Beobachtungsverfahren erklärt sind, beschreiben wir die Welt, wie sie nach unseren Annahmen vor 10 Milliarden Jahren aussah, als es weder menschliches Leben noch unsere Erde gab. Wir beschreiben sie so, wie sie ein menschlicher Beobachter erfahren hätte, wenn es damals schon solche Beobachter gegeben hätte. Wir systematisieren unsere Erfahrungen mithilfe von Naturgesetzen, und berechnen mit diesen Gesetzen das, was früher war, aus dem, was wir heute beobachten. Alle empirischen Aussagen gründen sich so auf unsere Erfahrungen. Jede Kosmogonie beruht auf der für uns nicht verifizierbaren Annahme der Konstanz von Naturgesetzen. Ihre Aussagen sind Extrapolationen dessen, was wir beobachten. Diese Extrapolationen sind nicht grundsätzlich problematischer als jene, mit denen wir von endlich vielen Beobachtungen zur Annahme genereller Naturgesetze übergehen, sie sind aber natürlich ebenso hypothetisch wie diese. ${ }^{7}$

"Unsere Erfahrungen implizieren z. B. nicht logisch, daß die Welt älter ist als der Mensch. Mit unseren heutigen Beobachtungen wäre auch die Hypothese verträglich, die Welt sei vor 6000 Jahren erschaffen worden in dem Zustand (z. B. mit all den Sedimentschichten und Fossilien), in dem sie sich nach unseren Annahmen vor 6000 Jahren befand; oder die Hypothese, die Welt sei erst gestern erschaffen worden und zugleich wir mit all unseren Erinnerun- 
$\mathrm{Da}$ jeder kontingente physikalische Satz einen nichtleeren psychologischen Gehalt hat (also nicht mit beliebiger konsistenten Satzmergen über rein Psychisches verträglich ist), besagt nur, daß er nicht mit beliebigen tatsächlichen oder möglichen Beobachtungen verträglich ist, nicht aber, daß wir nur über tatsächlich von uns beobachtete oder beobachtbare physikalische Phänomene reden können, also auch nicht, daß Aussagen über die Welt nur für den Zeitraum möglich sind, in dem es Menschen gab und geben wird. Wir vertreten hier nicht die These esse est percipi, nach der es keine unbeobachteten Dinge gibt.

Eine andere Frage ist es, ob sich im Sinn der Evolutionstheorie der Mensch als Teil der Natur ansehen läßt, als Produkt einer Entwicklung, die sich im Prinzip rein physikalisch beschreiben läßt. Nimmt man das an, so steht man vor dem Rätsel, von dem E. Schrödinger in (59), Kap. I. spricht: Die Natur ist ein Schauspiel, das sich vor einem Betrachter abspielt, der in diesem Schauspiel selbst vorkommt, dessen Geburt, Leben und Tod im Stück dargestellt wird. Kann der Betrachter mit dem Schauspieler identisch sein, der seine Rolle spielt? Offenbar nicht. ${ }^{78}$ Der Mensch, von dessen Evolution das Stück „,Kosmogonie" handelt, ist wie ein Schauspieler, dessen Rolle mit Persönlichkeit und Lebensgeschichte des Zuschauers - des Naturwissenschaftlers, der dieses Schauspiel betrachtet - gewisse Ähnlichkeiten aufweist, der gewisse Szenen aus seinem Leben spielt, unter denen aber die des Zuschauers nicht vorkommt. Wie wir im 6. Kapitel zu zeigen versucht haben, läßt sich das Psychische und damit der Mensch nicht vollständig als Teil der physikalischen Natur ansehen, von deren Evolution die Kosmogonie redet. In der Kosmogonie machen wir uns auf der Grundlage unserer Erfahrungen ein Bild davon, wie die Natur entstanden ist und sich entwickelt hat, und da der Mensch als leib-seelisches Wesen auch Teil der Natur ist, kommt in diesem Bild auch der Mensch vor, die Geschichte seiner Evolution. Da er aber nicht nur Teil der Natur ist, ist es keine vollständige Geschichte seiner Evolution. Wie weit die Theorie der Evolution auch immer fortschreitet, zumindest die Geschichte der Evolution dieser T'heorie selbst wird sie

gen an vermeintlich frühere 'Tage. Das wären nur Annahmen, die mehr Fragen aufwerfen, als sie beantworten.

${ }^{78}$ Schrödinger macht das Problem freilich dadurch noch komplizierter als es ist, daß er in phänomenalistischer Weise von der Welt als Konstruktion aus unseren Sinnesdaten redet (vgl. a.a.O. S. 1). 
nicht umfassen. ${ }^{79}$ Die Frage nach der Entstehung unseres Bewußtseins - nicht die nach primitiveren Formen des Bewußtseins, Denkens und Erfahrens - ist eine typische Grenzfrage: Sie ließe sich nur im Rahmen einer vollständigen Theorie des Menschen beantworten, und eine solche Theorie über uns selbst können wir nicht entwickeln, wie wir gesehen haben. Wir haben also keine philosophischen Einwände gegen die Evolutionstheorie anzumelden - sinnvolle Einwände können sich nur aus naturwissenschaftlicher Forschung ergeben. Soweit sie reicht, ist sie vermutlich korrekt. Aber sie reicht eben nicht weit genug und kann nicht weit genug reichen, um eine vollständige Antwort auf die Frage nach unserem eigenen Ursprung und unserer eigenen Natur zu geben. Den Wert der partiellen Informationen, die wir aus ihr über uns selbst erhalten, braucht man deshalb nicht zu bestreiten.

Existenz und Beschaffenheit der Natur sind also im Makrobereich, dem Bereich der normalen, alltäglichen Erfahrung insofern unabhängig von unseren Beobachtungen, als wir eine Beschreibung der Natur akzeptieren, nach der man in diesem Bereich Aussagen über unbeobachtete Dinge machen kann und es störungsfreie Beobachtungen gibt. In diesem Sinn läßt sich also ein Realismus vertreten, der mit unserer Behauptung verträglich ist, die physikalischen Terme würden unter Bezugnahme auf Beobachtungsverfahren und Erlebnisweisen erklärt, so daß die Aussagen der Physik die Natur so beschreiben, wie sie sich in unserer Erfahrung zeigt. Dieser Realismus bzgl. der Makrowelt ist, wie wir in 8.4 sahen, die methodische Voraussetzung für die Mikrophysik. Er wird also auch von dieser nicht aufgehoben. Im Mikrobereich, in dem es keine störungsfreie Beobachtbarkeit gibt, und in dem Aussagen über unbeobachtete physikalische Systeme, Aussagen über Wahrscheinlichkeiten von Meßergebnissen sind, ist hingegen kein Realismus haltbar, nach dem man den Objekten Eigenschaften unabhängig von Beobachtungen zuschreiben kann. Auch hier gilt aber, daß wir beobachteten Objekten, und nicht etwa nur den Beobachtungen oder nur dem aus beobachtetem Objekt und Beobachter bestehenden

79 Wie weit auch jemand sein Tagebuch über sein Leben fortschreibt, es wird doch unvollständig bleiben. Bis zu dem Punkt, wo er das beschreibt, was er gerade schreibt, kommt er nicht. Er müßte sonst den Satz hinschreiben „Ich schreibe jetzt gerade diesen Satz" und würde damit nur einen sinnlosen Ausdruck zu Papier bringen. 
System Eigenschaften zusprechen können, also nichts von der Art eines Idealismus.

Aus all dem ergibt sich ein ontologischer wie erkenntnistheoretischer Realismus, der die Natur nicht als Welt ,,an sich“, sondern als Gegenstand unserer Erfahrung begreift, ohne ihr damit Objektivität und Eigenständigkeit gegenüber dem Psychischen abzusprechen. Die Argumente für die Erkennbarkeit der Natur in 3.4 können wir beibehalten, denn sie waren unabhängig vom starken Objektivitätsbegriff. Und dasselbe gilt von den Argumenten für den ,,naiven“ Realismus.

Ein solcher Realismus weicht von dem common-sense-Realismus nicht allzu weit ab. Denn dieser ist naturgemäß ein Realismus bzgl. der normalen Erfahrung, der Erfahrung von Makrophänomenen, und die Deutung der Natur als ,Welt an sich" ist schon eine philosophische Interpretation. Im Alltagsverständnis ist die Unabhängigkeit von Existenz wie Beschaffenheit der Natur von menschlicher Erfahrung primär eine Unabhängigkeit von einzelnen konkreten Erfahrungen. $\mathrm{Daß}$ aber die Temperatur des Wassers unabhängig davon ist, ob jemand es als warm oder kalt empfindet, und daß die Existenz eines Sterns unabhängig davon ist, ob er beobachtet wurde, gilt natürlich auch nach unseren Überlegungen. Eine Übereinstimmung mit dem common sense haben freilich auch Kant und Berkeley für ihre voneinander wie von unserer weit divergierenden Theorie der Erfahrung behauptet, und daher wird man solchen Aussagen kein großes Gewicht beimessen. Tatsache ist, daß der Alltagsverstand sich die hier erörterten erkenntnistheoretischen Probleme gar nicht stellt, daß er darauf also auch keine Antwort gibt. Man kann ihn daher auch nicht für gewisse Antworten in Anspruch nehmen, sondern nur plausibel machen, daß sie unseren normalen Intuitionen in den Grenzen nicht widersprechen, bis zu welchen diese reichen.

Ein erkenntnistheoretischer und damit auch ein ontologischer Realismus im starken Sinn ist in der Philosophiegeschichte oft nicht auf der Ebene der Erfahrungserkenntnis vertreten worden, sondern auf der Ebene metaphysischer Erkenntnis. Wir haben schon in 1.4 auf die Rolle hingewiesen, die das Erkenntnisideal notwendigen Wissens in der Philosophie gespielt hat, die Ansicht, echte Erkenntnis läge nicht schon dann vor, wenn das Bestehen eines Sachverhalts richtig konstatiert wird, sondern erst dann, wenn seine Notwendigkeit eingesehen wird und damit jede Irrtumsmöglichkeit ausgeschlossen ist. Da 
uns Erfahrung immer nur zeigt, daß gewisse Sachverhalte bestehen, aber nie, daß sie notwendigerweise bestehen, vermittelt sie keine „echte“ Erkenntnis in diesem Sinn. Die Notwendigkeit einer Tatsache läßt sich nicht empirisch, sondern nur apriorisch begründen. Gibt es also echte Wirklichkeitserkenntnis, so muß es auch synthetische Erkenntnisse apriori geben. Mit Kant kann man sagen, daß die synthetischen Erkenntnisse apriori den Inhalt der Metaphysik ausmachen. Metaphysische Erkenntnis ist also eine über die Erfahrung hinausgehende Erkenntnis. Das wird oft so aufgefaßt, daß metaphysische Erkenntnis anderes zum Gegenstand hat als Erfahrungserkenntnis, daß sie Erkenntnis einer Realität über oder hinter der erfahrbaren Welt ist, wie z. B. in der platonischen Ideenlehre oder in einer rationalen Theologie. Metaphysik kann sich aber auch auf dieselbe Welt beziehen wie Erfahrung, wie z. B. in der rationalen Kosmologie. Ob eine Erkenntnis metaphysisch ist, hängt nicht von ihrem Gegenstand $a b$, sondern vom Modus ihrer Begründung, vom Modus der Geltung der erkannten Tatsache.

Sachverhalte, die notwendigerweise gelten, wurden nun vielfach als Tatsachen angesehen, die einer Wirklichkeit an sich zugehören. Metaphysische Erkenntnis ist dann Erkenntnis einer Wirklichkeit an sich. Während Erfahrung nicht bis zur Wirklichkeit selbst vordringt, sondern es mit einer der bloßen Meinung entsprechenden Scheinwelt zu tun hat, soll sich in metyphysischer Erkenntnis zeigen, wie die Wirklichkeit an sich beschaffen ist. Dieses Ideal einer unfehlbaren Erkenntnis einer Wirklichkeit an sich bezeichnen wir als platonisches Erkenntnisideal. Diese Konzeption ergibt sich nicht schon aus dem Begriff notwendigen Wissens. Die Annahme, mit Notwendigkeit geltende Sätze beschrieben eine Welt an sich, ist nicht zwingend: $\mathrm{Da}$ diese Sätze synthetisch sein sollen, handelt es sich nicht um eine analytische Notwendigkeit. Dann ist aber nicht gesagt, daß solche Sätze mit allen konsistenten Annahmen über Psychisches verträglich sind.

Auch Kant geht vom Ideal notwendiger Erkenntnis aus. Für ihn gibt es metaphysische Erkenntnis; sie bezieht sich aber nicht auf die Wirklichkeit an sich, sondern nur auf Erscheinungen. Nach seiner Ansicht gibt es eine Metaphysik der Erfahrung als Wissenschaft von den Strukturen der Erfahrungsgegenstände, die ihnen das Subjekt „,notwendigerweise“ aufgrund der Konstitution seiner Sinnlichkeit wie seines Verstandes aufprägt, aber keine Metaphysik als Erkenntnis der Wirklichkeit an sich. Bei Kant entfällt damit eine Komponente 
des platonischen Erkenntnisideals, daß Erkenntnis immer Erkenntnis einer Wirklichkeit an sich ist, während er die andere Komponente dieses Ideals beibehält: die Notwendigkeit des Erkannten. Er hält im Sinn der idealistischen Tradition am subjektiven Faktor des platonischen Ideals fest, dem Modus der Sicherheit, der Unfehlbarkeit, und streicht den objektiven Faktor: die Wirklichkeitserkenntnis. Erkenntnis ist nach Kant immer nur Erkenntnis von Erscheinungen, also Erkenntnis von Subjektivem.

Wir haben beide Komponenten aufgegeben: Nicht nur, wie Kant, das Ideal der Erkenntnis einer Wirklichkeit an sich, sondern auch das Ideal subjektiver Unfehlbarkeit. Wir halten aber am Ziel einer Wirklichkeitserkenntnis, einer objektiven Erkenntnis fest. Danach erscheint Erfahrungserkenntnis - obwohl sie keine Notwendigkeit begründet, kein perfektes Wissen liefert und keine Erkenntnis einer Welt an sich ist - als durchaus ,echte“ Erkenntnis. Wenn sich uns irgendwo objektive Wirklichkeit zeigt, so sicher in der Erfahrung. Wir wenden uns damit gegen die falschen Alternativen: Notwendige Erkenntnis oder gar keine (bloße Meinungen), Erkenntnis einer Wirklichkeit an sich oder Erkenntnis nur von Ideen. Es gibt in beiden Fällen ein sehr viel sinnvolleres Drittes: Erkenntnis als wahre Überzeugung, bzw. Erkenntnis einer im normalen Sinn des Wortes objektiven Wirklichkeit. Beide Komponenten des platonischen Erkenntnisideals sind aufzugeben, da sie nicht realisierbar sind: Es gibt, wie wir zu zeigen versucht haben, weder unproblematische Erkenntnis der Außenwelt noch Erkenntnis einer Wirklichkeit an sich. Diese Einsicht ist aber nicht nur destruktiv, sondern sie hat dadurch einen höchst konstruktiven Effekt, daß sie die philosophische Erkenntnisbemühung aus der unfruchtbaren Fixierung auf unerreichbare Ziele löst.

Kant nimmt hinter der Welt der Erscheinungen eine Wirklichkeit an sich an. Sie bleibt aber für ihn völlig unerkennbar, weil sie sich nicht in unseren Erfahrungen zeigt, und weil sich alle unsere Begriffe nur auf Erscheinungen anwenden lassen. Da die Wirklichkeit an sich nach Kant jedoch Ursache der Erscheinungen ist, drängen sich uns unabweislich Fragen über sie auf, die wir doch nicht beantworten können. Ihre Beschaffenheit - nicht ihre Existenz - ist so für Kant ein philosophisches Grenzproblem. Nach unserer Konzeption wie nach normalem Verständnis liegen, die Ursachen unseres Erlebens im Bereich des Erfahrbaren. Daher stellt sich für uns dieses Grenzpro- 
blem Kants nicht. Wir haben zunächst keinen Grund, eine Wirklichkeit an sich anzunehmen. Kants Grenzproblem ist jedoch nicht nur ein Produkt seines Systems, in ihm spiegelt sich vielmehr eine Frage, welche die Erkenntnistheorie seit ihren Anfängen begleitet. Der Mensch ist sich seiner selbst bewußt, er kann über sich selbst nachdenken. In der Reflexion auf uns selbst werden uns auch unsere Besonderheiten bewußt, unsere Bedingtheiten und Grenzen, unsere Endlichkeit. Mit dem Bewußtsein von der Begrenztheit unseres Lebens, von der kurzen Spanne zwischen Geburt und Tod, stellt sich uns die Frage nach dem, was jenseits dieser Grenzen liegt, die Frage vor allem, was jenseits des Todes liegt, und diese Frage hat die Menschen beschäftigt, soweit wir in der Geschichte zurückblicken können. Auch die Erkenntnistheorie ist nun eine Reflexion des Menschen auf sich selbst, eine Reflexion auf seine Erkenntnisfähigkeit, und indem in ihr die Bedingtheiten und Grenzen menschlicher Erkenntnis deutlich werden, stellt sich die Frage nach dem, was jenseits dieser Grenzen liegt, nach einer Wirklichkeit an sich; wie sich die Welt, wie sich der Mensch von einem Standpunkt aus darstellt, der den Bedingtheiten unseres Erkennens entrückt ist. Der Hinweis, daß wir keinen Begriff von einer Welt an sich machen können, daß wir keine Sprache zur Verfügung haben, in der wir sinnvolle und präzise Fragen über eine Welt an sich formulieren könnten, eliminiert dieses Problem noch nicht. Wie die Argumente, die wir im Abschnitt 1.7 gegen den universellen Erkenntnisskeptizismus vorgebracht haben, diesen nicht als sinnlos erwiesen haben, so erscheint auch die Frage, ob uns Erfahrungen etwas nicht nur für uns Bestehendes zeigen, noch nicht als abwegig, wenn man zugesteht, daß schon die Natur im normalen Sinn der Wörter nichts Subjektives, sondern etwas Objektives ist.

Das Sinnresiduum der Frage nach ,,echter" Erkenntnis haben wir in 1.7 durch das Beispiel des cartesischen Dämons verdeutlicht. Das Sinnresiduum der Frage nach ,echter" Objektivität der Erfahrungsgegenstände läßt sich in entsprechender Weise mit einem anderen Argument verdeutlichen, das Descartes in der ersten Meditation verwendet und das in der Philosophiegeschichte eine wichtige Rolle gespielt hat: dem Traumargument. Im Traum glauben wir Ereignisse zu erleben, Personen zu begegnen, Landschaften und Häuser zu sehen, Gespräche zu hören, zu handeln, um beim Erwachen zu erkennen, daß die Ereignisse und Dinge nichts anders als Produkte unserer Traumphantasie waren. Ist es nicht möglich, daß auch die Welt, die wir erle- 
ben, tatsächlich nichts anderes ist als ein Produkt von Vorgängen in uns selbst, eine furr Traumwelt? Auch das Traumerieben ist oft vivide, das Erlebte stellt sich als äußere Wirklichkeit dar, es erscheint überraschend und bedeutsam: auch 'Träume können kohärent sein, und auch im Traum erscheint es mir, als wenn die Welt von anderen Personen in gleicher Weise erfahren wird wie von mir. Die drei oben genannten Kriterien der Objektivität: Gegenständigkeit, Kohärenz und Intersubjektivität, bilden also keine Grundlage für die Behauptung, unser normales Erleben unterscheide sich grundsätzlich von dem in Träumen. Man hat of versuchr, das Traumargument dadurch zu widerlegen, daß man Kriterien angab, nach denen sich eine solche Unterscheidung durchführen lassen sollte. ${ }^{80}$ Demgegenüber ist aber zu betonen, daß im Argument nicht behauptet wird, es sei möglich, daß wir all das nur träumen, was wir zu erfahren meinen. Im normalen Sinn von „träumen" gibt es natürlich Kriterien, mit denen man feststellen kann, ob jemand träumt, und es mag auch Kriterien geben, nach denen jemand im Traum feststellen könnte, daß er träumt - obwohl das eher unwahrscheinlich ist: Überlegungen darüber, ob wir träumen, stellen wir im Traum nur selten an. Nicht die Unterscheidung von Wachen und Träumen ist das erkenntnistheoretische Problem, sondern die Frage, ob nicht das, was uns objektiv zu sein scheint, von einem höheren Standpunkt aus als bloß subjektiv erscheinen könnte, als etwas von der Art eines Traums, wobei die Wörter ,subjektiv“, „,objektiv" und ,Traum" nur metaphorisch verwendet werden. Diese Frage

${ }^{80}$ Descartes unterschied in (MP), 157 Träume vom wachen Erleben durch Kohärenz des Erlebten, mußte sich aber von Hobbes sagen lassen, man könne auch kohärent träumen. Auch für Leibniz ist die Kohärenz, die GesetzmäBigkeit des Beobachteten, die Voraussagen erlaubt, ein Kriterium für die $\mathrm{Zu}$ verlässigkeit von Beobachtungen und ihre Objektivität (vgl. (WP)I, 46 f.; II, 125; (T), 446f.). Sie begründet freilich nur eine ,moralische“, keine „,metaphysische" Gewißheit. Eine metyphysische Gewißheit würde erst eine vollständige Analyse der Phänomene ermöglichen. Diese moralische Gewißheit genügt aber, denn ,nihil autem aliud de rebus sensibilius aut scire possumus aut desiderare debemus, quam ut tam inter se quam cum indubitatis rationibus consentiant, atque adeo ut ex praeteritis praevideri aliquantenus futura possint. Alia in illis veritas aut realitas frustra expetitur, quam quae hoc praestat, nec aliud vel postulare debent sceptici vel dogmatici polliceri" ((WP)IV, 356). 
drängt sich uns zwar mit der Reflexion auf unsere Erkenntnisfähigkeit auf, ist aber nicht entscheidbar, da wir eben keinen Standpunkt außerhalb unserer selbst einnehmen, und uns von den Bedingungen unseres Erkennens befreien können. Sie ist also eine Grenzfrage. Jedes Argument für den in ihr angezielten ,objektiven“ Charakter der Erkenntnis muß von Aussagen über eine Wirklichkeit an sich ausgehen, stellt also eine petitio principii dar, und jeder Versuch einer Widerlegung muß das gleiche tun, führt also zu einem Selbstwiderspruch. Auch unmittelbare Evidenz können wir für eine Antwort nicht beanspruchen. Es ist uns evident, daß unsere (äußere) Erfahrung Erfahrung von einer objektiven Wirklichkeit im normalen, oben umrissenen Sinn ist, aber nicht, ob sich darin - insgesamt oder teilweise - etwas zeigt, das auch sub specie aeternitatis ,objektiv“ zu nennen wäre. Die Frage nach Existenz und Beschaffenheit einer Wirklichkeit an sich und ihrer Beziehung zu der von uns erfahrenen Welt ist also unentscheidbar, wenn man sie nicht von vornherein unter Hinweis darauf abweisen will, daß sie solange keinen präzisen Sinn hat, als offen bleibt, was ,objektive" Sachverhalte in diesem neuen Sinn sein sollen. Ebenso kann man die Frage nach der Unsterblichkeit mit dem Hinweis abtun, die Erfahrung zeige, daß alle Leute sterben; im normalen Sinn des Wortes lebe man nicht mehr, wenn man gestorben ist, und ein präziser anderer Sinn von ,leben“ sei nicht in Sicht. Nicht jede unpräzise Frage ist unsinnig. Andererseits ist aber auch nicht jede Frage, die so vage ist, daß man sie nicht beantworten kann, eine Grenzfrage. Wenn wir also die Frage nach einer Wirklichkeit ,an sich" als Grenzfrage bezeichnen, so deshalb, weil sich darin ein echtes Problem zumindest andeutet. Es ist wohl keinem, der sich damit ernsthaft beschäftigt hat, entgangen, daß dieses Problem auch an der Grenze sinnvoller Fragestellungen liegt.

\subsection{Objektivität und Idealismus}

Wir haben unsere erkenntnistheoretische Position als ,,realistisch“ bezeichnet. Es stellt sich aber die Frage, ob wir uns, nachdem wir in den Kapiteln 4 und 5 die idealistischen Thesen und Argumente verworfen haben, mit unseren Überlegungen nicht doch wieder idealistischen Gedanken genähert haben. Sind unsere eigenen Argumente ge- 
gen den absoluten Realismus und gegen den Dualismus nicht mit jenen für den Idealismus eng verwandt?

Der erkenntnistheoretische Idealismus geht von einem richtigen Grundgedanken aus: Die Welt, die wir erfahren, läßt sich nicht als eine Realität ,an sich“ verstehen. Wir können nur dann behaupten, unsere Erfahrung zeige uns, wie die Welt beschaffen ist, wenn wir die Beschaffenheit der Welt nicht als prinzipiell unabhängig von der Art und Weise ansehen, wie sie erfahren wird. ${ }^{81}$ Wir haben in diesem Sinn gesagt: Die Natur ist keine Welt an sich, sondern eine Welt, die dadurch charakterisiert ist, daß sie Gegenstand unserer Erfahrung ist. Aus diesem richtigen Gedanken zieht der Idealismus aber die nicht nur falsche, sondern abwegige Konsequenz, daß die Welt aus unseren Erfahrungen besteht; daß nur Sachverhalte des Erfahrens (Erscheinungen, Empfindungen, Ideen, Phainomena, Sinnesdaten, mentale Bilder, oder wie immer man das bezeichnet) Gegenstände der Erfahrung sind. Dieser Fehlschluß von der Aussage "Wir erfahren nicht Dinge an sich" auf die Behauptung ,Wir erfahren nur Ideen" und das Verständnis dieser Ideen als etwas Mentales oder Psychisches ist grundlegend für alle Formen des Idealismus und alle seine Argumente, wie wir gesehen haben, und diesen Schluß ziehen wir nicht. Die physische Natur besteht für uns nicht aus Erscheinungen, Erfahrungen sind nicht nur Erfahrungen von Eigenpsychischem, und äußere Erfahrung läßt sich nicht auf innere reduzieren. Die Unterscheidung von Sachverhalten des Erfahrens und erfahrenen Sachverhalten ist unverzichtbar, wenn man das Grundphänomen der Intentionalität der Erfahrung in den Blick bekommen will, das eine Grundlage der Unterscheidung von Psychischem und Physischem ist. ${ }^{82}$ Wir gehen also mit dem Idealismus einig in der Ablehnung des absoluten Realismus, unterscheiden uns aber von ihm in der Deutung der Erfahrung.

Anders als der Idealismus behaupten wir auch nicht, das Psychische (Erlebnisweisen, Empfindungen, Überzeugungen, Strebungen etc.) ließe sich unabhängig vom Physischen bestimmen und beschreiben. Aussagen über Psychisches sind ihrem Sinn nach auf Physisches bezogen, und daher ist der idealistische Versuch verfehlt, das Physische auf das Psychische zu reduzieren. Der Idealismus muß ja die norma-

${ }^{81}$ Vgl. dazu das Argument am Anfang des letzten Abschnitts.

${ }^{82}$ Vgl. dazu den letzten Abschnitt. 
len Aussagen über Physisches und die normale Unterscheidung innerer und äußerer Erfahrung sowie physischer und psychischer Phänomene im Rahmen seiner ontologischen Voraussetzungen rekonstruieren, und er muß dabei eine reduktionistische These vertreten. Wir haben dagegen betont, daß die These nicht haltbar ist. ${ }^{83}$ Physisches und Psychisches lassen sich nur in Abgrenzung von einander charakterisieren. Daher sind beide Realitätsbereiche gleich ursprünglich und in gleicher Weise relativ eigenständig.

Unsere Position unterscheidet sich also nicht nur in Formulierungen oder Details, sondern grundsätzlich von idealistischen. Mit dem Idealismus gehen wir nur in der Ablehnung des absoluten Realismus, des Dualismus und des Physikalismus einig. Anstelle des erkenntnistheoretischen Idealismus vertreten wir einen erkenntnistheoretischen Realismus, nach dem die Gegenstände (äußerer) Erfahrung nichts Psychisches oder auf Psychisches Reduzierbares sind, sondern etwas Objektives in dem im letzten Abschnitt skizzierten Sinn. Mit dem erkenntnistheoretischen Idealismus entfällt zugleich die Grundlage für den ontologischen Idealismus. ${ }^{84}$ Wir beziehen also eine Position zwischen Idealismus und absolutem Realismus, die sich von beiden deutlich abhebt.

Zur Verdeutlichung unserer Konzeption wollen wir sie mit den wichtigsten idealistischen Theorien vergleichen. Da wir auf Berkeley schon im 4. Kapitel eingegangen sind, bleibt noch etwas zu den Theorien des Deutschen Idealismus zu sagen.

Bei weitem den höchsten Rang hat dabei die Erkenntnistheorie Kants. Kant vertritt einen erkenntnistheoretischen Idealismus in Verbindung mit einem absoluten ontologischen Realismus. Für seine Begründung des erkenntnistheoretischen Idealismus ist vor allem die Argumentation in der Transzendentalen Ästhetik wichtig. ${ }^{85} \mathrm{Ihr}$ liegt -

${ }^{83}$ Vgl. dazu das Kapitel 5.

${ }^{84}$ Vgl. dazu den Abschnitt 4.3.

${ }^{85}$ Daneben findet sich bei Kant auch das übliche direkte Argument für den erkenntnistheoretischen Idealismus, das allerdings nur in recht dürftiger Weise angedeutet wird. Es beruht wieder auf der Verwechslung von Sachverhalten des Erscheinens mit erscheinenden Sachverhalten; auf dem Schluß von „Wir erfahren die Dinge nur so, wie sie sich uns aufgrund der Eigenart unserer Sinnlichkeit darstellen“ auf „Wir erfahren nicht die Dinge selbst, sondern 
trotz aller Unterschiede in der Durchführung - derselbe Gedanke zugrunde, den schon Berkeley verwendet hatte und auf den wir bereits in 8.3 hingewiesen haben. Berkeley ging aus von Lockes Unterscheidung der beobachteten Eigenschaften in primäre, die den Dingen selbst zukommen, und sekundäre, die sie lediglich aufgrund unserer Simnesorganisation zu haben scheinen, und wollte zeigen, daß auch die primären Qualitäten Lockes - speziell geometrische und zeitliche Merkmale sekundäre Qualitäten sind, um dann zu sagen: Wenn alle Eigenschaften nicht den Dingen selbst zukommen, sondern nur unsere Erfahrungsweise charakterisieren, so können wir auch nicht behaupten, daß wir die Dinge selbst wahrnehmen. Denn Gegenstände lassen sich nur vermittels ihrer Eigenschaften bestimmen und unterscheiden. Charakterisieren also die beobachteten Eigenschaften nur Erfahrungsweisen, so können auch die Gegenstände der Erfahrung nur Erscheinungen der Dinge, also Ideen sein. Kant unternimmt es in der Transzendentalen Ästhetik ebenfalls, die Idealität von Raum und Zeit, und damit die Idealität der Gegenstände der Erfahrung nachzuweisen. Er geht davon aus, daß die Sätze der euklidischen Geometrie - zu seiner Zeit war nur die euklidische Geometrie bekannt, so daß wir in seinem Sinn sagen können, die Sätze der Geometrie - apriorisch begründbar sind. Diese Annahme wird man Kant zugestehen müssen, wenn von Sätzen der mathematischen Geometrie die Rede ist. Kant unterscheidet nun aber nicht zwischen mathematischer Geometrie, deren Sätze von abstrakten, idealen Gebilden handeln, und physikalischer Geometrie, deren Sätze etwas über die Struktur des physikalischen Raums aussagen. Aus der Tatsache, daß alle Gegenstände der äußeren Erfahrung räumlich bestimmt sind, folgt für ihn daher ohne weiteres, daß der physikalische Raum auch euklidische Struktur hat.

nur unsere Art, sie wahrzunehmen" (vgl. dazu (KrV), 83 f.). Kant meint: Wären uns in der Wahrnehmung Gegenstände der Außenwelt selbst gegeben, so bliebe es unbegreiflich, ,wie die Anschauung einer gegenwärtigen Sache mir diese sollte zu erkennen geben, wie sie an sich ist, da ihre Eigenschaften nicht in meine Vorstellungskraft hinüberwandern können“. (W) V, 144. Ein ähnlicher Gedanke findet sich bei Leibniz, der damit begründen will, daß eine Wechselwirkung zwischen verschiedenen Objekten unbegreiflich sei. Für Kant stellt sinnliche Erfahrung die Dinge nicht so vor, wie sie sind, sondern nur die Art, wie sie unsere Sinne affizieren, und also ,werden durch sie ,bloß Erscheinungen', nicht die Sachen selbst dem Verstande zur Reflexion gegeben" ((W) $\mathrm{V}, 154$.) 
Damit sind aber die Sätze der Geometrie auch synthetische Sätze, Sätze über die von uns erfahrene Gegenstandswelt. ${ }^{86}$ Es gibt also apriorisch-synthetische Aussagen, und das ist nach Kant nur dann möglich, wenn die geometrische Struktur des Erscheinenden nicht von den Dingen selbst abhängt, sondern ihm vom Subjekt aufgeprägt wird; wenn der Raum nicht eine Realität an sich ist, sondern eine Anschauungsform. Er sagt: „Wollte man im mindesten daran zweifeln, $\mathrm{da} ß$ beide [Raum und Zeit] gar keine den Dingen an sich selbst, sondern nur bloße ihrem Verhältnisse zur Sinnlichkeit anhängende Bestimmungen sind, so möchte ich gern wissen, wie man es möglich finden kann, a priori und also vor aller Bekanntschaft mit den Dingen, ehe sie nämlich uns gegeben sind, zu wissen, wie ihre Anschauung beschaffen sein müsse, welches doch hier der Fall mit Raum und Zeit ist. Dieses ist aber ganz begreiflich, sobald beide für nichts weiter als formale Bedingungen unserer Sinnlichkeit, die Gegenstände aber bloß für Erscheinungen gelten; denn alsdann kann die Form der Erscheinung, d. i. die reine Anschauung, allerdings aus uns selbst, d.i. a priori vorgestellt werden " ${ }^{87}$

${ }^{86}$ Es kommt für das Argument nicht darauf an, ob die Sätze der mathematischen Geometrie analytische Sätze sind - etwa im Sinne der Begründung der Geometrie, die D. Hilbert in (99) angegeben hat - oder synthetische Sätze, die auf einer reinen Anschauung beruhen, wie das Kant meint.

${ }^{87}$ Kant (W)V, 146. - Kant hat hier, wie so oft, ein wichtiges Problem gesehen, wenn auch scire Antwort nicht befriedigt: ,Wieso kann man voraussetzen, daß logische und mathematische Gesetze auch für die Gegenstände der Erfahrung gelten?" Die Antwort, diese Sätze seien analytisch, genügt nicht. Unsere Logik ist die Logik der Sprache, mit der wir Erfahrungsinhalte beschreiben. Als analytische Sätze gelten logische Gesetze also für alle Aussagen über Erfahrungsinhalte. Die Frage ist aber, mit welchem Recht wir annehmen, daß diese Aussagen die Welt richtig darstellen. Können wir behaupten, die Welt bestehe ,an sich“ aus Substanzen und ihren Attributen, ihre ontologische Struktur werde durch die Subjekt-Prädikatstruktur unserer Aussagen nur abgebildet, oder müssen wir im Sinn Kants sagen, daß wir den Erfahrungsinhalten durch sprachlich-begriffliche Bestimmung diese Struktur aufprägen? Eine dritte Möglichkeit ist: Unsere Sprache ist zur Beschreibung der Erfahrungen entwickelt worden, und unsere Beschreibungsweise hat sich für die Systematisierung der Erfahrungen bewährt. Die grammatikalischen Universalien sind also weder angeboren (apriorisch), noch sind sie aus der Erfahrung abgelesen - die wir ja erst mit ihnen bestimmen - , sondern Sprache und Weltsicht haben sich zusammen entwickelt. Tatsächlich 
Entsprechend argumentiert Kant in Fällen zeitlicher Bestimmungen. Der Mangel dieser Arglimentation besteht darin, daß der synthetisct:-apriorische Charakter geometrischer Aussagen durch die naive Identifizierung von mathematischer und physikalischer Geometrie „,begründet" wird, und ebenso im Fall der Zeitlehre.

Kants transzendentaler Idealismus ist das Ergebnis seiner transzendentalen Ästhetik: ,Ich verstehe aber unter dem transzendentalen Idealismus aller Erscheinungen den Lehrbegriff, nach welchem wir sie insgesamt als bloße Vorstellungen und nicht als Dinge an sich selbst, ansehen, und demgemäß Zeit und Raum nur sinnliche Formen unserer Anschauung, nicht aber für sich gegebene Bestimmungen, oder Bedingungen der Objekte, als Dinge an sich selbst sind".

Erscheinungen oder Anschauungen sind nun noch nicht Erfahrungen im Sinne Kants. Erfahrung entsteht erst durch die begriffliche Bestimmung der Erscheinungen. Nach Kant lassen sich aber Begriffe auch die reinen Verstandesbegriffe - nur auf Erscheinungen beziehen; ihr Anwendbarkeitsbereich deckt sich mit dem Bereich möglicher Erfahrungen. Es gibt zwar bei ihm eine nicht auf Erfahrung bezogene Verwendung logischer Begriffe, aber mit den analytischen Sätzen der Logik können wir nichts über die Welt aussagen. Die mathematischen Begriffe - Mathematik bestand für Kant aus Geometrie und Arithmetik - beziehen sich dagegen schon auf Anschauungen, denn der Geometrie liegt die Raumanschauung, der Arithmetik die Zeitanschauung zugrunde. Sehen wir von logisch-mathematischen Begriffen $\mathrm{ab}$, so berühren sich unsere Argumente für den anschaulichen Gehalt von Erfahrungsbegriffen mit Kants Argumenten für diese These von den Grenzen der Anwendbarkeit solcher Begriffe, ${ }^{88}$ denn im Schematismus-Kapitel sagt er im Effekt, die Anwendungskriterien für Erfahrungsbegriffe seien letztlich anschauliche Kriterien, Kriterien, die auf räumliche oder zeitliche Anschauungen oder auf andere Erlebnisweisen Bezug nehmen. Ein eingehenderer Vergleich wäre allerdings schwierig, da die Aussagen Kants viele Interpretationsprobleme aufwerfen.

gibt es ja viele Phänomene, bei denen die Substanz-Akzidens-Analyse recht künstlich ist, und es gibt Sprachen, deren Grammatiken sich erheblich unterscheiden. Schon W. von Humboldt hat Kants Ideen in dieser Weise modifiziert.

${ }^{88}$ Erfahrungsbegriffe sind hier nicht nur „empirische Begriffe“ im Sinne Kants, d. h. aus Empfindungen abstrahierte Begriffe. 
Für Kant gab es auch in den Naturwissenschaften, insbesondere in der Physik, die für ihn durch die Newtonsche Mechanik repräsentiert wurde, apriorische Erkenntnisse. Das gilt nicht nur für die dieser $\mathrm{Me}-$ chanik zugrundeliegende Raum-Zeitlehre, sondern auch für die Newtonschen Gesetze selbst. Das läßt sich nach Kant wiederum nur so erklären, daß diese Sätze nicht Aussagen über eine Welt an sich sind, sondern Aussagen, die sich auf Erscheinungen beziehen. Sie ergeben sich für Kant aus den Grundsätzen des reinen Verstandes, und diese ergeben sich aus dem Schematismus der reinen Verstandesbegriffe - wir würden etwa sagen: Aus dem auf erlebnismäßige Unterschiede bezogenen Sinn von Erfahrungsbegriffen, oder: aus der Definition physikalischer Begriffe durch Bezugnahme auf Beobachtungsverfahren. ${ }^{89}$ Wir haben aber schon oben betont, daß man aus der Bezogenheit des Sinns von physikalischen Termen auf erlebnismäßige Kriterien oder Beobachtungsverfahren nicht darauf schließen kann, daß physikalische Aussagen nur über Erlebnisse, Beobachtungen oder Erscheinungen reden. Heute würde man auch den apriorischen Charakter der Grundgesetze der Newtonschen Mechanik bestreiten, schon deshalb, weil sie sich als nur in bestimmten Bereichen, in gewissen Näherungen gültig erwiesen hat. Man würde nicht leugnen, daß über die Definition der physikalischen Terme durch Bezugnahme auf Beobachtungsverfahren und durch Bedeutungspostulate viele apriorische Elemente in die Physik eingehen, deswegen aber noch nicht physikalische Theorien insgesamt als apriorisch begründbar ansehen. ${ }^{90}$

Seinen absoluten ontologischen Realismus begründet Kant so: „Wenn wir die Gegenstände der Sinne, wie billig, als bloße Erscheinungen ansehen, so gestehen wir hierdurch doch zugleich, daß ihnen ein Ding an sich selbst zum Grunde liege, ob wir dasselbe gleich nicht, wie es an sich beschaffen sei, sondern nur seine Erscheinung, d. i. die Art, wie unsere Sinne von diesem unbekannten Etwas affiziert

${ }^{89} \mathrm{Vgl}$. dazu die Abschnitte 7.4 und 8.3.

${ }^{90}$ Der Hinweis auf diese vorherrschende Meinung ist natürlich kein Argument gegen eine apriorische Begründbarkeit physikalischer Theorien. Es gibt auch heute Versuche, die Quantenmechanik oder eine noch tiefer liegende physikalische Grundtheorie apriorisch zu begründen, z. B. im Sinn einer Art „,Logik" von Beobachtungen. Auch aus einer apriorischen Geltung physikalischer Gesetze würde sich jedoch kein erkenntnistheoretischer Idealismus ergeben. 
werden, kennen" ${ }^{91}$ Erscheinungen sind also ihrem Begriff nach Erscheinungen von etwas, und das deutet Kant so: sie sind Erscheinungen von Dingen an sich, die in den Erscheinungen zwar nicht zur Gegebenheit gelangen, aber diese bewirken. Der Gedanke, der dahinter steht, ist wohl der: Sinnliche Empfindungen sind passiv. Wir bestimmen nicht selbst, was wir hören oder sehen. Sie sind also nicht Produkte des erfahrenden Subjekts allein, so daß es etwas außerhalb von uns geben muß, das sie in uns hervorruft. Diese Aussagen widersprechen dem normalen Sprachgebrauch: Erscheinungen sind intentional. Sie sind also Erscheinungen von etwas. Man kann aber nicht sagen: das, wovon sie Erscheinungen sind, erscheine uns nicht. Erscheinungen sind Erscheinungen von den erscheinenden Gegenständen - besser: Erfahrungen sind Erfahrungen von den erfahrenen Gegenständen. Wenn man also schon die Idealität der Gegenstände der Erfahrung annimmt, so läßt sich mit dem Hinweis auf die Intentionalität der Erfahrungen kein ontologischer Realismus begründen. Und umgekehrt beruht der erkenntnistheoretische Idealismus gerade auf der Mißachtung der Intentionalität der Erfahrungen.

Kant sieht sich nun vor die Aufgabe gestellt, im Bereich der Erscheinungen die übliche Unterscheidung von Subjektivem (wie z. B. Illusionen im Sinne von 5.2) und Objektivem zu rekonstruieren, zwischen einem Bereich subjektiven Erlebens und dem Bereich einer objektiven Natur. Für die Objektivität von Erfabrungsurteilen im Gegensatz zu den nur subjektiven Wabrnebmungsurteilen sind nach Kant zwei äquivalente Kriterien maßgebend:92 die intersubjektive Geltung der Aussagen und der gesetzmäßige Zusammenhang der Vorgänge in der Natur. Erfahrungsurteile sind allgemeingültig, d. h. für alle Menschen gültig. Nun unterscheiden sich die Menschen in den Empfindungen, die sie haben. Allgemeingültige Urteile können also nur auf den apriorischen Momenten der Erfahrung beruhen: auf den Anschauungsformen Raum und Zeit und auf den Grundsätzen des reinen Verstandes. Diese apriorischen Elemente bilden aber auch, wie Kant zu zeigen versucht, die Grundlagen der Newtonschen Physik. Nur jene Phänomene sind also der objektiven Natur zuzurechnen, die sich als Vorgänge im Einklang mit den Gesetzen der Newtonschen Mechanik deuten lassen. Die Natur wird also als Bereich der Erschei-

${ }^{91}$ Kant (W)V, 183.

${ }^{2} \mathrm{Vgl}$. dazu Kant (W)V, $163 \mathrm{ff}$. 
nungen bestimmt, die sich mit mechanischen Gesetzen beschreiben lassen; als Bereich dessen, was sich auf Mechanik reduzieren läßt. Ein Wahrnehmungsurteil ist ,Alle von mir bisher beobachteten $S$ waren P's“, ein Erfahrungsurteil hingegen ,Alle S sind P's“. Zur Begründung solcher universeller Erfahrungsurteile reichen einzelne Wahrnehmungen nie hin, sie ergeben sich nur aus apriorischen Prinzipien. ${ }^{93}$ Erst die Konstitution der Natur als einer durch apriorische Prinzipien, durch universelle Gesetzmäßigkeiten beherrschten Bereichs ermöglicht es so, sie als etwas Objektives anzusehen. Die Konstitution von Gegenständen als objektiven Phänomenen der Natur ist also für Kant eine Interpretation der Erscheinungen im Sinn der Newtonschen Mechanik. Dadurch wird der Mechanismus, die durchgängige Anwendbarkeit der Newtonschen Mechanik auf alle Naturvorgänge zu einer Tautologie, denn der Begriff der Natur wird von Kant gerade so bestimmt, daß diese Behauptung gilt. ${ }^{94}$

Auch bei unseren erkenntnistheoretischen Überlegungen sind wir auf das Problem der Objektivierung gestoßen, auf die Frage, nach welchen Kriterien wir Physisches und Psychisches, Subjektives und Objektives unterscheiden. ${ }^{95}$ Auch wir haben dabei auf Gesetzmäßigkeit und intersubjektive Geltung verwiesen. Wir haben aber im Unterschied zu Kant nicht angenommen, daß sich apriorische Gesetze angeben lassen, unter die sich alles Physische subsumieren lassen muß. Wir haben auch nicht behauptet, intersubjektive Geltung sei an Notwendigkeit gebunden. Und wir haben insbesondere im 5. Kapitel betont, daß das Programm einer Reduktion des Physischen auf Erscheinungen, Empfindungen oder Ideen nicht realisierbar ist. Auch Kant gibt nicht genau an, wie Erscheinungen als Erfahrungen ,zu buchstabieren" sind. Wir sind dagegen von der Intentionalität der Erfahrun-

${ }^{93}$ Für Kant fallen Universalität und Notwendigkeit zusammen. Die notwendige Geltung eines Satzes läßt sich aber nie empirisch, sondern immer nur apriorisch begründen.

${ }^{94}$ Das ist eine Rekonstruktion der Aussagen Kants, die im Detail nicht sehr klar sind, zumal er im Gebrauch der Wörter ,empirisch“, „Erfahrung“, „Erfahrungsurteil“ etc. im Übergang von der „Kritik der reinen Vernunft" zu den ,Prolegomena“ und der „Kritik der Urteilskraft" schwankt. Einmal sagt er z. B., daß Erfahrungsurteile nur kontingent gelten, dann aber sollen Erfahrungsurteile als Urteile über die Natur wieder mit Notwendigkeit gelten. Auf eine genauere Analyse müssen wir hier jedoch verzichten.

${ }^{95} \mathrm{Vgl}$. dazu den Abschnitt 8.6. 
gen ausgegangen, in der bereits die Differenzierung zwischen Psychischem und Physischem angelegt ist, und haben nur versucht, Gesichtspunkte für eine systematische und generelle Grenzziehung anzugeben. Diese Differenzierung haben wir als einen - im Rahmen von nur sehr lose umschreibbaren Kriterien wie Gegenständigkeit, Kohärenz und Intersubjektivität - historischen Prozeß begriffen, nicht als Produkt der Anwendung apriorisch angebbarer Regeln. Nach Kant wäre es in der Tat unverständlich, wie jemand, der die Newtonsche Mechanik nicht kennt, überhaupt zu Erfahrungen einer objektiven Natur gelangt, oder wie sich primitive Erfahrung so erheblich von unserer unterscheiden kann.

Kant glaubte mit seiner Theorie sowohl - gegenüber dem Dualismus - das Leib-Seele-Problem wie - gegenüber Hume - das Problem der Existenz und Erkennbarkeit der Außenwelt gelöst zu haben. Das Problem der Wechselwirkung zwischen Psychischem und Physischem verliert von einer idealistischen Position her natürlich seine Schrecken. Für Kant sind Physisches und Psychisches von gleicher Art, es sind Teilbereiche von Erscheinungen, und eine Wechselwirkung zwischen gleichartigen Phänomenen ist nicht verwunderlich. Wie wir im Abschnitt 8.5 gesehen haben, benötigt man für eine Lösung des Leib-Seele-Problems aber keinen Idealismus. In der Philosophie Kants tritt das Leib-Seele-Problem jedoch in verwandelter Gestalt auf als Frage, wie das Subjekt als Noumenon, als reines Vernunftwesen auf physisches Geschehen einwirken kann, das auch von Dingen an sich abhängt, wie z. B. in einem pflichtgemäßen Handeln. Dieses Problem - ebenso wie das allgemeine Problem der Vereinbarkeit einer kausalen Einwirkung der Dinge an sich auf die Welt der Erscheinungen mit der ihr immanenten kausalen Determiniertheit - erklärt Kant für unlösbar. Ein derartiges Problem tritt bei unserem Ansatz hingegen gar nicht auf.

Die Existenz einer Außenwelt (im Sinne der Natur) ist für Kant nicht problematischer als meine eigene Existenz - alles Erfahrbare ist nichts als Erscheinung, und der Erscheinungen bin ich gewiß, gleich ob ich sie mit dem inneren oder dem äußeren Sinn auffasse. Daher verbindet sich für Kant mit seinem transzendentalen Idealismus ein „empirischer Realismus“, nach dem die Außenwelt (die Natur) ebenso real ist wie das Eigenpsychische. ${ }^{96}$ Er lehnt den „empiri-

${ }^{96} \mathrm{Als}$ „transzendentalen Realismus" bezeichnet Kant die These des (absolu- 
schen Idealismus" Descartes ab, nach dem die Existenz der Außenwelt (der Welt äußerer Erfahrung) nicht beweisbar ist, sondern allenfalls plausibel gemacht werden kann. Diesen empirischen Idealismus widerlegt Kant so: Das empirische Bewußtsein meines eigenen Daseins in der Zeit, das Descartes als unproblematisch ansah, setzt etwas in der Zeit Beharrendes, Unverändertes voraus, an dem ich zeitliche Veränderungen messen kann. In mir selbst finde ich aber nur wechselnde Vorstellungen, nichts Konstantes. Das Beharrende kann also nur außer mir sein. Daher ist die Bestimmung meines Daseins in der Zeit nur durch die Existenz wirklicher Dinge, die ich außer mir wahrnehme, möglich. Innere Wahrnehmung gibt es also nur, wo es äußere gibt. Dieses Argument ist natürlich fragwürdig, man kann aber vielleicht im Sinne von Kant sagen: Die Selbsterfahrung ist nicht ursprünglicher als die äußere Erfahrung. Ich bestimme mich selbst wie das Eigenpsychische in der Abgrenzung von der Außenwelt. Und das würde sich dann mit unserer Aussage berühren, daß die Sprache über Psychisches auf die Sprache über Physisches bezogen ist, daß also das Psychische dem Physischen gegenüber nicht als unabhängig und primär angesehen werden kann. Die Erkennbarkeit der Außenwelt ergibt sich für Kant aus der Annahme apriorisch-synthetischer Erkenntnisse. Für uns ergibt sie sich hingegen aus der Annahme analytischer Beziehungen zwischen Sätzen über Physisches und solchen über Psychisches. Auch in diesem Fall ,löst" Kant ein Problem durch Umformu-

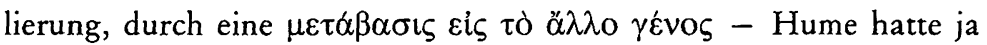
nicht die Existenz und Erkennbarkeit vor Erscheirungen bezweifelt. An der Stelle des alten Problems erscheint auch hier ein unlösbares neues: die Dinge an sich bleiben unerkennbar.

Es gibt also viele Beziehungen und Analogien zwischen unseren Überlegungen und denen von Kant. Ebenso gibt es aber deutliche Unterschiede im Grundsätzlichen wie im Detail. Sie ergeben sich vor allem daraus, daß wir im Gegensatz zu ihm keinen erkenntnistheoretischen Idealismus vertreten.

Der Idealismus Fichtes, Schellings und Hegels unterscheidet sich grundlegend von dem Kants. Den gemeinsamen Ausgangspunkt bildet nur der erkenntnistheoretische Idealismus. Während sich dieser aber bei Kant mit einem ontologischen Realismus verband, erklärte

ten) erkenntnistheoretischen Realismus, nach dem die Gegenstände der Erfahrung Dinge an sich sind. 
Fichte, wie schon Berkeley, beide für unvereinbar. Fichte erkannte aber auch, daß ein ontologischer Idealismus die Probleme richt löst. Er ging so von folgendem erkenntnistheoretischen Dilemma aus: Zwei Positionen stehen sich gegenüber, der ontologische Realismus, der eine Wirklichkeit an sich annimmt und den Fichte zugleich als einen Materialismus begreift, und der ontologische Idealismus, für den nur Intelligenzen mit ihren Vorstellungen existieren. ${ }^{97}$ Beide Positionen sind unhaltbar. Fichte bringt dafür zwei Argumente. Das erste lautet so: Im Sinn des erkenntnistheoretischen Idealismus ist ein $\mathrm{Ob}$ jekt immer Objekt für ein Subjekt, es gibt also keine Wirklichkeit an sich. Andererseits ist aber auch alles Bewußtsein intentional, Bewußtsein von Gegenständlichem, so daß auch ein Subjekt „für sich“ nicht denkbar ist. Fichte war der erste in der neuzeitlichen Philosophie, der die Intentionalität von Wahrnehmung und Denken klar herausgestellt hat. Als „Gesetz des Bewußtseins" formuliert er: „Kein Subjekt - kein Objekt; kein Objekt - kein Subjekt" ${ }^{98}$ - Eine brauchbare Ontologie muß also, so meint Fichte, von einer Realität ausgehen, in der (gegenständliches) Sein und Bewußtsein eine Einheit bilden. Diese Einheit suchte er im ,reinen Ich“, da in der Reflexion auf uns selbst Subjekt und Objekt unseres Denkens identisch sind. Über dieses ,reine Ich“ haben wir schon in 7.5 gesprochen. Das zweite Argument Fichtes besagt einmal, daß sich Psychisches nicht auf Physisches reduzieren läßt, daß also der Materialismus nicht haltbar ist. Zum anderen weist er darauf hin, daß unsere Sinnesempfindungen offenbar nicht von uns selbst produziert werden. Wir können nicht sehen, hören oder fühlen, was wir wollen. Nimmt man also nicht an, daß diese Empfindungen von äußeren Dingen verursacht werden, so bleibt nur die Berkeleysche Version des ontologischen Idealismus, nach der Gott die Rolle der Dinge an sich übernimmt, oder man muß eine Art Über-Ich annehmen, das die Sinnesempfindungen produziert. Beide Formen des ontologischen Idealismus operieren

97 Vgl. Fichte (W)I, $425 \mathrm{ff}$. - Der Dualismus ist für Fichte keine Alternative, da psycho-physische Wechselwirkungen danach unmöglich sind. Er sagt: „Man wird immer vergeblich nach einem Bande zwischen dem Subjekte und Objekte suchen, wenn man sie nicht gleich ursprünglich in ihrer Vereinigung aufgefaßt hat" ((W)I, 528).

${ }^{98}$ Fichte (W)I, 183. 
aber ebenso wie der ontologische Realismus mit einer bewußtseinstranszendenten Wirklichkeit, sind also für Fichte nicht akzeptabel.

Fichtes eigene Position bleibt weitgehend unklar. Seine Ausführungen sind in einem Maße konfus und wirr, für das es in der Philosophiegeschichte vorher kaum eine Parallele gibt. Seine Unfähigkeit zu elementaren logischen Unterscheidungen (wie z. B. jenen zwischen Objekten und Propositionen - ist a ein Objekt, so redet er ganz unbefangen von dem Nicht-a als einem bestimmten Objekt, als sei die Negation für Objekte definiert; nichtidentische Objekte ,,widersprechen" sich, usf. - zwischen Identität, Prädikation und Implikation oder Eigenschaft und Teil ${ }^{99}$ bewirkt, daß sich die Texte auch in den Grundgedanken nicht rational rekonstruieren lassen. Außerdem finden sich z. B. in den "Grundlagen der gesamten Wissenschaftslehre“ zwei einander widersprechende Konzeptionen: ein ontologischer Idealismus, in dem alle Erscheinungen dialektisch aus dem ,reinen Ich“ entwickelt werden sollen, und daneben, wie bei Kant, ein ontologischer Realismus, nach dem das „Nicht-Ich“ (die gegenständliche Welt) nun nicht vom Ich ,gesetzt" wird, sondern gleich ursprünglich mit ihm ist. ${ }^{100}$ Fichte meinte, man müsse zwischen Realismus und Idealismus ,mitten inne schweben“, und das hat er denn auch getan. Es lohnt also nicht, auf seine Systementwürfe näher einzugehen. Von Interesse ist allein die Zielsetzung, Realismus und Idealismus in einer Synthese zu vereinigen; weder ein Bewußtsein für sich noch ein Sein an sich als ontologisches Grunddatum anzunehmen, sondern eine Subjekt-Objekt-Einneit, als Einheit zweier gleich ursprünglicher, wesentlich auf einander bezogener Momente. Für diese Konzeption ist die Bezeichnung ",absoluter Idealismus" am passendsten. ${ }^{101}$ Dieser Idealismus, der in Schellings Identitätsphilosophie deutlicher wird, aber erst von Hegel konsequent entwickelt wurde, unterscheidet sich nun grundlegend vom ontologischen Idealismus, wie wir ihn früher charakterisiert haben. Dieser ist die letzte Konsequenz der erkenntnistheoretischen Entwicklung in der Aufklärung. Sie beginnt mit dem Dualismus von Descartes. Im 7. Kapitel haben wir die ontologische Seite dieses Dualismus erörtert, den psycho-physischen Dualismus.

\footnotetext{
${ }^{99} \mathrm{Vgl}$. z. B. Fichte (W),I, $101 \mathrm{ff}$; $106 \mathrm{ff}$; $92 \mathrm{f}$;; $108 \mathrm{ff}$. und $111 \mathrm{f}$. $100 \mathrm{Vgl}$. dazu z. B. Fichte (W)I, $279 \mathrm{ff}$.

101 Die Bezeichnung ,absoluter Idealismus“ verwendet zuerst Schelling für seine Identitätsphilosophie und dann Hegel für sein System.
} 
Ihm entspricht aber bei Descartes auch ein erkenntnistheoretischer Dualismus, nach den das Erkenntnissubjekt mit seinen cogitationes, seinen intellektuellen Anlagen und eingeborenen Ideen als autonome geistige Welt einer eigenständigen physischen Außenwelt gegenübersteht. Ontologisch sind beide Welten gleichberechtigt, erkenntnistheoretisch hingegen ist die subjektive Innenwelt die primäre Realität. Nur ihre Existenz ist unmittelbar evident, und nur ihre Beschaffenheit läßt sich unmittelbar erkennen. Existenz und Erkennbarkeit der Außenwelt sind hingegen problematisch und daher zu begründen. Die Welt ist nicht so, wie sie uns in der Erfahrung erscheint; wir können den Dingen nach Locke nur einige, nach Berkeley, Hume und Kant dann keine der Eigenschaften zuschreiben, mit denen sie uns erscheinen. Die Welt der Erscheinungen löst sich von der wirklichen Welt ab; diese wird so unerkennbar und schließlich zur bloßen Fiktion. Im ontologischen Idealismus wird die Annahme einer eigenständigen $\mathrm{Au}$ ßenwelt aufgegeben und damit der erkenntnistheoretische Dualismus, der den ontologischen voraussetzt. Dieser Idealismus, dem statt einer Außenwelt nur eine Welt von Erscheinungen verbleibt, kann konsequenterweise dann aber auch nicht an einer Autonomie des Subjekts festhalten. Da wir die Erscheinungen nicht selbst erzeugen, ist eine Realität anzunehmen, welche die Gegenstände unseres Bewußtseins und unser Bewußtsein von ihnen produziert. Mit der Natur wird so auch das Bewußtsein zu einem unselbständigen Moment eines übergreifenden Ganzen, des Absoluten des absoluten Idealismus. Am deutlichsten wird diese Konzeption bei Hegel, bei dem nun auch der schon bei Fichte und Schelling angelegte Entwicklungsgedanke zentrale Bedeutung gewinnt. Hegel entwirft eine Evolutionstheorie, die sich von der heute vertretenen vor allem in zwei Punkten unterscheidet: Sie ist erstens nicht physikalistisch. Wie für Fichte läßt sich auch für Hegel Psychisches nicht aus Physischem erklären. Physisches und Psychisches sind gleich ursprünglich; das eine gibt es nicht ohne das andere. In der Grundrealität (dem Absoluten), aus der sich im Prozeß der Evolution die Mannigfaltigkeit der physischen und psychischen Phänomene entwickelt, muß also beides schon angelegt sein. Eine Einheit von gegenständlichem Sein und Bewußtsein läßt sich nach Hegel wie nach Fichte nur als Selbstbewußtsein begreifen, als etwas Geistiges also, denn Geist ist für ihn das, was sich selbst zum Gegenstand wird. ${ }^{102}$ Mit der Bezeichnung.der Grundrealität als „Geist" verbindet sich aber bei Hegel nicht die Vorstellung eines handelnden 
Subjekts. Man könnte eher von einem evolutionären Spinozismus sprechen und die Grundrealität im Sinn eines neutralen Monismus verstehen. Zweitens deutet er die Evolution nicht als kausalen, sondern als teleologischen Prozeß, dessen Ziel die Selbsterkenntnis der Wirklichkeit ist, die sich in menschlicher Erkenntnis vollzieht. Aus dieser Konzeption ergibt sich für Hegel eine ähnliche Abwendung von den traditionellen erkenntnistheoretischen Fragestellungen, wie sie uns heute in der ,evolutionären Erkenntnistheorie“ begegnet. $\mathrm{Er}$ wendet sich gegen den erkenntnistheoretischen Dualismus. Ihm sind die erkenntnistheoretische Frage, wie wir mit unseren Anschauungsformen und Verstandesbegriffen die Wirklichkeit an sich erfassen können, und ihre Unlösbarkeit Produkte der falschen Voraussetzung einer Autonomie des Subjekts und der Außenwelt. Er versteht das Subjekt und seinen kognitiven Apparat als Teil der Gesamtwirklichkeit. Wir sind Produkte derselben Evolution, aus der auch unsere Umwelt entstanden ist; die Evolution menschlichen Bewußtseins folgt den gleichen dialektischen Gesetzen wie jene der gegenständlichen Welt. Die Welt ist vernünftig, sie ist erkennbar, denn sie ist Produkt derselben kosmischen Vernunft, die sich im menschlichen Denken begreift. Für Hegel entfällt damit die traditionelle erkenntnistheoretische Problematik. Mit der ,Gewißheit des Bewußtseins, alle Realität zu sein", ${ }^{103}$ erledigt sich die Frage, ob und wie eine dem Bewußtsein gegenüber (absolut oder relativ) eigenständige Wirklichkeit erkannt werden kann. Erkenntnistheorie wird bei ihm durch Geistesgeschichte ersetzt. Er nimmt eine Evolution menschlicher Erkenntnis an, Stufen des Bewußtseins (des Welt- und Selbstverständnisses), die zu einem immer vollständigeren und adäquateren Verständnis der gesamten Wirklichkeit führen. In der „Phänomenologie“ und dem 3. Teil der „Enzyklopädie“ beschreibt er eine solche Folge von Stufen des Bewußtseins, die, ausgehend von einer geringen Differenziertheit von Subjektivem und Gegenständlichem in der ,sinnlichen Gewißheit“

${ }^{102}$ Die Bestimmung der Grundrealität als „Geist" ergibt sich bei Hegel daneben auch noch aus anderen Überlegungen: Wie für Leibniz ist für ihn die Einheit einer Vielfalt von Gegenständen nur im Bewußtsein möglich. Wie für Fichte ist das Absolute als ens a se ferner für Hegel nur denkbar als Subjekt-Objekt-Einheit und als etwas, das sich selbst bestimmt. Und endlich gibt es für ihn wie für Kant echte Teleologie nur im Bereich des Geistigen.

${ }^{103} \mathrm{Vgl}$. Hegel (W) III, 178. 
über eine dualistische Konzeption bis hin zu idealistischen führt. Hegel siehi darin einen mit strenger Notwendigkeit ablaufender. ProzeP, in dem sich schrittweise das volle und richtige Bewußtsein - das Hegelsche - realisiert, und er betont, daß sich der absolute Idealismus nur auf dem Wege eines gedanklichen Nachvollzugs dieser historischen Entwicklung begründen läßt, daß man also nicht, wie Fichte und Schelling, das Absolute an die Spitze einer deduktiv zu entwikkelnden Philosophie stellen könne. Interessant an Hegels Analysen von Stufen des Bewußtseins sind weniger die Details als der Versuch, die Vielfalt der Erscheinungsformen von Kulturen aus dem in ihnen herrschenden Selbst- und Weltverständnis zu erklären, und dieses wiederum aus einer Konzeption der Epoche vom Subjekt-Objekt-Verhältnis. Es ist klar, daß man mit diesem Verhältnis allein viele Phänomene nicht erklären kann, aber Hegel hat damit sicher einen wichtigen Ansatzpunkt für geistesgeschichtliche Analysen aufgewiesen.

Der Vergleich dieser Ideen mit unseren Überlegungen zeigt eine Übereinstimmung in der Ablehnung von ontologischem und erkenntnistheoretischem Dualismus, von absolutem Realismus und ontologischem Idealismus. Auch im absoluten Idealismus ist von der Intentionalität des Bewußtseins die Rede - freilich nur des „,empirischen“, individuellen Bewußtseins - , und Bewußtsein und Außenwelt werden als aufeinander bezogene Momente einer polaren Einheit angesehen. ${ }^{104}$ Auch wir haben gesagt, das Verständnis des Subjekt-ObjektVerhältnisses ergebe sich nicht einfach aus der Natur der Sache, sondern sei Produkt einer Interpretation, die einem geschichtlichen Wandel unterliegt. Wir haben aber weder versucht, eine geschichtliche Abfolge von Bewußtseinsstufen anzugeben - das wäre Aufgabe einer Geistesgeschichte - noch haben wir eine solche Abfolge als einen notwendigen oder gesetzmäßigen Prozeß verstanden.

Diesen Verwandtschaften unserer Konzeption mit dem absoluten Idealismus steht vor allem folgender Unterschied gegenüber: Der Idealismus will Psychisches und Physisches und ihr gegenseitiges Verhältnis aus dem Absoluten, einer ursprünglichen Einheit von Bewußtsein und Sein ableiten und die Entfaltung dieser Einheit in die Mannigfaltigkeit der Erscheinungen ais einen notwendigen, dialekti-

${ }^{104}$ Auf Fichtes Äußerung zur Subjekt-Objekt-Polarität wurde schon in 8.1 hingewiesen. Von „Polarität“ spricht zuerst Schelling. Vgl. z. B. (W), Schriften $1799-1801$, S. 340. 
schen Prozeß begreifen. Er vertritt also einen Monismus, während wir eine - wenn auch nur relative - Eigenständigkeit von Psychischem und Physischem angenommen haben. Damit bleibt für uns erstens die erkenntnistheoretische Problematik erhalten, während sie für den absoluten Idealismus verschwindet. Denke nicht ich, sondern denkt die kosmische Vernunft in mir, so verschwindet mit der Eigenständigkeit des Denkens auch seine Fallibilität; es kann ebensowenig in die Irre gehen wie ein naturgesetzlich ablaufender Prozeß. Zweitens sollen die Intentionalität des Bewußtseins und das polare Verhältnis von Psychischem und Physischem, die wir als Gegebenheiten angesehen haben, hinter die wir nicht mehr zurückgehen können, im absoluten Idealismus aus einer übergeordneten Einheit erklärt werden; es wird so die Möglichkeit einer absoluten Erkenntnis der Gesamtwirklichkeit behauptet, die allen Bedingtheiten enthoben ist. Das ist auch ein Punkt, in dem sich der absolute Idealismus von der Philosophie Kants grundsätzlich unterscheidet. Während Kant in der „Kritik der reinen Vernunf" die Grenzen menschlicher Erkenntnis mit jenen des Erfahrbaren identifiziert und die Wirklichkeit an sich als unerkennbar erklärt - insbesondere Existenz und Wesen Gottes, des Absoluten -, lehnt der absolute Idealismus diese Begrenzung der Erkenntnisfähigkeit ab und versteht Philosophie schlicht als „Wissenschaft vom Absoluten“, wie Schelling sagt. Nur vom Absoluten, vom Unbedingten, gibt es nach Auffassung von Fichte und Schelling absolute, unbedingte und unfehlbare Erkenntnis. In der Reflexion auf das ,,reine Ich“ können wir uns zur Erkenntnis des Absoluten erheben und danit auch zu einer Erkenntnis der Wirklichkeit, wie sie an und für sich ist, und der Art und Weise, wie sich unser Bewußtsein und unser Erkennen sub specie aeternitatis darstellt. ${ }^{105}$ Auch nach Hegel gibt es ein Wissen des Absoluten, das zwar als inhaltliches Wissen durch die Reflexion auf die geistesgeschichtliche Entwicklung vermittelt ist, aber als Wissen von den formalen Strukturen in der „Logik“ direkt erreichbar ist, und

${ }^{105}$ Im Sinne der Adäquationstheorie der Wahrheit bestimmt Fichte Wahrheit als „Übereinstimmung von Denken und Sein“. Aufgrund einer falschen Deutung solcher Übereinstimmung meint er dann, je besser die Übereinstimmung, desto wahrer die Wahrheit, und da Identität den höchsten Grad der Übereinstimmung darstellt, bestehe die absolute Wahrheit in der Identität von Denken und Sein, von Bewußtsein und Sein, im ,absoluten Wissen", das dem reinen Ich entspricht. 
das auch von Hegel in seinen Analysen der Stufen des Bewußtseins immer schon vorausgesetzt vird. Damit steht der absolute Idealismus in der Tradition jener Metaphysik, deren Destruktion eines der zentralen Anliegen von Kants Vernunftkritik war. ${ }^{106}$ Er bildet zugleich die letzte Steigerung des Rationalismus, der freilich schon bei Fichte in einen mystischen Irrationalismus umschlägt. ${ }^{107}$

106 Die absoluten Idealisten haben die Kritik Kants an der traditionellen Metaphysik übernommen. Auch für sie war diese Metaphysik Produkt einer illegitimen Anwendung von Verstandeskategorien und -prinzipien, sie meinten aber, Vernunft könne die Grenzen des Verstandes überfliegen. Für sie gibt es Vernunfterkenntnis, die sehr viel weiter reicht als ,,bloße Verstandeserkenntnis“. Den Nachweis dafür bleiben sie aber schuldig, da sie nichts Vernünftiges von der Vernunft zu sagen wissen. Für Hegel ist z. B. die zweite Antinomie Kants, nach der materielle Dinge zugleich teilbar und unteilbar sind, ein Widerspruch, der sich dialektisch aufheben läßt. Was dabei aber herauskommen soll, bleibt offen. Der Versuch der Idealisten, jenseits der formalen Logik mit der Dialektik eine höhere, leistungsfähigere Logik aufzuweisen, ist gescheitert - nicht weil so etwas prinzipiell unmöglich wäre, sondern weil die Kritik des Idealismus an der formalen Logik auf einem völligen Mangel an logischem Unterscheidungsvermögen beruht und „Dialektik" nur ein Obertitel für ganz heterogene und vage Ideen bleibt. Wie sinnvolle Erweiterungen der traditionellen Logik aussehen, hat die Entwicklung seit Frege gezeigt. Damit ist natürlich nicht gesagt, daß diese Erweiterungen das Paradigma aller überhaupt möglichen Umformungen der Logik sein müssen.

${ }^{107}$ Man vergleiche Fichtes anfängliche Idee einer Wissenschaftslehre als einer deduktiven Wissenschaft (mit nur einem Axiom, aus dem zugleich die Logik ableitbar sein sollte, mit der man daraus etwas ableiten kann) im „Begriff der Wissenschaftslehre" von 1798 ((W)I, 27 ff.) mit den Äußerungen in der ,Einleitung in die Wissenschaftslehre" von 1813, wo es heißt, die Wissenschaftslehre sei dem gemeinen Menschenverstand unbegreiflich, denn ,diese Lehre setzt voraus ein ganz neues inneres Sinnenwerkzeug, durch welches eine neue Welt gegeben wird, die für den gewöhnlichen Menschen gar nicht vorhanden ist... Für die Menschen, wie sie sind, durch ihre Geburt und gewöhnliche Bildung, ist diese Lehre durchaus unverständlich; denn die Gegenstände, von denen sie redet, sind für dieselben gar nicht da, weil sie den Sinn nicht haben, durch und für welchen diese behandelten Gegenstände da sind. Es wird ihnen jene darum durchaus die Rede vom Nichts, von dem, was nicht da ist für sie“. ((W), IX, 4) - Bei Hegel heißt es: „Das Wahre ist so der bacchantische Taumel, an dem kein Glied nicht trunken ist". ((W)III, 46). 
Das Absolute des absoluten Idealismus ist, wie wir sahen, eine unsinnige Fiktion. Wenn man die Subjekt-Objekt-Beziehung als eine polare Einheit bezeichnet, heißt das nicht, daß damit jenseits der Mannigfaltigkeit psychischer und physischer Phänomene etwas aufgewiesen wäre, aus dem sie und ihre Wechselbeziehungen sich erklären oder ableiten ließen. Aussagen über die polare Struktur der Erfahrung ergeben sich aus einer immanenten Auslegung dieser Erfahrung, und im Prozeß dieser Auslegung sieht man sich nicht plötzlich auf einen Standpunkt versetzt, auf dem man von den Bedingtheiten empirischer Erkenntnis befreit ist und feststellen könnte, wie Mensch und Welt an sich beschaffen sind. Der absolute Idealismus mißachtet eine der wichtigsten Einsichten Kants, daß nämlich Aussagen über Bedingungen unseres Erkennens nur dann korrekt sind, wenn sie selbst diesen Bedingungen genügen, wenn sie, wie wir im Vorwort gesagt haben, selbstanwendbar sind. Auch in erkenntnistheoretische Untersuchungen gehen die Bedingtheiten, Voraussetzungen, Denkstrukturen unseres Erkennens ein. Wir können solche Strukturen zwar erhellen, uns aber nicht einfach von ihnen befreien. Sind Intentionalität und Polarität Grundstrukturen unseres Bewußtseins, so sind sie eben deswegen nicht ,erklärbar“, weil sie dann all unserem Denken und Erkennen zugrunde liegen. Für uns sind sie dann nicht mehr hintergehbare Grunddaten. Die Absurditäten, in die sich der absolute Idealismus in seinem Versuch verstrickt, das Absolute zu erkennen, illustrieren deutlich die Vergeblichkeit solchen Bemühens. Mit der erkenntnistheoretischen Problematik verschwindet in absoluten Idealismus auch das kritische Bewußtsein, das Bemühen um eine Prüfung der eigenen Erkenntnisansprüche.

Die Philosophie des absoluten Idealismus war der Versuch einer Kritik der Aufklärungsphilosophie, der Versuch ihre Einseitigkeiten zu überwinden, wie Rationalismus, Kritizismus und Szientismus, und die Gegensätze in ihr, wie jene zwischen Idealismus und Materialismus oder Determinismus und Freiheit. Sie wollte eine Konzeption entwickeln, in der nicht einer Einseitigkeit eine andere gegenübergestellt wird, dem Rationalismus ein Irrationalismus, dem Idealismus ein Realismus, sondern in der eine Synthese der verschiedenen Aspekte möglich wird. Dieser Versuch ist gescheitert, und er ist in seiner Mischung von analytischer Inkompetenz und maßlosem Anspruch so kläglich gescheitert, daß er seitdem nicht ernsthaft wiederholt wor- 
den ist. Szientismus und Naturalismus stehen nach wie vor in voller Blüte. Die Kluft zwischen Geistes- und Naturwissenschaften ist nicht nur geblieben, sondern hat sich vertieft, ebenso wie der Abstand zwischen wissenschaftlichem Weltbild und „Lebenswelt". Der Idealismus und der von ihm inaugurierte Denkstil haben wesentlich dazu beigetragen, daß Philosophie heute bei Laien wie Wissenschaftlern ihren Kredit weitgehend verloren hat und daher ihre nach wie vor wichtige kritische Funktion kaum mehr wahrnehmen kann. Der Idealismus hat manches richtig gesehen und viele seiner Ziele - wenn auch sicher nicht sein Bestreben, Philosophie zur ,W Wissenschaft vom Absoluten“" zu erheben, oder seine enzyklopädischen Ambitionen - sind immer noch aktuell. Man wird sich ihnen aber nur dann nähern, wenn man sich bemüht, den intellektuellen Maßstäben gerecht zu werden, die heute an wissenschaftliche Arbeiten angelegt werden. 



\section{Theorie und Wirklichkeit}

\subsection{Die Beobachtungssprache}

In den letzten Kapiteln haben wir uns mit der Frage beschäftigt, ob und in weichem Sinn wir es in der Erfahrung mit einer objektiven Realität zu tun haben. In diesem Kapitel wollen wir uns überlegen, mit welchem Recht und in welchen Grenzen wir sagen können, daß uns empirische Theorien ein korrektes Bild der Welt liefern. Auch das ist Teil der zentralen erkenntnistheoretischen Frage nach Existenz und Erkennbarkeit der Natur. Beschäftigen wir uns mit Aufbau, Begründung und Leistung von empirischen Theorien, so begeben wir uns auf das Gebiet der allgemeinen Wissenschaftstheorie, der allgemeinen Methodologie der Wissenschaften. Wir wollen also in diesem Kapitel jene wissenschaftstheoretischen Probleme diskutieren, die für unsere erkenntnistheoretische Frage relevant sind. Auf die Erörterung technischer Details können wir dabei weitgehend verzichten, da sie in der einschlägigen Literatur behandelt werden.

Um uns eine vorläufige Übersicht über die zu behandelnden Probleme zu verschaffen, gehen wir von der Zwei-Schichten-Theorie empirischer Sprachen aus, die R. Carnap entwickelt hat. Danach läßt sich das deskriptive Vokabular (die Menge der empirischen, d.h. nicht logischen oder mathematischen Grundterme) einer Wissenschaftssprache $S$ - die wir uns der Einfachheit halber als eine prädikatenlogische Sprache vorstellen wollen - in zwei Teile zerlegen: $V_{B}$, das Beobachtungsvokabular von $S$, soll Eigennamen für direkt beobachtbare Objekte (z. B. konkrete physische Dinge und Lebewesen) enthalten und Prädikate, die direkt beobachtbare Attribute ausdrükken (z. B. Farbattribute und Eigenschaften wie Schwer oder Hart). $\mathrm{V}_{\mathrm{T}}$, das theoretische Vokabular von $\mathrm{S}$, soll alle anderen Grundterme enthalten, also Eigennamen für nicht direkt beobachtbare Objekte (wie z. B. Elementarteilchen) und Prädikate für nicht direkt beobachtbare Attribute oder Größen (wie z. B. elektrische Ladung). Sätze von $S$, die deskriptive Ausdrücke nur aus $V_{B}$ enthalten, gehören zu jenem Teil von $S$, den wir als Beobact,tungssprache $S_{B}$ von $S$ bezeichnen. Ist $S$ 
die physikalische Sprache, so ist die Dingsprache die Beobachtungssprache $\mathrm{S}_{\mathrm{B}}$, während die Sprache der Physik das theoretische Vokabular enthält.

Die einfachsten Sätze von $S_{B}$, die also die Form haben ,Der Gegenstand a hat die Eigenschaft $F^{\prime \prime}$ - symbolisch ,F(a)“ - oder die Form „Die Gegenstände $a_{1}, \ldots, a_{n}$ stehen zueinander in der Beziehung $G^{\prime \prime}$ - symbolisch , $G\left(a_{1}, \ldots, a_{n}\right)^{\prime \prime}-$, nennen wir Basissätze. ${ }^{1}$ Solche Sätze sind durch direkte Beobachtungen entscheidbar, da die Objekte, von denen sie sprechen, direkt beobachtbar sind und ebenso das Zutreffen der Attribute, die diesen Objekten zugesprochen werden. Bezeichnen wir alle Sätze von S, die sich durch direkte Beobachtungen entscheiden lassen, deren Wahrheit oder Falschheit sich also durch direkte Beobachtung feststellen läßt, als Beobachtungssätze von $\mathrm{S}$, so gehören dazu nicht nur die Basissätze, sondern alle allein mit aussagenlogischen Verbindungen (wie ,nicht“, ,und“, ,oder") aus ihnen erzeugbaren Sätze. Das sind aber nicht alle Sätze von $S_{B}$, denn Aussagen von $S_{B}$ der Form „Es gibt ein Ding der Art F“ (symbolisch $\mathrm{VxF}(\mathrm{x})$ ) sind $\mathrm{zwar}$ durch direkte Beobachtung verifizierbar - die Beobachtung eines Objekts a mit der Eigenschaft $F$ reicht hin, um diesen Satz zu verifizieren - , sie sind aber nicht generell falsifizierbar. Umgekehrt sind Aussagen von $S_{B}$ der Form ,Alle Dinge haben die Eigenschaft F" (symbolisch $\Lambda \mathrm{xF}(\mathrm{x})$ ) zwar durch Beobachtung falsifizierbar - die Beobachtung, daß ein Objekt a die Eigenschaft $F$ nicht hat, reicht hin, um diesen Satz zu falsifizieren - , sie sind aber nicht generell verifizierbar. ${ }^{2}$ Entsprechend sind Sätze von $\mathrm{S}_{\mathrm{B}}$ der Form , $\mathrm{Zu}$ jedem $S$ gibt es ein $P$, das mit jenem in der Beziehung $R$ steht" - symbolisch $\Lambda \mathrm{x}(\mathrm{S}(\mathrm{x}) \supset \mathrm{Vy}(\mathrm{P}(\mathrm{y}) \Lambda \mathrm{R}(\mathrm{x}, \mathrm{y})))$ - oder ,,Es gibt ein $\mathrm{S}$, zu dem alle $\mathrm{P}$ in der Relation $\mathrm{R}$ stehen" - symbolisch $\mathrm{Vx}(\mathrm{S}(\mathrm{x}) \wedge \Lambda \mathrm{y}(\mathrm{P}(\mathrm{y}) \supset$ $\mathrm{R}(\mathrm{x}, \mathrm{y}))$ ) - durch direkte Beobachtungen im allgemeinen weder verifizierbar noch falsifizierbar.

Diese Bestimmungen von Beobachtungstermen und Beobachtungssätzen sind nun weder scharf, noch folgen sie allgemein anwendbaren

'Solche Basissätze sind von anderer Art als die Sätze der Form ,An der Raum-Stelle q gibt es zur Zeit t Objekte der Art F“, die Popper als „Basissätze" bezeichnet. (Vgl. Popper (66), $67 \mathrm{f}$.

${ }^{2}$ Durch Beobachtungen sind nur beschränkte Existenzsätze falsifizierbar, wie z. E. ,In diesem Raum befinden sich drei Personen“, und nur beschränkte Allsätze verifizierbar, wie z. B. ,Alle Personen in diesem Raum sind blond“. 
Kriterien. Der Schlüsselbegriff für die Unterscheidungen ist ,,direkt beobachtbar". Was aber ist ,direkt keobachtbar"? Das hängt offenbar davon $a b$, ob ich für Beobachtungen nur meine fünf Sinne gebrauche oder auch Meßinstrumente. Ohne Teleskope sind ferne Galaxien nicht beobachtbar, und in einer Nebelkammer kann ich auch Elementarteilchen beobachten. In einem Untersuchungskontext genügt es, Farben von Gegenständen dem Augenschein nach zu beurteilen, in anderen Kontexten müssen wir hingegen die Spektralverteilung von einfallendem und reflektiertem Licht physikalisch messen, um die erforderliche Genauigkeit zu erzielen. Die Unterscheidung versteht sich also nur relativ zum jeweiligen Untersuchungskontext, und sie ist auch dann nicht scharf. ${ }^{3}$ Für unsere Zwecke genügt es aber zunächst, direkte Beobachtbarkeit im Sinne prinzipieller Beobachtbarkeit ohne Zuhilfenahme von Meßinstrumenten zu verstehen. Die Zwei-Schichten-Theorie der Sprache ist eine für die Explikation methodologischer Unterscheidungen nützliche Fiktion, und nur in diesem Sinn verwenden wir sie hier. ${ }^{4}$

Mit ihrer Hilfe können wir nun die wichtigsten Probleme, um die es im folgenden geht, so formulieren:

1. Wie kommen wir zu den Beobachtungsbegriffen? Kann man im Sinn des Empirismus sagen, sie seien durch Abstraktion aus beobachteten Objekten gewonnen?

2. Wie lassen sich auf der Grundlage des Beobachtungsvokabulars die theoretischen Terme, d. h. die Terme des theoretischen Vokabulars einführen? Wie lassen sich empirische Unterscheidungen rechtfertigen, die sich nicht auf beobachtbare Unterschiede reduzieren lassen?

3. Gibt es eine definitive Verifikation oder Falsifikation von Beobachtungssätzen oder haben sie auch nur hypothetischen Charakter?5

${ }^{3} \mathrm{Vgl}$. dazu auch Stegmüller (70), 1. Halbbd., S. $189 \mathrm{ff}$. und S. $297 \mathrm{ff}$., sowie Kutschera (72), S. $258 \mathrm{ff}$.

${ }^{4}$ Auf die Kritik an der Zwei-Schichten-Theorie, die z. B. Feyerabend in (60), Kuhn in (62), Putnam in (62) und Hempel in (73) geübt haben, gehen wir im folgenden noch ein.

${ }^{5} \mathrm{Da}$ wir oben Beobachtungssätze als Sätze bestimmt haben, die sich durch direkte Beobachtungen entscheiden lassen, ist diese Frage genauer so zu formulieren: „Gibt es einfache empirische Sätze, die sich definitiv entscheiden lassen?“, oder: „Gibt es Beobachtungssätze im Sinne der obigen Bestimmung?"، 
4. Wie lassen sich Theorien oder Gesetzeshypothesen durch Beobachtungssätze begründen oder bestätigen? Das ist das Induktionsproblem.

5. Lassen sich Theorien, die theoretische Terme enthalten, realistisch als Aussagen über die Welt deuten, oder charakterisieren sie im Sinn des Instrumentalismus nur Verfahren, mit denen wir Beobachtungsaussagen aus anderen Beobachtungsaussagen ableiten?

6. Sind Begriffs- und Theorienbildung voneinander unabhängige Prozesse, oder muß man im Sinn des Holismus eine Theoriebeladenheit der Sprache annehmen?

7. Ergibt sich nicht eine relativistische Auffassung der Erfahrungserkenntnis, wenn man die Annahme verwirft, es gebe von hypothetischen Elementen unbelastete und von Interpretationen unabhängige Beobachtungsdaten als feste Grundlage all unserer empirischen Überzeugungen?

Wir werden sehen, daß die genannten Probleme alle eng miteinander zusammenhängen.

In diesem Abschnitt befassen wir uns mit dem ersten Problem, der Einführung von Beobachtungsbegriffen. Eine Antwort auf diese Frage gibt der Begriffsempirismus mit seiner Behauptung:

\section{EB: Alle empirischen Begriffe sind aus der Erfabrung abstrabiert.}

Als empiristisch bezeichnet man alle philosophischen Richtungen, die in der Erfahrung den einzigen Ursprungs- und Rechtfertigungsgrund aller empirischer Erkenntnis sehen. Alle Begriffe, mit denen wir die Welt beschreiben, sind nach dieser Auffassung der Erfahrung entnommen, und alle unsere Aussagen über die Welt sind aus Erfahrungen gewonnen. Wenn man von Philosophen wie John Stuart Mill absieht, die auch Logik und Mathematik zu den empirischen Wissenschaften rechnen, so erkennen die Empiristen zwar ein analytisches Apriori an, aber apriorische Sätze haben für sie keinerlei synthetischen Gehalt, d. h. aus ihnen folgen keine Sätze, die etwas über die Beschaffenheit der Welt aussagen. Die Sätze der Naturwissenschaften beruhen danach nicht auf irgendwelchen apriorisch-synthetischen Prinzipien, wie sie z. B. Kant annahm, sondern lassen sich nur aposteriorisch, d. h. durch Beobachtungen begründen. ${ }^{6}$ Die zweite Grundthese des Empirismus ist also: 
EU: Alle synthetischen Urteile beziehen ihre Berechtigung allein aus der Erfahrung.

Das ist die These des Urteilsempirismus, mit der wir uns in den Abschnitten 9.3 und 9.4 befassen. Sie ist von der These EB unabhängig, so daß z. B. Franz Brentano nur die erste These vertreten konnte, während in der heutigen Diskussion des Empirismus fast nur mehr die zweite These eine Rolle spielt, die auch als die wichtigere empiristische These angesehen werden muß.

Die These EB des Begriffsempirismus läßt sich in zwei Behauptungen aufspalten:

$\mathrm{EB}_{1}$ : Alle Beobachtungsbegriffe sind aus der Erfabrung abstrabiert. $\mathrm{EB}_{2}$ : Alle empirischen Terme lassen sich durch Beobachtungsterme definieren.

Auf die zweite Behauptung gehen wir im nächsten Abschnitt ein, hier befassen wir uns mit der ersten. Die erkenntnistheoretisch wichtigste Implikation dieser These ist, daß der Prozeß der Begriffsbildung sich danach von seinen Anfängen an rational rekonstruieren läßt.

Nun setzt aber jede Definition eines Begriffes durch Abstraktion bereits Begriffe voraus. Das gilt zunächst für jenes Verfahren der Definition durch Abstraktion, das in Logik und Mathematik verwendet wird. Hier geht man von einer Äquivalenzrelation $\mathrm{R}$ aus, die auf einer Menge $M$ von Objekten definiert ist. Mit ihr kann man zu jedem $\mathrm{Ob}$ jekt a aus $M$ die Klasse aller Objekte aus $M$ bilden, die zu a in der Relation R stehen. 'Ein Beispiel Freges mag das erläutern: Mit der Äquivalenzrelation der Parallelität zweier Geraden kann man den Begriff der Richtung einer Geraden a durch Abstraktion definieren als Klasse aller mit a parallelen Geraden. Ebenso setzen auch andere Typen von abstraktiven Definitionen Begriffe voraus. Bei der Einführung von

- Der Empirismus selbst ist, ebenso wie die Gegenposition des Rationalismus, sowohl mit realistischen als auch mit idealistischen Auffassungen der Erfahrung verträglich. So ist z. B. Humes Empirismus idealistisch, der logische Empirismus dagegen vorwiegend realistisch orientiert.

7 Die Beziehung $R(x, y)$ ist eine Äquivalenzrelation, wenn sie reflexiv, transitiv und symmetrisch ist, d. h. wenn gilt $\Lambda x R(x, x), \Lambda x y z(R(x, y) \wedge R(y, z) \supset$ $R(x, z))$ und $\Lambda x y(R(x, y) \supset R(y, x))$. Jedes Objekt aus $M$ gehört dann zu genau einer Äquivalenzklasse. 
Farbeigenschaften geht man z. B. von einer Relation der Farbähnlichkeit aus ${ }^{8}$ und auch die Abstraktionsprozesse, die Carnap im ,Logischen Aufbau der Welt" (28) diskutiert, setzen Begriffe voraus. ${ }^{9}$ Die Vorstellung, man könne allein aus Objekten Begriffe erzeugen, ist völlig unhaltbar. Objekte sind nicht ursprünglicher als Attribute, weil wir sie ja nur mithilfe von Attributen bestimmen und unterscheiden können. Die Einführung eines Begriffes durch Abstraktion stellt ferner eine Definition dar und setzt wie alle Definitionen definierende Begriffe voraus.

Die psychologische Abstraktionstheorie von Locke, nach der wir aus der Vorstellung von Einzeldingen durch Absehen von ihren Verschiedenheiten ,Allgemeinvorstellungen" bilden können, und nach der Begriffe solche Allgemeinvorstellungen sind, hat schon Berkeley vernichtend kritisiert. Soll z. B. die Allgemeinvorstellung eines Winkels alle Eigenschaften irgendwelcher Winkel enthalten, so ist sie unmöglich, da der vorgestellte Winkel dann zugleich spitz wie stumpf und rechtwinklig sein müßte; soll sie nur jene Eigenschaften haben, die allen Winkeln gemeinsam sind, so wäre sie nicht möglich, denn es wäre die Vorstellung eines Winkels, der weder spitz noch stumpf, noch rechtwinklig ist. ${ }^{10}$ Vorstellungen sind eben etwas anderes als Begriffe; Begriffe sind etwas Abstraktes, Vorstellungen sind hingegen immer Vorstellungen von konkreten Dingen (oder Ereignissen). Frege sagt dazu: „Da nun alles Vorstellung ist, können wir leicht die Gegenstände durch Hin- und Ablenkung der Aufmerksamkeit verändern. Besonders das letzte ist wirksam. Wir merken weniger auf eine Eigenschaft, und sie verschwindet. Indem wir so ein Merkmal nach dem andern verschwinden lassen, erhalten wir immer abstraktere Begriffe. Auch die Begriffe sind also Vorstellungen, nur weniger vollständige als die Gegenstände; sie haben noch die Eigenschaften jener, von denen nicht abstrahiert ist. Die Unaufmerksamkeit ist eine höchst wirk-

\footnotetext{
${ }^{8}$ Vgl. dazu Kutschera (75), 2.4.7.

${ }^{9}$ Zur Kritik dieses Verfahrens vgl. das Vorwort zur 2. Auflage von Carnap, sowie Goodman (51), S. $151 \mathrm{ff}$. Für eine kurze Darstellung vgl. auch Kutschera (72), $478 \mathrm{ff}$.

${ }^{10} \mathrm{Da}$ er ebensowenig wie Locke zwischen Vorstellungen und Begriffen unterscheidet, mündet Berkeleys Kritik an den „Allgemeinvorstellungen“ allerdings in einem Nominalismus. Vgl. die Einleitung zum ,Treatise Concerning the Principles of Human Knowledge“, (L), $93 \mathrm{ff}$.
} 
same logische Kraft; daher vermutlich die Zerstreutheit der Gelehrten. Nehmen wir z. B. an, es sitzen vor uns nebeneinander eine schwarze und eine weiße Katze. Wir achten nicht auf ihre Farbe: sie werden farblos, sitzen aber noch nebeneinander. Wir achten nicht auf ihre Körperhaltung: sie sitzen nicht mehr, ohne jedoch eine andere Haltung angenommen zu haben; aber jede ist noch an ihrer Stelle. Wir achten nicht mehr auf den Ort: sie werden ortlos, bleiben aber immer noch wohl geschieden. So haben wir vielleicht aus jeder einen allgemeinen Katzenbegriff gewonnen. Jeder Gegenstand verwandelt sich bei fortgesetzier Anwendung dieses Verfahrens in ein immer blutleereres Gespenst. Wir gewinnen so schließlich aus jedem Gegenstande ein Etwas, das gänzlich inhaltsentschränkt ist; aber das Etwas, das aus dem einen Gegenstande gewonnen ist, unterscheidet sich doch von dem aus einem andern Gegenstande gewonnenen, obwohl nicht leicht zu sagen ist, wodurch". ${ }^{11}$

Verfügen wir schon über Begriffe, so können wir damit durch Abstraktion oder auf andere Weise weitere Begriffe definieren. Woher bekommen wir aber die ersten empirischen Begriffe? Im Abschnitt 7.4 und im letzten Kapitel haben wir auf die Verankerung von Beobachtungsbegriffen im Erleben hingewiesen und betont, daß Unterscheidungen im Bereich der Dinge auf Unterschieden in der Art beruhen, wie wir diese Dinge erfahren. Von Begriffen kann man erst dort reden, wo Unterscheidungen sprachlich ausgedrückt werden. Es gibt aber vorsprachliche Unterscheidungen - wir haben betont: ohne die Fähigkeit zu solchen Unterscheidungen könnten wir keine Sprache erlernen - und speziell solche, die in der Art und Weise unseres Erlebens angelegt sind. Als Grundlage der Begriffsbildung im empirischen Bereich können wir angeborene Dispositionen annehmen, Dinge oder Vorgänge bestimmter Art in gleicher Weise zu erfahren. Es gibt eine vorrationale Abstraktion - in einem weiten Sinn dieses Wortes -, die sich auch bei Tieren belegen läßt, und die darin besteht, daß sie auf Gegenstände oder Reize einer gewissen Art in gleicher Weise reagieren. ${ }^{12} \mathrm{Im}$ Bereich des Erlebens haben wir schon auf die Phänomene der Gestaltwahrnehmung und der Form-, Größen- und Farbkonstanz hingewiesen, die auf einer Abstraktionsleistung unserer Wahrnehmungsorganisation beruhen. Wir haben auch schon gesehen,

11 Frege (KS), 181.

12 Vgl. dazu Lorenz (73), Kap. VII, 2 und Riedl (79), Kap. 3. 
daß die Umwelt eines Lebewesens primär aus dem besteht, was für es bedeutungsvoll ist. Auch uns fallen nicht beliebige Aspekte unserer Umgebung ins Auge, sondern zumeist nur das, was für uns bedeutungsvoll ist, und wir erleben Situationen als gleichartig, die für uns dieselbe Bedeutung haben. Es gibt also angeborene Dispositionen, bestimmte unter den beliebig vielen logisch möglichen Unterscheidungen im Bereich der Wahrnehmung zu machen, also natürliche Klassifikationen der Gegenstände. ${ }^{13}$ Wenn Pascal sagt, die Vernunft gehe von Voraussetzungen aus, die sie nicht rechtfertigen könne, ${ }^{14}$ so gilt das auch in dem Sinn, daß unser Begriffssystem auf vorrationalen Unterscheidungen beruht, nicht nur auf undefinierten Grundbegriffen, sondern auf angeborenen Erlebnisweisen. Diese biologische Grundlage unserer Begriffsbildung ist aber kein Apriori in dem Sinn, daß durch sie unsere begrifflichen Unterscheidungen determiniert oder begrenzt würden. Mit der Einführung sprachlicher Ausdrucksmittel werden die vorrationalen Unterscheidungen, wie wir in 7.4 sahen, modifiziert und erweitert. Mit der Sprache erlernen wir eine Fülle neuer Unterscheidungen, und in ihr sind wir in der Einführung neuer Begriffe durch Definitionen weitgehend frei. Erst diese Begriffsbildung auf der Grundlage einer Sprache ist ein rationaler Prozeß. Der Mensch ist auch in seiner Unterscheidungsfähigkeit kein spezialisiertes Lebewesen. Die artspezifischen Erlebnisformen sind für uns nicht mehr als eine Ausgangsbasis für die Einführung von Begriffssystemen, die sich von dieser Basis weit entfernen und eine kreative Leistung darstellen. Gegenüber dem Begriffsempirismus ist aiso zu betonen: Der Prozeß der Begriffsbildung ist nicht von Anfang an eine rein rationale Tätigkeit; wir sind in ihm weitgehend, aber nicht völlig frei - Grundlage unserer empirischen Begriffe bleiben Erlebnisweisen - , und die empirischen Klassifikationen der Dinge ergeben sich nicht aus diesen selbst durch rational beschreibbare Prozesse der Abstraktion, sondern sie beruhen auf erlebnismäßigen Unterschieden.

Mit Beobachtungsprädikaten reden wir nicht über Erlebnisweisen, sondern über Dinge und ihre Attribute. Die Bedeutung eines Beobachtungsprädikats besteht nicht allein in seinem anschaulichen, erlebnismäßigen Gehalt. Wir haben in den Abschnitten 7.4 und 8.6 gese-

${ }^{13} \mathrm{Vgl}$. dazu auch Quine (66), 218

${ }^{14} \mathrm{Vgl}$. dazu 1.7, S. 73. 
hen, daß von einem objektiven Sinn solcher Prädikate nur dann die Rede sein kann, wenn es für sie intersubjektive Gebrauchskriterien gibt; und wenn sie untereinander durch Sätze verbunden sind, welche die Bedeutung dieser Prädikate zusätzlich bestimmen. Was „,rot“ bedeutet, ergibt sich auch aus Sätzen wie:

1. Was (ganz) rot ist, ist nicht grün, gelb oder blau.

2. Reife Tomaten sind rot.

Satz (1) können wir als analytischen Satz ansehen. Es gibt also erstens Bedeutungspostulate, die das Verhältnis von Beobachtungsbegriffen zueinander bestimmen, im Beispiel die Bedingung des Konträrseins im Felde der Farbwörter. Solche Sätze ergeben sich nicht immer schon aus dem anschaulichen Gehalt der Prädikate. Zum Begriff der Ähnlichkeit gehört z. B., daß eine Ähnlichkeitsrelation transitiv ist, $\mathrm{da} ß$ also gilt: Ist a farbgleich mit $\mathrm{b}$ und $\mathrm{b}$ farbgleich mit $\mathrm{c}$, so ist auch a farbgleich mit c. Es kann aber sein, daß wir weder zwischen a und b, noch zwischen $b$ und $c$ einen Farbunterschied feststellen können daß also tatsächliche Farbunterschiede unter der Schwelle des Wahrnehmbaren bleiben - , wohl aber zwischen a und c. Solche Postulate können also zusätzliche, nicht im anschaulichen Gehalt begründete Bedeutungsfestlegungen liefern.

Sätze wie (2) sieht man gewöhnlich nicht als analytische Sätze an. Auch solche Sätze bestimmen aber die Bedeutung der in ihnen vorkommenden Wörter. „Rotsein“ heißt für uns auch soviel wie ,so ähnlich wie reife Tomaten aussehen". Sieht man nicht nur auf die Definitionen in Logik und Mathematik, sondern auf die Worterklärungen in Wörterbüchern, so findet man, daß sie meist Informationen über Tatsachen enthalten. So wird z. B. das Wort ,Tiger" durch den Satz erklärt "Tiger sind Großkatzen mit gelbbraunem Fell und schwarzen Streifen, die in den Waldgebieten Süd- und Ostasiens leben“ oder das Wort „Ukulele" durch ,, hawaische Gitarre, eine Nachbildung der in den 1870 er Jahren von Matrosen nach Hawai gebrachten, der Mandoline und Gitarre verwandten portugiesischen Cavaco (Machete), deren vier mit hinterständigen Wirbeln gespannte und in einem auf der Decke befindlichen Saitenhalter befestigte Saiten in d'fis'-a'-b' oder g'-c'-e'-a' gestimmt sind“'.15

${ }^{15}$ Beide Erklärungen sind dem „Großen Brockhaus“ entnommen. 
Daß es eine scharfe Grenze zwischen analytischen und synthetischen Sätzen gibt, ist eine der Voraussetzungen, eines der „Dogmen“, wie Quine sagt, des Empirismus. Nur unter dieser Bedingung ist die These, es gebe keine synthetisch-apriorischen Sätze, sinnvoll. Quine versucht demgegenüber zu zeigen, daß die Unterscheidung Synthetisch - Analytisch für natürliche Sprachen unscharf ist; daß es für sie allenfalls einen komparativen Begriff der Analytizität gibt, mit dem man Sätze als mehr oder minder analytisch einstufen kann. ${ }^{16}$ Er betont, daß der Sinn der Wörter unserer Sprache auch von unseren Annahmen über die Welt abhängt. Was das Wort „Zitrone“ bedeutet was Zitronen sind - ergibt sich auch aus solchen Sätzen wie ,Zitronen schmecken sauer", "Reife Zitronen sind gelb" oder „Zitronen wachsen auf Bäumen". Würden sich unsere Annahmen über Hunde so verändern, daß wir schließlich glauben, Hunde seien so klein wie Mäuse, Hunde bellten nicht, sondern piepsten, sie hätten sechs Beine und ernährten sich von Fliegen, so würde sich auch die Bedeutung des Wortes „Hund“ ändern. Was Hunde sind, ergibt sich aus ihren Eigenschaften und Verhaltensweisen. Und was das Wort „Hund“ bedeutet, hängt davon $a b$, was Hunde nach allgemeiner Auffassung sind. Man kann also nicht behaupten, das Wort „Hund“ bleibe in seiner Bedeutung konstant, wenn sich unsere Annahmen über die Eigenschaften von Hunden grundlegend ändern.

Für eine Kunstsprache $S$ kann man einen scharfen Begriff der Analytizität so festlegen, daß man genau jene Sätze von $S$ als analytisch bei einer Interpretation von $S$ bezeichnet, die in allen möglichen Welten gelten, welche dieser Interpretation zugrundeliegen. Natürliche Sprachen sind aber nicht über einer bestimmten Menge möglicher Welten interpretiert, der Gebrauch ihrer Prädikate ist nicht für beliebige Umstände erklärt, sondern nur für jene Umstände, die normalerweise vorkommen, also für Welten, die unserer hinreichend ähnlich sind. Das zeigt sich am Phänomen der Offenbeit empirischer Prädikate. Ob etwas als ,,Sessel“ bezeichnet werden kann, ist für die normalerweise vorkommenden Gegenstände hinreichend klar. Wenn aber ein Gegenstand, der wie ein Sessel aussieht, plötzlich immer kleiner würde, ganz verschwände, dann wieder erschiene, größer würde etc., so wüßten wir nicht mehr, ob er noch als "Sessel“ zu bezeichnen

${ }^{16} \mathrm{Vgl}$. dazu insbesondere Quine (51), und die ausführlichere Diskussion in Kutschera (75), 2.3.2. 
wäre. ${ }^{17}$ Die Alltagssprache ist für Unterscheidungen im Bereich normalerweise vorkommender Objekte und Ereignisse gemacht. Es gibt aber keine scharfe Grenze zwischen normalen und nichtnormalen Fällen, und daher auch keine scharfen Grenzen eines wohlbestimmten Sprachgebrauchs. So lassen sich auch für alle Standardbeispiele analytischer Sätze in natürlichen Sprachen entlegene Umstände angeben, in denen man nicht mehr behaupten kann, diese Sätze seien wahr. Wenn sich z. B. gewissen Elementarteilchen keine Ausdehnung mehr zuschreiben läßt, sollen wir dann sagen, der Satz ,Alle Körper sind ausgedehnt" sei nach wie vor wahr und diese Elementarteilchen seien eben keine Körper? Oder sollen wir sagen, sie seien Körper, aber nicht ausgedehnt? Man kann im Sinne Quines nur einen komparativen Begriff der Analytizität erklären, z. B. so, daß man sagt: Der Satz $\mathrm{A}$ ist höchstens so analytisch wie der Satz B, wenn für alle Mengen X von Welten, die mit einer Welt auch alle unserer Welt mindestens ebenso ähnlichen Welten enthalten, gilt: Ist $\mathrm{A}$ in allen X-Welten wahr, so auch B. ${ }^{18}$

Wenn also auch die Unterscheidung analytischer und synthetischer Sätze in vielen Fällen hinreichend klar ist, so gibt es doch neben eindeutig analytischen Sätzen wie „Primzahlen sind durch keine Zahl außer 1 ohne Rest teilbar" und eindeutig synthetischen Sätze wie „Hans ist verheiratet“ ein breites Spektrum von Sätzen, die weder eindeutig analytisch noch eindeutig synthetisch sind. Man kann nicht behaupten, nur eindeutig analytische Sätze bestimmten die Bedeutung der in ihnen vorkommenden Terme. Es gibt einen nicht scharf abgrenzbaren Bereich ,quasianalytischer" Sätze, die unter allen normalen Bedingungen gelten und die, da die Sätze nur für normale Umstände geklärt sind, die Bedeutungen der in ihnen vorkommenden Terme eingrenzen. ${ }^{19}$

${ }^{17}$ Dieses Beispiel bringt Wittgenstein in (53), 80.

${ }^{18} \mathrm{Vgl}$. dazu Kutschera (76), 7.2. Man kann mit einem komparativen Begriff der Analytizität einen klassifikatorischen natürlich so definieren, daß man sagt: Der Satz A ist analytisch genau dann, wenn der Satz , $1+1=2$ “ höchstens so analytisch ist wie A. Wegen der Unschärfe des Begriffs der Ähnlichkeit von Welten, würde das aber unser Problem nieht lösen.

${ }^{19} \mathrm{H}$. Putnam hat eine Konzeption der Bedeutung sprachlicher Ausdrücke entwickelt, die auf ähnlichen Gedanken beruht. Er geht davon aus, daß man ein 
Die Abhängigkeit der Bedeutung von Beobachtungstermen von unseren Annahmen über die Welt bezeichnet man als Theoriebeladenbeit der Beobachtungssprache. ${ }^{20}$ Diese Annahmen bilden zwar keine "Theorie", die Bezeichnung ist aber wegen der Ähnlichkeit dieses Phänomens mit der Bedeutungsabhängigkeit theoretischer Terme von den Theorien gerechtfertigt, in denen sie vorkommen. Darauf gehen wir im nächsten Abschnitt ein.

Diese Überlegungen zeigen noch einmal, daß eine enge Verbindung zwischen der Entwicklung bzw. dem Erlernen der Sprache und dem Erkennen der Welt besteht. Begriffliche Erkenntnis vollzieht sich in sprachlichen Urteilen und bewegt sich daher im Rahmen der Beschreibungs- und Unterscheidungsmittel, die uns die Sprache zur Verfügung stellt. Und umgekehrt beherrscht man die Sprache über einen Gegenstandsbereich nur dann, wenn man etwas über ihn weiß. Über Hunde kann man nur dann sinnvolle Aussagen machen, wenn man sie von anderen Dingen hinreichend sicher unterscheiden kann, wenn man weiß, was Hunde sind. Dazu bedarf es keiner Spezialkenntnisse, wie sie Hundezüchter oder Biologen haben, und nicht alles, was man von Hunden glaubt, muß richtig sein. Man beherrscht aber den $\mathrm{Ge}$ brauch des Wortes „Hund“ nur dann, wenn man es in der Regel richtig verwendet, wenn man also die Aussagen, die man über Hunde

Prädikat wie „,Tiger“ nur dann versteht (seine Bedeutung nur dann kennt), wenn man über ein (nicht scharf abgrenzbares) Minimum an alltäglichem Wissen über Tiger verfügt, wie es etwa in der oben zitierten Worterklärung enthalten ist. Dieses Minimum an Wissen bezeichnet Putnam als „Stereotype" des Wortes. Nur wer über dieses Wissen verfügt, kann sinnvoll über Tiger reden. Die Sätze dieses Minimalwissens sollen freilich keine analytischen Sätze sein - sie können sich z. B. auf Eigenschaften beziehen, die 'Tiger normalerweise haben; sie können gängige Meinungen über Tiger ausdrücken, welche wissenschaftlichen Ansichten nicht genau entsprechen, usf. Die Bedeutung eines Prädikats wird bei Putnam nicht nur durch diese Stereotype bestimmt, sondern auch durch fragwürdige semantische Charakterisierungen und durch seine Extension. Danach ändert sich aber die Bedeutung des Wortes „Tiger“, wenn sich der Bestand an lebenden Tigern ändert, was kaum sehr einleuchtend ist. Vgl. dazu die Aufsätze in Putnam (75).

${ }^{20}$ Von einer Theoriebeladenheit der Beobachtungssprache sprechen neben Quine auch viele andere Autoren, wie z. B. Kuhn in (62) und Feyerabend in (60). 
macht, in der Regel korrekt sind, und dazu benötigt man gewisse elementare Kenntnisse über Hunde. Je reicher unser empirisches Wissen wird, je mehr und je genauere Unterscheidungen wir machen können, desto reicher wird auch unsere Sprache; und je reicher die Sprache, desto mehr und desto genauere Unterscheidungen können wir machen. Unsere Sprache ist das Produkt einer langen Auseinandersetzung mit der Welt, das Ergebnis einer Fülle von Erfahrungen. Ihre Entwicklung läßt sich nicht rational rekonstruieren, und ihre Geschichte übersehen wir nur für eine relativ kleine Zeitspanne. Hypothesen über Ursprung und Ausbildung der Sprache sind höchst problematisch, weil sie in der Regel den Urvätern der Sprache Unterscheidungsmöglichkeiten unterstellen, für die erst die Sprache auf ihrem heutigen Entwicklungsstand die Grundlage bildet. Unsere Sprache ist also für uns eine Gegebenheit, hinter die wir kaum zurückgehen können. Mit ihr haben wir unsere Annahmen über die Welt erworben, haben wir gelernt, die Welt in gewisser Weise zu sehen. Unsere Sprache ist also für uns so etwas wie ein Apriori, wenn auch nicht im Sinn des Begriffsrationalismus, nach dem es eingeborene Begriffe gibt (ideae innatae), die, wie Kant sagt, im menschlichen Verstand angelegt sind. Denn es gibt Sprachen mit sehr unterschiedlichen Begriffssystemen und Grammatiken. Sprachen ändern sich, ihre Ausdrucksmittel lassen sich erweitern, aber alle Veränderungen der Sprache gehen von ihrem gegebenem Zustand aus. Wir können nicht unabhängig von ihr eine ganz neue Sprache konstruieren, sondern immer nur die gegebene umbauen.

\subsection{Theoretische Terme}

Auch bei der Erörterung von Einführung und Funktion theoretischer Terme gehen wir vom Begriffsempirismus aus. Seine zweite Teilthese haben wir in 9.1 so formuliert:

$\mathrm{EB}_{2}$ : Alle empirischen Terme lassen sich durch Beobachtungsterme definieren.

Danach gibt es keine theoretischen Terme, denn nach den Bestimmungen in 9.1 sind alle durch Beobachtungsterme definierbaren Terme zur Beobachtungssprache zu rechnen. Diese Auffassung wurde auch im frühen logischen Empirismus vertreten. Man stützte sich da- 
bei auf empirische Sinnkriterien, mit denen man den Bereich sinnvoller empirischer Aussagen abzugrenzen suchte. Wir wollen nicht näher auf diese Sinnkriterien eingehen, ${ }^{21}$ sondern nur bemerken, daß nach ihnen erstens Beobachtungssätze als empirisch sinnvoll ausgezeichnet werden, und zweitens Sätze A, die in gewissen deduktiven oder induktiven Relationen zu bereits als empirisch sinnvoll erkannten Sätzen $B_{1}, \ldots, B_{n}$ stehen. Alle diese Relationen bestehen nur dann, wenn in A nur solche deskriptiven Terme (wesentlich) vorkommen, die auch in den Sätzen $B_{1}, \ldots, B_{n}$ vorkommen. Daher werden durch die Sinnkriterien nur Sätze der Beobachtungssprache, und damit auch nur Beobachtungsterme als sinnvoll ausgezeichnet, so daß die These $E_{2}$ gilt. Alle vorgeschlagenen empiristischen Sinnkriterien haben sich jedoch, wie schon früher betont wurde, als unhaltbar erwiesen. Ausschlaggebend dafür war nicht nur, daß sich der Begriff des Beobachtungssatzes, und damit auch der des Beobachtungstermes, nicht generell, sondern nur in Abhängigkeit vom jeweiligen Untersuchungskontext in befriedigender Weise bestimmen läßt, sondern auch, daß alle Sinnkriterien ganz normale und zweifellos sinnvolle naturwissenschaftliche Aussagen aus dem Bereich empirisch sinnvoller Sätze ausschließen.

Es gibt nun viele empirische Terme, die sich nicht explizit durch Beobachtungsterme definieren lassen, die also als theoretische Terme anzusprechen sind. Die einfachsten Beispiele bilden Dispositionsterme, wie „,magnetisch“, ,elastisch“, ,zerbrechlich“ oder „,öslich“. Wie schon im Abschnitt 2.2 betont worden ist, läßt sich die Disposition $\mathrm{D}$, unter Bedingungen der Art $\mathrm{T}$ ein Verhalten der Art $\mathrm{R}$ zu zeigen (wo $T$ und $R$ Beobachtungsbegriffe seien) nicht mit extensionallogischen Mitteln durch $T$ und $R$ definieren. Für eine Definition der Dispositionsprädikate bleiben daher nur zwei Möglichkeiten: entweder man verwendet intensionslogisch definierte Konditionalsätze, oder man muß sich auf eine implizite Definition beschränken. Im ersten Fall setzt man

1. $\mathrm{D}(\mathrm{x}):=\mathrm{T}(\mathrm{x}) \rightarrow \mathrm{R}(\mathrm{x})$,

wobei das Symbol „ $\rightarrow$ “ für den Ausdruck „,wenn - dann“ steht. Auch wenn A und B Beobachtungssätze sind, ist jedoch der Konditionalsatz „,Wenn A, dann B“ kein Beobachtungssatz. Zeigen Beobach-

${ }^{21}$ Vgl. dazu die Literaturhinweise in der Anmerkurg 7 zu S. 254 sowie die Bemerkungen in 6.2. 
tungen, daß A wahr und B falsch ist, so ist damit zwar die Behauptung $A \rightarrow B$ falsifiziert, aber man kann diesen Satz nicht durch Beobachtungen widerlegen, wenn $A$ nicht gilt, und man kann ihn durch Beobachtungen auch nicht verifizieren. Der Satz A $\rightarrow$ B wird so interpretiert, daß er genau dann wahr ist, wenn $B$ unter all jenen Umständen (in all jenen Welten) gilt, von denen normalerweise einer besteht, wenn A gilt. Der Wahrheitswert des Satzes hängt daher nicht nur von den tatsächlich gegebenen und allein beobachtbaren Umständen ab, sondern auch von dem, was unter anderen Umständen gelten würde; er ergibt sich nicht nur aus dem Zustand unserer Welt, sondern aus unserer Einschätzung dessen, was unter der Bedingung A normalerweise gilt, also von unseren Erwartungen. ${ }^{22} \mathrm{Da}$ der Satz „Wenn A, dann B" auch dann kein Beobachtungssatz ist, wenn A und B Beobachtungssätze sind, kann der Ausdruck ,,wenn - dann" nicht zum logischen Vokabular der Beobachtungssprache gehören. Die Definition (1) ist also nicht mit dem empiristischen Programm vereinbar.

Im Sinn einer impliziten Definition wäre das Dispositionsprädikat ,D“ durch das Bedeutungspostulat

2. $\mathrm{T}(\mathrm{x}) \supset(\mathrm{D}(\mathrm{x}) \equiv \mathrm{R}(\mathrm{x}))$

einzuführen, oder durch ein System solcher oder ähnlicher Bedingungen. Auch damit geht man über die Beobachtungssprache hinaus, denn solche impliziten Definitionen lassen sich in der Regel nicht in explizite Definitionen umformen, und nur für diesen Regelfall interessieren wir uns hier. ${ }^{23}$

${ }^{22}$ Eine epistemische Interpretation von Konditionalsätzen, nach der ein Satz $A \rightarrow B$ genau dann wahr ist, wenn die Implikation $A \supset B$ gilt und es allgemeine Überzeugung ist, daß im Fall von A auch immer B gilt, liegt nahe, da sich sonst mit der Rede von ,normalen“ Umständen oder von der , Ähnlichkeit" von Welten kein genauer Sinn verbinden läßt. Vgl. dazu Kutschera (76), 4.5. Auch Hume hat betont, daß wir durch Beobachtungen keinen kausalen Zusammenhang zwischen zwei Ereignissen feststellen können. Nach seiner Ansicht gründen sich Kausalaussagen auf Erwartungen. Übersetzt man seine Aussagen über Erwartungen in die Sprache über subjektive Wahrscheinlichkeiten, so ergibt sich eine ähnliche Analyse von Kausalaussagen, wie wir sie hier für Konditionalsätze vorschlagen.

${ }^{23}$ Im Beispiel (2) folgt aus dem Postulat nur dann eine explizite Definition von $D$, wenn $T$ auf alle Objekte zutrifft. Dann trifft aber $D$ genau auf jene Dinge zu, auf die auch $R$ zutrifft; $D$ ist dann also kein Dispositionsprädikat. 
Theoretische Terme werden durch implizite Definitionen nur partiell interpretiert. Es gibt mehrere Interpretationen einer Sprache S, die allen Beobachtungstermen dieselben Bedeutungen zuordnen und die Postulate der impliziten Definitionen erfüllen, den oder die theoretischen Terme aber nicht in gleicher Weise deuten. Wäre das nicht der Fall, so wäre aus den Postulaten für jeden theoretischen Term eine Formel ableitbar, nach der er sich explizit durch Beobachtungsbegriffe definieren ließe. ${ }^{24}$ Gibt es aber mehrere Interpretationen der theoretischen Terme, so genügt die Deutung der Beobachtungssprache nicht, um allen Sätzen, in denen solche Terme vorkommen, einen bestimmten Wahrheitswert zuzuordnen. Selbst wenn wir also über alle mit der Beobachtungssprache beschreibbaren Fakten Bescheid wüßten, gäbe es Sätze der theoretischen Sprache, von denen wir nicht sagen könnten, sie seien wahr oder falsch.

Es gibt also theoretische Terme und daher ist auch der zweite Teil der begriffsempiristischen These EB nicht haltbar. Braucht man aber tatsächlich solche Terme? Daß theoretische Terme in den Naturwissenschaften tatsächlich eine wichtige Rolle spielen, ist unbestritten. Wenn sich aber zeigen ließe, daß sie nur prinzipiell entbehrliche Hilfsausdrücke sind, so wären sie jedenfalls für erkenntnistheoretische Erörterungen ohne Interesse. Das erscheint aber schon aus dem Grund unmöglich, daß z. B. alle physikalischen Größen, wie räumliche und zeitliche Distanz, Masse usw., durch operative Definitionen eingeführr weráen, wie wir im Abschnitr 8.3 gesehen haben.

Es gibt zwei Sätze über die Eliminierbarkeit theoretischer Terme. Das erste ist ein Theorem von W. Craig, nach dem man zu jeder Theorie $\mathrm{T}$ mit theoretischen Termen eine Theorie $\mathrm{T}$ ' ohne solche Terme angeben kann, die denselben empirischen Gehalt hat wie T. ${ }^{25}$

${ }^{24}$ Vgl. dazu Kutschera (72), Satz T3.3-1.

${ }^{25}$ Dieses Theorem wurde von Craig in (53) bewiesen; vgl. auch Craig (56) und die Darstellung in Kutschera (72), 4.1. - Wenn hier von der Einführung theoretischer Terme im Rahmen von Theorien die Rede ist, so ist dieses Wort in einem weiteren Sinn zu verstehen, in dem z. B. auch der Satz (2) eine "Theorie" darstellt. Auch die in 8.3 diskutierte Bestimmung physikalischer Größen durch Meßvorschriften sind insofern Theorien, als zu den operativen Definitionen auch noch empirische Annahmen hinzukommen müssen, die sicherstellen, daß die definierten Größen die gewünschten Eigenschaften haben. 
Als empirischen Gebalt einer Theorie $\mathrm{T}$ bezeichnet man entweder die Menge der aus $\mathrm{T}$ abieitbaren Beobachtunyssätze, oder die Menge der aus $T$ ableitbaren Sätze der Beobachtungssprache. Wir verwenden das Wort hier im letzteren Sinn, und sprechen im ersteren Sinn vom ,empirischen Gehalt i.e.S.". Aus dem Beweis des Theorems von Craig ergibt sich jedoch, daß 'T' eine wesentlich komplexere und unübersichtlichere Theorie ist als T. Damit wird deutlich, daß die Verwendung theoretischer Terme der Vereinfachung von Theorien dient. Einfachheit und Übersichtlichkeit sind aber zumindest praktisch unverzichtbare Forderungen an Theorien.

Ein sehr viel elementareres Argument für die Entbehrlichkeit theoretischer Terme hat F. P. Ramsey angegeben. Ist $T=T\left(t_{1}, \ldots, t_{n}\right)$ die Konjunktion der Axiome einer empirischen Theorie, in der die theoretischen Terme $t_{1}, \ldots, t_{n}$ vorkommen, so bezeichnet man den Satz $R(T)=V x_{1} \ldots x_{n} T\left(x_{1}, \ldots, x_{n}\right)$, der aus $T\left(t_{1}, \ldots, t_{n}\right)$ entsteht, indem man die Terme $t_{1}, \ldots, t_{n}$ durch passende Variablen $x_{1}, \ldots, x_{n}$ ersetzt und zu einer Existenzbehauptung übergeht, in der über diese Variablen quantifiziert wird, als Ramsey-Satz zur Theorie T. Die Theorie $R(T)$ hat nun denselben empirischen Gehalt wie T. ${ }^{26}$ Man kann daher eine Theorie $T$ einfach durch ihren Ramsey-Satz $R(T)$ ersetzen, der nach Konstruktion keine theoretischen Terme enthält, also ein Satz der Beobachtungssprache ist. $R(T)$ ist nicht wesentlich komplizierter als der Satz $\mathrm{T}$ selbst, so daß sich bei diesem Verfahren zur Elimination theoretischer Terme auch kein Verlust an Einfachheit ergibt.

Dem Ramsey-Verfahren liegt nun eine rein theorien-immanente Auffassung theoretischer Terme zugrunde. Danach erhalten sie nur im Rahmen einer fest umrissenen Theorie eine Bedeutung; sie lassen sich daher auch nur im Rahmen dieser Theorie sinnvoll verwenden. Kommt derselbe theoretische Term in verschiedenen Theorien vor, so liegt nur eine irrelevante syntaktische Identität vor, nicht aber eine relevante Bedeutungsgleichheit. So kann man etwa sagen, daß die Ausdrücke "Gerade" und ,parallel" in der euklidischen Geometrie eine andere Bedeutung haben als in der nicht-euklidischen. Andernfalls

${ }^{26}$ Es ist ein einfaches logisches Prinzip, daß ein Satz $A$, der aus $T\left(t_{1}, \ldots, t_{n}\right)$ folgt, auch aus $V x_{1} \ldots x_{n} T\left(x_{1}, \ldots, x_{n}\right)$ folgt, wenn die Terme $t_{1}, \ldots, t_{n}$ in $A$ nicht vorkommen. Folgt ein Beobachtungssatz $A$ aus $T$, so folgt er also auch aus $R(T)$. Da $R(T)$ aus $T$ logisch folgt, erhält man umgekehrt alle Beobachtungssätze, die aus $R(T)$ abgeleitet werden können, auch aus $T$. 
würden sich deren Aussagen widersprechen. Entsprechendes gilt für den Ausdruck ,,gleichzeitig“ in der klassischen und in der relativistischen Mechanik. Die theorienimmanente Deutung stützt sich auf die Tatsache, daß theoretische Terme erst durch die Postulate einer Theorie gedeutet werden. Das ist einerseits sicher richtig, andererseits werden aber theoretische Terme tatsächlich oft in einer theorienübergreifenden Weise verwender; derselbe Term kommt in derselben Bedeutung in einer anderen Theorie vor. Häufig wird untersucht, ob ein Satz A wahr ist, in dem theoretische Terme aus $\mathrm{T}$ vorkommen, der aber nicht aus $\mathrm{T}$ ableitbar ist. Nach der theorienimmanenten Deutung wäre das so zu verstehen, daß die Theorie $T \wedge A$ geprüft wird, in der nun die theoretischen Terme von $A$ und $T$ in einer neuen Weise gedeutet werden, und daß gefragt wird, ob die neue Theorie bei dieser Umdeutung der Terme wahr ist. Das entspricht aber nicht dem üblichen Verständnis, nach dem die theoretischen Therme in A so gedeutet werden sollen wie in T. Man kann z. B. metrische Größen wie Geschwindigkeit, Masse, elektrische Ladung etc. als theoretische Be. griffe ansehen. Die Masse, die man einem Körper im Rahmen einer Theorie zuschreibt, muß mit der Masse übereinstimmen, die man ihm in anderen Theorien zuordnet. „Masse" ist also kein theoretischer Term, der sich nur theorienimmanent deuten ließe.

Ein solcher Umgang mit theoretischen Termen, ihr intertheoretischer Gebrauch wäre bei einer theorienunabbängigen Deutung dieser Terme ohne weiteres möglich. Danach wird ihr Sinn durch gewisse Bedeutungspostulate festgelegt. Zu diesen Postulaten kommen in jeder empirischen Theorie, welche diese Terme enthält, rein synthetische Sätze hinzu, die den materialen Gehalt der Theorie bestimmen, nicht jedoch die Bedeutung der theoretischen Terme. Auch eine solche Auffassung ist jedoch nicht brauchbar. Denn ist eine Theorie $T\left(t_{1}, \ldots, t_{n}\right)$ mit den theoretischen Termen $t_{1}, \ldots, t_{n}$ rein synthetisch bzgl. der Deutung dieser Terme durch die analytischen Bedeutungspostulate $P\left(t_{1}, \ldots, t_{n}\right)$, so muß der Wahrheitswert von $T$ aufgrund der Deutung der theoretischen Terme durch $P$ (sowie der Interpretation der Beobachtungssprache) eindeutig festliegen. Dann gäbe es aber einen Satz A der Beobachtungssprache, so daß die Aussage $T \equiv A$ aus $P$ ableitbar ist. ${ }^{27} \mathrm{~T}$ wäre also bei der Deutung mit $\mathrm{P}$ durch eine Theorie

${ }^{27}$ Vgl. Kutschera (72), Theorem T3.3-2. 
A ohne theoretische Terme ersetzbar; diese Terme wären daher überflüssig.

Eine theorienübergreifende Deutung theoretischer Terme setzt jedoch keine theorienunabhängige Interpretation voraus. Gibt man den Gedanken einer strengen Grenze zwischen analytischen und synthetischen Sätzen auf, der sich schon im Bereich der Beobachtungssprache als unhaltbar erwiesen hat, so ist die Annahme, es gebe Aussagen über die Welt, die zugleich die Bedeutung der in ihnen vorkommenden Terme mitbestimmen, nicht mehr anstößig. Die Behauptung, eine Theorie vermittle uns ein Wissen über die theoretischen Konstrukte, die Objekte, Eigenschaften oder Größen, die durch die theoretischen Terme bezeichnet werden, ist dann mit der Aussage verträglich, die Theorie bestimme die Bedeutung dieser Terme. In der Regel wird der Sinn eines theoretischen Terms zunächst einmal durch die Gesetze einer allgemein akzeptierten Theorie $\mathrm{T}$ festgelegt, in denen er vorkommt. Andere Hypothesen über dieses theoretische Konstrukt kann man dann durch Beobachtung überprüfen wie eine synthetische Aussage, indem man Voraussagen mit $\mathrm{T}$ und $\mathrm{H}$ überprüft. Bewährt sich diese Hypothese, so kann sie als empirische Information über dieses Konstrukt aufgefaßt werden, die seine Eigenschaften und damit auch die Bedeutung des theoretischen Terms näher bestimmt.

Carnap hat folgenden Vorschlag zur Aufspaltung des Gehalts von Theorien in einen analytischen und einen synthetischen Teil gemacht. Den synthetischen Gehalt der Theorie $T$ identifiziert er mit ihrem Ramsey-Satz $R(T)$, den analytischen Gehalt von $T$ mit dem Satz $R(T) \supset T$. Dieser Satz soll die Bedeutungen der theoretischen Terme von $T$ festlegen. $D a R(T)$ ein Satz der Form $V x \ldots x_{n} T\left(x_{1}, \ldots, x_{n}\right)$ ist, läßt sich die Aussage $R(T) \supset T$ so lesen: ,Gibt es theoretische Begriffe $x_{1}, \ldots, x_{n}$, die $T$ erfüllen, so seien $t_{1}, \ldots, t_{n}$ solche Begriffe“. $T$ ist mit der Konjunktion von $R(T)$ und $R(T) \supset T$ logisch äquivalent. Und $R(T)$ hat, wie wir sahen, denselben empirischen Gehalt wie $T$. Aus $R(T) \supset \Gamma$ folgen hingegen keine synthetischen Sätze der Beobachtungssprache. ${ }^{28}$ Daher erfüllt das Bedeutungspostulat $R(T) \supset T$ das Kriterium der Nichtkreativität: Aus einer Definition dürfen keine

${ }^{28}$ Folgt ein Satz A der Beobachtungssprache aus $V_{x_{1}} \ldots x_{n} T\left(x_{1}, \ldots, x_{n}\right) \supset$ $T\left(t_{1}, \ldots, t_{n}\right)$, so folgt $A$ nach dem oben erwähnten logischen Theorem auch aus $V y_{1} . . y_{n}\left(V x_{1} \ldots x_{n} T\left(x_{1}, \ldots, x_{n}\right) \supset T\left(y_{1}, \ldots, y_{n}\right)\right)$, also aus dem logisch wahren Satz $V_{x_{1}} \ldots x_{n} T\left(x_{1}, \ldots, x_{n}\right) \supset V_{y_{1}} \ldots y_{n} T\left(y_{1}, \ldots, y_{n}\right)$. 
synthetischen Sätze folgen, die die definierten Zeichen nicht enthalten. Carnaps Vorschlag ist das einzige generell anwendbare Kriterium zur Unterscheidung von analytischem und synthetischem Gehalt von Theorien. Danach werden aber die theoretischen Terme durch das Bedeutungspostulat $R(T) \supset T$ nur unter der Voraussetzung erklärt, daß $R(T)$ richtig ist. Ist $R(T)$ dagegen falsch, so ist die Theorie $T$ sinnlos, da beliebige Interpretationen der theoretischen Terme zulässig sind. Es ist also nicht so, daß das Bedeutungspostulat die Bedeutung der theoretischen Terme von $T$ so festlegt, daß $T$ zu einem synthetischen Satz wird. Nach unserem Vorschlag ist es dagegen verfehlt, den analytischen Gehalt einer Theorie vom synthetischen trennen zu wollen.

Die theoretische Sprache ist nun in weit höherem Maß ,theoriebeladen" als die Beobachtungssprache, da theoretische Terme im Kontext von Theorien eingeführt werden. Die Theoriebeladenheit der empirischen Sprache widerspricht empiristischen Ideen, nach denen der Aufbau einer empirischen Sprache und die Begründung von Sätzen dieser Sprache zwei Vorgänge sind, die sich sauber voneinander trennen lassen. Mit der Einsicht, daß Theorienbildung und die Einführung von Termen miteinander verbunden sind, erweist sich auch die Unterscheidung von theoretischen und Beobachtungstermen als fragwürdig. Beobachtungsterme sollten Terme sein, die für direkt beobachtbare Dinge und Attribute stehen, während theoretische Terme begriffliche Konstrukte bezeichnen, die ihre Funktion im Rahmen einer theoretischen Interpretation von Beobachtungen haben. Wenn aber auch die Beobachtungsterme mithilfe von Gesetzesaussagen gedeutet werden, so unterscheiden sich theoretische Terme von Beobachtungstermen nur graduell, nicht aber prinzipiell. ${ }^{29}$ Unser Verständnis beider Arten von Termen beruht auf Annahmen über die Natur. Beobachtungsprädikate sind vor allem solche, die einen anschaulichen Gehalt haben, der es uns ermöglicht, sie in normalen Fällen Gegenständen recht zuverlässig nach dem direkten Augenschein zu- oder abzusprechen. Wir sind aber nur dann bereit, sie so zu gebrauchen, wenn wir das Vorliegen normaler Umstände voraussetzen. Auch sol-

${ }^{29}$ In diesem Sinn sagt Popper: " . . the customary distinction between 'observational terms' ... and 'theoretical terms' is mistaken, since all terms are theoretical to some degree, though some are more theoretical than others" $((63), 119)$. 
che Voraussetzungen sind Annahmen über die Welt, und wenn wir solche Annahmen zulassen - Annahmen z. B. nicht nur über das normale Funktionieren unserer Sinnesorgane, sondern auch solche über das normale Funktionieren von Meßinstrumenten --, so können wir nach dem Augenschein z. B. auch die Werte physikalischer Größen beurteilen. Dann besteht kein prinzipieller, sondern nur ein gradueller Unterschied zwischen Aussagen über die Nebelspuren, die wir in einer Wilsonschen Kammer beobachten, und solchen über Elektronen, die sie verursachen, obwohl die Deutung der Spuren als Elektronenbahnen sich auf Theorien über die Ablenkung von Elektronen in Magnetfeldern und die Abhängigkeit der Ionisierung von Ladung und Masse stützen.

\subsection{Beobachtungssätze}

Auch bei der Diskussion der Frage, wie sich empirische Aussagen begründen lassen, gehen wir vom empiristischen Erkenntnismodell aus. Die These des Urteilsempirismus, alle empirischen Urteile bezögen ihre Berechtigung allein aus der Erfahrung, kann man in verschiedener Weise deuten. Gehen wir von den Sätzen aus

A) Beobachtungen sind binreichend zur Begründung aller überhaupt begründbaren empirischen Sätze.

B) Beobachtungen sind notwendig zur Begründung aller empirischen Sätze.

C) Es gibt empirische Sätze, zu deren Begründung Beobachtungen notwendig sind.

D) Es gibt empirische Sätze, zu deren Begründung Beobachtungen binreichend sind.

so ergeben sich drei Versionen eines Urteilsempirismus, in denen die Behauptungen

a) $A$ und $B$,

b) A und $C$, oder

c) $B$ und $D$

vertreten werden. Wird weder A noch B behauptet, A ohne C, oder B ohne $\mathrm{D}$, so liegt keine empiristische These vor. Beobachtungen spielen dann keine ausgezeichnete Rolle gegenüber anderen Gründen für die Annahme synthetischer Sätze. Meist wird der Urteilsempirismus 
im Sinn von (a) bestimmt, also als stärkste der drei angegebenen Versionen.

Wir wollen im folgenden zeigen, daß die These D falsch ist. Da D aus A folgt, werden damit alle drei Versionen des Urteilsempirismus widerlegt. Im Sinn eines Urteilsrationalismus, wie ihn z. B. Kant vertrat, gibt es synthetische Sätze apriori, also Sätze, die sich ohne Bezugnahme auf Beobachtungen begründen lassen. Das ist die Negation der These B. Diese Behauptung werden wir uns hier nicht zu eigen machen. Wir vertreten einen "Apriorismus" oder „Rationalismus" nur in dem Sinn, daß D falsch ist, daß also kein synthetischer Satz allein durch Beobachtungen begründbar ist.

Man kann nun die angegebenen Thesen (a) - (c) des Urteilsempirismus, bzw. die Behauptungen A bis D, wie im Fall des Begriffsempirismus in zwei Teilthesen aufspalten. Im Fall der These A ergeben sich z. B. die beiden Behauptungen:

A 1: Es gibt einf́ache empirische Sätze, die sich allein durch Beobachtungen begründen lassen.

A 2: Alle anderen empirischen Sätze (die sich überhaupt begründen lassen, die also insbesondere wahr sind), lassen sich mithilfe solcher einfachen empirischen Sätze begründen.

Solche einfachen empirischen Sätze können wir nicht von vornherein als „Beobachtungssätze“ bezeichnen, da wir diesen Begriff in 9.1 so bestimmt haben, daß diese Sätze durch Beobachtungen entscheidbar sind. Hier geht es aber gerade um die Frage, ob es Beobachtungssätze in diesem Sinn gibt. Das ist also das erste Problem, das sich bei einer Diskussion der These A stellt. Das zweite Problem ist das Induktionsproblem: Vorausgesetzt es gibt Beobachtungssätze, lassen sich dann damit auch Gesetzesaussagen und Theorien begründen? Während die These A also mit dem Aufweis von Beobachtungssätzen noch nicht gerechtfertigt ist, steht und fällt die schwächere These D mit der Behauptung, daß es Beobachtungssätze gibt. Wir befassen uns also zunächst mit dieser Behauptung.

Wie wir in 2.1 gesehen haben, gibt es deduktive und induktive Begründungen. Eine deduktive Begründung von empirischen Sätzen mit Beobachtungen scheidet aus. Im Abschnitt 3.1 haben wir einen Unterschied gemacht zwischen sinnlichem Erleben, Beobachten und Wahrnehmen. Wir haben solche Sachverhalte ausgedrückt durch Sätze der Form ,Es erscheint der Person a, als ob p der Fall ist" - symbolisch 
$\mathrm{S}(\mathrm{a}, \mathrm{p})$ - , bzw. durch Sätze ,a beobachtet, daß p“ - symbolisch

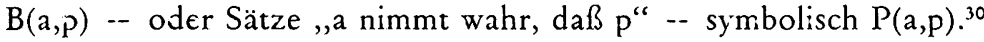
Diese Begriffe wurden so bestimmt, daß weder aus $S(a, p)$ noch aus $\mathrm{B}(\mathrm{a}, \mathrm{p})$ generell $\mathrm{p}$ folgt. Aus Aussagen über das, was ich erlebe oder beobachte, folgt also nicht, daß die erlebten oder beobachteten Sachverhalte tatsächlich bestehen. ${ }^{31}$ Ferner haben wir gesehen, daß es für alle empirischen Aussagen Gesetze gibt, die sie mit anderen empirischen Aussagen so verbinden, daß die letzteren uns als Kriterien für die Wahrheit der ersteren dienen. Auf der Geltung solcher Gesetze beruht der objektive Sinn der empirischer Aussagen. Aufgrund solcher Gesetze haben aber alle empirischen Aussagen - auch die einfachsten - hypothetischen Charakter: Die direkte Beobachtung, daß vor mir eine blaue Zündholzschachtel liegt, garantiert mir nicht, daß es tatsächlich so ist. Meine Aussagen über Farben implizieren z. B., $\mathrm{da} ß$ andere unter normalen Umständen sie ebenso beurteilen wie ich und daß bestimmte physikalische Messungen bestimmte Ergebnisse haben. Von einer "Schachtel" kann ich nur reden, wenn sich der beobachtete Gegenstand öffnen läßt und sich dabei innen als hohl erweist; von einer „,Streichholzschachtel“ nur, wenn sich beim Öffnen zeigt, daß sie Streichhölzer enthält; von ",Streichhölzern“" nur, wenn sie aus Holz bestehen und sich durch Reiben an der Seitenfläche der Schachtel entzünden lassen, usf. Die Behauptung „Hier liegt eine blaue Zündholzschachtel" , die ich aufgrund eines einzigen Blicks mache, ließe sich also nur durch sehr viele weitere Beobachtungen verifizieren. Selbst derart simple empirische Sätze haben also bei genauem Zusehen einen ähnlich hypothetischen Charakter, wie man ihn oft nur generellen Aussagen zuspricht. Von einer deduktiven Begründung von empirischen Sätzen mit Aussagen über Erlebnisse und Beobachtungen kann also nicht die Rede sein.

Eine induktive Begründung solcher Sätze gibr es aber zweifellos, denn wir sind ja meist aufgrund unseres Erlebens von der Geltung der

${ }^{30}$ In all diesen Kontexten ist $\mathrm{p}$ ein Sachverhalt, der durch einen einfachen empirischen Satz ausgedrückt wird.

${ }^{31}$ Aus dem Satz ,Ich nehme wahr, daß p“ folgt hingegen p. Das gilt aufgrund der Bestimmung des Wahrnehmungsbegriffs, hier geht es aber nicht um die Frage, ob als zuverlässig definierte Wahrnehmungen zuverlässig sind, sondern um die Frage der Begründung von Aussagen durch den direkten Augenschein, also durch Sätze der Form ,Es erscheint mir, als ob p“. 
erlebten Sachverhalte überzeugt. Aus dem anschaulichen Gehalt des Wortes ,,rot“ - um auf das Beispiel aus 7.4 zurückzugreifen - ergibt sich, daß in der Regel - unter normalen Beobachtungsbedingungen - die Dinge tatsächlich rot sind, die uns so erscheinen. Wenn es uns also so erscheint, als sei der Gegenstand rot, so ist es für uns prima facie sehr wahrscheinlich, daß er wirklich rot ist. Die Wahrscheinlichkeit, die wir dem Satz „Dies ist rot" aufgrund der Tatsache zuordnen, daß es uns so erscheint, hängt aber davon $a b$, was wir über die bestehenden Beleuchtungsverhältnisse annehmen und über das Funktionieren unserer Farbwahrnehmung. Da wir ferner, wie gerade noch einmal betont wurde, das Wort „rot" auch so verwenden, daß gewisse Zusammenhänge zwischen der Eigenschaft Rot und anderen empirischen Gegebenheiten bestehen, hängt diese Wahrscheinlichkeit auch von weiteren empirischen Annahmen ab. Formal können wir die Annahmen einer Person a im Zeitpunkt $t$ durch die subjektive Wahrscheinlichkeitsbewertung $w_{t}$ darstellen, die jedem Sachverhalt $p$ einen Wahrscheinlichkeitsgrad $w_{t}(p)$ zuordnet. Das Lernen aus der Erfahrung vollzieht sich dann durch die Bildung bedingter Wahrscheinlichkeiten. Stellt $a$ in einem auf $t$ folgenden Zeitpunkt $t$ ' fest, daß ein Sachverhalt $\mathrm{q}$ besteht, so ist $\mathrm{w}_{\mathrm{t}^{\prime}}(\mathrm{p})=\mathrm{w}_{\mathrm{t}}(\mathrm{p}, \mathrm{q})$. Erscheint es nun a in $\mathrm{t}^{\prime}$, als ob q gelte, so ist $\mathrm{w}_{\mathrm{t}^{\prime}}(\mathrm{p})=\mathrm{w}_{\mathrm{t}}(\mathrm{p}, \mathrm{S}(\mathrm{a}, \mathrm{q}))$ für alle Sachverhalte $\mathrm{p}$. Die Wahrscheinlichkeit $\mathrm{w}_{\mathrm{t}^{\prime}}(\mathrm{q})$, die a dem Sachverhalt $\mathrm{q}$ in $\mathrm{t}^{\prime}$ zumißt, ist also nicht nur durch die Tatsache bestimmt, daß es a in 't' so scheint, als ob q gelte, sondern sie hängt auch von der vorausgehenden Wahrscheinlichkeit $\mathrm{w}_{\mathrm{t}}$ ab und den Annahmen, die in dieser Bewertung stekken. Ergibt $w_{t}$ weder für $q$ noch für das Bestehen normaler Beobachtungsverhältnisse in ' $t$ ' eine geringe Wahrscheinlichkeit, so wird $\mathrm{w}_{\mathrm{t}}(\mathrm{q}, \mathrm{S}(\mathrm{a}, \mathrm{q}))=\mathrm{w}_{\mathrm{t}^{\prime}}(\mathrm{q})$ in unserem Beispiel eines hinreichend deutlichen Farberlebens groß sein. Ist hingegen s ein Sachverhalt - z. B. daß die Beleuchtungsverhältnisse so sind, daß wir Farben nicht zuverlässig beurteilen können - , für den $w_{t}(q, S(a, q) \wedge s)$ klein ist, und ist $w_{t}(s)$ groß, so wird $w_{t^{\prime}}(q)$ klein sein. ${ }^{32}$

${ }^{32}$ Dieses Wahrscheinlichkeitsmodell zur Darstellung der Annahmen einer Person und ihrer Änderung aufgrund von Erfahrung stellt natürlich eine starke Idealisierung dar, denn mit den Bedingungen für subjektive Wahrscheinlichkeiten gelten recht starke Kohärenzforderungen für derart charakterisierte Systeme von Annahmen und ihre Veränderungen. Vgl. dazu die Ausführungen in 1.1 . 
Aus dieser Überlegung ergeben sich folgende Konsequenzen:

1. Für dic Annahme einer Person a, daß cin beobachtbarer empirischer Sachverhalt $p$ besteht, ist nicht nur die Frage relevant, ob S(a,p) gilt, sondern diese Annahme beruht auch auf einer Reihe anderer Voraussetzungen. Die Vorstellung, es gäbe einfache empirische Sätze, über deren Annahme oder Verwerfung allein direktes Erleben entscheide (und allenfalls der Grad der Deutlichkeit dieses Erlebens), ist also nicht haltbar.

2. Die Wahrscheinlichkeit, die wir Beobachtungsverhalten zumessen, hängt von der apriori-Wahrscheinlichkeit $a b$, von der wir ausgehen. Beginnt die Person $a$ in $t$ mit einer Bewertung $w^{a}{ }_{t}$ und die Person $b$ mit der Bewertung $w_{t}^{b}$ und erleben a und $b$ zwischen $t$ und $t$ ' dasselbe - so daß also in diesem Zeitraum für alle Sachverhalte $p$ gilt $S(a, p)$ $\equiv S(b, p)-$, so können sich doch die Bewertungen $w^{a}{ }^{\prime}$ und $w_{t^{\prime}}^{b}$ erheblich unterscheiden. Unsere Annahmen über die Welt, die sich in Beobachtungssätzen ausdrücken, hängen also nicht nur von unserem Erleben $a b$, sondern auch von apriorischen Komponenten. Diese Komponenten sind keine synthetisch-apriorischen Sätze über die $\mathrm{Na}$ tur, wie bei Kant, sondern subjektive Wahrscheinlichkeitsannahmen. Wir wollen nicht behaupten, jeder Mensch verfügte über eine bestimmte apriori-Bewertung, die all seiner Erfahrung vorausgeht und aus der sich, im Sinne der Bildung bedingter Wahrscheinlichkeiten, all seine späteren Wahrscheinlichkeitsannahmen ergeben. Wir nehmen in unserem Modell nur an, daß wir in jedem Zeitpunkt bereits über Ausgangsbewertungen verfügen, die den Übergang vom Erleben zu Behauptungen über die Welt regeln. ${ }^{33}$ Diese Bewertungen werden aufgrund des Erlebens fortlaufend modifiziert. Man kann aber nicht behaupten, daß sie sich allein aus dem Erleben ergeben, denn bei jedem Übergang vom Erleben zu Annahmen über Beobachtungssachverhalte sind schon solche Bewertungen im Spiel. Qualitativ gesprochen: Jeder solcher Übergang beruht auf Voraussetzungen, auf Hintergrundannahmen. Die indukrive Begründung auch einfacher empirischer Sätze stützt sich also nicht nur auf direktes Erleben, sondern auch auf andere empirische Annahmen. Unsere Erfahrungsurteile beruhen weder

33 „Über eine Bewertung verfügen“" heißt nicht, sie explizit im Kopfe haben, sondern kann realistischerweise nur besagen, daß man eine Erwartungshaltung hat, die sich mit dieser Bewertung beschreiben läßt. 
nur auf direktem Erleben, noch auf der Gesamtheit des Erlebens, sondern auch auf apriori-Bewertungen.

3. In qualitativer - und damit ungenauerer - Sprechweise können wir den Gehalt einer apriori-Bewertung als System von Annahmen bezeichnen, in einem sehr weiten Sinn als ,apriori-Theorie“. Bei dieser Redeweise ergibt sich dann eine Theoriebeladenbeit auch einfachster empirischer Sätze. Empirische Urteile beruhen nicht immer auf Beobachtungen, sondern auch auf vorgängigen Annahmen über die Welt. Einen genaueren Sinn erhält der Ausdruck ,,Theoriebeladenheit" für Aussagen über die Werte physikalischer Größen. Wie wir in 8.3 gesehen haben, werden solche Größen durch Meßverfahren definiert. Die Verwendung dieser Meßverfahren beruht auf generellen empirischen Annahmen, also auf einer empirischen Theorie, nach der die Meßergebnisse Werte liefern, die den intendierten Eigenschaften der Größe entsprechen. Bei Messungen von Längen mit Maßsstäben setzen wir z. B. voraus, daß sie einen Längenbegriff ergeben, der die Eigenschaften einer Abstandsfunktion hat.

4. Beobachtungssätze im Sinn von 9.1 gibt es also nicht im Sinn von allein aufgrund von direktem Erleben definitiv entscheidbaren Sätzen, sondern nur im Sinn von Sätzen, die sich normalerweise aufgrund von mehr oder minder ,,direktem“ Erleben mit praktischer Sicherheit beurteilen lassen. Auch sie haben aber hypothetischen Charakter, und damit erweist sich auch der Unterschied zwischen Beobachtungssätzen und anderen empirischen Sätzen nur als ein gradueller. Alle empirischen Sätze sind hypothetisch, manche mehr, manche weniger, und Beobachtungssätze sind nur relativ wenig hypothetische, aufgrund von Erfahrung relativ direkt beurteilbare Aussagen.

Wenn sich nun schon einfache empirische Sätze nicht allein mit Beobachtungen begründen lassen, so gilt das erst recht für andere empirische Sätze, insbesondere für generelle Hypothesen. Damit ist also die These D bereits widerlegt. Erkenntnistheoretisch ist aber nicht nur die Frage nach der Begründung von Beobachtungssätzen relevant, sondern insbesondere auch die Frage nach der Rechtfertigung genereller Hypothesen und Theorien. Bei der Erörterung dieser Frage, der wir uns im folgenden Abschnitt zuwenden, sehen wir von der Begründungsproblematik der Beobachtungssätze ab. Unsere Frage ist also: 
Wie lassen sich mit Beobachtungssätzen generelle empirische Aussagen begründen?34

\subsection{Das Induktionsproblem}

Um uns nicht von vornherein mit dem Problem theoretischer Sätze zu belasten, betrachten wir zunächst nur Sätze der Beobachtungssprache. Wir unterscheiden wieder deduktive und induktive Begründungen. Da Beobachtungssätze molekulare Sätze sind, d. h. Basissätze oder mit aussagenlogischen Operatoren aus Basissätzen zusammengesetzte Aussagen, folgt aus ihnen kein genereller Satz. ${ }^{35}$ Aus endlich vielen Sätzen, die besagen, daß bestimmte Raben schwarz sind, folgt nicht einmal der Satz, daß ein weiterer Rabe schwarz ist, geschweige denn die Aussage, daß alle Raben schwarz sind. Synthetische generelle Sätze lassen sich also nicht deduktiv mit Beobachtungssätzen begründen.

Das hat schon Hume betont. Hume hat auch gesehen, daß sich keine induktiven Prinzipien rechtfertigen lassen, mit denen man Beobachtungssätze $F\left(a_{1}\right), \ldots, F\left(a_{n}\right)$ auf $F\left(a_{n+1}\right)$ schließen kann, wo $a_{n+1}$ ein noch nicht beobachtetes Objekt ist, oder gar auf den Satz $\Lambda x F(x)$. Denn wäre ein solches Prinzip $P$ analytisch begründbar, so wäre mit dem Schluß von $F\left(a_{1}\right), \ldots, F\left(a_{n}\right)$ und $P$ auf $F\left(a_{n+1}\right)$, bzw. $\Lambda x F(x)$, auch der Schluß von $F\left(a_{1}\right), \ldots, F\left(a_{n}\right)$ auf eine dieser Konklusionen analytisch gültig, was er, wie wir sahen, nicht ist. Für eine induktive Begründung von $\mathrm{P}$ müßte man aber entweder $\mathrm{P}$ selbst schon voraussetzen - also den Fehler einer petitio principii begehen - oder ein

${ }^{34}$ Es gibt natürlich viele Aussagen der Beobachtungssprache, die im Sinn von 9.1 keine Beobachtungssätze sind, deren Begründung durch solche Sätze aber keine weiteren Probleme aufwirft. So folgen z. B. die Aussagen VxF(x) und $F(a) \vee \Lambda x(F(x) \supset G(x))$ logisch unmittelbar aus dem Beobachtungssatz $\mathrm{F}(\mathrm{a})$.

${ }^{35}$ Genauer müßte man sagen: Aus endlich vielen Molekularsätzen folgt kein wesentlich genereller Satz, d. h. kein Satz der Gestalt $\Lambda x F(x)$, der nicht analytisch äquivalent ist mit einem Molekularsatz. Ist das Prädikat „,F“ z. B. dadurch definiert, daß es genau auf endlich viele Objekte $a_{1}, \ldots, a_{n}$ zutrifft, so folgt aus den Sätzen , $F\left(a_{1}\right)^{\prime \prime},, F\left(a_{n}\right)$ “ der Satz $\Lambda x F(x)$, da er mit ,F( $\left.a_{1}\right)$ $\wedge \ldots \wedge F\left(a_{n}\right)^{\prime \prime a ̈ q u i v a l e n t ~ i s t . ~}$ 
anderes Induktionsprinzip P'; dann geriete man aber in einen unendlichen Regreß. Alle Versuche, diese elementare Schwierigkeit zu umgehen, haben sich als vergeblich erwiesen. ${ }^{36}$ Induktive Prinzipien lassen sich nur im Rahmen der Theorie subjektiver Wahrscheinlichkeiten formulicron und da steller sie keine Scoblüsse dar, soncièn hussagen über bedingte Wahrscheinlichkeiten. ${ }^{37}$ In dieser Theorie wird ein Induktionsprinzip bewiesen, das besagt:

IP: Der Grenzwert von w $\left(F\left(a_{n}+1\right), F_{n}^{r}\right)$ für immer größere Zahlen $\mathrm{n}$ ist $\frac{\mathrm{r}}{\mathrm{n}}$.

Dabei sei $F_{n}^{r}$ die Aussage, daß von den $n$ Objekten $a_{1}, \ldots, a_{n}$ genau $r$ Objekte die Eigenschaft $F$ haben; daß also die relative Häufigkeit der F-Objekte unter den Objekten $a_{1}, \ldots, a_{n} \frac{r}{n}$ ist

Es gilt also insbesondere

IP': Der Grenzwert von $w\left(F\left(a_{n+1}\right), F\left(a_{1}\right) \wedge \ldots \wedge F\left(a_{n}\right)\right)$ für immer größere Zahlen $\mathrm{n}$ ist 1.

Die Bezugsperson, deren Wahrscheinlichkeitsbewertung $w$ ist, rechnet also sicher mit dem Eintreten des Ereignisses $F\left(a_{n+1}\right)$, wenn sie hinreichend vieie Objekte $a_{1}, \ldots, a_{n}$ untersucht hat und an ihnen alien die Eigenschaft $\mathrm{F}$ festgestellt hat.

IP gilt aber nur unter zwei Voraussetzungen:

a) Die Ereignisse $F\left(a_{i}\right)(i=1,2, \ldots)$ sind vertauschbar bzgl. w, d. h. es gilt $w\left(F\left(a_{i 1}\right) \wedge \ldots \wedge F\left(a_{i_{n}}\right)\right)=w\left(F\left(a_{j 1}\right) \wedge \ldots \wedge F\left(a_{j_{n}}\right)\right)$ für alle $n$-tupel von Objekten $a_{i 1}, \ldots, a_{i_{n}}$ und $a_{j 1}, \ldots, a_{j n}(n \geq 1)$.

$w\left(F\left(a_{i 1}\right) \wedge \ldots \wedge F\left(a_{i_{n}}\right)\right)$ darf also nur von der Zahl $n$, nicht aber von der Wahl der $\mathrm{a}_{\mathrm{il}}(1=1, \ldots, \mathrm{n})$ abhängen. In diesem Fall wollen wir auch sagen, das Prädikat F, bzw. die durch $\mathrm{F}$ ausgedrückte Eigenschaft sei vertauschbar bzgl. w.

${ }^{36} \mathrm{Vgl}$. dazu Kutschera (72), Kap. 2.

${ }^{37} \mathrm{Vgl}$. dazu 2.1. Schon Hume erklärt das induktive ,Schließen“ mit Erwartungen, die er als psychische Dispositionen versteht. Man kann die probabilistische Induktionstheorie als rationale Rekonstruktion der psychologischen Theorie Humes auffassen. 
b) Die Wahrscheinlichkeit w ist bzgl. F regulär, d. h. die unbedingte Wahrschıiniichkeit dafür, daß sich eine Ereignisfolge mit einem Grenzwert der relativen Häufigkeit $\frac{r}{n}$ ergibt, ist für keine Zahl $\frac{r}{n}$ mit $0 \leq \frac{r}{n} \leq 1$ Null.

Wir können (b) als eine Forderung der Vorurteilsfreibeit bzgl. der zu erwartenden Grenzwerte relativen Häufigkeiten der F-Objekte ansehen. ${ }^{38}$ Eine Vertauschbarkeit von $\mathrm{F}$ nehmen wir z. B. beim wiederholten Werfen einer Münze an. Besagt $\left(F\left(a_{i}\right)\right.$, daß sich beim i-ten Wurf mit der Münze „Kopf“ ergibt, so werden wir w so ansetzen, daß Vertauschbarkeit dieser Ereignisse besteht, d. h. wir werden es als ebenso wahrscheinlich ansehen, daß bei $n$ Würfen $r$ bestimmte Würfe Kopf ergeben, wie daß $r$ andere Würfe Kopf ergeben. In diesem Fall werden wir, solange wir nicht schon eine feste Meinung haben, welcher Prozentsatz. von Würfen auf lange Sicht „Kopf“ ergeben wird, auch die Regularitätsvoraussetzung machen. Und beides bewirkt, daß wir nach einer größeren Zahl $\mathrm{n}$ von Würfen, von denen $\mathrm{r}$ Kopf ergeben haben, mit großer Sicherheit annehmen werden, daß der Grenzwert der relativen Häufigkeiten bei unbegrenztem Fortsetzen der Würfe (die objektive Wahrscheinlichkeit von ,Kopf") nahe bei $\frac{\mathrm{r}}{\mathrm{n}}$ liegen wird.

Das Prinzip IP besagt, daß wir bzgl. der F-Ereignisse aus der Erfahrung lernen: Der Grad unserer Erwartung, daß das nächste, noch nicht geprüfte Objekt $a_{n+1}$, die Eigenschaft $F$ haben wird, wächst oder sinkt gegenüber der unbedingten oder apriori-Wahrscheinlichkeit $w\left(F\left(a_{n}+1\right)\right)$ für dieses Ereignis mit der beobachteten relativen Häufigkeit $\frac{r}{n}$ von $F$ in der Reihe $a_{1}, \ldots, a_{n}$ der bisher auf diese Eigenschaft hin geprüften Objekte. Ein solches Prinzip des Lernens aus der Erfahrung gilt nun keineswegs allgemein, d.h. die Bedingungen (a) und (b) sind nicht immer erfüllt, und die Forderung, nur solche apriori-Bewertungen $\mathrm{w}$ als ,,rational“ zuzulassen, für die sie für alle Eigenschaften F erfüllt sind - wie sie R. Carnap in seiner induktiven

${ }^{38}$ Auch unter Bezugnahme auf Wetten kann man die Regularitätsbedingung als Rationalitätsforderung begründen; vgl. Shimony (55), Kemeny (55) und Lehmann (55), sowie die Darstellung in Kutschera (72), 2.1.4. 
Logik aufgestellt hat ${ }^{39}$-, würde zu Widersprüchen führen. Für die Bedingung (a) ergibt sich das z. B. aus N. Goodmans ,Neuem Rätsel der Induktion““. ${ }^{40}$ Dieses ,,Rätsel“ geht von der traditionellen Formulierung induktiver Prinzipien aus, in der sie Schlüsse der Form sind:

1. Wenn $F\left(a_{1}\right)$ und $\ldots$ und $F\left(a_{n}\right)$, dann $F\left(a_{n}+1\right)$, oder

2. Wenn $F\left(a_{1}\right)$ und $\ldots$ und $F\left(a_{n}\right)$, dann $\wedge x F(x){ }^{41}$

Dabei sei $n$ eine nicht näher spezifizierte aber jedenfalls hinreichend große Zahl von Objekten $a_{1}, \ldots, a_{n}$, an denen bereits die Eigenschaft $F$ beobachtet wurde, und es wird vorausgesetzt, daß keine anderen Objekte, insbesondere nicht $a_{n+1}$, auf diese Eigenschaft hin untersucht worden sind. Läßt man nun z. B. in (1) für $F$ beliebige Eigenschaften zu, so ergibt sich nach Goodman folgender Widerspruch: $\mathrm{Zu}$ einer bestimmten Eigenschaft $\mathrm{F}$ läßt sich immer ein ,pathologisches“ Gegenstück F' definieren durch $F^{\prime}(x):=\left(x=a_{1} \vee \ldots \vee x=a_{n}\right) \wedge$ $F(x) \vee x \neq a_{1} \wedge \ldots \wedge x \neq a_{n} \wedge \neg F(x)$. F' soll also von den Objekten $a_{1}, \ldots, a_{n}$ genau auf jene zutreffen, auf die auch $F$ zutrifft, von den anderen Objekten aber genau auf jene, auf die F nicht zutrifft. Dann gilt aufgrund der Beobachtungen $F\left(a_{1}\right), \ldots, F\left(a_{n}\right)$ nach (1) $F\left(a_{n+1}\right)$. Es gilt aber auch $F^{\prime}\left(a_{1}\right), \ldots, F^{\prime}\left(a_{n}\right)$, also nach (1) $F^{\prime}\left(a_{n+1}\right)$, d. h. aber $\neg \mathrm{F}\left(\mathrm{a}_{\mathrm{n}+1}\right)$. Hat man $\mathrm{z}$. B. von allen bis heute auf ihre Farbe hin untersuchten Smaragden festgestellt, daß sie grün sind, so ergibt sich nach (1): Der erste morgen untersuchte Smaragd wird auch grün sein. Definiert man nun das pathologische Prädikat ,grot" durch „,bis heute auf die Farbe hin untersuchtes und grünes Objekt, oder bis heute noch nicht auf die Farbe hin untersuchtes und rotes Objekt", so folgt aus (1), da alle bisher untersuchten Smaragden auch grot waren, daß der erste morgen untersuchte Smaragd ebenfalls grot, d. h. aber rot sein wird.

39 Vgl. dazu Carnap (50), (52) und Carnap und Stegmüller (59), sowie die Darstellung und Kritik in Kutschera (72), 2.3.

${ }^{40}$ Vgl. dazu Goodman (55). Zur Kritik des Goodmanschen Lösungsversuchs für dieses Rätsel vgl. Kutschera (78).

${ }^{41}$ Daneben betrachtet man auch oft Schlüsse der Form: Wenn die relative Häufigkeit der Eigenschaft $F$ unter $n$ Objekten $r / n$ ist, so liegt die objektive Wahrscheinlichkeit von $F$ nahe bei dieser Zahl. Gegen diesen Schluß ergeben sich, wic ebenfalls schon Hume betor.t hat, dieselben Einvände wie gegen die beiden anderen. 
Wir wollen hier nicht die allesamt erfolglosen Bemühungen zur Unterscheidung von pathologischen und normalen Prädikaten referieren. ${ }^{42}$ Sofern sie das Ziel verfolgen, die Prinzipien (1) und (2) jedenfalls in Beschränkung auf normale Prädikate zu rechtfertigen, sind sie ohnehin vergeblich, da diese Prinzipien auch für normale Prädikate nicht gelten. Aus der Beobachtung noch so vieler schwarzer Raben folgt eben nicht, daß alle Raben schwarz sind; schon der nächste Rabe kann weiß sein. Von einer Folgebeziehung kann man aber nur reden, wo Wahrheit von den Prämissen auf die Konklusion vererbt wird. Es kann also von vornherein nur um die Frage gehen, für welche Prädikate das Prinzip IP gilt, für welche Prädikate wir also insbesondere Vertauschbarkeit annehmen und warum. Nach Goodmans Argument kann man das sicher nicht für alle Prädikate annehmen, für normale wie für ihre pathologischen Gegenstücke.

Bevor wir auf diese Frage eingehen, sei noch erwähnt, daß im Gegensatz zu einer verbreiteten Meinung auch generelle Sätze wie $\wedge \mathrm{xF}(\mathrm{x})$ induktiv bestätigungsfähig sind. Geht man im Fall des Münzwurfs z. B. von einer Wahrscheinlichkeit $w$ aus, für die gilt $\mathrm{w}(\wedge \mathrm{x} \neg \mathrm{Fx})=\mathrm{w}(\wedge \mathrm{xF}(\mathrm{x}))=\frac{1}{3}$, und ist auch die Wahrscheinlichkeit, daß die relative Häufigkeit von $F$ zwischen $O$ und 1 liegt, $\$$ (mit gleicher Wahrscheinlichkeit für jede solche statistische Hypothese), so besteht Vertauschbarkeit und Regularität, und $w\left(\wedge x F x, F_{n}\right)$ konvergiert schnell gegen $1 .^{43}$ Damit kann man der induktiven Praxis Rechnung tragen, in der oft die Annahme genereller Aussagen mit dem Aufweis einiger positiver Fälle begründet wird. Wird z. B. eine neue zoologische Spezies $S$ entdeckt und stellt man an einigen Exemplaren $a_{1}, \ldots, a_{n}$ fest, daß sie ein Merkmal $F$ haben, so wird oft damit schon die Hypothese als gut gesichert akzeptiert, daß alle Exemplare der Spezies $S$ die Eigenschaft $F$ haben. Voraussetzung ist dabei, daß man dieser Hypothese eine Anfangswahrscheinlichkeit größer Null zuord-

${ }^{42}$ Vgl. dazu Goodman (55), sowie Kutschera (72), 2.3.3.

${ }^{43}$ Das Beispiel geben Carnap und Jeffrey in (71), $205 \mathrm{ff}$. an. Solche Verteilungen sind nach den ursprünglichen Axiomen für die logische Wahrscheinlichkeit von Carnap ausgeschlossen. Daher hat Carnap zunächst den Bestätigungsgrad der generellen Hypothese $\wedge \mathrm{xF}(\mathrm{x})$ aufgrund von $\mathrm{F}^{\mathrm{n}_{n}}$ durch $w\left(F_{n+1}, F_{n}\right)$ bestimmt. Später hat er aber jene Axiome aufgegeben, die über jene der intersubjektiven Wahrscheinlichkeitstheorie hinausgehen, mit Ausnahme einer Regularitätsforderung. 
net. In vielen Fällen wird man auch schon davon ausgehen, daß entweder alle Exemplare der Spezies das Merkmal F haben oder keines; d. h. man wird von einer Bewertung w ausgehen, für die nur diese beiden Aussagen positive Wahrscheinlichkeit haben (die also nicht mehr iegulär isì). Dann genügi dic Ütëpr̈üfung sines ainziger Exemplars, um eine der beiden Annahmen zu bestätigen. Das ist dann gerechtfertigt, wenn F z. B. ein Merkmal des Körperbaus ist, dessen Vorhandensein oder Fehlen man als generelles Charakteristikum der Spezies $\mathrm{S}$ ansieht, so daß man, falls sich herausstellen sollte, daß nur einige Exemplare von $S$ die Eigenschaft $F$ haben, andere dagegen nicht, zwei verschiedene Spezies unterscheiden, also die Fragestellung ändern würde.

Induktive Begründungen hängen sehr stark von apriori-Wahrscheinlichkeiten ab. Das Induktionsprinzip IP besagt, daß unter den Bedingungen (a) und (b) die aufgrund von Beobachtungen modifizierten (bedingten) Wahrscheinlichkeiten gegen den Grenzwert der beobachteten relativen Häufigkeiten konvergieren. Damit wird der Einfluß der apriori-Bewertung zusehends schwächer. Gehen also zwei Personen von verschiedenen apriori-Bewertungen $\mathrm{w}_{1}$ und $\mathrm{w}_{2}$ aus und machen sie dieselben Beobachtungen, so werden sich ihre durch diese Beobachtungen bedingten Wahrscheinlichkeitsannahmen immer weniger unterscheiden; beide lernen also dasselbe aus gieicher Erfahrung. Voraussetzung dafür ist aber, $\mathrm{da} \beta \mathrm{w}_{1}$ und $\mathrm{w}_{2}$ die Bedingungen (a) und (b) erfüllen. Für die induktive Praxis spielen daher diese Bedingungen eine wichtige Rolle. ${ }^{44}$ (b) kann man, wie wir sahen, als Forderung der Vorurteilsfreiheit ansehen, also jedenfalls in vielen Fällen als Rationalitätsforderung rechtfertigen. Die Frage ist, ob man auch (a) begründen kann. Sätze über die Vertauschbarkeit einer Eigenschaft bzgl. einer subjektiven Wahrscheinlichkeitsbewertung sind nun sicher keine analytischen Sätze. Vertauschbarkeitsannahmen lassen sich aber auch nicht durch Beobachtungen begründen. Da in ihnen der Parameter w vorkornmt, der in Beobachtungssätzen nicht enthalten ist, folgen sie

${ }^{44}$ Am Beispiel vom Merkmal des Körperbaus einer Spezies haben wir gesehen, $\mathrm{daß}$ diese Bedingungen nicht notwendig sind, um zu einer Konvergenz der Annahmen zu gelangen. Sie sind aber allgemeine hinreichende Bedingungen, und daher beziehen wir uns bei der Diskussion der Frage, ob sich aprioriBewertungen begründen lassen, auf diese Bedingungen. 
nicht logisch aus Beobachtungssätzen, und durch Beobachtungssätze werden auch keine Wahrscheinlichkeitsaussagen induktiv legitimieri. Mar: kann nur mit Beobachtungsaussagen und apriori-Bewertungen andere Aussagen induktiv begründen, die nicht von Wahrscheinlichkeiten handeln. ${ }^{45}$ Apriori-Annahmen sind eine Voraussetzung, nicht ein. Ergebnis eines Lernens aus der Erfahrung. Das heißt natürlich nicht, daß wir uns bei solchen Annahmen nicht irgendwie auf Erfahrungen stützen, und daß wir sie nicht auch angesichts neuer Erfahrungen modifizieren könnten. Sie sind aber durch Beobachtungen weder verifizierbar noch falsifizierbar, hängen also nur indirekt und in einer sehr lockeren Weise mit Erfahrung zusammen. Wenn wir z. B. in einer längeren Reihe von Münzwürfen feststellen, daß auf „Kopf“ immer "Wappen" folgt und umgekehrt, so werden wir unsere ursprüngliche Vertauschbarkeitsannahme aufgeben und annehmen, daß hier eine Gesetzmäßigkeit vorliegt, nach der jedes Ergebnis das folgende determiniert; wir kommen dann zu anderen, in diesem Fall sehr viel spezielleren Erwartungen, nämlich nicht zu einer Wahrscheinlichkeit $\frac{1}{2}$ für das Ergebnis „Kopf“ beim nächsten Wurf, sondern zu einer Wahrscheinlichkeit 1 für „Kopf“, wenn der letzte Wurf „Wappen“ ergeben hat. Diese Modifikation ist aber nicht zwingend, denn es könnte sich bei der bisherigen Regularität um einen Zufall handeln. Es gibt also keine Folge von Ergebnissen, die uns zwingt, die Vertauschbarkeit aufzugeben und unsere apriori-Bewertung $\mathrm{zu}$ ändern. Man kann auch nicht behaupten, daß wir unsere apriori-Bewertungen nach der trial-and-error-Methode bilden: daß wir mit irgendeiner solchen Bewertung beginnen und damit aufgrund von Beobachtungen zu Voraussagen kommen und, wenn sich diese Voraussagen nicht bestätigen, die Bewertung zugunsten einer anderen aufgeben. Eine Beobachtung $A$ ist mit keiner subjektiven Wahrscheinlichkeitsaussage $w(A)=r$ unverträglich. Auch hohe Wahrscheinlichkeiten stellen keine Wahrheitsgarantie dar. Es gibt also keine rationalen Kriterien für die Aus-

${ }^{45}$ Demgegenüber lassen sich statistische Hypothesen als Sätze über objektive Wahrscheinlichkeiten durch Beobachtungen mithilfe subjektiver aprioriWahrscheinlichkeiten induktiv bestätigen. Es gilt unter den Bedingungen (a) und (b) auch, daß die Wahrscheinlichkeit dafür, daß der Grenzwert der relativen Häufigkeit von $F$ nahe bei $r / n$ liegt, unter der Bedingung, daß diese relative Häufigkeit bei den $\mathrm{n}$ bisher untersuchten Objekten festgestellt wurde, für große Zahlen n gegen 1 geht. 
wahl von apriori-Bewertungen. Das haben auch die Diskussionen über Kriterien zur Abgrenzung normaler von pathologischen Prädikaten im Zusammenhang mit Goodmans Rätsel der Induktion bestätigt sowie die Probleme, denen Carnap mit dem Versuch begegnet ist, ationale Kriterien für Wah.rscheinlichkeiten anzugeben, die über jene der Theorie subjektiver Wahrscheinlichkeiten hinausgehen.

Woher kommen apriori-Bewertungen? Wenn wir so fragen, dürfen wir darunter, wie schon betont wurde, sicher nicht nur Wahrscheinlichkeitsfunktionen verstehen, welche die Bezugsperson explizit angeben kann, sondern Erwartungen oder Erwartungshaltungen, die sich erst dort, wo sie hinreichend ausgeprägt und spezifiziert sind, durch solche Funktionen beschreiben lassen. Solche Erwartungen liegen allen induktiven Prozessen, allem Lernen aus der Erfahrung, also insbesondere auch dem Erlernen von Sprache zugrunde. Wie wir uns in 9.1 genötigt sahen, eine angeborene Abstraktionsfähigkeit anzunehmer, so wird man hier auch angeborene Erwartungshaltungen anzunehmen haben. Unser Lernen aus der Erfahrung hat also letztlich biologische Grundlagen. ${ }^{46}$ Auch hier geht also Vernunft von Voraussetzungen aus, die jenseits rationaler Begründbarkeit liegen. Daß aber auch dieses biologische „Apriori“ unsere rationale Tätigkeit weniger begrenzt als ermöglicht, ergibt sich daraus, daß unsere Erwartungen ihrerseits durch unsere Erfahrungen bestimmt werden - nicht nur durch jene, die wir selber machen, sondern auch durch die, welche uns mit der Weltansicht jener Kultur vermittelt werden, in die wir hineinwachsen.

Die Funktion der apriori-Annahmen kann man sich durch foigende Überlegung verdeutlichen: Unsere Wahrscheinlichkeitsannahmen haben nicht nur eine theoretische, sondern eine eminent praktische Bedeutung. Sie bestimmen zusammen mit unseren Präferenzen unser (rationales) Verhalten. ${ }^{47}$ Ist das Verhalten eines Organismus $\mathrm{O}_{1}$ so programmiert, daß er auf bestimmte Umweltreize (Sinnesreize) mit bestimmten Reaktionen antwortet, also feste Verhaltensdispositionen hat, so kann man auch sagen, ihm seien bestimmte "Überzeugungen“ und „Präferenzen“ angeboren - speziell Überzeugungen darüber, welche Umstände im Fall gewisser Beobachtungen bestehen oder eintreten werden - , die zusammen diese Dispositionen ergeben. Dieser

${ }^{46}$ Das beronen auch Lorenz in (73), Riedl in (79) und Popper in (72), $258 \mathrm{f}$. 47 Vgl. dazu den Abschnitt 2.4. 
Organismus ist nur dann seiner Umwelt angepaßt, wenn seine Reaktionen unter den entsprechenden Reizbedingungen für ihn zweckmäBig sind, d. h. wenn seine „Überzeugungen“ richtig sind. Einer veränderten Umwelt, in der die fest vorprogrammierten Reaktionen unter den Reizbedingungen nicht mehr zweckmäßig sind, kann er sich nicht mehr anpassen. Demgegenüber ist ein Organismus $\mathrm{O}_{2}$, dessen Reaktionen nicht fest mit Reizen verbunden sind, sondern der aus Erfahrung lernen kann, wesentlich anpassungsfähiger. Er bildet aufgrund von Beobachtungen gewisse Verhaltensdispositionen aus. In ihm sind also nur Dispositionen 2. Stufe oder Propensitäten eingebaut, aufgrund von gewissen Beobachtungen gewisse Verhaltensdispositionen auszubilden, nach denen er dann auf gewisse Reize in bestimmter Weise reagiert. Diese Propensitäten lassen sich - feste Präferenzen vorausgesetzt - als apriori-Wahrscheinlichkeiten darstellen. Sie führen aufgrund vergangener Beobachtungen zu (bedingten) Wahrscheinlichkeiten, die zusammen mit den Präferenzen das Verhalten bestimmen. Durch Beobachtungen bildet sich z. B. die Erwartung, daß eine gewisse Verhaltensweise unter gewissen beobachtungsmäßig feststellbaren Bedingungen optimal ist im Sinne der eigenen Präferenzen. Dieser Organismus $\mathrm{O}_{2}$ bildet also seine Verhaltensdispositionen erst aufgrund von Erfahrungen aus; er lernt aus Erfahrung, wie er sich zweckmäßigerweise zu verhalten hat. Damit ist sein Verhalten weniger sicher - Lernprozesse dauern eine gewisse Zeit - , aber er kann sich andererseits variierenden Umweltbedingungen anpassen. Den Menschen müssen wir in diesem Modell als einen Organismus $\mathrm{O}_{3}$ auffassen, dem nicht einmal feste Propensitäten angeboren sind, sondern der diese Propensitäten verändern kann. Das bewirkt einerseits eine abermals erhöhte Unsicherheit im Verhalten und eine noch losere Verbindung zwischen Beobachtungen und Überzeugungen, andererseits aber eine noch bessere Anpassungsfähigkeit und eine wesentlich erhöhte Lernfähigkeit. Wir haben ja schon oben gesehen, daß man je nach apriori-Bewertung schneller oder langsamer aus der Erfahrung lernen kann, und daß gewisse Beobachtungen es nahelegen, die ursprüngliche apriori-Bewertung zugunsten anderer aufzugeben, die genauere Informationen liefern. Das Induktionsproblem, wie wir gegebene Erfahrungen extrapolieren sollen, ist in dieser Sicht der Preis, den wir für eine prinzipiell unbegrenzte Fähigkeit der Anpassung unserer Überzeugungen und unseres Verhaltens an die Umwelt bezahlen. 
Man kann die Bewertungen, von denen wir ausgehen, als Ergebnis eines langen und komplexen Prozesses der Adaption an die Umwelt deuten. Dieser Prozeß ist ein kultureller Prozeß, in den der einzelne eintritt, und dessen Ergebnis er übernimmt, der aber durch individuelle und gemeinsrhaftliche Tärigkeit fortgesetzt vird. Jeder von uns wird in eine Sprache und eine Weltansicht hineingeboren; beides ist aber nicht unveränderlich, sondern wird laufend fortentwickelt. Entscheidend ist dabei, daß wir aus den Erfahrungen anderer lernen können, daß wir nicht alle Erfahrungen selber machen müssen, sondern sie uns mitteilen lassen können, und daß zweckmäßige Bewertungen, die zweckmäßige Art aus Erfahrung zu lernen, nicht genetisch, sondern durch Informationen übermittelt werden. Erfahrung ergibt sich aus unserem Erleben, das wir im Licht von Vor-Urteilen interpretieren; es handelt sich um den Prozeß einer wechselseitigen Adaption von Erleben und vorgängigen Annahmen mit dem Ziel einer erfolgreichen praktischen wie theoretischen Orientierung in unserem Verhalten. Obwohl also Erleben Grundlage unserer Aussagen über die Welt ist, ist es doch nicht alleinige Grundlage. Wir gewinnen neue Annahmen nicht nur aus unmittelbarem Erleben, sondern wir deuten und extrapolieren dieses Erleben schon immer im Sinn von Erwartungen oder Vorentwürfen; diese fungieren dabei nicht als unveränderliche Gegaberiheiteil, sonderiı wercien ihrerseiis aufyrund von Beobachtungen modifiziert.

Wir haben gesehen, daß sich auch (wesentlich) generelle Aussagen durch Beobachtungen induktiv begründen lassen. Wie steht es aber mit gesetzesartigen Aussagen? Einfache Aussagen dieser Art hab $\in \mathrm{n}$ wir in 2.2 als generelle Konditionalsätze charakterisiert und haben betont, daß nicht jede (wesentlich) generelle Aussage der Gestalt $\wedge \mathrm{x}(\mathrm{F}(\mathrm{x}) \supset \mathrm{G}(\mathrm{x}))$ den generellen Konditionalsatz $\wedge \mathrm{x}(\mathrm{F}(\mathrm{x}) \rightarrow \mathrm{G}(\mathrm{x}))$ impliziert. Eine induktive Begründbarkeit gesetzesartiger Aussagen durch Beobachtungen kann man nun nicht annehmen, auch nicht bei der oben erwähriten epistemischen Interpretation von Konditionalaussagen. Beobachtungen zeigen uns immer nur, was in unserer Welt der Fall ist, und die Kenntnis von Sachverhalten, die in unserer Welt bestehen, bedingt nur Erwartungen bzgl. dessen, was in unserer Welt der Fall sein wird, nicht aber Annahmen über das, was unter Bedingungen der Fall wäre, von denen wir wissen, daß sie nicht erfüllt sind. Beobachten wir, daß bei allen bisher untersuchten Objekten $a_{1}, \ldots, a_{n}$ gilt $F\left(a_{i}\right) \supset H\left(a_{i}\right)(i=1, \ldots, n)$, so können darunter $z$. B. Objekte $a_{i}$ 
sein, für die gilt $\neg \mathrm{F}\left(\mathrm{a}_{\mathrm{i}}\right) \wedge \neg \mathrm{H}\left(\mathrm{a}_{\mathrm{i}}\right)$. Die Überzeugung, daß das gilt, impliziert aber nicht, daß wir annehmen, daß $a_{i}$ die Eigenschaft $H$ haben würde, falls $a_{i}$ die Eigenschaft $F$ hätte. Aus den Annahmen über unsere Welt ergeben sich nur Annahmen über das, was unter Bedingungen gilt, die wir als in unserer Welt möglicherweise erfüllt ansehen. ${ }^{48} \mathrm{Da}$ man Konditionalsätze als empirisch ansehen wird - man kann Aussagen über Dispositionen und die gesetzesartigen Hypothesen der Naturwissenschaften kaum sämtlich als ,nichtempirisch“ bezeichnen - gibt es also empirische Sätze, die sich weder deduktiv noch induktiv durch Beobachtungen begründen lassen.

$\mathrm{Da}$ das auch für manche theoretischen Aussagen gilt, ${ }^{49}$ stellt sich die Frage, ob es nicht andere Formen der Überprüfung empirischer Aussagen durch Beobachtungen gibt als deduktive oder induktive Begründungen. Die gibt es sicher. In der wissenschaftstheoretischen $\mathrm{Li}$ teratur werden verschiedene Formen einer Bestätigung von Gesetzesaussagen oder Theorien durch Beobachtungssätze diskutiert, die weder deduktive noch induktive Begründungen darstellen. Nun muß man aber sagen: Ergibt die Bestätigung einer Hypothese durch Beobachtungen weder, daß sie wahr ist, noch daß sie aufgrund dieser Beobachtungen auch nur wahrscheinlicher ist als vorher, liefert sie also keine Begründung der Hypothese, so ist sie für unser Problem einer Begründbarkeit von Hypothesen durch Beobachtungen ohne Interesse; ist Bestätigung aber eine Form der Begründung, so muß sie eine deduktive oder induktive Begründung liefern. ${ }^{50}$

Wir wollen das anhand des Bewährungsbegriffs von K. Popper verdeutlichen, der in der gegenwärtigen wissenschaftstheoretischen Diskussion eine große Rolle spielt. ${ }^{51}$ Popper entwickelt folgende metho-

${ }^{48}$ Mit einer Wahrscheinlichkeit $w$ lassen sich nur solche bedingten Wahrscheinlichkeiten $w(A, B)$ bestimmen, für die $w(A)>0$ ist. Im Fall des bei der epistemischen Interpretation von Konditionalsätzen verwendeten bedingten Glaubensbegriffe gilt Entsprechendes. Vgl. dazu Kutschera (76), 4.3.

${ }^{49} \mathrm{Vgl}$. dazu den nächsten Abschnitt.

${ }^{50}$ Für eine Darstellung der verschiedenen Begriffe der Bestätigung vgl. z. B. Hempel (65), Kap. I, Kutschera (72), Kap. 5 oder Lenzen (73) und die dort angegebene Literatur.

51 Vgl. dazu vor allem Popper (66), und den Aufsatz „Conjectural Knowledge: My solution of the problem of induction" in (72). 
dologische Konzeption: Die wissenschaftliche Arbeit beginnt nicht mit Beobachtungen, sondern mit Hypothesen. Hypothesen ergeben sich nicht aus Beobachtungen, sondern sie stellen Fragen an die Natur dar, die erst systematische Erfahrung ermöglichen: Beobachtungen ohne Hypothesen sind blind, erst eine Hypnthese ermöglicht sinnvoll organisierte Experimente. ${ }^{52}$ „Hypothesen sind Netze: nur der wird fangen, der auswirft“ zitiert Popper Novalis als Motto seiner „Logik der Forschung". Hypothesen werden natürlich auf dem Hintergrund unseres Erfahrungswissens gebildet, aber sie sind kreative Entwürfe, schöpferische Einfälle, die weit über das hinausreichen, was wir schon wissen. ${ }^{53}$ Falls mehrere konkurrierende Hypothesen zur Auswahl stehen, soll man sich nach Popper an die informativste halten ${ }^{54}-$ an die unwahrscheinlichste, denn ein Satz ist um so informativer, je geringer seine Wahrscheinlichkeit ist; er geht um so weiter über das hinaus, was wir ohnehin schon annehmen, je unwahrscheinlicher er ist. Die informativsten Hypothesen, die präzisesten und am weitesten reichenden sind zugleich die für uns interessantesten wie die am leichtesten falsifizierbaren. Diese Hypothese wird dann strengen Tests unterworfen, in dem man aus ihr möglichst informative Voraussagen ableitet und diese dann durch Beobachtung überprüft. Besteht die $\mathrm{Hy}-$ pothese den Test, erweist sich die Voraussage als richtig, so bewährt sie sich an dieser Voraussage. Daraus fulyt nicht, daß sie wahe ist, ja nach Popper nicht einmal, daß sie wahrscheinlich ist. Wir halten aber solange an ihr fest, d. h. wir unterziehen sie immer weiteren strengen Tests, bis sie faisifiziert worden ist. Der Erkenntrisfortschritt besteht für Popper in der Falsifikation von Hypothesen, darin, daß wir erfahren, was nicht der Fall ist. Wir lernen aus unseren Fehlern, und, so zitiert Popper John Archibald Wheeler als Motto zu den „Conjectures and Refutations", ,Our whole problem is to make the mistakes as fast as possible". 55

52 Vgl. dazu Popper (66), S. 19. - Popper sagt: „Beobachtung ist stets Beobachtung im Lichte von Theorien“. ((66), 31, Anmerkung 1.) Und: „Die Natur antwortet nicht, wenn sie nicht gefragt wird" $((66), 225)$.

${ }^{53}$ So äußert sich auch Carnap in (50), $192 \mathrm{f}$. und in (46a), 520.

${ }^{54}$ Vgl. Popper (66), 373.

${ }^{55}$ Popper zitiert dort auch Oscar Wilde: ,Experience is the name everyone gives to his mistakes". 
Popper behauptet also nicht, eine Theorie - habe sie sich auch an noch so vielen Beobachtungen bewährt - sei aufgrund dieser Beobachtungen in irgendeinem Sinn gut begründet oder wahrscheinlich. In welchem Sinn dient dann aber das ganze Unternehmen dem Erkenntnisfortschritt? Es nützt uns nichts, immer nur zu erfahren, was nicht der Fall ist. Insbesondere nützt es uns nichts, zu erfahren, daß die Hypothesen falsch sind, die wir nach Popper für Falsifikationszwecke auswählen sollen, denn für sie ist es ja von vornherein unwahrscheinlich, daß sie gelten. Und ihre Falschheit ist nach Popper eine logische Folge von Beobachtungen, die wir machen; neue Informationen erhalten wir also nur durch Beobachtungen. Warum also überhaupt $\mathrm{Hy}-$ pothesen und Theorien entwerfen, wenn sie sich doch allenfalls als falsch erweisen können und der Erkenntnisfortschritt nur in neuen Beobachtungen besteht? Theorien wären dann nur als Anleitungen zu Beobachtungen nützlich, hätten aber als Hypothesen, die wir als unwahrscheinlich ansehen, kaum kognitiven Wert. Um das methodologische Verfahren, das er vorschlägt, doch als Weg zu erweisen, auf dem sich ein Erkenntnisfortschritt vollzieht, benützt Popper den Begriff der Wabrheitsäbnlichkeit (verisimilitude) und behauptet, unsere Hypothesen näherten sich bei Befolgung seiner methodologischen Regeln immer mehr der Wahrheit an, wenn sich die volle Wahrheit vielleicht auch nie erreichen ließe. Den Begriff der Wahrheitsähnlichkeit definiert er so: ${ }^{56}$ Der Wahrheitsgehalt $G_{W}(A)$ eines Satzes A ist die Menge aller wahren Sätze, die aus A folgen, der Falschheitsgehalt $\mathrm{G}_{\mathrm{F}}(\mathrm{A})$ die Menge der falschen Sätze, die aus A folgen. A soll nun wabrbeitsäbnlicher sein als $B$ genau dann, wenn gilt: $G_{W}(B)$ ist echt in $G_{W}(A)$ enthalten, aber nicht $G_{F}(B)$ in $G_{F}(A)$, oder $G_{F}(A)$ ist echt in $G_{F}(B)$ enthalten, aber nicht $G_{W}(A)$ in $G_{W}(B)$. Es sei nun $H_{1}$ eine Hypothese, die sich als falsch erwiesen hat durch die Beobachtung, daß

$56 \mathrm{Vgl}$. dazu Popper (63), 233. Auf den im Anhang eingeführten quantitativen Begriff der Wahrheitsähnlichkeit gehen wir hier nicht ein. Popper ordnet dort Satzmengen (oder Propositionen) mithilfe eines Wahrscheinlichkeitsmaßes einen Gehalt zu. Dieses Maß wird aber nicht definiert, sondern es werden nur Bedingungen (Wahrscheinlichkeitsaxiome) angegeben, die solche Maße erfüllen sollen. Nun gibt es aber ganz verschiedene solche Maße, und je nachdem, welches man wählt, ergeben sich verschiedene komparative Aussagen über den Gehalt und damit über die Wahrheitsähnlichkeit zweier Hypothesen. 
ein Satz $\mathrm{E}$, der aus $\mathrm{H}$ folgt, nicht gilt. $\mathrm{H}_{2}$ kommt als neue Hypothese nach Popper nur dann infrage, wenn (a) $\mathrm{H}_{2}$ das leistet, was die alte Hypothese $\mathrm{H}_{1}$ leistete - wenn nämlich die als richtig erwiesenen Voraussagen mit $\mathrm{H}_{1}$ sich auch mit $\mathrm{H}_{2}$ machen lassen - , und wenn (b) $\mathrm{H}_{2}$ $\neg$ E richtig voraussagt. $\left(\mathrm{H}_{2}\right.$ scll darüber hinaus veitere Voraussagen erlauben, die noch nicht getestet wurden, und sich an einigen davon bewähren.) Aus (b) folgt nun, daß $\mathrm{G}_{\mathrm{F}}\left(\mathrm{H}_{1}\right)$ nicht in $\mathrm{G}_{\mathrm{F}}\left(\mathrm{H}_{2}\right)$ enthalten ist - der falsche Satz $E$ ist ja Element von $G_{F}\left(H_{1}\right)$, aber nicht von $\mathrm{G}_{\mathrm{F}}\left(\mathrm{H}_{2}\right)$. Aus (a) und (b) folgt jedoch nicht, daß $\mathrm{G}_{W}\left(\mathrm{H}_{1}\right)$ echt in $\mathrm{G}_{\mathrm{W}}\left(\mathrm{H}_{2}\right)$ enthalten ist. Der wahre Satz $\neg \mathrm{E}-$ und evtl. weitere wahre Sätze - sind zwar in $G_{W}\left(H_{2}\right)$ enthalten und nicht in $G_{W}\left(H_{1}\right)$, $\mathrm{G}_{\mathrm{W}}\left(\mathrm{H}_{1}\right)$ kann aber bisher nicht getestete Voraussagen enthalten, die aus $\mathrm{H}_{2}$ nicht folgen. Poppers Vorschrift für die Auswahl von Hypothesen, die falsifizierte ersetzen können, garantiert also keinen Fortschritt der Wahrheitsähnlichkeit. Wir können darüber solange nicht urteilen, als wir nicht genau wissen, welche der aus $\mathrm{H}_{1}$ folgenden Sätze wahr sind.

Das Bild, das Popper vom Vorgehen der Wissenschaften zeichnet, ist nicht falsch, aber zumindest unvollständig. Es ist zweifellos richtig, daß Hypothesen oder Theorien kreative Entwürfe sind, die wir dadurch mit der Erfahrung konfrontieren, daß wir ihre Voraussagen durch Beobachtungen und Experimente überprüfen, und daßs wir uns vor allem für informative, für präzise und allgemeine Theorien interessieren. Es gibt, wie wir sahen, empirische Hypothesen, die sich durch Beobachtungen nicht begründen lassen, für deren Annahme Beobachtungen also nicht hinreichend, sondern nur notwendig sind. Es ist aber zu bemerken:

1. Nicht aus allen Hypothesen lassen sich Beobachtungsaussagen ableiten, auch dann nicht, wenn man weitere Annahmen hinzunimmt sofern natürlich die fraglichen Beobachtungsaussagen nicht schon aus diesen Annahmen selbst folgen. So haben wir schon früher bemerkt, daß Sätze, in denen All- und Existenzaussagen miteinander kombiniert sind, durch Beobachtungssätze nicht falsifizierbar sind. Eine große Klasse von naturwissenschaftlichen Hypothesen hat aber diese Gestalt. Das gilt insbesondere für alle Aussagen über Grenzwerte, also für. Differentialgleichungen und für statistische Hypothesen. Diese sind mit allen Beobachtungsreihen verträglich, können also an ihnen nicht scheitern. Betrachten wir z. B. den Satz: „,Die Halbwertszeit von Radium beträgt 1620 Jahre“, d.h. „Die objektive Wahr- 
scheinlichkeit dafür, daß ein Radiumatom in 1620 Jahren zerfällt, ist 1/2“. Die objektive Wahrscheinlichkeit eines Ereignistyps F, z. B. von „Kopf" bei den Würfen mit einer Münze, wird definiert als Grenzwert der relativen Häufigkeiten dieses Ereignistyps in einer unendlichen Reihe von (physikalisch unabhängigen) Wiederholungen eines Experiments, im Beispiel also als Grenzwert der Folge der relativen Häufigkeiten von „Kopf“ in n Würfen mit der Münze. Der Grenzwert einer Folge ist aber unabhängig von ihrem Verhalten in endlichen Anfangsabschnitten. Daher ist eine statistische Hypothese durch endlich viele Beobachtungen nicht falsifizierbar. Wenn wir z. B. eine große Menge von Radiumatomen untersuchen und nach 1620 Jahren feststellen, daß nur $5 \%$ von ihnen zerfallen sind, so widerlegt das unsere Aussagen über die Halbwertszeit nicht, es macht sie nur sehr unwahrscheinlich. Und die Hypothesen, daß $50 \%$ aller Würfe mit der Münze Kopf ergeben, wird auch durch 10000 Würfe nicht widerlegt, die alle nur „,Wappen“ ergaben. Es gibt also generelle Hypothesen, aus denen kein einziger Beobachtungssatz logisch folgt.

2. Beobachtungssätze lassen sich meist nicht aus einzelnen wissenschaftlichen Hypothesen ableiten, sondern nur aus Satzsystemen. Darauf hat zuerst P. Duhem hingewiesen. ${ }^{57}$ Da dieser Gedanke in der gegenwärtigen wissenschaftstheoretischen Diskussion vor allem von W. V. Quine zur Geltung gebracht wurde, ${ }^{58}$ spricht man auch vom Dubem-Quine-Argument. Aus dem Boyle-Mariotteschen Gesetz über den Zusammenhang von Volumen und Druck einer Gasmenge folgt z. B. kein einziger Beobachtungssatz. Will man dieses Gesetz durch Messungen testen, so sind dafür Annahmen über die Meßinstrumente erforderlich, über ihre Funktionsweise und ihre Zuverlässigkeit. Es ist ferner anzunehmen, daß die Druckänderungen so langsam vor sich gehen, daß die Temperatur des Gases durch Wärmeaustausch mit der Umgebung konstant bleibt, usw. Wenn aber ein Beobachtungssatz $B$ aus Sätzen $A_{1}, \ldots, A_{n}$ folgt, so ergibt sich aus $\neg B$ nicht, daß ein bestimmter Satz $A_{i}(i=1, \ldots, n)$ falsch ist - in unserem Fall also die getestete Hypothese - , sondern aus $\neg \mathrm{B}$ folgt nur, daß nicht alle Sätze $A_{1}, \ldots, A_{n}$ zugleich wahr sein können, daß also mindestens einer dieser Sätze falsch ist. In der Wahl, welchen Satz $A_{i}$ wir aufgeben wollen,

57 Vgl. Duhem (14).

${ }^{58}$ Vgl. z. B. Quine (51) und (62), Vorwort. 
sind wir zunächst frei. Wenn ein System von Annahmen mit der Erfahrung in Konflikt gerät, so können wir es irgendwie so modifizieren, daß der Konflikt verschwindet. Wir können insbesondere immer an der fraglichen Hypothese festhalten und das korrekte Funktionieren der Meßinstrumente bezweifeln cder Siörfaktcron anliehrnen. ${ }^{59}$

$\mathrm{Zu}$ diesem Argument sagt Popper: ${ }^{60}$ Solche Annahmen über Störfaktoren sind nur dann zulässig, wenn sie keine ad-hoc-Annahmen darstellen, $d$. h. wenn sie sich nicht nur auf die Beobachtung stützen, daß B nicht gilt, sondern wenn sie sich unabhängig davon überprüfen lassen; wenn sich also mit ihnen neue Voraussagen machen lassen. Dazu ein Beispiel: Man konnte die Beobachtung, daß die UranusBahn keine Ellipse ist, nur deshalb durch die Annahme erklären, es gebe einen bisher noch unbeobachteten Planeten, der diese Abweichung bewirkt - und konnte so an der Newtonschen Mechanik festhalten -, weil sich diese Annahme überprüfen ließ - man entdeckie dann auch den Planeten - den Neptun - an der berechneten Stelle. In einem parallelen Fall erwies sich eine solche Annahme als unhaltbar: Die Bahn des Merkur bildet keine Ellipse, sondern eine Rosette. Das konnte man durch die Annahme einer nicht kugelsymmetrischen Masseverteilung in der Sonne erklären. Nachdem man dafür keine anderen Anhaltspunkte fand, dieses Phänomen sich aber mit der Relativitätstheorie erkiären liełs, sah $\operatorname{man}$ es ais Faisifikikation der Newtonschen Mechanik an. Popper betont ferner, daß nicht in jedem Kontext die Gesamtheit unserer Annahmen zur Debatte steht. Wir problematisieren bei jeder Untersuchung nur einzeine Annahmen. Ist aiso $B$ eine Folge der Hypothese $H$ und der Hintergrundannahmen $A_{1}, \ldots, A_{n}$, so falsifiziert die Beobachtung, daß $B$ falsch ist, keine der Annahmen $A_{1}, \ldots, A_{n}$, sondern $H$. Beide Einwände Poppers lösen das Problem aber nicht. Auch neue Hypothesen lassen sich nicht völlig unabhängig von anderen Annahmen prüfen. Bei ihnen stellt sich also dasselbe Problem. Und im übrigen sollen wir ja nach Popper alle $\mathrm{Hy}-$ pothesen als problematisch ansehen; die Hintergrundannahmen $A_{1}, \ldots, A_{n}$ stehen dann aber ebenso zur Disposition wie $H$.

3. Wir sind überzeugt, daß gewisse Theorien, die sich gut bewährt haben, richtig sind. Wir wissen zwar, daß wir uns mit solchen Über-

${ }^{59}$ Vgl. dazu auch den Abschnitt 9.6.

${ }^{60} \mathrm{Vgl}$. Popper (63), $238 \mathrm{f}$. 
zeugungen irren können, daß es nicht logisch auszuschließen ist, daß wir künftig Beobachtungen machen werden, die nicht mit den Theorien verträglich sind, aber das heißt nicht, daß wir sie nicht für wahr oder doch für höchst wahrscheinlich richtig halten. Empirische Erkenntnis beschränkt sich nicht auf Beobachtungssätze. Diese Tatsache paßst aber nicht in Poppers Methodologie. Er gibt nicht an, wie sich Überzeugungen rechtfertigen lassen, die nicht aus Beobachtungssätzen folgen. Wie schon in 1.7 bemerkt wurde, vertritt Popper im Effekt den Begriff notwendiger Erkenntnis, der zu einer unangebrachten Skepsis führt. Er meint, da es immer möglich sei, daß sich Hypothesen als falsch erweisen, könnten wir nie sicher sein, daß sie gelten, das also auch nicht wissen. Aus „Es ist (alethisch) möglich, daß A falsch ist" folgt aber nicht ,Ich kann nicht sicher sein (ich kann nicht überzeugt sein), daß A gilt", also auch nicht „Ich kann nicht wissen, daß A gilt". Obwohl ich weiß, daß es (alethisch) möglich ist, daß A falsch ist - und das weiß ich von allen Sätzen, von denen ich weiß, $\mathrm{daß}$ sie kontingent sind - , kann ich doch davon überzeugt sein, daß A gilt; und wenn A tatsächlich gilt, weiß ich das dann auch.

4. Induktive Bestätigung und deduktive Bewährung schließen sich nicht aus, obwohl Popper immer wieder betont, Bewährung habe nichts mit induktiver Bestätigung zu tun. Bewährt sich eine Hypothese $H$ an einer Beobachtung $B$, so daß $B$ aus $H$ mit Hintergrundannahmen $A$ folgt, so ist die Wahrscheinlichkeit $w(H, B \wedge A)$ von $H$ aufgrund von $A$ und $B$ größer als die Wahrscheinlichkeit $w(H, A)$, die $H$ vorher hatte. Und ist, wie Popper annimmt, $B$ ein strenger Test von $H$, so daß die Wahrscheinlichkeit $w(B, A)$ von $B$ aufgrund von $A$ klein ist, so macht die Beobachtung $B$ die Hypothese $\mathrm{H}$ erheblich wahrscheinlicher, als sie vorher war. ${ }^{61}$ Von einer induktiven Bestätigung von $H$ durch $B$ relativ zu A spricht man aber, wenn gilt $w(H, B \wedge A)>$ $\mathrm{w}(\mathrm{H}, \mathrm{A})$. Bewährung hat also etwas mit induktiver Bestätigung zu tun, und allein darauf beruht es, daß wir gut bewährte Hypothesen anderen vorziehen. Davon, daß Popper das Induktionsproblem gelöst habe, wie er das beansprucht, kann also keine Rede sein. Das Induktionsproblem lautet: „Lassen sich generelle Hypothesen durch Beobachtungen begründen?" Diese Frage verneint Popper, weil er nur de-

${ }^{61}$ Es ist für $w(H, A)>0,0<w(B, A)<1$ und falls $B$ aus $H$ und $A$ folgt: $\mathrm{w}(\mathrm{H}, \mathrm{B} \wedge \mathrm{A})=\mathrm{w}(\mathrm{H} \wedge \mathrm{B} \wedge \mathrm{A}) / \mathrm{w}\left(\mathrm{B} \wedge \wedge^{\circ} \mathrm{A}\right)=\mathrm{w}(\mathrm{H} \wedge \mathrm{A}) / \mathrm{w}(\mathrm{B} \wedge \mathrm{A})=\mathrm{w}(\mathrm{H}, \mathrm{A}) /$ $w(B, A)>w(H, A)$. 
duktive Begründungen ins Auge faßt. Er ersetzt sie durch eine andere Frage: „Lassen sich generelle Hypothesen durch Beobachtungen begründen oder falsifizieren?" , die er dann positiv beantwortet. Seine „Lösung“ des Induktionsproblems besteht also in der Beantwortung einer anderer. Frage.

Abschließend sei noch einmal betont, daß unsere Argumente gegen den Urteilsempirismus nicht auf eine rationalistische Position hinauslaufen. Das „Apriori“ der empirischen Erkenntnis, das wir hier aufgewiesen haben, besteht in Wahrscheinlichkeitsbewertungen und Voraussetzungen, nicht in synthetischen Sätzen, die ohne Bezugnahme auf Erfahrung beweisbar wären. Es sind auch keine Annahmen, die unabhängig von aller Erfahrung wären, sondern solche, die über die jeweiligen Erfahrungen hinausgehen, aber durch sie auch modifiziert werden können. Damit erweist sich die traditionelle Unterscheidung apriorisch - aposteriorisch als fragwürdig. Das gleiche gilt, wie wir gesehen haben, für die Unterscheidung analytischer und synthetischer Sätze. Damit zeigt sich, daß die Kontroverse Empirismus - Rationalismus von falschen Voraussetzungen ausgeht.

\subsection{Kognitivistische und nichtkognitivistische Deu- tungen von Theorien}

Im Abschnitt 8.6 haben wir uns mit der Frage beschäftigt, ob und in welchem Sinn sich eine realistische Deutung der Erfahrung vertreten läßt. Dort ging es um die Realismus-Idealismus-Kontroverse. Das Vorkommen theoretischer Terme in empirischen Theorien wirft nun ein Problem auf, das ebenfalls unter der Bezeichnung "Realismus" diskutiert wird, bei dem es aber nicht um die Frage geht, ob und in welchem Sinn theoretische Aussagen von einer ,objektiven“ Natur handeln, sondern um die Frage, ob theoretische Aussagen einen kognitiven Sinn haben; ob sie Behauptungen über die Natur darstellen oder lediglich Hilfsmittel sind, mit denen man aus Beobachtungssätzen andere Beobachtungssätze ableiten kann. Es geht also um die Frage einer kognitiven oder einer instrumentalistischen Deutung von Theorien. Diese Kontroverse wird in der Literatur als „Realismus-Instrumentalismus-Kontroverse" bezeichnet, da ein ,Realismus“ in die- 
sem Sinn aber nichts mit den in 3.4 und 8.6 charakterisierten realistischen Positionen zu tun hat - er ist ebenso mit einer realistischen wie einer idealistischen Deutung der Erfahrung verträglich -, sprechen wir hier von kognitivistischen und nichtkognitivistischen Deutungen von Theorien.

Die kognitivistische Konzeption wird erstens durch die Quantenphysik infrage gestellt, deren Aussagen über physikalische Systeme nicht Informationen über diese Systeme selbst liefern, sondern nur beinhalten, welche künftigen Beobachtungsergebnisse wir aufgrund vergangener zu erwarten haben. Sie ist zweitens im Blick auf das Auftreten theoretischer Terme in Zweifel gezogen worden. Auf den ersten Punkt sind wir schon im 8. Kapitel eingegangen. Wir befassen uns daher hier nur mit dem zweiten Problem. Nichtkognitivistische Deutungen stützen sich dabei vor allem auf folgende beiden Argumente:

1. Theoretische Terme werden durch die Theorien, in denen sie vorkommen, nur implizit mir Ausdrücken der Beobachtungssprache definiert; sie werden damit nur partiell interpretiert, wie wir im Abschnitt 9.2 gesehen haben. Beobachtungsprädikate drücken also keine wohlbestimmten Attribute aus, und daher stellen Aussagen der theoretischen Sprache nicht eindeutig bestimmte Sachverhalte dar.

Dieses Argument spricht also theoretischen Aussagen einen kognitiven Sinn deshalb ab, weil ihr Sinn nicht hinreichend genau definiert ist, um sie als Aussagen über bestimmte Sachverhalte verstehen zu können. Man kann aber keinen prinzipiellen Unterschied zwischen dem kognitiven Status von Sätzen der Beobachtungssprache und dem theoretischer Sätze machen, denn wir haben schon gesehen, daß auch Beobachtungsprädikate nicht für alle möglichen Anwendungen erklärt sind, daß auch sie unscharf sind. Der Unterschied ist also nur graduell. Es hängt ferner nicht vom $\mathrm{Maß}$ seiner Präzision $\mathrm{ab}$, ob ein Satz kognitiven Sinn hat. Es gibt präzise Befehle oder Fragen und vage Behauptungen.

2. Im zweiten Argument geht es nicht um die semantische Unterdeterminiertheit theoretischer Sätze, sondern darum, daß solche Sätze in dem, was sich beobachten läßt, vielfach keine hinreichende Stütze finden. Man spricht in diesem Sinn von einer empirischen Unterdeterminiertheit von Theorien. Dabei geht es nicht um das Induktionsproblem. Das betrifft auch Sätze der Beobachtungssprache. Das Argument besagt vielmehr: Verschiedene Theorien können denselben em- 
pirischen Gehalt haben, so daß wir also mithilfe von Beobachtungen nicht entscheiden können, welche von ihnen richtig ist.

Um diesen Gedanken genauer darstellen zu können, müssen wir zunächst einige Begriffe einführen. Im folgenden sei $E^{*}(T)$ der empirische Gchali i.e.S. de: Theorie T, d. h. dic Merge der Beobachtungssätze, die aus $\mathrm{T}$ folgen. $\mathrm{E}(\mathrm{T})$ sei demgegenüber der empirische Gehalt (i.w.S.) von T, d. h. die Menge der Sätze der Beobachtungssprache, die aus $T$ folgen. $E^{*}(T)$ ist also Teilmenge von $E(T) .{ }^{62}$ Wir wollen sagen:

D 9.5-1: Zwei Theorien $T_{1}$ und $T_{2}$ sind empirisch äquivalent, bzw. empirisch äquivalent i.w.S. genau dann, wenn gilt $\mathrm{E}\left(\mathrm{T}_{1}\right)=\mathrm{E}\left(\mathrm{T}_{2}\right)$, bzw. $E^{*}\left(T_{1}\right)=E^{*}\left(T_{2}\right) \cdot{ }^{63}$

Die Theorien $T_{1}$ und $T_{2}$ seien in derselben Sprache $S$ formuliert, sie sollen sich aber bezgl. der theoretischen Terme unterscheiden, die in ihnen vorkommen. $V_{\mathrm{T} 1}$ sei das theoretische Vokabular von $\mathrm{T}_{1}, \mathrm{~V}_{\mathrm{T} 2} \mathrm{je}$ nes von $T_{2} . S_{1}$ (bzw. $S_{2}$ ) sei die Teilsprache von $S$, die neben den Beobachtungstermen nur die theoretischen Terme aus $V_{\mathrm{T} 1}$ (bzw. $V_{\mathrm{T} 2}$ ) enthält. Wir setzen eine bestimmte Interpretation für die Beobachtungssprache $S_{B}$ voraus; die Deutung der theoretischen Terme von $S$ ergibt sich dann aus den impliziten Definitionen, die insbesondere in $\mathrm{T}_{1}$ und $\mathrm{T}_{2}$ enthaiten sein können und die diese Terme ınic Termen der Beobachtungssprache verknüpfen. Die Beziehung logischer Folgen drücken wir wie in 3.3 durch das Symbol , $\Rightarrow$ " aus, so daß $A, B \Rightarrow C$ besagt, daß $C$ eirne logische Folge von $A$ und B ist. Das Symbol „«“ soll die Geltung einer logischen Folgebeziehung in beiden Richtungen ausdrücken. $A, B \Leftrightarrow C, D$ besagt dann soviel wie die Konjunktion der vier Sätze $A, B \Rightarrow C, A, B \Rightarrow D, C, D \Rightarrow A$ und $C, D \Rightarrow B$.

D 9.5-2: Die Theorien $T_{1}$ und $T_{2}$ heißen äquivalent genau dann, wenn es adäquate explizite Definitionen $D_{1}$ der Terme aus $V_{T 2}$ in $S_{1}$ gibt und adäquate explizite Definitionen $D_{2}$ der Grundterme von $S_{1}$ in $S_{2}$, so daß gilt $T_{1}, D_{1} \Leftrightarrow T_{2}, D_{2}$.

Eine Definition soll dabei adäquat heißen, wenn sie dem definierten Term seine normale Bedeutung (in S) zuordnet. Die Äquivalenz von

\footnotetext{
62 Vgl. dazu 9.2, S. 436.

${ }^{63}$ Aus $\mathrm{E}\left(\mathrm{T}_{1}\right)=\mathrm{E}\left(\mathrm{T}_{2}\right)$ folgt $\mathrm{E}^{*}\left(\mathrm{~T}_{1}\right)=\mathrm{E} *\left(\mathrm{~T}_{2}\right)$, aber nicht umgekehrt.
} 
$T_{1}$ und $T_{2}$ besagt also: $S_{1}$ läßt sich in $S_{2}$ übersetzen und $S_{2}$ in $S_{1}$, und dabei gehen die Sätze von $T_{1}$ in Theoreme von $T_{2}$ übar, bzw. die Sätze von $T_{2}$ in Theoreme von $T_{1} \cdot T_{1}$ und $T_{2}$ beinhalten also dasselbe.

D 9.5-3: Die Theorien $T_{1}$ und $T_{2}$ heißen äquivalent i.w.S. genau dann, wenn es explizite Definitionen $D_{1}$ der Terme von $S_{2}$ in $S_{1}$ und explizite Definitionen $D_{2}$ der Terme von $S_{1}$ in $S_{2}$ gibt, so daß gilt $T_{1}, D_{1} \Leftrightarrow T_{2}, D_{2}$.

Hier wird nun nicht vorausgesetzt, daß die Definition adäquat ist. Die Definitionen aus $D_{1}$ können also den Termen von $V_{T 2}$ andere Bedeutungen zuordnen als sie in $\mathrm{S}$ haben; dasselbe gilt für die Definition $D_{2}$ der Terme aus $V_{T 1}$. Äquivalenz i.w.S. impliziert wechselseitige Reduzierbarkeit der Theorien im Sinne von D 3.3-2. Die Umkehrung gilt jedoch nicht, da nach D 3.3-2 aus einer wechselseitigen Reduzierbarkeit mithilfe der Definitionen $D_{1}$ und $D_{2}$ weder $T_{1}, D_{1} \Rightarrow D_{2}$ folgt, noch $\mathrm{T}_{2}, \mathrm{D}_{2} \Rightarrow \mathrm{D}_{1}$.

Im Sinn des Modells deduktiver Bestätigung von Theorien kann eine Theorie empirisch nur so überprüft werden, daß die aus ihr ableitbaren Beobachtungssätze getestet werden - wenn man sich nicht schon auf die Geltung anderer Theorien oder Hypothesen stützen will. ${ }^{64}$ Die Theorie bezieht dann ihre empirische Legitimation allein aus ihrem empirischen Gehalt i.e.S., und man könnte nur behaupten, ihre Aussagen ließen sich empirisch legitimieren, wenn gelten würde: Im weiteren Sinn empirisch äquivalente Theorien sind äquivalent. Diese These ist u. a. von $\mathrm{H}$. Reichenbach, A. Grünbaum und H. Putnam vertreten worden.65 Sie ist aber offensichtlich falsch und zwar ganz unabhängig davon, ob in einer Theorie theoretische Terme vorkommen. Das zeigt schon das folgende simple Beispiel: $T_{1}$ sei die ,Theorie" $V x H(x) \wedge G(a), T_{2}$ die ,"Theorie" $G(a)$, wobei a, $G$ und $H$ Beobachtungsterme sind, und der Satz $\mathrm{VxH}(\mathrm{x})$ keine analytische

${ }^{64} \mathrm{Da}$ wir den Begriff des Beobachtungssatzes so definiert haben, daß auch der Satz A $\supset B$ ein Beobachtungssatz ist, falls das für A und B gilt, umfaßt diese Prüfung einer Theorie $\mathrm{T}$ auch den Fall, daß Voraussagen nicht aus $\mathrm{T}$ allein abgeleitet werden, sondern mithilfe anderer Beobachtungsaussagen. Läßt man dagegen bei der Überprüfung von $T$ auch Sätze zu, die aus $T$ nicht ableitbar sind und keine Beobachtungsaussagen darstellen, so kann man nicht mehr so argumentieren.

${ }^{65}$ Vgl. Reichenbach (61), 374 f., Putnam (63), Grünbaum (62), 446. 
Folge von $G(a)$ ist. Dann bildet der Satz G(a) mit den Beobachtungssätzen, die daraus folgen, den empirischen Gehalt i.e.S. der beiden Theorien, beide sind aber nicht äquivalent. Da die Menge der wahren Beobachtungssätze einer Sprache nicht einmal die Menge der wahren

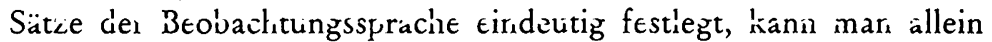
mit Beobachtungssätzen selbst Theorien ohne theoretische Terme nicht rechtfertigen. Die Behauptung einer empirischen Unterdeterminiertheit von Theorien ist sehr viel stärker als die Negation dieser These. Sie besagt, daß es empirisch äquivalente Theorien gibt, die nicht äquivalent sind, also Theorien, die nicht dasselbe beinhalten, $d$. h. verschiedene Aussagen über die Natur machen, zwischen denen wir aber weder durch Beobachtungen noch durch Sätze der Beobachtungssprache entscheiden können. Die Phänomene der geometrischen Optik kann man z. B. ebenso mit einer Wellenvorstellung des Lichts erklären, wie mit einer korpuskularen Vorstellung. In diesem Fall köınen wir z.wischen den beiden Vorstellungen entscheiden, indem wir untersuchen, welche Vorstellung sich bei der Erklärung anderer optischer Phänomene als fruchtbarer erweist. Das ist aber nicht immer möglich. Im Abschnitt 8.3 haben wir gesehen, daß der Übergang von der klassischen zur relativistischen Physik nicht allein durch empirische Tatsachen erzwungen wird, sondern daß er den Übergang zu einer Erklärung dieser Tatsachen mit anderen Begriffen bedeutet.

Diese Behauptung läßt sich so verstärken:

UD: $Z u$ jeder empirischen Theorie gibt es eine empirisch äquivalente, aber nicht äquivalente Theorie.

Der Beweis ist einfach: $\mathrm{Da}$ es nach den Theoremen von Ramsey und Craig zu jeder Theorie $T_{2}$ in der Sprache $S$ eine Theorie $T_{1}$ in der Beobachtungssprache $S_{B}$ von $S$ gibt, die mit $T_{2}$ empirisch äquivalent ist, und $\mathrm{da}$ die Äquivalenz von $\mathrm{T}_{1}$ und $\mathrm{T}_{2}$ Übersetzbarkeit der Sprache von $T_{2}$ in die von $T_{1}$ impliziert, würde aus der Negation von UD die These $\mathrm{EB}_{2}$ des Begriffsempirismus folgen, ${ }^{66}$ die wir schon als falsch erkannt haben. Aus derselben Überlegung ergibt sich sogar:

$\mathrm{UD}^{+}: \mathrm{Zu}$ jeder empirischen Theorie gibt es eine empirisch äquivalente, aber nicht i.w.S. äquivalente Theorie. ${ }^{67}$

${ }^{66} \mathrm{Vgl}$. den Abschnitt 9.2.

${ }^{67}$ Mit einer Theorie $T_{1}$ ist jene Theorie $T_{2}$, die aus dem Ramsey-Satz $R\left(T_{1}\right)$ besteht, empirisch äquivalent. Wäre $T_{1}$ mit $T_{2}$ i.w.S. äquivalent, so wäre $T_{1}$ 
Die empirische Unterdeterminiertheit von Theorien besagt also, daß es immer Theorien gibt, die verschiedene Ëklärungen derselben empirischen Phänomene liefern, zwischen denen wir aber durch Beobachtungen nicht entscheiden können. ${ }^{68}$ Die in solchen Theorien zur Erklärung der Phänomene eingeführten theoretischen Konstrukte lassen sich dann durch Erfahrungen nicht legitimieren. ${ }^{69}$

Die Thesen UD und UD ${ }^{+}$stützen sich also nicht auf die Tatsache, daß es Sätze der Beobachtungssprache gibt, die nicht aus endlich oder auch unendlich vielen Beobachtungssätzen folgen. Nach ihnen gibt es unterschiedliche Erklärungen nicht nur für alle beobachtbaren Tatsachen eines Gegenstandsbereichs, sondern auch für alle Tatsachen, die sich mit Sätzen der Beobachtungssprache beschreiben lassen. Die Erklärung dieser Tatsachen ist immer mithilfe verschiedener theoretischer Konstrukte möglich.

Die Geltung von UD hängt daran, daß wir in D9.5-2 explizite Definitionen der theoretischen Terme gefordert haben. Läßt man auch implizite Definitionen zu und geht von einer theorienimmanenten Deutung theoretischer Terme aus, so ist UD falsch: Der Ramsey-Satz $R\left(T_{1}\right)$ von $T_{1}$ ist im empirischen Gehalt $E\left(T_{1}\right)$ von ' $\Gamma_{1}$ enthalten; ebenso $R\left(T_{2}\right)$ in $E\left(T_{2}\right)$. Sind also $T_{1}$ und $T_{2}$ empirisch äquivalent, so gilt $T_{1} \Rightarrow R\left(T_{2}\right)$ und $T_{2} \Rightarrow R\left(T_{1}\right)$. Bei einer theorienimmanenten Deutung wird nun nach dem Gedanken von Carnap die Interpretation der Terme aus $V_{T 1}$ durch das analytische Postulat $R\left(T_{1}\right) \supset T_{1}$ festgelegt,

im Sinne von D3.3-2 auf ' $T_{2}$ reduzierbar. Es gäbe dann explizite Definitionen $D_{2}$ der theoretischen Terme von $T_{1}$ in der $T_{1}$ zugrundeliegenden Beobachtungssprache. Theoretische Terme sind aber nur solche, die sich nicht explizit in der Beobachtungssprache definieren lassen. Theorien mit theoretischen Termen lassen sich nie auf Theorien in der Beobachtungssprache reduzieren.

${ }^{68} \mathrm{Zu}$ jeder Theorie $T_{1}$, die nicht selbst schon theoretische Terme enthält, gibt es aus logischen Gründen immer eine empirisch äquivalente Theorie $\mathrm{T}_{2}$ mit theoretischen Termen. Man kann ja z. B., wo F ein theoretisches Prädikat ist, $T_{2}$ als den Satz $\operatorname{VxF}(x) \wedge\left(\operatorname{VxF}(x) \supset T_{1}\right)$ bestimmen.

${ }^{69} \mathrm{C}$. Glymour weist in (71) eine empirische Unterdeterminiertheit von Theorien für einige Einschränkungen des Theoriebegriffs nach, wobei er auch den Äquivalenzbegriff für Theorien erheblich liberalisiert. Er fordert nur, daß die Theorien ein gemeinsames Modell haben, $d . h$. daß es maximale und konsistente Satzmengen $m_{1}$ und $m_{2}$ über $S_{1}$, bzw. $S_{2}$ gibt, die $T_{1}$ bzw. $T_{2}$ enthalten, so daß zwischen $2 m_{1}$ und $2 m_{2}$ eine Äquivalenz besteht. 
jene der Terme aus $V_{T 2}$ durch $R\left(T_{2}\right) \supset T_{2}$. Es gilt also auch $R\left(T_{2}\right) \Rightarrow T_{2}$, also $T_{1} \Rightarrow T_{2}$, und $R\left(T_{1}\right) \Rightarrow T_{1}$, also $T_{2} \Rightarrow T_{1}$. Die beiden Theorien sind dann analytisch äquivalent, und man kann die Terme aus $V_{T 1}$ in $S_{2}$ implizit durch $R\left(T_{1}\right) \supset T_{1}$, jene aus $V_{T 2}$ in $S_{1}$ durch $\left.R_{(}^{\prime} T_{2}\right)=T_{2}$ defirieren. Eine thecrierimmancnte Dcuiung thecretischer Terme allein ist mit der These UD verträglich. Implizite Definitionen der Terme von $S_{2}$ in $S_{1}$ zuzulassen und umgekehrt entspricht jedoch nicht dem Gedanken, zu dessen Erläuterung die These von der empirischen Unterdeterminiertheit dienen soll. Es geht ja um die Frage, ob sich theoretische Aussagen empirisch legitimieren lassen. Eine gewisse Legitimation liegt darin, daß sich damit die empirischen Phänomene erklären lassen. Aber die Erklärbarkeit einer Tatsache E mit einer Annahme $\mathrm{H}$ besagt noch nicht, daß $\mathrm{H}$ richtig ist. Erst wenn sich zeigen ließe, daß $\mathrm{E}$ nur mit $\mathrm{H}$ erklärbar ist, ließe sich $\mathrm{H}$ durch $\mathrm{E}$ begründen. Dann wäre $\mathrm{H}$ aber (evtl. unter Benützung gewisser Hintergrundbedingungen) eine Folge von E. Hier ging es darum zu zeigen, daß theoretische Annahmen sich so nicht rechtfertigen lassen, daß sie keine Folgen empirischer Tatsachen sind. Es gibt immer alternative Erklärungen mit theoretischen Konstrukten, die nicht gleichwertig sind, und mit einer Erklärung setzt man nicht das Explanans einer anderen möglichen Erklärung voraus. Ließe man implizite Definitionen $\mathrm{zu}$, so würde man damit nicht zeigen, daßs die theoretischen Konstrukte, die eine Erklärung benützt, auch von der anderen vorausgesetzt werden, sondern man ginge nur von einer Erklärung zu einer anderen über. Niit dem Carnap-Postuiat, das man für $E\left(T_{1}\right)=E\left(T_{2}\right)$ auch als $R\left(T_{1}\right) \supset T_{2}$ formulieren kann, definiert man die theoretischen Begriffe von $T_{2}$ nicht durch jene von $T_{1}$, sondern sagt nur, daß sie irgendwie so zu interpretieren sind, daß $T_{2}$ wahr ist.

Kann man nun theoretischen Aussagen einen kognitiven Gehalt zusprechen, wenn sie sich empirisch nicht rechtfertigen lassen? Oder muß man eine instrumentalistische Auffassung vertreten, nach dem sich der kognitive Gehalt einer Theorie allein auf den Gehalt der aus ihr ableitbaren Beobachtungssätze oder Sätze der Beobachtungssprache bezieht?

In einer Version behauptet der Instrumentalismus, nur Beobachtungssätze und jene Aussagen, die aus ihnen folgen, hätten einen kognitiven Gehalt. Diese Auffassung, die im frühen Wiener Kreis vor al- 
lem von $L$. Wittgenstein und $M$. Schlick vertreten ${ }^{70}$ und von K. Popper von Anfang an scharf bekämpft worden ist, ergab sich aus dem Verifikationskriterium der Bedeutung. ${ }^{71}$ Alle universellen Hypothesen und Naturgesetze sind danach kognitiv sinnlos und nur als Regeln zur Gewinnung von Voraussagen zu verstehen. Nun ist aber erstens das. Verifikationskriterium unbrauchbar, wie wir schon früher gesehen haben. Zweitens ist eine Regel, wie z. B. ,Nimm für beliebige Objekte a immer an, daß das Ereignis $G(a)$ eintritt, falls du das Ereignis $F(a)$ beobachtest" nur dann akzeptabel, wenn man den Satz , $\wedge x(F(x) \supset$ $\mathrm{G}(\mathrm{x})$ )" für wahr hält. Drittens hat Hempel darauf hingewiesen, daß sich zwar alle Regeln zur Ableitung von singulären Aussagen aus anderen singulären Aussagen in Sätze übersetzen lassen, daß jedoch komplizierteren gesetzesartigen Aussagen wie z. B. $\wedge x \operatorname{Vy}(\operatorname{VzR}(x, y, z)$ $\supset \wedge \mathrm{zS}(\mathrm{x}, \mathrm{y}, \mathrm{z}))$ keine Regeln mit singulären Prämissen und Konklusionen entsprechen. Man kann also nicht alle Naturgesetze als Regeln auffassen. ${ }^{72}$ Daher wird diese erste Version des Instrumentalismus in der Wissenschaftstheorie heute kaum mehr vertreten. Sie klingt noch einmal bei Carnap an, wenn er in (50), $\$ 110 \mathrm{H}$ sagt, Gesetzesaussagen seien überhaupt entbehrlich: Eine generelle Hypothese akzeptieren wir nur aufgrund von Beobachtungen, die sie induktiv bestätigen; dieselben Beobachtungen, die diese Hypothese bestätigen, bestätigen aber auch die Voraussagen, die wir damit machen, so daß die Hypo-

${ }^{70} \mathrm{M}$. Schlick schreibt in (31), 57: „Es ist ja oft bemerkt worden, daß man von einer absoluten Verifikation eines Gesetzes eigentlich nie sprechen kann, da wir sozusagen stets stillschweigend den Vorbehalt machen, es aufgrund späterer Erfahrungen modifizieren zu dürfen. Wenn ich nebenbei ein paar Worte über die logische Situation sagen darf, so bedeutet der eben erwähnte Umstand, daß ein Naturgesetz im Grunde auch nicht den logischen Charakter einer ,Aussage' trägt, sondern vielmehr eine ,Anweisung zur Bildung von Aussagen "darstellt". Quine sagt in (64), 44: "As an empiricist I continue to think of the conceptual scheme of science as a tool, ultimately, for predicting future experience in the light of past experience". - Einen Instrumentalismus hat schon Berkeley vertreten. Vgl. dazu Popper (63), Kap. 6. Zum Instrumentalismus vgl. ebenda, Kap. 3, ferner Hempel (65), $354 \mathrm{ff}$. und Stegmüller (69), $97 \mathrm{ff}$.

${ }^{71} \mathrm{Vgl}$. dazu 6.2, S. 254.

${ }^{72}$ Zur Unentbehrlichkeit von Gesetzesaussagen und Theorien vgl. a. Popper (66), $11 \mathrm{f}$. 
these im Prinzip für Voraussagezwecke überflüssig ist. Voraussagen zu ermöglichen, ist aber nicht die einzige Funktion von Hypothesen und Theorien. Für die Systematisierung der Erfahrungen sind sie unentbehrlich und ebenso für Erklärungen. Carnap hat seine Ausführungen wohl auch nicht im Sinn eines Begrïndungsversuchs für eine extreme Version des Instrumentalismus verstanden. Jedenfalls ist er auf diese Gedanken später nicht mehr zurückgekommen. ${ }^{73}$

In einer liberalen zweiten Version bestreitet der Instrumentalismus nicht den Aussage-Charakter von Hypothesen und Gesetzen oder ihre Unentbehrlichkeit für die Systematisierung der Erfahrung, sondern er leugnet nur, daß man diese Theorien realistisch interpretieren kann; daß wir sie also als Aussagen über die Beschaffenheit der Welt ansehen können. Er bestreitet also die kognitive Leistung empirischer Theorien. Sieht man den kognitiven Gehalt, den deskriptiven Sinn und den Informationsgehalt von Sätzen der Beobachtungssprache als unproblematisch an, so läuft die Grundthese dieses Instrumentalismus auf die Behauptung hinaus: Der kognitive Inhalt einer empirischen Theorie $\mathrm{T}$ beschränkt sich auf den Inhalt der Sätze der Beobachtungssprache, die aus $T$ folgen, d. h. der Sätze aus dem empirischen Gehalt $E(T)$ von T. Der kognitive Gehalt von $T$ deckt sich also mit dem des Ramsey-Satzes R(T). Das wichtigste Argument für diese These naben der oben diskuticrten semartischen Unieideterminiertheit theoretischer Aussagen ist ihre empirische Unterdeterminiertheit. Aus dieser Unterdeterminiertheit folgt aber nicht, daß theoretische Aussagen keinen licgnitiver Sinn haben. Azuch unentscheidbäe Sätze können durchaus sinnvoll sein. Die Kontinuumshypothese, die im Rahmen der Zermelo-Fraenkelschen Mengenlehre weder beweisbar noch widerlegbar ist, ist dort trotzdem sinnvoll. Aus der empirischen Unentscheidbarkeit eines Satzes könnte man nur mithilfe irgendeines empiristischen Sinnkriteriums auf seine Sinnlosigkeit schließen, nach dem nur Sätze sinnvoll sind, die sich deduktiv oder induktiv durch empirische Sätze begründen oder widerlegen lassen. Diese Kriterien sind aber alle unbrauchbar, wie wir schon gesehen haben. Im übrigen ist wieder zu betonen: $\mathrm{Da}$ es bzgl. der semantischen Interpretation wie der Überprüfung durch Beobachtungen keine scharfe Grenze zwi-

${ }^{73}$ Popper hat in Carnaps Ausführungen jedoch einen Ansatz zur Wiederbelebung des schon tot geglaubten Instrumentalismus gesehen und hat entsprechend heftig darauf reagiert; vgl. (63), $215 \mathrm{ff}$. 
schen Beobachtungssätzen und theoretischen Aussagen gibt, entfällt die Voraussetzung des Instrumentalismus, es gebe einen Bereich von erfahrungsmäßig unmittelbar gegebenen Tatsachen. Man kann nicht verlangen, daß sich alle empirischen Behauptungen durch Beobachtungssätze oder Sätze der Beobachtungssprache rechtfertigen lassen, wenn auch diese hypothetisch sind. Man kann nicht annehmen, daß nicht theoretische Aussagen, sondern nur Sätze der Beobachtungssprache die Welt beschreiben, wenn auch diese schon Interpretationen mit begrifflichen Konstrukten darstellen.

Eine instrumentalistische Deutung von Theorien haben auch J. Sneed und W. Stegmüller vorgeschlagen. ${ }^{74}$ Die Charakterisierung empirischer Theorien als Axiomensysteme oder Mengen von Gesetzen, aus denen Sätze der Beobachtungssprache folgen, entspricht nicht dem üblichen Gebrauch dieses Wortes in der Physik. Physikalische Theorien, wie z. B. die Newtonsche Mechanik, sind rein mathematische Strukturen, die als solche sicher keine empirischen Aussagen sind. Empirische Aussagen ergeben sich aus der Theorie erst dann, wenn man die Begriffe der Theorie in Zusammenhang mit empirischen Begriffen bringt, mit denen sich gewisse empirische Phänomene beschreiben lassen, und behauptet, daß die Theorie bei dieser Zuordnung ein Modell dieser empirischen Phänomene ist, d.h. daß diese bei der fraglichen Zuordnung von der Theorie richtig beschrieben werden. So lassen sich die Grundgesetze der Newtonschen Mechanik auf verschiedenen Gebieten anwenden, auf die Bewegung der Planeten ebenso wie auf elastische Stoßvorgänge. Dabei können für spezielle Anwendungen oder auch Klassen von Anwendungen Spezialgesetze zur Theorie hinzugenommen werden, wie z. B. das Gesetz der Massenanziehung oder das Hookesche Gesetz. Ferner müssen für verschiedene Anwendungen der Theorie Kohärenzbedingungen gelten, nach denen z. B. denselben Objekten in verschiedenen Anwendungen dieselben Massen zugeordnet werden. Ohne solche Kohärenzbedingungen würde sich der Sinn der Relationsbegriffe und der metrischen Begriffe in verschiedenen Anwendungen ändern, und man könnte z. B. nicht die mit einer Federwaage bestimmte Masse eines Körpers in die Bewegungsgleichung für diesen Körper einsetzen. ${ }^{75}$

${ }^{74} \mathrm{Vgl}$. Sneed (71) und Stegmüller (70), 2. Halbbd.

${ }^{75}$ Sneed und Stegmüller bestimmen theoretische Terme so, daß ein metrischer Begriff $\mathrm{F}$ bzgl. einer Theorie $\mathrm{T}$ theoretisch heißt, wenn es Objekte a gibt, so 
Eine Theorie ist nach diesen Bestimmungen also keine Aussage und daher nicht wahr oder falsch. Wahr oder falsch sind nur die Aussagen, $\mathrm{da}$ es überhaupt empirische Modelle der Theorie gibt - das ist aber eine in ihrer Allgemeinheit kaum relevante Behauptung - oder daß gewisse Phänomenbereirhe (gewisse Mengen empirischer Obiekte und Begriffe) ein Modell der Theorie bilden, daß also die Theorie bei gewissen Interpretationen richtig ist. In diesem Sinn haben wir oben immer von Theorien gesprochen. Obwohl also das Sneed-Stegmüllersche Verständnis des Wortes „,Theorie“ seinen Verwendungen in der Physik besser angepaßt sein mag, wird doch mit diesem Theorienverständnis das Problem der Unterdeterminiertheit nicht beseitigt: Theorien als bloße mathematische Strukturen haben keinerlei empirische Relevanz. Relevant sind nur Behauptungen, daß man mit solchen Strukturen empirische Phänomene richtig beschreiben kann. Dabei stellt sich aber die Frage, wie sich die Annahme rechtfertigen läßt, es gebe etwas in der Welt, das den theoretischen Konstrukten der Theorie in der fraglichen Anwendung entspricht.

daß man $F(a)$ nur vermittels gewisser Anwendungen von $T$, also nicht ohne Zuhilfenahme von $T$ berechnen kann. Unsere semantische Auszeichnung theoretischer Terme ging davon aus, daß gewisse Terme, die Beobachtungscerme, unabhängig von $T$ eine fesie Bedeuiuing haberi. Die bzgl. T theoretischen Terme sind dann jene in $T$ vorkommenden Terme, für die das nicht gilt. Eine dezisionistische Bestimmung theoretischer Terme stützt sich endlich darauf, daß es für gewisse Sätze - die Beobachtungssätze - ein von T unabhängiges Entscheidungsverfahren (im weitesten Sinn des Wortes - bei metrischen Begriffen spricht man besser von einem Meßverfahren) gibt. Ein Term $F$ heißt dann theoretisch bzgl. T, wenn es Objekte a gibt, so daß F(a) sich nicht in einer von $T$ unabhängigen Weise bestimmen läßt. Dabei besteht folgender Zusammenhang: Ist $F$ ein Beobachtungsterm im semantischen Sinn, so liegt die Bedeutung von $F$ unabhängig von $T$ fest; es gibt dann von $T$ unabhängige Wahrheitsbedingungen, also auch Entscheidungskriterien für alle Anwendungen von $F$, so daß $F$ auch nach der dezisionistischen $\mathrm{Be}-$ stimmung ein Beobachtungssatz ist. Sind umgekehrt alle Anwendungen von $F$ ohne Bezugnahme auf $T$ entscheidbar, so ordnet das Entscheidungsverfahren $\mathrm{F}$ unabhängig von $\mathrm{T}$ eine Bedeutung zu; $\mathrm{F}$ ist dann also auch im semantischen Sinn ein Beobachtungsterm. Gibt es ferner ein von $T$ unabhängiges Entscheidungsverfahren für alle Anwendungen von $F$, so ist $F$ auch im Sinne von Sneed kein bzgl. T theoretischer Term. Setzt umgekehrt für jedes Argument a die Berechnung von $F(a)$ in einigen Anwendungen von $T$ nicht voraus, daß T gilt, so kann man $\mathrm{F}(\mathrm{a})$ in diesen Anwendungen ohne Bezug- 
Die empirische Unterdeterminiertheit von Theorien hat auch eine erkenntnistheoretisch wichtige Konsequenz, die mit der Frage eines kognitiven Gehalts theoretischer Aussagen nichts zu tun hat: Sie zeigt, daß sich empirisch begründete Annahmen nicht scharf von spekulativen oder metaphysischen Annahmen unterscheiden lassen. Eine solche Unterscheidung hatte der Empirismus mit seinen Sinnkriterien angezielt, und sie war eines seiner wichtigsten erkenntnistheoretischen Zielsetzungen. Das gilt nicht nur für den modernen logischen Empirismus, sondern ebenso für den Empirismus der Aufklärung. ${ }^{76}$ Im logischen Empirisrnus wurden Sinnkriterien entweder als Übersetzungs- oder als Begründungskriterien formuliert. Als Übersetzungskriterium besagen sie, daß nur solche Sätze empirisch sinnvoll sind, die sich in die Beobachtungssprache übersetzen lassen." $\mathrm{Da}$ sich aber nicht alle Aussagen der Naturwissenschaften so übersetzen lassen, ist dies Kriterium unbrauchbar. Es würde große Teile der Naturwissenschaften mitsamt den suspekten Aussagen der Metaphysik als sinnlos erklären. Als Begründungskriterium besagen Sinnkriterien, daß nur

nahme auf $\mathrm{T}$ berechnen, wegen den Kohärenzbedingungen also in allen Anwendungen von $T$. Alle drei Bestimmungen theoretischer Terme entsprechen sich also. Diese Überlegung macht die Äquivalenz der drei Bestimmungen freilich nur intuitiv plausibel. Ein strenger Äquivalenzbeweis ist wegen der Vagheit der in den drei Definitionen vorkommenden Ausdrücke (,von T unabhängiges Entscheidungs-, bzw. Berechnungsverfahren“ etc.) nicht möglich. Alle drei Bestimmungen des Unterschieds theoretischer von Beobachtungstermen beziehen sich auf eine Theorie T. Eine solche Relativierung der Begriffe hat auch Hempel in (73) vorgeschlagen. - Der Theoriebegriff von Sneed und Stegmüller unterstreicht noch einmal die Bedeutung eines theorienübergreifenden Verständnisses theoretischer Terme: In verschiedenen Anwendungen einer Theorie - in unserem Sinn: in verschiedenen Theorien - müssen die theoretischen Terme in gleicher Weise gedeutet werden. Die Messung von Größen, die in einer Anwendung der Theorie gebraucht werden, ist oft nur durch andere Anwendungen derselben Theorie möglich vielfach auch nur durch Anwendungen anderer Theorien. So läßt sich die Geschwindigkeit ferner Sterne, z. B. nur optisch durch die Spektralverschiebung des von ihm emittierten Lichts (den Doppler-Effekt) messen.

${ }^{76}$ Hume formuliert z. B. ein Sinnkriterium, das dem Postulat entspricht: Nur Sätze, die sich in eine Beobachtungssprache übersetzen lassen, sind sinnvoll. Vgl. Hume (EHU), 22.

${ }^{77}$ Vgl. dazu z. B. Carnap (36). 
solche Sätze empirisch sinnvoll sind, die sich mithilfe von Sätzen der Beobachtungssprache deduktiv oder induktiv begründen lassen. Die empirische Unterdeterminiertheit von Theorien zeigt, daß man auch damit anerkannte naturwissenschaftliche Theorien als empirisch sinnlos einstufen miißte. K. Popper, der sich ausdrücklich nicht als Empiristen versteht, hat versucht, eine Grenze, nicht zwischen empirisch sinnvollen und sinnlosen Aussagen zu ziehen, sondern zwischen wissenschaftlichen Hypothesen oder Theorien und nichtwissenschaftlichen Annahmen. Nach ihm sind nur solche Theorien als ,,wissenschaftlich" zu bezeichnen, die Voraussagen ermöglichen, die sich durch Beobachtungen falsifizieren lassen; die also einen nichtleeren empirischen Gehalt i.e.S. haben. ${ }^{78}$ Auch Theorien, in denen ,,metaphysische" Aussagen vorkommen, können aber einen nichtleeren empirischen Gehalt i.e.S. haben. So folgt z. B. aus der Theorie mit den beiden Axiomen $A$ und $A \wedge C \supset B$, wo $B$ und $C$ irgendwelche Beobachtungssätze sind und A der Satz „Das absolute Sein ist sich selbst transzendent", ein Beobachtungssatz, nämlich C $\supset \mathrm{B}$.

Nach Popper soll es ferner für je zwei konkurrierende Theorien ein experimentum crucis geben, das nur eine von beiden überleben kann. Wenn man die Rede von der „Konkurrenz" zweier Theorien dabei so versteht, daß auch zwei nicht-äquivalente, empirisch aber äquivalente Theorien miteinancier konkurrieren, so wäre die Behauptung also: „Nicht äquivalente Theorien sind nicht empirisch äquivalent". Das widerspricht aber der Feststellung der empirischen Unterdeterminiertrieit.

Die Ansätze zur Trennung Metaphysik-Empirie schließen also entweder zuviel oder aber nichts als ,,metaphysisch“ aus. Es gibt natürlich viele Fälle, in denen man gewisse Terme mit gutem Grund als nicht-empirisch oder unwissenschaftlich erklären kann. Wenn z. B. ein Term so vage ist, daß er sich von dem Präzisionsstandard der Wissenschaften zu weit entfernt, so wird man ihn in wissenschaftlichen Kontexten nicht verwenden. Aber allgemeine und exakte Präzisionstandards gibt es nicht, wie vor allem Wittgenstein in (53) betont hat: es gibt nur eine für gewisse Zwecke (in gewissen Kontexten) hinreichende Präzision, und der Präzisionsstandard ist in verschiedenen Wissenschaften und zu verschiedenen Zeiten verschieden. Im übrigen

${ }^{78}$ Vgl. Popper (66), $14 \mathrm{ff}$. und (63), $253 \mathrm{ff}$. 
sind alle theoretischen Terme mehr oder minder vage. Wenn ferner eine Theorie $T_{2}$ über der Sprache $S_{2}$ sich auf eine Theorie $T_{1}$ über einer Teilsprache $S_{1}$ von $S_{2}$ reduzieren läßt, wird man oft auf die bei dieser Reduktion herausfallenden Terme verzichten. Aber ein solcher Schnitt mit dem Occamschen Rasiermesser ist nicht immer gerechtfertigt: Definierbare Terme sind nicht einfach überflüssig, sondern sie dienen oft der Abkürzung und Vereinfachung von Theorien. Eine fruchtbare Definition kann einen wesentlichen Erkenntnisfortschritt bewirken.

Wir wolien hier natürlich nichı vage Terme mit präzisen oder rein spekulative Hypothesen mit naturwissenschaftlichen in einen Topf werfen. Wir wollen nur betonen, daß sich keine zugleich scharfe und generelle Grenze zwischen Empirie und Metaphysik oder Spekulation ziehen läßt. Alle Ansätze, das wissenschaftlich Rationale mit Empirie + Logik gleichzusetzen, sind gescheitert. Das ,,bloß“ Empirische gibt es nicht. Auch naturwissenschaftliche Theorien sind ,,spekulativ" und „metaphysisch"“ in dem Sinn, daß sie weit über das hinausgehen, was allein durch Beobachtungen mit logischen Mitteln legitimiert werden kann. Und schon die intentionale, gegenständliche Deutung und Beschreibung unseres sinnlichen Erlebens benützt Konstrukte - Objekte und Objektattribute - , die sich aus den Sinnesempfindungen nicht ableiten lassen.

Das Phänomen der empirischen Unterdeterminiertheit von Theorien weist endlich darauf hin, daß wir bei der Auswahl von Theorien auch Kriterien folgen, die nichts mit empirischen Begründungen zu tun haben. Schon Kant hat die Rolle des Vernunftinteresses bei der Annahme von Theorien betont. Er spricht von ,,regulativen Prinzipien", die mit allen möglichen Erfahrungen verträglich sind - also keinen empirischen Gehalt haben - , denen wir aber bei der theoretischen Systematisierung der Erfahrung folgen. Als Beispiele solcher Auswahlkriterien kann man etwa folgende anführen:

1. Informativität. Wir ziehen Theorien vor, die uns detailliertere Informationen liefern, die exaktere Voraussagen ermöglichen. Das ergibt sich einfach aus dem Zweck von Theorien, uns Informationen über die Welt zu geben. Auf dieses Kriterium hat Popper mit besonderem Nachdruck hingewiesen.

2. Einheitlichkeit. Wir sind an einer einheitlichen Beschreibung der Natur interessiert, wollen die Vielfalt der Erscheinungen aus möglichst allgemeinen Prinzipien verstehen. Obwohl z. B. die statistische 
Mechanik für die Erklärung thermodynamischer Phänomene nicht mehr leistet als die phänomenologische Thermodynamik, ziehen wir sie dieser vor, weil sie die Subsumption dieser Phänomene unter eine einheitliche Theorie ermöglicht.

3. Einfacbleeit. W'ir sird an einfachen und übcrsichtlicheri Theorien interessiert, mit denen man z. B. auf möglichst einfache Weise Voraussagen ableiten kann. Einfachheit ist dabei ein Kriterium für Begriffswie für Satzsysteme. Beides hängt zusammen, denn oft ermöglicht es die Einführung neuer Begriffe, Theorien einfacher zu formulieren. Einfachheit ist ein Erfordernis der Denkökonomie. Sie ist eine Eigenschaft von Naturbeschreibungen, nicht eine Eigenschaft der Natur selbst: dieselbe Sache läßt sich einfach und komplex beschreiben. Je nach Wahl des Koordinatensystems kann man z. B. eine Kurve durch eine einfache oder eine komplexe Gleichung beschreiben. Man kann daher nicht sagen, Einfachheit sei ein ,Siegel der Wahrheit“ - simplicitas sigillum veri. ${ }^{79}$

4. Erkenntnisideale. Die Wissenschaftsgeschichte zeigt, daß man in verschiedenen Epochen verschiedene Ziele wissenschaftlicher Erkenntnis verfolgt hat und dementsprechend verschiedene Kriterien für Wissenschaftlichkeit verwendete. In der aristotelischen Philosophie und der von ihr geprägten Wissenschaftskonzeption spielt z. B. das teleologische Element eine zentrale Rolie: das Ideal eines Verständnisses der Welt als einer zweckmäßigen Organisation. Bei Leibniz zeigt sich, wie dieses Ideal zu einer Auswahl anderer - auch physikalischer - Theorien führt, als die Zielsetzung eines kausalen Verständnisses der Natur.

Solche Auswahlkriterien können in Konkurrenz miteinander stehen, so daß Prioritäten gesetzt werden müssen. Das gilt offensichtlich

${ }^{79}$ Newton meinte: „Nature is pleased with simplicity and effects not the pomp of superfluous causes" (Newton's Philosophy of Nature, hrsg. H. S. Thayer, New York 1953, S. 3) und Galilei sagt: „Endlich hat uns zur Untersuchung der natürlich beschleunigten Bewegung gleichsam mit der Hand geleitet die aufmerksame Beobachtung des Geschehens und der Natur in allen ihren Verrichtungen, bei deren Ausübung sie die allerersten, einfachsten und leichtesten Hilfsmittel zu verwenden pflegt." (,Unterredungen und mathematische Demonstrationen über zwei neue Wissenszweige ...", hrsg. A. v. .Oettingen, Darmstadt 1964, S. 147). Zur Bestätigungschance einfacher Hypothesen vgl. Quine (63). 
dann, wenn verschiedene Erkenntnisideale miteinander in Wettstreit treten. Es gilt aber auch für die ersten drei Auswahlkriterien. Weniger informative oder umfassende Theorien sind oft einfacher als informative und umfassende.

Die Vorstellung, es gäbe nur ein Auswahlkriterium für wissenschaftliche Theorien: die Wahrheit, ist zu einfach. Wissenschaftliche Theorien sind Antworten auf Fragen, die aus gewissen Interessenlagen heraus gestellt werden. Wir sind nicht an beliebigen Wahrheiten interessiert, sondern an bestimmten. Theorien sollen natürlich richtig sein, und in der wissenschaftlichen Arbeit geht es sicher um Erkenntnis der Wahrheit, aber gerade dann, wenn man an Wahrheit und Objektivität interessiert ist, wird man sich auch Rechenschaft über die Reichweite von Wahrheitskriterien geben und über den Einfluß von kognitiven und praktischen Interessen auf die Gestaltung der Wissenschaft; über die eigenen Erkenntnisinteressen und über die der wissenschaftlichen Tradition, in der man steht.

Kant hat den Unterschied zwischen Aussagen, die sich rein theoretisch begründen lassen, und jenen, die sich auf ein Interesse der Vernunft stützen, entschieden durchgehalten, glaubte aber an eine praktische, d.h. moralische Legitimierbarkeit theoretischer Überzeugungen. Unabhängig davon, ob man ihm in diesem Punkt folgen will, muß man jedenfalls sagen, daß er die Bedeutung praktischer Fragen für theoretische klar erkannt hat. Die Rolle, die dieses Problem in seinem Denken spielte, ergibt sich schon daraus, daß er sich in allen drei Kritiken damit auseinandergesetzt hat.

\subsection{Holismus}

Wir haben in den letzten Abschnitten - wenn auch nur in sehr groben Zügen - ein Bild der Erfahrungserkenntnis entworfen. Die wichtigsten Aussagen wollen wir uns nun noch einmal im Zusammenhang vor Augen stellen. Sie lassen sich sämtlich als Gegenthesen zum Empirismus auffassen.

1. Wir haben gegenüber dem Empirismus die Kreativität wissenschaftlicher Arbeit betont, den kreativen Charakter der Begriffsbildung wie des Entwurfs von Theorien. Das historische Verdienst des Empirismus ist es, mit seinem Insistieren auf Erfahrung und Logik als Kontrollinstanzen des Denkens der ,Wolken-Philosophie“ unbe- 
gründeter und unpräzisierter Meinungen, wie A. Einstein sie nannte, kräftig entgegengewirkt $\mathrm{zu}$ haben. Als wissenschaftstheoretische Konzeption mit Monopolanspruch bedeutet er aber eine nicht minder große Gefahr für den Erkenntnisfortschritt als diese Wolken-Philosophie. Darauf hat Einstein ebenfalls hirgewiesen, und zwar zu siner Zeit, in welcher der logische Empirismus noch in voller Blüte stand. Er schrieb: ,... die in unserem Denken und in unseren sprachlichen Äußerungen auftretenden Begriffe sind alle - logisch betrachtet frei Schöpfungen des Denkens und können nicht aus den Sinnes-Erlebnissen induktiv gewonnen werden. Dies ist nur deshalb nicht so leicht zu bemerken, weil wir gewisse Begriffe und Begriffs-Verknüpfungen (Aussagen) gewohnheitsmäßig so fest mit gewissen Sinnes-Erlebnissen verbinden, daß wir uns der Kluft nicht bewußt werden, die - logisch unüberbrückbar - die Welt der sinnlichen Erlebnisse von der Welt der Begriffe und Aussagen trennt. - So ist z. B. die Reihe der ganzen Zahlen offenbar eine Erfindung des Menschengeistes, ein selbstgeschaffenes Werkzeug, welches das Ordnen gewisser sinnlicher Erlebnisse erleichtert. Aber es gibt keinen Weg, um diesen Begriff aus den Erlebnissen selbst gewissermaßen herauswachsen zu lassen. Ich wähle hier gerade den Begriff der Zahl, weil er dem vorwissenschaftlichen Denken angehört und an ihm der konstruktive Charakter trotzdem noch leicht erkennbar ist. Je mehr wir uns aber den primitivsten Begriffen des Alltags zuwenden, desto mehr erschwert es uns die Masse eingewurzelter Gewohnheiten, den Begriff als selbständige Schüpfung des Denkens zu erkenrren. So kornnce die für das Verständnis der hier obwaltenden Verhältnisse so verhängnisvolle Auffassung entstehen, daß die Begriffe aus den Erlebnissen durch ,Abstraktion", d. h. durch Weglassen eines Teils ihres Inhaltes, entstehen. Ich will nun zeigen, warum mir diese Auffassung so verhängnisvoll erscheint. - Hat man sich einmal Humes Kritik zu eigen gemacht, so kommt man leicht auf den Gedanken, es seien aus dem Denken alle jene Begriffe und Aussagen als ,,metaphysische“ zu entfernen, welche sich nicht aus dem sinnlichen Roh-Material herleiten lassen. Denn alles Denken erhält materialen Inhalt durch nichts anderes als durch seine Beziehung zu jenem sinnlichen Material. Letzteres halte ich für völlig wahr, die darauf gegründete Vorschrift für das Denken aber für falsch. Denn dieser Anspruch - wenn er nur völlig konsequent durchgeführt wird - schließt überhaupt jedes Denken als ,,metaphysisch“ aus. - Damit Denken nicht in „Metaphysik“, bzw. in leeres 
Gerede ausarte, ist es nur notwendig, daß genügend viele Sätze des Begriffssystems mit Sinnes-Erlebnissen hinreichend sicher verbunden seien und daß das Begriffssystem im Hinblick auf seine Aufgabe, das sinnlich Erlebte zu ordnen und übersehbar zu machen, möglichste Einheitlichkeit und Sparsamkeit zeigte... Dies alles gilt in gleicher Weise für das Denken des Alltags wie für das mehr bewußt systematisch gestaltete Denken in den Wissenschaften. - Es wird nun klar sein, was gemeint ist, wenn ich Folgendes sage: Hume hat durch seine klare Kritik die Philosophie nicht nur entscheidend gefördert, sondern ist ihr auch ohne seine Schuld zur Gefahr geworden, indem durch diese Kritik eine verhängnisvolle „Angst vor der Metaphysik“ ins Leben trat, die eine Krankheit des gegenwärtigen empirizistischen Philosophierens bedeutet; diese Krankheit ist das Gegenstück zu jenem früheren Wolken-Philosophieren, welches das sinnlich Gegebene vernachlässigen und entbehren zu können glaubte ". 80

Die Kreativität der Theorienbildung hat, wie wir schon sahen, auch Popper besonders betont. Theorien oder Hypothesen sind für ihn nie Folgerungen aus Beobachtungen, sondern Vorbedingungen für systematische experimentelle Untersuchungen. Auf die Kreativität der Begriffsbildung hat auch Quine hingewiesen. Er sagt: "Physical objects are conceptually imported into the situation as convenient intermediaries - not by definition in terms of experience, but simply as irreducible posits comparable, epistemologically, to the gods of Homer. For my part I do, qua lay physicist, believe in physical objects and not in Homer's gods; and I consider it a scientific error to believe otherwise. But in point of epistemological footing the physical objects and the gods differ only in degree and not in kind. Both sorts of entities enter our conception only as cultural posits. The myth of physical objects is epistemologically superior to most in that it has proved more efficacious than other myths as a device for working a manageable structure into the flux of experience. - Positing does not stop with macroscopic physical objects. Objects at the atomic level are posited to make the laws of macroscopic objects, and ultimately the laws of experience, simpler and more manageable; and we need not expect or demand full definition of atomic and subatomic entities in terms of macroscopic ones, any more than definition of macroscopic things in terms of sense data. Science is a continuation of common sense, and it ${ }^{80}$ Einstein (44), $286 \mathrm{f}$. 
continues the common-sense expedient of swelling ontology to simplify theory". ${ }^{81}$

Gegenüber der phänomenalistischen Konzeption, die in diesen Aussagen zum Ausdruck kommt, ist freilich zu betonen, daß Sinnesempfindungen keine von begrifflichen Interpretationen und hypothetischen Annahmen freie Basis empirischer Erkenntnis darstellen; daß sie nicht primär gegenüber den physikalischen Objekten sind oder etwas unmittelbar Gegebenes darstellen. Quine selbst hat ja betont, daß solche Sinnesdaten nur Abstraktionen aus Wahrnehmungen sind, also Konstrukte, und daß wir über sie mit Begriffen reden, die der Dingsprache entnommen sind. ${ }^{82}$

Der Hinweis auf den kreativen Charakter von Begriffs- und Theorienbildung in den empirischen Wissenschaften unterstreicht noch einmal unsere allgemeinen Aussagen zur Kreativität des Wirklichkeitsverständnisses im 8. Kapitel.

2. Gegen den Empirismus haben wir ferner die enge Verbindung zwischen Sprachkonstruktion und Theorienbildung hervorgehoben. Der Aufbau und die semantische Charakterisierung einer Sprache ist nicht ein Prozess, der sich von der Formulierung von Aussagen über die Welt in dieser Sprache abtrennen ließe. Darauf hat vor allem wieder Quine mit seiner Kritik an der Unterscheidung analytischer und synthetischer Säize hingewieseiı. Dié Theoriebeladenheii der Sprache zeigt sich besonders deutlich bei der Einführung theoretischer Terme, sie wird aber auch in der semantischen Charakterisierung der Beobachtargssprache deutlich. Umgckehrt lïnnite mar alich von einer „Sprachbeladenheit von Theorien“" oder von einer „Sprachbeladenheit der Erfahrung" reden: Sprachen sind keine Beschreibungsmittel für vorgegebene Objekte, Attribute und Sachverhalte, sondern Mittel der Unterscheidung, wie wir in 7.4 gesehen haben. Die Grenzen der Ausdrucksfähigkeit einer Sprache bestimmen nicht nur die Grenzen dessen, worüber wir reden können, sondern auch die Grenzen dessen, was wir begreifen können, und in gewissem Maße auch die Grenzen unserer Erfahrung. Theorien als Systeme von Aussagen können natürlich nur Informationen enthalten, die sich in der Sprache ausdrükken lassen, in der sie formuliert sind. Darüber hinaus gehen in sie

${ }^{81}$ Quine (51), $44 \mathrm{f}$.

${ }^{82} \mathrm{Vgl}$. dazu den Abschnitt 5.3. 
aber auch die Sätze ein, welche die Bedeutungen der Terme bestimmer, und das sind nicht immer nur Aussagen, die sich als ,,rein analytisch" bezeichnen ließen.

3. Wir haben der empiristischen Ansicht widersprochen, in der Erfahrung ließe sich etwas unmittelbar Gegebenes isolieren, es gäbe mit hypothetischen Elementen nicht belastete Beobachtungsdaten, die als feste Basis für die Begründung aller hypothetischen Aussagen dienen können. Eine phänomenalistisch verstandene Basis würde zur Begründung von Aussagen der Dingsprache nicht ausreichen, und selbst einfachste Beobachtungssätze sind schon ,theoriebeladen“, d. h. sie beinhalten mehr als sich durch Beobachtungen definitiv verifizieren läßt. Das hat schon Popper betont, für den Beobachtungssätze, bzw. seine Basissätze, nur relativ unproblematisch sind, sich grundsätzlich aber immer als falsch erweisen können. Für ihn ergibt sich das auch daraus, daß die experimentellen Befunde in den Naturwissenschaften beliebig reproduzierbare Effekte sein müssen, also streng genommen selbst schon universelle Aussagen sind: Immer, wenn man im Experiment die und die Anfangs- und Randbedingungen herstellt, wird man das und das beobachten. ${ }^{83}$ Zwischen Beobachtungen und Hypothesen besteht kein einseitiges Begründungsverhältnis, so daß jene immer nur als begründende, diese als begründete Aussagen auftreten, sondern ein Verhältnis wechselseitiger Begründung, eine Rückkopplung zwischen Beobachtungen und Theorien. Das gilt insbesondere dann, wenn Beobachtungen mithilfe von Meßinstrumenten vorgenommen werden und die Deutung der Meßergebnisse sich auf Theorien stützt. 4. Gegen die empiristische These, Beobachtungen seien hinreichend für die Begründung von Theorien haben wir ferner eingewendet, daß induktive Begründungen auf subjektiven apriori-Wahrscheinlichkeiten beruhen, daß also unsere empirischen Annahmen sich nicht nur auf Beobachtungen stützen, sondern auch auf Voraussetzungen und Erwartungen. Das gilt zunächst für generelle Hypothesen und andere Aussagen, die sich durch vergangene Beobachtungen nicht verifizieren lassen. Wegen des hypothetischen Charakters der Beobachtungssätze gilt es aber auch für diese: Auch das, was wir aufgrund unseres Erlebens über dessen Gegenstand annehmen, beruht auf vorgängigen Überzeugungen und Erwartungen. Diese Erwartungen werden ihrer-

${ }^{83} \mathrm{Vgl}$. dazu (66), 19; diese Ausführungen haben wir schon auf S. 287 zitiert. 
seits aber durch Beobachtungen modifiziert, so daß auch hier ein Prozess wechselseitiger Adaption vorliegt.

5. Im letzten Abschnitt haben wir gegenüber instrumentalistischen Begrenzungen kognitiv sinnvoller Aussagen betont, daß man auch Theorien und Aussagen mit thecretischen Termer einen kognitiven Gehalt nicht absprechen kann, wenn man nicht alle Aussagen über die Natur als kognitiv sinnlos erklären will. Eine zugleich scharfe und allgemeine Abgrenzung zwischen empirischen und ,metaphysischen“ Aussagen erwies sich damit als unmöglich. Für die Auswahl der theoretischen Konstrukte, mit denen empirische Phänomene zu erklären sind, gibt es keine rein empirischen oder logischen Kriterien. Empirische Theorien müssen, wie Popper betont hat, an der Erfahrung scheitern können, sie müssen durch Beobachtungen überprüft werden können, sie dürfen nicht immun gegenüber aller Erfahrung sein; aber zugleich präzise und allgemeine Kriterien dafür, wann eine Theorie durch Beobachtungen falsifiziert ist, gibt es nach dem Duhem-QuineArgument nicht. Auch hier gilt: Die Schlüsse, die wir aus unseren Beobachtungen ziehen, hängen vom Gesamtsystem unserer Annahmen und Erwartungen ab.

Dieses Bild von der Struktur der Erfahrungserkenntnis ist sehr viel komplexer als jenes, das der Empirismus entwirft. Es ist in der Tat so komplex, daß danach alies mit allem zusammenzuhängen scheint und man nicht mehr sieht, wie diese Interdependenzen sich entwirren und in eine geordnete Systematik bringen lassen sollen. Uns ging es hier

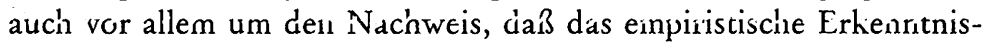
modell zu einfach ist. Bestehen die von uns behaupteten Interdependenzen, so ist eine systematische Rekonstruktion des wissenschaftlichen Erkenntnisprozesses inadäquat, nach der er sich als Folge von Schritten darstellt, bei der jeder Schritt von den späteren unabhängig und in sich gesichert ist. Präzisere Analysen, als wir sie gegeben háben, sind natürlich erwünscht und sicher auch möglich. Dabei wird man aber sehr viel genauer auf die einzelnen Theorien eingehen müssen und auf ihre Entwicklung; man wird weniger pauschale Aussagen machen können als das in der allgemeinen Wissenschaftstheorie üblich ist.

Die Konzeption der Struktur der Erfahrungserkenntnis, die wir hier skizziert haben, kann man als bolistisch bezeichnen. Ohne nähere Erläuterung besagt das freilich wenig, denn „Holismus“ ist eine Sam- 
melbezeichnung für verschiedene Behauptungen, insbesondere

a) für die These von der Theoriebeladenheit der Sprache, speziell der Beobachtungssprache,

b) für die These von der Theoriebeladenheit der Beobachtungssätze, sei es als Behauptung, es gebe keine Sätze, deren Wahrheitsbedingungen unabhängig von allen Theorien sind, sei es in Form der stärkeren Behauptung, es gebe für keine Theorie für sie relevanten Sätze, deren Wahrheitsbedingungen unabhängig von ihr selbst sind. ${ }^{84}$

c) für die Dubem-Quine-These, nach der sich nicht einzelne Hypothesen oder Theorien durch Beobachtungen falsifizieren lassen, sondern nur größere Komplexe von Theorien und Annahmen über die Welt, und nach der es daher auch kein experimentum crucis für Theorien gibt, kein definitives Scheitern von Theorien an der Erfahrung.

d) für die These vom Einfluß nicht empirischer Kriterien bei der Auswahl von Theorien.

Diese vier Behauptungen hängen inhaltlich miteinander zusammen. Meist wird als zentrale Aussage des Holismus die Duhem-QuineThese angesehen. So unterscheidet I. Lakatos in (70) zwischen einem scbwachen und einem starken Holismus, der sich aus einer schwachen, bzw. starken Interpretation dieser These ergibt. Bei der scbwachen Deutung besagt sie, ein System von Annahmen, die zur Voraussage eines Beobachtungsergebnisses notwendig sind, könne nur in seiner Gesamtheit an dieser Voraussage scheitern. Das ist eine logische Trivialität und wird auch von Lakatos als solche anerkannt; der einzig interessante Gedanke besteht im Hinweis, daß Voraussagen sich nie allein auf einzelne Hypothesen oder Theorien stützen, sondern immer auch auf andere Theorien oder auf Hintergrundannahmen. Nach der starken Deutung der Duhem-Quine-These gibt es keine rationalen Auswahlkriterien für mögliche Modifikationen des Annahmesystems, die es mit widersprechenden Beobachtungstatsachen in Einklang bringen. Diese Behauptung versucht Lakatos zu widerlegen, indem er Poppers ,naiven“ Falsifikationsbegriff durch eine ,sophistizierte“ Version ersetzt: Danach wird, kurz gesagt, eine Theorie immer nur

${ }^{84}$ Zwischen den Thesen (a) und (b) wird oft nicht unterschieden. Sie hängen natürlich inhaltlich zusammen, sind aber nicht identisch. Beide Thesen haben z. B. Feyerabend in (60), Kuhn in (62), Putnam in (62) und Hempel in (73) vertreten. Für sie entfällt damit die Voraussetzung für die Unterscheidung Beobachtungssprache - Theoretische Sprache. 
durch eine andere Theorie falsifiziert. Gerät also eine Theorie $\mathrm{T}$ in Konflikt mit Beobachtungen, so gilt $T$ erst dann als durch diese Beobachtungen falsifiziert, wenn schon eine andere Theorie T' bereitsteht, die T ersetzen kann. Dazu muß T' erstens dasselbe leisten, was T bisher geleistet hat, $d$. h. aus $T$ ' müsser sich jenc VCraussager mit $T$ ergeben, die sich als richtig erwiesen haben. Zweitens muß T' die fraglichen Beobachtungen erklären können, und drittens muß T' neue Voraussagen ermöglichen und einige davon müssen sich bereits als richtig erwiesen haben; T' muß sich also schon bewährt haben. Die Ersetzung von $T$ durch $T^{\prime}$ 'ist dann rational gerechtfertigt, weil sich $T^{\prime}$ bewährt hat und zwar auch dort, wo das für $T$ nicht gilt. Es ist nun zwar richtig, daß man eine Theorie in der Regel nur dann aufgeben wird, wenn eine Alternativtheorie verfügbar ist. Aber das Duhem-Quine Problem bestand darin, daß es mehrere Alternativen zur Änderung eines Systems von Annahmen gibt, wenn es an der Erfahrung scheitert. Man kann die Theorie ändern oder die Hintergrundannahmen. Die Änderung muß sich natürlich bewähren, aber das ist trivial. Der nichttriviale Inhalt des Hinweises von Lakatos - der sich freilich auch bei Popper findet - besteht darin, daß es in der Regel nicht so einfach ist, hinreichend allgemeine und plausible Theorien zu entwerfen. In vielen Fällen bietet sich nur eine Alternative an. In dem in 9.4 erwähnten Beispiel der nach der Newtonschen Mechanik nicht erwarteten Perihelbewegung des Merkur versuchte man es so lange mit ad-hocAnnahmen, bis in der Relativitätstheorie eine echte theoretische Alternative zur klassischen Mechanik zur Verfügung stand. ${ }^{8 ;}$

Auch Stegmüller geht bei seiner Diskussion des Holismus ${ }^{86}$ von der Duhem-Quine-These aus. Er unterscheidet, z. T. in Anlehnung an Sneed (71), vier holistische Thesen: Die Behauptungen, daß eine

${ }^{85}$ Lakatos hat in (70) auch eine Methodologie wissenschaftlicher Forschungsprogramme entwickelt. Danach müssen alle vorgeschlagenen Änderungen einer Theorie in ein ,fortschrittliches Forschungsprogramm" hineinpassen, in eine Folge von Theorien, von denen jede empirisch gehaltvoller ist als die vorhergehende. Wegen der vielen Einwände, die gegen diesen Gedanken vorgebracht wurden, wollen wir hier nicht näher darauf eingehen. Vgl. dazu z. B. Feyerabend (70), Kuhn (72) und Smart (72). Insbesondere ist zu betonen, daß auch Lakatos keine Kriterien dafür angibt, wie lange es sinnvoll ist, an einem Forschungsprogramm festzuhalten.

${ }^{86}$ Vgl. Stegmüller (70), 2. Halbbd. Kap. IX, 8. 
Theorie nur als Ganzes akzeptiert oder verworfen wird, und daß es keine Verwerfung von Theorien aufgrund eines experimentum crucis gibt, charakterisieren für ihn den gemäßigten Holismus. ${ }^{87}$ Der Holismus im strengen Sinn wird darüber hinaus erstens durch die Behauptung charakterisiert, daß es keine von der Theorie unabhängigen Entsch€idungskriterien für die Geltung jener Beobachtungssätze gibt, die diese Theorie stützen. Die empirischen Daten, die für eine Theorie relevant sind, werden im Licht dieser Theorie selbst interpretiert. Mit den Worten Kuhns: „... theories do not evolve piecemeal to fit the facts that were there all the time. Rather, they emerge together with the facts they fit...". ${ }^{88}$ So etwas kommt z. B. vor, wenn die Werte physikalischer Größen durch eine Anwendung der Theorie bestimmt werden und dann in einer anderen Anwendung derselben Theorie zu Erklärungszwecken herangezogen werden. Zweitens wird der strenge Holismus dadurch charakterisiert, daß sich die Bedeutungen der theoretischen Terme einer Theorie mit deren Anwendungsbereich ändern. ${ }^{89}$

Die entscheidenden Anstöße zur Durchsetzung holistischer Ideen sind von Quine und von T.S. Kuhn ausgegangen. Nach Quine können wir nicht einzelne Sätze oder Theorien mit Beobachtungen konfrontieren, sondern im Prinzip immer nur das Gesamtsystem unserer Annahmen über die Welt. Die Annahmen in diesem System sind durch ein dichtes Netz von Abhängigkeiten so eng miteinander verbunden, daß eine Beobachtung, die im Konflikt zu diesem System steht, nie nur einen bestimmten Satz daraus in Frage stellt. Quine sagt: "The totality of our so-called knowledge or beliefs, from the most casual matters of geography and history to the profoundest laws of atomic physics or even of pure mathematics and logic, is a man-made fabric

${ }^{87}$ Den starken wie den schwachen Holismus im Sinn von Lakatos rechnet er dabei zu diesem gemäßigten Holismus.

${ }^{88}$ Kuhn (62), 140.

${ }^{89}$ Beide Aussagen beziehen sich auf den Sneed-Stegmüllerschen Theoriebegriff. Stegmüller interpretiert sie so, daß sie richtig werden. Sieht man verschiedene Anwendungen derselben Theorie als verschiedene Theorien an, so würde die erste Bestimmung nur auf Theorien zutreffen, die immun gegenüber Erfahrungen sind, denen also nach Popper der Charakter der Wissenschaftlichkeit fehlt. Die zweite Bestimmung gilt dann bei einer theorienübergreifenden Interpretation theoretischer Terme. 
which impinges on experience only along the edges. Or, to change the figure, total science is like a field of force whose boundary conditions are experience. A conflict with experience at the periphery occasions readjustments in the interior of the field. Truth values have to be redistributed over some of our statements. Reevaluation of some statements entails reevaluation of others, because of their logical interconnections - the logical laws being in turn simply certain further statements of the system, certain further elements of the field. Having reevaluated one statement we must reevaluate, some others, which may be statements logically connected with the first or may be the statements of logical connections themselves. But the total field is so underdetermined by its boundary conditions, experience, that there is much latitude of choice as to what statements to reevaluate in the light of any single contrary experience. Not particular experiences are linked with any particular statements in the interior of the field, except indirectly through considerations of equilibrium affecting the field as a whole ... For vividness I have been speaking in terms of varying distances from a sensory periphery. Let me try now to clarify this notion without metaphor. Certain statements, though about physical objects and not sense experience, seem peculiarly germane to sense experience - and in a selective way: some statements to some experiences, orhers to others. Such statements, especially gcrmane to particular experiences, I picture as near the periphery. But in this relation of "germaneness" I envisage nothing more than a loose association reflecting the relative likelihood, in practice, of our choosing one statement rather than another for revision in the event of recalcitrant experience...

A recalcitrant experience can, I have urged, be accommodated by any of various alternative reevaluations in various alternative quarters of the total system; but, in the cases which we are now imagining, our natural tendency to disturb the total system as little as possible would lead us to focus our revisions upon ... specific statements ... These statements are felt, therefore, to have a sharper empirical reference than highly theoretical statements of physics or logic or ontology. The latter statements may be thought of as relatively centrally located within the total network, meaning merely that little preferential connection with any particular sense data obtrudes itself". 90

${ }^{90}$ Quine (51), 42 - 44. Vgl. auch Quine (74). 
Wenn Quine auch logische Gesetze und sprachliche Konventionen zu jenen Annahmen rechnet, die für eine Modifikation angesichts widerstreitender Erfahrungen infrage kommen, so ist das zwar fragwürdig, es tangiert aber seinen Grundgedanken nicht. ${ }^{91}$ Danach richten wir uns bei solchen Modifikationen nach Kriterien der Einfachheit und.dem Gesichtspunkt, keine zu radikalen Änderungen vorzunehmen. Andere Kriterien, wie jene von Popper und Lakatos sind damit nicht ausgeschlossen. Auswahlkriterien - oder genauer: Kriterien für eine Neubewertung unserer Annahmen im Licht neuer Erfahrungen ergeben sich auch, wenn man das Annahmesystem nicht als Menge von Überzeugungen versteht, sondern im Sinn früherer Bemerkungen als bedingte Wahrscheinlichkeitsbewertung auf einer Menge empirischer Sachverhalte. Eine solche Bewertung ändert sich mit neuen Beobachtungen, und aus ihr ergibt sich, welche Annahmen dabei wahrscheinlicher und welche weniger wahrscheinlich werden. Ein solches Modell ermöglicht erheblich genauere Unterscheidungen als das Quinesche.

Kuhn hat in (62) anhand historischer Beispiele gezeigt, daß die übliche Vorstellung von der Entwicklung der Wissenschaften als einem kumulativen Prozeß der Vermehrung von Wissen durch Sammlung von Fakten und durch Formulierung immer exakterer und umfassenderer Theorien nicht haltbar ist. Perioden normaler Wissenschaft, auf die ein solches Modell zutrifft, werden vielmehr immer wieder von wissenschaftlichen Revolutionen unterbrochen, auf die es nicht anwendbar ist. Ein normales Stadium wissenschaftlicher Entwicklung ist nach Kuhn gekennzeichnet durch das Vorhandensein eines Paradig-

${ }^{91}$ Kommt unter unseren Annahmen der Satz vor „Alle Schwäne sind weiß“, so werden wir sie nicht dadurch in Übereinstimmung mit einem Beobachtungssatz „Dieser Schwan ist schwarz" bringen, daß wir ,,alle“ in ,einige“ oder „,schwarz" in ,,weiß" umdeuten. Logik und Sprache definieren den Rahmen, in dem man erst Annahmen und Beobachtungen ausdrücken und von Beziehungen der Folge und des Konflikts reden kann. Das Problem widersprechender Beobachtungen und einer Modifikation der Annahmen, die diese damit in Einklang bringt, stellt sich erst in einem solchen Rahmen; man würde es also verfehlen, wenn man auch ihn als disponibel ansähe. Das heißt freilich nicht, daß wir nicht auch unsere Logik oder unsere Sprache ändern könnten oder daß solche Änderungen nicht gelegentlich sinnvoll wären. Sie werden aber nie durch einzelne Beobachtungen motiviert. 
mas. Ein Paradigma umfaßt eine Theorie, Modellfälle ihrer erfolgreichen Anwendung, metaphysische Hintergrundannahmen über den Gegenstandsbereich der Theorie und methodologische Normen für Begründungen und experimentelle Untersuchungen. Hintergrundannahmen und Normen sind dahei, wie Kuhn betont; nicht explizit formuliert und die intendierten Anwendungen der Theorie sind nicht exakt abgegrenzt, sondern nur paradigmatisch bestimmt durch die Modellfälle erfolgreicher Anwendungen.92 Wenn hier von Theorien und intendierten Anwendungen die Rede ist, hat man an einen Theoriebegriff wie den von Sneed und Stegmüller zu denken. Ein solches Paradigma definiert Methoden zur Lösung von Problemen, die sich in einigen wichtigen Fällen bewährt haben, und die normale Wissenschaft besteht darin, neue Probleme mithilfe dieser Methoden zu lösen, die Methoden zu verfeinern oder mithilfe neuer Spezialgesetze den Anwendungsbereich der Theorie zu vergrößern. Sie ist also, wie Kuhn sagt, eine Tätigkeit des puzzle solving, nicht jedoch eine Konfrontation der Theorie mit der Erfahrung, eine Prüfung ihrer Gültigkeit. In der normalen Wissenschaft wird die Theorie nicht als eine zu testende Hypothese angesehen, sondern als ein Instrument zur Lösung von Problemen. Sie ist nicht Gegenstand, sondern Grundlage der Untersuchungen. ${ }^{93}$

Für jedes Paradigina gibt es ungelöste Probleme, d. 1. Phänurıene, die sich einer erfolgreichen Beschreibung oder Erklärung mithilfe der Theorie entziehen - Kuhn spricht von Anomalien. In der normalen $\mathrm{W}^{\prime}$ issenschaft werder sie jedoch nicht ais Irıdizien für die Unzulänglichkeit des Paradigmas gewertet, sondern als prinzipiell lösbare Probleme. Ungelöste Probleme sind in diesem Stadium nicht Herausforderungen an das Paradigma, sondern an die Geschicklichkeit der Wissenschaftler bei seiner Anwendung. Probleme oder Problemgruppen, die sich hartnäckig einer Lösung mithilfe des Paradigmas entziehen, können aber mit der Zeit das Bewußtsein wecken, daß das Para-

92 Vgl. Kuhn (62), 44. - Eine Reflexion auf die Normen und Hintergrundannahmen und ihre explizite Formulierung stellen nach Kuhn meist schon Schritte auf dem Weg zu ihrer Kritik, zu ihrer Ablösung dar, oder ein Symptom für eine Krise (vgl. a.a.O. S. 88).

${ }^{93}$ Eine Theorie im Sinne von Sneed ist in der Tat keine Aussage, die man falsifizieren könnte. Aussagen sind nur Behauptungen, daß gewisse intendierte Modelle tatsächlich Modelle der Theorie sind. 
digma für ihre Erklärung unbrauchbar ist. Dadurch gerät es in eine Krisc. In dieser Krise erhalten neue Theorien, bzw. Paradigmen eine Chance, die sich für eine Lösung der Probleme anbieten. Die Krise bewirkt eine Bereitschaft unter den Wissenschaftlern, eine neue Theorie zu akzeptieren, mit einem neuen Paradigma zu arbeiten. ${ }^{94}$

Einen solchen Paradigmenwechsel bezeichnet Kuhn als eine wissenschaftliche Revolution, denn solche Vorgänge haben für ihn eine gewisse Ähnlichkeit mit politischen Revolutionen. Eine politische Revolution entsteht aus dem wachsenden Gefühl, daß die alten Institutionen, politischen Lebensformen und Wertvorstellungen neuen sozialen oder wirtschaftlichen Bedingungen oder veränderten Zielvorstellungen nicht mehr angemessen sind. Ihnen wird dann der Entwurf einer neuen politischen Ordnung entgegengestellt, der mit den alten Vorstellungen unvereinbar ist. Damit stellt sich eine tiefgreifende politische Polarisierung ein. Es gibt keine gemeinsame Basis mehr für die Entscheidung, welche von beiden Ordnungen besser ist. Jede Partei argumentiert von ihrem politischen Paradigma aus und so kann sich die neue Ordnung gegenüber der alten nur durch Konversion oder Gewalt durchsetzen. ${ }^{95}$ Ähnlich sieht Kuhn den wissenschaftlichen $\mathrm{Pa}$ radigmawechsel. Zunächst ist die Krise des alten Paradigmas die Vorbedingung für die Entwicklung eines neuen. Es breitet sich das Gefühl aus, daß das alte Paradigma gewisse wichtige Probleme nicht lösen kann, daß eine bloße Evolution oder Modifikation, wie sie sich auch im Rahmen der normalen Wissenschaft vollziehen kann, nicht geeignet ist, mit den Problemen fertig zu werden, sondern daß neu angesetzt werden muß, daß nur ein neues Paradigma die Anomalien lösen kann. Im Sinn des Kuhnschen Paradigmabegriffs bedeutet dabei ein Paradigmenwechsel nicht nur den Übergang von einer Theorie zu einer anderen, sondern oft auch eine Änderung der wissenschaftlichen Sprache, der Hintergrundannahmen oder der methodologischen Normen. ${ }^{96}$ Für Kuhn stellt ein Paradigmenwechsel insgesamt eine grund-

${ }^{94}$ Kuhn betont, daß keine Bereitschaft besteht, eine neue Theorie anzunehmen, solange die alte funktioniert; daß also neue Ideen nur in einer Krise eine Chance bekommen. Vgl. a.a.O. S. $75 \mathrm{f}$.

${ }^{95}$ Vgl. a.a.O. S. $92 \mathrm{f}$.

${ }^{96}$ Ein Paradigmenwechsel kann auch eine Änderung der Vorstellungen darüber bewirken, was, ,wissenschaftlich“ ist. Vgl. dazu a.a.O. S. $104 \mathrm{ff}$. 
legende Änderung der Auffassungen dar, die er mit dem psychologischen Phänomen des Gestaltwandels vergleicht. Die Phänomene werden dabei anders gesehen als vorher, so wie man ein Bild einmal als Darstellung einer Ente, das andere mal als Darstellung eines Hasen auffassen kann 97 Der Paradigmenwechse! öffnet den Blick für neue Phänomene. Man sieht nun die Welt anders und beschreibt die Fakten in nicht äquivalenten Weisen. Es handelt sich dabei nach Ansicht Kuhns nicht nur um eine neue Interpretation derselben Beobachtungsdaten, denn Beobachtungen sind nicht theorienunabhängig; es gibt nicht das neutrale, identische Gegebene. ${ }^{98}$ Zwischen den beiden Paradigmen kann man also nicht durch Berufung auf theorienunabhängige Beobachtungen entscheiden. Ja es gibt oft nicht einmal eine gemeinsame methodologische Basis für diese Entscheidung, keine gemeinsamen Begründungsmaßstäbe. Eine Revolution wird nicht durch Experimente und Logik entschieden: "The competition between paradigms is not the sort of battle that can be resolved by proofs". 99

Kuhn betont gegenüber Popper, daß Theorien oder Paradigmen nicht durch Erfahrung falsifiziert werden. ${ }^{100}$ Jede Theorie hat ihre ungelösten Probleme: Phänomene, die man mit ihr (noch) nicht erklären kann oder die man mit ihr (noch) nicht in Einklang bringen kann. Im Zustand der normalen Wissenschaft wird das jedoch nicht als Arguınerit yegen die Theorie gewerce, sondern man spricht von noch ungelösten Problemen. Erst von einem neuen Paradigma aus werden solche Fälle als Widerlegungen des alten angesehen. Die Ablehnung cires Paradiginas beruht nicht auf eineri Vergleich mit der Naiur als solcher, sondern auf dem Vergleich mit der Natur, wie sie ein anderes Paradigma darstellt. ${ }^{101} \mathrm{Kuhn}$ betont auch, daß wissenschaftliche Re-

${ }^{97}$ Vgl. a.a.O. Kap. X. So ein Bild findet sich z. B. in Wittgenstein (53), S. 228.

${ }^{98}$ A.a.O. S. $120 \mathrm{ff}$. Kuhn bezieht sich dabei auf die These von der Theoriebeladenheit von Beobachtungen, vgl. a.a.O. S. 7, 52. Für ihn beruht die Idee vom wissenschaftlichen Fortschritt als einem kumulativen Prozess auf dem empiristischen Erkenntnismodell, nach dem unser Wissen eine geistige Konstruktion auf der Basis nackter, von Interpretationen und Hypothesen unbelasteter Sinnesdaten ist (vgl. dazu a.a.O. S. 96).

${ }^{99} \mathrm{Vgl.} \mathrm{a.a.O.} \mathrm{S.} 148$.

${ }^{100}$ Vgl. a.a.O. Kap. VIII.

${ }^{101}$ A.a.O. S. 77. Kuhn sagt, es sei oft erst die Art und Weise, wie sich ein Phänomen in einer neuen Theorie darstellt, die es zum Gegenbeispiel für eine andere macht. Ferner trifft jedes Paradigma eine Auswahl unter den empiri- 
volutionen sich nicht im Sinne von elementaren Kriterien wie Einfachkeit oder höhere Leistungsfähigkeit als Fortschritt deuten lassen. Die neue Theorie ist zunächst oft weniger exakt formuliert als die alte, ist nicht einfacher und hat oft weniger Probleme gelöst als diese. ${ }^{102}$ Die alte Theorie läßt sich auch in der Regel nicht auf die neue reduzieren. Wenn man sagt, die Newtonsche Mechanik lasse sich approximativ (für Geschwindigkeiten, die gegenüber der Lichtgeschwindigkeit klein sind) auf die relativistische Mechanik reduzieren, so verkennt man, meint Kuhn, daß dabei Terme wie „Raum“, „Zeit“ und ,Masse“ umgedeutet werden. ${ }^{103}$ Insofern sind die konkurrierenden Paradigmen inkommensurabel. Daher gibt es keinen Punkt, an dem der Widerstand gegen ein neues Paradigma unlogisch oder unwissenschaftlich würde. ${ }^{104}$

Das Fazit Kuhns ist also, daß es keine objektiven empirischen oder logischen Kriterien gibt, nach denen man einen Paradigmenwechsel als Fortschritt deuten kann, sondern daß er sich immer nur vom Standpunkt des Siegers aus als Fortschritt darstellt. Fortschritt im kumulativen Sinn gibt es insbesondere nur innerhalb eines Paradigmas, d. h. in der normalen Wissenschaft, nicht jedoch, wenn man wissenschaftliche Revolutionen einbezieht. Daher muß man die Vorstellung vom wissenschaftlichen Fortschritt als einem teleologischen Prozeß aufgeben, der immer näher zur Wahrheit hinführt. Kuhn zeichnet

schen Phänomenen nach ihrer Wichtigkeit. Das Interesse konzentriert sich auf gewisse Phänomene, die man als lösbar ansieht, und läßt andere unbeachtet. Diese können aber in einem neuen Paradigma zu zentralen Problemen werden. Die Beurteilung eines Paradigmas hängt also auch davon ab, was als wichtig angesehen wird. Vgl. dazu a.a.O. S. $14 \mathrm{f}, 110$.

${ }^{102}$ A.a.O. S. 157. Kuhn verweist auf den Fall der kopernikanischen Astronomie, die anfangs weder einfacher noch präziser war als die ptolemäische (vgl. S. 75 f.). Es war die Krise, in welche die ptolemäische Astronomie geraten war, als ihre Komplexität schneller wuchs als ihre Exaktheit, die die Bereitschaft schuf, die kopernikalische zu akzeptieren und ihr einen Wechsel auf die Zukunft auszustellen.

${ }^{103}$ A.a.O. S. $98 \mathrm{ff}$. An anderer Stelle schreibt Kuhn: "Copernicus' innovation was not simply to move the earth. Rather, it was a whole new way of regarding the problems of physics and astronomy, one that necessarily changed the meaning of both 'earth' and 'motion'."

${ }^{104}$ A.a.O. S. 151 f., 159. 
demgegenüber ein darwinistisches Bild des Erkenntnisfortschritts, ${ }^{105}$ wobei allerdings der für die Evolutionstheorie Darwins entscheidende Gedanke des ,,survival of the fittest", der natürlichen Auslese fehlt, der sich nach Ansicht Kuhns für die wissenschaftliche Evolution auch nicht präzisieren läßt, da uns objektive, faradigmenurabhängigc Kriterien zur Beurteilung der Frage fehlen, wie gut die verschiedenen $\mathrm{Pa}$ radigmen den tatsächlichen Gegebenheiten angepaßt sind. ${ }^{106}$ Für Kuhn ergibt sich aus diesen Ideen nicht, daß wissenschaftliche Revolutionen einen ,,irrationalen“ Charakter hätten: Was ,,wissenschaftlich" oder ,,rational“ ist, bestimmen die Wissenschaftler. Sie bestimmen es je nach ihrem Standpunkt, und dieser Standpunkt ist per definitionem der des Paradigmas, das sich durchgesetzt hat. Die Rede von „Irrationalität" würde voraussetzen, daß es feste, paradigmenunabhängige Kriterien für Wissenschaftlichkeit und Rationalität gäbe, was nach Kuhn nicht der Fall ist.

Kuhn kommt so aufgrund wissenschaftsgeschichtlicher Analysen so $\mathrm{zu}$ einem ähnlichen Bild von Status, Leistung und Überprüfung von Theorien und ihrem Zusammenhang mit der Erfahrung, wie es sich für uns aufgrund wissenschaftstheoretischer und erkenntnistheoretischer Überlegungen ergab. Diese abstrakten Überlegungen erhalten durch seine konkreten historischen Anralysen ein erhebiich größeres Gewicht. Das Neue bei Kuhn ist die Anwendung dieser Ideen auf die Wissenschaftsentwicklung, insbesondere auf das Problem des wissenschaftlichen Fcrtschritis.

Die Kritik an den Aussagen Kuhns bezieht sich - sofern sie von den wissenschaftsgeschichtlichen Fakten absieht und sich auf deren Deutung beschränkt - vor allem auf folgende Punkte:107

${ }^{105}$ A.a.O. S. $170 \mathrm{ff}$. Ähnliche Ideen finden sich bei Popper, vgl. (72), 261.

${ }^{106}$ Die Welt kann nach Kuhn nicht vorurteilsfrei, sondern immer nur durch die Brille von Paradigmen erfaßt werden, die durch Beschränkung und Systematisierung Ordnung in die chaotische Komplexität der sinnlichen Erfahrung bringen. Vgl. a.a.O. S. 109. Darwins Prinzip vom survival of the fittest wird so zum Prinzip des survivals of the survivor trivialisiert.

$107 \mathrm{Zu}$ einigen wissenschaftsgeschichtlichen Details vgl. das Postscript zur 2. Auflage von (62), 174. Zur Kritik an Kuhn vgl. Lakatos und Musgrave (70), Stegmüller (70), 2. Halbbd. Für Kuhns Reaktionen vgl. das Postskript zur 2. Auflage von (62), sowie (70), (72) und (76). 
1. Der Begriff des Paradigmas ist unklar. Kuhn verwendet den Begriff zum Teil in einem engeren Sinn, in dem ein Paradigma eine Theorie - etwa im Sinne Sneeds - ist, verbunden mit einer paradigmatisch beschriebenen Menge intendierter Anwendungen, und einer theoretischen Sprache, in der die Theorie formuliert ist. An anderen Stellen wird der Ausdruck ,Paradigma“ aber in einer wesentlich weiteren Bedeutung verwendet, so daß auch ein oder mehrere der folgenden $\mathrm{Be}$ standteile dazuzurechnen sind: eine Beobachtungssprache, Hintergrundannahmen (die nicht Teil der Theorie sind, aber in ihren Anwendungen verwendet werden), Auswahlkriterien für die Theorie, die weder empirisch noch logisch sind, eine Wissenschaftskonzeption, Normen wissenschaftlichen Experimentierens und Begründens. Ein erheblicher Teil dessen, was Kuhn über Paradigmenwechsel sagt, gilt nur für einen Wandel von Paradigmen in diesem umfassenden Sinn. Andererseits wird aber häufig auf historische Fälle Bezug genommen, bei denen man wohl nur von einem Theorienwechsel sprechen kann. Für eine Präzisierung der Analysen Kuhns ist also eine wesentlich schärfere Bestimmung des Paradigmenbegriffs unerläßlich. Diesen Einwand hat er im Postscript zur 2. Auflage von (62) anerkannt, und sich für den engeren Paradigmenbegriff entschieden.

2. Kubns Thesen sind oft überzogen. Viele seiner Aussagen über Paradigmenwechsel ließen sich nur dann aufrecht erhalten, wenn es sich um einen Paradigmenwechsel im umfassenden Sinn handelte. Derartige Paradigmenwechsel sind aber viel seltener als bloße Theorienwechsel, und die historischen Hinweise Kuhns reichen kaum aus, sie eindeutig zu belegen. Der Übergang von der klassischen Newtonschen zur relativistischen Mechanik oder zur Quantenmechanik bedeutet z. B. keine Änderung der Wissenschaftskonzeption; keine dieser Theorien ist vom Standpunkt der anderen aus ,unwissenschaftlich". Man kann auch nicht sagen, die Vertreter der verschiedenen Theorien hätten keinerlei gemeinsame empirische oder wissenschaftstheoretische Basis zur Beurteilung der Theorien gehabt. Daß die gemeinsame Basis nicht hinreicht, eine der Theorien definitiv zu falsifizieren, ist dagegen eine sehr viel bescheidenere Behauptung. Es war z. B. unumstritten, daß die Quantenmechanik und die relativistische Mechanik gewisse Phänomene erklären konnten, die sich im Rahmen der klassischen Physik nicht befriedigend erklären ließen. Wenn Kuhn sich in seiner Argumentation auf holistische Thesen stützt, so ist zu betonen, daß diese zum Teil nur bei Bezugnahme auf sehr umfassende 
Systeme von Annahmen gelten, nicht jedoch bei Bezugnahme auf einzelne Theorien. Daher wird man vieles von dem, was er über wissenschaftliche Revolutionen sagt, nicht generell auf die Ablösung einer, wenn auch fundamentalen Theorie durch eine andere anwenden können, sondern nur auf Revolutionen in der Wissenschaftsknnzeption. 3. Die Aussagen Kubns enthalten Widersprüche. Zunächst stellt seine Auffassung selbst einen Paradigmenwechsel innerhalb der Wissenschaftsgeschichte dar, eine Revolution gegenüber der Auffassung vom kumulativen Fortschritt der Wissenschaften und ihrer ,teleologischen" Entwicklung. Wären seine Aussagen über Revolutionen richtig, so gäbe es keine gemeinsame Basis historischer Fakten und methodologischer Regeln der Geschichtswissenschaft, auf deren Grundlage er seine Konzeption gegenüber der alten in einer objektiven Weise rechtfertigen oder diese gar falsifizieren könnte. Es würde sich dann einfach um eine andere Weise handeln, die historischen Ereignisse zu sehen, die man nicht rechtfertigen, sondern nur durch Propaganda durchsetzen könnte. Sie wäre auch nicht in irgendeinem objektiven Sinn besser als die alte Konzeption. Kuhn will aber sein Werk selbst nicht so verstanden wissen, sondern vielmehr die Richtigkeit seiner Auffassung durch historische Fakten begründen und zeigen, $\mathrm{daß}$ sie adäquater ist als die alte. ${ }^{108}$

Eir anderer Widerspruch steckt in der $\Lambda$ ussage Kuhrs, in cirer wissenschaftlichen Revolution stünden sich Theorien gegenüber, die zugleich inkommensurabel und unverträglich sind. Zwei Theorien sind aber nur dann unverträglich, venn aus der eiren folgt, daß die ardere falsch ist und sie sind nur dann inkommensurabel, wenn die Aussagen, die aus ihnen folgen, voneinander logisch unabhängig sind. Beides zugleich kann also nicht gelten.

4. Kubns Rationalitätsbegriff ist $z u$ eng. Seine Aussage, rational sei, was die Wissenschaftler einer Epoche so nennen, ist nur eine andere Formulierung dafür, daß es keine allgemeinen Rationalitätsstandards gibt, daß ein Paradigmenwechsel sich nicht rational verstehen läßt. Es ist natürlich richtig, daß wir keine Garantie dafür haben, daß der historische Prozeß der Wissenschaftsentwicklung in Richtung auf eine immer umfassendere und vollkommenere Wirklichkeitserkenntnis hin verläuft. Tatsächlich zeigt ja ein Blick in die Geschichte, daß Epochen

${ }^{108} \mathrm{Zu}$ diesem Selbstanwendungsproblem vgl. auch die Kritik Schefflers in (72). 
wissenschaftlicher Blüte, wie z. B. die der griechischen Klassik und des Hellenismus, durch Perioden des Verfalls abgelöst werden. Darum geht es aber bei der Frage nach der Existenz allgemeiner Rationalitätskriterien nicht -- bei dieser historischen Beurteilung werden sie ja schon vorausgesetzt. Erkenntnis hat ferner viele Aspekte, und Fortschritt ist meist nur Fortschritt unter einem Aspekt, der mit einem Rückschritt unter anderen Aspekten gekoppelt sein kann. ${ }^{109}$ Daraus erklären sich oft widersprechende Beurteilungen einer Entwicklung. Ist das neue Paradigma unter einem Aspekt besser als das alte, unter einem anderen Aspekt hingegen schlechter, so folgt daraus nicht, daß es keine rationalen Maßstäbe für eine Beurteilung gäbe. Wenn es auch oft schwierig ist, die Bedeutung verschiedener Aspekte gegeneinander abzuwägen, so ist doch nicht einzusehen, warum das prinzipiell unmöglich sein sollte. Bei umfassenden Paradigmen, bei verschiedenen Wissenschaftskonzeptionen, genügen die empiristischen Kriterien Logik und Empirie sicher nicht mehr; hier müßte man Erkenntnisziele bewerten, und das gelingt nur mit praktischen Kriterien. ${ }^{110}$ Solche Kriterien müssen aber nicht notwendig immer bloß subjektiv oder irrational sein.

Wenn auch aufgrund dieser Einwände die Thesen Kuhns erheblich einzuschränken sind, und man wohl sagen muß, daß er keinen einzi-

${ }^{109}$ So kann auch eine Reihe von Fortschritten insgesamt zu einem Rückschritt führen.

${ }^{110}$ Sneed und Stegmüller haben versucht, rationale Kriterien für einen wissenschaftlich-revolutionären Fortschritt anzugeben. (Vgl. dazu Stegmüller (70), 2. Halbbd., Kap. VIII, $\$$ 9.) Sie beziehen sich dabei nur auf einen $\mathrm{Pa}$ radigmenwechsel i.e.S., d. h. auf die Ersetzung einer Theorie durch eine andere (in ihrem Sinn von ,Theorie"), wobei weder die Wissenschaftskonzeption noch die Beobachtungssprache sich ändert. Ein Fortschritt liegt in ihrem Sinn genau dann vor, wenn sich die verdrängte Theorie (in ihren richtigen Anwendungen) auf die verdrängende reduzieren läßt. Da sie dabei aber einen zu weiten Reduktionsbegriff benützen - im Effekt den in 3.3 auf S. 169 erwähnten - ergibt das kein brauchbares Kriterium. Auch wenn sich ein passender Reduktionsbegriff angeben ließe, der diese Mängel vermeidet, so könnte man damit doch keinen Paradigmenwechsel im umfassenderen Sinn beurteilen. Vgl. auch die Kritik Kuhns an diesem Kriterium für fortschrittliche wissenschaftliche Revolutionen in (76), $190 \mathrm{ff}$., der betont, Reduzierbarkeit erfordere Übersetzbarkeit, die im Fall inkommensurabler Theorien nicht gegeben sei. 
gen Paradigmenwechsel im umfassenden Sinn dieses Wortes aufgewiesen hat, so widerlegen doch seine wissenschaftsgeschichtlichen Analysen die empiristischen und kritizistischen Auffassungen wissenschaftlichen Fortschritts.

\subsection{Relativismus}

Für den Empirismus gibt es etwas erfahrungsmäßig unmittelbar Gegebenes und damit eine feste, objektive, von allen hypothetischen Elementen und Deutungen freie Basis empirischer Erkenntnis. Alle überhaupt sinnvollen empirischen Sätze lassen sich in Aussagen über unmittelbar Gegebenes übersetzen, und alle empirischen Fragen lassen sich im Prinzip allein durch Bezugnahme auf unmittelbar Gegebenes beantworten. Aufgrund unserer Kritik am unmittelbar Gegebenen lassen sich diese Behauptungen nicht mehr aufrechterhalten. Gibt es nichts unmittelbar Gegebenes, hat jede Bestimmung dessen, was uns in einer Erfahrung gegeben ist, hypothetischen Charakter, beruhen alle Begründungen empirischer Sätze mit Beobachtungen auf Annahmen oder Erwartungen, sind unsere Aussagen über die Natur nicht nur Beschreibungen gegebener Sachverhalte, sondern Deutungen in einem Rahmen vorgängiger Annahmen, kommen in unseren 'Theorien über die Natur Konstrukte vor, denen nichts direkt Beobachtbares entspricht, so hängt das Ergebnis empirischer Untersuchungen entscheidend auch von dem ab, was wir in die Erfahrung hineintragen. Es liegt der Schluß nahe, die holistische Konzeption der Erfahrungserkenntnis, die wir dem empiristischen Erkenntnismodell entgegengestellt haben, führe in einen Relativismus, nach dem empirische Erkenntnis immer Erkenntnis im Rahmen vorgängiger Annahmen und Deutungen ist und verschiedene Ansätze ganz verschiedene Ergebnisse liefern. Es kann dann miteinander unverträgliche Weltbilder oder Paradigmen des Naturverständnisses geben - in dem umfassenden Sinn, in dem Th. Kuhn dieses Wort oft gebraucht -, zwischen denen wir weder mit logischen noch mit empirischen Mitteln entscheiden können, weil es keine von diesen Paradigmen unabhängigen Entscheidungskriterien gibt.

Eine ähnliche Konzeption hat Wittgenstein in (74) vertreten. Erkenntnis ist für ihn immer Erkenntnis im Rahmen eines Systems. Zu einem solchen System gehören eine Sprache, allgemeine Hintergrund- 
annahmen, Argumentationsweisen und empirische Untersuchungsmethoden - Kriterien also für die Beurteilung von wahr und falsch. Ein solches umfassendes System - Wittgenstein redet auch von ,,Sprachspielen" - ist nicht Ergebnis von Erfahrungen, sondern der Rahmen, der erst festlegt, was als Erfahrung gilt und was aus Erfahrungen folgt. Er sagt: „Aber mein Weltbild habe ich nicht, weil ich mich von seiner Richtigkeit überzeugt habe; auch nicht, weil ich von seiner Richtigkeit überzeugt bin. Sondern es ist der überkommene Hintergrund, auf welchem ich zwischen wahr und falsch unterscheide“. „Unser Wissen bildet ein großes System. Und nur in diesem System hat das Einzelne den Wert, den wir ihm beilegen“. „Alle Prüfung, alles Bekräften und Entkräften einer Annahme geschieht schon innerhalb eines Systems. Und zwar ist dies System nicht ein mehr oder weniger willkürlicher und zweifelhafter Anfangspunkt aller unserer Argumente, sondern es gehört zum Wesen dessen, was wir ein Argument nennen. Das System ist nicht so sehr der Ausgangspunkt, als das Lebenselement der Argumente"."111

Die zentrale These des empirischen Relativismus ist:

ER: Es gibt miteinander konkurrierende, aber inkommensurable empirische Theorien oder Weltbilder.

Daß zwei Theorien miteinander konkurrieren, heißt, daß nicht beide zugleich richtig sein können, daß sie unverträglich sind. Mit dieser Behauptung wird ein Wahrheitsrelativismus in dem Sinn ausgeschlossen, daß die Festlegung der Begriffe Wahr und Falsch in verschiedenen Theorien in verschiedener Weise vorgenommen wird, daß derselbe Satz in einer Theorie wahr, in einer anderen dagegen falsch sein kann. Andernfalls gäbe es keinen theorienunabhängigen Wahrheitsbegriff, mit dem man die Aussage über die Unverträglichkeit der Theorien sinnvoll formulieren könnte. ${ }^{112}$ In der These ER geht es nicht um

111 Wittgenstein (74), $\$ 94,410,105$. Wittgenstein betont dabei, daß die Annahmen, Regeln und Begründungsweisen, die ein solches Weltbild bestimmen, nicht explizit formuliert sein müssen. Sie sind, so könnte man sagen, in den Erwartungen enthalten, mit denen wir an die Einzelphänomene herangehen, wie in der Sprache, mit der wir sie beschreiben.

${ }^{112}$ Für einen solchen Wahrheitsrelativismus gibt es kein einziges stichhaltiges Argument. Er ist meist das Resultat einer der drei folgenden Verwechslungen: a) Derselbe Satz - verstanden als ein syntaktisches Gebilde - kann 
Fragen des Wahrseins, sondern um Fragen der Erkennbarkeit von Wahrheit.

Konkurrieren zwei Theorien nicht miteinander, so können beide wahr sein. Sie können von verschiedenen Phänomenen handeln, oder dieselben Phänoınene unter verschiedenen fspekıeı bescinreïuen. Thre Inkommensurabilität wirft dann noch kein erkenntnistheoretisches Problem auf. Sind sie aber unverträglich, so stehen wir vor dem Problem, daß wir uns für eine von ihnen entscheiden müssen, da wir nicht beide zugleich akzeptieren können. In diesem Fall besagt aber ihre Inkommensurabilität, daß sich eine solche Entscheidung nicht rechtfertigen läßt. Es gibt dann keine von ihnen unabhängigen rationalen oder empirischen Kriterien, mit denen sich eine von ihnen als besser begründet auszeichnen ließe. Alle Kriterien, über die wir verfügen, setzen die eine oder die andere Theorie voraus.

Eine Inkommensurabilität von Theorien ergibt sich nicht schon aus der Einsicht, daß alle unsere empirischen Urteile hypothetisch sind, $\mathrm{da}$ sie sich als falsch erweisen können; daß es im empirischen Bereich keine definitiven oder Letztbegründungen gibt. In der These ER geht

bei verschiedenen Interpretationen (aufgrund verschiedener semantischer Konventionen) verschiedene Wahrheitswerte erhalten. Aus dieser trivialen Bemerkung folgt aber nicht, daß derselbe Satz im normalen Sinn, d. h. derselbe Satz in derselben Bedeutung bei gewissen Annahmen wahr, bei anderen aher falsch. ist. $\mathrm{Da}$ in verschiedenen Thecrien ein Satz verschieden gedeutet wird und dabei einmal wahr, das andere mal falsch ist, begründet keine Unverträglichkeit der Theorien. b) Aus gewissen Annahmen kann sich ergeben, daß ein Satz wahr ist - wenn er nämlich daraus folgt - aus anderen, daß er falsch ist. Auch damit erhält man keinen Relativismus. Denn die Tatsache, daß ein Satz aus gewissen Annahmen folgt, besagt noch nicht, daß er wahr ist. Dazu ist vielmehr erforderlich, daß die Annahmen wahr sind, aus denen er folgt. c) Ein Satz kann nach gewissen Kriterien wahr sein, nach anderen aber falsch. Aus der Tatsache, daß ein Satz nach besrimmten Kriterien wahr ist, folgt aber wiederum noch nicht seine Wahrheit. Das gilt nur dann, wenn die Kriterien korrekt sind. Die Behauptung, die Unterscheidung Wahr - Falsch sei relativ zum System von Annahmen wird in diesem letzteren Sinn meist - insbesondere auch von Wittgenstein - fälschlich damit begründet, daß unsere Urteile über wahr und falsch sich auf solche Systeme stützen. Unsere Urteile hängen natürlich von unseren Annahmen und Wabrheitskriterien ab; daraus folgt aber nicht, daß die Wahrheit der Urteile von diesen Annahmen und Kriterien abhängt. 
es nicht um die Frage, ob sich eine der beiden Theorien definitiv als wah:, bzw. als falsch erkennen läßt, sondern nur um die Frage, ob sich eine von ihnen als besser begründet erweisen läßt, ob sich Gründe für eine Entscheidung für eine von ihnen finden lassen.

Wir wollen nun die wichtigsten Argumente für die These ER diskutieren, die sich auf die Ergebnisse der früheren Abschnitte dieses Kapitels stützen.

1. Die Theoriebeladenbeit der Beobachtungssätze. Sind im Sinne der Ausführungen in 9.3 auch die einfachsten empirischen Aussagen, die Beobachtungssätze, theoriebeladen, so fehlt eine theorienunabhängige empirische Grundlage für den Vergleich von Theorien. Erfahrung ist dann immer Erfahrung im Lichte von Theorien. Dieselben Experimente können im Rahmen verschiedener Theorien verschieden interpretiert werden und so miteinander unverträgliche Theorien stützen.

Aus der Theoriebeladenheit der Beobachtungssätze ergibt sich nur, $\mathrm{da} ß$ es inkommensurable Theorien geben kann, Theorien, für die es entweder gar keine gemeinsame empirische Basis gibt, von der her sie sich beurteilen lassen, oder bei denen die gemeinsame empirische $\mathrm{Ba}$ sis nicht ausreicht, eine von ihnen als besser begründet auszuzeichnen. Die These ER ist damit aber noch nicht gerechtfertigt, denn dafür wäre nicht nur zu zeigen, daß es inkommensurable Theorien geben kann - das ist ohnehin trivial - , sondern daß es Theorien geben kann, die zugleich inkommensurabel und unverträglich sind. Eigentlich wären sogar solche Theorien anzugeben, nicht nur Argumente für ihre Möglichkeit. Nun ergibt sich die Theoriebeladenheit der Beobachtungssätze nur aus der Theoriebeladenheit der Beobachtungssprache. Sind aber die Beobachtungssprachen mit verschiedenen Theorien beladen, so sind sie - trotz evtl. syntaktischer Gleichheiten - verschiedene Sprachen, zwischen deren Sätzen keine Unverträglichkeit bestehen kann. Überlegen wir uns das etwas genauer: Die Theorie $T_{1}$ sei in der Sprache $S_{1}$ formuliert, die Theorie $T_{2}$ in der Sprache $S_{2} . S_{1 B}$ sei die Beobachtungssprache von $S_{1}, S_{B 2}$ jene von $S_{2}$. Eine Unverträglichkeit zwischen $T_{1}$ und $T_{2}$ kann man nur dann behaupten, wenn sich zumindest Teile von $S_{1}$ und $S_{2}$ in eine Sprache $S$ übersetzen lassen, und wenn dabei ein $S a t z$ von $S_{1 B}$, der aus $T_{1}$ folgt, in einen Satz $A$ von $S$ übersetzt wird, und ein Satz von $S_{2 B}$, der aus $T_{2}$ 
folgt, in den Satz $\neg$ A. ${ }^{113}$ Wir haben dann aber in dem Satz A einen Satz einer Beobachtungssprache vor uns, der durch unterschiedliche Annahmen der beiden Theorien nicht belastet, also ihnen gegenüber neutral ist, und die Beobachtungssätze von $\mathrm{S}$, die für oder gegen $\mathrm{A}$ sprechen, sind dann neutrale Argumente für eine der beiden Theorien und gegen die andere. Eine Unverträglichkeit von $T_{1}$ und $T_{2}$ läßt sich also nir dann behalipien, weun sie empirisch richi vöilig inkommensurabel sind. Umgekehrt: Besteht eine vollständige empirische Inkommensurabilität zwischen $T_{1}$ und $T_{2}$, so sind die Theorien nicht unverträglich. Es kann dann zwar ein Satz aus $T_{1}$ folgen, dessen Negation aus $T_{2}$ folgt, aber dann handelt es sich nur um syntaktisch, nicht aber inhaltlich gleiche Sätze. Im Fall völliger Inkommensurabilität ist es auch fraglich, ob man von ,gleichen Experimenten“ sprechen kann, gleichen Erlebnissen etc., die in beiden Theorien in verschiedener Weise gedeutet werden, oder ob man nicht konsequenterweise von verschiedenartigen Erfahrungen reden muß.

\section{Alles Lernen aus der Erfabrung berubt auf apriori-Bewertungen. Im} Sinne der Ausführungen in 9.3 und 9.4 ergeben sich all unsere empirischen Annahmen nicht allein nach logischen Prinzipien aus dem, was wir erleben, sondern einfache Erfahrungsurteile beruhen ebenso wie generelle Hypothesen auf ycrgängigen Arnahmen urd Erwarturgen. Es kann also sein, daß zwei Personen aufgrund gleichen Erlebens wegen ihrer verschiedenen Ausgangsbewertungen zu verschiedenen, miteinander unverträglichen Theorien kommen; daß keiner den anderen auf Tatsachen hinweisen kann, aus denen sich die Unhaltbarkeit seiner Theorie ergibt.

Wir haben nun schon in 9.4 betont, daß unsere apriori-Bewertung aufgrund von Erfahrungen modifiziert werden. Wir passen Erfahrungen und Erwartungen einander so an, daß wir mit unseren Erwartungen möglichst richtig liegen. Ob wir richtig liegen, erweist das Zutreffen oder Nichtzutreffen unserer Vorhersagen. Auf längere Sicht zeigt

${ }^{113}$ Durch $T_{1}$ und die Deutung von $S_{1 B}$ wird der Wahrheitswert eines Satzes $B$, der theoretische Terme enthält, nur dann eindeutig festgelegt, wenn es einen $S a t z C$ aus $S_{1 B}$ gibt, so daß $B \equiv C$ aus $T_{1}$ folgt. (Vgl. dazu Kutschera (72), 3.3, Theorem $T 3.3-2$.) Ebenso für $T_{2}$. Widersprechen sich also $T_{1}$ und $T_{2}$ bzgl. theoretischer Sätze, so auch bzgl. Sätzen ihrer Beobachtungssprachen. 
sich also, welche Erwartungen angemessen sind. Man könnte einwenden, auch Aussagen über das Zutreffen oder Nichtzutreffen von Vorhersagen stützten sich auf Erwartungen. Das ist zwar im Prinzip richtig, wir haben aber auch gesehen, daß die Beobachtungssprache nur dann eine intersubjektive Sprache ist, wenn wir in unseren Erfahrungsurteilen in der Regel übereinstimmen. Eine gemeinsame Sprache beruht so auch auf gemeinsamen Erwartungen. In ein und derselben Sprachgemeinschaft spielen daher unterschiedliche Erwartungen jedenfalls bzgl. der Beobachtungssätze keine signifikante Rolle. Zwischen zwei Sprachgemeinschaften mag das anders sein. Dann sprechen beide aber nicht dieselbe Beobachtungssprache, und die Annahme der Inkommensurabilität läßt sich dann wie im ersten Argument nicht mit jener einer Unverträglichkeit der Theorien vereinbaren.

3. Die Dubem-Quine-These. Nach dieser These können wir nicht einzelne Hypothesen oder Theorien mit der Erfahrung konfrontieren, sondern nur das Gesamtsystem unserer Annahmen - oder jedenfalls umfangreichere Systeme von Annahmen über die Natur. Ein Konflikt des Systems mit der Erfahrung läßt sich immer so bereinigen, daß man an einer bestimmten Theorie festhält. Theorien lassen sich also gegenüber Erfahrungen immunisieren; es gibt keine Erfahrung, an der eine Theorie definitiv scheitern würde. Ebenso gibt es für zwei miteinander unverträgliche Theorien kein experimentum crucis, das nur eine der beiden überlebt. Durch passende Modifikation der Hintergrundannahmen kann man an jeder von ihnen festhalten, egal was die Erfahrung zeigt.

Dieses Argument erinnert ein wenig an die Paradoxie des Sorites: Ein Sandkorn ist kein Sandhaufen; sind n Sandkörner kein Haufen, so auch $n+1$ Körner nicht. Also bilden auch 10 Millionen Sandkörner keinen Sandhaufen. Eine Theorie, die - zusammen mit gewissen Hintergrundannahmen - einer Beobachtung widerspricht, läßt sich retten durch eine Modifikation der Hintergrundannahmen. Schon ein solcher Rettungsversuch ist nicht immer einfach: Die Annahme einer Störung der Beobachtung ist z. B. zu rechtfertigen. Dazu auf die Geltung der Theorie zu rekurrieren, wäre offenbar eine petitio principii, und man könnte damit auch nicht bestimmte Störfaktoren aufweisen. Nimmt man aber eine bestimmte Störung an, so läßt sich diese An- 
nahme in der Regel prüfen. ${ }^{114}$ Wenn sich nun auch eine Theorie in einem Fall durch Änderung der Hintergrundannahmen retten läßt, so doch nicht immer. Erfordert das Festhalten an der Theorie ständig neue Annahmen über nicht erklärbare Störungen, oder immer neue Modifikationen der Hintergrundannahmen, gerät also die Theorie auch mit den modifizierten Annahmen immer wieder in Konflikte mit der Erfahırung, so wird man sie aufgeben. Sie ieistet dann nichts für Voraussagezwecke. Wir haben betont: Es gibt keine zugleich präzisen und allgemeinen Kriterien dafür, wann eine Theorie als gescheitert anzusehen ist, aber daraus folgt nicht, daß sich beliebige Theorien mit beliebigen Erfahrungsdaten vereinbaren lassen - ebenso wenig wie aus der Tatsache, daß wir keine bestimmte $\mathrm{Zahl} n$ angeben können, so $\mathrm{da}$ eine Menge von Sandkörnern genau dann ein Haufen ist, wenn sie mindestens n Körner enthält, folgt, daß wir nicht manche Mengen definitiv als Haufen bezeichnen können.

4. Die empirische Unterdeterminiertheit von Theorien. In 9.5 haben wir gesehen, daß es empirisch äquivalente, aber nicht äquivalente Theorien gibt. Zwei solche Theorien entwerfen verschiedene Bilder der Natur oder von Teilbereichen der Natur. Wir können aber mit empirischen oder logischen Mitteln entscheiden, welches von beiden Bildern richtig ist.

Ein Argument für die Relativitätsthese ER ergäbe sich aus der empirischen Unterdeterminiertheit von Theorien erst, wenn man zeigen könnte, daß es empirisch äquivalente, miteinander aber unverträgliche Theorien gibt. Das wurde aber weder bewiesen, noch läßt es sich beweisen. Stimmen zwei Theorien $T_{1}$ und $T_{2}$ in ihrem empirischen Gehalt überein, so sind ihre Ramseysätze $R\left(T_{1}\right)$ und $R\left(T_{2}\right)$ analytisch äquivalent. Denn $R\left(T_{1}\right)$ gehört zum empirischen Gehalt von $T_{1}$ und $R\left(T_{2}\right)$ zu dem von $T_{2}$; der gesamte empirische Gehalt von $T_{1}$ folgt aber aus $R\left(T_{1}\right)$ und der von $T_{2}$ aus $R\left(T_{2}\right)$. Die Theorie $T_{1}$ ist daher bei jeder zulässigen Deutung ihrer theoretischen Terme mit der Theorie $T_{2}$ bei jeder zulässigen Deutung ihrer theoretischen Terme verträg-

${ }^{114}$ Auf Quines Behauptung, man könne sich widersprechender Beobachtungen einfach durch eine Änderung von Sprache oder Logik entledigen, sind wir schon in der Anmerkung 91 zu S. 503 eingegangen. 
lich, und umgekehrt. ${ }^{115}$ Zwischen $T_{1}$ und $T_{2}$ kann dann höchstens ein irrelevanter syntaktischer, jedoch kein relevanter semantischer Widerspruch bestehen. Ein bloß syntaktischer Widerspruch zwischen $T_{1}$ und $T_{2}$ liegt $\mathrm{z}$. B. vor, wenn $T_{1}$ aus den Axiomen $F(a) \supset G(a)$ und $F(a)$ besteht, $T_{2}$ aus den Axiomen $\neg F(a) \supset G(a)$ und $\neg F(a)$, wobei $F$ ein thecretischer Term, $G$ und a Beobachtungsterme seien. Der gemeinsame empirische Gehalt von $T_{1}$ und $T_{2}$ besteht dann aus den Sätzen der Beobachtungssprache, die aus $G(a)$ folgen. Zwischen $T_{1}$ und $T_{2}$ besteht ein syntaktischer Widerspruch, weil aus $T_{1} F(a)$, aus $T_{2}$ aber $\neg \mathrm{F}$ (a) folgt. Das ist jedoch ohne Interesse, da der theoretische Term $F$ in $T_{1}$ anders gedeutet wird als in $T_{2}$, nämlich so, daß der Satz $F(a)$ wahr ist, während dieser Satz nach der Deutung von $F$ in $T_{2}$ falsch ist. Kommen in $T_{1}$ und $T_{2}$ dieselben theoretischen Terme vor, so werden sie durch $T_{1}$ und $T_{2}$ nur dann in derselben Weise gedeutet, wenn alle ihre Interpretationen, die $T_{1}$ erfüllen, auch $T_{2}$ erfüllen und umgekehrt, d. h. wenn $T_{1}$ und $T_{2}$ dasselbe besagen. In unserem früheren Beispiel der Erklärbarkeit derselben optischen Phänomene mit einer Wellen- und einer Korpuskulartheorie des Lichts ergibt sich der Anschein eines Widerspruchs aus der Verwendung der Terme "Welle“ und „Teilchen“, für die im normalen Sinn gilt, daß Wellen keine Teilchen sind, für die es also Bedeutungsbestimmungen gibt, die in den Theorien selbst nicht verwendet werden, die man also entweder zu den Theorien hinzunehmen muß - wobei sie dann einen anderen empirischen Gehalt bekommen - oder von denen man im Kontext der Theorien abzusehen hat.

Stimmen die beiden Theorien $T_{1}$ und $T_{2}$ nur in ihrem empirischen Gehalt i.e.S. überein, so kann es einen Satz A der (gemeinsamen) Beobachtungssprache geben, so daß $A$ aus $T_{1}$ ableitbar ist, aus $T_{2}$ dage-

115 Ist $R\left(T_{1}\right)$ wahr, so ist eine Deutung der theoretischen Terme von $T_{1}$ zulässig, wenn sie $T_{1}$ erfüllt; ist $R\left(T_{1}\right)$ falsch, so ist jede Deutung der theoretischen Terme von $T_{1}$ zulässig, und alle Deutungen machen $T_{1}$ falsch. Ebenso für $T_{2}$. (Bei Theorien in dem hier betrachteten umfassenden Sinn kann man von einer theorienimmanenten Deutung der theoretischen Terme ausgehen, also auch das Carnapsche Bedeutungspostulat für sie verwenden; vgl. dazu S. 453.) Ist nun $T_{1}$ wahr, so auch $R\left(T_{1}\right)$, also $R\left(T_{2}\right)$, also auch $T_{2}$ bei jeder zulässigen Interpretation. Ist $R\left(T_{1}\right)$ falsch, so auch $T_{1}$ bei jeder Interpretation der theoretischen Terme; auch $R\left(T_{2}\right)$ ist dann falsch, also $T_{2}$ bei allen Interpretationen, so daß $T_{2}$ bei allen Interpretationen mit $T_{1}$ verträglich ist. $\mathrm{Da}$ auch die Umkehrung gilt, ist unsere Behauptung damit bewiesen. 
gen $\neg$ A. Die Theorien sind dann nicht inkommensurabel, sondern es gibt entweder ein experimentum crucis - hier können wir gleiche Hintergrundannahmen voraussetzen - oder Beobachtungsdaten, die eine von beiden Theorien besser stützen als die andere. Theorien, von denen wir keine als durch Beobachtungen besser begründet bezeichnen können, sind nicht unverträglich. Sie können nur in dem Sinne konkurrieren, daß jede von ihren für gewisse Systemıacisierungšwecke ausreicht, so daß es genügt, mit einer von ihnen zu arbeiten. Man kann dann aber nicht sagen: „Nur eine von ihnen kann richtig sein, wir können jedoch nicht erkennen, welche".

Neben diesen wissenschaftstheoretischen Argumenten für die These ER gibt es noch eine Reihe von Argumenten, die auf einen Relativismus in einem anderen - nicht immer klar bestimmten - Sinn abzielen. Ein solches Argument ist das von der Pluralität der Welten.116 Nach der linguistischen Relativitätsthese, die zuerst von W. von Humboldt entwickelt wurde, oder nach der Quineschen These von der Indeterminiertheit der Übersetzungen ${ }^{117}$ gibt es Sprachen, die sich nicht ineinander übersetzen lassen. Ihre empirischen Aussagen reden also über verschiedene Sachverhalte. Wenn man die Welt als Menge von Sachverhalten versteht, wie wir das in 3.2 getan haben, so redet man also in diesen Sprachen über verschiedene Welten. Vor eirer bestimmten Welt kann man also nur relativ zu einer bestimmten Sprache reden, und damit ist auch empirische Erkenntnis als Erkenntnis der Welt immer nur Erkenntnis eines durch eine Sprache erfaßten Gegenstandsbereichs. Empirische Theorien, die in Sprachen formuliert sind, von denen sich keine in die andere übersetzen läßt, sind also schon deswegen inkommensurabel, weil sie von verschiedenen Welten handeln.

Dazu ist zunächst einmal zu sagen, daß nach diesem Argument nur dann zwei ganz verschiedene Welten vorlägen, wenn sich kein einziger empirischer Satz der einen Sprache in die andere übersetzen ließe. $\mathrm{Da}$ es solche Sprachen gibt, ist aber nirgends belegt worden. Selbst wenn man das annimmt, kann man aber sagen: Ist $W\left(S_{1}\right)$ die Menge der durch die wahren Sätze der Sprache $S_{1}$ beschriebenen Sachver-

${ }^{116}$ Vgl. dazu Goodman (75) und Kuhn (62), Kap. X.

${ }^{117}$ Vgl. dazu den Abschnitt 7.4, S. $381 \mathrm{ff}$. 
halte und $\mathrm{W}\left(\mathrm{S}_{2}\right)$ die Menge, die in gleicher Weise durch $\mathrm{S}_{2}$ bestimmt wird, so kann man W( $\left.\mathrm{S}_{1}\right)$ und $\mathrm{W}\left(\mathrm{S}_{2}\right)$ immer dann als Teilmengen derselben Welt ansehen, wenn sie mit einander verträglich sind. Völlige Inkommensurabilität schließt aber, wie wir oben sahen, Unverträglichkeit aus. Goodman behauptet: Das, was ein Satz beschreibt, ist der Sachverhalt, den er ausdrückt; die wahren Sätze einer Sprache beschreiben so die Menge der durch sie ausgedrückten Sachverhalte. ${ }^{118}$ Enthalten zwei Sprachen also keine synonymen Sätze, so beschreiben sie verschiedene Welten. Diese Identifizierung des Inhalts eines Satzes mit dem, was er beschreibt oder worüber er redet, entspricht aber nicht normalem Sprachgebrauch. Der Satz „,Fritz ist groß und dick“ drückt den Sachverhalt aus, daß Fritz groß und dick ist; das ist der Inhalt dieses Satzes. Der Satz beschreibt aber nicht diesen Sachverhalt, sondern mit ihm beschreiben wir Fritz; mit ihm reden wir über Fritz. Ein Satz spricht über den oder die Gegenstände, die sein Subjektterm bezeichnet. Kein normaler empirischer Satz redet in diesem Sinn über die Welt oder beschreibt sie. Empirische Sätze beziehen sich nur auf die Welt in dem Sinn, daß aus ihnen Aussagen über die Welt folgen. Das, worauf sich die Sätze einer Sprache beziehen, ist in ihnen nicht immer ausdrückbar. Aus den Axiomen einer Theorie der reellen Zahlen, die in einer Sprache $S$ formuliert sind, folgen z. B. jnhaltlich auch Aussagen über reelle Zahlen, für die es in $S$ keine Namen gibt. Die empirischen Sätze zweier Sprachen, die keine synonymen Sätze enthalten, können sich in diesem Sinn auf die gleiche Welt beziehen. Tatsächlich wäre es im Sinn des normalen Sprachgebrauchs unsinnig zu behaupten, Völker mit verschiedenartigen Sprachen bezögen sich mit ihren empirischen Aussagen auf verschiedene Welten. Sie behaupten Verschiedenes, aber es ist kaum plausibel anzunehmen, daß sie über verschiedene Realitäten reden, zumal wir die Wörter „Wirklichkeit" und ,Welt" immer in einem umfassenden Sinn verstehen. Man kann natürlich eine Terminologie einführen, in der die Aussage „Nicht ineinander übersetzbare Sprachen reden über verschiedene Welten" richtig ist, aber das besagt noch nichts über die Sache, die hier zur Diskussion steht. Aus der Verschiedenheit von Sprachen und Weltansichten folgt nicht, daß es verschiedene Welten gibt.

${ }_{118} \mathrm{Vgl}$. Goodman (75), 59. 
Neben philosophischen Argumenten für relativistische Deutungen der Erfahrungserkenntnis gibt es auch viele einzelwissenschaftliche: wissenschafts- und geistesgeschichtliche, ethnologische, soziologische, psychologische, linguistische und biologische. Die wissenschaftsgeschichtlirhen Credanken von Thomas Kuhn haben wir schon erwähnt. Von Seiten der Geschichte, der Ethnologie und Psychologie ergaben sich relativistische Konzeptionen, als man in früheren oder anderen Bewußtseinsformen - Formen des Welt- und des Selbstverständnisses, des Denkens und Erfahrens - nicht mehr lediglich primitive Vorstufen unseres eigenen Bewußtseins sah, sondern ihre Eigenart und ihre spezifische Leistung besser zu verstehen lernte. Die Biologie hat auf die entwicklungsgeschichtlichen Voraussetzungen und Bedingtheiten unseres Erlebens und Verhaltens hingewiesen. Soziologen haben sich unter dem von Max Scheler geprägten Titel einer "Wissenssoziologie" um die Aufhellung von Zusammenhängen zwischen Weltansicht und sozialen Strukturen bemüht. Die Sprachwissenschaften endlich haben seit Wilhelm von Humboldt auf die $\mathrm{Zu}$ sammenhänge von Sprache und Weltauffassung hingewiesen und haben Kants These von der Prägung der Erfahrung durch die menschliche Vernunftorganisation durch die linguistische Relativitätsthese, die These von der Abhängigkeit der Erfahrung von der Sprache ersetzt.

$\Lambda$ uf all diese Ideen können wir hiei nichi näher eingehen. ${ }^{119}$ Wir wollen nur betonen, daß sie zwar eine Relativität des Erkennens belegen, nicht aber einen Relativismus rechtfertigen. Nach ihnen kann man annekmen:

1. Eine Pluralität von in sich geschlossenen, verschiedenartigen Weltansichten.

2. Eine Inkommensurabilität solcher Weltansichten in dem Sinn, daß es keine voraussetzungslosen rationalen oder empirischen Kriterien gibt, mit denen sie sich sämtlich nach dem Grad ihrer Begründetheit oder Leistungsfähigkeit vergleichen lassen. Vergleiche unter speziellen Aspekten sind möglich, aber eine bestimmte Gewichtung dieser Aspekte läßt sich nicht theoretisch, sondern nur praktisch rechtfertigen.

${ }^{119}$ Aus der Vielzahl einschlägiger Arbeiten nennen wir nur einige wenige: Zur Biologie, Psychologie und Ethnologie Lorenz (73), Riedl (79), Furth (69), Werner (59) und Lévi-Strauss (62); zur Soziologie Berger und Luckmann (66). Zur linguistischen Relativitätsthese vgl. Kutschera (75), Kap. 4. 
3. Zusammenhänge zwischen Erfahrung, Denken, Sprache und Lebensform.

4. A.bhängigkeiten des individuellen Denkens und Erfahres von den überlieferten und in der Gesellschaft praktizierten Denk- und Erfahrungsweisen.

5. Abhängigkeiten der Weltansichten von biologischen, psychologischen, historischen und sozialen Gegebenheiten.

Was also belegt wird, ist eine Relativität unseres Denkens und Erfahrens im Sinn einer Bedingtheit und Systembezogenheit. Es ergibt sich aber kein Relativismus im Sinn der These ER, nach der es miteinander unverträgliche und zugleich inkommensurable Weltansichten gibt. Der Anschein der Unverträglichkeit verliert sich oft, wenn man beachtet, daß Übersetzungen einzelner Aussagen aus Sprachen fremder Kulturen insofern problematisch sind, als wir die Übersetzung auf dem Hintergrund unserer Anschauungen lesen, während sich mit dem übersetzten Satz eine ganz andere Vorstellungswelt verbindet. Weltansichten sind Systeme von Annahmen, und Annahmen können sich natürlich widersprechen; wo aber ein echter Widerspruch vorliegt, da gibt es in der Regel auch Argumente pro und contra. So etwas wie eine völlige Inkommensurabilität von Weltansichten ist ferner nie nachgewiesen worden; gerade die Möglichkeit eines Vergleichs von Denk- und Erfahrungsweisen, der ja die Grundlage von Aussagen über ihre Verschiedenheit ist, spricht dagegen: Eine vollständige Inkommensurabilität besteht nur dort, wo kein Vergleich möglich ist.

Unter den einzelwissenschaftlichen Argumenten findet sich auch keines, das einen Relativismus im Sinn einer Behauptung der Systemgebundenbeit alles Denkens und Erfahrens rechtfertigen würde, der Behauptung, wir würden in einen kognitiven Horizont hineingeboren, den wir nicht überschreiten oder verändern können. Auch dagegen spricht schon die bei der Begründung in Anspruch genommene Fähigkeit, sich andere Weltansichten zu erschließen. Der einzelne übernimmt Sprache, Weltansicht, Denk- und Erfahrungsformen von der Gesellschaft, in die er hineingeboren wird, und diese Tradition bildet die Grundlage seiner eigenen kognitiven Leistungen. Wenn er auch zu diesem Erbe nur wenig hinzufügen kann, so beweist doch der geschichtliche Wandel der Weltansichten, daß die Tradition das individuelle Denken nicht determiniert, daß die überkommene Weltansicht nicht so sehr Schranke als Bedingung seiner Möglichkeit ist. Wie der einzelne keine von Grund auf neue Sprache entwickeln, sondern 
allenfalls einen kleinen Beitrag zur Entfaltung oder Umgestaltung der ihm überkommenen Sprache liefern kann, so kann er auch keine von Grund auf neue Weltansicht entwerfen. Er kann aber Anstöße zu kleineren oder größeren Veränderungen geben.

Die Einsicht, daß eine bisher fraglos akzeptierte Annahme sich auf eine nicht oder nicht ausreichend begründete Voraussetzung stützt, stellt an sich schon einen Erkenntnisgewinn dar und liefert die Chance zu neuen Erkenntnissen. Alternative Hypothesen können entworfen und auf ihre Tragfähigkeit hin untersucht werden, und selbst wenn sie scheitern, so ergibt sich damit doch meist eine neue Stütze für die alte Annahme. Ebenso hat die Einsicht, daß die eigene Weltansicht nicht so selbstverständlich ist, wie man meinte, daß sie auf vielen Voraussetzungen und Annahmen beruht, und daß es Alternativen dazu gibt, nicht nur den negativen Effekt der Verunsicherung. Eine relativistische Auffassung der Erkenntnis führt zu einer skeptischen Haltung; die Einsicht, daß unsere Weltansicht durch mannigfache Voraussetzungen begrenzt und bedingt ist und $\mathrm{da} ß$ es Alternativen dazu gibt, erweitert dagegen unseren Erkenntnishorizont.

In der Einleitung haben wir betont, daß auch die Erkenntnistheorie nicht den Bedingungen enthoben ist, unter denen alle menschliche Erkenntnis steht, und daß sie deshalb nur als immanente Selbstkritik des Erkennens möglich ist. Die Tatsache, daß unsere Erfahrung und unser Denken kulturellen Bedingungen unterliegt und daß es daneben noch andere Formen des Erfahrens und Denkens gibt, grenzt ihre Zielsetzung noch enger ein: Erkenntnistheorie ist für uns nur als immanente Selbstkritik des Weltverständnisses unserer Zeit und unserer Kultur möglich. Auch der Erkenntnistheoretiker steht auf dem Boden einer überkommenen Weltansicht, auch er kann sich nicht völlig von ihren Annahmen lösen. Er kann die Sprache nicht neu schaffen, in der er seine Aussagen formulieren will, sondern er muß im wesentlichen die vorhandene verwenden - sonst könnte er sich auch kaum anderen mitteilen. Wenn er seine Behauptungen begründen will, so muß er sich auf gemeinsame Überzeugungen stützen. Diese Immanenz der Kritik bedeutet nicht, daß sie immer nur die Weltansicht bestätigen könnte, von der sie ausgeht. Aber um Teile dieser Ansicht begründet in Zweifel ziehen zu können, muß die Kritik anderes unbezweifelt stehen lassen. Das Ziel der Erkenntnistheorie der Aufklärung, eine radikale Neubegründung menschlichen Wissens, ist nicht realisierbar. Ohne 
uns auf Überkommenes zu stützen, auf das, was sich in der Vergangenheit bewährt hat, können wir nichts Neues schaffen. Otto Neurath sagt: „Wie Schiffer sind wir, die ihr Schiff auf offener See umbauen müssen, ohne es jemals in einem Dock zerlegen und aus besten Bestandteilen neu errichten zu können" .120

${ }^{120}$ O. Neurath (32), 206. 


\section{Literatur}

Abel, Th. (48): The operation called Verstehen, American Journal of Sociology 54 (1948/49), abgedr. in H. Feigl und M. Brodbeck (53), $677-687$

Aqquist, L. (74): A new approach to the logical theory of actions and causality, in S. Stenlund (Hrsg.): Logical Theory and Semantic Analysis, Dordrecht $1974,73-91$

Armstrong, D. (68): A Materialist Theory of the Mind, London 1968

Austin, J. L. (46): Other Minds, Proceedings of the Aristotelian Society, Suppl. vol. 20 (1946), $148-187$

Austin, J. L. (62): Sense and Sensibilia, hrsg. von G. J. Warnock, Oxford 1962; dt: Sinn und Sinneserfahrung, Stuttgart 1975

Ayer, A. J. (36): Language, Truth, and Logic, London 1936

Ayer, A. J. (40): Foundations of Empirical Knowledge, London ${ }^{1} 1940,{ }^{2} 1964$

Ayer, A. J. (63): Philosophical Essays, London 1963

Ayer, A. J. (63a): The Concept of a Person, and Other Essays, London 1963

Benaceraff, P. (68): God, the devil, and Gödel, Monist 51 (1968), 9-33

Berger, P. L. und Luckmann, Th. (66): The Social Construction of Reality, New York 1966; dr. Frankfurt ^. M. 1969

Berkeley, G. (D): Three Dialogues between Hylas and Philonous in Opposition to Sceptics and Atheists, 1713, zitiert nach (L)

Berkeley, G. (L): A New Theory of Vision and Other Writings, hrsg. A. D. Lindsay, London 1910, Nachdruck 1969

Berkeley, G. (T): A Treatise Concerning the Principles of Human Knowledge, 1710, zitiert nach (L)

Betti, E. (54): Zur Grundlegung einer allgemeinen Auslegungslehre, in: Festschrift für E. Rabel, Tübingen 1954, Bd. II, 79-168

Betti, E. (55): Teoria generale della interpretatione, Mailand 1955. dt: Allgemeine Auslegungslehre als Methodik der Geisteswissenschaften, Tübingen 1967

Birkhoff, G. und v. Neumann, J. (36): The logic of quantum mechanics, Annals of Mathematics 37 (1936), 823-43

Black, M. (52): Phenomenalism, American Philosophical Association, Eastern Division, vol. 1: Science, Language, and Human Rights, Philadelphia 1952

Brentano, F. (30): Wahrheit und Evidenz, hrsg. von O. Kraus, Leipzig 1930

Broad, C. D. (54): Berkeley's denial of material substance, The Philosophical Review 63 (1954). Abgedr. in Martin und Armstrong (74), 255-283 
Butler, J. (W): The Works of Joseph Butler, 2 Bde., hrsg. S. Halifax, Oxford $1849 / 50$

Carnap, K. (28): Der logische Aufbau der Welt, 'Leipzig 1928, ${ }^{2} \mathrm{Hamburg} 1961$

Carnap, R. (32): Die physikalische Sprache als Universalsprache der Wissenschaft, Erkenntnis 2 (1932), 432-465

Carnap, R. (32a): Psychologie in physikalischer Sprache, Erkenntnis 3 (1932), $107-142$

Carnap, R. (36): Testability and Meaning, Philosophy of Science 3 (1936), 419-471; 4 (1937), 1-40; ${ }^{2}$ New Haven/Conn. 1954

Carnap, R. (50): Logical Foundations of Probability, ${ }^{1} 1950$, Chicago ${ }^{2} 1967$

Carnap, R. (50a): Empiricism, semantics, and ontology, Revue Internationale de Philosophie 4 (1950), 20-40; Abgedruckt in Carnap (56)

Carnap, R. (52): The Continuum of Inductive Methods, Chicago 1952

Carnap, R. (56): Meaning and Necessity, ${ }^{2}$ Chicago 1956

Carnap, R. (66): Philosophical Foundations of Physics, New York 1966, dt. München 1969

Carnap, R. und Jeffrey, R. (Hrsg.) (71): Studies in Inductive Logic and Probability, Bd. 1, Berkeley 1971

Carnap, R. und Stegmüller, W. (59): Induktive Logik und Wahrscheinlichkeit, Wien 1959

Cartwright, R. L. (54): Ontology and the theory of meaning, Philosophy of Science 21 (1954), 316-325

Chihara, C. S. und Fodor, J. A. (65): Operationalism and ordinary language: A critique of Wittgenstein, American Philosophical Quarterly 2 (1965), abgedruckt in Pitcher (66), 384-419

Chisholm, R. (42): The problem of the speckled hen, Mind 51 (1942), $368-373$

Clark, M. (64): Knowledge and grounds: A comment on Mr. Gettier's paper, Analysis 24 (1964), 46-48

Couturat, L. (02): Sur la métaphysique de Leibniz, Revue de Métaphysique et de Morale 10 (1902), $1-25$

Craig, W. (53): On axiomatizability within a system, Journal of Symbolic Logic, 18 (1953), 30-32

Craig, W. (56): Replacement of auxiliary expressions, The Philosophical Review 65 (1956), $38-55$

Davidson, D. (63): Actions, reasons, and causes, The Journal of Philosophy 60 (1963), 685-700

Descartes, R. (MP): Meditationes de prima Philosophia, lat.-dt., hrsg. von E. Schröder, Hamburg 1956. (WVII). Dt. Ausgabe mit den Einwänden und Erwiderungen, hrsg. A. Buchenau, 1915, Nachdruck Hamburg 1972

Descartes, R. (PP): Principia philosophiae (W VIII,1), dt. hrsg. A. Buchenau, Nachdruck Hamburg 1965 
Descartes, R. (W): Oeuvres, hrsg. von Ch. Adam und P. Tannery, 12 Bde., Paris $1897-1913$

Diels, H. (51): Die Fragmente der Vorsokratiker, hrsg. von W. Kranz, 3 Bde., Dublin/Zürich 61951, Nachdruck 1971

Dilthey, W. (W): Gesammelte Schriften, 12 Bde., hrsg. B. Groethuysen u. a., ${ }^{1}$ Leipzig 1914-36, ${ }^{2}$ Stuttgart 1957-60

Ditfurth, H. von (74): Zusammenhänge, Hamburg 1974

Dodds, E. (66): The Greeks and the Irrational, ${ }^{5}$ Berkelcy 1966, dt: Darmstadt 1970

Dray, W. (57): Laws and Explanation in History, Oxford 1957

Duhem, P. (14): La Théorie Physique: Son Objet, Sa Structure, Paris 1914; dt: Ziel und Struktur der physikalischen Theorien, Hamburg 1978

Einstein, A. (20): Über die spezielle und die allgemeine Relativitätstheorie, Braunschweig ${ }^{6} 1920$

Einstein, A. (44): Bemerkungen zu Bertrand Russells Erkenntnistheorie, in Schilpp (44), $278-290$

Feigl, H. (53): Notes on Causality, in Feigl und Brodbeck (53), 408-418

Feigl, H. (58): The „Mental“ and the „Physical“, in Minnesota Studies in the Philosophy of Science, Bd. II (hrsg. H. Feigl u. a.), Minneapolis 1958, $370-497$

Feigl, H. (63): Physicalism, Unity of Science, and the Foundations of Psychology, in Schilpp (63), $227-267$

Feigl, H. und Brodbeck, M. (Hrsg.) (53): Readings in the Philosophy of Science, New York 1953

Feigl, H. und Sellars, W. (Hrsg.) (49): Readings in Philosophical Analysis, New York 1949

Feyerabend, P. (60): Das Problem der Existenz theoretischer Entitäten, in E. Topitsch (Hrsg.): Probleme der Wissenschaftstheorie, Festschrift für Victor Kraft, Wien 1960, 35-72

Feyerabend, P. (63): Materialism and the Mind-Body Problem, Review of Metaphysics 17 (1963), 49-66

Feyerabend, P. (70): Consolations for the specialist, in Lakatos und Musgrave (70), $197-230$

Fichte, J. G. (W): Fichtes Werke, hrsg. von I. H. Fichte, Bonn 1834/35 und Berlin 1845/46, Nachdruck, 11 Bde., Berlin 1971

Fichte, J.G. (WLO1): Darstellung der Wissenschaftslehre 1801/02, hrsg. R. Lauth, Hamburg 1977

Fine, K. (75): Vagueness, truth, and logic, Synthese 30 (1975), 265-300

Finetti, B. de (37): La prévision: ses lois logiques, ses sources subjectives, Annales de l'Institut Henri Poincaré 7 (1937), 1-68

Finetti, B. de (70): Teoria delle Probabilitá, 2 Bde., Turin 1970

Finetti, B. de (72): Probability, Induction, and Statistics, London 1972

Fraassen, B. C. van (69): Presuppositions, supervaluations, and free logic, in 
K. Lambert (Hrsg.): Philosophical Problems in Logic, Dordrecht 1970, $67-91$

Frege, G. (GGA): Grundgesetze der Arithmetik, 2 Bde., 1893/1903, ${ }^{2}$ Hildesheim 1966

Frege, G. (KS): Kleine Schriften, hrsg. von J. Angelelli, Darmstadt 1967

Furth, H. G. (69): Piaget and Knowledge - Theoretical Foundations, Englewood Cliffs/N. J. 1969; dt: Intelligenz und Erkennen - Die Grundiagen der genetischen Erkenntnistheorie Piagets, Frankfurt a. M. 1972

Gadamer, H.-G. (60): Wahrheit und Methode, Tübingen ${ }^{1} 1960,{ }^{2} 1965$

Gettier, E. (63): Is justified true belief knowledge?, Analysis 23 (1963), $121-123$

Glymour, C. (71): Theoretical realism and theoretical equivalence, Boston Studies in the Philosophy of Science VIII, Dordrecht 1971, 275-288

Goethe, J. W. (W): Goethes Werke, 14 Bde., Hamburg ${ }^{6} 1971$

Gödel, K. (31): Über formal unentscheidbare Sätze der Principia Mathematica und verwandter Systeme I, Monatshefte für Mathematik und Physik 38 (1931), $173-198$

Goodman, N. (51): The Structure of Appearance, 11951, 2New York 1966 Goodman, N. (55): Fact, Fiction, Forecast, 11955, 2Indianapolis 1965 Goodman, N. (75): Words, works, worlds, Erkenntnis 9 (1975), 57-73 Grice, H. P. (57): Meaning, Philosophical Review 66 (1957), 377-388 Grice, H. P. (68): Logic and Conversation (William James Lectures), Unveröffentlichtes Manuskript, Harvard 1968

Grünbaum, A. (62): Geometry, chronometry, and empiricism, in H. Feigl und G. Maxwell (Hrsg.): Minnesota Studies in the Philosophy of Science, Bd. 3, Minneapolis 1962, 405-526

Grünbaum, A. (63): Philosophical Problems of Space and Time, New York 1963 Hardie, W. F. R. (45): The paradox of phenomenalism, Proceedings of the Aristotelian Society, 46 (1945/46), $127-154$

Hegel, G. W. F. (W): Werke, 20 Bde., Frankfurt a. M. 1971

Heisenberg, W. (30): Die physikalischen Prinzipien der Quantentheorie, Leipzig ${ }^{1} 1930,{ }^{4} 1944$

Heitsch, E. (74): Parmenides, München 1974

Helmholtz, H. (83): Über den Ursprung und die Bedeutung der geometrischen Axiome, Wiss. Abhandlungen Bd. II, Leipzig 1883

Helmholtz, H. (96): Über die Tatsachen, die der Geometrie zum Grunde liegen, Reden und Vorträge, Bd. II, Braunschweig 1896

Helmholtz, H. (21): Schriften zur Erkenntnistheorie, hrsg. von P. Hertz und M. Schlick, Berlin 1921

Hempel, C. G. (35): Analyse logique de la psychologie. Revue de Synthèse 10 (1935); engl. Übersetzung in Feigl und Sellars (49), 373-384

Hempel, C. G. (52): Fundamentals of Concept Formation in Empirical Science, Chicago 1952 
Hempel, C. G. (65): Aspects of Scientific Explanation and Other Essays in the Philosophy of Science, New York 1965

Hempel, C. G. (73): The meaning of theoretical terms: a critique of the standard empiricist construal, in: P. Suppes, L. Henkin, A. Joja und G. C. Moisil, (Hrsg.): Logic, Methodology, and Philosophy of Socience IV, Amsterdam 1973, 367-378

Hempel, C. G. und Oppenheim, P. (48): Studies in the logic of explanation, Philosophy of Science 15 (1948), abgedruckt in Hempel (65), $245-290$

Hermes, H. (61): Aufzählbarkeit, Entscheidbarkeit, Berechenbarkeit, Berlin 1961

Hertz, H. (94): Die Prinzipien der Mechanik, 1894 (Gesammelte Werke, Bd. III), Nachdruck Darmstadt 1963

Hilbert, D. (99): Grundlagen der Geometrie, 1899, ${ }^{11}$ Stuttgart 1972

Hintikka, J. J. (62): Knowledge and Belief, Ithaca/N.Y. 1962

Hume, D. (T): A Treatise of Human Nature, hrsg. von L. A. Selby-Bigge 1888, Nachdruck Oxford 1941

Hume, D. (EHU): An Enquiry Concerning Human Understanding, 1748, hrsg. L. A. Selby-Bigge, ${ }^{2} 1902$, Nachdruck Oxford 1955

Huxley, T.H. (98): Method and Results: Collected Essays, Bd. I, London 1898

Jeffrey, R. C. (65): The Logic of Decision, New York ${ }^{1} 1965,{ }^{2} 1967$, dt. München 1967

Kalke, W. (69): What is wrong with Fodor and Putnam's functionalism? Nous 3 (1969), $83-94$

Käsbauer, M. (76): Definitionen der wissenschaftlichen Erklärung, Erkenntnis 10 (1976), 255-273

Kant (KrV): Kritik der reinen Vernunft, hrsg. R. Schmidt, Hamburg 1952

Kant (W): Werke, 10 Bde., hrsg. W. Weischedel, Darmstadt 1968-71

Katz, D. (48): Gestaltpsychologie, Basel 21948

Kemeny, J. G. (55): Fair bets and inductive probabilities, Journal of Symbolic Logic 20 (1955), 263-273

Kemeny, J. G. und Oppenheim, P. (56): On reduction, Philosophical Studies 7 (1956), 6-19

Kenny, A. (68): Descartes - A Study of His Philosophy, New York 1968

Kim, J. (66): On the psycho-physical identity theory, American Philosophical Quarterly 3 (1966), $227-235$

Kochen, S. und Specker, E. P. (65): Logical structures arising in quantum theory, in: J. W. Addison, L. Henkin, A. Tarski: The Theory of Models, Amsterdam 1965, $177-189$

Krantz, D. H., Luce, R. D., Suppes, P. und Tversky, A. (71): Foundations of Measurement, Bd. I, New York 1971

Kretschmer, E. (44): Körperbau und Charakter, Berlin ${ }^{18} 1944$ 
Kripke, S. (71): Identity and necessity, in M. K. Munitz (Hrsg.): Identity and Individuation, New York 1971, 135-164

Kripke, S. (72): Naming and necessity, in G. Harman und D. Davidson (Hrsg.): Semantics of Natural Language, Dordrecht 1972, 253-355, $763-769$

Kuhn, T.S. (62): The Structure of Scientific Revolution, Chicago ${ }^{1} 1962$, 21970, dt: Frankfurt a. M. 1967

Kuhn, T. S. (70): Reflections on my critics, in Lakatos und Musgrave (70), $231-278$

Kuhn, T.S. (72): Notes on Lakatos, Boston Studies in the Philosophy of Science 8 (1972), $137-146$

Kuhn, T.S. (76): Theory-change as structure-change, Erkenntnis 10 (1976), $179-199$

Kutschera, F. v. (67): Elementare Logik, Wien 1967

Kutschera, F. v. (72): Wissenschaftstheorie, 2 Bde., München 1972

Kutschera, F. v. (75): Sprachphilosophie, München ${ }^{2} 1975$

Kutschera, F. v. (76): Einführung in die intensionale Semantik, Berlin 1976

Kutschera, F. v. (77): Humes Gesetz, Grazer Philosophische Studien 4 (1977), $1-14$

Kutschera, F. v. (78): Goodman on Induction, Erkenntnis 12 (1978), 189-207

Kutschera, F. v. (79): Grundbegriffe der Metaphysik von Leibniz im Vergleich zu Begriffsbildungen der heutigen Modallogik, Studia Leibnitiana, Sonderheft 8 (1979), 93-107

Kutschera, F. v. (80): Grundbegriffe der Handlungslogik, in H. Lenk (Hrsg.): Handlungstheorien interdisziplinär, Bd. 1: Handlungslogik, München $1980,67-106$

Kutschera, F. v. (81): Grundlagen der Ethik, erscheint 1981

Kutschera, F. v. und Breitkopf, A. (71): Einführung in die moderne Logik, Freiburg ${ }^{1} 1971,{ }^{4} 1979$

Lakatos, J. (70): Falsification and the methodology of scientific research programs, in Lakatos und Musgrave (70), $91-195$

Lakatos, J. und Musgrave, A. (Hrsg.) (70): Criticism and the Growth of Knowledge, Cambridge 1970

Lecat, M. (35): Erreurs de Mathématiciens, des origines à nos jours, Brüssel 1935

Lehmann, R. S. (55): On confirmation and rational betting, Journal of Symbolic Logic 20 (1955), 251-262

Lehrer, K. (74): Knowledge, Oxford 1974

Leibniz, G.W. (WP): Philosophische Schriften, hrsg. von C. I. Gerhardt, 7 Bde., Berlin 1875 - 90, Nachdruck Hildesheim 1960/61

Leibniz, G. W. (WM): Mathematische Schriften, hrsg. von C. I. Gerhardt, 7 Bde., Berlin 1849-63. Nachdruck Hildesheim 1962

Leibniz, G. W. (OF): Opuscules et fragments inédits de Leibniz, hrsg. L. Couturat, Paris 1903, Nachdruck Hildesheim 1961 
Leibniz, G. W. (NE): Nouveaux Essays sur l'Entendement Humain, 1765, dt. von E. Cassirer, Hamburg ${ }^{2} 1968$

Leibniz, G. W. (T): Essais de Théodicée, 1710, dt. von A. Buchenau, Hamburg 21968

Leibniz, G. W. (DM): Discours de Métaphysique, frz.-dt., Hamburg 1958

Lenzen, W. (73). Theorien cier Bestätigung wissenschafdicher Hypothesen, Stuttgart 1973

Lenzen, W. (78): Recent work in epistemic logic, Acta Philosophica Fennica 30, No. 1, Dordrecht 1978

Lenzen, W. (80): Glauben, Wissen und Wahrscheinlichkeit, Wien 1980

Lenzen, W. (80a): Wittgensteins Zweifel über Wissen und Gewißheit, Grazer Philosophische Studien 10 (1980), 43-52

Lenzen, W. (81): On the representation of classificatory valuestructures, erscheint in Theory and Decision

Lersch, Ph. (54): Der Aufbau der Person, München 61954

Lévi-Strauss, C. (58): Anthropologie structurale, Paris 1958, dt. Frankfurt a. M. 1971

Lévi-Strauss, C. (62): La pensée sauvage, Paris 1962; dt. Frankfurt a. M. 1968 Lewis, C. I. (45): An Analysis of Knowledge and Valuation, La Salle/Ill. 1945

Lewis, D. (68): Counterpart theory and quantified modal logic, The Journal of Philosophy 65 (1968), $113-126$

Lewis, D. (69): Convention, Cambridge/Mass. 1969

Lewis, D. (70): How to define theoretical terms, The Journal of Philosophy 67 (1970), $427-446$

Lewis, D. (72): Psychophysical and theoretical identifications, Australasian Journal of Philosophy 50 (1972), 249-258

Lewis, D. (73): Counterfactuals, Oxford 1973

Locke, j. (E): An Essay Concerning Human Understanding, hrsg. von J. W. Yolton, 2 Bde., London 1961

Lorenz, K. (73): Die Rückseite des Spiegels, München 1973

Lorenzen, P. (62): Metamathematik, Mannheim 1962

Lucas, J. (61): Minds, machines, and Gödel, Philosophy 36 (1961), 122-127

Luce, R. D. und Raiffa, H. (57): Games and Decisions, New York 1957

Ludwig, G. (53): Der Meßprozeß, Zeitschrift für Physik 135 (1953), 483-511

Ludwig, G. (54): Die Grundlagen der Quantenmechanik, Berlin 1954

Ludwig, G. (54a): Die Stellung des Subjekts in der Quantentheorie, in: Veritas, Justitia, Libertas - Festschrift zur 300. Jahrfeier der Columbia-Universität, Berkeley 1954

Martin, C. B. und Armstrong, D. M. (74): Locke und Berkeley, London o. J.

Meggle, G. (81): Grundbegriffe der Kommunikation, (Regensburger Dissertation 1979), Berlin 1981

Mittelstaedt, P. (59): Untersuchungen zur Quantenlogik, Sitzungsbericht der 
Bayerischen Akademie der Wissenschaften, Math.-Naturw. Klasse, 1959, 321-386

Mitteistaedt, P. (61): Quantenlogik, Fortschritte der Physik 9 (1961), $106-147$

Mittelstaedt, P. (66): Philosophische Probleme der modernen Physik, Mannheim 1966

Moore, G.E. (39): Proof of an External World, Proceedings of the British Academy 26 (1939); abgedruckt in Philosophical Papers, London 1959

Moore, G. E. (42): Reply to my critics, in Schilpp (42), 533-677

Nelson, L. (08): Über das sogenannte Erkenntnisproblem, Göttingen 1908

Neumann, J. yon (32): Mathematische Grundlagen der Quantenmechanik, Berlin 1932

Neurath, O. (32): Protokollsätze, Erkenntnis 3 (1932/33), 204-214

Nietzsche, F. (W): Werke, 20 Bde., Leipzig 1901-1917

Nilson, D. R. (76): Bibliography on the history and philosophy of quantum physics, in Suppes (76), 457-520

O'Connor, D. (69): Modern Materialism: Readings on Mind-Body Identity, New York 1969

Pap, A. (49): Elements of Analytic Philosophy, New York 1949

Pascal, B. (W): Oeuvres complètes, hrsg. L. Brunschwieg und B. Boutroux, 14 Bde., Paris 1908-14

Passmore, J. (62): Explanations in everyday life, in science, and in history, in History and Theory, Bd. 2 (1962), 105-125

Peirce, Ch. S. (65): Collected Papers, hrsg. von Ch. Hartshorne und P. Weiss, Cambridge/Mass. 1965

Pitcher, G. (Hrsg.) (66): Wittgenstein - The Philosophical Investigations. A Collection of Critical Essays, New York 1966

Popper, K. (50): Indeterminism in quantum physics and in classical physics, The British Journal for the Philosophy of Science 1 (1950/51), $117-133,173-195$

Popper, K. (63): Conjectures and Refutations, London ${ }^{1} 1963,{ }^{3} 1969$

Popper, K. (66): Logik der Forschung, ${ }^{1}$ Wien 1934, ${ }^{2}$ Tübingen 1966

Popper, K. (72): Objective Knowledge, Oxford '1972, ${ }^{2} 1979$

Popper, K. und Eccles, J. C. (77): The Self and Its Brain, Berlin 1977

Price, H. H. (40): Hume's Theory of the External World, Oxford 1940

Price, H.H. (41): Review of ,The Foundations of Empirical Knowledge, Mind 50 (1941), 280-293

Putnam, H. (60): Minds and machines, in S. Hook (Hrsg.): Dimensions of Mind, New York 1960, 138-164

Putnam, H. (62): What theories are not, in E. Nagel, P. Suppes, A. Tarski (Hrsg.): Logic, Methodology, and Philosophy of Science, Stanford $1962,240-251$

Putnam, H. (63): An examination of Grünbaum's Philosophy of Geometry, in 
B. Baumrin (Hrsg.): Philosophy of Science, The Delaware Seminar, Bd. 2, New York 1963, 205-255

Putnam, H. (66): The mental life of some machines, in H. Castaneda (Hrsg.): Intentionality, Minds, and Perception, Detroit 1966, 177-200

Putnam, H. (75): Mind, Language, and Reality, Philosophical Papers Bd. II, Cambridge/Mass. 1975

Quine, W. V. (48): On what there is, Review of Metaphysics (1948), abgedruckt in Quine (64), 1 -- 19

Quine, W. V. (51): Two dogmas of empiricism, Philosophical Review 60 (1951), abgedruckt in Quine (64), 20-46

Quine, W. V. (51a): Carnaps views on ontology, Philosophical Studies 2 (1951), 65-72, abgedruckt in Quine (66)

Quine, W. V. (60): Word and Object, Cambridge/Mass. 1960

Quine, W. V. (62): Methods of Logic, London 21962; dt. Frankfurt 1969

Quine, W. V. (63): On simple theories of a complex world, Synthese 15 (1963), $103-106$, abgedruckt in Quine (66)

Quine, W. V. (64): From a Logical Point of View, ${ }^{2}$ Cambridge/Mass. 1964

Quine, W. V. (66): The Ways of Paradox and other Essays, New York 1966

Quine, W. V. (74): On Popper's negative methodology, in P. A. Schilpp (74), $218-220$

Reichenbach, H. (24): Axiomatik der relativistischen Raum-Zeit-Lehre, Braunschweig 1924, Nachdruck 1965

Reichenbach, H. (28): Philcscphic der Raum-Zeit-Lchre, Perlin 1928, engl.: The Philosophy of Space and Time, New York 1957

Reichenbach, H. (44): Philosophical Foundations of Quantum Mechanics, Berkeley/Cal. 1944, dt. Basel 1949

Reichenbach, H. (49): The philosophical significance of the theory of relativity, in Schilpp (49), $287-311$

Reichenbach, H. (61): Experience and Prediction, Chicago 1961

Rescher, N. (63): Discrete state systems, Markov chains, and problems in the theory of scientific explanation and prediction, Philosophy of Science 30 (1963), 325-345

Rescher, N. (67): The Philosophy of Leibniz, Englewood Cliffs/N. J. 1967

Riedl, R. (79): Biologie der Erkenntnis, Berlin 1979

Rosser, J. B. (36): Extensions of some theorems of Gödel and Church, Journal of Symbolic Logic, 1 (1936), 87-91

Rothacker, E. (19): Einleitung in die Geisteswissenschaften, ${ }^{1} 1919,{ }^{3}$ Tübingen 1948, Nachdruck 1965

Rothacker, E. (27): Logik und Systematik der Geisteswissenschaft, in: Handbuch der Philosophie II, C, 1-171, München 1927, Nachdruck München 1965

Russell, B. (18): Mysticism and Logic, London 1918 
Russell, B. (26): Our Knowledge of the External World, ${ }^{1}$ Chicago 1914, ${ }^{2}$ London 1926

Ryle, G. (49): The Concept of Mind, London 1949

Scheffler, I. (72): Vision and revolution: a postscript on Kuhn, Philosophy of Science 39 (1972), 366-374

Scheffler, I. und Chomsky, N. (58): What is said to be, Proceedings of the Aristotelian Society 59 (1958/59), $71-82$

Scheibe, E. (67): Bibliographie zu Grundlagenfragen der Quantenmechanik, Philosophia Naturalis 10 (1967), 249-290

Schelling, F. W. J. (W): Ausgewählte Werke, 10 Bde., Darmstadt 1966-73 (Nachdruck von „Sämtliche Werke“, hrsg. von K. F. A. Schelling 1856-61)

Schilpp, P. A. (Hrsg.) (42): The Philosophy of G. E. Moore, Evanston/Ill. $1942,{ }^{3} 1968$

Schilpp, P. A. (Hrsg.) (44): The Philosophy of Bertrand Russell, La Salle/Ill. ${ }^{1} 1944,{ }^{4} 1971$

Schilpp, P. A. (Hrsg.) (49): Albert Einstein - Philosopher - Scientist, 2 Bde., 1949/1951, Nachdruck New York 1959

Schilpp, P. A. (Hrsg.) (63): The Philosophy of R. Carnap, La Salle/Ill. 1963 Schilpp, P. A. (Hrsg.) (74): The Philosophy of K. Popper, La Salle/Ill. 1974 Schlick, M. (18): Allgemeine Erkenntnislehre, Berlin 1918

Schlick, M. (31): Die Kausalität in der gegenwärtigen Physik, Naturwissenschaften 19 (1931), abgedruckt in Schlick: Gesammelte Aufsätze, Wien $1938,41-82$

Schrödinger, E. (35): Die gegenwärtige Situation in der Quantenmechanik, Naturwissenschaften 23 (1935), 807-812, 823-828, 844-849

Schrödinger, E. (58): Mind and Matter, London 1958

Shimony, A. (55): Coherence and the axiom of confirmation, Journal of Symbolic Logic 20 (1955), $1-28$

Smart, J. J. C. (62): Sensations and brain processes, in V. C. Chappell (Hrsg.): The Philosophy of Mind, Englewood Cliffs/N. J. 1962, 160-172

Smart, J. J. C. (72): Science, history and methodology, The British Journal for the Philosophy of Science 23 (1972), 266-274

Sneed, J. (70): Quantum mechanics and classical probability theory, Synthese 21 (1970), 34-64

Sneed, J. (71): The Logical Structure of Mathematical Physics, Dordrecht 1971

Snell, B. (55): Die Entdeckung des Geistes, Göttingen ${ }^{1} 1955,{ }^{4} 1975$

Stegmüller, W. (58): Der Phänomenalismus und seine Schwierigkeiten, Archiv für Philosophie 8 (1958), 36-100, Neudruck Darmstadt 1969

Stegmüller, W. (65): Glauben, Wissen und Erkennen - Das Universalienproblem einst und jetzt, Darmstadt 1965

Stegmüller, W. (69): Wissenschaftliche Erklärung und Begründung, Berlin 
1969 (Probleme und Resultate der Wissenschaftstheorie und analytischen Philosophie, Bd. I)

Stegmüller, W. (69a): Metaphysik, Skepsis, Wissenschaft, ${ }^{2}$ Berlin 1969

Stegmüller, W. (70): Theorie und Erfahrung, 2 Halbbände, Berlin 1970 und 1973 (Probleme und Resultate der Wissenschaftstheorie und analytischen Philosophie Bd. II)

Stegmüller, W. (73): Personelle und statistische Wahrscheinlichkeit, 2 Halbbände, Berlin 1973 (Probleme und Resultate der Wissenschaftstheorie und analytischen Philosophie Bd. IV)

Stout, G. F. (38): Phenomenalism, Proceedings of the Aristotelian Society 34 $(1938 / 39), 1-18$

Strawson, P. F. (49): Truth, Analysis 9 (1949), $83-97$

Strawson, P. F. (50): Truth, Proceedings of the Aristotelian Society, Suppl. vol. 24 (1950), $260-277$

Süßmann, G. (58): Über den Meßvorgang, Abh. d. Bayer. Akademie d. Wiss., Math.-Naturwiss.Klasse 88 (1958), 5-41

Suppes, P. (63): The role of probability in quantum mechanics, in B. Baumrin (Hrsg.): Philosophy of Science, The Delaware Seminar, Bd. II, New York 1963, 319-337

Suppes, P. (66): The probabilistic argument for a non-classical logic of quantum mechanics, Philosophy of Science 33 (1966), 14-21

Suppes, P. (Hrsg.) (76): Logic and Probability in Quantum Mechanics, Dordrecht 1976

Suppes, P. und Zanciti, M. (76): Stochâstic irccmpleteness of quantum mechanics, in Suppes (76), 303-322

Tarski, A. (35): Der Wahrheitsbegriff in den formalisierten Sprachen, Studia Philosophica 1 (1935/36), 261-405

Tarski, A. (44): The semantic conception of truth and the foundations of semantics, Journal of Philosophy and Phenomenological Research 4 (1944), 341-375. Abgedruckt in Feigl und Sellars (49)

Wach, J. (26): Das Verstehen - Grundzüge einer Geschichte der hermeneutischen Theorie im 19. Jahrhundert, 3 Bde., Tübingen 1926-33; Nachdruck Hildesheim 1966

Warden, J. (69): A semantic analysis, Phoenix 23 (1969), 148

Webb, J. (68): Metamathematics and the philosophy of mind, Philosophy of Science 35 (1968), 156- 178

Weber, M. (66): Soziologische Grundbegriffe (Sonderdruck aus „Wirtschaft und Gesellschaft"), Tübingen ${ }^{2} 1966$

Weber, M. (68): Gesammelte Aufsätze zur Wissenschaftslehre, ${ }^{3}$ Tübingen 1968

Weizsäcker, C. F. v. (55): Komplementarität und Logik, Naturwissenschaften 42 (1955), $521-29,545-55$

Werner, H. (48): Comparative Psychology of Mental Development, New York 21948 
Werner, H. (59): Einführung in die Entwicklungspsychologie, ${ }^{4}$ München 1959 Weyl, H. (23): Raum - Zeit - Materie, Berlin ${ }^{5} 1923$, Nachdruck Darmstadt 1961

Wigner, E. P. (61): Remarks on the mind-body question, in I. J. Good (Hrsg.): The Scientist Speculates, London 1961. (dt: Phantasie der Wissenschaft, Düsseldorf 1965.)

Wittgenstein, L. (22): 'Tractatus logico-philosophicus, London 1922, Nachdruck Frankfurt a. M. 1963

Wittgenstein, L. (53): Philosophische Untersuchungen, hrsg. von G. Anscombe und R. Rhees, Oxford 1953

Wittgenstein, L. (74): Über Gewißheit, hrsg. von G.F. M. Anscombe und G. H. von Wright, Oxford 1974

Wright, G. H. von (71): Explanation and Understanding, London 1971. dt: Frankfurt a. M. 1974 
Namen

Abel, Th. 138,526

Allport. G. W. 345

Aquist, L. 102,526

Aristoteles 68, 492

Armstrong, D. 196, 253, 526, 532

Arnauld, A. 298

Augustinus 73

Austin, J. L. 159 f., 207, 229, 251, 259,526

Ayer, A. J. 158 f., 227, 231 f., 238, $240 \mathrm{ff} ., 301,526$

Bacon, F. $\quad 68,118$

Benaceraff, P. 283, 526

Berger, P. L. $\quad 404,522,526$

Berkeley, G. XIV, 154, 189, 192, $197 \mathrm{f} ., 200,203,213 \mathrm{ff} ., 237,346$, $354,379,409,416 f ., 424,427,440$, 485,526

Betti, E. $138,147,526$

Birkhoff, G. $\quad 375,526$

Black, Ní. i59f., 22j, j26

Blau, U. 93

Block, N. 271

Bohr, N. 371

Bolyai, J. 360

Born, M. 371

Boswell, J. 220

Bourdin 50

Boyle, R. 196

Breitkopf, A. 4, 163, 166, 531

Brentano, F. 43, 243f., 439, 526

Broad, C. D. 231, 526

Butler, J. 328, 527

Carnap, R. $\quad 97,163 \mathrm{ff} ., 180,225$, 236, 242, 252, 254 ff., 262 ff., 366,
$435,440,453$ f., 463 ff., 468, 472, $483 \mathrm{ff} ., 489.519,527$

Cartwright, R. L. $\quad 165,527$

Caterus 298

Chihara, C. S. 258,527

Chisholm, R. 232, 527

Chomsky, N. 165, 535

Church, A. 284

Cicero XII

Clark, M. 22, 527

Couturat, L. $\quad 307,527$

Craig, W. 450 f., 527

Darwin, Ch. VII, 250, 508

Davidson, D. $128,130,527$

Demokrit 249

Descartes, R. $\quad 10,38 \mathrm{f}$., 50, $68 \mathrm{f}$., 72 , $118,154,177$ f., 189 ff., 198 f., 214, $220 \mathrm{f}$., $291 \mathrm{ff}$., $297 \mathrm{f}$., $305 \mathrm{f}$., $309 \mathrm{f}$., 325 f., 354, 412f., 424, 426 f., $527 f$.

Diels, H. $63,68,77,528$

Dilthey, W. $132 \mathrm{f}$., 137 ff., 149, 395, 528

Ditfurth, H. von 185,528

Dodds, E. 346, 352, 528

Dray, W. 126,528

Duhem, P. $\quad 475,498 \mathrm{ff} ., 517,528$

Eccles, J. C. $295,393,533$

Einstein, A. $358 f ., 362,365,379$, 382, $494 \mathrm{f}$., 528

Epikur 249

Feigl, H. $\quad 100,264,310$ f., 528

Feyerabend, P. 159, 437, 446, 499 f., 528

Fichte, J. G. 71, 180, 189, 205, 243, 335 f., 339, 424 ff., 429 ff., 528 
Fine, K. $\quad 167,528$

Finetsi, B. de. 2, 528

Fodor, J. A. 258, 527

Fraassen, B. van $167,528 \mathrm{f}$.

Frege, G. 45, 56, 70, 169, 195, 199, $241,247,332,439$ ff., 529

Freud, S. $250,391 \mathrm{f}$.

Furth, H. G. 522, 529

Gadamer, H. G. $\quad 138,529$

Galilei 492

Gassendi, P. 298

Gauß, C. F. 360 ff.

Gehlen, A. 136, 393

Gettier, E. 20 ff., 28, 529

Geulincx, A. 306

Glymour, C. 483,529

Goethe, J. W. 142, 144, 529

Gödel, K. XI, 282 f., 529

Goodman, N. $96 \mathrm{ff} ., 171,207 \mathrm{f}$., 225, 236, 242, 440, 464 f., 468, 520 f., 529

Grelling, K. 282

Grice, H. P. 13, 84, 112, 296, 320, 529

Grünbaum, A. $\quad 358$ f., 369, 481, 529

Hanfmann, E. 345

Hardie, W.F.R. 242, 529

Harsanyi, J. 125

Harvey, W. 249

Hegel, G.W.F. $119,142,189,220$, $330,332,335,340$ f., 392, 424, $426 \mathrm{ff} ., 529$

Heidegger, M. 65

Heisenberg, W. 371, 529

Heitsch, E. 349, 529

Helmholtz, H. 178, 200 f., 362, 529

Hempel, G. $76,81,90,95 \mathrm{f} ., 104 \mathrm{f}$., $126,128,138,147,152,172,254$, $279,437,471,485,489,499,529 f$.

Heraklit 351

Hermes, H. 284, 530

Hertz, H. 178, 201 f., 530
Hilbert, D. 418,530

Hintikka, J. J. 1, 530

Hobbes, Th. 39, 68, 118, 298, 413

Humboldt, W. v. 155, 210, 419, 520,522

Hume, D. 54 ff., 66 f., 95, 198 ff., 215 f., $221,305,330,423 \mathrm{f}$., 427 , $439,449,462 \mathrm{f}$., 464, 490, $495 \mathrm{f}$., 530

Husserl, E. 243

Huxley, T. H. 308 f., 530

Jackson, R. 196

James, W. 290

Jeffrey, R. C. $89,122,465,527,530$

Johnson, S. 220, 224

Jung, C. G. 392

Kalke, W. 272, 530

Käsbauer, M. 94, 530

Kant, I. VII, XI, 32, 51 f., 70, 108, $120,143,152,155,180,192,197$, 199, 202, 210, 216, 287, 318, $333 \mathrm{ff} ., 354,379,392,396 \mathrm{ff} ., 403$, 409 ff., 416 ff., 426 ff., 430 ff., 438 , $447,456,459,491,493,530$

Kasanin, J. 345

Katz, D. 246, 530

Kemeny, J. G. $168,463,530$

Kennedy, R. J. 358

Kenny, A. $\quad 194,305,530$

Kim, J. 269, 530

Kochen, S. 375,530

König, J. 282

Krantz, D. H. 176, 530

Kretschmer, E. 338,530

Kripke, S. 266, 531

Kuhn, T. S. VIII, $437,446,499 \mathrm{ff}$., $503 \mathrm{ff}$., $511,513,520,522,531$

Lakatos, J. 499 f., 503, 508, 531

Lamettrie, J. 271

Laplace, P. S. 280

Lavoisier, A. L. 250 
Lecat, M. 56, 531

Lehmann, R. S. 463, 531

Lehrer, K. 28, 531

Leibniz, G. W. XV, 10, 31, 34, 68, 116, 118 ff., 132, 151, 212 f., 217, $221,285,287,300,305 \mathrm{ff}$., 326 , $328,390,392,413.417,428,492$, $531 \mathrm{f}$.

Lenzen, W. 1 f., 5 f., 11, 15, 60, 254, 471,532

Lersch, Ph. 246, 532

Lévy-Bruhl, L. 343

Lévi-Strauss, C. 345, 522, 532

Lewis, C. I. 239, 531

Lewis, D. 98, 112, 132, 267, 320, 532

Lobatschewsky, N. 360

Locke, J. 154, 189, 192 ff., 200, $213 \mathrm{f} ., 217,220,293,326 \mathrm{ff} ., 354$, $417,427,440,532$

Lorenz, K. VIII, IXf., 304, 391, 402, 441, 468, 522, 532

Lorenzen, P. 284, 532

Loreniz, H. A. 357

Lucas, J. 283, 532

Luce, R. D. $122,176,530,532$

Luckmann, Th. 404, 522, 526

Ludwig, G. 376, 380, 532

Malebranche, N. 306

Martin, C. B. 196, 532

Maupertuis, P.L.M. de 116

Meggle, G. 112, 532

Mersenne, M. 178

Michelson, A. A. 357,365

Mill, J. St. $\quad 95,237,438$

Miller, D. C. 24

Mittelstaedt, P. $359,371,375$, $532 \mathrm{f}$.

Moore, G. E. 224, 231, 233, 533

Morley, E. W. 357,365

Musgrave, A. 508, 531

Nelson, L. XII, $48 \mathrm{ff} ., 75,533$
Neumann, J.v. $169,371,375$, $383,526,533$

Neurath, O. 525, 533

Newton, I. $\quad 355,360,492$

Nietzsche, F. 393, 533

Nilson, D. R. 371,533

Novalis 472

Occam 164

O'Connor, D. 253, 264, 533

Oppenheim, P. 95 f., 168, 172, 279

Pap, A. 306, 533

Parmenides 68, 266

Pascal, B. 21, 67, 72f., 442, 533

Passmore, J. 80, 88, 533

Peirce, Ch. S. 319 f., 533

Piaget, J. VIII

Platon 16f., 20 ff., 131, 142, 220 , 295

Popper, K. 24, 284, 287, 295, 393, 436, 454, 468, $471 \mathrm{ff}$., $473 \mathrm{ff}$., $476 \mathrm{f} ., 485 \mathrm{f} ., 490 \mathrm{f} ., 495,497 \mathrm{f}$., $500 f ., 503,506,508,533$

Price, H. H. 232, 238, 239, 533

Priestley, J. 250

Protagoras 63

Putnam, H. 269, 271 ff., 437, 445 f., $481,499,533 \mathrm{f}$.

Pyrrho 67

Pythagoras 178

Quine, W. V. $4,162 \mathrm{ff} ., 174 \mathrm{f}$., 234 f., 244, 268, 312, 315, $319 \mathrm{ff}$., 404, 442, 444 ff., 475, 485, 492, 495 f., 498 ff., 501 ff., 517 f., 520 , 534

Raiffa, H. $\quad 122,532$

Ramsey, F. P. 451

Reichenbach, H. 359, 365, 369, $375 \mathrm{ff} ., 481,534$

Rescher, N. 34, 120, 534

Richard, J. 282 
Rickert, H. 133, 135

Riedl, R. 391, 441, 468, 522

Riemann, B. 36

Rosser, J. B. 282, 534

Rothacker, E. 133, 534

Russell, B. 231, 238, 290, $534 \mathrm{f}$.

Ryle, G. 153, 157, 216, 235, 257, $261 \mathrm{ff} ., 295,301 \mathrm{ff} ., 329,535$

Sapir, E. 210

Scheffler, I. $165,510,535$

Scheibe, E. 371,535

Scheler, M. 522

Schelling, F.W.J. 119, 142, 424, 426 f., 429 f., 535

Schilpp, P. A. $379,382,535$

Schlick, M. $39 \mathrm{ff} ., 485,535$

Schrödinger, E. $382,407,535$

Shimony, A. 463,535

Smart, J. J. 266, 500, 535

Sneed, J. 375,487 ff., 500 f., 504, $509,511,535$

Snell, B. 312, 346, 349 ff., 535

Specker, E. P. $\quad 375,530$

Spinoza, B. $68,154,289,428$

Stalnaker, R. 98

Stegmüller, W. 2, 40 f., 61, 86, 90 f., $93 \mathrm{f} ., 96,100,104 \mathrm{ff} ., 115,120$, $126,128,134,138,147,152,159$, $164,168,176,236,238,241,254$, $279,281,437,464,485,487 \mathrm{ff}$., $500 \mathrm{f}$., 504, 509, 511, 527, $535 \mathrm{f}$.

Stout, G. F. 242,536
Strawson, P. F. $\quad 45,536$

Süßmann, G. $381 \mathrm{f} ., 536$

Suppes, P. $\quad 176,375,530,536$

Tarski, A. XI, 46, 277, 331, 536

Thorndike, E. M. 358

Tversky, A. 176,530

Uexküll, J. von 347

Wach, J. 133, 536

Warden, J. 352, 536

Watson, J. B. 261

Webb, J. 283 f., 536

Weber, M. 147, 536

Weizsäcker, C. F. von 375,536

Werner, H. $344 \mathrm{ff}$., 403, 522, $536 \mathrm{f}$.

Weyl, H. 359, 537

Wheeler, J. A. 472

Whorf, B. 155, 210

Wigner, E. P. 295, 537

Wilde, O. 472

Windelband, W. 135

Wittgenstein, L. $41 \mathrm{f} ., 59 \mathrm{f} ., 66,71$, $153,160,183,211,255,257 \mathrm{ff}$., $313,316,319 f$., 382, 445, 485, 490, 506, 512 f., 537

Wright, G. H. von 127,537

Xenophanes 77

Zanotti, M. 375,536 


\section{Stichwörter}

Abjiidtheorien $19 \mathrm{i} \hat{\mathrm{f}}$.

Absicht $110 \mathrm{ff} ., 112$

Abstraktion $439 \mathrm{ff}$.

Adäquationstheorie (der

Wahrheit) $42 \mathrm{f}$., $45 \mathrm{f}$.

Additionstheorem der

Geschwindigkeiten 357

Analytisch 3 f., 29 f., 56 f., 151, $444 \mathrm{f}$.

Antezedensbedingung 96

Antinomien, logische 331

semantische XI, 277, $281 \mathrm{f}$.

Aposteriorisch 152

Apriorisch 152

Äquivalenz (von Theorien) $480 \mathrm{ff}$.

Äquivalenzrelation 439

Ausdruck 341

Aussage, wesentlich generelle 97

Basissatz 436

Bedeutungserklärung, vgl. Erklärung

Bedeutungsverstehen, vgl. Verstehen

Begriffsempirismus $438 \mathrm{ff} ., 447$

Begründung $36,50 \mathrm{f}$., $87 \mathrm{ff}$.

deduktive, modale 87

formal korrekte 93

induktive 89

kausale, epistemische $91 \mathrm{f}$.

Behaviorismus 250, $261 \mathrm{ff}$., $302 \mathrm{f}$.

Beobachtung $156 \mathrm{ff}$.

Beobachtungssatz 155, $254 \mathrm{f}$., 436

Beobachtungssprache 435

Beschreibung 86

Bestätigung 98, 471, 477

Bestätigungskriterium $254 \mathrm{ff}$.

Bewährung $471 \mathrm{f}$.

Beweggründe $127 \mathrm{f}$.

Beweis 94
Bewußtsein 334

Bewußt-Unbewußt $390 \mathrm{ff}$.

Bewußtseinspsychologie 390

Cogitatio 69, 200, $291 \mathrm{f}$.

Cognitio rei - circa rem $140 \mathrm{ff}$.

Deskriptiv 137

Determinismus $279 \mathrm{ff}$.

Dingsprache 180, 224, 251

Dispositionen $104 \mathrm{ff} ., 130,448$

DN-Modell (wissenschaftlicher

Erklärung) 94, 96, 101, 106, 172, $279,285,388 \mathrm{f}$.

Doxastische Logik 1

Dualismus 154, $290 \mathrm{ff}$., 337, 353, 367,385 ff., 394

Duhem-Quine-These $475 \mathrm{f}$., $498 \mathrm{ff}$., $517 \mathrm{f}$.

Eigenschaften, manifeste-dispositionelle $104 \mathrm{f}$., 302

Einfühlung $138 \mathrm{f} ., 146 \mathrm{f}$.

Emergenz 300

Empfindung $234 \mathrm{ff}$., $243 \mathrm{ff}$.

Empirisch 152

Empirismus (vgl. a. Begriffs- und Urteils-E.) 438 f., 489 f., $493 \mathrm{f}$., 512

Entität 329

Entscheidung $122 \mathrm{ff}$.

Entscheidungstheorie $122 \mathrm{ff}$.

Epiphänomenalismus $307 \mathrm{f}$.

Epistemische Logik 1

Erfahrung, innere $152 \mathrm{f}$., $199 \mathrm{f}$.

Erfahrungserkenntnis $151 \mathrm{ff}$.

Erfolgsverbum 128, 157, 292 
Erkenntnis (vgl. a. Wissen) 9, $140 \mathrm{ff}$.

Erkenntnisgrund $91 \mathrm{f}$.

Erkenntnisideal, platonisches $410 \mathrm{f}$.

Erkenntnisskepsis $52 \mathrm{ff}$.

Erklärung $86 \mathrm{ff}$. deduktiv-nomologische 96 determinativ-teleologische 107 dispositionelle $104 \mathrm{ff}$. finale $115 \mathrm{f}$.

funktionale $86,107 \mathrm{ff}$. intentionale $109 \mathrm{ff}$.

kausale $86,95 \mathrm{ff} ., 118,130$

rationale $86,121 \mathrm{ff}$.

statistische 90

teleologische $86,107 \mathrm{ff}$.

Erscheinung 192

Evidenz 22, $36 \mathrm{ff} ., 51,70 \mathrm{f}$. perfekte 39,230

Evidenzskepsis $40 \mathrm{f}$., 62,71

Evidenztheorie (der Wahrheit) 43

Existenz $162 \mathrm{ff}$.

Existenzfragen, interne-externe $162 \mathrm{f} ., 224,251$

Explanandum 96

Explanans 96

Explikation 76

Fallibilität 55,59

Finalitätsprinzip 120

Folgebeziehung, logische-analytische $167 \mathrm{f}$.

Freiheit 280, 286

Fundiertheit $17 \mathrm{ff}$.

Funktion $82,107 \mathrm{f}$.

Funktionalismus $271 \mathrm{ff}$.

Funktionsverstehen, vgl. Verstehen

Galilei-Transformation 357

Gegenständigkeit 401

Gehalt, anschaulicher (erlebnismäßiger) $\quad 318,419$ empirischer 451,480
Geisteswissenschaften $121 \mathrm{ff}$, $132 \mathrm{ff}$.

Genidentität 377 ff.

Gesetze, deterministische 99, 279

finale $116 \mathrm{ff}$.

Kausal- 96, 100, 130, 305

Koexistenz- 99

statistische, indeterministische 99

Sukzessions-, Verlaufs- 99

Gesetzesartigkeit (von

Aussagen) $96 \mathrm{ff}$.

Gestalthaftigkeit der

Wahrnehmung $246 \mathrm{f}$.

Glauben $1 \mathrm{ff}$.

Glaubensgründe 91

Gleichzeitigkeit $363 \mathrm{ff}$.

Grenzfragen 70 ff., 286, 408, 414

Grund $91 \mathrm{f}$.

Handlung $128 \mathrm{ff}$.

Harmonie, prästabilierte $306 \mathrm{f}$.

Hermeneutik 138

Holismus 155, $493 \mathrm{ff}$., $498 \mathrm{ff}$.

Ich $331 \mathrm{ff}$.

Idealismus $33,69,415 \mathrm{f}$. absoluter $426 \mathrm{ff}$. erkenntnistheoretischer $154 \mathrm{f}$., $191 \mathrm{f}$., $202 \mathrm{ff} ., 219,415 \mathrm{ff}$. ontologischer $154 \mathrm{f}$., $213 \mathrm{ff}$., $218 f ., 416$

Idee 192, $194 \mathrm{f} ., 198 \mathrm{f}$.

Identität, personale $327 \mathrm{f}$.

Identitätsthese $264 \mathrm{ff}$.

Idiographisch 135, 139, 144

Illusion 226

Indeterminiertheit (der Übersetzung) $221,321 \mathrm{f}$.

Induktionsprinzipien $462 \mathrm{ff}$.

Induktionsproblem $461 \mathrm{ff}$., $516 \mathrm{f}$.

Inertialsystem 356

Inkommensurabilität $\quad 507,514$

Instrumentalismus $484 \mathrm{ff}$.

Intentionalität $243 \mathrm{ff}$., $300 \mathrm{f}$. 
Internalisierung 341

Interpretation $82 \mathrm{f}$.

Kausalgesetz, vgl. Gesetz

Kausalitätsprinzip $\quad 102,120,280 \mathrm{f}$.

Kausalsatz $101 \mathrm{ff}$.

Kenntnis $141 \mathrm{ff}$.

Koexistenzgesetz, vgl. Gesetz

Kognitivistische-nichtkognitivistische

Deutung von Theorien $478 \mathrm{ff}$.

Kohärenz 403

Kohärenztheorie (der Wahrheit) 44

Konditionalsätze $98,238,448 \mathrm{f}$., $470 f$.

Konstanzphänomene $245 \mathrm{ff}$., $401 \mathrm{ff}$.

Konventionstheorie (der

Wahrheit) $44 \mathrm{f}$.

Kopenhagener Deutung 371

Leibnizprinzip $326 \mathrm{f}$.

Leib-Seele-Problem 154, 223, 297, $303 \mathrm{ff} ., 384 \mathrm{ff}$.

Lorentz-Transformation 359

Materialismus $154,249 \mathrm{ff}$.

Materiell 250f., 299

Meßprozeß, quantenmechanischer $380 \mathrm{ff}$.

Metaphysik 410

Metrisierung 176

Modell $175 \mathrm{ff}$.

algebraisches $176 \mathrm{ff} ., 185,192$ semantisches $176 \mathrm{ff}$.

Möglichkeit, alethische-doxastische $59 \mathrm{f}$.

Momentanobjekte $377 \mathrm{f}$.

Monismus, neutraler 154, 289

Naturgesetz $96 \mathrm{ff}$.

Newtonsche

Raum-Zeit-Lehre $355 \mathrm{ff}$.

Nominalisierung $330 \mathrm{ff}$.

Nomothetisch 135 f., 144

Normativ 137

Nutzenswert 122
Objekt $267 \mathrm{f}$.

Objektivität (von Sachverhalten) $180,186 \mathrm{ff}$.

Offenheit (empirischer Prädikate) 444

Okkasionalismus 306, 346

Paradigma $503 \mathrm{ff}, 512$

Parallelismus, psycho-physischer $306 \mathrm{f}$.

Phänomenale Entitäten $191 \mathrm{ff}$., $203 \mathrm{ff}$., $331 \mathrm{ff}$.

Phänomenalismus $223 \mathrm{ff} ., 337$

Physik, klassische $355 \mathrm{ff} ., 399$

Physikalismus 154, $249 \mathrm{ff}$., 261, 295, 310

Platonismus 179

Polarität 338 f., 344 f., 349 ff., 429

Präferenz 123

Präsupposition 11

Prinzip vom zureichenden

Grund 31

Privatsprache 255, 260

Problemlos 6, 18, 38

Proposition $1 \mathrm{f}$.

Psychisches $290 \mathrm{ff}$.

Qualitäten, primäre-sekundäre $189 \mathrm{ff}$., 354 , 417

Quantenlogik $374 \mathrm{f}$.

Quantenmechanik 275, $371 \mathrm{ff} ., 399$

Ramsey-Satz 451

Ratiomorpher Apparat 391, 393

Rationalismus $10,33,69,439,447$, 456

Rationalität 125 f., 389

Raum $354 \mathrm{ff}$.

Realgrund 91

Realismus $154 \mathrm{ff}$., $395 \mathrm{ff}$., $478 \mathrm{f}$. erkenntnistheoretischer $154 \mathrm{f}$., $180,219,400$ 
Realismus, naiver $154,184 \mathrm{ff} ., 409$ ontologischer $155,179 \mathrm{f} ., 219$, $295,313,396 \mathrm{ff}$. schwacher $188,396,400 \mathrm{ff}$. starker (absoluter) 188, 370, $396 \mathrm{ff}$.

Reduktion, von Sprachen $165 \mathrm{f}$., 226

von Theorien $168 \mathrm{ff}$., $178 \mathrm{f}$., 226, 266

W-Reduktion 170ff., 179, 226, 241, 252f., 266, $274 \mathrm{ff}$.

Regularität 463

Relationen $195 \mathrm{f}$.

Relativismus $154,156,512 \mathrm{ff}$.

Relativitätsprinzip, mechanisches 356

Relativitätstheorie $358 \mathrm{ff}$.

Repräsentationstheorien $191 \mathrm{ff}$.

Sachverhalt $1 \mathrm{f}$., 97

Scheinevidenz 38

Seele $198 \mathrm{f}$, $292 \mathrm{ff} ., 299,313,325 \mathrm{ff}$.

Seinsgrund 91

Selbstanwendbarkeit X, 432

Sinn 82

Sinnesdatum 192, $223 \mathrm{ff}$., $228 \mathrm{f}$., $231 \mathrm{ff}$., 299

Sinnkriterien, empiristische 152, $254,256,448,489 \mathrm{f}$.

Sinnskepsis 65

Sinnverstehen, vgl. Verstehen

Skepsis $52 \mathrm{ff}$.

empirische $181 \mathrm{ff}, 206,313$

Solipsismus 216, 223

Spielraum 386

Sprache, der Physik 180, 224, $251 \mathrm{f}$. phänomenalistische $224 \mathrm{ff}$.

physikalische $224,323 \mathrm{f}$.

Standardnamen 267

Störungsfreiheit (von

Beobachtungen) $372 \mathrm{ff}$.

Subjekt 336

Subjektiv - Objektiv 339,400ff.
Synthetisch 4, 152

System 279

Tatsache 2

Teleologieprinzip 120

Terme, theoretische $435,447 \mathrm{ff}$., $487 \mathrm{f}$.

Theorie $167 \mathrm{f} ., 279,487 \mathrm{f}$.

Theoriebeladenheit $446,454,460$, $515 \mathrm{f}$.

Traumargument $412 \mathrm{f}$.

Typenbegriff 338

Übersetzung $166,224 \mathrm{f}$.

Überzeugung 2

Universe of discourse $163 \mathrm{f}$.

Unproblematisch 18,397

Unterdeterminiertheit, empirische $479 \mathrm{ff}$., $518 \mathrm{f}$.

Ursache $91 \mathrm{f} ., 95 \mathrm{ff}$., $100 \mathrm{ff}$., $128 \mathrm{ff}$.

Urteilsempirismus $439,455 \mathrm{ff}$.

Verifikationskriterium (der

Bedeutung) $254,256,485$

Verläßlich 19,37

Verlaufsgesetz, vgl. Gesetz

Vermuten 2

Vernunftgrund 91

Verstehen $79 \mathrm{ff} ., 121 \mathrm{ff}$., $132 \mathrm{ff}$., $136 \mathrm{f}$.

anschaulich-erlebnismäßiges 139 , $144 \mathrm{ff}$. einfühlendes $138 \mathrm{f}$.

Vertauschbarkeit 462

Wahrheit $42 \mathrm{ff}$.

Wahrheitsähnlichkeit $473 \mathrm{f}$.

Wahrheitskriterien $43,47,51 \mathrm{f}$.

Wahrheitsrelativismus $63 \mathrm{ff}, 513 \mathrm{f}$.

Wahrheitsskepsis $62 \mathrm{f}$.

Wahrnehmung $156 \mathrm{ff}$.

Wahrscheinlichkeit $2,89,123 \mathrm{f}$, 256 
Wechselwirkung,

Wollen 111, 125

psycho-physische 296, $303 \mathrm{ff}$., W-Reduktion, vgl. Reduktion $384 \mathrm{ff}$.

Welt 4, 160, $210 \mathrm{f}$., $520 \mathrm{ff}$.

Zeit $354 \mathrm{ff}$.

Wirklichkeit $160 \mathrm{ff}$.

Wirkung $96 \mathrm{ff} ., 100 \mathrm{ft}$.

Ziel 112

Wissen $9 \mathrm{ff}$., $74 \mathrm{ff}$.

Zirkelfreiheit $93 \mathrm{f}$.

als wahre Überzeugung $16,76 \mathrm{ff}$.

fundiertes $17 \mathrm{ff}$., $58,74 \mathrm{f}$.

Zwang 129

notwendiges $30 \mathrm{f} ., 58,60,68$,

Zweck 82, 108

Zweck-Erklärung 108

$409 \mathrm{ff}$.

perfektes $29 \mathrm{ff} ., 51,55 \mathrm{f}$., 58 ,

$69 f ., 74,221$

Zweiaspektenlehre 289

Zwei-Schichten-Theorie (der Sprache) $435 \mathrm{ff}$.

rationales-deskriptives

11

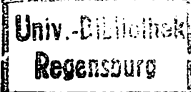

